\title{
XII National Congress SISMES Padua, 8-10 October, 2021
}

(C) Springer-Verlag Italia S.r.l., part of Springer Nature 2022

\section{KEYNOTE LECTURES AND ORAL PRESENTATIONS \\ SESSIONE DI APERTURA: ESERCIZIO, PREVENZIONE E TERAPIA 1 - COVID}

\section{1}

Impact of the covid-19 lockdown on changes in physical activity levels, eating habits and psychological wellbeing of the florentine academic population: role of socio-demographic factors

Gabriele Mascherini ${ }^{1}$, DoloresCatelan ${ }^{2}$, DomenicoPellegrini $^{3}$, CristianPetri $^{I}$, CristinaScaletti ${ }^{1}$, MassimoGulisano ${ }^{1}$

${ }^{1}$ Università Degli Studi Di Firenze, Dipartimento Di Medicina Sperimentale E Clinica, Firenze, Italy;

${ }^{2}$ Università Degli Studi Di Firenze, Dipartimento Di Statistica, Informatica, Applicazioni 'G. Parenti', Firenze, Italy;

${ }^{3}$ Università Degli Studi Di Firenze, Dipartimento Di Scienze Della Salute, Firenze, Italy

Purpose: The confinement and lockdown imposed by the COVID-19 pandemic have produced restrictions in the lifestyle of Italian citizens with variations in their psychological well-being. The aim of the study was to identify changes and relationship with socio-demographic parameters.

Methods: An online survey was administered to 1383 subjects (1007 females and 307 males) working in the University of Florence, Italy. Three validated questionnaires were used for the survey: the Global Physical Activity Questionnaire, the Med Diet Score and the Psychological General Well-Being Index-A. All the subjects were asked to complete the questionnaires twice, in order to attain a picture of the habits before and a later time point during confinement.

Results: Our results show that work-related physical activity was decreased, along with an increase in sedentary behaviour (from 07:22 $\pm 03: 20$ to $08: 49 \pm 03: 41 \mathrm{~h}: \mathrm{min} ; \mathrm{p}<0.001$, ES $=0.38$ ), whereas recreational physical activity was increased (vigorous exercise varied from $568.5 \pm 838.6$ to $833.7 \pm 1263.0$ METs; $\mathrm{p}<0.002$, ES $=0.25$ ). Eating habits changed according to the place where meals were eaten, with an increased habit for breakfast and snacks and a slight increase in alcohol consumption. Psychological well-being decreased (Index from $21.4 \pm 3.9$ to $18.0 \pm 5.3 ; \mathrm{p}<0.001$, $\mathrm{ES}=0.723$ ), especially in terms of vitality and positive thinking. The socio-demographic variables affecting these variations were mostly represented by age, gender and working conditions.

Conclusions: Young age and self-employment conditions can be considered factors for the changes in daily habits induced by confinement that may affect psychological well-being.

\section{References}

1. Fornili M, Petri D, Berrocal C, Fiorentino G, Ricceri F, Macciotta A, Bruno A, Farinella D, Baccini M, Severi G, Baglietto L. Psychological distress in the academic population and its association with socio-demographic and lifestyle characteristics during COVID-19 pandemic lockdown: results from a large multicenter Italian study. PLoS One. 2021;16(3):e0248370. https://doi.org/10.1371/journal.pone.0248370.

\section{2}

An in-home low-intensity structured exercise program improved mobility and strength after intensive care hospitalization for COVID-19

Nicola Lamberti ${ }^{1}$, Giovanni Piva ${ }^{2}$, Anna Crepaldi ${ }^{1}$, Giulia Fregna ${ }^{3}$, Nicola Schincaglia ${ }^{3}$, Giada Milani ${ }^{3}$, Sofia Straudi ${ }^{1}$, Fabio Manfredini ${ }^{l}$

${ }^{1}$ Università Degli Studi Di Ferrara, Neuroscienze E Riabilitazione, Ferrara, Italy;

${ }^{2}$ Università Degli Studi Di Ferrara, Studi Umanistici, Ferrara, Italy; ${ }^{3}$ Azienda Ospedaliero-universitaria Di Ferrara, Unità Di Medicina Riabilitativa, Ferrara, Italy

Purpose: Prolonged hospital stay due to Covid-19 infection resulted in patients' deconditioning. In this prospective pragmatic trial in Covid-19 patients after discharge from intensive care unit, we studied the effects on exercise capacity of a structured in-home exercise program compared to a traditional walking prescription.

Methods: Between January and May 2021 we enrolled Covid-19 patients with the following criteria: aged $>18$ years old, both sexes, capacity to walk for at least $20 \mathrm{~m}$; absence of clinical conditions contraindicating exercise. Patients, 3-month after the first positive swab (T0), were evaluated with 6-min walking test (6MWT), 30-s sit- 
to-stand test (30STS) and SF-12 questionnaire. After evaluation, all patients were proposed to perform a home-based progressively-increasing interval walking (walk-rest ratio $1: 1$ ) for $10 \mathrm{~min}$ per day [1-2], or to perform the physical activity recommended by the ACSM guidelines (20 min per 6-day a week at moderate intensity). Both programs lasted 3 months, and they were followed by the same outcome measures session (T1, 26 weeks). Patients were therefore classified upon their choice into two groups: home-based exercise (HB) or usual care (UC).

Results: In June 2021, 73 patients were assessed at T0, but only 23 yet concluded the 3-month program $(\mathrm{HB}$ group $\mathrm{n}=12, \mathrm{UC} \mathrm{n}=11)$. At baseline, the two groups did not differ for demographics, clinical characteristics or exercise capacity. All patients performed the programs, with a mean weekly walking time of 60 and $85 \mathrm{~min}$ for $\mathrm{HB}$ and UC respectively. No adverse events were recorded. After 3 months, patients of HB group significantly improved 6MWT (327 \pm 101 to $431 \pm 86 \mathrm{~m} \mathrm{p}=0.031), 30$ STS $(10 \pm 4$ to $14 \pm 4$ reps; $\mathrm{p}=$ 0.005 ) and PCS domain of SF-12 (PCS $43 \pm 8$ to $50 \pm 6 \mathrm{p}=0.007$; MCS $51 \pm 8$ to $53 \pm 4 \mathrm{p}=0.43$ ). On the contrary, patients of UC group showed a stable performance for 6MWT (358 \pm 109 to $365 \pm$ $105 \mathrm{~m} \mathrm{p}=0.37$ ), and improvements in 30STS ( $9 \pm 6$ to $11 \pm 6$ reps; $\mathrm{p}$ $=0.044$ ) and PCS domain of SF-12 (PCS $43 \pm 11$ to $47 \pm 9 \mathrm{p}=0.06$; MCS $49 \pm 8$ to $50 \pm 10 \mathrm{p}=0.70$ ). The between-group comparison showed significant greater variations for $\mathrm{HB}$ compared to UC for 6MWT $(\mathrm{p}=0.004)$, but not for the other parameters.

Conclusions: An in-home low-intensity progressively increasing walking program was more effective at improving mobility than unstructured walking in patients after Covid-19 infection with prolonged hospitalization. These preliminary data need to be confirmed with the continuation of the study.

\section{References}

1. Manfredini F, et al. Training rather than walking: the test in -train out program for home-based rehabilitation in peripheral arteriopathy. Circ J. 2008 Jun;72(6):946-52. https://doi.org/10.1253/ circj.72.946.

2. Lamberti N, et al. Don't stop walking: the in-home rehabilitation program for peripheral artery disease patients during the COVID19 pandemic. Intern Emerg Med. 2021 Jan 7:1-9. https://doi.org/ 10.1007/s11739-020-02598-4.

\section{3}

Supervised and unsupervised home-based exercise
program for women with osteoporosis: focus
on adherence during COVID-19 pandemic

Erika Pinelli $^{1}$, Giuseppe Barone ${ }^{2}$, Francesco Benvenuti ${ }^{1}$, Giuseppe Audino $^{I}$, Raffaele Zinno ${ }^{1}$, Laura Bragonzoni ${ }^{I}$

${ }^{1}$ University of Bologna, Department of Life Quality Studies, University of Bologna, Italy, Bologna, Italy;

${ }^{2}$ University of Bologna, Department of Life Quality Studies, University of Bologna, Italy, Bologna, Italy

Purpose: Exercise program is important for maintaining or increasing bone mineral density in people with low bone mass. But home physical activity during covid-19 pandemic was the only alternative to keeping active people with. This study aims at investigating the adherence to an exercise program specifically designed for women with postmenopausal osteoporosis when administered as individual home training (IHT) or online group training (OGT).

Methods: 40 women, aged between $67 \pm 6.5$ years were recruited for the study. A randomization was performed to divide the women in two groups: IHT or OGT. The OGT group were supervision by the trainers during exercise session utilizing a video conferencing software. The IHT group instead received a book with the exercises explained, a diary and a training program, every 6 weeks the program was changed and the people met the trainers for explain in videocall. They were also monitored with a call every 15 days.

Results: In both groups did 24 weeks of training, twice a week for 1-h session. The total of the training sessions was $48(100 \%)$. The adherence was registered with a diary for the IHT and with a register for the OGT. The adherence in both groups was 65\% (26 women), while $35 \%$ was not adherent (14 women:7 OGT and 7 IHT). The causes of not adherence depend on: family problems $50 \%$, health issue $14 \%$, work duties $14 \%$ and IT ability and possibility to have broadband connection $21 \%$ (only OGT).

Conclusions: The relationship between trainers and participants is important in maintaining adherence to a physical activity program. When physical contact is not possible, such as the period of the pandemic, frequent contact by telephone or other appropriate technological tools can help maintain the relationship and adhesion. Even if home environmental sometimes prevents the participation in a physical activity program, online training promotion is important because it could be an opportunity to achieve elderly people.

\section{References}

1. Rodrigues IB, Armstrong JJ, Adachi JD, MacDermid JC. (2017) Facilitators and barriers to exercise adherence in patients with osteopenia and osteoporosis: a systematic review. Osteoporos Int.; 28(3):735-745.

\section{4} "COVIDENTARY": an online exercise training
program to reduce sedentary behaviours in children
with type 1 diabetes during the COVID-19 pandemic

Valeria Calcaterra ${ }^{1}$, Dario Iafusco ${ }^{2}$, Vittoria Carnevale Pellino ${ }^{3}$, Agnese Pirazzi ${ }^{3}$, Chiara Mameli ${ }^{4}$, Gianluca Tornese ${ }^{5}$, Antonietta Chianese $^{2}$, Crescenzo Cascella ${ }^{2}$, Maddalena Macedoni ${ }^{4}$, Francesca Redaelli ${ }^{4}$, Gian Vincenzo Zuccotti ${ }^{4}$, Matteo Vandoni ${ }^{3}$

${ }^{1}$ Università Di Pavia, Pediatric and Adolescent Unit, Department of Internal Medicine, University of Pavia, Pavia, Pavia, Italy;

${ }^{2}$ University of Campagna, Regional Center of Pediatric Diabetology "G. Stoppoloni" Department of Pediatrics, University of Campania "luigi Vanvitelli", Napoli, Italy, Napoli, Italy;

${ }^{3}$ University of Pavia, Laboratorio Di Attività Motoria Adattata, Dipartimento Di Salute Pubblica, Medicina Sperimentale E Forense, Pavia, Italy;

${ }^{4}$ Università Degli Studi Di Milano, Dipartimento Di Pediatria Ospedale Dei Bambini Vittore Buzzi E Dipartimento Di Scienze Biomediche E Cliniche, Milan, Italy;

${ }^{5}$ Irccs "burlo Garofolo", Institute For Maternal and Child Health, Trieste, Italy

Purpose: The present study explored the physical activity (PA) level and the variation in glycaemic control in children with type 1 diabetes (T1D) before and during the lockdown. Then an online training program supported by sport-science specialists was proposed.

Methods: Parents of children with T1D $(<18$ years) filled out an online survey about anthropometric characteristics, PA, play, sport, sedentary time and the medical related outcomes. An adapted online program "Covidentary" was proposed through full-training (FT) and active breaks $(\mathrm{AB})$ modality.

Results: 280 youth $(11.8 \pm 3.3$ years $)$ were included in the analysis. A decline in sport $(-2.1 \pm 2.1 \mathrm{~h} /$ week $)$ and outdoor-plays $(-73.9 \pm$ $93.6 \mathrm{~min} /$ day) was reported. It was founded an increase in sedentary 
time $(+144.7 \pm 147.8 \mathrm{~min} /$ day $)$, in mean glycaemic values $(+25.4$ $\pm 33.4 \mathrm{mg} / \mathrm{dL})$ and insulin delivery (71.8\% of patients). $37 \%$ of invited patients attended the training program, $46 \%$ took part in $\mathrm{AB}$ and $54 \%$ in FT. The AB was carried out for $90 \%$ of the total duration, while the FT for $31 \%$, both perceived as moderate intensity effort.

Conclusions: A decline of participation in sport activities and a subsequent increase of sedentary time influence the management of T1D of children, increasing the risk of acute/long-term complications. Online exercise program may contrast the pandemic's sedentary lifestyle.

References

1. Riddell MC, Gallen IW, Smart CE, Taplin CE, Adolfsson P, Lumb AN, et al. Exercise management in type 1 diabetes: a consensus statement. Lancet Diabetes Endocrinol 2017;5:377-90. https://doi.org/10.1016/S2213-8587(17)30014-1.

2. Saunders TJ, Chaput JP, Tremblay MS. Sedentary behaviour as an emerging risk factor for cardiometabolic diseases in children and youth. Can J Diabetes 2014;38:53-61.

5

\section{An international pilot study on physical exercise habits and intention to practice before,} during and after the COVID-19 pandemic

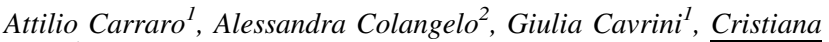 \\ Conti $^{1}$ \\ ${ }^{1}$ Libera Università Di Bolzano, Facoltà Di Scienze Della \\ Formazione, Bressanone, Italy; \\ ${ }^{2}$ Università Degli Studi Di Padova, Dipartimento Di Filosofia, \\ Sociologia, Pedagogia E Psicologia Applicata, Padova, Italy
}

Purpose: This pilot study explores physical exercise (Ex) habits and enjoyment before and during the COVID-19 pandemic, as well as future intentions to practice and action planning after the pandemic. Methods: A total of 270 adults (male $=100$; female $=168$; unspecified $=2$; age range $18-70$ years.) living in Europe $(78,1 \%)$, USA $(16,3 \%)$ and Canada $(5,6 \%)$ completed in June 2021 an online survey investigating type, modality and settings of Ex practice before and during the pandemic. Frequency, duration and enjoyment (Motl et al., 2001) of a typical session, before and during the pandemic, were considered. We also investigated intention and action planning regarding future practice (Dombrowski \& Luszczynska, 2009).

Results: The majority of respondents described themselves as physically active people (82.6\%). Results revealed a significantly lower frequency of moderate-intensity Ex during the pandemic than before $(\mathrm{p}<0.01)$; a similar trend was found for the frequency of vigorousintensity Ex $(p<0.001)$. The duration of a typical session decreased significantly during respect to before the pandemic $(p<0.001)$. As expected, in this period, participants reported a boost of at home and body weight Ex, also supported by the use of digital technologies. Despite the fact that respondents showed a significantly lower enjoyment in a typical Ex session during, compared with the before period $(p<0.001)$, they reported a high level of intention to practice after the pandemic. The majority of participants planned very often or often, when $(67.4 \%)$, how often $(65.2 \%)$, where $(70 \%)$, with whom $(51.5 \%)$ and how $(70 \%)$ they would like to practice. For the future, participants expressed interest in digital training. However, a strong preference for small group and in-presence training supervised by a professional emerged. While the interest in home training remained, a greater preference for practicing outdoors was also shown.

Conclusions: The social restrictions imposed by the COVID-19 pandemic seem to have influenced Ex habits and enjoyment, as well as preferences towards certain types, modalities and settings. According to the aims and results of the present pilot study, a further extended study will be launched in September 2021, adopting a shorter survey and modified criteria for participants' recruitment.

\section{References}

1. Dombrowski S, and Luszczynska A (2009) The interplay between conscious and automatic self-regulation and adolescents' physical activity: The role of planning, intentions, and lack of awareness. Appl Psychol 58: 257-273.

2. Motl R, Dishman R, Saunders R, Dowda M, Felton G, and Pate R (2001) Measuring enjoyment of physical activity in adolescent girls. Am J Prev Med 21: 110-117.

\section{6}

Long-term impact of COVID -19 on children's physical activity

Ilaria Tosi $^{1}$, Francesco Casolo $^{1}$, Christel Galvani ${ }^{2}$

${ }^{1}$ Università Cattolica Del Sacro Cuore, Pedagogia, Milan, Italy;

${ }^{2}$ Università Cattolica Del Sacro Cuore, Psicologia, Milan, Italy

Purpose: The WHO provided guidelines on physical activity (PA) and sedentary (SED) behaviour for children (WHO, 2020). The emergency pandemic situation and related restrictions that the population has experienced since February 2020 has greatly changed the habits and time spent on extracurricular sports (Stockwell, 2021).

Methods: The study was designed as a school-based controlled trial targeting primary schoolchildren and has been carried out between November 2019 and December 2020. 121 subjects attending the first and second years of primary school were involved. The assessment was performed three times, November 2019 (Nov19), October 2020 (Oct20) and December 2020 (Dec20). PA level and SED habits in leisure time was evaluated using 3 Day Physical Activity Recall (3DPAR) questionnaire (Weston, 1997), filled out by the parents of the subjects. During the first assessment the surrounding reality was in a condition of normality for what concerns the socio-sanitary situation. The second monitoring was carried out during the resumption of activities following a general lockdown period. The last one was carried out during a period of partial lockdown characterized by the full suspension activities of gyms, swimming pools and sports centers. Results: On average, less than half $(39,1 \%)$ of children undertook the recommended $60 \mathrm{~min}$ of $\mathrm{PA}$ and all children spent more than $2 \mathrm{~h}$ (285 $\mathrm{min}$ ) each day in SED activities. During the period of restrictions, children carried out alternative activities in the domestic context, which resulted in a reduction of sedentariness (Nov19: $304 \pm$ 40, Oct20: $273 \pm 40$, Dec20: $278 \pm 41 \mathrm{~min} ; \mathrm{p}<0,0001$ ), but also in a net reduction of vigorous activities (Nov19: $25 \pm 27$, Oct20: $33 \pm 31$, Dec20: $9 \pm 14$ min; $\mathrm{p}<0,0001)$.

Conclusions: The pandemic situation has changed the extracurricular habits of children. Ensuring children can access to indoor or outdoor non-structured vigorous physical activities, and parents' support are important prerequisites for enabling children to practice healthy movement behaviours during lockdown.

\section{References}

1. WHO. WHO guidelines on physical activity and sedentary behaviour. Geneva: World Health Organization; 2020. Licence: CC BY-NC-SA 3.0 IGO.

2. Stockwell S. Changes in physical activity and sedentary behaviours from before to during the COVID-19 pandemic lockdown: a systematic review. BMJ Open SEM. 2021;7:e000960. 
3. Weston AT. Validation of an instrument for measurement of physical activity in youth. Med Sci Sports Exerc. 1997 Jan;29(1):138-43.

\section{ESERCIZIO, PREVENZIONE E TERAPIA 2}

\section{7}

\section{Effects of lifestyle interventions on cardiorespiratory fitness and metabolic control in breast cancer survivors: evidence from movis clinical trial}

\author{
Valentina Natalucci ${ }^{l}$, Carlo Ferri Marini ${ }^{1}$, Francesco Lucertini ${ }^{1}$, \\ Giosuè Annibalini ${ }^{I}$, Luciana Vallorani ${ }^{l}$, Davide Sisti ${ }^{1}$, Roberta \\ Saltarelli $^{1}$, Anna Villarini ${ }^{2}$, Silvia Monaldi, Simone Barocci ${ }^{4}$, \\ Vincenzo Catalano ${ }^{3}$, Marco Bruno Luigi Rocchi ${ }^{1}$, Piero Benelli, \\ Vilberto Stocchi ${ }^{5}$, Rita Emili ${ }^{3}$, Elena Barbieri ${ }^{1}$
}

University of Urbino Carlo Bo, Department of Biomolecular Sciences, Urbino, Italy ${ }^{1}$;

Fondazione Irccs Istituto Nazionale Dei Tumori, Epidemiology Unit, Department of Research, Milan, Italy,

U.o.c. OnScologia Medica, Asur Area Vasta 1, Ospedale Santa Maria Della Misericordia Di Urbino, Urbino, Italy ${ }^{3}$;

U.o.c. Patologia Clinica, Area Vasta N. 1, Ospedale Santa Maria Della Misericordia Di Urbino, Urbino, Italy ${ }^{4}$;

University San Raffaele, Department of Human Sciences For The Promotion of Quality of Life, Rome, Italy

Purpose: Lifestyle interventions (LI) in breast cancer (BC) survivors (BCSs) have a high health and social impact on both health-related outcome and disease-free survival rate. This study aimed to evaluate the cardiometabolic effects of LI in BCSs.

Methods: 30 BCSs (women; stages 0-II; non-metastatic; age $53.5 \pm$ 7.6 years; non-physically active) participating in the MoviS trial (NCT04818359) were randomly allocated to an intervention (IA) and a control (CA) arm. Both groups received a 3-month LI based on nutritional and exercise counselling. In addition, the IA was supposed to receive a 3-month remotely (1 day/week) and on-site ( 2 day/week) supervised aerobic exercise training program having progressive increases in exercise intensity (40\%-70\% of heart rate reserve) and duration (20-60 min). Due to the imposed COVID-19 pandemic restrictions, from the 4th week the type of the exercise supervision of IA was adapted to solely remotely supervised. Before the LI (t0), and 3 (t1), 6 (t2), and 12 (t3) months from LI start participants' body mass index (BMI), physical activity level (PAL), cardiorespiratory fitness (VO2max), glycemia (GLY), low-density lipoprotein cholesterol (LDL) and triglycerides (TRI) were assessed. A general linear model (multiple analysis of variance for repeated measures) was used to compare the dependent variables (BMI, VO2max, GLY, LDL, and TRI) between groups (IA and CA-between factor) over time ( $\mathrm{t} 0, \mathrm{t} 1$, $\mathrm{t} 2$, and $\mathrm{t} 3$-within factor). For each dependent variable, simple contrasts between baseline ( $\mathrm{t} 0)$ and post intervention ( $\mathrm{t} 1$, $\mathrm{t} 2$, and $\mathrm{t} 3$ ) values were performed. At each timepoint, Pearson's $r$ between the PAL and the dependent variables were evaluated. Alfa $=0.05$.

Results: There was no difference between IA and CA. There was a significant effect $(\mathrm{p}<0.001)$ of time on VO2max and GLY but not on BMI, LDL, and TRI. Compared to t0 $(30.6 \pm 5.6) \mathrm{VO} 2 \mathrm{max}$ increased at $\mathrm{t} 1(34.0 \pm 6.0 ; \mathrm{p}<0.001), \mathrm{t} 2(33.6 \pm 6.3 ; \mathrm{p}<0.001)$, and $\mathrm{t} 3(32.8$ $\pm 6.0 ; \mathrm{p}=0.001)$. Whereas, compared to t0 $(101.0 \pm 11.6)$ GLY decreased $(\mathrm{p}<0.001)$ at $\mathrm{t} 1(91.9 \pm 11.1), \mathrm{t} 2(90.3 \pm 10.8)$, and $\mathrm{t} 3$ $(94.7 \pm 10.6)$. PAL was correlated positively with $\mathrm{VO} 2 \max (\mathrm{r}: \mathrm{t} 0=$ $0.58, \mathrm{t} 1=0.45, \mathrm{t} 2=0.56, \mathrm{t} 3=0.71)$ and negatively with BMI $(\mathrm{r}: \mathrm{t} 0=-$ $0.65, \mathrm{t} 1=-0.69, \mathrm{t} 2=-0.68, \mathrm{t} 3=-071), \mathrm{GLY}(\mathrm{r}: \mathrm{t} 0=-0.23, \mathrm{t} 1=-0.36$, $\mathrm{t} 2=-0.35, \mathrm{t} 3=-0.34)$, TRI $(\mathrm{r}: \mathrm{t} 0=-0.30, \mathrm{t} 1=-0.32, \mathrm{t} 2=-0.22, \mathrm{t} 3=-$ 0.33 ), and LDL ( $\mathrm{r}: \mathrm{t} 0=-0.42, \mathrm{t} 1=-0.45, \mathrm{t} 2=-0.37, \mathrm{t} 3=-0.30$ ).

Conclusions: LI improved cardiometabolic health in BCSs, and these improvements were correlated with PAL.

References

1. Bull FC, Al-Ansari SS et al. (2020) World Health Organization 2020 guidelines on physical activity and sedentary behaviour. $\mathrm{Br}$ J Sports Med 54(24):1451-1462.

2. Natalucci V, Ferri Marini C et al. (2021) Effects of a HomeBased Lifestyle Intervention Program on Cardiometabolic Health in Breast Cancer Survivors during the COVID-19 Lockdown. J Clin Med 10(12):2678.

\section{8}

Mediating effect of physical activity on sleep and fatigue in haematological cancer patients

Lucia Castelli $^{I}$, Antonino Mule ${ }^{l}$, Letizia Galasso ${ }^{I}$, Fabio Esposito $^{I}$, Angela Montaruli ${ }^{1}$, Eliana Roveda ${ }^{1}$, Philipp Zimmer ${ }^{2}$

Università Degli Studi Di Milano, Dipartimento Di Scienze Biomediche Per La Salute, Milan, Italy ${ }^{1}$; Technical University Dortmund, Department For Performance and Health, Institute For Sport and Sport Science, Dortmund, Germania $^{2}$

Purpose: Haematological cancer patients (HCP) usually report reduced physical activity (PA) and increased sleep deficiency. Sleep deficiency and PA's emerging and beneficial roles are intensely studied in solid cancer patients. However, there has been less attention to these issues in HCP. HCP's sleep deficiency is usually described as one of the five symptoms affecting these patients, and it is linked to higher levels of cancer-related fatigue (C-RF). C-RF also prevent HCP from being physically active. Indeed, C-RF and PA act in a vicious circle in these subjects, and the reduced PA could negatively interact with the side effect of the disease and its treatment. Moreover, most of the studies are focused on patients' conditions during or after chemotherapy. The present study assesses the differences in sleep by comparing PA and fatigue among HCP at the onset of chemotherapy. Furthermore, it investigates the relationship between sleep, PA and fatigue.

Methods: The sample consisted of 58 newly diagnosed HCP $(47.1 \pm$ 15.4 years; $51.7 \%$ males). Participants filled in five questionnaires within two weeks from starting treatment: PSQI, a visual analogue scale, MFI-20, HADS, and EORTC QLQ-C30 to assess sleep, PA, fatigue, anxiety, depression, and quality-of-life, respectively. ANCOVA analysis investigated the differences between good and bad sleepers, while Mediation analysis described the relation between sleep, PA and fatigue. SPSS software was used to run the analysis. Results: HCP reported more sleep problems than the German population norm even at the chemotherapy onset. The HCP classified as good sleepers were less represented than those classified as bad sleepers (25\% and $75 \%$, respectively) and showed less frequent PA (p $=0.04)$, higher fatigue $(\mathrm{p}=0.032)$, anxiety $(\mathrm{p}=0.003)$, depression $(\mathrm{p}$ $=0.011)$ and pain $(\mathrm{p}=0.011)$. The mediation analysis disclosed PA as a mediating factor between sleep and fatigue; in other words, it revealed significant indirect effects of sleep on fatigue through PA. Conclusions: Haematological cancer patients are described as predominantly sleep impaired even at the onset of chemotherapy. Bad sleep quality is associated with less frequent PA and higher fatigue. In addition, it highlights the combined action of sleep and PA on fatigue: an increment in PA frequency could be beneficial for fatigue improvements. PA and sleep could represent meaningful intervention 
targets to improve a patient's status before and at the onset of chemotherapy.

\section{References}

1. Miladinia M, Baraz S, Ramezani Monir, Malehi AS. (2018) The relationship between pain, fatigue, sleep disorders and quality of life in adult patients with acute leukaemia: During the first year after diagnosis. Eur J Cancer Care 27.

2. Kreutz C, Schmidt ME, Steindorf K. (2019) Effects of physical and mind-body exercise on sleep problems during and after breast cancer treatment: a systematic review and meta-analysis. Breast Cancer Res Treat 176:1-15.

\section{9}

The choice (choose health, oncological patients centered exercise): a phase II randomized controlled trial.

Alice Avancini $^{1}$, Giuliano Scrivano ${ }^{2}$, Lorenzo Belluomini $^{1}$, Ilaria Trestini $^{I}$, Daniela Tregnago ${ }^{1}$, Mauro Valentini ${ }^{2}$, Francesco Leone ${ }^{3}$, Michele Milella ${ }^{1}$, Sara Pilotto ${ }^{1}$, Massimo Lanza ${ }^{4}$

University of Verona, Department of Oncology, Verona, Italy ${ }^{1}$; Lega Italiana Per La Lotta Contro I Tumori, Lega Italiana Per La Lotta Contro I Tumori, Biella, Italy ${ }^{2}$;

Asl Biella, Medical Oncology, Biella, Italy ${ }^{3}$;

University of Verona, Department of Neuroscience, Biomedicine, and Movement, Verona, Italy ${ }^{4}$

Purpose: Post-diagnosis physical activity is linked with improvement in survival and treatment-related side effects in cancer patients. Nevertheless, most cancer patients are insufficiently active. The CHOiCE is a phase II trial designed to establish the safety, feasibility, and preliminary efficacy of exercise (EX) in cancer patients (pts).

Methods: Three months of bi-weekly training, including aerobic and resistance activities, was proposed. Aerobic training consisted of cardiovascular exercises, progressively increasing from 10 to $30 \mathrm{~min}$ at moderate intensity, i.e., 3-5 of the 10-point Borg Rating of the Perceived Exertion Scale (RPE). Strength included six bodyweights or elastic band exercises to perform in 2-3 sets of 8-12 repetitions. The primary endpoint was EX feasibility, defined as recruitment rate, adherence, withdrawals, and adverse events (AEs). Secondary endpoints were functional capacity, assessed using 6 mins walking test, muscular strength, measured with handgrip, and leg press strength. Pts' quality of life (QoL) was assessed using the European Organization for Research and Treatment of Cancer Quality of Life and Core Questionnaire (EORTC- QLQ-C30).

Results: Out of 80 eligible pts, 34 pts with mixed cancer types were randomized to the EX-intervention $(n=19)$ or the control one $(n=$ 15). At the post-CHOiCE timepoint, $88 \%$ of the participants completed the assessments. The median EX-attendance rate during $\mathrm{CHOiCE}$ was 93\%, and no serious AEs were reported. Compared to the controls, the EX-intervention group exhibited significative improvement in functional capacity, $(\mathrm{p}<0.001)$, upper $(\mathrm{p}=0.002)$ and lower $(p=0.006)$ limb flexibility. From baseline to post-intervention, handgrip strength increased in EX-group $(p=0.002)$ as well as leg press strength $(\mathrm{p}=0.003)$, while in the controls remained stable. Regarding QoL, improvements in physical function, social function, and fatigue were reported in the EX-group, whereas no significant changes were found in other domains.

Conclusions: Our preliminary findings suggest that the EX-intervention proposed in the $\mathrm{CHOiCE}$ trial was safe and well-tolerated in cancer pts. EX training created improvements in health-related skills as well as in some QoL domains. Further pts' enrollment is currently ongoing.

\section{References}

1. Campbell KL, Winters-Stone KM, Wiskemann J, May AM, Schwartz AL, Courneya KS, et al. Exercise Guidelines for Cancer Survivors: Consensus Statement from International Multidisciplinary Roundtable. Med Sci Sports Exerc. 2019 Nov; 51(11):2375-90.

\section{0}

\section{Adapted physical activity protocol for the management of breast cancer-related upper limb lymphedema} Giuditta Carretti
Grazia Muraca $^{1}$, Daniela Mirandola

Department of Experimental and Clinical Medicine,

Section of Anatomy and Histology, University of Florence, Florence, Italy $^{1}$;

The Italian League Against Tumors (lilt), Department of Experimental and Clinical Medicine, Section of Anatomy and Histology, University of Florence, Florence, Italy ${ }^{2}$;

Oncological Rehabilitation Centre (cerion), Oncological Network, Prevention and Research Institute (ispro), Florence, Italy ${ }^{3}$; Oncological Network, Prevention and Research Institute, (ispro), Florence, Italy ${ }^{4}$

Purpose: Secondary upper limb lymphedema (LE), which is among the most dreaded chronic complications in breast cancer (BC) treatment's outcomes, with risk proportional to the extent of axillary lymph node dissection and radiotherapy, results from lymphatic system obstruction or disruption. In BC survivors, chronic LE can cause psychophysical sequelae jeopardizing quality of life (QoL) and, owing to the lack of effective therapies, represents a major challenge for healthcare professionals. Recent researches support the positive effects deriving from regular participation in structured adapted physical activity (APA) programs in preventing or attenuating cancer treatment-related impairments with an overall improvement of QoL. In this context, the aim of our study was to investigate the possible effects of an APA intervention in reducing LE and improving both upper limb's strength and mobility, as well as QoL of BC survivors. Methods: $\mathrm{BC}$ survivors $(\mathrm{n}=16)$ with chronic moderate/severe LE attending the Cancer Rehabilitation Center in Florence were assessed before and after 8-week APA. The protocol, consisting of both APA specialist-supervised and self-leaded sessions, was centered on a specific motor gesture performed using an innovative purpose-designed proprioceptive board named "Hand Walk". Body mass index and bioimpedance parameters were measured. Indirect upper limb volume measurement and ultrasonography were also performed and functional tests (i.e. wrist flexion/extension and hand strength) were executed. QoL, depression/anxiety and pain intensity were evaluated by ULL27, HADS, distress thermometer and NRS questionnaires, respectively.

Results: Although the bioimpedance measurements unchanged, the ultrasound and volumetric measures revealed a trend toward a LE reduction after the APA protocol. of note, wrist mobility, hand strength, pain perception, depression and QoL were all significantly ameliorated after APA.

Conclusions: Our findings suggest that a multidisciplinary treatment approach, also involving APA professionals, is required in the management of BC-related LE in order to improve patient psychophysical outcomes and QoL. 


\section{1}

Physical activity and oxidative stress modulations in breast cancer survivors: a comparison between online supervised combined training and usual care

\section{Elisa Grazioli ${ }^{1}$, Ivan Dimauro ${ }^{2}$, Guglielmo Duranti ${ }^{2}$, Eliana} Tranchita $^{I}$, Claudia Cerulli ${ }^{1}$, Arianna Murri ${ }^{1}$, Cristina Fantini ${ }^{2}$, Sveva Maria Nusca ${ }^{3}$, Marilena Colangelo ${ }^{4}$, Annalisa Piccinetti, ${ }^{5}$, Andrea Palmieri ${ }^{5}$, Patrizia Pellegrini ${ }^{6}$, Mariachiara Vulpiani ${ }^{4}$, Francesco Cavaliere ${ }^{5}$, Attilio Parisi ${ }^{7}$

University of Rome Foro Italico, Laboratory of Physical Exercise and Sport Sciences, Department of Movement, Human and Health Sciences, Rome, Italy ${ }^{I}$;

University of Rome Foro Italico, Unit of Biology, Genetics and Biochemistry of Human Movement, Department of Movement, Human and Health Sciences, Rome, Italy ${ }^{2}$;

Sant'andrea University Hospital, Phd Course In "translational Medicine and Oncology", Department of Medical and Surgical Sciences and Translational Medicine, "sapienza" University, Rome, Italy $^{3}$;

Sant'andrea University Hospital, Physical Medicine and Rehabilitation Unit, Department of Medical and Surgical Sciences and Translational Medicine, "sapienza" University of Rome, Rome, Italy ${ }^{4}$;

Asl Viterbo, Breast Surgery Unit and Integrated Center of Breast Care, Belcolle Hospital, Viterbo, Italy ${ }^{5}$;

Sant'andrea University Hospital, Department of Clinical and Molecular Medicine, "sapienza" University of Rome, Rome, Italy ${ }^{6}$;

University of Rome Foro Italico, Laboratory of Physical Exercise and Sport Sciences, Department of Movement, Human and Health Sciences, Rome, Italy ${ }^{7}$

Purpose: Breast cancer (BC) is one of the most common cancers worldwide and the leading cause of cancer death in women. Evidence suggests that a regular physical activity (PA) either after the diagnosis of $\mathrm{BC}$ or in the early steps post-surgery improves survival outcomes $(1,2)$. Moreover, it is known that the beneficial association between $\mathrm{PA}$ and $\mathrm{BC}$ survival are partially related to its influence on several hormones (i.e. sex hormones, insulin, IGF-1) and on the reduction of systemic oxidative stress levels, which appear to be directly involved in the pathogenesis and progression of this disease $(3,4)$. Indeed, high levels of oxidative stress may contribute to cancer aetiology through several mechanisms involving damage to DNA, proteins and lipids, as well as neoangiogenesis, leading to genetic instability, tumor growth and metastasis development (5).

Methods: The aim of this project is to evaluate in post-surgical BC patients undergoing treatment (mean age $52 \pm 7,2$ ) the efficacy of a 16-weeks online supervised combined and well-structured exercise training $(\mathrm{CT}, \mathrm{n}=5)$, and of the Usual Care Protocol $(\mathrm{UC}, \mathrm{n}=5)$ on physiological and psychological parameters (i.e., functional capacity, strength, Body composition, Quality of life, Fatigue). Moreover, in order to explore the impact of physical activity on redox homeostasis, CAT and SOD activity, TBARs, protein carbonylation (PrCar), as well as total thiol and glutathione will be evaluated.

Results: Our preliminary results highlight a general improvement of 6 min walking test $(+6,5 \%)$ and Fatigue perception $(-59 \%)$, as well as an increase in lower limb muscular strength $(+22,8 \%)$ and in shoulder flexibility (right: $+24,1 \%$; left: 18,8\%) in the online supervised CT. No differences were found in pre and post assessment of UC group. Ongoing analyses are investigating the influence of physical activity on redox-balance.

References
1. De Luca V,Minganti C,et al.Effects of concurrent aerobic and strength training on breast cancer survivors: a pilot study.Public Health.2016.

2. Nourazarian AR,Kangari P,et al. Roles of oxidative stress in the development and progression of breast cancer.Asian Pac J Cancer Prev 2014.

3. Kruk J.Overweight, obesity, oxidative stress and the risk of breast cancer.Asian Pac J Cancer Prev 2014.

4. Behrend L,Henderson G,et al.Reactive oxygen species in oncogenic transformation.Biochem Soc Trans 2003.

12

Effects of 12 weeks of walking and nordic walking practice, in breast cancer survivors and postmenopausal women, on daily physical activity. The "Allenarsi per la salute" research project

Andrea Di Blasio ${ }^{1}$, Teresa Morano ${ }^{1}$, Ines Bucci ${ }^{1}$, Anna D'eugenio ${ }^{1}$, Laura Masini $^{I}$, Federica Lancial, Saveria Tavoletta ${ }^{2}$, Ettore Cianchetti $^{2}$, Massimo Rinaldi ${ }^{1}$, Simona Grossi ${ }^{2}$, Pasquale Valentini ${ }^{3}$, Giorgio Napolitano ${ }^{l}$

Università “G. D’annunzio” Di Chieti-pescara, Dipartimento Di Medicina E Scienze Dell'invecchiamento, Chieti, Italy ${ }^{l}$;

Asl02 Di Lanciano-vasto-chieti, Ospedale "G. Bernabeo", Ortona, Italy $^{2}$;

Università “G. D'annunzio" Di Chieti-pescara, Dipartimento Di Economia, Pescara, Italy

Purpose: Aerobic exercise is the most studied and practiced activity for health, both for breast cancer survivors (BCS) and menopausal women (MW). The most common aerobic activity is walking training (W), found widespread because it is a low-cost activity wherever practicable. Therefore, increasing literature report the usefulness of Nordic Walking (NW) for BCS and female health. Based on Epstein and Wing (1980) suggestion, Gomersall and colleagues hypothesized that when physical activity (PA) is increased or decreased in one domain, there will be a compensatory change in another domain, to maintain an overall stable level of PA, over time. Therefore, the aim of the study was to investigate the effects of $\mathrm{W}$ and NW training, having the same duration, intensity and frequency, on PA of BCS and MW.

Methods: Two hundred and seventeen BCS (45.57 6.21 years) and 245 MW (54.80 6.93 years), enrolled for the "Allenarsi per la salute" research project, were randomly assigned to $\mathrm{W}(\mathrm{BCS} \mathrm{n}=127$; MW $\mathrm{n}$ $=138)$ or NW (BCS $n=90 ;$ MW $n=107)$ group, and were trained for 12 weeks. Daily PA was recorded, over 7 consecutive days, using a multisensory device. Both $\mathrm{W}$ and $\mathrm{NW}$ groups were trained with a 3-times per week cadence. Each workout lasted $75 \mathrm{~min}$, including warm-up, central phase, and cool down. Daily PA was recorded before the beginning of the workouts and during the last two weeks of training. Physical activity variables were considered as $\%$ of a day.

Results: Statistical analysis showed a decrease of daily sedentary time (SED) and an increase of light intensity physical activities (LIGHT), while no significant variations have been shown on other physical activity variables. Both baseline mean daily METs and typology of training explained SED and LIGHT variations. Low mean daily METs and NW practice determined the best reduction of SED and increase of LIGHT. On the contrary, moderate intensity physical activity variation has been shown linked with baseline mean daily METs and BMI, in a negative manner.

Conclusions: Twelve weeks of aerobic training can modify daily SED and LIGHT. NW seems more able than W, both in BCS and 
MW, to determine this positive daily physical activity recomposition, even if it is important to consider baseline physical activity level, as it can counteract the described results. High baseline mean daily METs and BMI can negatively address moderate intensity physical activity recomposition, irrespectively from training (W/NW) and health status.

\section{References}

1. Epstein LH, Wing RR (1980). Aerobic exercise and weight. Addict Behav 5:371-388.

2. Gomersall SR, Rowlands AV, English C, Majer C, Olds TS (2013). The ActivityStat hypothesis: the concept, the evidence and the methodologies. Sports Med 43:135-149.

3. Di Blasio A et al. (2018). Aerobic physical exercise and negative compensation of non-exercise physical activity in post-menopause: a pilot study. J Sports Med Phys Fitness 58:1497-1508.

\section{ANATOMIA E SPORT E POSTURA E RECUPERO FUNZIONALE}

13

\section{Postural changes through step frequency and metronome training to enhance knee peak force reduction in non-professional runners.}

\section{Federico Roggio $^{1}$, Antonino Bianco ${ }^{2}$, Antonio Palma $^{2}$, Giuseppe Musumeci ${ }^{3}$}

Phd Program In Health Promotion and Cognitive Sciences, Sport and Exercise Sciences Research Unit, University of Palermo.,

University of Catania, Research Center On Motor Activities (cram)., Catania, Italy ${ }^{I}$, University of Palermo, Department of Psychology, Educational Science and Human Movement, Palermo, Italy ${ }^{2}$, University of Catania, Research Center On Motor Activities (cram), Department of Biomedical and Biotechnological Sciences, Human Anatomy and Histology Section, School of Medicine, Catania, Italy ${ }^{3}$

Purpose: Runner's postural biomechanics is a qualitative aspect of sports performance that must be perfected to increase performance and reduce joint trauma incidence. Specifically, a motor pattern that prefers the rearfoot run over the forefoot is common among western runners. From a dynamic perspective, the heel generates a ground reaction force of about $1.5-3$ of the body weight in $50 \mathrm{~ms}$, affecting the lower limb joints [1]. In the forefoot run, the ground reaction force develops more slowly, in about $200 \mathrm{~ms}$, to reduce the shock absorbed by the joints[2]. The purpose of this study is twofold: to investigate the biomechanical aspects of non-professional runners and to understand if, following a training based on the acquisition of a correct forefoot posture in conjunction with the learning of the correct running frequency set at 180 steps per minute, spm, they can improve sports and physical performance.

Methods: The study involved 30 non-professional runners, average age $45.7 \pm 6.7$, and with the support of a motion analysis system, a treadmill with a markerless 3D camera, the joint biomechanical relationship of running posture was evaluated. A specific 4-week training protocol was then administered to a pilot group aiming to induce the correct lower limb kinematic, learn forefoot running posture, train muscles through the isoinertial system, and run on the correct step frequency at $180 \mathrm{spm}$ with a digital metronome aid.

Results: Standard values of non-professional runners' joint biomechanical relationships were obtained. The 4-week training showed changes in joint values, such as the foot's dorsiflexion from $26^{\circ}$ to $2.7^{\circ}$, force spike reduction in the load kinematics, and spatio-temporal parameters related to running as contact time from $0.26 \mathrm{~s}$ to $0.23 \mathrm{~s}$.
Conclusions: A 4-week training based on posture management, step frequency training, and targeted muscle training can induce significant changes in non-professional runners whose running posture is rearfoot and therefore determine a knee injury reduction, reactive force increase, and correct running biomechanics.

\section{References}

1. Willson JD, Bjorhus JS, Williams DS 3rd, Butler RJ, Porcari JP, Kernozek TW. Short-term changes in running mechanics and foot strike pattern after introduction to minimalistic footwear. PM R. 2014;6(1):34-43. https://doi.org/10.1016/j.pmrj.2013.08.602

2. Lieberman DE, Venkadesan M, Werbel WA, et al. Foot strike patterns and collision forces in habitually barefoot versus shod runners. Nature. 2010;463(7280):531-535. https://doi.org/10. 1038/nature08723

\section{4}

\section{Epidemiological study of stress fractures in élite Italian track and field athletes}

\author{
Davide Grigoletto $^{1}$, Gioi Spinello ${ }^{1}$, Nicole Casali ${ }^{2}$, Silvia Cerea ${ }^{2}$, \\ Marta Ghisi $^{2}$, Giuseppe Marcolin ${ }^{1}$, Antonio Paoli ${ }^{1}$, Tatiana Moro ${ }^{1}$ \\ University of Padua, Department of Biomedical Sciences, Padova, \\ Italy $^{1}$; \\ University of Padua, Department of General Psychology, Padova, \\ Italy $^{2}$
}

Purpose: Lower-limb stress fractures are one of the most common injuries in track and field athletes. Stress fractures result from the action of various factors that expose the bone to microtraumas and therefore to fractures. This epidemiological study investigated the risk factors leading to stress fractures and bone overload diseases in Italian elite track and field athletes.

Methods: 135 high-level athletes $(M=52 ; F=83$; mean \pm SD: 23.67 \pm 3.39 years) present in the 2019 top 50 positions of the Italian national rankings in the speed, middle $(800 \mathrm{~m}$ and $1500 \mathrm{~m})$ and long (3000 m, $5000 \mathrm{~m}$, and $3000 \mathrm{~m}$ hedges) distances, and obstacles specialties, were interviewed through a telephone questionnaire. The first part included general close-ended questions, while the second part presented specific close-ended questions on stress fractures.

Results: $38.52 \%$ of the athletes suffered at least one stress fracture (males: 28.30\%; female: $45.12 \%$ ). Injured athletes showed differences in the probability of incurring fractures, with an effect of the training ground $(\chi 2=12.43, \mathrm{p}=0.03)$, with higher probability when training on indoor surfaces or asphalt. Similarly, differences in the number of fractures were detected considering the training ground $(\mathrm{F}$ $(5,125)=2.85, \mathrm{p}=0.02)$, with fewer fractures among athletes running on trucks built with new materials. Females showed differences between specialties in the probability of incurring fractures $\left(\chi^{2}=\right.$ $7.63, p=0.02$ ), with a higher probability in long distances. Moreover, the presence of menstrual irregularities affected the number of fractures $(\mathrm{t}(1,80)=-2.54, \mathrm{p}=0.01)$ with the higher incidences in middle and long-distance athletes $(\mathrm{t}(1,80)=-2.90, \mathrm{p}=0.005)$. Logistic regression highlights middle distance as a significant predictor of fractures $(B=1.77, p=0.01)$. Multiple linear regression shows the age of menarche as the only significant predictor of fractures $(\mathrm{B}=$ $0.29, \mathrm{p}=0.01)$. Males showed differences in the training ground $(\mathrm{F}(4$, $46)=5.88, p<0.001)$, with more fractures among athletes that run on old and hard tracks.

Conclusions: Results of the current epidemiological study highlight how the training surface represents a high risk factor of incurring stress fractures. Menarche age and menstrual disorders are the main 
risk factors in women, mainly when competing in $800 \mathrm{~m}$ and $1500 \mathrm{~m}$ specialties.

\section{References}

1. Lambert C, Reinert N, Stahl L, Pfeiffer T, Wolfarth B, Lachmann $\mathrm{D}$, Shafizadeh S, Ritzmann R. Epidemiology of injuries in track and field athletes: a cross-sectional study of specific injuries based on time loss and reduction in sporting level. Phys Sportsmed. 2020 Dec 8:1-10. https://doi.org/10.1080/00913847. 2020.1858701.

2. Fredericson M, Jennings F, Beaulieu C, Matheson GO. Stress fractures in athletes. Top Magn Reson Imaging. 2006 Oct;17(5):309-25. https://doi.org/10.1097/RMR. 0b013e3180421c8c.

\section{5}

\section{Effect of unexpected platform shifting on postural balance control in young healthy subjects}

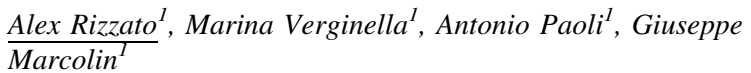 \\ University of Padua, Department of Biomedical Sciences, Padova, \\ Italy $^{l}$
}

Purpose: Postural balance control is the ability of a subject to maintain the center of pressure $(\mathrm{CoP})$ within the base of support to prevent falling. When referring to dynamic condition, balance control is the ability of the subject to react efficiently to the base of support displacements (i.e., platform shifting) or to external mechanical stimuli. The aim of this cross-sectional study was to evaluate the dynamic postural responses during platform shifts at different velocities and displacements.

Methods: 11 healthy subjects $(M=6 ; F=5 ;$ mean $\pm S D: 23.18 \pm 2.1$ years; $65.2 \pm 10.1 \mathrm{~kg} ; 1.731 \pm 0.10 \mathrm{~m}$ ) were enrolled for the study. Subjects' dynamic postural control was tested on a dynamometric platform (AMTI BP6040-AMTI force and motion) positioned over a shifting platform (Shaker Table 100-LiTem Life Testing Machines) that could generate sudden perturbations in two directions: anteriorposterior (AP) and posterior-anterior (PA). During the experimental protocol, composed by 18 trials, velocities $(100 \mathrm{~mm} / \mathrm{s}, 200 \mathrm{~mm} / \mathrm{s}$ e $400 \mathrm{~mm} / \mathrm{s})$ and displacements $(25 \mathrm{~mm}, 50 \mathrm{~mm} \mathrm{e} 100 \mathrm{~mm})$ of the shifting platform were randomly combined. Three trials without perturbations were randomly administered to avoid an expected perturbation every trial. The whole protocol was repeated after a 5-min break. Each trial lasted $60 \mathrm{~s}$ and the perturbations were randomly produced between seconds 20 and 40 . The displacement between the maximum and minimum peak of the $\mathrm{CoP}$ anterior-posterior trajectory (CoP_ $\square \square \mathrm{P}$ ) following the perturbation was considered for the analysis.

Results: In the AP direction, two-way ANOVA analysis revealed no significant effects of the displacement $(p=0.650)$ but a significant effect of the velocity $(p=0.001)$ and interaction between the two factors $(p=0.003)$. In the PA direction, two-way ANOVA analysis showed a significant effect of the velocity $(p=0.011)$ but no significant effects of the displacement $(\mathrm{p}=0.588)$ nor interaction between them $(p=0.653)$. Overall, observed oscillations were higher when perturbations were administered in the AP rather than PA direction.

Conclusions: Outcomes from this study demonstrated a greater effect of velocity on a perturbation-induced destabilization. Conversely, the extent of the displacement seemed to be less important in affecting subjects' dynamic postural balance control. Finally, further investigations are needed to deepen the mechanisms underlying the greater oscillations detected in the AP than PA direction.

\section{References}

1. Zemková E, Kováčiková Z, Jeleň M, Neumannová K, Janura M. Postural and trunk responses to unexpected perturbations depend on the velocity and direction of platform motion. Physiol Res. 2016 Nov 23;65(5):769-776.

2. Johnson, T. K., \& Woollacott, M. H. (2011). Neuromuscular responses to platform perturbations in power-versus endurancetrained athletes. Perceptual and motor skills, 112(1), 3-20.

16

\section{Postural threat influences the coupling between anticipatory and compensatory postural adjustments in response to an external perturbation}

Francesco Piscittelli ${ }^{1}$, Francesco Pascucci ${ }^{I}$, Paola Cesari ${ }^{l}$, Matteo Bertucco $^{I}$

University of Verona, Department of Neurosciences, Biomedicine and Movement Sciences, Verona, Italy ${ }^{I}$

Purpose: It is well known that fear of falling influence the control of postures and postural threat increase the conscious control of postures, the general strategy for providing protection against a loss of balance is to limiting large body movements. The aim of the present study was to verify how subjects exposed to conditions that presented different levels of postural threat respond to an external perturbation. We investigated how the CNS adjusts the central motor commands through the modulation of APA (anticipatory postural adjustments) and CPA (compensatory postural adjustments) for optimizing body stability under postural threat.

Methods: Eighteen healthy subjects participated in this study. Subjects stood in front of a pendulum at two different heights above ground level: $2 \mathrm{~cm}$ (Low) and $80 \mathrm{~cm}$ (High). They were instructed to receive a series of perturbations, induced by a pendulum, and to maintain their balance for at least $5 \mathrm{~s}$ after the impact. EMG signals were recorded from 6 muscles on the dominant side of the body: tibialis anterior (TA), medial gastrocnemius (GM), rectus femoris (RF), biceps femoris (BF), erector spinae (ES), rectus abdominis (RA). EMG signals were used to quantify APAs and CPAs, as well as the co-activation (C-Index) and reciprocal changes (R-Index) in the activity of agonist-antagonist pairs.

Results: In the APA phase, RF was greater with High compared to Low threat. GM muscle showed a larger deactivation with High compared to Low condition. During the compensatory (CPA) phase, the activity of TA muscle was found higher with Low compared High, while GM muscle showed larger activity with High than Low postural threat. Overall, higher C-indexes values than R-indexes were observed during the CPA phase, while R-indexes showed higher values during APA phase. Interestingly, R-Index was found higher with Low than High condition for the TA/GM pair muscles during the APA phase, while R- and C-index for RF/BF pair muscles showed higher values with High compared Low threat condition.

Conclusions: The results suggest that the CNS sends central commands for anticipating postural adjustments by adopting primarily a muscle reciprocal activation instead of a muscle co-contraction strategy. More importantly, when in condition of postural threat, this latter strategy is further emphasized. For CPAs mainly co-contractions strategies took place, and particularly for the muscles pairs that were presenting less involvement in the APAs.

References 
1. Latash ML. Muscle coactivation: definitions, mechanisms, and functions. J Neurophysiol. 2018;120(1):88-104.

2. Santos, MJ, Kanekar N, and Aruin AS (2010). The role of anticipatory postural adjustments in compensatory control of posture: 1. Electromyographic analysis. J Elecromyogr 24(3), 338-397.

3. Chen B, Lee YJ, and Aruin AS. (2017) Role of point of application of perturbation in control of vertical posture. Exp Brain Res. 235(11):3449-3457.

\section{7}

\section{Morphological evaluation of the effect of exercise and sedentary behaviour on interstitial telocytes in skeletal muscles of rats}

\author{
Silvia Ravalli $^{1}$, Federico Roggio $^{1}$, Giovanni Lauretta ${ }^{1}$, Bruno \\ Trovato $^{I}$, Benedetta Magri ${ }^{l}$, Giuseppe Musumeci ${ }^{l}$ \\ Università Degli Studi Di Catania, Dipartimento Di Scienze \\ Biomediche E Biotecnologiche, Catania, Italy ${ }^{I}$
}

Skeletal muscle atrophy, resulting from states of hypokinesis or immobilization, leads to morphological, metabolic, and functional changes within the muscle tissue, a large variety of which are supported by the stromal cells populating the interstitium. Telocytes represent a recently discovered population of stromal cells, that has been increasingly identified in several human organs and seems to participate in sustaining cross-talk, promoting regenerative mechanisms, and supporting local stem cell niche differentiation. The aim of this morphologic study was to investigate the presence of Telocytes in the tibialis anterior muscle of healthy rats who underwent a protocol of endurance training for either 4 weeks or 16 weeks in comparison to sedentary rats. Histomorphometric analysis of the diameter of the muscle fibers highlighted muscle hypotrophy in sedentary rats. Telocytes were identified by CD34/CD117 and CD34/vimentin double-positive immunofluorescence staining. Results showed that Telocytes were significantly reduced in sedentary rats at 16 weeks, while rats subjected to regular exercise maintained a stable TCs population after 16 weeks. Understanding the relationship between TCs and exercise offers new chances in the field of regenerative medicine, suggesting possible triggers for TCs in sarcopenia and other musculoskeletal disorders, promoting adapted physical activity and rehabilitation programs in clinical practice.

References

1. Popescu et al. Identification of tel-ocytes in skeletal muscle interstitium: Implication for muscle regeneration. J. Cell. Mol. Med. 2011.

2. Manetti et al. Morphological evidence for telocytes as stromal cells supporting satellite cell activation in eccentric contractioninduced skeletal muscle injury. Sci. Rep. 2019.

\section{8}

\section{Postural assessment in adolescent idiopathic scoliosis: relations between trunk and spine morphology, static balance and self-perceived body image}

Guido Belli $^{1}$, P Maietta Latessa $^{l}$, F Rigliaco $^{2}$, M Mauro $^{l}$, S Toselli $^{3}$ Department of Sciences For Quality of Life, University of Bologna, Italy $^{l}$;
Master Degree In Sport Sciences, University of Bologna ${ }^{2}$; Department of Biomedical and Neuromotor Sciences, University of Bologna, Italy ${ }^{3}$

Purpose: Adolescent idiopathic scoliosis (AIS) is a 3-D deformity of the spine that affects aesthetic and quality of life during growth. Since AIS can progressively increase and be responsible for several diseases, the regular screening and monitoring are necessary in order to obtain early diagnosis and treatment. The aim of the study is to compare different methods of postural evaluation commonly used for non-invasive assessment in people with AIS.

Methods: Fifteen people with diagnosis of AIS were recruited from a physiotherapy centre (age range: 12-22 years). After medical evaluation, they were tested during a single session time with following tools: Trunk Appearance Perception Scale (TAPS: body image), Scoliosis Research Society Questionnaire - Italian Version (SRS-22: quality of life and body image), Spinal Mouse (SPINAL: spine morphology and trunk inclination in sagittal and frontal plane), Nintendo Balance Board with MyPerfectPosture ${ }^{\circledR}$ software (BALANCE: centre of pressure position and static balance), Photogrammetry with APECS App (PHOTO: body alignment in frontal and sagittal plane). Data from each tools were selected and compared in order to correlate them. In particular, SPINAL was assumed as reference tools for multiple analysis.

Results: BALANCE data are significantly correlated with SPINAL inclination on sagittal plane (adj R2 $=0.43 ; \mathrm{p}<0.05$ ) but not with inclination on frontal plane $(\operatorname{adj} \mathrm{R} 2=0.13 ; \mathrm{p}>0.05)$ and spinal curvature (adj R2 $=0.31 ; \mathrm{p}=0.08$ ). PHOTO data are significantly correlated with SPINAL inclination on frontal plane (adj R2 $=0.43 ; \mathrm{p}$ $<0.05)$. No significance was found between TAPS, SRS-22 and SPINAL. Simple correlation analysis between selected variables evidenced poor to moderate correlation for all tools.

Conclusions: SPINAL has been previously validated as an effective tools for spine evaluation in frontal and sagittal plane; although correlation with it ranged from poor to moderate, BALANCE can be a simple, low cost and quick methods to assess posture in people with AIS. Furthermore, TAPS, SRS-22 and PHOTO could be easily added to globally monitor subjects during growth.

References

1. Livanelioglu A, Kaya F, Nabiyev V, Demirkiran G, Firat T (2016), The validity and reliability of "Spinal Mouse" assessment of spinal curvatures in the frontal plane in pediatric adolescent idiopathic thoraco-lumbar curves, Eur Spine J; 25(2): 476-82.

2. Clark RA, Mentiplay BF, Pua YU, Bower KJ (2018), Reliability and validity of the Wii Balance Board for assessment of standing balance: a systematic review, Gait Posture; 61: 40-54.

\section{ALLENAMENTO E VALUTAZIONE SPORTIVA 1}

\section{9}

\section{The effects of strength training on triathlon race and physiological determinants of cycling and running performance}

Simone Villanova $^{1}$, Marta Colosio ${ }^{2}$, Raffaele Mazzolari ${ }^{3}$, Andrea Pilotto $^{4}$, Alessio Marciano ${ }^{1}$, Simone Porcelli ${ }^{5)}$.

University of The West of Scotland, Institute For Clinical Exercise and Health Science, Glasgow, Regno Unito, Italy ${ }^{l}$;

University of Milan, Department of Biomedical Sciences For Health, Milan, Italy ${ }^{2}$;

University of The Basque Country, Department of Physical Education 
and Sport, Vitoria-gasteiz, Spagna, Italy ${ }^{3}$;

University of Udine, Department of Medicine, Udine, Italy;

University of Pavia, Department of Molecular Medicine, Milan, Italy ${ }^{5}$

Purpose: Triathlon is a multidisciplinary endurance sport that includes swimming (S), cycling $(\mathrm{C})$ and running $(\mathrm{R})$ in a single race. Maximal oxygen consumption ( $\left.\dot{V}^{\prime} \mathrm{O} 2 \mathrm{max}\right)$, anaerobic threshold (AT), and steady-state oxygen consumption ( $\left.\dot{V}^{\prime} \mathrm{V}^{\prime} \mathrm{O} 2\right)$ at given running speed (RE) or cycling power (CE) are the main physiological determinants of triathletes'performance ${ }^{1}$. Strength training (ST) may affect at least some of these variables ${ }^{2}$ but it can also represent a detrimental stimulus for endurance performance if not adequately combined to endurance training $(\mathrm{ET})^{3}$. We investigated the effects of ST, in combination to ET, on physiological responses to exercise, muscle force and race performance in an Olympic triathlon(RACE).

Methods: Twenty-six triathletes (age:31 \pm 4 years) were assigned to either ET-ST $(N=14)$ or ET $(N=12)$. Training volume for $S, C$ and $\mathrm{R}$ was similar between groups. ET-ST performed two ST sessions per week, at least $6 \mathrm{~h}$ from last ET session. Before (PRE) and after (POST) 8 weeks of intervention, subjects performed: i) incremental maximal tests, followed by a verification trial to assess $\dot{V}^{\prime} \mathrm{O} 2 \mathrm{max}$ and AT for both R and C; ii) 6-min moderate constant speed/work-rate exercises to assess $\mathrm{RE}$ and $\mathrm{CE}$ respectively; iii) RACE $(1.5 \mathrm{~km} \mathrm{~S}$, $40 \mathrm{~km} \mathrm{C}$, and $10 \mathrm{~km} \mathrm{R}$ ). Maximal voluntary isometric force of knee extensors muscles (MVICKE) was assessed before the race (T0), after $\mathrm{S}$ (T1), after C (T2), and at the end (T3).

Results: A significant timexgroup interaction was reported for V'O2max, AT, CE and RE. In POST, ET-ST showed larger improvements in $\mathrm{CE}$ and RE whereas ET showed a larger improvement in V'O2max and AT. RACE time was lower than PRE for both ET-ST and ET $(\mathrm{P} \leq 0.01)$ and performance in $\mathrm{C}+\mathrm{R}$ sections improved of about $2 \%$ only in ET-ST $(\mathrm{P}=0.03)$. At T0, MVICKE increased only in ET-ST $(\mathrm{P}=0.04)$ with a force loss along RACE similar between groups (about 20\%).

Conclusions: In well-trained triathletes, the addition of ST to traditional ET improves exercise economy, MVICKE and $\mathrm{C}+\mathrm{R}$ race time, although a detrimental effect on some physiological parameters related to performance was reported. Coaches should consider the benefits of ST to maximize lower-limb strength and exercise economy, without disregarding potential negative interference effects.

\section{References}

1. Millet G, Vleck V, Bentley $\mathrm{D}(2011)$ Physiological requirements in triathlon.Journal of Human sport exercise6:2

2. Beattie K, Kenny I, Lyons M, Carson P(2014)The effects of strenght training on Performance in endurance athletes. Sport Medicine45:845-65.

3. Berryman N, Mujika I, Bousquet L(2019)Concurrent Training for Sports Performance:The 2 sidles of the medal. International journal of sport physiology and performance14:279-85.

\section{0}

\section{Acute effects of prismatic adaptation on shot accuracy} of penalty kicks in young soccer players: a pilot study

\author{
Valerio Giustino $^{I}$, Rosario Emanuele Bonaventura ${ }^{I}$, Guglielmo \\ Pillitteri $^{I}$, Simona Pajaujiene ${ }^{2}$, Antonino Bianco ${ }^{1}$, Antonio Paoli ${ }^{3}$, \\ Massimiliano Oliveri ${ }^{1}$, Antonio Palma ${ }^{1}$, Giuseppe Battaglia ${ }^{1}$ \\ University of Palermo, Department of Psychology, Educational \\ Science and Human Movement, Palermo, Italy ${ }^{1}$; \\ Lithuanian Sports University, Department of Coaching Science, \\ Kaunas, Lituania ${ }^{2}$;
}

University of Padova, Department of Biomedical Sciences, Padova, Italy $^{3}$

Purpose: In soccer, a successful penalty kick requires kicking accuracy towards the side to shoot. Prismatic Adaptation (PA) is a procedure performed using prismatic lenses that shift the visual field during a movement task. PA is closely related to the visual perception system and can have a direct effect on the accuracy required for the penalty kick. Hence, the aim of this pilot study was to investigate any effects of PA on penalty kick accuracy.

Methods: A number of seven young male soccer players (16 years old), right-handedness and right-footedness, was recruited for the study. All participants performed three experimental sessions held a week from each other. Each session included a sequence of 30 penalty kicks, without goalkeeper, aimed at one of the three lines marked on a football goal. The sequence of 30 penalty kicks was performed before and immediately after PA in which lens deviation side was different between the three sessions (i.e., right deviation, left deviation, neutral).

Results: A significant interaction was found between lens deviation side and error from the target on the football goal $(\mathrm{p}<0.05)$. Although not statistically significant, an interesting result concerning the penalty kick accuracy and lens deviation side was revealed. No significant interaction was found between lens deviation side, before and after PA, and error from the target on the football goal. The penalty kick aimed towards the central target on the football goal and the after effect of the rightward PA were significantly correlated positively $(\mathrm{r}=0.98, \mathrm{p}<0.001)$.

Conclusions: Our results suggest that PA can have a positive effect on the accuracy of penalty kicks. These findings hypothesize the use of PA as an integrative training program in order to improve penalty kicks performance in soccer.

\section{References}

1. Martin TA, Norris SA, Greger BE, Thach WT. (2002) Dynamic coordination of body parts during prism adaptation. J Neurophysiol 88(4):1685-1694.

2. Navarro M, van der Kamp J, Schor P, Savelsbergh GJP. (2018) Implicit learning increases shot accuracy of football players when making strategic decisions during penalty kicking. Hum Mov Sci 61:72-80.

\section{1}

\section{Effects of pyramidal and polarized training intensity distributions over a 16-week periodization in well- trained endurance runners}

\author{
$\underline{\text { Luca Filipas }}^{1}$, Matteo Bonato $^{1}$, Gabriele Gallo $^{2}$, Roberto Codella ${ }^{1}$ \\ Università Degli Studi Di Milano, Department of Biomedical \\ Sciences For Health, Milan, Italy ${ }^{1}$; \\ Università Degli Studi Di Genova, Department of Experimental \\ Medicine, Genova, Italy ${ }^{2}$
}

Purpose: The aim of this study was to investigate the effects of modifying training intensity distribution throughout a 16-week periodization in well-trained endurance runners, comparing four different periodization of training intensity distribution.

Methods: Sixty well-trained male runners completed a 16-week training intervention divided into four groups: a pyramidal periodization (PYR); a polarized periodization (POL); a pyramidal + polarized periodization (PYR + POL); a polarized + pyramidal periodization (POL + PYR). Groups PYR and POL trained according to a pyramidal or polarized distribution for 16 weeks. The 16 -week 
intervention was split into two 8-week phases to allow for an alternating periodization in the groups PYR + POL and POL + PYR, starting with pyramidal or polarized distribution and then switching to the other. The periodization patterns were isolated manipulations of training intensity distribution, while keeping training load clamped. Participants were tested pre-, mid- and post-intervention for body mass, speed at 2 and 4 mmol-L-1, VO2peak and 5-km running time trial performance.

Results: There were significant group $\mathrm{x}$ time interactions for VO2peak $(\mathrm{P}<0.0001)$, speed at $2(\mathrm{P}<0.0001)$ and $4 \mathrm{mmol} \cdot \mathrm{L}-1 \quad(\mathrm{P}<$ $0.0001)$ and $5-\mathrm{km}$ running time trial performance $(\mathrm{P}=0.0001)$. Specifically, participants in PYR + POL group showed the largest improvement in all these variables. No significant interactions were observed for body mass, HRpeak, La-peak and RPE.

Conclusions: Each intervention effectively improved endurance surrogates and performance in well-trained endurance runners. However, the alteration of pyramidal followed by polarized distribution was superior in maximizing physiological and performance improvements.

\section{References:}

1. Kenneally M, Casado A, Santos-Concejero J. The effect of periodization and training intensity distribution on middle- and long-distance running performance: a systematic review. Int $\mathbf{J}$ Sports Physiol Perform. 2018;13(9):1114-1121.

2. Stöggl TL, Sperlich B. The training intensity distribution among well-trained and elite endurance athletes. Front Physiol. 2015;6:295

\section{2}

\section{Changes in hamstring tightness: variation of hamstring flexibility in prepubertal soccer}

\section{Federico Abate Daga ${ }^{1}$, Samuel Agostino ${ }^{1}$, Marco Panzolini ${ }^{1}$, Luca Baseggio $^{I}$, Luca Beratto ${ }^{I}$ \\ Adapted Training and Performance-Research Group, Università Degli Studi Di Torino, Torino, Italy ${ }^{1}$}

Purpose: Soccer demands a lot of running, sprinting, rapid change of direction and jumping where the hamstrings are strongly stressed. (Turner et al., 2014) Therefore, optimal performance in these actions depends on hamstring health. On the other hand, hamstrings muscles tend to tightness throughout biological maturation (Zakas et al., 2002). This study aimed to investigate the hamstring flexibility rate among prepubertal soccer players from U8 to U12 and the role of age and soccer years of practice on the course of hamstring flexibility.

Methods: Six hundred eleven (611) young Italyn soccer players from a local soccer school in Turin were recruited for this research and assigned to each group according to their chronological age (U8 $=124$ players; $\mathrm{U} 9=130$ players; $\mathrm{U} 10=151$ players; $\mathrm{U} 11=89$ players; $\mathrm{U} 12$ = 65 players). Hamstring flexibility was measured using the Sit and Reach Test (SAR), while data analysis was run using a one-way Analysis of Variance (one-way ANOVA). Furthermore, Tuckey's post hoc was used to determine differences among the classes of age. Finally, a linear regression model was calculated to identify the possible relationship between age and flexbibility.

Results: The results of the regression indicated that the model explained $14 \%$ of the variance and that the relationship between age and flexibility is very strong $F=(1,609)=96,669, p<0,0001 ; R 2=$ 0,137 . Meanly, flexibility decrease of $1,405 \mathrm{~cm}$ each year. The oneway ANOVA showed significant differences in flexibility among groups $(\mathrm{F}=32,76, \mathrm{P}<0,0001)$. Tuckey's post hoc identified significant differences between U8 and U10 $(\mathrm{p}<0,01 ;-2,39 \mathrm{~cm}$ of hamstring stretching), U8 and U11 ( $p<0,05 ;-2,19 \mathrm{~cm}), \mathrm{U} 8$ and U12 ( $<<0,0001 ;-5,90)$, U9 and U12 ( $<<0,0001 ;-4,98 \mathrm{~cm}), \mathrm{U} 10$ and U12 (p $<0,0001 ;-3,5 \mathrm{~cm}), \mathrm{U} 11$ and U12 (p $<0,001 ;-3,70 \mathrm{~cm})$.

Conclusions: This study underlines changes in hamstring flexibility across different age groups of prepubertal soccer players. The older and more experienced in soccer are less flexible than the younger, considering the hamstring muscles. Thus, appropriate stretching protocols should be included in prepubertal soccer training to avoid the risk of lead players to excess hamstring tightness.

\section{References}

1. (2014). Hamstring strain prevention in elite soccer players. Strength and Conditioning Journal, 36(5), 10-20. https://doi.org/ 10.1519/SSC.0000000000000076

2. Zakas, A., Galazoulas, C., Grammatikopoulou, M. G., \& Vergou, A. (2002). Effects of stretching exercise during strength training in prepubertal, pubertal and adolescent boys. Journal of Bodywork and Movement Therapies, 6(3), 170-176. https://doi.org/10. 1054/jbmt.2001.0275

\section{3}

\section{Effects of different strength training protocols on sprint performance in youth soccer: horizontal vector} exercises vs vertical vector exercises

\author{
Valerio Bonavolontà ${ }^{l}$, Francesca Latino ${ }^{l}$, Federico Colonna ${ }^{l}$, \\ Francesco Fischetti ${ }^{I}$, Stefania Cataldi ${ }^{1}$
}

Università Degli Studi Di Bari, Dipartimento Di Scienze Mediche Di Base, Neuroscienze E Organi Di Senso, Bari, Italy ${ }^{1}$

Purpose: To investigate the influence of different strength training protocols (horizontal vector exercises vs vertical vector exercises) on sprint performance in young soccer players.

Methods: Thirty-eight adolescents (mean age $14 \pm 3.5$ years) divided into three groups of training characterized by horizontal vector exercises $(\mathrm{HV}, \mathrm{n}=17)$, vertical vector exercises, $(\mathrm{VV}, \mathrm{n}=17$; ) and a control group $(\mathrm{CG}, \mathrm{n}=14)$ were involved. Sprint performance was assessed before and after 10 weeks of training with 10-m, 30-m and 60-m test. Squat test ( $\mathrm{n}$ of maximal repetitions) was also administered to assess lower limbs strength.

Results: After the intervention, significant improvements were found for squat test in $\mathrm{HV}$ and VV ( $\mathrm{p} \leq 0.01)$; differences were also found for $10-\mathrm{m}$ test in HV, for $30-\mathrm{m}$ test in VV and HV $(\mathrm{p} \leq 0.01)$ and for $60-\mathrm{m}$ test in VV $(\mathrm{p} \leq 0.01)$.

Conclusions: HV training seems to have a positive effect on sprint distance between 10- and 30-m while VV exercised seems to affect longer distance sprint performance, such as $60-\mathrm{m}$. HV strength production resulted most associated with maximal acceleration performance while VV appears to play a crucial role during the transition from lower to higher speeds.

\section{References}

1. Abade, E, Silva, N, Ferreira, R, Baptista, J, Gonçalves, B, Osório, S, and Viana, J (2019) Effects of Adding Vertical or Horizontal Force- Vector Exercises to In-season General Strength Training on Jumping and Sprinting Performance of Youth Football Players. J Strength Cond Res 13(3): 230-235.

2. Styles, WJ, Matthews, MJ, and Comfort, P (2016) Effects of strength training on squat and sprint performance in soccer players. J Strength Cond Res 30(6): 1534-1539. 


\section{4}

Correcting procedure to remove relative age effect from jumper performances: a practical tool to improve talent identification

Paolo Riccardo Brustio $^{1}$, Paolo Moise ${ }^{2}$, Antonio La Torre ${ }^{3}$, Alberto Rainoldi $^{4}$, Gennaro Boccia ${ }^{5}$

University of Verona, Department of Neuroscience, Biomedicine and Movement, Verona, Italy ${ }^{1}$;

University of Turin, School of Exercise \& Sport Sciences, Suism, Torino, Italy ${ }^{2}$;

Università Degli Studi Di Milano, Department of Biomedical Sciences for Health, Milan, Italy ${ }^{3}$;

University of Turin, Department of Medical Sciences, Torino, Italy ${ }^{4}$; University of Turin, Department of Clinical and Biological Sciences, Torino, Italy

Purpose: Asymmetry in birth-date distribution (i.e., Relative Age Effect, RAE) is a bias observed in sports competitions, especially during adolescence and youth. The RAE reflects the possible advantages/disadvantages in early sport success of those born at the beginning/end of the calendar year. In the context of Italyn jumpers' disciplines, this study aimed to investigate whether corrective adjustment procedures based on decimal age may remove or at least reduce $\mathrm{RAE}$.

Methods: Male and female athletes competing in the high and long jump from 12 to 19 years of age were included in the study. Longitudinal quadratic trendline equations across ages 12-19 years were calculated considering athletes' exact age (i.e. day of competition minus day of birth) and respective performance. Corrective adjustment calculations from estimated longitudinal quadratic equations were applied. This means that all raw performance times were adjusted using expected within annual-age performance differences generated from the quadratic line. For example, two males in the 13 years age-group, one being 13.00 years and the second who was 13.99 years on the day of competition, had their long jump performance increased by $1.28 \mathrm{~m}$ and $0 \mathrm{~m}$, respectively. Afterwards, RAE distributions (Quartile 1-4) for "All", "Top 25\%" and "10\%" of jumper's performance were examined based on raw and corrected performance for each age group.

Results: The uncorrected performance showed small to large RAE. When re-examining the data using the corrective adjustment calculations, the RAE disappeared, especially in top-level athletes in both gender and jumper disciplines.

Conclusions: Using longitudinal reference data, corrective adjustment procedures removed relative age advantages of early birth athletes. From a talent identification perspective this approach may provide practical strategies to create solutions to minimize disadvantages of late-born athletes in the early steps of their career.

\section{References}

1. Brustio PR, Kearney PE, Lupo C, Ungureanu AN, Mulasso A, Rainoldi A, and Boccia G. (2019) Relative Age Influences Performance of World-Class Track and Field Athletes Even in the Adulthood. Front Psychol. 10:1395.

2. Cobley S, Abbott S, Eisenhuth J, Salter J, McGregor D and Romann M. (2019) Removing relative age effects from youth swimming: The development and testing of corrective adjustment procedures. J Sci Med Sport: 22(6).
EDUCAZIONE FISICA E PEDAGOGIA DELLO SPORT

25

\section{Non-linear didactic technology-based intervention to enhance basic motor competencies with mobak-5-6: a pilot study in primary school}

\section{Domenico Monacis ${ }^{I}$, Dario Colella}

Università Di Foggia, Dipartimento Di Studi Umanistici. Lettere, Beni Culturali, Scienze Della Formazione, Foggia, Italy ${ }^{I}$

Purpose: Technologies in physical education (PE) represent a useful tool to increase levels of physical activity and reduce sedentary behavior, but their effectiveness on motor competence development is still unclear. The aim of the study is to assess the effects of physical education intervention based on linear and non-linear didactic approach integrated with technology on motor competencies development.

Methods: The sample consisted of 120 primary school children (F: 54; M: 66), ranged from 10 to 11 years, divided in Normal weight $(\mathrm{Nw})$ and Overweight-Obese (Ow-Ob) according to BMI. Children and assigned to Experimental Group (EG) and Control Group (CG). The intervention protocol provided the proposal of different executive variants of motor tasks based on MOBAK-5-6 basic motor competencies, concerning self-movement areas and object control. Both the EG and the CG have planned activities with the use of the video projector in the gym: for each video-exercise were structured 3 levels of difficulty and increasing intensity. EX followed a non-linear didactic approach, using different teaching styles, proposing different variants of motor tasks, and soliciting various learning methods, while in CG teachers explained and demonstrated only one variant of the proposed task, with children practicing and performing sequentially what was proposed. The intervention protocol was proposed for 8 weeks, while the assessment was carried with the MOBAK-5-6 one week pre- and post- intervention.

Results: Results evidenced positive effects on both $\mathrm{Nw}$ and $\mathrm{Ow}-\mathrm{Ob}$ groups in EG, and on total sample, improving scores in all MOBAK subtest and total motor qualifications, while less positive effects could be observed in CG for Normal-weight sample.

Conclusions: The integration of technology into physical education lessons, combined with non-linear didactics approaches represents an innovative and effective way to develop motor competencies in primary school. Technological tools and devices are instruments used by teachers to enhance motor experience, re-oriented the traditional setting in physical education, contextualizing and adapting motor tasks, expanding, and strengthening the relationship between motor learnings in different ways (i.e. active breaks, exergames, active videogames, hybrid learning environment, etc.). However, further studies are needed to confirm the results obtained.

\section{References}

1. Mosston, M., \& Ashworth, S. (2008). Teaching physical education (first online edition).

2. Chow, J. Y., \& Atencio, M. (2014). Complex and nonlinear pedagogy and the implications for physical education. Sport, Education and Society, 19(8), 1034-1054.

3. Quintas, A., Bustamante, J.-C., Pradas, F., \& Castellar, C. (2020). Psychological effects of gamified didactics with exergames in Physical Education at primary schools: results from a natural experiment. Computers and Education, 152. 


\section{6}

Effects of an outdoor cardio-dance program on selfesteem and their impact on academic performance in overweight school-girls

\section{Francesca Latino $^{I}$, Valerio Bonavolonta ${ }^{l}$, Francesco Fischetti $^{1}$, Stefania Cataldi ${ }^{I}$ \\ Università Degli Studi Di Bari “aldo Moro”, Dipartimento Di Scienze Mediche Di Base, Neuroscienze E Organi Di Senso, Bari, Italy $^{l}$}

Purpose: To explore self-esteem as mediators between physical activity and academic performance in overweight schoolgirls, based on the assumption that high self-esteem encourages high performance. Moreover, it was aimed to find out the relationship between BMI and academic performance, starting from the premise that high self-concept led students to achieve academically, as they proved optimistic feelings for themselves on account of previous achievements obtained.

Methods: 140 schoolgirls (14-15 years) were randomly assigned to an experimental group $(\mathrm{n}=70)$ that performed a cardio-dance program, or a control group $(n=70)$. At baseline and after training, a battery of standardized assessment motor tests (Standing long jump test, Harvard step test, sit and reach test, and butt kicks test), and Rosenberg self-esteem scale were administered. BMI was used as indicator of correlation with body fat and academic achievement. Academic performance had been measured through scholastic grades. Results: In comparison to the control group, experimental group's self-esteem scores were higher after the intervention program $(\mathrm{F} 1,138$ $=271.43, \mathrm{p}<0.001, \eta 2 \mathrm{p}=0.66)$. They also reported considerably lower BMI $(\mathrm{F} 1,138=199.77, \mathrm{p}<0.001, \eta 2 \mathrm{p}=0.59)$ as consequence of the intervention. Students who improved self-esteem and BMI were also those who reported best grades $(\mathrm{p}<0.001)$.

Conclusions: The results indicated that physical activity, self-esteem and academic performance were positively related. In particular, selfesteem seemed to play a significant role in mediating the relationship between physical activity and academic performance. Lastly, the results also indicated that BMI may affected academic performance. References

1. Prezza, M., Trombaccia, F.R., \& Armento, L. (1997). The Rosenberg Self-Esteem Scale: Italyn translation and validation. Giunti Organizzazioni Speciali, 223, 35-44.

2. Sani, S.H.Z., Fathirezaie, Z., Brand, S., Pühse, U., HolsboerTrachsler, E., Gerber, M., Talepasand, S. (2016). Physical activity and self-esteem: Testing direct and indirect relationships associated with psychological and physical mechanisms. Neuropsychiatr. Dis. Treat., 12, 2617-2625.

\section{7}

\section{Sport, physical activities, bodily practices and welfare policies}

\section{Antonio Borgogni ${ }^{1}$, Silvia Sangalli ${ }^{1}$}

Università Di Bergamo, Dipartimento Di Scienze Umane E Sociali, Bergamo, Italy ${ }^{l}$

Purpose: Sport, physical activities, and bodily practices are more and more recognized as part of the new welfare system. An enhancement on the project development and studies, has been given by the EU White Paper on sport in 2007 in which the social aspects of sport have been emphasized at political level. Recently, the Work Plan for sport
2021-2024 and the Italyn National Resilience Plan 2021 have empowered the matter also in connection with school's drop-out, sport overall accessibility, and urban regeneration. However, despite the growth of programs and activities, the lack of scientific literature does not allow to assess the long-lasting effects in terms of sport participation, education, and social inclusion. Frequently, studies present an acritical view of sport as cure-all for every social problem. Focusing on youth discomfort, the research will in-depth investigate the literature, the programs, and the activities carried out at national and international level aiming to find the success criteria.The hypothesis is that, to be successful, an intervention should be durable, integrated with the opportunities given by the welfare system, managed by educators and sport operators sharing competences and common backgrounds. The latest aspect is also challenging the academic disciplinary formation and the continuing education.

Methods. A systematization - more than a review - of the scientific literature, as well the projects and programs report, is a methodological keystone in a so dispersed field. The research will be idiographic and qualitative. The contexts of investigation (sports organizations, social cooperatives, and schhols) will be chosen according to the meaningfulness of the cases. Interviews, observations, focus groups, shadowing will be the main tools.

Results. The expected results are to identify success criteria and limits of the interventions in relation with the congruences with the interdisciplinary education, the support given by organizations and the welfare integrated system allowing the sustainability of the outcomes.

Conclusions. The research, due to its not-so-explored, anyway relevant, subject, is expected to offer a novel view and a basis for further research and a platform to guide decision making processes.

\section{References}

1. Coalter F. (2015). Sport-for-challenge: some thoughts from a sceptic. Social inclusion, 3, 3, 19-23.

2. European Commission (2007). White Paper on Sport.

3. European Council (2020). Work Plan for Sport 2021-24.

4. Hartmann D. (2012). Theorizing Sport as Social Intervention, Quest, 55, 2, 118-140.

5. Henry, I. (2015). The role of sport in fostering open and inclusive societies. Research for CULT Committee.

6. Presidenza del Consiglio dei Ministri (2021). Piano Nazionale di Ripresa e Resilienza.

\section{8}

An internet-supported continuing professional development training with secondary school physical education teachers: study protocol for the physical education for moving (PE4MOVE) trial

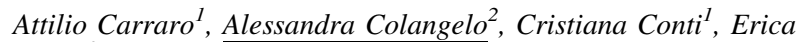
Gobbi $^{3}$, Marco Petrini ${ }^{4}$

Libera Università Di Bolzano, Facoltà Di Scienze Della Formazione, Bressanone, Italy ${ }^{1}$;

Università Degli Studi Di Padova, Dipartimento Di Filosofia, Sociologia, Pedagogia E Psicologia Applicata, Padova, Italy ${ }^{2}$; Università Degli Studi Di Urbino Carlo Bo, Dipartimento Di Scienze Biomolecolari, Urbino, Italy;

Ufficio Scolastico Regionale Per Le Marche, Coordinamento Per L'educazione Fisica E Sportiva, Ancona, Italy ${ }^{4}$

Purpose: Despite the well-recognized, multiple benefits of physical activity (PA), it is estimated that a four-fifths of European adolescents 
are insufficiently physically active (WHO, 2018). The role of physical education (PE) in promoting students' PA has been widely recognized and one of the most frequently reported suggestion is that PE teachers need to be specifically trained in guiding and motivating students to be more physically active. The aim of this study is to present the protocol of the PE4MOVE project, developed (1) to monitor Marche Region's secondary-school students' PA practice, factors related to engagement in PA and physical fitness; (2) to train PE teachers on strategies to foster students' PA participation; (3) to evaluate the effectiveness of a Continuing Professional Development training (CPDt), with both teachers and their students.

Methods: The PE4MOVE project is inspired by the Erasmus + Sport Project IMPACT (Papaioannou et al., 2020). Through the collaboration with the USR Marche, 65 secondary schools and 148 teachers have been recruited, they will then invite a group of approx. 7.000 students to participate in the study. Using a cluster randomized design, PE teachers and their students will be allocated to either an intervention group (IG) or a waitlist-control group (CG). PE teachers of both groups will attend the same CPDt, but on different time periods. The CPDt will consist of a series of webinars and online laboratories, based on the concept of physical literacy and on the most popular theories, models and techniques used in youth PA promotion. Teachers' motivations, intentions, attitudes, self-efficacy, behaviours and teaching practices to foster PA will be analysed. Students' selfreported and objectively measured (with Actigraphs GT3X) PA, physical fitness (adopting the FitBack test battery: 6-MWT, handgrip and seat-and-reach) and factors associated with PA participation and intention to practice will also be studied. Data will be collected before and after the CPDt, during the 2021/22 and 2022-23 school years.

Conclusions: The study will add knowledge about PA of a large sample of Italyn secondary-school students and the effectiveness of a CPDt on teachers and students. Results will be useful to design and plan intervention in other Italyn and European regions and to advise educational Authorities and stakeholders on the importance of PE to promote out-of-school students' PA.

\section{References}

1. Papaioannou A, Carraro A, Sarazzin P, Demirhan G, Ramis Y, Duda J, Scheuer C, Gortsila E, Özdurak H, Holzweg M, Eid L, Kerreres D, and Krommidas C. (2020). Identifying and motivating youth who mostly need physical activity: IMPACT project. Brussel, European Commission.

2. WHO (2018). Physical activity factsheets for the 28 European union member states of the WHO European region. Geneva: World Health Organization.

3. www.fitbackeurope.eu/en-us/

\section{9}

\section{The gross motor coordination of northeast young Italyns}

Matteo Giuriato ${ }^{1}$, Valentina Biino ${ }^{2}$, Marianna Bellafiore ${ }^{3}$, Giuseppe Battaglia $^{3}$, Antonio Palma ${ }^{3}$, Carlo Baldari ${ }^{4}$, Laura Guidetti ${ }^{5}$, Maria Chiara Gallotta ${ }^{6}$, Federico Schena ${ }^{7}$, Massimo Lanza ${ }^{7}$.

Faculty of Physical Culture, Department of Health and Natural Sciences, Unit of Molecular Biology, University of Physical Education and Sport, Gdansk, Polonia ${ }^{I}$, Department of Human Sciences, University of Verona, Verona, Italy ${ }^{2}$, Sport and Exercise Sciences Research Unit, University of Palermo, Palermo, Italy, Department of Theoretical and Applied Sciences, Ecampus University, Novedrate (co), Italy ${ }^{4}$, University Nicolo Cusano, University Niccolo Cusano, Rome, Italy ${ }^{5}$, Department of Physiology and Pharmacology “vittorio Erspamer", Sapienza University of Rome, Rome, Italy ${ }^{6}$, Department of Neuroscience, Biomedicine and Movement, University of Verona, Verona, Italy ${ }^{7}$

Purpose: Despite a mass of evidence that assigns motor coordination a significant role in movement education and health promotion (Robinson, 2015; Fransen, 2012; Vandorpe, 2012; Pesce, 2016; Faigenbaum, 2012), there are some serious obstacles to study and structurally promote this aspect of motor skills. The main purpose of our cross-sectional research was to verify the current level of gross motor coordination, measured with the "Körperkoordinations Test für Kinder (KTK)" (Kiphard, 1974) of northeast Italyn boys and girls between 6 and 13 years. Secondary purposes were to study the genders differences and the four KTK subtests trends with ages.

Methods: KTK data were collected from 1135 schoolchildren (girls: $\mathrm{n}=1050$; boys: $\mathrm{n}=1156$ ). Results were compared with the German References proposed by Kiphard (1974) and with similar research carried out in Belgium (Vandorpe, 2011).

Results: KTK Raw score (RS) increase with the age of the subjects (F $=203.794 ; \mathrm{p}<0.001)$, but it does not show differences between genders and interaction gender $\mathrm{x}$ age. Motor Quotient (MQ), the KTK's standardized value, tend to decrease with age $(\mathrm{r}=-0.176 ; \mathrm{p}<$ $0.001)$ and it show differences by gender $(\mathrm{F}=42.164$; $\mathrm{p}<0.001)$, age $(\mathrm{F}=19.105 ; \mathrm{p}<0.001)$ without interaction gender $\mathrm{x}$ age. Males results are better than girls in the raw scores of two of four sub-tests (JS: $\mathrm{F}=12.183$; HH: $\mathrm{F}=10.554$; $\mathrm{p}$ for all $<0.001$ ). Girls show better performances than boys in the WB $(\mathrm{F}=10.911)$. Differences among our results and those of Kiphard and Vandorpe was found $(\mathrm{F}=$ 103.639; $p<0.001)$ : both the results of Belgium and ours were lower than the German references (Belgium $=-3.5 \%$; Italy $=-8.75 \% ; \mathrm{p}<$ 0.001). The northeast Italyn values are significantly lower than Belgium $(-5.25 \% ; \mathrm{p}<0.001)$.

Conclusions: Overall, RS and MQ indicate low levels of GMC in the Italyn youth population, particularly for girls. The decrease in standardized values (MQ) as age increases must be studied to identify the possible causes. The knowledge of the updated parameters of the KTK can provide helpful References for the development of policies to support physical activity, sport and physical education in youth.

References:

1. Kiphard, E. J., \& Schilling, F., 1974. Körperkoordinationstest für Kinder. Weinheim: Beltz Test GmbH.

2. Vandorpe B, Vandendriessche J, Lefevre J, et al., 2011. The KörperkoordinationsTest für Kinder: reference values and suitability for 6-12-year-old children in Flanders Scand J Med Sci Sports,; 378-388 https://doi.org/10.1111/j.1600-0838.2009. 01067.x

\section{0}

\section{Psychomotor activity and english learning: a study in primary school}

\author{
Alessandra Nart ${ }^{1}$, Cristiana Lucchetti ${ }^{1}$, Vincenzo Biancalana ${ }^{1}$ \\ University of Urbino, Department of Biomolecular Sciences, Urbino, \\ Italy $^{l}$
}

Purpose: Learning is a behavioural modification resulting from interaction with the environment: it is the result of new experiences. In childhood, during the learning process the body plays a crucial role in the acquisition of new knowledge, necessary for the development of cognitive processes (Kubesch, 2004). Physical activity has a positive impact on the ability to concentrate as well as on the curricular 
learning performance (Biancalana, 2018). The aim of this study is to enhance English learning through psychomotor activity.

Methods: The study included 36 students (18 females; 18 males) aged between 6 and 7 years, attending first grade primary school, divided into 2 groups: the gold group work group ( 9 females; 9 males) and the silver group control group ( 9 females; 9 males). An entrance test and an exit test (which involved translating English words into Italyn) were used for the initial and final assessment of the level of knowledge of the English language, respectively. The psychomotor activity protocol included 3 psychomotor games aimed at facilitating the learning of English words (parts of the human body, colours and animals). The games were proposed as the final moment of the weekly English lesson, for a total of 6 lessons.

Results: The exit test (average value of $97.5 \pm 3.9$ and $59.6 \pm 18.8$ for the gold group and silver group, respectively) showed an increase in knowledge of English words for both groups compared to the entrance test (average value of $54.3 \pm 19.7$ and $54.6 \pm 25.6$ for the gold group and silver group, respectively); the increase of the gold group was higher. The average values of the exit test showed a greater and highly significant difference of the gold group compared to the silver group $(\mathrm{p}<0.001)$. Having regard to the gender difference, the exit test revealed higher values among females than among males, both in the gold group (average value equal to $98.3 \pm 3.4$ and $96.5 \pm$ 4.1 for the females and males, respectively) and in the silver group (average value equal to $64.6 \pm 23.7$ and $55.6 \pm 10.8$ for the females and males, respectively).

Conclusions: The results demonstrated that psychomotor activity could provide an effective support tool for school learning.

References

1. Biancalana V, Lucchetti C. (2018) Educazione motoria e miglioramento della disponibilità attentiva in età evolutiva. PEDAGOGIA PIU' DIDATTICA, Erickson 4 (2).

2. Kubesch S. (2004) Das bewegte Gehirn, «Schnittstelle von Sport und Neurowissenschaft» 34 (2):135-144.

3. MullenderWijnsma Marijke J, Hartman E, de Greeff Johannes W, Doolaard S, Roel J, Bosker Roel J, Visscher C. (2016) Physically active math and language lessons improve academic achievement. A cluster randomized controlled trial. Am Acad Pedia.

\section{ESERCIZIO, PREVENZIONE E TERAPIA 3}

\section{1 \\ Interrelationship among thigh intermuscular adipose tissue, cross-sectional area, muscle strength and functional mobility in older subjects}

\author{
Stefano Borghi ${ }^{1}$, Matteo Bonato ${ }^{I}$, Antonio La Torre ${ }^{I}$, Giuseppe \\ Banfi ${ }^{2}$, Jacopo Vitale ${ }^{3}$ \\ Università Degli Studi Di Milano, Dipartimento Di Scienze \\ Biomediche Per La Salute, Milan, Italy ${ }^{1}$; \\ Irccs Istituto Ortopedico Galeazzi, N.a., Milan, Italy ${ }^{2}$; \\ Irccs Istituto Ortopedico Galeazzi, Lamss, Milan, Italy ${ }^{3}$
}

Purpose: The human aging is one of the phenomena of greatest impact globally during twenty-first century. Aging is associated with a decline in skeletal muscle mass and function, causing changes in strength, physical performance and movement. The aim of this crosssectional study was to investigate the possible association between lower limb strength, muscle mass and composition and balance ability in elder subjects.

Methods: 16 older participants (Age: $66 \pm 6$ years; BMI: \pm ; male $=$ 4 and female $=12$ ) were assessed for muscle strength (maximum isometric strength of knee extensors by digital dynamometer and one repetition maximum by leg-press, 1RM), balance and gait capacity (Mini-BESTest), body composition by dual energy X-ray absorptiometry (obtaining Appendicular Skeletal Muscle Mass Index, ASMMI), magnetic resonance imaging of thigh muscles to evaluate Intermuscular Adipose Tissue (IMAT) and muscle Cross Sectional Area (CSA).

Results: A significant positive correlation between 1RM leg press and ASMMI $(\mathrm{r} 2=0.30 ; \mathrm{p}=0.028)$ and thigh CSA $(\mathrm{r} 2=0.28 \mathrm{p}=0.036)$ was detected whereas no significant correlation was observed between $1 \mathrm{RM}$ leg press and IMAT $(\mathrm{r} 2=0.15 ; \mathrm{p}=0.1435)$. Significant positive correlations between knee extensors strength and ASMMI ( $22=0.34$; $\mathrm{p}=0.0185)$ and thigh CSA $(\mathrm{r} 2=0.26 ; \mathrm{p}=0.0447)$ were observed. Conclusions: The positive correlation between lower limbs muscle mass and strength highlights that muscle size is an important factor to generate force in elders. Improving muscle health in subjects over 60 years of age could reduce frailty and risk of falls.

References

1. Vitale JA, Messina C, Albano D, Fascio E, Galbusera F, Corbetta S, Sconfienza LM, Banfi G. (2021) Appendicular Muscle Mass, Thigh Intermuscular Fat Infiltration, and Risk of Fall in Postmenopausal Osteoporotic Elder Women. Gerontology 5:1-10. https://doi.org/10.1159/000513597.

2. Vitale JA, Bonato M, Borghi S, Messina C, Albano D, Corbetta S, Sconfienza LM, Banfi G. (2020) Home-Based Resistance Training for Older Subjects during the COVID-19 Outbreak in Italy: Preliminary results of a 6-Months RCT. Int J E.

\section{2}

Facilitators and barriers to practicing physical activity and complying who guidelines during pregnancy: a survey to investigate women' and midwives' perception through focus group

Sofia Marini ${ }^{1}$, Alice Masini ${ }^{1}$, Francesca Scognamiglio ${ }^{1}$, Isotta Caravita $^{2}$, Rossella Messinal, Giorgia Soldă ${ }^{1}$, Vincenza Leccese ${ }^{3}$, Virginia Bertini $^{3}$, Dila Parma ${ }^{3}$, Laura Dallolio ${ }^{1}$

University of Bologna, University of Bologna, Department of Biomedical and Neuromotor Science, Bologna, Italy ${ }^{1}$; University of Bologna, University of Bologna, Department of Medical and Surgical Science, Bologna, Italy ${ }^{2}$,

University of Bologna, University of Bologna, Department of Medical and Surgical Sciences, Bologna, Italy ${ }^{3}$

Purpose: Regular physical activity (PA) is a protective factor and represents a key challenge for pregnant women in a view of their own and baby's health. Moreover, acute physiological responses to PA are generally increased during pregnancy compared to non-pregnancy. Therefore, according to the World Health Organization (WHO) guidelines, pregnant women (PW) without contraindications should practice $150 \mathrm{~min}$ of moderate PA per week. Nonetheless, PA levels are worryingly low among pregnant women. The aim of this survey was to investigate pregnant women and midwives' points of view towards PA practice and recommendations during pregnancy, in a view to tackle the situation providing future PA promotion strategies. Methods: Pregnant women and midwives recruited at the University Hospital of Bologna, were involved in focus groups carried out online in the period between July 2020 and April 2021. Focus groups, were audio-recorded, transcribed verbatim and anonymized before being coded using a thematic approach. According to the COM-B model framework, data analysis was conducted. 
Results: A total number of 4 focus group were conducted, involving 10 pregnant women (aged 30-46) and 10 midwives (aged 43-61). The majority of the sample had already experience in practicing PA $(10 / 10$ women and 5/10 midwives) and overall reported to be aware about the importance of PA during pregnancy. Moreover, WHO recommendations were considered to be feasible by both groups. However, PW expressed the following barriers related to PA practice: lack of specific indications and opportunities concerning PA practice, low levels of self-efficacy and motivation. Similarly, midwives reported a lack of specific information and knowledge related to PA promotion in pregnancy and lack of confidence in managing PA, as main obstacles.

Conclusions: Both groups perceptions indicate that PA represents a positive element for the mother's and baby's well-being. However, findings suggest that both midwives and women need to be trained in order to tackle barriers and support PA promotion during pregnancy, in a sustainable view. In light of this, the COM-B model can be used to implement new intervention strategies involving midwives to reach the goal, given that their role is crucial, especially in enhancing women' self-efficacy and empowerment. Finally, these results can be used as a started point to target specific PA interventions in order to improve PA among pregnant women.

\section{References}

1. The World Health Organization. WHO Guidelines on Physical Activity and Sedentary Behaviour; WHO: Geneva, Switzerland, 2020.

2. Mottola MF, Davenport MH, Ruchat SM, Davies GA, Poitras VJ, Gray CE, Jaramillo Garcia A, Barrowman N et al. 2019 Canadian guideline for physical activity throughout pregnancy. Br J Sports Med. 2018; 52(21):1339-1346 https://doi.org/10.1136/bjsports2018-100056.

\section{3}

\section{Exercise training in kidney transplant recipients: comparison between a supervised and a home-based program}

Luca Innella ${ }^{1}$, Giovanni Piva ${ }^{2}$, Piero Fumarola ${ }^{1}$, Nicola Lamberti ${ }^{2}$, $\overline{\text { Fabio Manfredini }}{ }^{2}$, Valentina Totti ${ }^{3}$, Yuri Battaglia ${ }^{4}$, Alda Storari ${ }^{4}$, Luca Pomidori ${ }^{1}$, Michele Felisaatti ${ }^{1}$, Gabriela Sangiorgi ${ }^{5}$

Esercizio Vita Medical Fitness, R\&s, Ferrara, Italy ${ }^{1}$;

Università Di Ferrara, Neuroscienze E Riabilitazione, Ferrara, Italy $^{2}$;

Centro Nazionale Trapianti, Prescrizione Esercizio Fisico, Rome, Italy $^{3}$;

Ospedale Universitario Di Ferrara, Unità Di Nefrologia E Dialisi, Ferrara, Italy ${ }^{4}$

Centro Trapianti Emilia Romagna, Centro Riferimento Trapianti, Bologna, Italy ${ }^{5}$

Purpose: Physical inactivity and poor physical fitness are important targets to address in order to improve clinical outcomes after kidney transplantation [1]. Emilia-Romagna Region has promoted an exercise network to improve physical fitness in kidney transplant recipients (KTr) [2]. In this study we aimed to compare adherence and outcomes after a prescribed exercise program conducted with an exercise specialists supervision in a selected gym classes or at home. Methods: Twenty-one KTr being treated at a Nephrology Unit were contacted from the Unit of Rehabilitation Medicine for possible inclusion. Patients underwent a baseline testing sessions including a Balke-Ware treadmill test for the evaluation of the maximal oxygen consumption estimation (VO2peak), 6-min walking test (6MWT), 5-time sit-to-stand test (5STS). Thereafter, patients were proposed to perform the prescribed exercise under supervision in a specialized physical activity center (SUP) or at home (HB). KTr who choosed SUP underwent a 3 weekly 60 -min training sessions including warmup, aerobic (treadmill or cycling) exercise, strength training (isotonic machines) stretching and cool down. Those selecting HB performed an inside-home progressively-increasing interval walking training (walk-sit ratio 1:1) for $10 \mathrm{~min}$ per day [3]. At the end of 6-month program, the testing session was readministered for both groups.

Results: Seventeen patients $(81 \%)$ adhered, 8 selecting SUP and 9 HB. At baseline, KTr of SUP group were younger $(55 \pm 11$ vs $63 \pm$ $2 ; \mathrm{p}=0.04)$ with greater $6 \mathrm{MWT}(480 \pm 62$ vs $356 \pm 112 \mathrm{~m} ; \mathrm{p}=0.03)$ than HB subjects. After 6-month, 6 subjects of SUP group (two dropped out for health reasons) and all 9 subjects of HB group safely completed the program, with a high adherence (exercise sessions executed $>80 \%$ respect to the prescribed). Both groups significantly improved all outcomes, with greater variations observed in HB for 6MWT (SUP: +15 vs HB $+52 \mathrm{~m}$ ), 5 STS (SUP: -1.2 vs HB $-5.0 \mathrm{~s}$ ) and VO2 (SUP: +1.2 vs HB $+3.1 \mathrm{ml} / 100 \mathrm{~g} / \mathrm{min}$ ). The betweengroup comparison corrected for baseline covariates highlighted a significant difference in favor of HB for 6MWT variations $(\mathrm{p}=$ 0.046).

Conclusions: Physical activity is effective in improving cardiovascular fitness in KTr both supervised in specialized gyms and carried out at home through prescribed structured programs for subjects unable or unwilling to attend certified gyms.

\section{References}

1. De Smet S, Van Craenenbroeck AH. Exercise training in patients after kidney transplantation. Clin Kidney J. 2021;14(Suppl 2):ii15-ii24.

2. Totti V, et al. Promotion of Pre- and Post-Transplant Physical Exercise in the Emilia-Romagna Region: The Network of the Program "Transplantation, Physical Activity, and Sport". Transplant Proc. 2019 Nov;51(9):2902-2905.

3. Manfredini F, et al. Exercise in Patients on Dialysis: A Multicenter, Randomized Clinical Trial. J Am Soc Nephrol. 2017 Apr;28(4):1259.

\section{4}

Classic pilates method for the hip joint stability in older adult: cross sectional study applied to the updated ACSM guidelines American College of sport medicine

\section{Cesare Cavalli ${ }^{1}$, Matteo Quarantelli ${ }^{1}$}

Università Telematica San Raffaele Roma, Università Telematica San Raffaele Roma, Rome, Italy ${ }^{l}$

Purpose: The aim of the study is to verify the hypothesis that the hip joint can be strengthened through the classic Pilates method adapted to the new updated guidelines of the ACSM American College of Sport Medicine.

Methods: The experimental hypothesis is linked to the lifestyle of the asymptomatic sedentary subject with mild diseases of the musculoskeletal system in relation to the hip and the ACSM recommendations on exercise testing and prescription for the elderly population. The structure of the survey originates in the latest WHO World Health Organization 2018 report on frailty, sarcopenia and prevention of falls in the elderly $3-4$ and is based on the effects of an 8-week program, administered from September to October 2020, lasting $60 \mathrm{~min}$, one meeting a week, for the practice of 21 classic Pilates exercises which aim to increase the flexibility and muscular endurance of the abdominal region and the deep muscles of the trunk, 
lumbar spine and hip, and improve control of the posture, static and dynamic balance, and breathing. Ten volunteer individuals were recruited with mean age $64.4 \pm$ standard deviation 4.5 years, mean weight $63.25 \pm 5.75 \mathrm{~kg}$, mean height $164.39 \pm 1.58 \mathrm{~cm}$, of which five men and five women without previous experience with the method.

Results: During the sessions of the Pilates method (MP), heart rate (HR) and rate of perceived exertion according to Borg scale 0-10 (RPE-CR10) were monitored at each exercise to determine the estimate of the maximum volume of oxygen consumed per minute (VO2Max) and energy expenditure (EE) with linear regression equation. VO2Max was estimated using the submaximal AstrandRyhming step test. The mean values and SD for HR and RPE were calculated for each exercise and the percentages of the mean values for HRmax and RPEmax in each exercise showed a significant, strong and positive correlation $(\mathrm{r}=0.82 ; \mathrm{p}=0.001)$. In 10 exercises the HR value was $60 \%$ higher than HRmax, the mean values of $\mathrm{EE}$ and VO2Max are respectively $216.91 \pm(78.91) \mathrm{kcal}$ and $34.49 \mathrm{~mL} \bullet(\mathrm{Kg} \bullet$ $\min )-1 \pm(3.5)$

Conclusions: The dose response effect of the classical Pilates Method reaches the levels of cardio vascular and respiratory fitness and EE required by the new ACSM guidelines, if practiced 5 times/week, by promoting positive changes on the quality of life and on the levels of physical inactivity that can be easily raised with a structured program of physical activity from mild to moderate intensity, with safety.

References

1. Joseph Pilates, Miller JM., 1945. Return to Life Through Contrology, Boston Publishing House;

2. ACSM Guidelines for Exercise Testing and Prescription, 11th Ed. 2021;

3. Oliveira JS et. Al. Evidence on Physical Activity and the Prevention of Frailty and Sarcopenia Among Older People: A Systematic Review to Inform the World Health Organization Physical Activity Guidelines. J Phys Act Health. 2020 Aug 11;

4. WHO Global Strategy on Diet, Physical Activity and Health 2010;

\section{5}

\section{Muscle carnitine palmitoyl2 transferase II (CPTII) deficiency: a precious model for the new application of exercise - nutrition integrated approach in metabolic diseases}

\author{
Massimo Negro ${ }^{1}$, Giuseppe Cerullo ${ }^{2}$, Fausto Feletti ${ }^{3}$, Hellas Cena ${ }^{4}$, \\ Giuseppe D'antona ${ }^{I}$ \\ Università Di Pavia, Criams Sport Medicine Centre Voghera, \\ Voghera, Italy ${ }^{1}$; \\ Uniparthenope, Department of Movement Sciences and Wellbeing, \\ Napoli, Italy ${ }^{2}$; \\ University of Pavia, Department of Internal Medicine, Pavia, Italy ${ }^{3}$; \\ University of Pavia, Department of Public Health, Experimental \\ and Forensic Medicine, Pavia, Italy ${ }^{4}$
}

Carnitine palmitoyltransferase II (CPTII) deficiency, the most frequent inherited disorder regarding muscle fatty acid metabolism, leads to a reduced mitochondrial long-chain fatty acid oxidation during endurance exercise. This condition results in a clinical syndrome characterized by muscle fatigue and/or muscle pain with a variable annual frequency of severe rhabdomyolytic episodes. While since the CPTII deficiency discovery remarkable scientific advancements have been reached in genetic analysis, pathophysiology and diagnoses, the same cannot be said for the methods of treatments. The current recommendations remain those of following a carbohydratesrich diet with a limited fats intake and reducing, even excluding, physical activity, without, however, taking into account the long-term consequences of this approach. Suggestions to use carnitine and medium chain triglycerides remain controversial; conversely, other potential dietary supplements able to sustain muscle metabolism and recovery from exercise have never been taken into consideration. Clarification of biochemical mechanisms related to nutrition and physiological aspects of muscle metabolism in CPTII deficiency allows to propose new theoretical bases of its treatment and opens new avenues onto the application of exercise-nutrition integrated approach in metabolic muscular diseases.

\section{6}

\section{Anthropometry and physical exercise: new insights} on bone loss prevention

\author{
Alba Pasini ${ }^{I}$, Anna Strada ${ }^{1}$, Andrea Samuele Marani ${ }^{1}$, Enrico \\ Bortolotto $^{I}$, Natascia Rinaldo ${ }^{l}$
}

Università Degli Studi Di Ferrara, Dipartimento Di Neuroscienze E Riabilitazione, Ferrara, Italy ${ }^{l}$

Purpose: Osteoporosis is a multietyhiological skeletal disorder characterized by an increased bone fragility. Researches showed the influence of mechanical strain on bone metabolism, indicating physical exercise as a preventive tool against osteopenia and osteoporosis. Nevertheless, there is still lack of information concerning the influence of each sport category on Bone Mineral Density (BMD), especially when considering sex, age and lifestyle of a subject. Aim of this preliminary study is to analyse the relationship between sport categories and bone mineral density (BMD) in a group of Italyn men and women, by also considering anthropometric variables.

Methods: 160 volunteers ( 81 men, 79 women) practicing different sports underwent anthropometric and BMD measurements. Body composition, Handgrip Strength (HS) and weight status were collected following the standards proposed in Lohman et al. (1988). BMD was collected by Phalangeal Quantitative Ultrasonometry (Sonic Bone Profiler IGEA). A new questionnaire concerning information on sport practice and lifestyle behaviours that could increase the risk of low BMD was also administered.

Results: Preliminary results showed good correlations between BMD and anthropometric variables (HS, Fat Mass percentage, Waist to Hip Ratio, Waist to Height Ratio); all correlations were stronger especially in the women group. The comparison of BMD between athletic and sedentary was significant in women and in the younger age group (20-30). Moreover, BMD in women practicing high-impact/intensity sports was significantly higher than BMD in those practicing sports characterized by lower impact levels. Concerning lifestyle-related risk factors, women represent the most sedentary group $(54,4 \%)$, especially in the 50-59 (72,8\%) and $60+$ groups $(78,6 \%)$; women also showed to be more prone to smoking (21,5\% vs $9,9 \%)$. Alcohol consumption was similar in the two groups, with a slightly higher frequency in men $(75,3 \%$ vs $69,6 \%)$. Nevertheless, men tend to be less sedentary $(12,3 \%)$ and to practice high-intensity sports $(39,5 \%$ vs $16,4 \%)$, as well as high-impact sports $(30,9 \%$ vs $8,9 \%)$ and resistance training $(39,5 \%$ vs $16,4 \%)$.

Conclusions: Preliminary results showed that anthropometric variables can be directly associated to BMD levels. Moreover, the importance of lifestyle-related factors is highlighted, along with the need of a specific questionnaire to consider a proper association between sport practice and risk factors.

\section{References}


1. Lohman TG, Roche AF, Martorell R (1988). Anthropometric Standardization Reference Manual. Champaign, IL: Human Kinetics Books.

2. Marini S, Barone G, Masini A, Dallolio L, Bragonzoni L, Longobucco Y, Maffei F (2020). The effect of physical activity on bone biomarkers in people with osteoporosis: a systematic review. Front Endocrin 11.

\section{CONTROLLO MOTORIO E APPRENDIMENTO}

\section{7}

\section{Neuromuscular activations but not impact absorption capacity in single-leg landing tasks are restored following 5 months of sport practice after ACL reconstruction}

\author{
Jacopo Emanuele Rocchi ${ }^{1}$, Luciana Labanca ${ }^{1}$, Luca Laudani ${ }^{2}$, \\ Arrizza Alice $^{I}$, Carlo Minganti ${ }^{1}$, Pierpaolo Mariani ${ }^{l}$, Andrea \\ Macaluso $^{l}$ \\ Università, Università Degli Studi Di Roma "foro Italico", Rome, \\ Italy $^{l}$; \\ Università, Cardiff Metropolitan University, Cardiff, Regno Unito ${ }^{2}$
}

Purpose: It has been shown that for an athlete undergoing anterior cruciate ligament reconstruction (ACL-R) the most critical phase with respect to the risk of non-contact re-injury is the early return to play. Nevertheless, the underlying mechanisms of motor programming alterations leading to abrupt and uncoordinated movements are not clear yet. Subjects with ACL-R in the first 5 months of sport repractice would show improvements in electromyographic and kinematic patterns performing landing tasks compared to healthy individuals.

Methods: Ten ACL-R male athletes were recruited. 11 Healthy male athletes formed the control group. All the participants underwent two testing sessions. ACL-R subjects were tested at T0 $(6.0 \pm 1.3$ months after surgery) and $\mathrm{T} 1$ (12.2 \pm 2.5 months after surgery). Healthy controls were tested in season, at baseline and 5 months apart from baseline. Four different single-leg-landing tasks were performed. Normalized vertical ground reaction force, knee angular displacement on the sagittal plane and Electromyographic (EMG) activity of Vastus Lateralis, Vastus Medialis, Rectus Femoris, Biceps Femoris and Semitendinosus muscles were recorded during the landing phase.

Results: ACL-R subjects significantly reduced pre-impact EMG duration from T0 to T1 for knee extensors (T0: $110.8 \pm 22.1$, T1: 65.8 $\pm 5.5 \mathrm{~ms}$ ) and flexors (T0: $194.6 \pm 28.9, \mathrm{~T} 1: 172.3 \pm 7.4 \mathrm{~ms})$ compared to healthy participants (extensors T0: $73.2 \pm 7.5$, T1: 67.5 $\pm 6.5 \mathrm{~ms}$; flexors T0: $165.5 \pm 10.0$, T1: $172.5 \pm 8.6 \mathrm{~ms})$. Initial Contact knee angle did not show significant changes in time in both groups. Maximum knee flexion angle significantly improved for ACL-R subjects (T0: $40.4 \pm 0.8^{\circ}, \mathrm{T} 1: 53.2 \pm 5.2^{\circ}$ ) compared to controls (T0: $51.8 \pm 6.7^{\circ}, \mathrm{T} 1: 55.2 \pm 5.2^{\circ}$ ). ACL-R subjects showed significantly lower normalized peak vGRF after 5 months of sport repractice (T0: $3.4 \pm 0.25, \mathrm{~T} 1: 3.1 \pm 0.1$ ) but not as much as to approach the values of healthy participants (T0: $2.7 \pm 0.2, \mathrm{~T} 1: 2.6 \pm$ $0.1)$.

Conclusions: After 5 months of sport re-practice ACL-R subjects compared to healthy controls did improve motor programming of single leg landings. On the contrary, impact absorption capacity remained impaired.

References

1. Aerts I, Cumps E, Verhagen E, Wuyts B, Van De Gucht S, Meeusen R. The Effect of a 3-Month Prevention Program on the
Jump-Landing Technique in Basketball: A Randomized Controlled Trial. J Sport Rehabil. 2015;24(1):21-30.

2. Agel J, Arendt EA, Bershadsky B. Anterior Cruciate Ligament Injury in National Collegiate Athletic Association Basketball and Soccer: A 13-Year Review. Am J Sports Med. 2005;33(4):524-531.

38

Peripheral impairments of oxidative metabolism after 10 days of complete inactivity are upstream of mitochondrial respiration

\section{Lucrezia Zuccarelli $^{1}$, Giovanni Baldassarre ${ }^{1}$, Bruno Grassi ${ }^{1}$}

University of Udine, Department of Medicine, Udine, Italy ${ }^{l}$

Purpose and methods: In order to identify peripheral biomarkers of impaired oxidative metabolism during exercise following a 10-day bed rest (BR), 10 males performed an incremental exercise (to determine peak pulmonary $\mathrm{VO} 2[\mathrm{VO} 2 \mathrm{p}]$ ) and moderate-intensity exercises, before (PRE) and after (POST) BR. Blood flow response was evaluated in the common femoral artery by Eco-Doppler during 1-min passive leg movements (PLM). The intramuscular matching between $\mathrm{O} 2$ delivery and $\mathrm{O} 2$ utilization was evaluated by near-infrared spectroscopy (NIRS). Mitochondrial respiration was evaluated ex vivo by high-resolution respirometry in isolated muscle fibers, and in vivo by NIRS by the evaluation of skeletal muscle $\dot{V} \mathrm{O} 2(\dot{V} \mathrm{O} 2 \mathrm{~m})$ recovery kinetics. Resting $\dot{V} \mathrm{O} 2 \mathrm{~m}$ was estimated by NIRS.

Results: Peak $\dot{V}$ O2p was lower in POST vs. PRE. The area under the blood flow vs. time curve during PLM was smaller $(\mathrm{P}=0.03)$ in POST $(274 \pm 233 \mathrm{~mL})$ vs. PRE $(427 \pm 291)$. An increased $(\mathrm{P}=0.03)$ overshoot of muscle deoxygenation during a metabolic transition was identified in POST. Skeletal muscle citrate synthase activity was not different $(\mathrm{P}=0.11)$ in POST $(131 \pm 16$ nmol.min-1.mg-1) vs. PRE (138 \pm 19 ). Maximal ADP-stimulated mitochondrial respiration (66 \pm 18 pmol.s-1.mg-1 [POST] vs. $72 \pm 14$ [PRE], P = 0.41) was not affected by BR. Apparent Km for ADP sensitivity of mitochondrial respiration was reduced in POST vs. PRE $(\mathrm{P}=0.04)$. The VO2m recovery time-constant was not different $(\mathrm{P}=0.79)$ in POST $(22 \pm$ 6 s) vs. PRE $(22 \pm 6)$. Resting VO2m was reduced by $25 \%$ in POST vs. PRE $(\mathrm{P}=0.006)$.

Conclusions: Microvascular-endothelial function was impaired following a 10-day BR, whereas mitochondrial mass and function (both in vivo and ex vivo) were unaffected or slightly enhanced. Peripheral impairments of oxidative metabolism after 10 days of complete inactivity are mainly upstream of mitochondrial respiration.

Acknowledgements: Support by the Italyn Space Agency (ASI, MARS-PRE Project, Grant No. DC-VUM-2017-006).

\section{9}

How different spatiotemporal constraints influence gaze behaviour in predicting the ball direction

Alessandro Piras ${ }^{I}$, Matthew Timmis ${ }^{2}$, Aurelio Trofe ${ }^{3}$, Milena Raff ${ }^{1)}$.

University of Bologna, Department of Biomedical and Neuromotor Sciences, Bologna, Italy ${ }^{1}$;

Anglia Ruskin University, Cambridge Centre For Sport and Exercise Sciences, School of Psychology and Sport Science, Cambridge, Regno Unito $^{2}$; 
University of Bologna, Department For Life Quality Studies, Rimini, Italy $^{3}$

Purpose: The aim of the present study was to demonstrate how visual search behaviours are designed in a dynamic manner by the different constraints imposed by the task, the environment, and the individual characteristics of the performer (Williams et al. 2004). We investigated eye movements of soccer goalkeepers during the prediction of a ball kicked from different distances ( $11 \mathrm{~m}$ vs. $6 \mathrm{~m})$, different players (left-footed vs. right-footed) and different environment (soccer pitch vs. futsal pitch).

Methods: Eight intermediate-level male goalkeepers, one right and one left-footed male kicker volunteered for the experiment. Placed in front of a screen, in which videos of penalties were back projected, goalkeepers, wearing an eye-tracker and two inertial sensors for eye and body movement recordings, were instructed to predict the ball future direction. Response accuracy, reaction time, and gaze behaviour were collected for analysis.

Results: During penalties kicked from $11 \mathrm{~m}$, goalkeepers successfully predicted than missed penalties (60\% vs 40\%), while no significant difference was found from $6 \mathrm{~m}$. They were slower when predicted compared to missed penalties (4706 ms vs. $4646 \mathrm{~ms}$ ). Analysis of gaze behaviour showed greater microsaccade rates and a lower saccade rates during penalties kicked from $6 \mathrm{~m}$ than from $11 \mathrm{~m}$. Pupils size increased as the goalkeepers' perception of the penalty taker's intention developed, reaching the greatest value just before the goalkeepers' final movement initiation.

Conclusions: When the spatiotemporal constraint is less severe (far, at $11 \mathrm{~m}$ ), the goalkeepers choose a visual search strategy with more fixations and small saccades. When the spatiotemporal constraint is more severe (near, at $6 \mathrm{~m}$ ), goalkeepers rely on peripheral vision to monitor kicker's movements through the use of microsaccades.

\section{References}

1) Williams, A. M., Janelle, C. M., \& Davids, K. (2004). Constraints on the search for visual information in sport. International Journal of Sport and Exercise Psychology, 2(3), 301-318.

2) Piras, A., Timmis, M., Trofè, A., \& Raffi, M. (2020). Understanding the underlying mechanisms of Quiet Eye: The role of microsaccades, small saccades and pupil-size before final movement initiation in a soccer penalty kick. European Journal of Sport Science, 1-10.

\section{0}

\section{Impact of cognitive training on motor control skills and brain function in high school students}

\author{
Alessandra Scarton ${ }^{1}$, Marco Daprä ${ }^{l}$, Alan Mansi ${ }^{2}$, Cecilia Paini ${ }^{2}$, \\ Federico Gori ${ }^{I}$, Silvia Pogliaghi ${ }^{3}$ \\ Microgate Srl, Microgate Srl, Bolzano, Italy ${ }^{I}$; \\ Liceo Sportivo Attilio Bertolucci, Liceo Sportivo Attilio Bertolucci, \\ Parma, Italy ${ }^{2}$; \\ Università Di Verona, Dipartimento Di Neuroscienze, Biomedicina E \\ Movimento, Verona, Italy ${ }^{3}$
}

Purpose: Mental retardation (MR) and disorders of psychological, behavioral or emotional development (DPBE) represent heterogeneous conditions that share cognitive deficits and motor control difficulties. The use of computerized cognitive training (CTT) and cognitive motor training (CMT) is receiving increasing interest as a possible treatment for people with neurological diseases. However, evidence of effectiveness is still limited and controversial [1]. This research aims to assess if and to what extent CCT and CMT impact on cognitive deficits and motor control ability in students affected by MR and DPBE compared to healthy, age-matched controls.

Methods: 25 high school students $(17.7 \pm 0.9$ years $)$ participated to the study: 16 healthy controls (CS) (6 females) and 9 students (4 females) diagnosed with either MR (ICD F70-F79) or DPBE (ICD F80 - F98)[2] (DS) undertook a 2-months training consisting of 4 sessions per week ( $30 \mathrm{~min} \times 3$ of CCT $+30 \mathrm{~min}$ of CMT per week). CCT and CMT were performed with the online platform BRAIN HQ (Posit Science, USA) and the platform WITTY SEM (Microgate Srl, Bolzano), respectively. Before (T0) and at the end of the trainings (T1) the motor control was evaluated with a march in place test and the cognitive function by comparing the results of 3 tests performed with WITTY SEM in the areas of visual precision, memory and attention. Values at T0 and T1 in CS and DS were compared by 2-way ANOVA.

Results: CS and DS differed significantly at baseline in all the 3 cognitive tests $(\mathrm{p}<0.01)$. The biggest differences were found in the level of attention (average stimulus time, $2.11 \pm 2.65 \mathrm{~s}$ DS vs $0.12 \pm$ $0.04 \mathrm{~s}$ CS) and visual precision (average stimulus time, $2.50 \pm 2.52 \mathrm{~s}$ DS vs $0.26 \pm 0.11 \mathrm{~s}$ CS). Both groups improved significantly with training and at T1 no significant differences were found between the two groups. DS and CS differed significantly at baseline in the motor control (as indicated by the area of motion of the march in place, 516 \pm 457 vs $180 \pm 76 \mathrm{~cm} 2$ ). No significant improvement was detected in either group following training.

Conclusions: Our data confirm that 3 sessions/week of CCT significantly improved cognitive function in students affected by MR/DPBE and in healthy controls. It remains to be evaluated if the extent and the retention of the improvements is sufficient to impact on everyday life. However, one session/week of CMT was not sufficient to impact on the motor control in both MR/DPBE and healthy controls.

\section{References}

1. Harvey PD, McGurk SR, Mahncke H, and Wykes T. (2018) Controversies in Computerized Cognitive Training. Biol. Psychiatry Cogn. Neurosci. Neuroimaging 3(11):907-915.

2. “ICD-10 Version:2019." https://icd.who.int/browse10/2019/en\#/.

\section{1}

Muscle synergies during isometric maintenance of upright standing posture under directional pulling forces

Andrea Monte ${ }^{1}$, Anna Benamati ${ }^{1}$, Agnese Pavan ${ }^{1}$, Andrea D'avella ${ }^{2}$, Matteo Bertucco

University of Verona, Department of Neurosciences, Biomedicine and Movement Sciences, Verona, Italy ${ }^{1}$;

University of Messina, Department of Biomedical, Dental, Morphological and Functional Imaging Sciences, Messina, Italy ${ }^{2}$

Purpose: Muscle synergies have been defined as coordinated recruitment of groups of muscles with specific activation balances and time profiles. They have been proposed as building blocks employed by the CNS to simply the generation of task-specific forces and movements with a redundant neuromuscular system. Muscle synergies capture muscle coordination during reactive postural responses under mechanical perturbations and across different biomechanical contexts. However, the characterization of muscle synergies during static upright standing posture has not been investigated yet. Here we explore a novel experimental paradigm to characterize muscle synergies during isometric maintenance of upright standing posture while 
directional pulling loads are applied to modulate EMG (electromyography) activity in leg and lower-back muscles.

Methods: Eleven healthy adults $(24 \pm 2.4$ years $), 7$ males and 4 females, participated in the experiment. Participants were asked to stand on a force platform while feedback on the 2-D position of center of pressure (COP) was provided as a circular cursor $(0.5 \mathrm{~cm}$ dimeter $)$ displayed on a monitor. Pulling forces of two different magnitudes (5\% and $10 \%$ of body weight) were applied at the level of the waist in 8 evenly spaced directions in the horizontal plane. As soon as the pulling force was applied the participant was asked to maintain the COP within a circular target $(2.5 \mathrm{~cm}$ diameter $)$ for at least $15 \mathrm{~s}$. The target represented the participant's COP position in the steady-state upright standing posture. Surface EMG signals were recorded from 16 postural muscles of the participant's dominant side. Five trials for each of the 16 conditions were recorded ( 8 horizontal directions $\times 2$ pulling forces). Muscle synergies and activation coefficients were extracted for each condition using the non-negative matrix factorization.

Results: Results show that isometric contractions of postural muscles under small external forces during static upright standing posture can be characterized by a set of muscle synergies. The synergies recruitment coefficients demonstrate a subject-specific tuning related to the direction and magnitude of pulling forces.

Conclusions: These results lay the foundations for further studies investigating the use of myoelectric control and muscle synergies for the development of novel rehabilitation tools for recovering functional postural control.

\section{References}

1. Torres-Oviedo G, and Ting LH. (2007) Muscle synergies characterizing human postural responses. J Neurophysiol 98: 2144-56.

2. Torres-Oviedo G, Macpherson JM, and Ting LH. (2006) Muscle synergy organization is robust across a variety of postural perturbations. J Neurophysiol 96: 1530-46,

3. Berger DJ, and d'Avella A. (2014) Effective force control by muscle synergies. Front Comput Neurosci 8: 46.

\section{2}

\section{Cognitive-motor interference assessment in military personnel: validity and reliability of two dual-task tests}

\author{
Chiara Gattoni ${ }^{1}$, Borja Martinez Gonzalez ${ }^{2}$, Samuele M. Marcora ${ }^{2}$ \\ School of Sport and Exercise Sciences, University of Kent, Chatham, \\ Regno Unito ${ }^{1}$; \\ Dipartimento Di Scienze Biomediche E Neuromotorie, Università Di \\ Bologna, Bologna, Italy ${ }^{2}$
}

Purpose: Cognitive-motor interference can be defined as a decrease in cognitive and/or physical performance due to concurrent cognitive and physical tasks (dual-tasking), when compared to the same tasks performed in isolation (single-tasking). The aim of the study was to investigate the validity and reliability of two novel cognitive-motor interference tests in military personnel.

Methods: 24 participants from the Royal Engineers and Royal Air Force Cadets attended four experimental visits. In visits 1 and 3 they performed a 10-min loaded marching, a 10-min Psychomotor Vigilance Test (PVT), and the two tasks combined. Step length, step frequency, reaction time, and number of lapses were measured. In visits 2 and 4 they performed a 5-min running time trial, a 5-min Word Recall Task, and the two tasks combined. Running distance and number of words recalled were recorded.
Results: In comparison to single-task condition, significant higher step frequency and shorter step length were found in dual-task $(\mathrm{p}<$ $0.001)$. No differences were observed for reaction time and number of lapses $(\mathrm{p}>0.05)$. Running distance and number of words recalled were reduced in dual-task condition, compared to single-task $(\mathrm{p}<$ 0.001 ). Good to excellent reliability was found for all cognitive and physical variables in both, single and dual-task conditions, apart from the number of lapses, with low reliability in both conditions.

Conclusions: The main findings of the study suggest that Running + Word Recall Task test is a valid and reliable dual-task test which could be used to assess cognitive-motor interference. Although the difficulty of the Marching + PVT test may need to be increased to show interference on the cognitive side.

\section{References}

1. Albuquerque, I., Tiwari, A., Parent, M., Cassani, R., Gagnon, J. F., Lafond, D., Falk, T. H. (2020). Wauc: a multi-modal database for mental workload assessment under physical activity. Frontiers in Neuroscience, 14.

2. Radomski, M. V., Weightman, M. M., Davidson, L. F., Finkelstein, M., Goldman, S., McCulloch, K., Stern, E. B. (2013). Development of a measure to inform return-to-duty decision making after mild traumatic brain injury. Military medicine, 178(3), 246-253.

\section{FISIOLOGIA DELLO SPORT 1}

\section{3}

\section{Functional impairment of skeletal muscle oxidative metabolism in response to 35 days of complete inactivity}

\section{Simone Porcelli ${ }^{1}$, Desy Salvadego ${ }^{2}$, Bruno Grassi $^{3}$}

Dipartimento Di Medicina Molecolare, Università Di Pavia, Pavia, Italy $^{1}$

X, Jožef Stefan Institute, Ljubljana, Slovenia ${ }^{2}$;

Dipartimento Di Medicina, Università Di Udine, Udine, Italy ${ }^{3}$

Purpose: Complete inactivity, usually simulated by bed-rest (BR), is known to lead to a reduction in maximal O2 uptake (V'O2max) [1] which appears to be quantitatively related to the duration of the BR period [2]. Decrease in V'O2max has been classically associated to "central factors" (reduced maximal cardiac output (Q'max) and $\mathrm{O} 2$ delivery), whereas substantially unchanged maximal capacity of $\mathrm{O} 2$ extraction has been described [3]. Nevertheless, complete inactivity induces significant impairments at skeletal muscle level, such as lower microvascular blood flow, reduced volume density of mitochondria, and impaired oxidative enzyme activities [4]. This project aimed to investigate "central" and "peripheral" limitations to oxidative metabolism during exercise in response to 35 days of complete inactivity.

Methods: Ten male young volunteers underwent to 35 days of BR. At baseline (BR0) and post-BR (BR35), pulmonary gas exchange, stroke volume (SV) and Q' by impedance cardiography, and skeletal muscle (vastus lateralis) fractional $\mathrm{O} 2$ extraction $(\triangle \mathrm{HHbMb})$ by nearinfrared spectroscopy were determined during both whole-body incremental (IE) and constant moderate intensity work-rate (CWR) cycling and incremental dynamic knee-extension (KE).

Results: During IE, peak work-rate, V'O2peak, SVpeak, Q'peak, and $\Delta \mathrm{HHbMbpeak}$ were decreased from BR0 to BR35 (about $-20 \%$, respectively). During CWR, VO2 kinetics were significantly slower in BR35 than BR0 (time constant from $31.1 \pm 2.0 \mathrm{~s}$ to $40.0 \pm 2.2 \mathrm{~s}$ ) and a more pronounced "overshoot" of $\triangle \mathrm{HHbMb}$ was observed. During $\mathrm{KE}$, an exercise paradigm which significantly reduces any constraint 
to oxidative function deriving from cardiovascular $\mathrm{O} 2$ delivery, both VO2peak, normalized per unit of quadriceps muscle mass $(56.9 \pm$ 11.0 vs. $46.5 \pm 6.4 \mathrm{ml} \cdot \mathrm{min}-1 \cdot 100 \mathrm{~g}-1)$, and $\Delta$ HHbMbpeak (66.5 \pm 11.2 vs. $46.3 \pm 12.1 \%$ ) were reduced from B0 to BR35.

Conclusions: This project suggests 35 days of complete inactivity impair muscle oxidative function not only at "central" level but also "downstream" with respect to bulk blood flow to the exercising muscles, including blood flow distribution/O2 utilization inside the muscle, peripheral $\mathrm{O} 2$ diffusion, and intracellular oxidative metabolism. Future studies will have to investigate the effects of shorter BR on those functional biomarkers of impairment of oxidative metabolism.

\section{References}

1. Convertino VA. Cardiovascular consequences of bed rest: effect on maximal oxygen uptake. Med Sci Sports Exerc 29: 191-196, 1997.

2. Capelli $\mathrm{C}$ et al. Factors determining the time course of VO2 max decay during bedrest: implications for $\mathrm{VO} 2 \mathrm{max}$ limitation. Eur $\mathrm{J}$ Appl Physiol 98: 152-160, 2006.

3. Ferretti $\mathrm{G}$ et al. The interplay of central and peripheral factors in limiting maximal $\mathrm{O} 2$ consumption in man after prolonged bed rest. J Physiol 501: 677-686, 1997.

4. Bloomfield SA. Changes in musc.

\section{4 \\ Neuromuscular alterations in response to 10 days of complete inactivity}

\author{
Martino Franchi ${ }^{l}$, Elena Monti ${ }^{l}$, Fabio Sarto ${ }^{l}$, Giuseppe Sirago ${ }^{l}$, \\ Luana Toniolo $^{I}$, Luana Nogara ${ }^{l}$, Sandra Zampieri ${ }^{l}$, Bostian \\ Simunic $^{2}$, Rado Pišot ${ }^{2}$, Carlo Reggiani ${ }^{1}$, Marco Narici ${ }^{1}$ \\ University of Padova, Department of Biomedical Sciences, Padova \\ Italy ${ }^{I}$, Institute For Kinesiology Research, Science and Research \\ Centre Koper, Koper, Slovenia ${ }^{2}$
}

Purpose: Inactivity induces skeletal muscle atrophy, alterations in muscle architecture and a significant decline in neuromuscular function1. Such "signatures" of disuse are already detectable after 2-3 days of complete unloading2. Since muscle force is lost to a much greater extent than muscle mass 1,3, the aim of this study was to investigate the early determinants of such disproportionate loss of muscle force with respect to the decrease in muscle size in response to 10 days of complete inactivity.

Methods: Ten male volunteers $(22.9 \pm 4.7 \mathrm{y})$ were recruited for 10 days of a bed rest (BR) intervention. At baseline (BR0) and postBR (BR10), quadriceps femoris (QF) and vastus lateralis (VL) volume, together with knee-extensors force (MVT) were obtained by MRI and dynamometry, respectively. A time-course of QF and VL changes in muscle cross-sectional area and architecture was assessed by ultrasound images. At BR0 and BR10 blood samples and a biopsy from the vastus lateralis (VL) were collected. Neuromuscular junction (NMJ) stability (serum levels of C-terminal Agrin Fragment -CAF) and myofibre innervation status (positivity for neural cell-adhesion molecule -NCAM- staining) were assessed, together with single fibre mechanical properties and sarcoplasmic reticulum (SR) calcium handling.

Results: From BR0 to BR10, QF volume and MVT respectively decreased by $5.2 \%(\mathrm{p}<0.01)$ and $14.3 \%(\mathrm{p}<0.01)$. QF and VL midthigh CSA decreased by $3.3 \%$ and $5.8 \%$, respectively, whereas VL pennation angle showed a significant decrease already after 8 days of BR $(5.7 \%, p<0.01)$. A significant increase of NCAM positive fibres was found compared to baseline at BR10 (26.1-fold, p $<0.01$ ), accompanied by a $19.2 \%$ increase in serum CAF levels at BR10 (p < $0.05)$, reflecting increased instability of the NMJ. Fast fibre CSA decreased by $15 \%(\mathrm{P}=0.055)$ at $\mathrm{BR} 10$, while single fibres maximal tension (force/CSA) was unmodified. Nonetheless, the handling of $\mathrm{Ca} 2+$ by the sarcoplasmic reticulum was altered as calcium release induced by caffeine decreased at BR10 by $35.1 \%(\mathrm{p}<0.01)$ and $30.2 \%(\mathrm{p}<0.01)$ in fast and slow fibres, respectively.

Conclusions: Our data show that the early onset of neuromuscular junction instability and impairment in calcium dynamics involved in E-C coupling precede the detection of morphological alterations of skeletal muscle. Such mechanisms are proposed as determinants to the greater decline in muscle force than in muscle size during unloading.

\section{References}

1. M. D. de Boer, C. N. Maganaris, O. R. Seynnes, M. J. Rennie, M. V. Narici, J. Physiol. 583, 1079-1091 (2007).

2. R. Demangel, et al., J. Physiol. 595, 4301-4315 (2017).

3. B. T. Wall, et al., Acta Physiol. 210, 600-611 (2014).

\section{5}

\section{Effects of age on neuromuscular fatigue: comparison} between isometric, dynamic and cycling tasks

\author{
Giorgio Varesco $^{l}$, Eric Luneau ${ }^{l}$, Guillaume Y Millet $^{1}$, Leonard \\ Feasson $^{2}$, Thomas Lapole ${ }^{I}$, Vianney Rozand ${ }^{l}$
}

Laboratoire Interuniversitaire De Biologie De La Motricité, Université De Lyon, Université Jean Monnet, Saint-etienne, Francia ${ }^{1}$; Unité De Myologie, Service De Physiologie Clinique Et De

L'exercice, Centre Hospitalier Universitaire De Saint-étienne, Saintetienne, Francia ${ }^{2}$

Purpose: The effects of ageing on neuromuscular fatigue has been extensively studied during local (i.e. single-joint) isometric and dynamic fatiguing tasks. We aimed to investigate performance fatigability during isometric, dynamic and locomotor fatiguing tasks performed at similar duty-cycle and workload to provide a direct comparison between tasks and ages.

Methods: Preliminary data were used for the present Thirteen young men $(<35 \mathrm{yr})$, four older men $(>60 \mathrm{yr})$ and five very old men $(>$ $80 \mathrm{yr}$ ) randomly performed three testing sessions: an isometric (ISO) and a dynamic (DYN) incremental intermittent task for the kneeextensors on an isokinetic dynamometer, and a similar test on a validated ergometer (BIKE). Increments were calculated relatively to the participants' body weight using device-specific equations (ISO and DYN: $5 \%$ of body weight; BIKE: $0.3 \mathrm{~W} \cdot \mathrm{kg}-1$ ). Performance was quantified as test duration and performance fatigability as the loss in maximal isometric force. Voluntary activation (VA\%) and contractile function (potentiated resting twitch amplitude, TwPOT) were evaluated using percutaneous nerve stimulation and the interpolated twitch technique.

Results: Performance decreased with age for all the tests $(\mathrm{P}<0.05)$. Performance fatigability was significantly higher in young men $(-30$ $\pm 13 \%)$ than very old men $(-20 \pm 10 \% ; \mathrm{P}=0.006)$, but not old men $(-$ $26 \pm 11 \% ; \mathrm{P}=0.176)$, independent of the test. Decrease in TwPOT showed significant age effect, but the post-hoc analysis showed no differences between the young $(-44 \pm 25 \%)$, old $(-32 \pm 15 \%)$ and very old $(-33 \pm 13 \%)$. Finally, difference in VA\% was similar across sessions and ages $(4 \pm 7 \%, 3 \pm 5 \%$ and $1 \pm 6 \%$ for young, old and very old men, respectively). Those results were probably influenced by the low number of participants included in this preliminary analysis. 
Conclusions: Those preliminary results suggest different performance and fatigability across ages, with young adults performing better and tolerating a larger amount of fatigability, mainly from peripheral contractile factors. The completed dataset with a sufficient number of participants will elucidate the interaction between age and fatiguing task.

\section{References}

1. Krüger, R., Aboodarda, S., Samozino, P., Rice, C., \& Millet, G. (2018). Isometric versus Dynamic Measurements of Fatigue: Does Age Matter A Meta-analysis. Medicine \& Science in Sports \& Exercise, 50(10), 2132-2144.

2. Varesco, G., Royer, N., Singh, B., Parent, A., Féasson, L., Lapole, T., ... Rozand, V. (2021). Reliability and agreement of a dynamic quadriceps incremental test for the assessment of neuromuscular function. Journal of Electromyography and Kinesiology, 56, 102,503.

\section{6}

\section{Effects of respiratory muscle training on diaphragm muscle structure and function: a novel electromyographic, mechanomyographic and ultrasound approach}

\section{Christian Doria ${ }^{1}$, Marta Borrelli ${ }^{1}$, Susanna Rampichini ${ }^{1}$, Giuseppe Coratella $^{I}$, Stefano Longo ${ }^{l}$, Eloisa Limonta ${ }^{l}$, Johnny Padulo ${ }^{l}$, Emiliano C $e^{I}$, Fabio Esposito ${ }^{I}$ \\ Università Degli Studi Di Milano, Dipartimento Di Scienze Biomediche Per La Salute, Milan, Italy ${ }^{l}$}

Purpose: Respiratory muscles can be trained like other skeletal muscles by different types of respiratory muscle training (RMT) methods. Isocapnic hyperpnoea, by allowing partial $\mathrm{CO}_{2}$ rebreathing, ensures normo-capnia during high ventilatory loads, inducing positive effects on respiratory function. This study sought to assess the effects of an isocapnic hyperpnoea training on diaphragm muscle structure and function assessed by an EMG, mechanomyographic (MMG) and ultrasound integrated approach.

Methods: Eleven healthy subjects (age $26.1 \pm 5.2$ years., body mass $72.4 \pm 5.1 \mathrm{~kg}$, stature $1.68 \pm 0.06 \mathrm{~m}$, mean $\pm \mathrm{SD}$ ) were evaluated before and after eight weeks of RMT under isocapnic hyperpnea (3 days/week) for respiratory functionality (maximum voluntary ventilation, MVV; maximum inspiratory pressure, MIP; and maximum expiratory pressure, MEP), diaphragm EMG and MMG activity during phrenic nerve stimulation (PNS), and diaphragm thickness and excursion at rest, during paced respiratory activity and during PNS by ultrasound.

Results: After RMT, MVV, MIP and MEP increased $(+6 \%,+22 \%$, and $+24 \%$, respectively; $\mathrm{P}<0.05$ ). During PNS, only diaphragm MMG amplitude increased, while the EMG remained unchanged. A reduction in diaphragm thickness during maximal inspiration $(-18 \%, \mathrm{P}$ $<0.05)$ and an increase in the excursion of the diaphragm during paced modality $(+61 \%$; $\mathrm{P}<0.05)$ occurred.

Conclusions: Eight weeks of RMT likely induced a diaphragm structure remodelling that lead to an increased excursion and strength. These findings suggest that this EMG, MMG and ultrasound integrated approach may provide better insight on diaphragm muscle structure and function.

\section{7}

Neural and contractile determinants of purely explosive isometric contractions of knee extensors

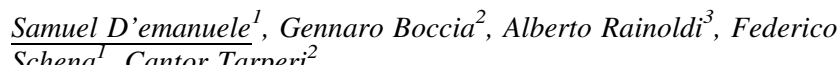
Schena $^{I}$, Cantor Tarperi ${ }^{2}$

University of Verona, Department of Neurosciences, Biomedicine and Movement Sciences, Verona, Italy ${ }^{l}$;

University of Turin, Department of Clinical and Biological Sciences, Turin, Italy ${ }^{2}$;

University of Turin, Department of Medical Sciences, Turin, Italy ${ }^{3}$

Purpose: The aim of this work is to identify the contractile and neural determinants (Folland et al. 2014) of purely explosive contractions (i.e. contractions without a plateau phase and lasting $<200 \mathrm{~ms}$ ), using the means of high-density surface electromyography (HDsEMG) and electrically evoked stimuli of knee extensors.

Methods: A total of 25 healthy subjects ( $25 \pm 2$ years; $5 \mathrm{~F})$ performed 10 purely explosive isometric knee extension interspersed of $3 \mathrm{~s}$ rest. HDsEMG was recorded from vastus lateralis (VL) and medialis (VM) with two matrixes of 64 electrodes. We evaluated the contractile and EMG (root mean square, M-Wave amplitude and conduction velocity) responses voluntary and electrically evoked stimulation with octets (8 stimuli delivered at $300 \mathrm{~Hz}$ ). Linear stepwise regression was adopted to assess the determinants of force after 50,100 , and $150 \mathrm{~ms}$ of voluntary contractions.

Results: The force expressed at $50 \mathrm{~ms}$ was determined only by root mean square (36\% of variance explained) from VM and VL, at $100 \mathrm{~ms}$ was determined by the octet contractile response $(65 \%$ of variance explained), at $150 \mathrm{~ms}$ was determined by maximal voluntary force ( $71 \%$ of variance explained) and by octet contractile response (6\% of variance). The muscle fiber conduction velocity did not emerge a significant determinant in any time interval.

Conclusions: pure explosive isometric force production exhibited different neural and contractile determinants depending on the considered force-time intervals. According to previous evidence, the force in the first $50 \mathrm{~ms}$ of contraction depends on muscle activation (central mechanism), while the force at $100 \mathrm{~ms}$ depends on contractile (peripheral) mechanism. Contrary to previous evidence on biceps brachii (Del Vecchio et al. 2018), the muscle fiber conduction velocity did not affect the force in any of the periods considered.

\section{References}

1. Folland JP, Buckthorpe MW, Hannah R. (2014) Human capacity for explosive force production: neural and contractile determinants. Scand J Med Sci Sports 24(6):894-906.

2. Del Vecchio A, Negro F, Falla D, Bazzucchi I, Farina D, Felici F. (2018) Higher muscle fiber conduction velocity and early rate of torque development in chronically strength trained individuals. J Appl Physiol 125(4), 1218-1226.

48

Alterations in the control of motor units studied with high-density emg after 10 days of unilateral suspension of the lower limb in humans

\author{
Giacomo Valli ${ }^{I}$, Fabio Sarto ${ }^{I}$, Martino V Franchi ${ }^{l}$, Andrea Casolo ${ }^{1}$, \\ Marco V Narici ${ }^{l}$, Giuseppe De Vito ${ }^{1}$ \\ Dipartimento Di Scienze Biomediche, Università degli Studi di \\ Padova, Padova, Italy ${ }^{P}$
}


Purpose: Reduced skeletal muscle activation, due to physical inactivity, disease and space flights can deeply affect the contractile properties of the muscle tissue. In this study we investigated the functional alterations of the skeletal muscle after 10 days of unilateral lower limb suspension (ULLS, walking with crutches) at the level of the single motor units (MUs).

Methods: To investigate MUs properties, the electrical activity of the muscle vastus lateralis of 11 young males $(22.1 \pm 2.9$ years $)$ was recorded with high-density electromyography (HD-EMG), using a grid of 64 electrodes, during isometric contractions at 10, 25 and 50\% of maximum voluntary contraction (MVC) at baseline (BLS0) and immediately after the suspension period (LS10).

Results: After the HD-EMG decomposition and the removal of duplicated MUs, a total of 926 unique MUs were identified. MVC decreased at LS10 (from 816.18 \pm 97.22 to $573.20 \pm 104.69 \mathrm{~N}$ ) as did the ability of the participants to maintain the isometric contraction with a reduction of the steadiness at $25 \%$ MVC. The neuromuscular impairment was confirmed by reduced absolute MUs recruitment and de-recruitment thresholds at 10, 25 and 50\% MVC while the relative thresholds increased at 10 and $25 \%$ but not at $50 \%$. The discharge rate of the MUs decreased at 10 and 25\% MVC across almost all the points of evaluation (e.g., recruitment, de-recruitment and steady state) but not at $50 \%$. The coefficient of variation of interspike interval of the MUs (marker of irregular firing pattern) increased at the start and end of the steady state phase at $25 \% \mathrm{MVC}$ while slightly decreased at $50 \%$.

Conclusions: Altogether, these results highlight that 10 days of ULLS can induce significant alterations of the skeletal muscle function at the level of the single MUs and that these impairments are relative to intensity of contraction and neuromuscular effort.

Acknowledgements: Funding by ASI, MARS-PRE Project, n. DCVUM-2017-006, is acknowledged.

\section{References}

1. E. Monti, et al. (2021) Neuromuscular junction instability and altered intracellular calcium handling as early determinants of force loss during unloading in humans. $\mathrm{J}$ Physiol 599(12):3037-3061.

2. E.L. Campbell, et al. (2013) Skeletal muscle adaptations to physical inactivity and subsequent retraining in young men. Biogerontology 14(3):247-59.

\section{ATTIVITÀ MOTORIA ADATTATA ALLE DISABILITÀ}

\section{9}

\section{Bone mineral density in the impaired and in the non- impaired hip of athletes with unilateral lower limb amputation}

\section{Valentina Cavedon $^{1}$, Carlo Zancanaro ${ }^{1}$, Chiara Milanese ${ }^{1)}$.}

Università Di Verona, Dipartimento Di Neuroscienze, Biomedicina E Movimento, Verona, Italy ${ }^{1}$

Purpose: People with a lower limb amputation (LLA) are at risk of osteoporosis in the impaired limb. To date there is no data about the bone health in the impaired hip of athletes with amputation/s. In this study we enrolled a sample of athletes with LLA with a twofold aim: first, to investigate the difference between the bone mineral density (BMD) in the total hip region of the impaired lower limb (I_HIP) and that in the non-impaired lower limb (NI_HIP); second, to explore the impact of the level of amputation on BMD in the I_HIP.

Methods: Twenty-six male athletes with LLA (above-knee amputation $[\mathrm{AKA}], \mathrm{n}=10$; below-knee amputation $[\mathrm{BKA}], \mathrm{n}=16$ ) aged
$35.31 \pm 9.32$ years were enrolled in this study. The average duration of injury was $12.81 \pm 9.78$ years and the weekly volume of training was $5.00 \pm 1.83 \mathrm{~h}$. Areal BMD (aBMD, g/cm2) in the I_HIP and in the NI_HIP was assessed by means of Dual-Energy X-Ray Absorptiometry. A mixed-design 2 (Severity of injury: AKA and BKA) $\times 2$ (Injury: I_HIP and NI_HIP) Analysis of Variance with repeated measures on the second factor was performed to assess the impact of both the injury in itself and the severity of injury on bone health. The level of statistical significance was set at $\mathrm{P}<0.05$.

Results: A statistically significant main effect for Injury $(\mathrm{F}=283.741$, $\mathrm{P}<0.001)$ and Injury by Severity of Injury interaction $(\mathrm{F}=37.412, \mathrm{P}$ $<0.001)$ was found. Athletes with AKA and athletes with BKA had similar aBMD values in the NI_HIP $(0.97 \mathrm{~g} / \mathrm{cm} 2$ and $1.01 \mathrm{~g} / \mathrm{cm} 2$, respectively). Athletes with AKA and BKA had $-39.20 \%$ and $-17,58 \%$ aBMD in the I_HIP versus the NI_HIP, respectively $(\mathrm{P}<0.001$ for both).

Conclusions: In athletes with LLA the injury has a negative impact in itself on the bone health of the I_HIP, with athletes with AKA having a higher risk of demineralization than those with BKA. These results suggest that the practice of an adapted sport may not be sufficient to prevent the reduction in aBMD in the I_HIP of athletes with LLA and support the prescription of specific exercises aimed at improving the aBMD in this athletic population.

\section{References}

1. Sherk, VD, Bemben, MG and Bemben, DA (2008) BMD and bone geometry in transtibial and transfemoral amputees. J Bone Miner Res 23(9): 1449-1457.

\section{0}

\section{Shots' distribution in wheelchair para table tennis matches}

Alessandro Guarnieri $^{1}$, Ileana Ramazzina ${ }^{1}$, Ivan Malagoli Lanzoni ${ }^{2}$

University of Parma, Department of Medicine and Surgery, Parma, Italy $^{1}$;

University of Bologna, Department of Biomedical and Neuromotor Sciences, Bologna, Italy ${ }^{2}$

Purpose: In Para Table Tennis the players that compete in a wheelchair are classified within class 1-5 (C1-C5). The most used technical and tactical indicators in Table Tennis are related to shot types used by the players during the game. Thus, the purpose of this study was to compare the shot distribution in the five wheelchair classes (C1-C5) during elite competitions.

Methods: Five matches for every class $(5 \times 5=25$ matches $)$ were randomly selected from Elite Para Table Tennis international tournaments. All the athletes were male players and right-handed. An expert coach analyzed game video footages in slow motion $(0.2 \mathrm{X})$ with the software Kinovea and collected data about the shot types used by the players across the whole games. Shot's classification (forehand or backhand) included: service, drive, top, lob, push, block, top counter top, lob, smash, and chop.

Results: Results showed significant differences $(p<0.05)$ among different classes in the distribution of shots. Indeed, $\mathrm{C} 1$ used more backhand drive $(26 \%)$ and backhand serve $(21 \%)$. C2 used also backhand drive (17\%) and backhand push (17\%). Conversely, C3 preferred backhand push $(25 \%)$ followed by backhand drive $(15 \%)$. Moreover, C4 played backhand push (26\%), backhand drive (14\%) and serve (14\%). C5 used backhand push (27\%) and backhand serve $(10 \%)$. Specific differences were detected for: forehand drive $(\mathrm{C} 1$ : $16 \%$ ), backhand block (C3: 11\%), and backhand top (C5: 10\%). 
Conclusions: In conclusion, this study can be useful for coaches, athletes, physical trainers, and performance analysts, in order to design specific training sessions for Para Table Tennis players from different classes (C1-C5).

\section{References}

1. Fuchs, M., Liu, R., Malagoli Lanzoni, I., Munivrana, G., Straub, G., Tamaki, S., Yoshida, K., Zhang, H. and Lames, M. (2018) Table Tennis Match Analysis: A Review. J Sport Sci 36(23): 2653-26.

2. Fuchs, M., Faber, I., Lames, M. (2019) Game characteristics in elite para table tennis. Ger J Exerc Sport Res 49: 251-258.

3. Malagoli Lanzoni I., Di Michele R. and Merni F. (2014) A notational analysis of shot characteristics in top-level table tennis players, Eur J Sport Sci 14(4): 309-317.

\section{1}

\section{Relationship between strength and sprint ability in Italyn soccer amputee players}

\author{
$\underline{\text { Luca Beratto }}^{1}$, Caterina Biasiolo ${ }^{2}$, Andrea Cavedon $^{3}$, Chiara \\ Milanese $^{3}$, Valentina Cavedon ${ }^{3}$ \\ Neuromuscularfunction | Research Group, University of Turin, \\ Torino, Italy ${ }^{1}$; \\ School of Exercise and Sport Sciences, University of Turin, Torino, \\ Italy $^{2}$; \\ University of Verona, Department of Neuroscience, Biomedicine \\ and Movement Sciences, Verona, Italy ${ }^{3}$
}

Purpose: Amputee soccer is a variation of conventional soccer in which athletes with lower and upper (goalkeeper) limb amputation participate. The athlete with lower limb amputation plays using crutches. Sprinting during the match on crutches represents one of the crucial motor tasks in amputee soccer1,2. This study aims to determine the relationship between upper and lower limb strength and sprint performance in Italyn amputee soccer players.

Methods: Twelve amputee soccer players recruited from two different teams participated in this study. Seated medicine ball and handgrip strength tests were used to assess the upper limb strength, while countermovement jump (CMJ) was used to evaluate lower limb strength. In addition, sprint tests $(10,20$ and $30 \mathrm{~m})$, T-square and L-drill tests were measured to assess sprint and agility performance. The goalkeepers were also evaluated but not included in the statistical analysis. Spearman correlation coefficient was used to evaluate the correlation between upper and lower limb strength on sprint and agility performance. Moreover, to determine the impact of the amputee level on performance, the participants were divided into athletes with above-knee amputation (AKA) and below-knee amputation (BKA), respectively. Mann-Whitney test was used to analyze the performance outcomes difference between AKA and BKA groups. Results: No significant correlation was found between upper limb strength and sprint performance. Significant correlations were found between L-drill and T-square tests $(r=0.60, p=0.04)$, L-drill and $\mathrm{CMJ}(\mathrm{r}=-0.57, \mathrm{p}=0.05)$ and $\mathrm{T}$-square and $20 \mathrm{~m}$ sprint $(\mathrm{r}=-0.59, \mathrm{p}=$ 0.04). Significant difference between AKA and BKA athlete in $10 \mathrm{~m}$ sprint $(\mathrm{p}=0.02)$.

Conclusions: The study underlines that upper limb strength does not influence sprint effectiveness in amputee soccer players. Otherwise, lower limb strength and $20 \mathrm{~m}$ sprint can affect agility outcomes. Finally, the amputee level of the athlete can impact on $10 \mathrm{~m}$ sprint performance.

References
1. Aytar, A., Pekyavas, N. O., Ergun, N., \& Karatas, M. (2012). Is there a relationship between core stability, balance and strength in amputee soccer players? A pilot study. Prosthetics and orthotics international, 36(3), 332-338.

2. Wieczorek, M., Wiliński, W., Struzik, A., \& Rokita, A. (2015). Hand Grip Strength Vs. Sprint Effectiveness in Amputee Soccer Players. Journal of human kinetics, 48, 133-139.

52

A supervised walking program for the amelioration of cognitive functions in schizophrenic subjects even during Covid-19 pandemic

\author{
Simona Mandini ${ }^{l}$, Valentina Zerbini ${ }^{1}$, Tommaso Piva ${ }^{l}$, Erica \\ Menegatti $^{2}$, Andrea Raisi ${ }^{1}$, Giovanni Grazzi ${ }^{1}$, Gianni Mazzoni ${ }^{1}$ \\ Università Degli Studi Di Ferrara, Neuroscienze E Riabilitazione, \\ Ferrara, Italy ${ }^{1}$; \\ Università Degli Studi Di Ferrara, Medicina Traslazionale Per La \\ Romagna, Ferrara, Italy ${ }^{2}$
}

Purpose: People with mental illnesses tend to engage in less physical activity than the rest of the population and have higher cardiovascular risk factors. Recently they were associated to an increased risk for Covid-19 mortality. Aim of this study was to enrol schizophrenic subjects in a long-term moderate-intensity physical activity program and to evaluate its effects on their cognitive functions and cardiovascular risk factors. An additional outcome of the study has been the comparison of the adherence to the physical activity program before and during the Covid-19 pandemic.

Methods: Forty sedentary patients diagnosed with schizophrenia (mean age $46.4 \pm 9.6$ ) followed by the Local Public Mental Health Department were included and allocated to a 1-year walking program (WG $\mathrm{n}=28$ ), consisting of two guided walking sessions/week, or to a control group ( $\mathrm{CG} n=12)$ basing on their willingness. VO2peak, walking speed, blood pressure and anthropometric variables were assessed only in the subjects following the walking program. In all subjects, cognitive functions were assessed with the Screen for Cognitive Impairment in Psychiatry (SCIP) and the Frontal Assessment Battery (FAB) questionnaires.

Results: After 1-year, the 20 subjects completing the walking program displayed significant improvements of cognitive functions. A positive correlation between SCIP score and the number of attended sessions $(\mathrm{R}=0.86, \mathrm{p}<0.0001)$ was evident in the patients attending at least 75 of the 100 walking sessions. Walking speed and VO2peak increase significantly and an increase of body weight, BMI, systolic and diastolic blood pressure was also observed. All the 12 CG patients completed the program. They did not display significant improvements of cognitive functions. The adherence to the walking program registered during Covid-19 period did not differ from that observed before the pandemic. To note that during Covid-19 period 21 additional subjects attended to the walking program.

Conclusions: The main finding of this study is the improvement in cognitive functions documented in the subjects participating to the walking program with a significant correlation between this improvement and the number of walking sessions, evident only in the patients who attended to at least 1.5 sessions/week. The walking program, guided by exercise specialist, proved to be an effective and enjoyable activity for people with mental disorder, even during the physical activity pandemic due to Covid- 19 .

References 
1. WHO. Motion for your mind: Physical activity for mental health promotion, protection and care. Geneva, World Health Organization, 2019.

2. Vancampfort D et al. Sedentary behavior and physical activity levels in people with schizophrenia, bipolar disorder and major depressive disorder: a global systematic review and metaanalysis. World Psychiatry. 2017;16:308-15.

\section{3}

\section{Effect of covid-19 related lockdown on physical fitness in top level wheelchair basketball athletes}

\author{
Stefania Della Porta ${ }^{1}$, Ilaria Peluso ${ }^{2}$, Andrea De Rito ${ }^{1}$, Riccardo \\ Capitani $^{I}$, Valentina Cavedon ${ }^{3}$, Chiara Milanese ${ }^{3}$, Fabio \\ Castellucci $^{4}$, Sheila Bellito ${ }^{4}$, Elisabetta Toti ${ }^{5}$, Anna Raguzzini ${ }^{5}$, \\ Tommaso Sciarra ${ }^{6}$, Marco Bernardi ${ }^{1}$
}

Scuola Di Specializzazione In Medicina Dello Sport E Dell'esercizio Fisico, Department of Physiology and Pharmacology "v. Erspamer", Sapienza University of Rome., Rome, Italy ${ }^{I}$;

Crea-an, Research Centre For Food and Nutrition, Council

For Agricultural Research and Economics, Rome, Italy ${ }^{2}$; Laboratory of Anthropometry and Body Composition, Department of Neurosciences, Biomedicine and Movement Sciences, University of Verona, Verona, Italy, Verona, Italy ${ }^{3}$;

Federazione Italiana Pallacanestro In Carrozzina (fipic), Health and Fitness Department, Rome, Italy ${ }^{4}$.

Crea-an, Research Centre For Food and Nutrition, Council For Agricultural Research and Economics, Rome, Italy;

Centro Veterani Della Difesa, Dipartimento Scientifico-Celio, Policlinico Militare Di Roma, Rome, Italy ${ }^{6}$

Purpose: The present study, part of a major health assessment project to allow wheelchair basketball athletes (WBA) to have a safe return to play after the Covid-19 related lockdown (LD), was aimed to examine the effects of the possible LD forced sedentary behaviour (1) on all components of physical fitness (PF) in the WBA of the Italyn National Team.

Methods: Twelve male WBA (3 with spinal cord injury, 2 with spina bifida, 5 with amputation and 2 with other health conditions), competing in the Italyn major league and recruited in the National Team to participate in the European Championship, were evaluated at the peak of their PF status, i.e. at the end of the Italyn Championship (PRE) and after the LD time period (POST). PF assessment (Bernardi et al., 2012) included: (1) a cardiopulmonary incremental maximal exercise tests (CPET) carried out with an arm cranking ergometer (ACE, ER 800, ergoline Gmbh, Bitz, Germany), to measure oxygen uptake peak (VO2peak) through a stationary metabolimeter (Quark CPET, Cosmed, Italy) and power peak (PP); (2) a supramaximal (130\% of the CPET PP) ACE test carried out up to exhaustion (130\%ExTest), to measure total mechanical work and blood lactate peak (Lactate Pro2 LT-1730 Arkray, Japan), (3) a 10-s-long Wingate Anaerobic Test (Monark $891 \mathrm{E}$, Vansbro, Sweden), to assess mean explosive power (MEP); and (4) two upper body isometric strength tests, to assess maximal voluntary contraction of handgrip and shoulder lowering muscles (SLM). During the 69 day long LD, WBA trained at home as much as possible (especially strength training with free weights).

Results: Comparing LD PRE vs POST Results we found a significant decrease in the aerobic fitness: VO2peak and PP decreased from 2675 \pm 507.8 to $2388.7 \pm 564.96 \mathrm{~mL} \cdot \mathrm{min}-1,(\mathrm{p}=0.01)$ and from $167.8 \pm$ 36.97 to $158.2 \pm 34,65 \mathrm{~W}(\mathrm{p}=0.04)$, respectively. Controversial results were obtained in the strength evaluation because WBA showed a significant reduced strength $(\mathrm{p}=0.01)$ only in SLM $(1324 \pm 155.7$ vs $1223 \pm 179.7 \mathrm{~N})$. No significant differences were found in the PRE vs POST $130 \%$ ExTest and MEP results .s

Conclusions: Our study shows the negative impact of forced sedentary behaviour on aerobic fitness and upper body strength in athletes with a locomotor impairment, thus reaffirming the fundamental role of exercise training as a fundamental tool to promote and maintain cardiovascular health as well as optimal athletic performance.

\section{References}

1. Narici M, Vito G, Franchi M, Paoli A, Moro T et al. (2021) Impact of sedentarism due to the COVID-19 home confinement on neuromuscular, cardiovascular and metabolic health... Eur J Sport Sci. 2021 Apr;21(4):614-635

2. Bernardi M., Carucci S., Faiola F., Egidi F., Marini C., Castellano V., Faina M. (2012) Physical Fitness Evaluation of Paralympic Winter Sports Sitting Athletes. Clin J Sport Med Jan;22(1):26-30.

\section{4}

\section{Has COVID-19 pandemic lasting effects on young adults with down syndrome physical activity levels?}

\author{
Erica Gobbi $^{1}$, Stefano Amatori ${ }^{I}$, Christian Ciferni ${ }^{I}$, Fabrizio \\ Perroni $^{1}$, Davide Sisti ${ }^{1}$, Marco Bruno Luigi Rocchi ${ }^{1}$
}

Università Di Urbino Carlo Bo, Dipartimento Di Scienze Biomolecolari, Urbino, Italy ${ }^{l}$

Purpose: COVID-19 pandemic has caused unprecedented social restrictions. It was more challenging than usual to maintain habitual physical activity (PA) patterns (Jiménez-Pavón et al., 2020), significantly affecting the more vulnerable groups in society, such as people with Down Syndrome (DS) that are characterized by a range of medical conditions in addition to intellectual disability (Villani et al., 2020). The aim of this study was to investigate PA, sedentary behavior, and screen time of young adults with DS, over time and in relation to parental PA levels.

Methods: Parents, on behalf of their child, were invited to complete a 15-min-long online questionnaire, available from the mid of May 2021 (corresponding to the slowly re-opening of gyms and sports activities). The survey investigated sociodemographic aspects and weekly PA levels of both parents and youths with DS; moreover, it included questions on youths' daily sedentary behavior and screen time. Lifestyle habits were asked referring to three-time points: before the pandemic, during the lockdown restrictions, and referring to the situation of slowly re-opening. A total of 57 parents voluntarily proxy reported information about a group of young adults with DS $(M=41$, $\mathrm{F}=16,21.4 \pm 7.7$ years old). Changes on the lifestyle factors were investigated using a repeated measures multivariate analysis of variance. Association between parents' and their child PA was assessed by Pearson's correlation.

Results: Participants reported negative effects of restrictions, with a reduction in PA levels, and an increase in sedentary and screen time ( $\mathrm{p}$ $<0.05$ for all), independently from sociodemographic characteristics. Moreover, post-lockdown PA resulted worsened in comparison to before the pandemic $(p<0.05)$. A significant positive correlation between parents' and their child PA was detected in the before the pandemic $(r=0.38 ; p<0.05)$, while this was no longer reported in the re-opening phase.

Conclusions: The results show the persistence of the negative impact of the COVID-19 related restrictions on the lifestyle of young adults with DS. Moreover, these findings highlight the importance of addressing the needs of the disabled community in the phase of restrictions' easing, including family support interventions. 


\section{References}

1. Jiménez-Pavón, D, Carbonell-Baeza, A, and Lavie, CJ (2020) Physical exercise as therapy to fight against the mental and physical consequences of COVID-19 quarantine: Special focus in older people. Prog Cardiovasc Dis 63(3):386.

2. Villani, ER, Vetrano, DL, Damiano, C, Paola, AD, Ulgiati, AM, Martin, L, ... and Carfî, A (2020) Impact of COVID-19-Related Lockdown on Psychosocial, Cognitive, and Functional WellBeing in Adults With Down Syndrome. Front Psychiatry 11:1150.

Fitness, alimentazione e nutrizione nello sport

\section{5}

Acute effects of self-selected music on muscular fitness in middle-aged adults

Francesca Greco $^{1}$, Elisa Grazioli ${ }^{2}$, Attilio Parisi ${ }^{2}$, Attilio Carraro ${ }^{3}$, Maurizio Bertollo $^{4}$, Luca Rotundo ${ }^{5}$, Antonio Paoli ${ }^{5}$, Giuseppe Marcolin $^{5}$, Antonio Aversa ${ }^{1}$, Gian Pietro Emerenziani ${ }^{1}$

"Magna Graecia" University of Catanzaro, Department of Experimental and Clinical Medicine, Catanzaro, Italy ${ }^{l}$, University of Rome "foro Italico", Department of Movement, Human and Health Sciences, Rome, Italy ${ }^{2}$, Free University of Bozen, Faculty of Education, Bozen, Italy ${ }^{3}$, University G. D'annunzio of Chietipescara, Department of Medicine and Aging Sciences, Chieti, Italy ${ }^{4}$, University of Padova, Department of Biomedical Sciences, Padova, Italy $^{5}$

Purpose: Strength training plays a crucial role in promoting healthy ageing. Music stimuli might affect how individuals perform and perceive strength exercises and how they feel while exercising. Therefore, this research aimed to investigate the effects of self-selected music (SSM) on muscular fitness components and affective valence during a strength exercise.

Methods: Fifteen healthy middle-aged males (mean age: $51.9 \pm$ 8.0 years) performed an isometric exercise on their dominant lower limb under three different test conditions (SSM, research-selected music (RSM) and no music (CC). SSM was chosen by participants, while RSM was selected from a list of motivational songs (mean music tempo: $128.8 \pm 4.0 \mathrm{bpm}$ ). In each test session, maximal strength and time to max, Rate of Force Development (RFD), mean endurance strength and fatigue index value were evaluated. Moreover, the Rate of Perceived Exertion (RPE) and the Feeling Scale (FS) were administered.

Results: Maximal strength was significantly higher $(\mathrm{p}<0.01)$ in SSM than in the RSM condition. Mean endurance strength was significantly higher $(\mathrm{p}=0.02)$ in SSM than in the RSM condition. A significant condition $\mathrm{x}$ time interaction $(\mathrm{p}=0.01)$ for FS was found. FS at the end of the exercise was significantly higher in SSM than RSM ( $p=0.03)$ and CC $(p=0.02)$ conditions. FS was significantly higher $(\mathrm{p}=0.01)$ at the end of the exercise than at the beginning in the SSM condition. No significant differences were found for time to max, RFD, fatigue index value and RPE.

Conclusions: Listening to SSM while performing an isometric strength exercise led to a greater muscular performance also enhancing participants' affective valence. On the contrary, listening to RSM led to the worst muscular performance.

\section{References}

1. Terry PC, Karageorghis CI, Curran ML, Martin OV, ParsonsSmith RL. (2020) Effects of music in exercise and sport: A metaanalytic review. Psychol Bull 146: 91-117.
2. Feiss R, Kostrna J, Scruggs JW, Pangelinan M, Tenenbaum G. (2021) Effects of music tempo on perceived exertion, attention, affect, heart rate, and performance during isometric strength exercise. J Sports Sci 39:161-169.

56

Time-efficient, space-saving, and interactive platform to assess physical capacities in adult employees

Gennaro Boccia $^{1}$, Anna Mulasso ${ }^{2}$, Simone Cuomo ${ }^{2}$, Paolo Riccardo Brustio $^{3}$, Corrado Lupo ${ }^{2}$, Alexandru Ungureanu ${ }^{2}$, Alberto Rainoldi ${ }^{2}$

University of Turin, Department of Clinical and Biological Sciences, Torino, Italy ${ }^{1}$;

University of Turin, Department of Medical Sciences, Torino, Italy ${ }^{2}$; University of Verona, Department of Neurosciences, Biomedicine and Movement Sciences, Torino, Italy ${ }^{3}$

Purpose: In the context of corporate welfare initiatives, we developed a platform (hardware and software) that allow the assessment of the most critical physical capacities of employees in a time-efficient (less than $15 \mathrm{~min})$, space-saving $(1.5 \times 1.5 \mathrm{~m})$, and interactive way. The present study aimed to characterize the metrics' features and assess the protocol's feasibility and acceptability.

Methods: A total of 402 employees (272 women) of the University of Turin, aged 24 to 68 years, executed six validated tests (including finger tapping, sit and reach, handgrip strength, single-leg stance, five times sit-to-stand, YCMA step test) on a sensorized and semi-automatic platform. To detect latent physical-capacity variables, we performed a principal component analysis (PCA) on the outcomes metrics of the physical tests. In addition, we recorded adverse events and tests completion rate to assess feasibility. Acceptability was measured with ad hoc questionnaires.

Results: The PCA found four components (explaining 68\% of variance): strength (including the handgrip strength, sit-to-stand estimated power); hand dexterity (including the number of finger tapping); cardiorespiratory fitness (including the outcome of the YCMA step test); balance (including single-leg stance outcome measures). The sit and reach test was positively associated with cardiorespiratory fitness $(r=0.45)$ and negatively associated with the strength component $(r=$ -0.68). No adverse events were recorded. The initiative was found acceptable and useful in $95 \%$ of the participants. Except YCMA step test, which showed $90 \%$ of completion rate, all other tests were completed $100 \%$ of the occasions.

Conclusions: The platform could safely and efficiently assess the most critical physical capacities in an interactive way. Identifying the four components allows to characterize the employees' physical capacities and helps in suggesting the exercise programs most appropriate for their current functional state.

\section{7}

What are the factors associated with physical capacities in adult employees? Preliminary results of the project wellness@work for unito

Anna Mulasso ${ }^{1}$, Simone Cuomo ${ }^{1}$, Gennaro Boccia ${ }^{2}$, Raffaella Degan $^{3}$, Alessia Moroni ${ }^{3}$, Margherita Micheletti Cremasco ${ }^{4}, \underline{\text { Alberto }}$ Rainoldi $^{1}$

University of Turin, Department of Medical Sciences, Turin, Italy ${ }^{1}$; University of Turin, Department of Clinical and Biological Sciences, 
Turin, Italy ${ }^{2}$;

University of Turin, Suism University Service Center In Hygiene and Sport Sciences, Turin, Italy ${ }^{3}$;

University of Turin, Department of Life Science and Systems Biology, Turin, Italy ${ }^{4}$

Purpose: Physical capacities (PC) are important predictors of life expectancy (Clausen et al., 2018) and functional abilities (Rantanen et al., 1999) in adult population. To know the factors associated with PC is crucial in order to implement targeted interventions able to increase PC. Therefore, this preliminary study aims to assess factors associated with PC in a population of adult employees.

Methods: 402 Employees (mean age 47 years, SD $=10 ; 67.7 \%$ of women) participated in the project Wellness@Work for UniTO. PC were measured using validated tests: Finger Tapping for manual dexterity, YMCA Step for cardiorespiratory fitness, hand-grip and Sit to Stand for strength, One Leg Stance for balance, and Sit and Reach for flexibility. Moderate to vigorous intensity physical activity (MVPA) was evaluated using the Global Physical Activity Questionnaire. Previous sports activities, health and sociodemographic characteristics were collected with ad-hoc questions. An overall index of the PC was created using Z scores for men and women. Data were treated through linear regression, adjusted for age and BMI, and correlation analyses.

Results: Regression models showed that PC index was influenced by previous sports activities $(\beta=0.108 ; p=0.025)$, with an explained variance of $11.3 \%$. Taking into consideration the single PC, cardiorespiratory fitness was explained by MVPA $(\beta=0.126 ; p=0.011$; $\mathrm{R} 2=0.041)$, while strength was influenced by previous sports activities, both considering the hand-grip strength $(\beta=0.111 ; p=$ $0.027 ; \mathrm{R} 2=0.035)$ and the Sit to Stand $(\beta=0.124 ; \mathrm{p}=0.011 ; \mathrm{R} 2=$ 0.095). Lastly, perception of good health, absence of chronic disease and to be a non-smoker were correlated to high PC index.

Conclusions: These preliminary findings recognize the role of sports activities performed during the whole life cycle for developing and maintaining satisfying levels of PC. In line with the WHO recommendations, actual level of MVPA is specifically associated to cardiorespiratory fitness. The promotion of PC requires a broad-based approach that fosters active lifestyle and addresses health factors.

References

1. Clausen JS, Marott JL, Holtermann A, Gyntelberg F, and Jensen MT. (2018). Midlife cardiorespiratory fitness and the long-term risk of mortality: 46 years of follow-up. J Am Coll of Cardiol 72(9): 987-995.

2. Rantanen T, Guralnik JM, Foley D, Masaki K, Leveille S, Curb JD, and White L. (1999). Midlife hand grip strength as a predictor of old age disability. Jama 281(6): 558-560.

\section{8}

Erasmus + sport eumove project "Let's move

Europa": results from teacher's focus groups to outline a learning unit about healthy lifestyle intervention in primary and secondary school children

\author{
Andrea Ceciliani ${ }^{1}$, Alice Masini ${ }^{2}$, Alessandra Anastasia ${ }^{2}$, Aurelia \\ Salussolia $^{2}$, Giorgia Solda ${ }^{2}$, Giorgia Zanutto ${ }^{2}$, Raffaela Mulato ${ }^{3}$, \\ Stephan Riegger ${ }^{3}$, Sofia Marini ${ }^{2}$, Laura Dallolio ${ }^{2}$ \\ Universittà Di Bologna, Dipartimento Scienze Per La Qualità Della \\ Vita Unibo, Bologna, Italy ${ }^{I}$; \\ Universittà Di Bologna, Dipartimento Di Scienze Biomediche E
}

Neuromotorie, Bologna, Italy ${ }^{2}$; Onluss, Moving School 21, Treviso, Italy ${ }^{3}$

Purpose: Prevention of obesity is one of the most important public health priorities considering the prevalence's increase of overweight and obesity in young people in the last decades. The Eumove Project aims to design, develop, implement and evaluate a set of resources for teachers, parents, school leaders and stakeholders in order to promote healthy lifestyles in children and adolescents to prevent risk factors for non-communicable disease. We manage an intellectual output focused on creating learning units (LUs) about healthy lifestyle (physical activity, nutrition and sleep) for teachers to promote extracurriculum activities. We conducted three focus groups (FC) with teachers to outline concrete and sustainable guidelines for the construction of LUs based on aged groups.

Methods: One conductor and one observer from research's group handled the meetings with $\mathrm{n}=17$ primary and secondary school teachers, $($ aged $=49.5 \pm 11,6 ; 15$ female vs 2 male). The FC were structured in four domains: feasibility of learning units, implementation strategies, space-time factors, stakeholders. FC were audiorecorded, transcribed verbatim and anonymzed before being coded using a thematic and inductive approach.

Results: The generalizable categories highlighted some common elements to be included in LUs, both in primary and secondary schools: multidisciplinary approach, open and dynamic teaching programs, proposing challenge tasks in extracurricular hours, monitoring of activities (daily diaries, tests, questionnaires). Regarding primary school the following aspects are added: involvement of families through challenging tasks to be carried out with the children. Concerning secondary school, the following elements are outlined: involving extracurricular work groups monitored by teachers, use of digital technology and Internet platforms (apps, social, etc.).

Conclusions: Our findings suggest that the construction of LUs on healthy lifestyles requires the determination of an educational network between family, school and social environment. The school should encourage children to carry out extracurricular activities in groups or with their parents using challenges helpful in monitoring the activities. Without this educational network, which starts from the school and return to it, it would not seem possible to obtain time and space for an effective education in healthy lifestyles involving children, adolescents and families.

\section{References}

1. Zannini, L. et al. (2016) La coerenza tra metodo di raccolta dati e modalità della loro analisi nella ricerca qualitativa. Encyclopaideia XX (44), 89-105.

2. Gobo, G. (2005) L'analisi semeiotica del Focus Group. Il caso della comunicazione pubblicitaria. Milano: Franco Angeli.

\section{9}

\section{The role of vitamins in athlete's muscle metabolism}

Cristina Mennitti ${ }^{1}$, Mariarita Brancaccio ${ }^{1}$, Alessandro Gentile ${ }^{I}$, Mariella Calvanese ${ }^{I}$, Luca Gentile ${ }^{I}$,Jacopo Ulisse ${ }^{l}$, Giovanni D'alicandro $^{2}$, Giulia Frisso ${ }^{1}$, Olga Scudiero ${ }^{1}$

Università Degli Studi Di Napoli Federico Ii, Dipartimento Di Medicina Molecolare E Biotecnologie Mediche, Napoli, Italy ${ }^{1}$, Aorn, Santobono-pausillipon, Dipartimento Di Neuroscienze E Riabilitazione, Centro Di Medicina Dello Sport E Disabilità,, Napoli, Italy $^{2}$

Purpose: In recent years, laboratory medicine has become important in the clinical evaluation of athletes. Strenuous exercise can determine changes in serum concentrations of biochemical parameters. In 
particular, creatine kinase (CK) and lactate dehydrogenase (LDH) have been identified as markers of muscle damage. Moreover, nutrition also plays a key role in sport performance, indeed a correct intake of vitamins helps bone and muscle metabolism, reduces inflammation and oxidative stress, and protects against injuries. The purpose of this study is to identify any changes in serum levels of vitamins $\mathrm{A}$ and $\mathrm{E}, \mathrm{CK}$ and $\mathrm{LDH}$ respectively as markers of oxidative stress and muscle damage.

Methods: 15 Professional basketball athletes and 15 sedentary controls were recruited. Serum parameters were measured at time 0 (September), before the start of the sports season, and at time 1, 2 months later (November). Vitamin A and E levels were measured with the HPLC method, whereas the serum determination of CK and LDH was carried out with spectrophotometric method.

Results: Serum levels of Vitamin A in both september $(\mathrm{p}<0.01)$ and november $(\mathrm{p}>0.001)$ are significantly higher than in controls; similarly, serum levels of vitamin $E$ in both september $(p<0.1)$ and november $(\mathrm{p}<0.1)$ are significantly increased compared to controls. Levels of $\mathrm{CK}$ and LDH are increased but are within the reference ranges.

Conclusions: The variations in the concentrations of the parameters analyzed proportionally reflect the type and intensity of physical exercise to which athletes undergo. Although the effects on athletic performance have not yet been clarified, vitamin supplementation could represent a valid tool to counteract inflammation, muscle damage and the harmful effects of oxidative stress caused by intense physical exercise.

\section{References}

1. Mennitti C; Brancaccio M; Gentile L; Ranieri A; Terracciano D; Cennamo M; La Civita E; Liotti A; D’Alicandro G; Mazzaccara C; Frisso G; Pero R; Lombardo B; Scudiero O (2020) Athlete's Passport: Prevention of Infections, Inflammations, Injuries and Cardiovascular Diseases. J. Clin. Med. 9, 2540.

2. Lukaski HC (2004) Vitamin and mineral status: effects on physical performance Nutrition 20(7-8):632-44.

\section{0}

\section{Physical performance in adults affected by sarcopenic obesity: the effects of strength training alone or combined with different dosages of amino acids supplementation. preliminary results}

\author{
Laura Ghiotto $^{1}$, Valentina Muollo $^{2}$, Chiara Milanese $^{1}$, Elena Zoico $^{2}$, \\ Mauro Zamboni ${ }^{2}$, Andrea P Rossi ${ }^{2}$, Federico Schena ${ }^{1}$ \\ Università Di Verona, Dipartimento Di Neuroscienze, Biomedicina E \\ Movimento, Verona, Italy ${ }^{1}$; \\ Università Di Verona, Dipartimento Di Medicina, Verona, Italy ${ }^{2}$
}

Purpose: Sarcopenic obesity (SO) is characterized by the co-presence of low muscle mass and high body fat 1 . This risk and prevalence increase with age, particularly in adults aged 65 and older2. Furthermore, the intervention that combined strength training and nutrition intake may be a useful strategy to counteract SO, even if the literature is conflicting. We investigated whether 5 months of strength training (ST) alone or combined with amino acids supplementation might give positive effects on physical performance and muscle strength in individuals affected by SO.

Methods: 29 women and men (67.6 \pm 4.7 years) affected by SO were recruited into a supervised ST with upper and lower body exercises at $70-80 \%$ of 1-repetition maximum (1-RM), 3 times/week, 60 minsession and followed a moderately hypocaloric diet. Participants were randomized into three double-blind groups with different dosages of oral amino acids: placebo $(\mathrm{n}=11)$, AA-2 $(4 \mathrm{~g} /$ day, $\mathrm{n}=8)$ and AA-2 $(8 \mathrm{~g} /$ day, $\mathrm{n}=10)$ amount of amino acids. Before and after intervention, we examined: physical performance with Short Physical Performance Battery (SPPB), muscle strength with a handgrip (HG) dynamometer and 1-RM at lat machine, chest and leg press.

Results: During the 5 months of ST training, adherence was above $80 \%$ and did not differ between the three groups $(\mathrm{p}>0.05)$. After the intervention, there was a significant change over time for gait speed as a score $(\mathrm{p}=0.032)$, chair stand in seconds $(\mathrm{p}=0.004)$, total score of SPPB $(\mathrm{p}=0.048)$ and peak handgrip $(\mathrm{p}=0.002)$. Within-group analyses revealed an improvement in P and AA-1 groups for chair stand as a second $(-23.3 \%,-11.3 \%$, respectively) and in $\mathrm{P}$ group for chair stand as a score (19.4\%). Regarding muscle strength, there was a significant change over time for 1-RM in all groups, with an increase in $1-\mathrm{RM}$ at chest press (ranging from 39 to $42 \%, \mathrm{p}<0.001$ ), lat machine (ranging from 30 to $37 \%, \mathrm{p}<0.001$ ) and leg press (ranging from 37 to $43 \%, p<0.001)$. Statistical analysis showed that there was no time $\mathrm{x}$ group interaction and no significant differences between groups $(\mathrm{p}>0.05)$.

Conclusions: Our preliminary results suggest that, after 5 months of supervised ST, both exercises alone or combined with amino acid supplementation might be an effective strategy to improve physical performance and overall muscle strength. However, nutritional supplements appeared to not further enhance physical function in SO population.

\section{References}

1. Baumgartner RN (2000). Body composition in healthy aging. Ann N Y Acad Sci 904, 437-448.

2. Batsis JA \& Villareal DT (2018). Sarcopenic obesity in older adults: aetiology, epidemiology and treatment strategies. Nat Rev Endocrinol 14, 513-537.

\section{ESERCIZIO, PREVENZIONE E TERAPIA 4}

\section{1}

Regular physical activity is associated with improvement of anthropometric parameters in adult patients with cystic fibrosis: 3 years of controlled program

Marta Mallardo ${ }^{1}$, Ausilia Elce ${ }^{2}$, Rita Polito ${ }^{3}$, Paola Iacotucci ${ }^{4}$, Vincenzo Carnovale ${ }^{4}$, Federica Zarrilli ${ }^{5}$, Giuseppe Castaldo ${ }^{5}$, Aurora Daniele $^{5}$, Ersilia Nigro

Università Della Campania "l. Vanvitelli", 1dipartimento Di Scienze E Tecnologie Ambientali, Biologiche, Farmaceutiche, Napoli, Italy' Università Telematica Pegaso, Dipartimento Di Scienze

Umanistiche, Naples, Italy ${ }^{2}$;

Ceinge-biotecnologie Avanzate Scarl, Napoli, Italy; Ceingebiotecnologie Avanzate Scarl, Napoli, Italy; Naples, Italy ${ }^{3}$; Università Degli Studi Di Napoli Federico Ii, Dipartimento Di Scienze Mediche Traslazionali, Naples, Italy ${ }^{4}$;

Università Degli Studi Di Napoli Federico Ii, Napoli, Italy, Dipartimento Di Medicina Molecolare E Biotecnologie Mediche,, Naples, Italy ${ }^{5}$

Purpose: Cystic fibrosis (CF) is one of the most commonly inherited diseases characterized by a severe decline in lung function and associated with both metabolic features and an increased inflammatory status. Recently, we found that physical activity (PA) improves general health status of CF patients, reducing lung function decline and counteracting inflammation. In this study we analyze the effects 
of a 3-years physical activity program on anthropometric parameters of CF patients, in particular on body composition including body mass index, fat mass, fat-free mass, phase angle and body water.

Methods: The parameters were measured through bioelectrical impedance analysis. As such, we examined CF patients classified on the basis of lifestyle: physically active patients and no-physically active patients.

Results: We observed that, after 3-years, CF patients performing regular PA have an improved phase angle $(5,8$ vs. 5,3$)$, fat-free mass $(27,4$ vs. $26,8 \mathrm{~kg} / \mathrm{m})$, muscular mass $(29,5$ vs. $27,1 \mathrm{~kg})$ and fat mass $(9,2$ vs. $11,3 \mathrm{~kg} / \mathrm{m})$ compared to non-active patients. Furthermore, active $C F$ patients have less extracellular water $(15,0$ vs. $16,8 \mathrm{~L})$. No differences were found in BMI and total body water.

Conclusions: even if preliminaries, these data confirm that PA is an useful strategy to utilize in the management of $\mathrm{CF}$ patients. PA, indeed, contributes to improve inflammatory state, lung functions, and anthropometric parameters. Modifications in lifestyle (PA and nutrition) need to be anticipated to obtain improvements in quality of life of $\mathrm{CF}$ patients; the implementation and promotion of PA must become more actively promoted in the future for $\mathrm{CF}$ patients management.

\section{2}

\section{The effects of different meal-exercise combinations on postprandial glycaemia in healthy individuals}

\author{
Alessio Bellini $^{1}$, Andrea Nicolo ${ }^{I}$, Ilenia Bazzucchi $^{1}$, Massimo \\ Sacchetti $^{I}$ \\ Università Degli Studi Di Roma “foro Italico", Dipartimento Di \\ Scienze Motorie Umane E Della Salute, Rome, Italy ${ }^{l}$
}

Purpose: High postprandial glycaemic excursions have been associated with an increased risk of developing cardiometabolic disorders (Ceriello et al., 2008). Several exercise and nutritional strategies may be adopted for improving the post-meal glycaemic response (Gillen et al., 2021). However, our current understanding of how postprandial exercise should be prescribed according to the characteristics of the meal is limited. Hence, this study was designed to evaluate the effects of different meal-exercise combinations on postprandial glycaemia. Methods: Twenty-one healthy active young individuals performed one of two studies (10 participants in Study 1 and 11 in Study 2), consisting of four protocols each. All protocols lasted $2 \mathrm{~h}$ and were preceded by the consumption of a standardized meal high in carbohydrate $(\mathrm{CHO})$ content. In Study $1,0.75 \mathrm{~g}$ or $1.5 \mathrm{~g}$ of $\mathrm{CHO}$ per $\mathrm{kg}$ of body weight were consumed. In Study 2, $75 \mathrm{~g}$ of $\mathrm{CHO}$ were consumed as dextrose solution (100\% of energy from $\mathrm{CHO}$ ) or mixed meal ( $71 \%$ of energy from $\mathrm{CHO}, 14 \%$ from protein, $15 \%$ from fat). For each study, on two occasions, participants remained seated for the entire experimental period $(0.75 \mathrm{CON}, 1.5 \mathrm{CON}$, OGTT_CON, MEAL_CON). During the other experimental trials, 30 min of brisk walking (120 steps per minute) were performed starting 15 min after the beginning of the meal (0.75EX, 1.5EX, OGTT_EX, MEAL_EX). Glycaemia was assessed every $15 \mathrm{~min}$ throughout the experimental sessions. The positive incremental area under the curve (iAUC) at $2 \mathrm{~h}$ was calculated for both studies.

Results: Study 1 revealed a significant attenuation of the glucose peak at $30 \mathrm{~min}$ in $0.75 \mathrm{EX}$ and $1.5 \mathrm{EX}$ compared with $0.75 \mathrm{CON}$ and $1.5 \mathrm{CON}(\mathrm{p}<0.009)$. Similarly, in Study 2 both OGTT_EX and MEAL_EX significantly reduced glucose levels at 30 min compared with OGTT_CON and MEAL_CON ( $p<0.004)$. No differences between the two exercise conditions were found in any of the two studies. Study 1 did not show differences between conditions in the iAUC at $2 \mathrm{~h}$. Conversely, in Study 2 both MEAL_CON and
MEAL_EX significantly attenuated iAUC compared to OGTT_CON and OGTT_EX $(\mathrm{p}<0.025)$.

Conclusions: Thirty min of postprandial walking are effective in reducing the glucose peak even when increasing the absolute and relative content of $\mathrm{CHO}$ in a meal. However, the effect on glycaemic response was reduced throughout the first $2 \mathrm{~h}$ post meal when the absolute content of CHO increases.

References

1. Ceriello A, Esposito J, Piconi L, Ihnat MA, Thorpe JE, Testa R, Boemi M, Giugliano D (2008) Oscillating glucose is more deleterious to endothelial function and oxidative stress than mean glucose in normal and type 2 diabetic patients. Diabetes 57:1349-54.

2. Gillen JB, Estafanos S, Govette A (2021) Exercise-nutrient interactions for improved postprandial glycemic control and insulin sensitivity. Appl Physiol Nutr Metab.

\section{3}

Comparison of two exercise testing protocols to assess maximal fat oxidation in obese subjects

Dafne Ferrari ${ }^{1}$, Gian Pietro Emerenziani ${ }^{2}$, Lavinia Falcioni $^{3}$,
Ludovica Cardinali $^{3}$, Matteo Campanella ${ }^{1}$, Silvia Migliaccio
Davide Curzi

Ecampus, Ecampus University, Novedrate, Italy ${ }^{1}$;

University of Magna Graecia of Catanzaro, Department

of Experimental and Clinical Medicine, Catanzaro, Italy ${ }^{2}$;

University of Rome "foro Italico", Department of Movement, Human and Health Sciences, Rome, Italy ${ }^{3}$;

Niccolò Cusano University, Niccolò Cusano Department, Rome, Italy $^{4}$

Purpose: For their relevance in the physical activity (PA), numerous studies investigated different approaches to evaluate maximal fat oxidation (MFO) during exercise and the exercise intensity inducing MFO. The aim of this study was to investigate the use of a short exercise protocol compared to a larger state-to-stage increments protocol on treadmill in obese subjects.

Methods: Thirty-six obese adult subjects were involved in this study. Sixteen male $($ age $=51.5 \pm 10.5$ years, $\mathrm{BMI}=36.7 \pm 4.8 \mathrm{~kg} / \mathrm{m} 2)$ and twenty female subjects (age $=42.4 \pm 12.4$ years, BMI $=36.5 \pm$ $4.7 \mathrm{~kg} / \mathrm{m} 2$ ) performed two exercise testing protocols. In two separate testing sessions, after the body composition assessment, subjects performed a sub-maximal incremental graded test on treadmill. Depending on testing sessions, the workload was increased every 2or 3-min, evaluating maximal fat oxidation (MFO) and Fatmaxzone variables.

Results: No significant differences were found in heart rate (HR), $\mathrm{VO} 2$ (oxygen uptake $\mathrm{ml} / \mathrm{kg}$ ) and rate of perceived exertion (RPE) at MFO in the 2-min protocol compared with 3-min protocol $(105.9 \pm$ 22.3 vs $105.8 \pm 14.4 ; 14.5 \pm 2.4$ vs $13.7 \pm 3.2 ; 3.0 \pm 1.4$ vs $3.0 \pm$ 2.0). Significant differences were found in MFO in the 2-min protocol compared to 3 -min protocol $(576.3 \pm 162.9$ vs $506.0 \pm 148.1)$. From the analysis of the Fatmaxzone, no significant differences were found in the VO2 and HR in high and low borders. Moreover, a statistically significant difference was found in the fat oxidation in low and high borders.

Conclusions: Even if the amount of fat oxidation is stage-time dependent, the exercise intensity at which the MFO and the Fatmaxzone occur is not influenced by the stage-time of the test protocol. Thus, the two-exercise testing protocols could be considered 
interchangeable to assess the HR and RPE at MFO and at Fatmaxzone, as variables commonly used to prescribe PA.

\section{References}

1. Amaro-Gahete FJ et al., (2019) Optimizing Maximal Fat Oxidation Assessment by a Treadmill-Based Graded Exercise Protocol: When Should the Test End? Front Physiol. 23;10:909.

2. Chávez-Guevara IA, Hernández-Torres RP, Trejo-Trejo M, González-Rodríguez E, Moreno-Brito V, Wall-Medrano A, Pérez-León JA, Ramos-Jiménez A. (2021) Exercise Fat Oxidation Is Positively Associated with Body Fatness in Men with Obesity: Defying the Metabolic Flexibility Paradigm. Int J Environ Res Public Health. 29;18(13):6945.

\section{4}

\section{Acute effects of whole body electromyostimulation in early parkinson's disease}

\author{
$\underline{\text { Andrea Buonsenso }}^{1}$, Giovanni Fiorilli $^{1}$, Giulia Di Martino ${ }^{2}$, Marco \\ Centorbi $^{I}$, Attilio Parisi ${ }^{2}$, Giuseppe Calcagno ${ }^{1}$, Alessandra Di \\ Cagno $^{2}$ \\ Università Degli Studi Del Molise, Dipartimento Di Medicina E \\ Scienze Della Salute, Campobasso, Italy ${ }^{I}$; \\ Università Degli Studi Di Roma Foro Italico, Dipartimento Di \\ Scienze Motorie, Umane E Della Salute, Rome, Italy ${ }^{2}$
}

Purpose: Parkinson's disease (PD) patients lead a sedentary lifestyle, being unable or unwilling to exercise conventionally, due both to physical and mental limitations. Physical activity has been identified as a possible treatment for cognitive impairment [1] and could upregulate neurotrophic factor levels, counteracting dyskinesia [2]. Whole-Body Electromyostimulation (WB-EMS) seems a promising exercise modality for this population inducing a global-body electrical myostimulation activating up to 8-10 different muscle groups synchronously, while the patient performs functional movements during the stimulation. WB-EMS are not previously applied to an exercise program for PD patients. The aim of this study was to assess the acute effects of a single session of WB-EMS on physical and cognitive performances and neurotrophic factor levels in PD patients. Methods: Ten subjects (aged $72.60 \pm 6.82$ ) underwent 20-min physical activity with superimposed WB-EMS (Miha Bodytec equipment-GmbH, Augsburg, Germany) and, after four weeks, the same protocol with no WB-EMS. WB-EMS was conducted with intermittent stimulation $4 \mathrm{~s}$ WB-EMS $/ 4 \mathrm{~s}$ rest, at $85 \mathrm{~Hz}, 350 \mu \mathrm{s}$.

Results: The RM-ANOVA showed significant improvements in Sitto-Stand ( $p<0.01)$, Arm Curl $(\mathrm{p}<0.01)$, Handgrip $(\mathrm{p}<0.01)$ and Soda-POP test $(\mathrm{p}<0.01)$ after WB-EMS intervention. Significant improvements were found in Rey auditory verbal learning test ( $\mathrm{p}=$ $0.002)$ and Trail making test $(\mathrm{p}=0.010)$ after control group condition. Higher proNFG serum levels were observed in the WB-EMS condition compared to the no WB-EMS after 60 min post intervention $(\mathrm{p}=$ 0.0163).

Conclusions: WB-EMS could be a promising strategy to improve motor and non-motor symptoms in PD patients. Such as time-efficient solution this strategy may improve their adherence to physical activity programs and to increase their wellbeing. The decrease of cognitive performances after WB-EMS intervention could be due to the greater fatigue effects induced by physical activity superimposed with electrical myostimulation.

\section{References}

1. Amara AW, Memon AA. (2018) Effects of exercise on nonmotor symptoms in Parkinson's disease. Clin Ther 40: 8-15.
2. Lauzé, M.; Daneault, J.F.; Duval, C. The effects of physical activity in Parkinson's disease: A review. J. Parkinsons dis. 2016, 6, 685-698.

\section{5}

\section{Effect of 12-week physical activity on muscle and bone health in people living with HIV}

\author{
Matteo Bonato $^{1}$, Laura Galli ${ }^{2}$, Simona Bossolasco ${ }^{2}$, Cecilia \\ Bertocchi $^{3}$, Giuseppe Balconi ${ }^{3}$, Marco Borderi ${ }^{4}$, Gaspare Pavei ${ }^{5}$, \\ Giampiero Merati ${ }^{6}$, Antonio La Torre ${ }^{1}$, Adriano Lazzarin ${ }^{2}$, Giuseppe \\ Banfi $^{7}$, Paola Cinque ${ }^{2)}$.
}

Università Degli Studi Di Milano, Department of Biomedical Sciences For Health, Milan, Italy ${ }^{1}$;

Department of Infectious Diseases, Irccs San Raffaele Scientific Institute, Milan, Italy ${ }^{2}$;

Departement of Radiology, Irccs San Raffaele Scientific Institute, Milan, Italy ${ }^{3}$;

Infectious Disease Unit, Sant'orsola Hospital, University of Bologna, Bologna, Italy ${ }^{4}$;

Department of Pathophysiology and Transplantation, Università

Degli Studi Di Milano, Milan, Italy ${ }^{5}$;

Department of Biotechnology and Life Sciences, University

of Insubria, Varese, Italy ${ }^{6}$;

Laboratory of Movement and Sport Science, Irccs Istituto Ortopedico Galeazzi, Milan, Italy

Purpose: We previously showed in a pilot study that a 12-week protocol consisting of 3 sessions per week of 60 min brisk walking with (strength-walk group) or without (walk group) 30 min resistance training, improved physical performance, lipid profile and inflammatory markers in people living with HIV (PLWH)1. The aim of this study was to assess the effects on body composition, bone mineral density and bone remodeling biomarkers in PLWH.

Methods: Twenty-five cART-treated participants [19/25 male; age: 51 (48-56) years; CD4 + : 576 (463-701) median (25\%-75\% quartiles)] were assessed: 15 in the walk group and 10 in the strength-walk group. Assessments at baseline (BL) and week 12 (W12) included fat mass (FM), fat free mass (FFM) at arms, limbs, and total body, appendicular skeletal muscle mass index (ASMMI), bone mineral density (BMD), t-score, and z-score at spine, femoral neck and trochanter by total body dual-energy X-ray absorptiometry (DEXA), and bone turnover biomarkers, including osteoprotegerin (OPG), receptor activator of NF-kappaB ligand (RANKL), serum crosslaps (CTX) and bone alkaline phosphatase (BAP). Changes between BL and W12 were assessed by Wilcoxon match-paired signed rank test, BL values, and percent change differences between groups by Mann-Whitney test.

Results: All participants completed the 12-week program with a median adherence of $64 \%(59 \%-75 \%)$. Eight $(32 \%)$ participants had sarcopenia (cut off points ASSMI: $<5.5 \mathrm{~g} / \mathrm{m} 2$ women; $6.5 \mathrm{~kg} / \mathrm{m} 2$ men) at BL, $3(12 \%)$ had osteoporosis and $20(80 \%)$ osteopenia. Concerning body composition, significant W12 improvements from BL were observed in legs FM, total FM, and ASMMI in both groups; in arms FM in the walk group and in arms FFM, legs FFM, and total FFM in the strength-walk group only. Percent increases from BL differed significantly between the two groups for BMI, ASMMI, total FFM, arms FFM and legs FFM. At W12, 6 of the 8 participants with sarcopenia, normalized ASMMI. As for bone parametres, significant changes of BMD, T-score and Z-score at spine, femoral neck and trochanter, at W12 vs. BL were neither observed in the whole group nor in the two training groups. Significant W12 increases from BL 
were observed in CTX in both training groups, but no changes of OPG, RANKL and BAP.

Conclusions: This pilot study provides relevant information for designing larger interventional controlled studies for increasing muscle mass and potentially reducing sarcopenia in PLWH.

\section{References}

1. Bonato M, Galli L, Passeri L, Longo V, Pavei G, Bossolasco S, Bertocchi C, Cernuschi M, Balconi G, Merati G, Lazzarin A, La Torre A, Cinque P. A pilot study of brisk walking in sedentary combination antiretroviral treatement (cART)- treated patients: benefit on soluble and cell inflammatory markers. BMC Infect Dis. 2017 Jan 11;17(1):61.

\section{6}

\section{"Palestra della salute": the 10 year's experience of "esercizio vista medical fitness"}

\section{Michele Felisatti $^{I}$, Piero Fumarola ${ }^{1}$, Luca Innella ${ }^{I}$, Luca Pomidori ${ }^{1}$}

Esercizio Vita Medical Fitness, R\&s, Ferrara, Italy ${ }^{1}$

Purpose: Through interactions between health and non-health facilities called "Palestra della Salute", the Supervised Exercise Training (SExT) can be adapted for elder individuals and those with chronic diseases by exercise physiology (LM-67), in order to improve or maintain health. The aim of the current study is to describe the characteristics of the facility's clients, how they were referred to the gym and their level of satisfaction with the proposed SExT programmes.

Methods: The clients referred to the Esercizio Vita (EV) (Ferrara, Italy) facility between September 2011 and June 2021 were enrolled in the present study, an innovative start-up in the field of health gyms. Customers info such as personal data, medical condition at the time of admission and method of access to the facility were analyzed in an internal database. The level of Satisfaction was assessed through an anonymous survey administered after 2 month of training. The collected data were then analyzed retrospectively.

Results: 5447 clients were enrolled in the study (M:F, 2238:3209), mean age was 63 years old (SD: $\pm 18 \mathrm{y})$. The mean amount of pathologies per patient was 1.8 (range:0-8). Regarding the modality of access to the gym, $27 \%$ of patients were referred by healthcare providers (specialist physician $18 \%$, general practitioner $2 \%$, physiotherapist $7 \%$ ). The remaining $73 \%$ of clients were referred by wordof-mouth $(25 \%)$, advice of a previous client $(19 \%)$ or a relative $(8 \%)$, advertising (4\%), organizations $(2 \%)$ and others $(15 \%)$. The level of satisfaction was excellent in $95.3 \%$ of cases and almost half of the patients $(48.4 \%)$ continued to train for at least 2 years.

Conclusions: The number of patients enrolled in this study highlights the importance of EV design for the elderly population and those with comorbidities. The rate of referral to the facility by healthcare providers shows an interest in managing risk factors and maintaining residual abilities through SExT. The "Palestra della Salute" act as a link between healthcare facilities and the territory in the field of chronic diseases, with programs offered by highly trained and qualified professionals in the field of exercise physiology.

References

1. Caspersen CJ, Powell KE, Christenson GM. Physical activity, exercise, and physical fitness: definitions and distinctions for health-related research. Public Health Rep. 1985;100(2):126-131.

2. Lucini D, Pagani M. Exercise Prescription to Foster Health and Well-Being: A Behavioral Approach to Transform Barriers into
Opportunities. Int J Environ Res Public Health. 2021 Jan 22;18(3):968.

\section{PSICOLOGIA DELL'ESERCIZIO E DELLO SPORT}

\section{7}

\section{Predictors of muscle dysmorphia symptoms in male and female competitive powerlifters}

\author{
Silvia Cerea $^{l}$, Hillary Pontalto ${ }^{1}$, Antonio Paoli ${ }^{2}$, Marta Ghisi ${ }^{1}$ \\ Università Degli Studi Di Padova, Dipartimento Di Psicologia \\ Generale, Padova, Italy ${ }^{1}$; \\ Università Degli Studi Di Padova, Dipartimento Di Scienze \\ Biomediche, Padova, Italy ${ }^{2}$
}

Purpose: Muscle Dysmorphia (MD) is a psychological disorder characterized by the preoccupation with the idea that one's body is not sufficiently lean and muscular. Athletes with MD are at high-risk of developing unhealthy behaviors (e.g., over-training; anabolic-androgenic steroid use and abuse) to achieve the desired physique (Olivardia, 2001); such unhealthy behaviors may have a detrimental effect on performance and lead to sport withdrawal. MD is prevalent among males practicing strength training sports (Cerea et al., 2018); however, recent evidence highlighted that MD is growing in prevalence also among females, because of the recent emphasis places on muscularity for the female body (Hale, 2013). Given the negative impact of MD symptoms on athletes' physical and mental health, the aim of the current study is to assess predictors of MD symptoms in a sample of competitive male and female powerlifters.

Methods: 180 Competitive male $(n=127)$ and female $(n=53)$ powerlifters took part in the study and completed self-report questionnaires aimed at assessing MD symptoms, associated psychological features (i.e., self-esteem, perfectionism, social phobia, and general distress), and social network use. Performance was also investigated with the Ipf Gl Point (International Powerlifting Federation; IPF, 2020). Mean months of training was $42.46(\mathrm{SD}=37.07)$ for males and $44.48(\mathrm{SD}=51.12)$ for females. Mean hours of training per week was $12.07(\mathrm{SD}=3.71)$ for males and $11.26(\mathrm{SD}=2.68)$ for females.

Results: Controlling for general distress, multiple regressions highlighted that physical appearance comparisons on Instagram were predictive of MD symptoms in male competitive powerlifter $(\mathrm{t}=2.04$; $\mathrm{p}=0.04)$, whereas self-esteem approached significance $(\mathrm{t}=-2.00 ; \mathrm{p}=$ $0.05)$; the overall model explained the $28 \%$ of the variance in the questionnaire assessing MD symptoms. With respect to females, controlling for general distress, self-esteem emerged as a significant predictor of MD symptoms $(\mathrm{t}=-3.36 ; \mathrm{p}=0.002)$, whereas Ipf $\mathrm{Gl}$ Point approach significance $(\mathrm{t}=-1.98 ; \mathrm{p}=0.05)$; the overall model explained the $61 \%$ of the variance.

Conclusions: Different predictors of MD symptoms emerged for male and female competitive powerlifters. In particular, appearance comparisons on Instagram, as well as self-esteem, should be target in male powerlifters, because they may have an impact on MD symptoms. Concerning females, self-esteem and performance indicators should be address.

\section{References}

1. Cerea S, Bottesi G, Pacelli QF, Paoli A, and Ghisi M (2018) Muscle dysmorphia and its associated psychological features in three groups of recreational athletes. Sci Rep 8: 1-8.

2. Hale BD, Diehl D, Weaver K, and Briggs M (2013) Exercise dependence and muscle dysmorphia in novice and experienced female bodybuilders. J Behav Addict 2: 244-248. 
3. Olivardia R (2001) Mirror, mirror on the wall, who's the largest of them all? The features and phenomenology of muscle dysmorphia. Harv Rev Psychiatr 9: 254-259.

\section{8}

\section{The mediating role of stress in the relationship between mood and performance in elite canoe polo players}

\author{
Donatella Di Corrado ${ }^{1}$, Andrea Buscemi ${ }^{2}$, Paola Magnano ${ }^{I}$, Nelson \\ Mauro Maldonato ${ }^{3}$, Matej Tusak ${ }^{4}$, Marinella Coco ${ }^{2}$ \\ Department of Human and Social Sciences, Kore University, Enna, \\ Italy $^{l}$; \\ Department of Biomedical and Biotechnological Sciences, University \\ of Catania, Catania, Italy ${ }^{2}$; \\ Department of Neuroscience and Reproductive \\ and Odontostomatological Sciences, University of Naples Federico Ii, \\ Naples, Italy ${ }^{3}$; \\ Department of Social and Humanistic Sciences In Sport, University \\ of Ljubljana, Ljubljana, Slovenia ${ }^{4}$
}

Purpose: Canoe polo is a physiologically and technically complex sport of interval activities. Sport performance is characterized by competitive stressful conditions that elicit emotional states. The purpose of the study was to verify the role that stress plays in the linkage between performance and the six mood dimensions (tension, depression, anger, vigor, fatigue, and confusion).

Methods: Thirty-two elite canoe polo players from four different Italyn teams (16 senior males and 16 senior females), aged between 29 and 38 years old $(\mathrm{M}=32.3, \mathrm{SD}=2.71)$, participated in the study. Measures included level of psychological stress, six mood dimensions, and performance outcome. The assessment took place one day before the first game of the national competition "ITALY CUP".

Results: Male athletes reported lower scores on stress and on dysfunctional emotion-related states $(\mathrm{p}<0.001)$ than female athletes. The Results of the mediation analysis showed that psychological stress plays a mediating role between moods and performance.

Conclusions: Overall, given the limited literature, the findings supported an integrative approach to the study of the linkage between emotion and action in canoe polo. The goal of each athlete is to improve the performance parameters of sport and to minimize and control interferences. Education programs for coaches should constantly integrate psychological preparation as a fundamental element of an athlete's good performance.

References

1. Bali A. (2015) Psychological factors affecting sports performance. Int. J. Phys. Educ. Sports Health 1: 92-95.

2. Di Corrado D. (2017) Biological underpinnings of mood and the role of physical exercise. Sport Sci. Health 13: 461-468.

3. Lazarus R.S. (2001) Stress and Emotion: A new synthesis. Hum. Relat. 54: 792-803.

\section{9}

Effects of regular pa during covid19 home confinement on mood, motivation, readiness to train, and sleep quality in master cyclists

Francesca Vitali ${ }^{1}$, Elisabetta Campesan ${ }^{I}$, Francesco Chiappero ${ }^{2}$, $\overline{\text { Antonio La Torre }}^{3}$, Jacopo Antonino Vitale ${ }^{4}$

University of Verona, Dep. of Neurosciences, Biomedicine and Movement Sciences, Verona, Italy ${ }^{l}$;

Founder of Reaction, Founder of Reaction, Saluzzo (cn), Italy ${ }^{2}$; University of Milan, Dep. of Biomedical Sciences For Health, Milan, Italy $^{3}$;

Irccs Istituto Ortopedico Galeazzi, Irccs Istituto Ortopedico Galeazzi, Milan, Italy ${ }^{4}$

Purpose: The home confinement due to the Covid-19 pandemic has exposed most people to unprecedented stressful situations. Staying home, while was a safe measure, have unintended negative consequences since such efforts to avoid human-to-human transmission of the virus may have led to reduced mood, motivation to train, sleep quality and physical activity (PA). Recent studies have underlined a strong health rationale for continuing PA while taking precautions. In this context, a study was conducted to analyze the effect of the first lockdown (March-April 2020) on mood, motivation, readiness to train, sleep quality and PA in cyclists.

Methods: 9 male master cyclists (Mage: $45 \pm 5$ years) were evaluated before and during the first lockdown: they continued to train safely at home under the tele-supervision of a trainer with a master's degree in Sport Sciences. They filled daily the POMS questionnaire and two ad hoc items assessing motivation and readiness to train for a month (M: $35 \pm 9$ days). Sleep was continuously monitored by actigraphy and a sleep diary throughout the entire study protocol.

Results: Repeated-measures ANOVA and bivariate correlation analysis were conducted on considered parameters. Actigraphy-based sleep parameters did not change across the study weeks. Anaerobic exercises were positively associated with positive mood and negatively associated with negative moods. Motivation and readiness to train remain positive and stable along the analyzed period. Sleep Efficiency (SE) and normalized athletic efficiency were positively correlated. SE and Training Stress Balance (TSB, a measure of fatigue) were negatively correlated: a higher SE was associated with lower TSB.

Conclusions: The results indicate that maintaining the same training volume and intensity during home confinement under the tele-supervision of a trainer can lead to minimal variations in mood, motivation, readiness to train, sleep quality and PA. The sleep quality of the participants was good along the analyzed period which coincided with the first lockdown. They showed positive mood (high vigor and low negative moods), a medium-high motivation and readiness to train while continuing exercise safely. Regular PA had a positive influence on mood, motivation to train and sleep quality showing that even during the Covid19 pandemic maintaining a regular PA routine was a key strategy to protect physical and mental health.

\section{References}

1. Chirico A, Lucidi F, Galli F, Giancamilli F, Vitale J, Borghi S, La Torre A, Codella R. COVID-19 Outbreak and Physical Activity in the Italyn Population: A Cross-Sectional Analysis of the Underlying Psychosocial Mechanisms. Front Psychol. 2020; 11:2100. 


\section{0}

\section{Paralympic athletes' stress and resilience in the age of COVID-19}

\author{
Marco Centorbi ${ }^{1}$, Alessandra Di Cagno ${ }^{2}$, Andrea Buonsenso ${ }^{I}$, Giulia \\ Di Martino $^{2}$, Sebastiano Riccio ${ }^{l}$, Giovanni Fiorilli ${ }^{1}$, Giuseppe \\ Calcagno ${ }^{l}$
}

Università Degli Studi Del Molise, Dipartimento Di Medicina E Scienze Della Salute, Campobasso, Italy ${ }^{I}$;

Università Degli Studi Di Roma Foro Italico, Dipartimento Di

Scienze Motorie, Umane E Della Salute, Rome, Italy ${ }^{2}$

Purpose: The 2019 Coronavirus (COVID-19) outbreak caused home confinement, as well as training and sport competitions withdrawals. The prolonged inactivity impact, and lack of in-person interactions among teammates-coaches, could have negative affected athletes. Disabled people are most vulnerable and could have drastic negative impacts of lockdown on varied aspects of their life. The aim of the study was to assess the level of psychological distress and the consequent reactions such as intrusion, avoidance and hyperarousal using the Event Scale-Revised (IES-R) questionnaire. The second aim was to compare athletes and Paralympic athletes.

Methods: Data were collected from one hundred and forty-six participants, 73 athletes (40.23 \pm 13.73 age) and 73 Paralympic athletes $(42.11 \pm 13.70$ aged). Differences by age, type of sport (individual vs. team) and competitive level (high, medium and low) were examined. Results: Referring to the perceived stress impact total score (TS), Paralympians resulted less stressed by Covid-19 lockdown $(8,22 \%)$, comparing with athletes $(50 \%)$. Significant differences between athletes and Paralympics- athletes was found $(\mathrm{p}<0.001)$. One-way ANOVAs showed, in young Paralympic athletes, significantly higher scores for TS $(p=0.05)$ and avoidance behaviors $(p=0.003)$ than adults. Between individual and team sport, significant differences were found in hyperarousal $(\mathrm{p}=0.037)$, with higher results in individual sport. Comparing athletes and Paralympic athletes, all athletes showed higher values in all subscales and total score. $(\mathrm{p}<0.001)$.

Conclusions: High psychological distress due to the quarantine restrictions was assessed for the general Italyn sport population, more than athletes with disabilities. Previous findings suggested that Paralympians benefited from exposure to highly demanding situations and this helped develop individualized cognitive coping strategy1. Resilience arises as a psychological factor of paramount importance, since it assists athletes in the process of adapting and overcoming such stressful demands2.

\section{References}

1. Powell, A. J., \& Myers, T. D. (2017). Developing mental toughness: lessons from Paralympians. Frontiers in Psychology, 8,1270 .

2. Sarkar M, Fletcher D. Psychological resilience in sport performers: A review of stressors and protective factors. J. Sports Sci 2014;32(15):1419-1434. https://doi.org/10.1080/02640414.2014. 901551

\section{1}

\section{The influence of physical activity and sedentary time on mood states: a survey conducted on the just the woman I am participants}

\author{
Anna Mulasso ${ }^{1}$, Emanuela Rabaglietti ${ }^{2}$, Luca Scolfaro ${ }^{3}$, Raffaella \\ Palumbo $^{3}$, Raffaella Motta ${ }^{3}$, Omar Capuzzo ${ }^{4}$, Alberto Rainoldi ${ }^{1}$
}

University of Turin, Department of Medical Sciences, Turin, Italy ${ }^{1}$, University of Turin, Department of Psychology, Turin, Italy ${ }^{2}$, Links Foundation, -, Turin, Italy ${ }^{3}$, Centro Universitario Sportivo Di Torino, -, Turin, Italy ${ }^{4}$

Purpose: the enhancing effects of physical activity (PA) on mood states of people are supported by many evidences (Berger and Motl, 2000), while little is know about the role of sedentary time (ST) on mood states. Our aim is to investigate the relationship between physical activity, sedentarism and mood states, corresponding to the factors of Anger, Confusion, Depression, Fatigue, Tension and Vigor in a sample of adult sport participants.

Methods: 228 participants (mean age 39 years, $\mathrm{SD}=14 ; 189$ women) in the event Just The Woman I Am (Torino, Edition 2021) filled out the survey. The Global Physical Activity Questionnaire (GPAQ; Bull et al., 2009) measures quantity of PA and ST; the Italyn Mood Scale (ITAMS; Quartiroli et al., 2017) assesses mood states considering six subscales related to Anger, Confusion, Depression, Fatigue, Tension and Vigor. PA is quantified as MET-minutes/week of moderate and vigorous PA. Data were treated through linear regression analyses, controlling for age, gender and education.

Results: regression models are statistically significant, specifically: Confusion $(\mathrm{R} 2=0.13 ; \mathrm{F}(5,222)=7.94 ; \mathrm{p}<0,001)$ is influenced by both PA and ST; Depression $(\mathrm{R} 2=0.11 ; \mathrm{F}(5,222)=6.44 ; \mathrm{p}<0,001)$ and Vigor $(\mathrm{R} 2=0.12 ; \mathrm{F}(5,222)=7.23 ; \mathrm{p}<0,001)$ by PA; Fatigue $(\mathrm{R} 2$ $=0.10 ; \mathrm{F}(5,222)=5.86 ; \mathrm{p}<0,001)$ and Tension $(\mathrm{R} 2=0.10 ; \mathrm{F}(5,222)$ $=5.98 ; \mathrm{p}<0,001)$ by ST. On the contrary, PA and ST do not act on Anger. Findings show that an increase of PA reduces both Confusion and Depression and acts positively on Vigor; similarly, whereas a decrease of ST counteracts Confusion, Fatigue and Tension.

Conclusions: these results notice that the promotion of PA, and specifically PA at moderate and vigorous intensity, in association with a reduction of the time spent in sedentary activities can be a practical and effective approach to ameliorate the mood states in adult population.

\section{References}

1. Berger BG, Motl RW. (2000). Exercise and mood: A selective review and synthesis of research employing the profile of mood states. J Appl Sport Psychol 12(1): 69-92.

2. Bull FC, Maslin TS, Armstrong T. (2009). Global physical activity questionnaire (GPAQ): nine country reliability and validity study. J Phys Act Health 6:790-804.

3. Quartiroli A, Terry PC, Fogarty GJ. (2017). Development and initial validation of the Italyn Mood Scale (ITAMS) for use in sport and exercise contexts. Front Psychol 8:1483.

72

The impact of noisy information processing in the evaluative and decisional dimensions. the role of heuristics, cognitive biases, and noise

\section{Franco Viviani $^{I}$}

Università Di Padova, Dipartimento Scienze Biomediche, Padova, Italy $^{l}$

Purpose: In the last sixty years, many empirical studies have focused on the systematic deviations that human beings produce with respect to what is normally expected regarding their decisions. The aim of the present paper is to highlight the importance of recognizing the main and variegated "mental shortcuts" affecting our evaluations and decisions with emphasis in the motor sciences context. 
Methods: After a short revision of recent literature, an exemplifying paper on the role of persuasion with deceit in 84 tennis player professionals is discussed. To half of them, incorrect information has been instilled in the athletes' thinking, aiming to understand whether their evaluation processes complied with the hypothesized previsions or not.

Results: In the sub-group of suggested tennis players their evaluations were in accordance to the suggestions preliminary provided. The degree of persuasion turned out to be inversely proportional to their level category.

Conclusions: We cannot precisely be aware of our hidden mental and unconscious shortcuts because they are easier to ignore, but we can still overcome them. Even if a lot of judgement has no connection with reality, increased awareness of our mental pitfalls could help. If our system is indisputably noisy our aim is to let it be more disciplined.

References

1. Kahneman D, Sibony O, \& Sunstein CR. (2021) Noise. A flaw in human judgement. London: Little Brown Sparks.

2. Sibony O, Lovallo D, \& Powell TC. (2017). Behavioral Strategy and the Strategic Decision Architecture of the Firm. Cal Man Rev https://doi.org/10.1177/0008125617712256

3. Viviani F, \& Fenza M. (2018) Persuasion with deceit elicits the "Trojan Horse Effect" in tennis players. Antrocom J Anthrop 14(1): $55-59$

FISIOLOGIA DELL'ESERCIZIO E DELLO SPORT 2

73

\section{Estimates of muscle fiber cross-sectional area from the decomposition of high-density surface emg signals}

\author{
Andrea Casolo ${ }^{1}$, Sumiaki Maeo ${ }^{2}$, Thomas Balshaw ${ }^{3}$, Jonathan \\ Folland $^{3}$, Dario Farina ${ }^{4}$, Alessandro Del Vecchio ${ }^{5}$ \\ Università Degli Studi Di Padova, Dipartimento Di Scienze \\ Biomediche, Padova, Italy ${ }^{1}$; \\ Ritsumeikan University, College of Sport and Health Sciences, \\ Kusatsu, Giappone, Italy ${ }^{2}$; \\ Loughborough University, School of Sport, Exercise \& Health \\ Sciences, Loughborough, Regno Unito, Italy ${ }^{3}$, Imperial College \\ London, Department of Bioengineering, London, Regno Unito ${ }^{4}$; \\ Friedrich-alexander University, Department of Artificial Intelligence \\ In Biomedical Engineering, Erlangen, Germania ${ }^{5}$
}

Purpose: Motor unit conduction velocity (MUCV) is considered a good predictor of motor unit (MU) recruitment $(1,2)$ and a "size principle" parameter, due to its linear relation with muscle fiber diameters (3). However, this association has been previously assessed in animal models (3), in human patients with intramuscular EMG (4) and healthy individuals (5) during electrically stimulated contractions. Therefore, the aim of this study was to explore the relation between MUCV assessed during volitional contractions and muscle fiber cross-sectional area (CSA) in healthy individuals.

Methods: 16 strength-trained (ST) athletes (age: $22.9 \pm 3.5$ yr.; body mass: $88.2 \pm 12.9 \mathrm{~kg}$ ) and 13 untrained (UT) individuals (age: $20.5 \pm$ 5.9 yr.; body mass: $74.6 \pm 11.4 \mathrm{~kg}$ ) completed a series of linearly increasing isometric ramp contractions at forces up to $70 \%$ of maximum volitional force (MViF) with elbow flexors. Concurrently, highdensity surface electromyography (HDsEMG) signals were recorded from biceps brachii (BB) muscle, and in turn decomposed into individual motor unit discharge timings. MU characteristics (recruitment thresholds, MU RT; discharge rates, MU DR) and MUCV
(MUCVAVERAGE, MUCVMIN and MUCVMAX) were estimated. Muscle fiber type composition and cross-sectional area (CSA) of BB were evaluated through muscle biopsies.

Results: Positive linear associations between MUCV and MU RT were observed for all participants $(\mathrm{R} 2=0.78 \pm 0.09, \mathrm{P}<0.001)$. When all participants were considered as one single group, significant linear associations were observed between MUCVAVERAGE and muscle fiber type I $(\mathrm{R} 2=0.34, \mathrm{P}=0.001)$ and type II CSA $(\mathrm{R} 2=0.27$, $\mathrm{P}=0.003$ ). Similarly, MUCVMAX was significantly associated with muscle fiber type I $(\mathrm{R} 2=0.40, \mathrm{P}=0.001)$ and to a larger extent to type II CSA ( $22=0.57, \mathrm{P}=0.001)$. MUCVMIN was linearly associated with muscle fiber type I CSA $(\mathrm{R} 2=0.23, \mathrm{P}=0.008)$ only. No significant associations were observed between any MUCV variable and muscle fiber type composition.

Conclusions: The main finding of the current study is that MUCV of BB muscle, here assessed during volitional submaximal contractions for the first time, was linearly associated with muscle fiber CSA of BB muscle in healthy individuals. Accordingly, considering that MUCV can be non-invasively estimated from the surface electromyogram, it could be readily adopted as an alternative to the more invasive bioptic sampling for muscle fiber CSA estimation.

\section{References}

1. Del Vecchio et al., J Appl Physiol, 2017.

2. Andreassen and Arendt-Nielsen, J Physiol, 1987.

3. Hakansson, Acta Physiol Scand, 1956.

4. Blijham et al., J Appl Physiol, 1985.

5. Methenitis et al., Med Sci Sports Exerc, 2016.

\section{4}

\section{Deficits in knee extension strength following anterior cruciate ligament reconstruction are explained by a reduced neural drive to the quadriceps muscle}

$\underline{\text { Stefano Nuccio }}^{1}$, Alessandro Del Vecchio ${ }^{2}$, Andrea Casolo ${ }^{3}$, Luciana Labanca $^{1}$, Jacopo Emanuele Rocchi ${ }^{4}$, Francesco Felici ${ }^{1}$, Andrea Macaluso $^{1}$, Pier Paolo Mariani ${ }^{4}$, Deborah Falla ${ }^{5}$, Dario Farina ${ }^{6}$, Paola Sbriccoli ${ }^{1}$

Università Degli Studi Di Roma "foro Italico", Dipartimento Di Scienze Motorie, Umane E Della Salute, Rome, Italy"

Friedrich-alexander University, Department of Artificial Intelligence In Biomedical Engineering, Erlangen-nuremberg, Germania ${ }^{2}$; Università Degli Studi Di Padova, Dipartimento Di Scienze Biomediche, Padova, Italy ${ }^{3}$; Villa Stuart Sport Clinic, Villa Stuart Sport Clinic -fifa Medical Centre of Excellence, Rome, Italy ${ }^{4}$;

University of Birmingham, Centre of Precision Rehabilitation For Spinal Pain (cpr Spine), School of Sport, Exercise and Rehabilitation Sciences, Birmingham, Regno Unito ${ }^{5}$; Imperial College London, Department of Bioengineering, London, Regno Unito ${ }^{6}$

Purpose: Persistent quadriceps strength deficits are common following anterior cruciate ligament reconstruction (ACLR) and are related to increased risk of reinjury. The underlying adaptations occurring in the activity of spinal motoneurons are still unexplored. This study examined the discharge characteristics of large populations of motor units (MUs) in the vastus lateralis (VL) and vastus medialis (VM) muscles following ACLR.

Methods: Nine ACLR soccer players and ten controls performed unilateral isometric knee extensions at $35 \%, 50 \%$ and $70 \%$ of the maximal voluntary isometric force (MVIF). High-density surface electromyography (HDsEMG) was used to record the myoelectrical 
activity of the VL and VM muscles of both lower limbs. HDsEMG signals were decomposed with a convolutive blind source separation method and MU properties were extracted and compared between sides and groups.

Results: The reconstructed side of the ACLR group showed a lower MVIF compared to the contralateral side $(\mathrm{P}<0.001)$. This deficit in MVIF was accompanied by reduced MU discharge rates $(\mathrm{P}<0.05)$, lower absolute MU recruitment and derecruitment thresholds $(<0.05)$ and lower input-output gain of motoneurons $(\mathrm{P}=0.009)$. The side-toside difference in MVIF was associated with the side-to-side difference in MU discharge rates $(\mathrm{P}<0.05)$ and with the side-to-side difference in absolute MU recruitment and derecruitment thresholds $(\mathrm{P}<0.05)$. A strong between-side correlation was found for $\mathrm{MU}$ discharge rates of the VL of ACLR individuals $(\mathrm{P}<0.01)$. There were no significant between-group differences $(\mathrm{P}>0.05)$.

Conclusions: These results indicate that mid-to-long term quadriceps strength deficits after ACLR may be explained by a reduced neural drive to the vasti muscles, with potential changes occurring in both excitatory and inhibitory synaptic inputs. These findings provide novel insights on the neural underpinnings of quadriceps weakness following ACLR. A full understanding of this impairment can help to develop effective rehabilitation protocols to regain muscle strength and reduce the risk of reinjury.

References

1. Nuccio S, Del Vecchio A, Casolo A, Labanca L, Rocchi JE, Felici F, .. \& S Sbriccoli P (2020). Muscle fiber conduction velocity in the vastus lateralis and medialis muscles of soccer players after ACL reconstruction. Scand J Med Sci Sports 30, 1976-1984.

2. Lepley AS, Gribble PA, Thomas AC, Tevald MA, Sohn DH \& Pietrosimone BG (2015). Quadriceps neural alterations in anterior cruciate ligament reconstructed patients: A 6-month longitudinal investigation. Scand J Med Sci Sport 25, 828-839.

\section{5}

\section{The effect of mental fatigue on endurance performance at different exercise-intensity domains}

\author{
Alessio Marciano $^{1}$, Chiara Gattoni $^{2}$, Simone Villanova ${ }^{1}$, Giulia \\ Papetti $^{3}$, Beatrice Banfo ${ }^{3}$, Samuele Maria Marcora ${ }^{4}$, Simone \\ Porcelli \\ University of West Scotland, Institute For Clinical Exercise \\ and Health Science, Glasgow, Regno Unito ${ }^{1}$; \\ University of Kent, School of Sports and Exercise Sciences, \\ Canterbury, Regno Unito ${ }^{2}$; \\ University of Pavia, Department of Molecular Medicine, Pavia, \\ Italy $^{3}$; \\ University of Bologna, Department of Biomedical Science \\ and Neuromotor Sciences Dibinem, Bologna, Italy ${ }^{4}$
}

Purpose: Mental Fatigue (MF) is a psychobiological state caused by prolonged periods of demanding cognitive activity ${ }^{1}$ that can lead to both cognitive and physical performance impairments. Some authors have demonstrated detrimental effects of MF on endurance performance $^{2}$ but the results are controversial. Different studies ${ }^{34}$ have suggested the higher the exercise intensity the lower the impact of MF on performance, but no studies have directly investigated whether ergolytic effects of MF on endurance performance are modulated by exercise-intensity domains. Thus, we evaluated the effect of MF on time to exhaustion during cycling exercise at heavy- and severeintensity.
Methods: Fifteen young athletes (12 M; $3 \mathrm{~W}$; age $24 \pm 4$ years) performed an incremental cycling test to exhaustion followed by a verification trial. Critical power (CP) was estimated from a 2 min allout test ${ }^{5}$. Following a randomized double-blind cross-over placebocontrolled study design, subjects underwent four testing sessions, each of them composed by either 45-min documentary watching (CTRL) or 45-min Flanker Task (MT) followed by time to exhaustion (TTE) tests at heavy $(90 \% \mathrm{CP} ; \mathrm{H})$ or severe $(110 \% \mathrm{CP}, \mathrm{S})$ intensity. Pulmonary gas exchange, lactate concentration ([La]) in the capillary blood and heart rate (HR) were recorded during exercise. Subjective rating of MF was assessed by VAS scale after both MT and exercises. Physical workload (PW) was evaluated by NASA TLX questionnaire. Motivation was assessed after MT by Intrinsic motivation scale.

Results: Maximal oxygen uptake (V'O2max) and Maximal Workrate were $53.0 \pm 10 \mathrm{ml} * \mathrm{~min}-1 * \mathrm{~kg}-1$ and $366 \pm 82 \mathrm{w}$, respectively. $\mathrm{CP}$ was $284 \pm 72 \mathrm{w}$. TTE was significantly lower after MT in both $\mathrm{H}$ $(2262 \pm 1237 \mathrm{~s}$ vs $2037 \pm 1308 \mathrm{~s}, \mathrm{p}=0.042)$ and $\mathrm{S}(537 \pm 239 \mathrm{~s}$ vs $408 \pm 309 \mathrm{~s}, \mathrm{p}=0.045$ ). During $\mathrm{H}$, physiological responses to exercise were not affected by MT whereas, during S, V'O2, [La] and HR were lower in MT vs CTRL. Motivation was not different among conditions $(p>0.112)$. VAS scale was significantly higher after MT than CTRL, both before $(14.0 \pm 3.6$ vs $5.0 \pm 5.1, \mathrm{p}=0.001)$ and after exercise $(12.9 \pm 3.3$ vs $5.0 \pm 5.1, \mathrm{p}=0,01)$.

Conclusions: A prolonged MT significantly impaired exercise performance to a similar extent (about 9\%) in both heavy and severe domains. The ergolytic effects of MT were associate to higher subjective levels of mental fatigue, despite no changes in motivation. Thus, performance impairments induced by mental fatigue seem not to be exercise-intensity dependent.

\section{References}

1. Boksem \& Tops (2008) Mental fatigue: Costs and benefits. Brain Res.

2. Marcora et al. (2009) Mental fatigue impairs physical performance in humans. J. Appl. Physiol.

3. Martin et al. (2018) Mental Fatigue Impairs Endurance Performance. Sport. Med.

4. Van Cutsem et al. (2017) The Effects of Mental Fatigue on Physical Performance. Sport. Med.

5. Murgatroyd et al. (2014) A 'ramp-sprint' protocol to characterise indices of aerobic function and exercise intensity domains $[\ldots]$. Eur. J. Appl. Physiol.

76

\section{The effect of leg preference on mechanical efficiency} during single-leg extension exercise

Massimo Venturelli ${ }^{1}$, Cantor Tarperi ${ }^{2}$, Chiara Milanese ${ }^{I}$, Luca Festa $^{I}$, Luana Toniolo ${ }^{3}$, Carlo Reggiani ${ }^{3}$, Federico Schena ${ }^{1}$

Università Di Verona, Neuroscienze Biomedicina E Movimento, Verona, Italy ${ }^{1}$; Uiversità Di Torino, Scienze Cliniche E Biologiche, Torino, Italy ${ }^{2}$; Università Di Padova, Scienze Biomediche, Padova, Italy

Purpose: To investigate how leg preference affects net efficiency ( $\eta$ net), we examined central and peripheral hemodynamics, muscle fiber type, muscle activation and force of preferred (PL) and nonpreferred (NPL) leg. Our hypothesis was that PL greater efficiency could be explained by adaptations and interactions between central, peripheral factors and force.

Methods: Fifteen young participants performed single-leg extension exercise at absolute $(35 \mathrm{~W})$ and relative $(50 \%$ peak power-output (Wpeak)) workloads with PL and NPL. Oxygen uptake, 
photoplethysmography, Doppler ultrasound, near-infrared-spectroscopy (NIRS), integrated electromyography (iEMG), maximal isometric force (MVC), rate of force development (RFD) and muscle biopsies of both vastus lateralis, were studied to assess central and peripheral determinants of $\eta$ net.

Results: During exercise executed at $35 \mathrm{~W}$, ๆnet was $17.5 \pm 5.1 \%$ and $11.9 \pm 2.1 \%(\mathrm{p}<0.01)$ in NP and NPL respectively, while during exercise at the $50 \%$ of Wpeak, was in PL $=18.1 \pm 5.1 \%$ and in NPL $=$ $12.5 \pm 1.9(\mathrm{p}<0.01)$. The only parameter correlated with $\eta$ net was iEMG which showed an inverse correlation for absolute $(r=-0.83$ and -0.69 for PL and NPL) and relative workloads $(r=-0.92$ and -0.79 for PL and NPL). MVC and RFD were higher in PL than in NPL but not correlated to $\eta$ net.

Conclusions: This study identified a critical role of leg preference in the efficiency during single-leg extension exercise. The whole spectrum of the central and peripheral, respiratory, circulatory and muscular determinants of $\eta$ net did not explain the difference between PL and NPL efficiency. Therefore, the lower muscle activation exhibited by the PL is likely the primary determinant of this physiological phenomenon.

\section{References}

1. Poole DC, et al. Pulmonary and leg VO2 during submaximal exercise: implications for muscular efficiency. J Appl Physiol (1985) 72: 805-810, 1992.

2. Carpes FP, et al. Influence of leg preference on bilateral muscle activation during cycling. Journal of sports sciences 29: 151-159, 2011.

3. Arevalo JA, et al. Lower-Limb Dominance, Performance, and Fiber Type in Resistance-trained Men. Med Sci Sports Exerc 50: 1054-1060, 2018.

\section{7}

\section{The effects of a 12-week stretching training program on the plantarflexor muscles mechanical properties}

\author{
Stefano Longo ${ }^{l}$, Emiliano Ce ${ }^{l}$, Angela Valentina Bisconti ${ }^{2}$, Susanna \\ Rampichini $^{I}$, Christian Doria ${ }^{l}$, Marta Borrelli ${ }^{l}$, Eloisa Limonta ${ }^{1}$, \\ Giuseppe Coratella ${ }^{l}$, Fabio Esposito ${ }^{1}$ \\ Università Degli Studi Di Milano, Department of Biomedical \\ Sciences For Health, Milan, Italy ${ }^{1}$; \\ The University of Utah, Department of Internal Medicine, Salt Lake \\ City, $U S A^{2}$
}

Purpose: There is no consensus about the effects of passive stretching training (PST) on the mechanical properties of the muscle-tendon complex (MTC), particularly due to short-time low-volume interventions. Moreover, an acute bout of PS induces maximum voluntary contraction (MVC) depression. However, the effects long-term PST on MVC are controversial. Therefore, we investigated the effects of long-time high-volume PST on passive stiffness and MVC of plantarflexor muscles.

Methods: Thirty healthy adults participated in the study. Fifteen participants (STR; $\mathrm{N}=15,6$ women, 9 men; age $22.3 \pm 0.8$ years, body mass $68.5 \pm 9.4 \mathrm{~kg}$, stature $1.74 \pm 0.08 \mathrm{~m}$ ) underwent 12-week plantarflexor muscles PST $[(5 \times 45 \mathrm{~s}$-on/15 s-off $) \times 2$ exercises $] \times$ 5times/week (duration: $2250 \mathrm{~s} /$ week), while 15 participants (CTRL: $\mathrm{N}=15,6$ women, 9 men; age $23.4 \pm 0.8$ years, body mass $67.4 \pm$ $9.5 \mathrm{~kg}$, stature $1.73 \pm 0.08 \mathrm{~m}$ ) served as control (no PST). Range of motion (ROM), maximum passive resistive torque (PRTmax), passive MTC and muscle stiffness, and plantarflexors maximum voluntary contraction (MVC), were calculated Pre, at the 6th (Wk6), and the 12th week (Wk12) of the protocol in both groups.
Results: Compared to Pre, in STR ROM increased $(\mathrm{P}<0.05)$ at Wk6 $(8 \%)$ and $\mathrm{Wk} 12$ (23\%). PRTmax increased at $\mathrm{Wk} 12(30 \%, \mathrm{P}<0.05)$, while MTC stiffness decreased $(16 \%, \mathrm{P}<0.05)$. Muscle stiffness decreased $(\mathrm{P}<0.05)$ at $\mathrm{Wk6}(11 \%)$ and $\mathrm{Wk} 12(16 \%)$. No changes in MVC was found in STR (P $>0.05$ ). Percentage changes in ROM correlated with percentage changes in PRTmax $(\rho=0.62, P=0.01)$ and MTC stiffness $(\rho=-0.78, P=0.001)$. In CTRL, no changes $(P>$ 0.05 ) occurred in any variables at any time point.

Conclusions: The expected long-term PST-induced changes in ROM were associated with modifications in the whole passive mechanical properties of the ankle joint, while maximum force-generating capacity characteristics were preserved.

\section{References}

1. Medeiros DM, and Lima CS. (2017) Influence of chronic stretching on muscle performance: Systematic review. Hum Mov Sci 54: 220-229.

2. Longo S, Cè E, Bisconti AV, Rampichini S, Doria C, Borrelli M, Limonta E, Coratella G, Esposito F. (2021) The effects of 12 weeks of static stretch training on the functional, mechanical, and architectural characteristics of the triceps surae muscletendon complex. Eur J Appl Physiol 121(6):1743-1758.

\section{8}

\section{Changes in hamstrings volume and architecture in response to 10-day bed rest}

Fabio Sarto ${ }^{1}$, Elena Monti ${ }^{1}$, Bostjan Šmunic ${ }^{2}$, Rado Pišot ${ }^{2}$, Marco V. Narici $^{1}$, Martino V. Franchi ${ }^{1}$

University of Padova, Department of Biomedical Sciences, Padova, Italy $^{1}$;

Science and Research Centre Koper, Science and Research Centre Koper, Koper, Slovenia ${ }^{2}$

Purpose: The hamstrings (HS) muscle group plays a fundamental role in maintaining knee stability, thus contributing to the prevention and rehabilitation of lower limb musculoskeletal injuries1. However, little is known about HS structural adaptations after periods of disuse/ inactivity. The purpose of this study was to investigate the morphological changes in the HS during 10 days of bed rest (BR).

Methods: Ten young healthy males underwent a 10-d BR. Before (BR0) and after (BR10) the BR period, HS muscle volume was assessed by MRI, while biceps femoris long head (BFlh) muscle architecture was evaluated by ultrasound (US). BFlh fascicle length (Lf) was obtained using 1) panoramic US (extended-field-of-view EFOV-technique), 2) the manual linear extrapolation (MLE) method, and 3) two trigonometric equations (Eq A and B) from a single B-Mode US snapshot.

Results: A significant reduction in muscle volume was observed in BFlh $(\mathrm{p}=0.0019 ; \Delta=-3.53 \%)$, biceps femoris short head $(\mathrm{p}=$ $0.0023 ; \Delta=-3.54 \%)$, semitendinosus $(\mathrm{p}=0.0023 ; \Delta=-2.63 \%)$, semimembranosus $(\mathrm{p}=0.0019 ; \Delta=-2.01 \%)$ and HS pooled together $(\mathrm{p}<0.001 ; \Delta=-2.78 \%)$. No differences were detected in BFlh MT e PA, while we found a little reduction in Lf for EFOV ( $<<$ $0.001 ; \Delta=-2.84 \%)$ and MLE $(\mathrm{p}=0.0082 ; \Delta=-1.94 \%)$ techniques. Differences between $\mathrm{Eq} \mathrm{A}$ and the other US methods were observed at both time points for BFlh Lf evaluation, while BlandAltman analyses highlighted relevant average absolute biases between EFOV and other methods. A significant correlation $(r=0.83 ; p=$ 0.0033 ) in percentage of change in BFlh Lf values was reported only between EFOV and MLE.

Conclusions: Despite being a non-postural muscle group, the HS exhibited a moderate, but significant, reduction in muscle volume in 
response to a short period of unloading. Instead, only limited architectural changes in the BFlh were observed. Noteworthy, we showed that different US methods lead to different results in the assessment of BFlh Lf adaptations, similarly to what previously reported for a single time point2. The implementation of EFOV scans (or the MLE method with a large field of view) to assess BFlh Lf changes is warranted by the present results.

Acknowledegements: The present study was funded by ASI, MARSPRE, Project n. DC-VUM-2017-006.

References

1. Baratta, R. et al. (1988) Muscular coactivation. The role of the antagonist musculature in maintaining knee stability. Am. J. Sports Med. 16: 113-122.

2. Franchi, M. V., Fitze, D. P., Raiteri, B. J., Hahn, D. \& Sporri, J. (2019) Ultrasound-derived biceps femoris long head fascicle length: extrapolation pitfalls. Med. Sci. Sport. Exerc. 52: 233-243.

\section{FISIOLOGIA DELLO SPORT 3}

\section{9}

\section{The effect of endurance training on pulmonary VO2 kinetics in solid organs transplanted recipients}

Alessio Del Torto ${ }^{1}$, Carlo Capelli ${ }^{2}$, Roberto Peressutti ${ }^{3}$, Adriana Di $\overline{\text { Silvestre }^{3}, \text { Ugolino Livi }}{ }^{4}$, Chiara Nalli ${ }^{4}$, Sandro Sandro ${ }^{4}$, Gianpaolo Amici $^{5}$, Umberto Baccarani ${ }^{6}$, Stefano Lazzer ${ }^{7}$

University of Udine, Department of Medical Area, Udine, Italy ${ }^{1}$; University of Verona, Department of Neurosciences, Biomedicine and Movement Sciences, Udine, Italy,

University Hospital of Udine, Regional Transplantation Centre, Friuli Venezia Giulia Region, Udine, Italy ${ }^{3}$;

University Hospital of Udine, Cardiac Surgery Unit, Udine, Italy ${ }^{4}$;

San Daniele Del Friuli Hospital, Nephrology and Dialysis Unit, San Daniele Del Friuli, Italy ${ }^{5}$;

University Hospital of Udine, Liver-kidney Transplant Unit, Udine, Italy

University of Udine, School of Sport Sciences, Udine, Italy ${ }^{7}$

Purpose: Heart, kidney, and liver transplant recipients (HTx, KTx, and LTx, respectively) are characterized by poor level of cardiorespiratory fitness; in fact, they suffer from reduced peak pulmonary $\mathrm{O} 2$ uptake and slower pulmonary uptake $(\mathrm{VO} 2 \mathrm{p})$ kinetics when compared to healthy mates of similar age. For a given constant work rate of moderate intensity (CWR-MOD), the slower the VO2p kinetics response, the greater the time for the VO2p to attain the steady state, and the higher intracellular perturbation (e.g., accumulation of $\mathrm{H}+$ and depletion of $\mathrm{PCr}$ ) will occur, indicating an impaired muscular metabolic stability. Hence, sluggish kinetics would herald a reduced exercise capacity and tolerance. Exercise training is known to improve VO2p kinetics in healthy subjects and in clinical populations; therefore, we investigated the effects of single and double leg cycling endurance training (SL-ET and DL-ET, respectively) on VO2p kinetics in 33 transplanted recipients $(\mathrm{HTx}=13, \mathrm{KTx}=11$, and LTx =9). The O2 deficit (O2Def) and the mean response time (MRT) of VO2p kinetics during CWR-MOD and the amplitude of VO2p slow component (SCamp), during heavy intensity constant work rate exercise (CWR-HVY) were studied.

Methods: Patients performed a double leg cycling incremental step test to exhaustion, two CWR-MOD tests, and a CWR-HVY trial before and after the training program. SL-ET group $(n=17)$ and DLET group $(\mathrm{n}=16)$ performed 24 sessions of SL-ET and DL-ET, respectively. $\mathrm{VO} 2 \mathrm{p}$ was measured breath-by-breath during each experiment

Results: After DL-ET, O2Def, MRT and SCamp decreased (i.e. improved) by $24.9 \% \pm 16.2(\mathrm{P}<0.0001), 25.9 \% \pm 13.6(\mathrm{P}<0.0001)$ and $38 \% \pm 52(\mathrm{P}=0.0003)$, respectively. In response to SL-ET, O2Def, MRT and SCamp dropped by $16.4 \% \pm 13.7(\mathrm{P}=0.008)$, $15.6 \% \pm 13.7(\mathrm{P}=0.004)$ and $35 \% \pm 31(\mathrm{P}=0.002)$, respectively. The O2Def, MRT, and SCamp improved by the same order of magnitude and were not significantly different between SL-ET and DL-ET following the training program.

Conclusions: The results indicate that SL-ET is as effective as DLET in transplanted recipients to improve VO2p kinetics during moderate intensity exercise and to lower the SCamp during heavy intensity exercise. The findings suggest that the slower VO2p kinetics might be caused by the impairment of peripheral gas exchanges.

\section{References}

1. Tomczak CR, Warburton DER, Riess KJ, et al. Pulmonary oxygen uptake and heart rate kinetics during the six-minute walk test in transplant recipients. Transplantation. 2008;85(1):29-35. https://doi.org/10.1097/01.tp.0000296056.00863.f0

2. Tomczak CR, Tymchak WJ, Haykowsky MJ. Effect of exercise training on pulmonary oxygen uptake kinetics in heart transplant recipients. Am J Cardiol. 2013;112(9):1489-1492. https://doi. org/10.1016/j.amjcard.2013.06.037

\section{0}

The effect of cigarette smoking on the cardiorespiratory and metabolic kinetics in young trained males

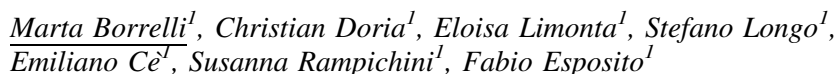

Università Degli Studi Di Milano, Department of Biomedical Sciences For Health, Milan, Italy ${ }^{1}$

Purpose: Cigarette smoking is a well-known risk factor for cardiopulmonary disease and a major cause of mortality, leading to systemic modifications that affect the cardiorespiratory response to exercise. Indeed, nicotine stimulates the sympathetic nervous system, elevating the cardiac work. Likewise, tar impairs oxygen diffusion across the alveolar-capillary interface and carbon monoxide causes a less efficient oxygen transport and extraction. Despite the impact of acute cigarette smoking on the cardiorespiratory and metabolic response to exercise has been already assessed, the effect of long-term cigarette smoking has not been yet investigated.

Methods: Six trained male smokers (SM; $13 \pm 5$ cigarette/day; age: $21 \pm 2$ yr., body mass: $77 \pm 6 \mathrm{~kg}$; mean \pm SD) and five non-smokers (CTRL; age: $26 \pm 4$ yr., body mass: $81 \pm 11 \mathrm{~kg}$ ) were enrolled. After assessing the maximum pulmonary oxygen uptake (V'O2max) on a cycle ergometer, the participants performed in different days two sets, each consisting in two step transitions to a 6-min moderate exercise (90\% of gas exchange threshold) separated by a 30-min resting recovery. Cycling cadence was constrained to remain between 60-70 rpm. Expiratory ventilation (V'E), carbon dioxide production (V'CO2), V'O2 and heart rate $(\mathrm{fH})$ responses were fitted by a monoexponential function. The amplitude (AMP), starting value (Y0) the time constant $(\tau)$ of all parameters were calculated during the increasing and decreasing phases.

Results: Despite a similar V'O2max between the two groups, the $\mathrm{fH}$ $\mathrm{Y} 0$ was higher in SM than in CTRL in the increasing ( $89 \pm 8$ vs $77 \pm$ $6 \mathrm{bpm}$, respectively; $\mathrm{P}=0.02)$ and decreasing phase ( $146 \pm 11$ vs 132 $\pm 10 \mathrm{bpm}$, respectively; $\mathrm{P}=0.06$ ). No differences in the metabolic parameters were observed. 
Conclusions: These findings suggest that the long-term cigarette smoking slowed down only the off-phase of the cardiac response to a moderate exercise transition. On the contrary, the respiratory and metabolic kinetics seemed to be unaffected by chronic smoking, likely because of the high fitness level of the participants.

References

1. Murias, J.M., Spencer, M.D., DeLorey, D.S., et al. (2011). Speeding of VO2 kinetics during moderate-intensity exercise subsequent to heavy-intensity exercise is associated with improved local $\mathrm{O} 2$ distribution. Journal of applied physiology, 111(5), 1410-1415.

2. Rotstein, A., Sagiv, M., Yaniv-Tamir, A., Fisher, N., \& Dotan, R. (1991). Smoking effect on exercise response kinetics of oxygen uptake and related variables. International journal of sports medicine, 12(03), 281-284.

\section{1}

Acute physiological and metabolic responses to a highintensity continuous training and three high-intensity interval training regimes in active university students

\section{Marco Panasci $^{I}$, Emanuela Faelli ${ }^{1}$, Ambra Bisio ${ }^{1}$, Piero Ruggeri ${ }^{l}$ Vittoria Ferrandol \\ Università Degli Studi Di Genova, Dipartimento Di Medicina Sperimentale, Genova, Italy ${ }^{I}$}

Purpose: The aim of this study was to compare the acute cardiorespiratory and metabolic effects induced by a High-Intensity Continuous Training (HI-CT) and three High-Intensity Interval Training regimes (HIITs) in young active students. The assessment of internal training load through the rate of perceived exertion (RPE) at iso-time was also a focus.

Methods: Fifteen active university students (age: $21.0 \pm 1.1 \mathrm{ys,}$ height: $1.74 \pm 0.9 \mathrm{~m}$, weight: $64.8 \pm 12.9 \mathrm{~kg}$, VO2max $48.1 \pm$ $7.5 \mathrm{~mL} \bullet \mathrm{kg}-1 \bullet \mathrm{min}-1)$ performed an incremental treadmill test to assess VO2max and maximal aerobic speed (MAS). Then, each participant performed, on testing sessions, separated by a minimum of $72 \mathrm{~h}$ and in a randomized order, one HI-CT session at 95\% MAS for $8 \mathrm{~min}$ and three different HIIT sessions $(10 \mathrm{~s}-20 \mathrm{~s}, 30 \mathrm{~s}-30 \mathrm{~s}, 50 \mathrm{~s}-$ $30 \mathrm{~s}$ ) with a work phase at $95 \%$ MAS and an active recovery at $40 \%$ MAS for 16 min. Oxygen consumption (VO2), heart rate (HR), time spent near or above 90\%VO2max (T@90\%VO2max) and blood lactate concentration $([\mathrm{La}]+)$ were measured. The rating of perceived exertion (RPE), by the Borg's CR 10 scale, was used to assess the subjective level of fatigue experienced at the end of each testing session.

Results: The statistical analysis revealed that VO2peak, HRpeak, T@90\%VO2max, and RPE values in 10-20 HIIT were significantly lower compared to 30-30 and 50-30 HIIT regimes (p always < 0.001). In HI-CT session VO2peak, HRpeak, T@90\%VO2max were significantly higher than in $10-20$ and $30-30$ sessions $(\mathrm{p}<0.0001$ and $\mathrm{p}<0.05$ ). Blood lactate values were significantly higher in HI-CT and in 50-30 and 30-30 HIITs, compared to $10-20$ protocol. Finally, internal workload in HI-CT and 50-30 HIIT was significantly higher than those in $30-30(\mathrm{p}<0.01)$ and $10-20(\mathrm{p}<0.0001)$ HIIT sessions. Conclusions: Our results show that in active young subjects a HI-CT and a long work interval HIIT regime (50-30) represent an optimal training stimulus to elicit higher physiological and metabolic responses compared to short work interval HIIT regimes (30-30 and 10-20), being the most effective for stressing both the aerobic and anaerobic energy systems. Coaches can choose and balance the level of aerobic/anaerobic energy requirements and neuromuscular engagement associated with the three HIIT regimes and a HI-CT protocol based on the expected physiological and metabolic adaptations as well as neuromuscular characteristic and ability level of the athlete/subject individual profile.

82

Effects of 3-month polarized training vs. high intensity interval training on cardiorespiratory fitness, body composition and fat metabolism in obese adults

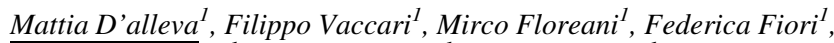
Michela Marinoni ${ }^{l}$, Maria Parpinel ${ }^{1}$, Stefano Lazzer ${ }^{1}$

Università Degli Studi Di Udine, Dipartimento Di Area Medica, Udine, Italy ${ }^{1}$

Purpose: The positive effects of High Intensity Interval Training (HIIT) and Moderate Intensity Continuous Training (MICT) on peak $\mathrm{O} 2$ uptake (V'O2peak) and body composition are well documented in obese adults. However, the combination of HIIT and MICT, also called Polarized Training (POL), often used in highly trained endurance athletes, have not been verified in adult males with obesity. The purpose of this study was to investigate changes in body composition, physical capacities and fat oxidation rate, induced by a 3-month either POL or HIIT in a real world setting.

Methods: Forty male patients (mean age $39 \mathrm{y}$; mean body mass index [BMI] $33 \mathrm{~kg} \bullet \mathrm{m}-2$ ) participated in this study (n: 20 POL, n: 20 HIIT), attending $\sim 36$ sessions of training, online supervised by researchers. At baseline (PRE), at the end of the training period (POST) body composition, $\mathrm{V}^{\prime} \mathrm{O} 2$ peak, and fat oxidation rate were measured. The POL group performed $\sim 45$ min of training per session, while HIIT group performed $\sim 33 \mathrm{~min}$ of training per session. The two training programs were iso-caloric.

Results: At POST, body mass (BM) and fat mass (FM) decreased by 5 and $-11 \%,(\mathrm{P}<0.05)$ in POL and HIIT, respectively, $\mathrm{V}^{\prime} \mathrm{O} 2$ peak increased in both groups by +12 and $+9 \%$, respectively $(\mathrm{P}<0.05)$. Maximal fat oxidation rate increased in POL and HIIT by 4 and $9 \%$, respectively $(\mathrm{P}<0.05)$, without significant difference between the two groups.

Conclusions: POL was more effective in improving V'O2peak than HIIT, without difference in fat oxidation. The guidelines of American College and Sport Medicine (ACSM) for physical activity in adult obese males (age 18-75) advise to perform a minimum of $150 \mathrm{~min}$ per week of MICT, or 75 min per week of HIIT, without giving details of their combination. The results of this study could provide the basis for a correct combination of HIIT and MICT during a single training session, in a weekly schedule, for optimize aerobic fitness in obese subjects.

References

1. Su, L., Fu, J., Sun, S., Zhao, G., Cheng, W., Dou, C., \& Quan, M. 0.2019.. Effects of HIIT and MICT on cardiovascular risk factors in adults with overweight and/or obesity: A meta-analysis. PloS One. 14:1

2. Stöggl, T., and Sperlich, B. 2014. Polarized training has greater impact on key endurance variables than threshold, high intensity, or high volume training. Front. Physiol. 5: 33. 
83

\section{Is heart rate a sensitive indicator of aerobic demand during shuttle run?}

Susanna Rampichini ${ }^{I}$, Eloisa Limonta ${ }^{1}$, Marta Borrelli ${ }^{l}$, Emiliano $\bar{C}^{I}$, Giuseppe Coratella ${ }^{l}$, Stefano Longo ${ }^{I}$, Angela Valentina Bisconti $^{1}$, Matteo Zago ${ }^{2}$, Filippo Bertozzi ${ }^{1}$, Chiarella Sforza ${ }^{1}$, Fabio Esposito $^{l}$

Università Degli Studi Di Milano, Dipartimento Di Scienze Biomediche Per La Salute, Milan, Italy ${ }^{1}$;

Politecnico Di Milano, Department of Electronics, Information and Bioengineering (deib), Milan, Italy ${ }^{2}$

Purpose: The relationship between heart rate (HR) and pulmonary oxygen uptake (VO2), namely HRvsVO2, has been already extensively evaluated to assess the validity of $\mathrm{HR}$ as a sensitive indicator of the metabolic demands during different physical activities. The assessment of $\mathrm{HRvsVO} 2$ as an indicator of the aerobic system engagement has been demonstrated during soccer-specific exercises, whereas it has been rejected during badminton simulations and indoor climbing. Whether or not shuttle runs present a HRvsVO2 similar to that obtained during standard forward running test is still unknown. Therefore, this study aimed at assessing the validity of HR as a valid indicator of aerobic demand by comparing $\mathrm{HRvSVO} 2$ obtained during traditional forward running to that obtained during shuttle runs at different speeds.

Methods: Thirteen participants (age: $21 \pm 2$ years.; body mass: 71.3 $\pm 5.9 \mathrm{~kg}$; stature: $1.73 \pm 0.07 \mathrm{~m}$; mean \pm standard deviation) performed a discontinuous incremental test (IIAT) on a treadmill for maximum VO2 and maximum aerobic velocity (VAM) assessment, during which metabolic and HR data were collected. On a different day, the HRvsVO2 was evaluated during two 5-min shuttle runs sets at different speeds: $50 \%$ (low speed, LS) and 75\% (high speed, HS) of the VAM, and compared with the values determined during IIAT. A paired t-test was used to check for possible differences in cardiorespiratory and metabolic parameters between HS and LS, and between slopes and intercepts of HRvsVO2 obtained during the IIAT and shuttle tests.

Results: VO2 and HR were significantly higher at HS than LS (+ $15 \%$ and $+16 \%$, respectively; $p<0.05$ ). Both slope and intercept of HRvsVO2 during shuttle tests were similar to those obtained during the IIAT $(\mathrm{p}=0.942$ and $\mathrm{p}=0.526$ for slope and intercept, respectively).

Conclusions: A similar HRvsVO2 was observed during shuttle runs and standard incremental test, suggesting the use of HR as a sensitive indicator to monitor the aerobic demand during shuttle tests. These findings provide support for all those sport activities with frequent occurrence of changes of direction, such as small-sided games.

\section{References}

1. Esposito et al.Validity of heart rate as an indicator of aerobic demand during soccer activities in amateur soccer players.EJAP(2004)93:167-172.

2. Rampichini et al.Heart rate and pulmonary oxygen uptake response in professional badminton players: comparison between on-court game simulation and laboratory exercise testing.EJAP(2108)118:2339-2347.

3. Limonta et al.Cardiovascular and metabolic responses during indoor climbing and laboratory cycling exercise in advanced and élite climbers.EJAP(2018)118:371.
ESERCIZIO, PREVENZIONE E TERAPIA 4

84

\section{Did children's physical activity change differently in boys and girls before and after school closures during COVID-19 pandemic? Results from the I-move study}

Alice Masini $^{1}$, Sofia Marini ${ }^{1}$, Giuseppe Barone ${ }^{2}$, Laura Bragonzoni ${ }^{2}$, Stefania Toselli $^{1}$, Alessia Tessari ${ }^{3}$, Maria Cristina Bisi ${ }^{4}$, Rita Stagni ${ }^{4}$, Alessandra Sansavini ${ }^{3}$, Marcello Lanari ${ }^{5}$, Andrea Ceciliani ${ }^{2}$, Laura Dallolio $^{1}$

University of Bologna, Department of Biomedical and Neuromotor Sciences, Bologna, Italy ${ }^{1}$;

University of Bologna, Department For Life Quality Studies, Rimini, Italy $^{2}$;

University of Bologn, Department of Psychology, Bologna, Italy ${ }^{3}$; University of Bologna, Department of Electrical, Electronic, and Information Engineer, Bologna, Italy ${ }^{4}$;

University of Bologna, Paediatric Emergency Unit, Irccs Azienda Ospedaliero-universitaria Di Bologna, Bologna, Italy ${ }^{5}$

Purpose: The World Health Organization (WHO) recommend children to perform at least $60 \mathrm{~min}$ of Physical Activity (PA) per day to obtain benefits in terms of general health, quality of life and mental wellbeing. Globally children did not comply with this guidelines and the SARS-CoV-2 pandemic could have intensified this gap affecting negatively the children's PA habits. The aim of this study was to investigate gender differences in PA levels before and after school closures during Covid-19 using a randomized convenience sample from the Imola Active Break Study (I-MOVE).

Methods: Pre post change $(\Delta)$ in PA levels from November 2019 and January 2021 was calculated using Actigraph accelerometers considering gender differences. We analysed respectively Weekly and daily time spent in Moderate to Vigorous PA (W-MVPA; D-MVPA), Weekly times spent in Sedentary Behaviour (W-SB) and type of PA intensity divided in Moderate and Vigorous PA (respectively MPA and VPA). Statistical analysis was conducted, through SPSS, using Paired T-test and ANOVA.

Results: Our convenience sample was $\mathrm{N}=77$ children (48 boys and 29 girls, mean age $=7.83 \pm 1.41)$. Weekly and daily time spent in moderate to vigorous PA significantly decreased $(\triangle \mathrm{W}-\mathrm{MVPA}=$ $30.99 \pm 104.93 ; \Delta \mathrm{D}-\mathrm{MVPA}=-15.32 \pm 16.21 ; \mathrm{p}<0.05)$. Total time spent in MPA and VPA significantly dropped $(\triangle \mathrm{MPA}=-15.80 \pm$ 65.86; $\Delta \mathrm{VPA}=-15.19 \pm 46.06 ; \mathrm{p}<0.05)$. On the contrary total time spent in sedentary activity increased $(\Delta \mathrm{W}-\mathrm{SB}=1196.01 \pm 381.49 ; \mathrm{p}$ $<0.05)$. Considering gender differences, the most significant results were highlighted in decreasing of daily MVPA and Vigorous intensity of PA in boys rather than girls $(\triangle \mathrm{D}-\mathrm{MVPA}$ boys $=-19.54 \pm 16.55 \mathrm{vs}$ girls $=-8.32 \pm 13.13 . \mathrm{p}<0.05)(\Delta \mathrm{VPA}:$ boys $=24.12 \pm 49.00 \mathrm{vs}$ girls $=-0,4195 \pm 36.96 ; p<0.05)$. Regarding sedentary habit, boys raised more minute spent in sedentary activity during Covid-19 than girl (DW-SB: boys $=+1262.42 \pm 387.60$ vs girls $=+1086.39 \pm$ 350.39; $\mathrm{p}<0.05)$.

Conclusions: PA levels in our children sample worsen during the pandemic and children were more sedentary. In November 2019 boys used to have higher PA levels, especially VPA, compared to girls and consequently, during Covid-19, boys seemed to have suffered more from the restrictions imposed in terms of PA levels reduction.

In this scenario, classroom-based PA program, such as Active Breaks in the I-MOVE study, could represent a proposal to re-increased the recommended level of PA and reduce the sedentary behaviour, especially in boys. 


\section{References}

1. WHO. WHO Guidelines on Physical Activity and Sedentary Behaviour; WHO: Geneva, Switzerland. (2020).

2. Ten Velde, G. et al. (2021) Physical activity behaviour and screen time in Dutch children during the COVID-19 pandemic: Pre-, during- and post-school closures. Pediatric obesity, e12779.

\section{5}

\section{Online physical activity and nutritional supplementation: effects on quality of life and disease progression in chronic kidney disease patients}

\author{
Arianna Murri ${ }^{1}$, Elisa Grazioli ${ }^{1}$, Giulia Marrone ${ }^{2}$, Manuela Di \\ Lauro $^{3}$, Silvia Urciuoli ${ }^{4}$, Claudia Cerulli ${ }^{1}$, Eliana Tranchita, \\ Annalisa Romani ${ }^{4}$, Nicola Di Daniele ${ }^{3}$, Attilio Parisi ${ }^{1}$, Annalisa \\ Noce $^{3}$
}

University of Rome "foro Italico", Department of Exercise, Human and Health Sciences, Rome, Italy ${ }^{1}$;

University of Rome Tor Vergata, Phd School of Applied Medical, Surgical Sciences, Rome, Italy ${ }^{2}$;

University of Rome Tor Vergata, Uoc of Internal Medicine-center of Hypertension and Nephrology Unit, Department of Systems Medicine, Rome, Italy ${ }^{3}$;

University of Florence, Phytolab (pharmaceutical, Cosmetic, Food Supplement, Technology and Analysis), Disia, Florence, Italy ${ }^{4}$

Purpose: Chronic kidney disease (CKD), pathological condition that induces or aggravates sarcopenia, is characterized by the loss of muscle mass and strength. CKD decreases the physical capacity (PC) and the quality of life and increases the cardiovascular mortality. Literature data suggest that regular physical activity (PA) in combination with dietetic-nutritional treatments are effective to counteract CKD progression and comorbidities. This pilot study evaluates the potential beneficial effects of 12-weeks online PA protocol, combined with functional bars supplementation on quality of life, functional parameters and CKD progression.

Methods: $10 \mathrm{CKD}$ patients, aged $60 \pm 5.1$ years, were enrolled and randomized in 2 homogeneous groups for age and gender: A) PA protocol combined with functional bars that were analysed for active metabolites and total antioxidant capacity; B) only PA protocol. Quality of life using the 36-item Short Form Health Survey (SF-36), $\mathrm{PC}$, strength and laboratory parameters were evaluated at baseline (T0) and after 12 weeks (T2).

Results: Currently, we analysed preliminary data. 6-min walking test reported an increase of $9.9 \%$ in Group A and of $0.2 \%$ in Group B, whereas only Group B showed an improvement in the strength test (Handgrip right $+0.1 \%$; Handgrip left $+4.1 \%$ ). SF-36 Physical functioning domain increased of $2.8 \%$ and $2.1 \%$ in Group A and Group B respectively. The Role limitations and the Health change scores were higher of $23.1 \%$ and $25 \%$ compared with $\mathrm{T} 0$ outcomes in Group B only. In both groups, we observed an increase of estimatedglomerular filtration rate and a reduction of creatinine and, only in the group A, a decrease of azotemia.

Conclusions: Our preliminary data suggest that the study promises to improve not only the knowledge about the impact of PA and nutritional therapy on CKD, but also their combined effect.

\section{References}

1. GBD Chronic Kidney Disease Collaboration.(2020)Global, regional, and national burden of chronic kidney disease, 1990-2017:a systematic analysis for the Global Burden of Disease Study 2017. Lancet. 29;709-733.
2. Hirai K,Ookawara S,Morishita Y.(2016)Sarcopenia and Physical Inactivity in Patients With Chronic Kidney Disease.Nephrourol Mon.26;8(3).

3. Cupisti A,D'Alessandro C,Fumagalli G,Vigo V,Meola M,Cianchi C,Egidi MF.(2014)Nutrition and physical activity in CKD patients. Kidney Blood Press Res.39(2-3).

86 Efficacy of thermal aquatic exercise program
on physical fitness in chronic venous disease patients

Erica Menegatti ${ }^{1}$, Simona Mandini ${ }^{2}$, Gianni Mazzoni ${ }^{2}$, Anselmo Pagani $^{I}$, Giampiero Avruscio ${ }^{3}$, Paolo Zamboni ${ }^{1}$, Sergio Gianesini ${ }^{1}$

Vascular Diseases Center, Department of Translational Medicine, University of Ferrara, Ferrara, Italy ${ }^{1}$;

Center For Exercise Science and Sport, Department of Neuroscience and Rehabilitation, University of Ferrara, Ferrara, Italy ${ }^{2}$; Angiology Unit, Department of Cardiac, Thoracic and Vascular Sciences, Padova University Hospital, Padova, Italy ${ }^{3}$

Purpose: Activation of lower limb muscle pump together with ankle joint mobilization play fundamental role in enhancing venous and lymphatic drainage. Recently has been demonstrated the effectiveness of a thermal aquatic exercise program on clinical outcomes related to chronic venous disease (CVD) compared to dry-land environment.The lack of physical activities is numbered among the CVD risk factors for this reason the aim of the present investigation is to assess the potential effect of the proposed thermal water exercise program on physical fitness and QoL in CVD patients.

Methods: Sixteen (16) consecutive CVD patients (11F/5 M, mean age $61 \pm 11$ years; BMI $27 \pm 5 \mathrm{~kg} / \mathrm{m} 2$ ) were included in the study. The cohort underwent an adapted physical activity program aimed to activate all the lower limb muscle pumps, to mobilize the main lower limb joint and to stimulate balance. Water pletismography, senior fitness test, resting pulse (HR) and blood pressure (BP), validated questionnaire were used to assess leg volume, physical fitness and QoL respectively, following 5 standardized exercise sessions in thermal water pool.

Results: At the end of the exercise program a significant improvement of the outcomes related to the lower limb strength $(\mathrm{p}<0.0001)$, endurance $(p<0.006)$, rapidity and balance $(p<0.05)$ together with a significant decrease in resting HR $(-1.8 \%, \mathrm{p}<0.0001)$ and systolic BP $(-1.1 \%, \mathrm{p}<0.04)$ was recorded. Moreover, a significant leg volume reduction was found $(-16 \% ; \mathrm{p}<0.002)$ following the 5 exercise sessions. Finally, a significant improvement of bodily pain $(\mathrm{p}<$ $0.0005)$ and social function $(\mathrm{p}<0.002)$ QoL items was found.

Conclusions: The thermal water exercise program demonstrates to be an effective conservative treatment option for CVD patient, impacting also on cardiovascular and musculoskeletal outcomes. The reported data can be helpful in clinical CVD disease management, as well as in further research development on the topic.

\section{References}

1. Goddard AA, et al. Reversal of lower limb edema by calf muscle pump stimulation. J Cardiopulm Rehabil Prev 2008; 28:174-179.

2. Menegatti E, et al. Randomized controlled trial on Dryland and Thermal Aquatic standardized exercise protocol for chronic venous disease (DATA study). J Vasc Surg Venous Lymphat Disord. 2021. Epub ahead of print.

3. Jawien A. The influence of environmental factors in chronic venous insufficiency. Angiology. 2003;54 Suppl 1:S19-31. 
87

\section{Adherence to healthy lifestyle in sport sciences university students during COVID-19 pandemic}

\author{
Antonino Mulè $^{l}$, Letizia Galasso ${ }^{1}$, Lucia Castelli $^{1}$, Fabio Esposito $^{1}$, \\ Angela Montaruli ${ }^{l}$, Eliana Roveda \\ Università Degli Studi Di Milano, Dipartimento Di Scienze \\ Biomediche Per La Salute, Milan, Italy ${ }^{l}$
}

Purpose: The COVID-19 pandemic has changed our daily habits. The lockdown and other related restrictions have exposed people to sedentary behaviors and unhealthy nutrition, increasing the risk to develop diseases (1). The present study aimed to investigate physical activity, nutrition, and sleep habits in university students.

Methods: 533 university students from the University of Milan, Italy, were recruited. Students filled out a questionnaire concerning anthropometric characteristics (weight, height), age and their habits in different areas of life: physical activity was evaluated by the GodinShephard Leisure Time Physical Activity Questionnaire; nutrition was investigated by the Mediterranean Diet Quality Index for children and adolescents; sleep was evaluated by the Pittsburgh Sleep Quality Index proposed for both weekday (WD) and weekend (WE). Moreover, we investigated the consumption of cigarettes and the consume wine and alcohol. The students were divided into two groups according to the degree course followed: Sport Sciences (S) (275 students) and Nursing (N) (258 students) and compared for all variables.

Results: The two groups showed statistically significant difference in weight $(\mathrm{p}=0.001)$ and height $(\mathrm{p}<0.001)$, but not in age and BMI. S showed higher levels than $\mathrm{N}$ in physical activity $(\mathrm{p}<0.001)$ and adherence to the Mediterranean diet $(\mathrm{p}<0.001)$. By contrast, $\mathrm{S}$ slept better compared to $\mathrm{N}$ during both WD $(\mathrm{p}<0.001)$ and WE $(\mathrm{p}=$ 0.002 ). These results suggest that $S$ are more active, sleep better and have great adherence to the Mediterranean diet compared to $\mathrm{N}$ students. Moreover, the smokers were significantly higher in N $(28 \%)$ compared to $\mathrm{S}(15 \%)(\mathrm{p}<0.001)$, while no differences were found for the consumption of alcohol (S: $26 \%$ and N: $25 \%$ ) or wine (S: $31 \%$ and N: $35 \%$.

Conclusions: Results showed how Sport Sciences university students, who usually more active, adopt a healthy lifestyle even during the COVID-19 lockdown. The present study showed the importance to promote strategies to maintain a healthy lifestyle even in situations of restrictions like during the lockdown to reduce the onset of diseases.

\section{References}

1. Martinez-Ferran M, Guia-Galipienso F, Sanchis-Gomar F, Pareja-Galeano H, (2020) Metabolic Impacts of Confinement during the COVID-19 Pandemic Due to Modified Diet and Physical Activity Habits. Nutrients. 12:1549.

\section{8 \\ Population perception exercise oncology: the popcorn study}

Anita Borsati $^{1}$, Ilaria Trestini ${ }^{2}$, Daniela Tregnago ${ }^{2}$, Massimo Lanza $^{1}$, Michele Milella $^{2}$, Sara Pilotto ${ }^{2}$, Alice Avancini ${ }^{2}$

University of Verona, Department of Neuroscience, Biomedicine and Movement Science, Verona, Italy,

University of Verona, Department of Oncology, Verona, Italy ${ }^{2}$

Purpose: Exercise (EX) has been recognized as an important complementary supportive care for cancer patients. Despite the benefits, a large percentage of patients are not sufficiently active in Italy. Among the barriers, the perception of the behavior by the population can influence the chance to practice it. This survey aimed to explore the perception of the Italyn population about exercise oncology.

Methods: The POPCORN survey included 20 items regarding sociodemographic characteristics, educational and occupational levels, health conditions and current exercise levels estimated using the Leisure Score Index (LSI) from Godin's Leisure Time Exercise Questionnaire. The items about the perception of exercise in oncology, drawn from previous studies, were based on the Theory of Planned Behavior and concerned the safety, importance and beneficial effects of exercise, the capability of patients to practice it and the role of support by family/caregivers or oncologists for patients. Descriptive and correlation analyses were performed.

Results: 224 subjects anonymously completed the questionnaire. The majority were male $(52 \%)$ and had at least a high school degree (43\%). Only $7 \%$ of participants had a cancer diagnosis, and $6 \%$ were healthcare providers. Only $31 \%$ of participants were sufficiently active (LSI > 24). The items concerning the opinion of the respondents showed that the majority agreed or strongly agreed that exercise is beneficial $(65 \%)$ and important $(67 \%)$ for cancer patients. About $55 \%$ believe that the oncologist should advise patients to exercise, and $57 \%$ think that caregivers should encourage patients to exercise. Only $12 \%$ strongly agreed that exercise is safe for patients and only $6 \%$ think that patients are able to practice during the period of treatments. Most (60\%) of the subjects reported that less than $20 \%$ of cancer patients engage in a sufficient level of physical activity. A significant correlation was found between higher EX levels of participants and the importance given to exercise for patients with cancer $(\mathrm{p}=0.048)$. Socio-economics conditions did not influence the perception, whereas it seems that healthcare providers are more conscious about the benefits of EX in oncology.

Conclusions: This study shows that a large percentage of subjects has a positive opinion about EX-oncology, even if safety issues and concerns about patients' capability of exercising have emerged.

\section{9}

Adapted physical activity for women with osteoporosis and vertebral fracture: the point of view of the OSTEOAFA/2014 study participants

$\underline{\text { Sofia Marini }}^{1}$, Alice Masini ${ }^{1}$, Andrea Mascolo ${ }^{2}$, Angela Buffa ${ }^{3}$, Nazzarena Malavolta ${ }^{4}$, Giuseppe Barone ${ }^{2}$, Laura Bragonzoni ${ }^{2}$, Pasqualino Maietta Latessa ${ }^{2}$, Laura Dallolio ${ }^{1}$

University of Bologna, University of Bologna, Department of Biomedical and Neuromotor Sciences, Bologna, Italy ${ }^{l}$; University of Bologna, University of Bologna, Department For Life Quality Studies, Rimini, Italy ${ }^{2}$;

Ausl, Metropolitan Network of Rheumatology Ausl of Bologna, Bologna, Italy ${ }^{3}$;

University Hospital of Sant'orsola Malpighi, Departmental Program For Rheumatic Diseases, Mother Fortunata Toniolo Nursing Home, Bologna, Italy ${ }^{4}$

Purpose: Osteoporosis (OP) and consequent fractures represent a major health concern worldwide, having a substantial impact on quality of life (QoL). Thus, a multi-modal approach including exercise is required. However, exercise alone, even if in the right dosage, seems to be not enough to improve QoL. For this reason, the aim of the present analysis was to investigate the effect of an adapted physical activity (APA) program specifically tailored for women with OP and vertebral fractures, on QoL and participants' experience of illness. 
Methods: A convenient sample of women from the OSTEO-AFA/ 2014 study were asked to be interviewed to assess the QoL, the experience of illness and the role of the APA program carried out 1-h session twice a week for 6 months. Semi-structured interviews, have been conducted according to the methodology of qualitative research McGill Illness Narrative Interview (MINI). The analysis was performed using Nvivo software, version 12 plus.

Results: Seven women (aged 60-75) with at least one vertebral fracture (range: 1-3), were interviewed and included in the qualitative analysis. All the participants reported to be fully aware about the fact that some daily life actions were no longer executable, after the illness and particularly fracture. The role of APA, as the women indicated, was fundamental not only for the reacquisition of some autonomy at physical level, but also as psychological support, reflecting an improvement in QoL. In addition, all of them claim to feel better after performing the APA, especially because it was carried out in a group. Moreover, the majority states that has learned to control and move their body safely. Finally, after the conclusion of the OSTEO-AFA/ 2014 study intervention, 5 out of 7 women decided to continue the APA program paying a fee. The main motivations emerged from those who have not extended the participation were distance from the gym and lack of time.

Conclusions: Overall, the words of the participants confirmed that APA, conducted by trainers able to deal with this condition, is a valuable means of improving the QoL through an educational approach to the movement that allowed them to acquire awareness of their body, regain autonomies in performing daily life activities and finally a better state of psycho-physical well-being.

\section{References}

1. Gibbs, J. C., et al. (2019). Exercise for improving outcomes after osteoporotic vertebral fracture. The Cochrane database of systematic reviews, 7(7).

\section{ALLENAMENTO E VALUTAZIONE SPORTIVA 2}

\section{0}

\section{Coherence between rating of perceived exertion and training intensity zones in elite racewalkers}

\author{
Claudio Quagliarotti $^{1}$, Silvia Assenza ${ }^{1}$, Patrizio Parcesepe ${ }^{2}$, Gaspare \\ Pavei $^{3}$, Antonio La Torre ${ }^{4}$, Maria Francesca Piacentini ${ }^{I}$ \\ University of Rome 'foro Italico', Department of Movement, Human \\ and Health Sciences, Rome, Italy; \\ G.s. Fiamme Gialle, Sez. Atletica Leggera, Rome, Italy ${ }^{2}$; \\ University of Milan, Department of Pathophysiology \\ and Transplantation, Milan, Italy ${ }^{3}$; \\ University of Milan, Department of Biomedical Sciences For Health, \\ Milan, Italy
}

Purpose: Coaches normally prescribe training based on a 3 zone intensity model (Z) [1]. Z1 normally corresponds to intensities below the first ventilatory or lactate threshold (LT1), Z2 between LT1 and the second threshold (LT2) and Z3 above LT2. Previous studies have identified specific RPE breakpoints corresponding to the 3 intensity zones on the CR10 scale[2] to help coaches in monitoring training sessions for cross-country skiers [1] and open-water swimmers[3]. However, the RPE breakpoints were different between these 2 modes of locomotion leading to conclude that the RPE breakpoints corresponding to the LT could be sport-specific. Race walking is an endurance activity showing a specific locomotor pattern different from both walking and running $[4,5]$, that may affect the relationships between RPE and Zs. This study aimed to evaluate the correspondence between Zs, race-pace speeds and RPE in elite racewalkers. Methods: Six elite racewalkers (3 males and 3 females), all part of the National Olympic Team, performed a total of 16 incremental tests on track: $7 \times 1600 \mathrm{~m}$. Lactate concentration (Lactate Pro 2, Kyoto, Japan), HR and RPE (CR-10 Italyn language[6]) were collected at the end of each step. Lactate, HR, speed and RPE values were interpolated through a third-degree polynomial to determine the estimated value for each RPE scale point. LT1 was estimated by the highest workload not associated with a rise in lactate concentration (estimated by visual and Wilcoxon test) from the average baseline lactate at rest; LT2 was estimated by a rise of $3 \mathrm{mM}$ above the first rise in lactate concentration[7].

Results: Z1 lies between 0 and 2 on the CR10 scale and up to 77-81\% HRmax, Z2 lies between 2.5 and 6 RPE and up to 91-93\% HRmax and $\mathrm{Z} 3 \geq 7 \mathrm{RPE}$ and above $93 \%$ HRmax. The $50 \mathrm{~km}$ race pace speed corresponds to a CR10 value of 3 and $84 \%$ HRmax and the $20 \mathrm{~km}$ race pace speed to a value of 5 and $90 \%$ HRmax.

Conclusions: Prescription of training based on the RPE scale needs to rely on correct intensity zone breakpoints calculated by physiological tests. Compared to other sports, in racewalking $\mathrm{Z} 1$ seems to lie in a very narrow range of RPE values. In fact, RPE values corresponding to $\mathrm{Z} 1 \leq 4$ were found in cross-country skiers [1], and $\leq 3$ in openwater swimmers[3]. Probably the increase of race walking speed leads to a sharp increase in muscle metabolism, for the decreasing energysaving role of the tendons[5], determining a substantial increase in energy cost and fatigue.

\section{References}

1. Seiler KS, Kjerland GØ. Scand J Med Sci Sport. 2006;16(1):49-56.

2. Foster C. Med Sci Sport Exerc. 1998;30(7):1164-1168.

3. Ieno C, et al.. J Funct Morphol Kinesiol. 2020;5(1):21.

4. Pavei G, et al.. Eur J Sport Sci. 2014;14(7):661-670.

5. Cronin NJ, et al. Mechanical and neural function of triceps surae in elite racewalking. J Appl Physiol. 2016;121(1):101-105.

6. Impellizzeri FM, et al. Sc Dello Sport. 2009;28(82):11-18.

7. Cerezuela-Espejo V, et al. Front Physiol. 2018;9(SEP).

\section{1}

\section{The energetic cost of forward vs shuttle running in marathon runners and soccer players}

\author{
Johnny Padulo $^{1}$, Antonio Buglione ${ }^{2}$, Christian Doria ${ }^{1}$, Marta \\ ${\text { Borrelli }{ }^{I}, \text { Fabio Esposito }}^{l}$ \\ Università Degli Studi Di Milano, Department of Biomedical \\ Sciences For Health, Milan, Italy ${ }^{1}$; \\ San Raffaele Roma Open University, Department of Human Sciences \\ and Promotion of The Quality of Life, Rome, Italy ${ }^{2}$
}

Purpose: In the last decades, the energetic cost assessment provided new insight on shuttle or forward running as training modalities. No study, though, quantified the benefit of forward/shuttle running in soccer players and marathon runners.

Methods: To this aim, 8 marathon runners (age $34 \pm 7$ years; training experience $5.7 \pm 0.8$ years) and 8 soccer players (age $18 \pm 1$ years; training experience $5.8 \pm 1.8$ years) participated in this study. Each participant was assessed randomly on shuttle running $[80 \%$ VO2MAX, on 20-22 m for 6' (Buglione and di Prampero 2013)] or continuous running (60\% VO2MAX for 6') with three days of recovery in-between. For each condition, the blood lactate (BL) and the energetic cost of forward $(\mathrm{Cr})$ and shuttle running (CSh) was determined. To assess differences for metabolic demand in terms of 
$\mathrm{Cr}, \mathrm{CSh}$ and $\mathrm{BL}$ over the two running conditions on the two groups a MANOVA was used. Rejection level was set at $\alpha<0.05$.

Results: VO2MAX was $67.9 \pm 4.5$ and $56.8 \pm 4.3 \mathrm{ml} \cdot \mathrm{min} \cdot-1 \cdot \mathrm{kg}-1$ (p $=0.0002$ ) for marathon runners and soccer players, respectively. On forward running, the marathon runners had a lower $\mathrm{Cr}$ compared to soccer players $(3.86 \pm 0.16 \mathrm{~J} \cdot \mathrm{kg}-1 \cdot \mathrm{m}-1$ vs $4.19 \pm 0.26 \mathrm{~J} \cdot \mathrm{kg}-1 \cdot \mathrm{m}-1 ; \mathrm{F}$ $=9.759$, respectively; $-8 \% ; \mathrm{p}=0.007)$. On shuttle running, marathon runners had a higher CSh compared to soccer players (8.66 \pm $0.60 \mathrm{~J} \cdot \mathrm{kg}-1 \cdot \mathrm{m}-1$ vs $7.86 \pm 0.51 \mathrm{~J} \cdot \mathrm{kg}-1 \cdot \mathrm{m}-1 ; \mathrm{F}=8.282$, respectively; $+10 \%$; with $\mathrm{p}=0.012$ ). BL on forward running was lower in marathon runners compared to soccer players $(1.06 \pm 0.07 \mathrm{mmol} \cdot \mathrm{L}-1$ vs $1.56 \pm 0.42 \mathrm{mmol} \cdot \mathrm{L}-1$, respectively; $-32 \%$; with $\mathrm{p}=0.005$ ). Conversely, BL on shuttle running was higher in marathon runners compared to soccer players $7.99 \pm 1.49 \mathrm{mmol} \cdot \mathrm{L}-1$ vs $6.04 \pm$ $1.69 \mathrm{mmol} \cdot \mathrm{L}-1$, respectively; $+32 \%$; with $\mathrm{p}=0.028$ ).

Conclusions: The energetic cost optimization on forward or shuttle running is strictly related to the sport practiced. These two running modalities elicited two different physiological responses that must be considered during training sessions and competitions.

\section{References}

1. Buglione A, and di Prampero PE. (2013) The energy cost of shuttle running. Eur J Appl Physiol 113:1535-1543.

\section{2}

\section{Home advantage is influenced by the timing of the events during rugby union domestic competition}

\author{
${ }_{\text {Alexandru Nicolae Ungureanu }}{ }^{1}$, Luca Mattina $^{2}$, Corrado Lupo ${ }^{1}$, \\ Paolo Riccardo Brustio \\ University of Turin, Neuromuscularfunction Research Group, \\ Department of Medical Sciences, Turin, Italy ${ }^{1}$; \\ University of Turin, School of Exercise \& Sport Sciences, Suism, \\ Turin, Italy ${ }^{2}$; \\ University of Verona, Department of Neuroscience, Biomedicine \\ and Movement, Verona, Italy ${ }^{3}$
}

Purpose: Although performance analysis was extensively implemented in rugby, it has primarily only focused on discrete, descriptive, and comparative statistics. Therefore, using a dynamical system analysis approach (Colomer, 2020) the present study aimed to identify patterns for successful game results in rugby union from sequences outcomes.

Methods: A 234 performances of the 2019-20 Top14 competition (French top-level clubs) were analyzed. Data were obtained from the Ultimate Rugby web domain (https://www.ultimaterugby.com/\#). In particular, the regular 80 min playing time was divided into different periods according to 3 different scenarios (i.e., $8 \times 10$-min, $4 \times$ 20-min, $2 \times 40$-min periods). Events (i.e., try, kick-at-goal, conver sions, substitution, cards) were then coded in relation to the period (e. g., try scored in the first quarter), the fixture (i.e., home vs away), and the power balance between teams. A 3 Exhaustive CHAID decision trees (DT) were grown using win/loss as the binary response variable using a 10 sample folds training cross-validation. Minimum cases in parent and child node were set to 100 and 50, respectively. The DT with the higher classification reliability and with more than 1 node was considered.

Results: $12 \pm 3$ events occurred during each of the 234 performances. DT in the $4 \times 20$-min scenario was more reliable compared to the others one and produced more than 1 node. The $4 \times 20$-min scenario DT was able to correctly classify $76.6 \%$ of wins and $74 \%$ of loses (vs 55.9 and $98.4 \%$ for $8 \times 10$-min and vs 76.6 and $74 \%$ for the $2 \times$ 40-min). Playing home led to $72.6 \%$ winning performances compared to $22.2 \%$ away. In the playing home scenario lower winning occurrences (53.2\%) happened within the first 3 events of the game when failing a kick-at-goal attempt, or when a substitution was made in the second and the third quarter, or when opponents scored a try in the first or the second quarter.

Conclusions: In line with other studies on international rugby union (Thomas et al., 2008, Vaz et al., 2012), home advantage occurred in the national club competition. From the perspective of a dynamical system analysis, this advantage is associable to specific match periods in which the game events observed in this study occur. In particular, failing to score points (i.e., kick-to-goal attempt failed) and conceding a try in the 1 st or 2 nd quarter of the game may reduce the home advantage.

\section{References}

1. Colomer, C. M., Pyne, D. B., Mooney, M., McKune, A., \& Serpell, B. G. (2020) Performance analysis in rugby union: a critical systematic review. Sports medicine-open, 6.1: 1-15.

2. Thomas, S., Reeves, C., \& Bell, A. (2008) Home advantage in the six nations rugby union tournament. Perceptual and Motor Skills, 106.1: 113-116.

3. Vaz, L., Carreras, D., \& Kraak, W. (2012) Analysis of the effect of alternating home and away field advantage during the Six Nations Rugby Championship. International Journal of.

\section{3}

\section{Training session monitoring in female cross-country skiers of olympic interest}

\author{
Chiara Zoppirolli ${ }^{l}$, Roberto Modena ${ }^{1}$, Federico Schena ${ }^{2}$, Barbara \\ Pellegrni $^{1}$, Lorenzo Bortolan ${ }^{1}$ \\ Cerism, Università Degli Studi Di Verona, Rovereto, Italy ${ }^{1}$; \\ Cerism, Università Degli Studi Di Verona, Verona, Italy ${ }^{2}$
}

Purpose: Elite cross-country skiers are usually tested in laboratory settings, for both physiological and biomechanical aspects. However, on-field measurements during races or training sessions could be useful to assess athletes' performance during real sport conditions. Here we present methodology employed and data collected during a training session aimed at evaluating performance during a roller skiing sprint simulation.

Methods: Five female members of the Italyn team and belonging to the project Milano-Cortina 2026 were monitored during a roller skies sprint race simulation with the classical technique, consisting of three $990 \mathrm{~m}$ all out laps with $20 \mathrm{~m} \Delta+$, separated by $150 \mathrm{~s}$ of active rest. A Polar Team Pro device was used to instantaneously collect athletes' position on the track, velocity and heart rate. Moreover video clips were taken on two $15 \mathrm{~m}$ long flat sections, after the starting line and before the arrival, and analysed (Tracker 5.1.5) to monitor the gross kinematics of the double poling technique. Mean and maximal values of heart rate and velocity were compared across the three laps with a one-way ANOVA RM, while a two-way ANOVA RM was used to evaluate the effect of laps and section on kinematic parameters.

Results: Both mean and maximal speed did not changed across laps ( $\mathrm{P}$ $>0.05$ ), while for mean and maximal heart rate increased significantly $(\mathrm{P}<0.05)$. Gross cycle kinematics exhibited a clear effect of section, with reduced double poling sectional speed $(\mathrm{P}<0.001)$, cycle length $(P=0.006)$ and augmented duty cycle $(P=0.003)$. On the other hand, no general effect of laps on gross cycle kinematics was found (all $\mathrm{P}>0.05$ ). Confirming previous findings, cycle frequency tended to be constant for all the measurements $(\mathrm{P}>0.05$ for both laps and section effect). 
Discussion: Elite female cross-country skiers maintained an even pacing strategy across the laps of the simulated race, even though metabolic work increaseed significantly. The effect of fatigue seems not to alter the gross-cycle kinematics across the laps, but an acute effect of fatigue appeared within each lap. Such type of evaluations offer interesting data to athletes and coaches for a general and individualized performance evaluation during real training session. Athletes' monitoring on the field should be further developed and considered as an integral part of elite athlete evaluation, in addition to standardized lab tests, in order to better address training.

\section{References}

1. Zory R, Vuillerme N, Pellegrini B, Schena F, Rouard A (2009). Effect of fatigue on double pole kinematics in sprint crosscountry skiing, Human Movement Science 28: 85-98.

2. Zoppirolli C, Bortolan L, Stella F,Boccia G, Holmberg H-C, Schena F, Pellegrini B (2018). Following a long-distance classical race the whole-body kinematics of double poling by elite cross-country skiers are altered. Front. Physiol 9:978.

\section{4}

\section{Effect of mental fatigue and subsequent 20-min daytime napping on sleepiness and cognitive performance in endurance athletes}

\author{
Jacopo Vitale $^{1}$, Luca Filipas ${ }^{2}$, Luigi Mininno $^{2}$, Antonio La Torre ${ }^{2}$ \\ Irccs Istituto Ortopedico Galeazzi, Laboratorio Del Movimento E \\ Scienze Dello Sport, Milan, Italy'; \\ Università Degli Studi Di Milano, Dipartimento Di Scienze \\ Biomediche Per La Salute, Milan, Italy ${ }^{2}$
}

Purpose: The aim of this study was to assess the effect of mental fatigue (MF) on 20-min daytime napping characteristics and the subsequent effects on sleepiness, perceptions of MF, and cognitive performance in endurance athletes.

Methods: Nine amateur master athletes (age: $38.4 \pm 9.1$ years; BMI: $20.1 \pm 1.1$; VO2peak: $62 \pm 5 \mathrm{~mL} \cdot \mathrm{kg}-1 \cdot \mathrm{min}-1)$ performed in a randomized, counterbalanced, and crossover order, two identical experimental sessions separated by an interval of one week: a mental fatigue (MF) and a control session (CON). In CON, the participants underwent a 20-min nap between 14.00 and 15:00 and, in MF, also a 30-min pre-nap battery of cognitive tasks was included. Sleepiness was monitored by the Karolinska Sleepiness Scale (KSS) and a visual analogue scale (VAS) was used to assess the perceptions of MF before (PRE), immediately after (POST1) and 30-min after the day time nap (POST30). Further, cognitive performance was evaluated at POST30 with a 5-min flanker task. Sleep was continuously monitored by actigraphy and a sleep diary throughout the study protocol.

Results: MF did not negatively influence the actigraphy-based sleep characteristics of daytime nap whereas subjects reported higher subjective sleep quality of the nap in CON compared to MF (7.3 \pm 1.7 vs $5.6 \pm 2.6, \mathrm{p}<0.05)$. VAS was higher in MF $(51.0 \pm 26.2)$ than CON $(20.1 \pm 11.4)$ at PRE $(\mathrm{p}<0.01)$ and decreased at POST30 only in MF $(13.5 \pm 10.8 ; \mathrm{p}<0.001)$. Similarly, KSS was higher in MF $(4.9 \pm$ 1.7) than CON $(4.2 \pm 2.0)$ at PRE $(\mathrm{p}<0.05)$ and decreased at POST30 in MF $(3.3 \pm 0.6 ; \mathrm{p}<0.05)$. Response time in the cognitive task was lower at POST30 in CON than MF (416 $\pm 39 \mathrm{~ms}$ vs $566 \pm$ $34 \mathrm{~ms}, \mathrm{p}<0.01)$, while accuracy remained similar in the two conditions.

Conclusions: MF did not affect the objective sleep characteristics of the 20-min daytime nap. Nonetheless, in the MF experimental session, endurance athletes had higher VAS and KSS scores before the nap, reported lower subjective sleep quality of the nap and had a lower cognitive performance at POST30.

\section{References}

1. Lastella VM, Halson SL, Vitale JA, Memon AR, Vicent GE. (2021). To Nap or Not to Nap? A Systematic Review Evaluating Napping Behavior in Athletes and the Impact on Various Measures of Athletic Performance. Nat Sci Sleep. Epub ahead of print.

2. Filipas L, Ferioli D, Banfi G, La Torre A, Vitale JA. (2021). Single and Combined Effect of Acute Sleep Restriction and Mental Fatigue on Basketball Free-Throw Performance. Int J Sports Physiol Perform 16(3):415-420.

\section{5}

\section{Bioimpedance vector analysis of young male and female team handball players}

\author{
$\underline{\text { Pascal Izzicupo }}^{1}$, Andrea Di Credico ${ }^{I}$, Giulia Gaggi ${ }^{1}$, Barbara \\ Ghinassi $^{I}$, Angela Di Baldassarre ${ }^{I}$
}

Dipartimento Di Medicina E Scienze Dell'invecchiamento, Università Degli Studi “G. D'annunzio”, Chieti, Italy ${ }^{l}$

Purpose: Team handball is a dynamic sport game played at professional and youth levels in numerous countries. Although empirically, it is observable that elite team handball players are generally taller and heavier. However, there is a lack of reference body composition bioelectrical-impedance values. Therefore, this study aims a) to characterize a sample of young male and female elite team handball players in relation with a nonathletic reference population; and b) to generate their $50 \%, 75 \%$, and $95 \%$ percentiles of the bioelectrical variables.

Methods: The study included 55 young players (Males, n: 37, age: $17.0 \pm 1.2 \mathrm{yr}$, height: $185.8 \pm 7.3 \mathrm{~cm}$, weight: $82.0 \pm 11.0 \mathrm{~kg}$, BMI: $23.7 \pm 2.5$; Females, n: 18, age: $17.8 \pm 0.9 \mathrm{yr}$, height: $171.2 \pm$ $6.4 \mathrm{~cm}$, weight: $67.4 \pm 7.2 \mathrm{~kg}$, BMI: $23.0 \pm 2.0$ ) selected for the Italyn Handball National Teams in the following age categories: males U17 (n: 19), males U19 (n: 18) and females U19. Stature and bioelectrical variables $(\mathrm{R}$, resistance; $\mathrm{Xc}$, reactance; PA, phase angle; and $\mathrm{Z}$, impedance module) were assessed in a state of euhydration and standard conditions. Bioelectrical-impedance vector analysis (BIVA) was used to characterize the bioelectrical vector (BIA vector) distribution pattern for each group.

Results: Most players were mostly located outside the $75 \%$ and some beyond the $95 \%$ percentile of the bioelectrical tolerance ellipses of the age-matched general population. Compared to the reference values BIA vector showed statistically significant differences in males $\mathrm{U} 17(\mathrm{~T} 2=51.0, \mathrm{p}<0.0001)$, males U19 $(\mathrm{T} 2=82.0, \mathrm{p}<0.0001)$ and females U19 $(\mathrm{T} 2=85.8, \mathrm{p}<0.0001)$. Male groups were also bioelectrically different $(\mathrm{T} 2=13.7, \mathrm{p}=0.0036)$.

Conclusions: BIVA showed specific bioelectrical characteristics in young male and female elite team handball players. This study provides an original data set of bioelectrical-impedance reference values parameters of young male and female elite team handball players. Our result might help interpret individual bioimpedance vectors and define target regions for young handball players.

Acknowledgments: The authors thank the Italyn Team Handball Federation. 


\section{BIOMECCANICA}

\section{6}

\section{The transition between walking and running: muscle and tendon behavior can explain the transition between gaits?}

\author{
Andrea Monte $^{1}$, Paolo Tecchio ${ }^{1}$, Francesca Nardello ${ }^{1}$, Beatriz \\ Bachero-mena $^{2}$, Luca Paolo Ardigó ${ }^{\prime}$, Paola Zamparo ${ }^{1}$ \\ Università Di Verona, Università Di Verona, Dipartimento Di \\ Neuroscienze, Biomedicina E Movimento, Verona, Italy ${ }^{1}$; \\ Universidad De Sevilla, Department of Physical Education and Sport, \\ Siviglia, Spagna ${ }^{2}$
}

Purpose: the spontaneous transition between walking and running occurs at about $7-8 \mathrm{~km} / \mathrm{h}$ and the underpinning mechanism of this transition is still partially understood. The aim of this study was to investigate the possible role of the lower limbs muscle-tendon mechanical behavior in explaining the walk to run transition.

Methods: we combined ultrasound data of Gastrocnemius Medialis (GM) with kinematic and metabolic data during walking and running at matched speeds $(6,7$ and $8 \mathrm{~km} / \mathrm{h})$. In addition, the F-L and F-V relationships of GM was experimentally assessed by means of dynamometric measurements (Monte et al., 2020). Combining the ultrasound data collected during these experiments with those obtained during the locomotion trials, the F-L and F-V potential as a function of speed was obtained (Bohm et al., 2019). Belly ( $\mathrm{Gb}=$ belly length/fascicle length) and tendon ( $\mathrm{Gt}=\mathrm{MTU}$ length/Belly length) gearing were calculated for each locomotor trials as well as energy cost $($ Cnet $=\mathrm{VO} 2$ net $/ \mathrm{v})$.

Results: Cnet increased as a function speed in walking (from 2.6 to $4.2 \mathrm{~J} / \mathrm{kg} / \mathrm{m})$ and was unaffected by speed in running $(4.2 \mathrm{~J} / \mathrm{kg} / \mathrm{m}) . \mathrm{Gb}$ decreased with speed and was larger in running than in walking; $\mathrm{Gt}$ was unaffected by speed in walking and increased with speed in running, the two functions crossing at $8 \mathrm{~km} / \mathrm{h}$, as is the case for Cnet. The F-L and F-V potentials decreased as a function of speed and were higher for running than walking (i.e. muscles fascicles operate at higher F-L and F-V potentials during running).

Conclusions: The present data indicate a clear gap between muscle and tendon behaviour in the two locomotion modes; tendon, but not muscle, behaviour could help in explaining the walk to run transition in terrestrial locomotion.

References

1. Monte A, Blatzopoulos V, Maganaris CN, Zamparo P (2020) Gas- trocnemius Medialis and Vastus Lateralis in vivo muscletendon behaviour during running at increasing speeds. Scand J Med Sci Sports. https://doi.org/10.1111/sms.13662

2. Bohm S, Mersmann F, Santuz A, Arampatzis A (2019) The force-length-velocity potential of the human soleus muscle is related to the energetic cost of running. Proc Biol Sci 286(1917):20,192,560

97

\section{Use of pressure sensors to determine the hand thrust force in swimming: preliminary results of a comparative validation study}

\author{
$\frac{\text { Matteo Cortesi }}{{ }^{1}}$, Riccardo Zoboli $^{1}$, Riccardo $^{2}$ Olivato $^{1}$, Cheong-yui \\ Wong \\ University of Bologna, Department For Life Quality Studies, \\ Bologna, Italy ${ }^{1}$;
}

Hong Kong University of Science and Technology, 2department of Electronic and Computer Engineering, Hong Kong, Hong Kong ${ }^{2}$

Purpose: The capability to exert useful force in water is a key factor for swimming performance. For this Purpose, tethered swimming is the gold standard method to calculate the thrust force of the swimmer. Moreover, the dynamic pressure approach has been used in swimming research to characterize hands and feet thrust force. In this study, an assessment of the accuracy and reliability of pressure sensors to estimate the hand thrust force of the swimmer was performed.

Methods: Eleven young swimmers at the national level $(75.0 \pm 6.1 \%$ of 50-m World Record) including five males $(16.0 \pm 0.0$ y) and six females $(15.0 \pm 0.0 \mathrm{y})$ performed a single bout of $15 \mathrm{~s}$ fully tethered swimming only arms. The tethered force (Ftet) was assessed using a load-cell system. The swimmers wore four wireless pressure sensors (SEAL, Platysens) to measure the average differential hydrodynamic pressures (Phand). The sensors were placed between the third and fourth metacarpals on the palm and back of each hand. The product of the average pitch angle of the hand $\left(\alpha=47^{\circ}\right.$ based on previous literature) and hand's surface area (HSA) was calculated to obtain the average force of the hands (Fhand) as Phand * (HSA * $\alpha$ ). Accuracy level was evaluated comparing Phand and Fhand with Ftet. Reliability of the pressure sensors was expressed as level of agreement between Phand of ten stroke cycles. Hydrostatic and hydrodynamic pressure calibrations of the sensors were applied to the data.

Results: Average Phand, Fhand, Ftet and HSA were $3.208 \pm 0.714$ $\mathrm{Kpa}, 34.7 \pm 9.6 \mathrm{~N}, 77.8 \pm 15.5 \mathrm{~N}$ and $157.5 \pm 7.8 \mathrm{~cm} 2$, respectively. Almost perfect correlations $(\mathrm{r}=0.91, \mathrm{p}<0.001 ; \mathrm{r}=0.90, \mathrm{p}<0.001)$ were observed for Phand vs Ftet and for Fhand vs Ftet. Pairwise $\mathrm{t}$-tests showed a significant difference between Fhand and Ftet $(\mathrm{p}<$ $0.001)$. Statistics reveal considerable bias $(-43.1 \mathrm{~N} ; 95 \% \mathrm{CI}-27.2$ to $-59.0 \mathrm{~N})$ and considerable RMSE $(43.8 \mathrm{~N})$ for Fhand vs Ftet comparison. The test-retest reliability coefficient between cycles for Phand was almost perfect (ICC always higher than 0.95).

Conclusions: These preliminary results demonstrate an acceptable level of accuracy and reliability for hand swimmer's force calculation using pressure sensors. Despite the high level of agreement between pressure sensors and tethered system, the force measured in all-out swimming sprints by pressure sensors was significantly lower. This stable inter-individual difference could be partly imputable to the forearm contribution in thrust force.

\section{References}

1. Morouço PG, Marinho DA, Keskinen KL, et al. (2014). Tethered swimming can be used to evaluate force contribution for shortdistance swimming performance. J Strength Cond Res 28, 3093-3099.

2. Sanders RH, Andersen JT, Takagi H et al. (2017). The segmental movements in front crawl swimming. Handbook Hum Mot 1-15.

3. Samson M, Monnet T, Bernard A, et al. (2018). Comparative study between fully tethered and free swimming at different paces of swimming in front crawl. Sports Biomech 18, 571-586.

98

Front vs back overhead press: an electromyographic analysis and possible implications for resistance training

Giuseppe Coratella $^{l}$, Gianpaolo Tornatore ${ }^{l}$, Stefano Longo ${ }^{1}$, Susanna Rampichini, ${ }^{1}$, Marta Borrelli ${ }^{l}$, Christian Doria ${ }^{l}$, Emiliano Ce ${ }^{I}$, Fabio Esposito ${ }^{I}$

Università Degli Studi Di Milano, Dipartimento Di Scienze Biomediche Per La Salute, Milan, Italy ${ }^{1}$ 
Purpose: The current study compared the muscles excitation during overhead press performed with the barbell passing in front or behind the head or using a shoulder press device.

Methods: Ten competitive bodybuilders performed in random order front (front-BMP) or back barbell military press (back-BMP), and front (front-MSP) or back machine shoulder press (back-MSP). Normalized surface electromyographic root mean square (RMS) of anterior, medial and posterior deltoid, upper trapezius, pectoralis major and triceps brachii was recorded during both the ascending and descending phases.

Results: During the ascending phase, anterior deltoid showed greater RMS in back-BMP than back-MSP [Effect size (ES): 1.50(0.45/ 2.42)]. Medial deltoid showed greater RMS in back-BMP than frontBMP [ES: 1.28(0.27/2.18)] and front-MSP [ES: 2.71(1.41/3.79)], and front-BMP showed greater RMS than front-MSP [ES: 1.51(0.46/ 2.43)]. Moreover, front-BMP showed greater RMS than front-MSP [ES: 2.80(1.48/3.89)]. Posterior deltoid showed greater RMS in backBMP than front-BMP [ES: 2.08(0.92/3.06)], front-MSP [ES: 3.75(2.18/5.01)] and back-MSP [ES: 2.32(1.10/3.33)]. Pectoralis major showed greater RMS in front-BMP than back-BMP [ES: 4.30(2.57/5.66)] and back-MSP [ES: 4.63(2.80/6.06)], and in frontMSP than back-BMP [ES: 3.52(2.01/4.73)] and back-MSP [ES: 3.97(2.33/5.27)]. No between-exercise difference was observed for upper trapezius and triceps brachii. Similar findings were observed during the descending phase, albeit more differences were retrieved comparing barbell vs machine exercises.

Conclusions: Performing back overhead press enhances the recruitment of medial and posterior deltoid, while front overhead favors pectoralis major. Overhead performed using barbell excites muscles more than using machine. Front and back overhead press could be selectively used to stimulate the muscles surrounding the shoulders. References

1. Escamilla RF, Yamashiro K, Paulos L, Andrews JR. Shoulder muscle activity and function in common shoulder rehabilitation exercises. Sport Med. 2009;39: 663-685. https://doi.org/10.2165/ 00007256-200939080-00004

2. Cools AM, Johansson FR, Borms D, Maenhout A. Prevention of shoulder injuries in overhead athletes: A science-based approach. Brazilian J Phys Ther. 2015;19: 331-339. https://doi.org/10.1590/ bjpt-rbf.2014.0109

\section{9}

\section{Kinematics of backward standing starts in elite cyclists: the effect of initial crank angle}

\section{Francesca Nardello ${ }^{1}$, Riccardo Bernabe $e^{l}$, Simone Bettega ${ }^{I}$, Marco Zandomeneghi $^{I}$, Paola Zamparo ${ }^{I}$ \\ Università Degli Studi Di Verona, Dipartimento Di Neuroscienze, Biomedicina E Movimento, Verona, Italy ${ }^{I}$}

Purpose: In modern sprint-cycling competitions, the athletes perform a preparatory counter movement that consists in reaching the backmost standing position, quickly accelerating the body forward at the starting signal. The purpose of this study was to investigate the kinematics of backward standing starts in elite cyclists, as well as the effect of initial crank angle.

Methods: The kinematics of cycling starts was investigated in 7 male elite cyclists belonging to the Italyn track cycling team $(23.4 \pm$ 4.1 years; $1.84 \pm 0.05 \mathrm{~m}$ stature; $78.4 \pm 5.5 \mathrm{~kg}$ body mass; $15.1 \pm$ 4.0 years of practice). They were asked to perform $30 \mathrm{~m}$ sprints (indoor velodrome) from 3 starting positions: i) seated, with the crank of the pushing leg at the self-selected position (S-ss); ii) backward standing, with the crank at the self-selected position (BSt-ss); iii) backward standing, with the crank angle at $90^{\circ}$ with the vertical axis $\left(\mathrm{BSt}-90^{\circ}\right)$. Average velocity after 5 and $30 \mathrm{~m}$ was also measured by means of a photocell system.

Results: The starting angle was the same in BSt-ss and S-ss (about $65^{\circ}$ ) and the fastest speed after 5 and $30 \mathrm{~m}$ was attained in BSt-ss. In these starts peak downstroke velocity was the highest and the counter movement occurred earlier than in BSt $-90^{\circ}$. The advantage of a quick start was limited to the first downstroke: the following pedaling cycles were not affected by changes in the initial crank position or by the preparatory counter movement.

Conclusions: In backward standing starts, a quick start is associated with an earlier backward movement. Moreover, when the initial crank position is closer to the bottom dead centre (BDC), less time is available to exert force (and hence to increase velocity) in the first pedaling cycle. Thus, in backward starting starts, it is more advantageous to start with an initial crank position closer to $65^{\circ}$ than to $90^{\circ}$. References

1. Gianikellis K, Skiadopolous A, and Bote A. (2011) 3D Kinematics applied to the study of individual BMX gate start technique. Portuguese Journal of Sport Sciences 11: 251-254.

2. Merkes PFJ, Menaspà $P$, and Abbiss CR (2019) Power output, cadence, and torque are similar between the forward standing and traditional sprint cycling positions. Scand J Med Sci Sports 00: $1-10$.

\section{0}

\section{Typical risk pattern for anterior cruciate ligament injury is largely present in competitive athletes: biomechanical screening through wearable sensors}

$\frac{\text { Stefano Di Paolo }}{\text { Zaffagnini }^{2}}$, Laura Bragonzoni ${ }^{1}$, Alberto Grassi ${ }^{2}$, Stefano

Università Di Bologna, Dipartimento Di Scienze Per La Qualità Della Vita, Bologna, Italy ${ }^{I}$;

Istituto Ortopedico Rizzoli, Clinica Ortopedica E Traumatologica Ii, Bologna, Italy ${ }^{2}$

Purpose: The incidence of acute knee injuries, e.g., Anterior Cruciate Ligament (ACL) injury is growing with regards to young and female athletes' populations. The identification of specific biomechanical patterns involving multiple joints is increasingly claimed in prevention and rehabilitation programs [1]. It is not clear whether such patterns are present in the healthy athletes' population. The aim of the present study was to investigate the presence of biomechanical risk patterns for ACL injury in a healthy population during the execution of high-dynamics movements.

Methods: Thirty-four competitive young, healthy athletes $(22.8 \pm$ 4.1 years, 18 males and 16 females, Tegner Level 9) were enrolled. Every athlete performed a frontal deceleration (DEC) and a change of direction at $90^{\circ}$ (COD). Three valid repetitions per leg were collected. Kinematical data of ankle, knee, hip, and trunk joints were collected through a set of 15 wearable inertial sensors (Awinda, Xsens Technologies). According to the current literature [1], nine ACL risk factors were identified across four categories: limited lower limb flexion (knee, hip, ankle), valgus collapse (knee valgus, hip abd/adduction and internal rotation), foot rotation, non-neutral trunk posture (ispilateral bending, contralateral rotation). A movement was considered "at-risk" in the presence of at least 5 simultaneous risk factors. The central strike of each task was isolated, and risk factors were assessed at initial contact and at the maximum knee flexion angle. The rate of athletes with at-risk movements was assessed and a 
multivariate regression with gender, age, approaching speed, limb dominance was performed $(\mathrm{p}<0.05)$.

Results: The presence of the injury risk pattern (at least 5 risk factors) was identified in 24 athletes $(71 \%)$ in at least one movement. Five athletes $(15 \%)$ exhibited the risk pattern in at least three movements. The overall rate of injury patterns was between 9 and $12 \%$. The COD at maximum knee flexion showed significant odds ratio for approaching speed and gender female $(\mathrm{OR}=4.3$ and $\mathrm{OR}=4.8$, respectively).

Conclusions: The present study underlined a large presence of biomechanical patterns typical of ACL injury in young and healthy athletes during the execution of complex dynamic movements. Such movements should be investigated in sport-specific environments for a wider comprehension of the actual risk level and preventative trainings might be deployed in at-risk athletes.

References

1. Della Villa F, Buckthorpe M, Grassi A, et al. Br J Sports Med. 2020;54(23):1423-1432.

\section{1}

\section{Biomechanical analysis of different device positions on backstroke start in élite swimmers}

\author{
Emanuele Chirico $^{I}$, Giancarlo Cervino $^{I}$, Sabrina Demarie ${ }^{I}$, \\ Giuseppe Vannozzi \\ Università Degli Studi Di Roma "foro Italico", Scienze Del \\ Movimento Umano E Dello Sport, Rome, Italy ${ }^{1}$
}

Purpose: In competitive swimming, backstroke start is widely considered as the most different and challenging start among the other swimming styles [2]. A new backstroke start equipment (Omega OBL2) has been approved by Federation Internationale de Natation (FINA) since 2014. Through this device, five different adjustable positions may be set [3]. The aim of this study was to verify the effect of the device position on start biomechanics.

Methods: Nine élite swimmers (age 22,7 $\pm 1,9$; height $179.4 \pm$ $7.3 \mathrm{~cm}$; body mass $70.5 \pm 8.9 \mathrm{~kg}$; FINA points $811 \pm 80.85$; percentage PB/WR 93.2\% \pm 2.64 ) performed ten backstroke starts in random order, two for each device position, separated by 2 min of passive rest. A body-worn inertial sensor and a video camera were used to analyse the start biomechanical variables [3]. Only the best performance for each position (best time in $5 \mathrm{~m}$ ) was further investigated through statistical analysis. A repeated measure ANOVA was performed with Bonferroni correction.

Results: Significant differences were observed among different device positions in the following video-based parameters: take-off velocity, head entry distance, and entry difference between head and centre of mass $(p<0.005)$. Peak to Peak values for antero-posterior linear acceleration and medio-lateral angular velocity were significantly different only between the two extreme device positions $(\mathrm{p}<$ 0.005). No significant difference was found in the $5 \mathrm{~m}$ time duration and in other biomechanical variables.

Conclusions: The Backstroke start is deeply affected by the use of the newly adopted device. Although significant differences in starting biomechanics were highlighted for different device positions, $5 \mathrm{~m}$ time duration was similar in each condition; notably, the results obtained are comparable with previous studies. However, the high level of the subjects analysed may have influenced the final results due to possible compensation strategies they might have adopted. Nevertheless, when the device was set in a higher position, a better time was performed by all athletes. It can be speculated that, with a higher device setting, a more comfortable position could be achieved and a higher propulsion force be applied by swimmers.

\section{References}

1. Barkwell GE, et al. Backstroke start performance: the impact of using the Omega OBL2 backstroke ledge. Sports Biomech. 2018;17(4):429-441.

2. De jesus, $\mathrm{K}$, et al. Are the new starting block facilities beneficial for backstroke start performance?. J.S.S., 2016, 34.9: 871-877.

3. Vannozzi, G., et al. "Analysis of swim turning, underwater gliding and stroke resumption phases in top division swimmers using a wearable inertial sensor device." Biomech. and Med. in Swimming XI; Eds (2010): 178-180.

ASPETTI BIOMOLECOLARI DELLE ATTIVITÀ SPORTIVE

\section{2}

\section{MIR-1303 and MIR-548A2 are down-regulated in muscle tissue of veteran football players: effects} on longevity

Annamaria Mancini ${ }^{1}$, Daniela Vitucci ${ }^{2}$, Francesca Maria Orlandella $^{3}$, Alessia Terracciano ${ }^{2}$, Stefania Orrü ${ }^{2}$, Peter Krustrup ${ }^{4}$, Giuliana Salvatore $^{2}$, Pasqualina Buono ${ }^{2}$

University Parthenope, Naples, Italy, Movement Sciences and Wellness, Napoli, Italy ${ }^{1}$;

University Parthenope, Naples, Italy, Movement Sciences and Wellness, Naples, Italy; ${ }^{2}$, 3irccs Sdn Naples, Italy, 3irccs Sdn Naples, Italy, Naples, Italy;

University of Southern Denmark, Department of Sports Science and Clinical Biomechanics, Odense, Danimarca ${ }^{4}$

Purpose: The aims of the present research were to analyse the effects of long-term football training on the miRNAs skeletal muscle expression in veterans football players (VPG) of and on the identification of their putative targets involved in the longevity pathways.

Methods: miRWalk database was used to generate predicted and validated miRNA-binding sites of known human genes1. The expression of the identified putative targets were analyzed and validated by western blot analysis both in muscle biopsies from VPG and in myoblast LHCN-M2 cells.

Results: We previously identified 14 miRNAs differently expressed in VPG compared to active untrained subjects (control group; CG) muscle $(\mathrm{p}<0.05) 2$; among them, miR-1303 and miR-548a2 showed the highest significant score $(p<0.01)$. Based on the intersection of different algorithms runned simultaneously by miRWalk we identified many putative targets of miR-1303 and miR-548a2. Among them, we characterized $\mathrm{BAG}-2$, a protein involved in ubiquitination of misfolded protein by CHIP, as a down-regulated species by miR-1303 in human muscle and in LHCN-M2 cells3. Then, we identified PSMDs, components of the $26 \mathrm{~S}$ proteasome, involved in the ATP-dependent degradation of ubiquitinated proteins as miR-468a 2 target.

Conclusions: In conclusion, we demostrated, for the first time, that miR-1303 and miR-548a2 expressions are regulated by long-term football training in veterans' muscle tissues and that their putative targets are involved in protein quality control pathways. These results contributes to the enlargement of knowledge on the molecular mechanisms linking long-term training and longevity, through autophagy pathways.

\section{References}


1. Sticht C. et al. (2018) miRWalk: An online resource for prediction of microRNAbinding sites. PLoS One. 13(10): $\mathrm{e} 0206239$.

2. Mancini A. et.al. (2019) Lifelong Football Training: Effects on Autophagy and Healthy Longevity Promotion. Front Physiol. 10:132.

3. Mancini A. et al. (2021) Regular football training down-regulates miR-1303 muscle expression in Veterans. EJAP https://doi.org/ 10.1007/s00421-021-04733-1

\section{3}

\section{Angiogenic and myogenic response to exercise in healthy and diabetic older adults}

\author{
$\underline{\text { Tatiana Moro }}^{1}$, Antonio Paoli ${ }^{1}$, Giorgia Schiavinotto $^{l}$, Lisa Vigo $^{l}$, \\ Elena Volpi ${ }^{2}$, Blake B. Rasmussen ${ }^{3}$, Christopher S. Fry ${ }^{4}$ \\ University of Padua, Department of Biomedical Sciences, University \\ of Padova, Padova, Italy ${ }^{1}$; \\ University of Texas Medical Branch, Department of Internal \\ Medicine/geriatrics, Galveston, $U_{S A}^{2}$; \\ University of Texas Medical Branch, Department of Nutrition \\ and Metabolism, Galveston, $U S A^{3}$; \\ University of Kentucky, Department of Athletic Training and Clinical \\ Nutrition, Lexington, USA ${ }^{4}$
}

Purpose: Aging induces an overall decline in muscle quality and function and impaired tissue regenerative capacity. This physiological process advances slowly with healthy aging and can be rushed by concurrent diseases resulting in risk of developing disability, frailty, and institutionalization in later life. Recently, capillaries have taken on a leading role on the hypertrophic response to resistance exercise (RET), both in young and older individuals. Type 2 diabetes (T2DM) is a metabolic disease associated with a decrease in capillary density and function, which may reduce the hypertrophic capacity following exercise. Thus, the aim of this study was to indagate the angiogenic and myogenic response to RET in a group of healthy and diabetic older adults.

Methods: 19 healthy older adults $(71.1 \pm 4.3$ years $)$ and 11 diabetic older adults $(69.8 \pm 3.4$ years $)$ underwent to 12 weeks of RET. Muscle biopsies from vastus lateralis were obtained before and after 12 weeks of training. Immunohistochemical analysis was used to quantify myosin heavy chain isoform expression, cross-sectional area (CSA), satellite cell abundance (SC), capillary and pericytes content. Pre and post study days also included a stable isotope infusion trial wherein subjects ingested an essential amino acid (EAA) to test their anabolic response.

Results: Following RET, only healthy older adults increased total fiber CSA $(\mathrm{p}<0.05)$, and the results was driven by a specific increase in fiber type II size $(+23 \%$; $p<0.05)$. Healthy older adults also presented a higher capillarization compared to the T2DM pairs ( $\mathrm{p}<$ 0.05 ), but training did not affect this parameter. Pericytes counts increased in the healthy group, but the difference wasn't significant. SC was significant affected by T2DM condition a no effect of training was detected. Ultimately, T2DM didn't show any anabolic response to EAA compared to healthy counterpart.

Conclusions: T2DM seems to be characterized by an anabolic resistance and this may be due to a lower endothelial and SC function. Unfortunately, 12 weeks of RET doesn't seems to be sufficient to reverse this condition.

\section{4}

Muscle mitochondrial uncoupling and cardiac output predict VO2PEAK during single-leg knee extensor exercise $\frac{\text { Gaia Giuriato }^{I}}{\text { Venturelli }^{I}}$, Elisa Calabria $^{l}$, Federico Schena $^{l}$, Massimo $^{2}$

Università Di Verona, Neuroscienze, Biomedicina E Del Movimento, Verona, Italy ${ }^{l}$

Purpose: The dynamic single-leg knee extensor ergometer (SLKE) is a model used to isolate the quadriceps exercising muscle. Accordingly, even at peak exercise, the cardiac responses are not maximized and the peripheral components (muscle mitochondria $\mathrm{O} 2$ extraction) play a crucial role. In healthy populations, the maximal exercise is usually limited by the cardiac output (CO), but during the SLKE exercise, muscular exhaustion overcomes the cardiorespiratory limits. This study aims to understand the relative contribution of the $\mathrm{CO}$ and the muscle mitochondrial respiration during a maximal SLKE exercise. We hypothesize peripheral components to predominantly influence VO2peak during SLKE.

Methods: During a maximal incremental test $(+10 \mathrm{~W} / \mathrm{min})$ at a SLKE, VO2peak of 20 young healthy subjects $(10 \mathrm{M}+10 \mathrm{~F})$ was assessed using a metabolic cart. Moreover, a muscle biopsy sample from the vastus lateralis was taken from each participant, and mitochondrial respiration was analyzed by high-resolution respirometry. $\mathrm{CO}$, and mitochondrial Electron Transport Coupling Efficiency (ETCE), Net Oxphos Capacity (NOC), Electron Transport Excess Capacity (EC) were calculated.

Results: A multiple linear regression was calculated to predict VO2peak based on CO, ETCE, NOC, and EC. A significant regression equation was found $(\mathrm{F}(4,13)=64.26, \mathrm{p}<0.001)$, with an $\mathrm{R} 2$ of 0.9519. Participants' predicted VO2peak is equal to $3.308+$ 0.1034 (CO, 4.02(ETCE) + 0.01226(NOC, 0.0001476(EC), where CO is expressed in $\mathrm{L} / \mathrm{min}$. CO ( $\mathrm{p}<0.0001)$, ETCE $(\mathrm{p}=0.001)$, and NOC ( $p=0.027$ ) were significant predictors of VO2peak.

Conclusions: During SLKE, the VO2peak is highly influenced by CO and muscle mitochondria $\mathrm{O} 2$ extraction capacity. Indeed, the increase in $\mathrm{VO} 2$ is linearly associated with increases in $\mathrm{CO}$ and mitochondrial respiration in the OXPHOS/ET state. Most importantly, we assessed that VO2peak is inversely associated with the mitochondrial coupling efficiency, usually influenced by ROS accumulation, protein secretion, leak/slip partial coupling. The last point confirms in vivo studies and suggests that mitochondrial uncoupling, as well as CO, play a key role in VO2 consumption during maximal SLKE exercise.

\section{References}

1. J. R. Gifford et al., Symmorphosis and skeletal muscle VO2 max: in vivo and in vitro measures reveal differing constraints in the exercise-trained and untrained human. J Physiol 594, 1741-1751 (2016).

2. M. F. Bartlett, L. F. Fitzgerald, R. Nagarajan, J. A. Kent, Oxidative ATP synthesis in human quadriceps declines during 4 min of maximal contractions. J Physiol 598, 3789 (2020). 
105

\section{Different types of training protect muscles against reactive oxygen species injury in $\mathrm{LHCN}-\mathrm{M} 2$ human myoblast cells}

\author{
Annamaria Mancini ${ }^{1}$, Daniela Vitucci ${ }^{2}$, Giuseppe Labruna ${ }^{2}$, \\ Pasqualina Buono ${ }^{3}$ \\ University Parthenope, Naples, Italy, Movement Sciences \\ and Wellness, Napoli, Italy ${ }^{l}$; \\ Ceinge-biotecnologie Avanzate, Naples, Italy., Ceinge-biotecnologie \\ Avanzate, Naples, Italy' \\ University Parthenope, Naples, Italy, Movement Sciences \\ and Wellness, Naples, Italy
}

Purpose: We investigated the effects of different types of aerobic/ anaerobic training on oxidative metabolism in the human LHCN-M2 myoblast cell line (1) and on their ability to protect from $\mathrm{H} 2 \mathrm{O} 2$ injury. Methods: Long-term trained ( $>3$ years; $\geq 180 \mathrm{~min} /$ week) healthy young males (19-28 years) were recruited: swimmers (SW n.6), bodybuilders (BB n.6), soccer (SO n.6) and volleyball players (VB n.6). Blood samples were collected $8 \mathrm{~h}$ after the last training bout. Human sera pool (HC) was used as untrained control. LHCN-M2 were cultured in growth (GM $15 \% \mathrm{FBS}$ ) or in differentiation medium, $\mathrm{DM}$, supplemented with $0.5 \%$ serum from trained subjects or $\mathrm{HC}$ respectively, for $4 \mathrm{~d}$ (2). The extracellular acidification rate (ECAR) and the oxygen consumption rate (OCR), markers of glycolysis and mitochondrial respiration, respectively, were measured by Seahorse XFe96 analyser. OxPhos: basal respiration, ATP-linked respiration, maximal respiration; Glycolysis: basal glycolysis and glycolytic capacity indexes were calculated. LHCN-M2 cells were treated with 5 and $10 \mathrm{mM} \mathrm{H} 2 \mathrm{O} 2$ in $\mathrm{DM}$, supplemented with $0.5 \%$ serum from trained subjects. ROS concentration was estimated by DCFDA/ H2DCFDA-Cellular ROS Assay Kit.

Results: ECAR and OCR rates were higher in LHCNM2 cells cultured with GM compared to DM, supplemented with different sera (p $<0.001)$. Cells treated with sport trained sera showed a lower rate of basal glycolysis compared to HC $(\mathrm{p}<0.05)$. Moreover, in the DM cultured cells the ATP-linked respiration was higher in "mixed" (VB $+\mathrm{SO})$ and aerobic SW sports compared to HC-treated cells $(\mathrm{p}<$ 0.001 and $\mathrm{p}<0.01$, respectively). After, $\mathrm{H} 2 \mathrm{O} 2$ injury (5 and $10 \mathrm{mM}$ $\mathrm{H} 2 \mathrm{O} 2$ windows) $\mathrm{ROS}$ production was lower in $\mathrm{VB}+\mathrm{SO}, \mathrm{BB}$ and $\mathrm{SW}$ treated cells compared to $\mathrm{HC}$.

Conclusions: Our results suggested that the treatment with sport trained sera induces a reduction in basal glycolysis and glycolytic capacity in LHCN-M2. Furthermore, ATP-linked respiration is increased by mixed-sport and $\mathrm{SW}$ treatment suggesting that a decrease in proton leak may be an important cytoprotective mechanism against ROS in LHCN-M2.

References

1. Zhu $\mathrm{CH}$. et al. Cellular senescence in human myoblasts is overcome by human telomerase reverse transcriptase and cyclindependent kinase 4: Consequences in aging muscle and therapeutic strategies for muscular dystrophies 2007 Aging Cell. 6:515.

2. Vitucci et al. Serum from differently exercised subjects induces myogenic differentiation in LHCN-M2 human myoblasts $2018 \mathrm{~J}$ Sports Sci.36:1630-1639.

\section{6}

The effect of dexamethasone and tumor necrosis factorA on gene expression, protein synthesis and sarcomere arrangement in myotubes: shedding light on in vitro models of skeletal muscle wasting

${ }_{\text {Andrea di Credico }}{ }^{1}$, Giulia Gaggi ${ }^{1}$, Barbara Ghinassi ${ }^{1}$, Pascal Izzicupo $^{I}$, Angela Di Baldassarre ${ }^{I}$

Università, Università Degli Studi “G. D'annunzio”, Chieti, Italy ${ }^{I}$

Purpose: Physiological and pathological conditions may lead to muscle wasting, a condition of unbalanced muscle protein degradation. In vitro models of muscle wasting are fundamental to study stimuli and molecules able to reverse such a condition. Generally, treatment with dexamethasone (DEX) or tumor necrosis factor- $\alpha$ $(\mathrm{TNF}-\alpha)$ are used as muscle wasting models; however, results are controversial. Aim of this study was to describe the effect of DEX and $\mathrm{TNF}-\alpha$ alone and in combination on structural integrity, protein synthesis and gene expression of myotubes after $24 \mathrm{~h}$ of treatment. Methods: Differentiated C2C12 cells were treated with DEX $(1 \mu \mathrm{M})$, TNF- $\alpha(20 \mathrm{ng} / \mathrm{ml})$ alone and in combination for $24 \mathrm{~h}$. Modifications of Myh1, Myh2, Mhy7, ACTA1 and DMD gene expression were evaluated by qPCR, while $\alpha$-actinin and myosin protein expressions were analyzed by western blot and immunofluorescence.

Results: The muscle fibers diameter, sarcomere banding and sarcomere protein expression were significantly affected by TNF- $\alpha$, but not by DEX treatment. These structural changes were not observed in DEX + TNF- $\alpha$ samples. At molecular levels, TNF- $\alpha$ treatment determined a decrease of Myh2 and Myh1 mRNA levels, while DEX significantly increases Myh7, Myh1 and DMD gene expression. In DEX + TNF- $\alpha$, Myh1 expression was significantly higher than in TNF- $\alpha$ treated myotubes. ACTA1 gene expression was not affected by the treatments.

Conclusions: TNF- $\alpha$, but not DEX, affected the skeletal muscle morphology and sarcomere integrity. These negative effects were not observed when cells underwent the combined DEX + TNF- $\alpha$ treatment, suggesting a protective role for DEX. The observation that DEX increased the expression of genes encoding for structural proteins, while TNF- $\alpha$ led to opposite trend, supports this hypothesis. These results show that TNF- $\alpha$, but not short term DEX treatment may represent a model of muscle wasting. Nevertheless, further studies are needed to investigate the molecular responses to these molecules and to optimize models of skeletal muscle wasting.

\section{References}

1. Sartori, R., Romanello, V. \& Sandri, M. (2021) Mechanisms of muscle atrophy and hypertrophy: implications in health and disease. Nat Commun 12, 330.

2. Morrison-Nozik A, Anand P, Zhu H, et al. (2015) Glucocorticoids enhance muscle endurance and ameliorate Duchenne muscular dystrophy through a defined metabolic program. Proc Natl Acad Sci USA. 112(49):E6780-E6789.

\section{7}

Whole-body cryotherapy treatment modulates the innate immune response in non-professional football players

Valentina Selleri $^{1}$, Marco Mattioli ${ }^{2}$, Domenico Lo Tartaro $^{3}$, Sara De Biasi $^{2}$, Annamaria Paolini ${ }^{3}$, Marcello Pinti ${ }^{1}$, Roberta D'alisera ${ }^{4}$, Laura Roli ${ }^{5}$, Pasqualino Maietta ${ }^{6}$, Ferdinando Tripi $^{7}$, Emanuele 
Guerra $^{4}$, Anna Vittoria Mattioli ${ }^{8}$, Gustavo Savino ${ }^{4}$, Tommaso Trenti ${ }^{5}$, Andrea Cossarizza ${ }^{2}$, Milena Nasi $^{8}$

Università Degli Studi Di Modena E Reggio Emilia (unimore), Dipartimento Di Scienze Della Vita, Modena, Italy ${ }^{1}$;

Università Degli Studi Di Modena E Reggio Emilia (unimore),

Dipartimento Di Scienze Mediche E Chirurgiche Materno-infantili E Dell'adulto, Modena, Italy ${ }^{2}$;

Università Degli Studi Di Modena E Reggio Emilia (unimore), Dipartimento Di Scienze Biomediche, Metaboliche E Neuroscienze, Modena, Italy ${ }^{3}$;

Azienda Usl Di Modena, Dipartimento Di Salute Pubblica, Medicina Dello Sport, Modena, Italy ${ }^{4}$;

Azienda Usl Di Modena, Dipartimento Di Medicina Di Laboratorio E Patologia, Modena, Italy ${ }^{5}$;

Alma Mater Studiorum Università Di Bologna (unibo), Dipartimento Di Scienze Per La Qualità Della Vita, Bologna, Italy";

Associazione Sportiva No Profit A.s. La Fratellanza 1874, A.s. La

Fratellanza 1874, Modena, Italy ${ }^{7}$;

Università Degli Studi Di Modena E Reggio Emilia (unimore), Dipartimento Chirurgico, Medico, Odontoiatrico E Di Scienze Morfologiche, Modena, Italy ${ }^{8}$

Purpose: Whole-body cryotherapy (WBC) is a recently widely strategy used for muscle recovery after injury that can to activate inflammatory response. WBC consists of short exposure, of about 2-3 min, to dry air at cryogenic temperatures up to $-190{ }^{\circ} \mathrm{C}$. The aim of our study is to analyze WBC effects on metabolic, hormonal, and immunological responses of non-professional football players (NPFPs).

Methods: nine male NPFPs (age: $20 \pm 2$ years) on the same team are recruited and, in particular, they played and trained each day before, during, and after WBC treatment. We collected NPFPs blood samples immediately before WBC and after 5 once-day sessions, then we evaluated a set consisting of fifty analytes, including hormones profile, hematologic parameters, and serum chemistry. We proceeded with monocytes (Mo) phenotyping and then we investigated the concentration of some plasmatic markers with anti-inflammatory effects (IL2RA, IL1RN) or typically increased during inflammation [CCL2, IL-18, free mitochondrial DNA (mtDNA)].

Results: WBC treatment (WBC-t) leads to a decrease not only in mean platelet volume, mean corpuscular hemoglobin, and ferritin levels, but also in testosterone and estradiol levels, even if they remain within the normal ranges. This treatment is also responsible for total Mo increased and, in particular, a reduction of classical Mo has been demonstrated in parallel with an increase of non-classical ones. Moreover, each Mo subset shows lower expression of CXCR4. Considering pro-inflammatory molecules, IL1RA showed a tendency to decrease, while IL1RN and IL18 decreased in plasma after WBC-t. Conversely, circulating mtDNA levels appeared unaltered by treatment.

Conclusions: The differences detected in monocyte subset after WBC-t suggest that, in this condition, Mo could be redistributed into the surrounding tissue. In addition, CXCR4 reduction in Mo subsets could be due to their differentiation process. Hence, WBC could promote Mo differentiation through a mechanism that is still unknown. Since WBC seems to regulate the innate immune system in the enrolled NPFPs, it could have a role in tissue repair beyond a beneficial anti-inflammatory effect.

References

1. Patel K, Bakshi N, Freehill MT, and Awan TM (2019) WholeBody Cryotherapy in Sports Medicine. Curr Sports Med Rep 18:136-140.

2. Nasi M, Bianchini E, Lo Tartaro D, De Biasi S, Mattioli M, Paolini A, Gibellini L, Pinti M, De Gaetano A, D'Alisera R, Roli L, Chester J, Mattioli AV, Polverari T, Maietta P, Tripi F, Stefani
O, Guerra E, Savino G, Trenti T, and Cossarizza A (2020) Effects of whole-body cryotherapy on the innate and adaptive immune response in cyclists and runners. Immunol Res. 68: 422-435.

\section{ALLENAMENTO E VALUTAZIONE SPORTIVA 3}

\section{8}

\section{Fast or slow start? The role of running strategies in triathlon}

$\underline{\text { Kristina Skroce }}^{I}$, Cantor Tarperi $^{2}$, Iacopo Brasi ${ }^{I}$, Federico Schena ${ }^{1)}$.

Università Di Verona, Dip. Neuroscienze, Biomedicina E Movimento, Verona, Italy ${ }^{1}$;

Università Di Torino, Dip. Scienze Cliniche E Biologiche, Torino, Italy $^{2}$

Purpose: To investigate the impact of different running intensity distributions on the physiological parameters after a variable cycling fraction (triathlon-specific) in sprint triathlon format.

Methods: Thirteen well-trained triathletes (age $36.4 \pm 10.8$ yy, height $174.8 \pm 7.9 \mathrm{~cm}$, mass $70.6 \pm 11.1 \mathrm{~kg}$; V'O2max $62.4 \pm 8.9$ mil min$1 \mathrm{~kg}-1$; mean $\pm \mathrm{SD}$ ) attended the laboratory five times in order to complete two incremental tests and three subsequent experimental sessions that mimed different intensities distribution in triathlon race. Experimental sessions were realized in a randomized order and differentiated by the speed of the 1st running kilometer: SS (slow-start) starting at $95 \%$ of vVT2; C (constant) starting at vVT2 and FS (faststart) starting at $105 \%$ of $\mathrm{vVT} 2$. Measurement of physiological variables like V'O2 and HR were recorded throughout the whole cycle-run session. BLa- and ratings of perceived exertion (RPE) were recorded at end-bike, 1st, 3rd, 5th and end-run time point.

Results: A meaningful difference was found between the 1 st $\mathrm{Km}$ of SSRP and FSRP in V'O2 ( $\mathrm{SE}=0.58, \mathrm{P}=0.0005)$, BLa- $(\mathrm{SE}=0.21, \mathrm{P}$ $=0.0097), \mathrm{HR}(\mathrm{SE}=1.23, \mathrm{P}=0.0011)$ and $\mathrm{RPE}(\mathrm{SE}=2.83, \mathrm{P}=$ $0.0047)$ values. BLa- had a decreasing trend in all conditions from the end-bike to the end-run time point. No differences in these parameters in-between protocols was found at the end of the running bout whatever the condition.

Conclusions: Significant differences in physiological parameters were found mainly on the 1st kilometer of the running bout. These findings are important for athletes (in particular triathletes) to determine the optimal racing strategy and to focus on the best training Methods to perform in medium duration efforts.

\section{References}

1. Hausswirth C, Le Meur J, Bieuzen F, Brisswalter J, Bernard T. Pacing strategy during the initial phase of the run in triathlon: influence on overall performance. Eur J Appl Physiol. 2010; 108 (6): $1115-1123$.

2. Suriano R, Vercruyssen F, Bishop D, Brisswalter J. Variable power output during cycling improves subsequent treadmill run time to exhaustion. J Sci Med Sport. 2007; 10 (4): 244-251.

3. Bailey SJ, Vanhatalo A, Dimenna FJ, Wilkerson DP, Jones AM. Fast start strategy improves VO2 kinetics and high.

\section{9}

Perception of velocity during free-weight exercises: difference between back squat and bench press 
Università, Università Degli Studi Di Roma 'foro Italico', Rome, Italy $^{1}$

Purpose: Velocity-based training(VBT) is an objective method of autoregulation utilized to monitor the intensity of a lift(1).Perception of bar velocity $(\mathrm{PV})$ is a subjective parameter shown to be valid in estimating velocity during resistance exercises $(2,4)$ and useful when training without specific devices. The aim of this study was to investigate if $\mathrm{PV}$ can be improved through specific training sessions and if it differs between exercise modalities.

Methods: Eleven resistance trained participants(27 \pm 5 years; $78 \pm$ $9 \mathrm{~kg} ; 180 \pm 6 \mathrm{~cm}$ ) completed two 1-RM tests (bench press(BP) and back squat( $(\mathrm{Sq})$ ). Thereafter, before(pre-test) and after(post-test) 5 -week of specific training (10 sessions, 2 day/week) their PV was assessed with 5 blinded loads in random order.For each load athletes were asked to perform 2 reps and report their PV using the velocity scale validated by Bautista et al.(Vp)(3).During the 5-week of training they received visual and auditory feedback while performing different loads and viewed the scale at the end of each set.Mean propulsive velocity(Vr) of the barbell was recorded by a linear position transducer(Vitruve).Spearman's rho correlation coefficients(rs) were used to determine interrelationships between variables.A simple linear regression analysis was performed using $\mathrm{Vr}$ as the criterion variable and $\mathrm{Vp}$ as the predictor variable.Delta score(ds) represents the difference between $\mathrm{Vp}$ and Vr.Bland-Altman plots were constructed to explore the agreement between variables. Friedman test and Wilcoxon signed-rank tests were used to identify any differences in ds between exercises and loads.

Results: Positive correlations between $\mathrm{Vp}$ and $\mathrm{Vr}$ found in pre-test (Sq:rs $=0,820, \mathrm{p}<0,01, \mathrm{BP}: \mathrm{rs}=0,860, \mathrm{p}<0,01)$ increased in the post-test (Sq:rs $=0,930, \mathrm{p}<0,01, \mathrm{BP}: \mathrm{rs}=0,881, \mathrm{p}<0,01$ ).In the pretest, $\mathrm{Vp}$ and $\mathrm{Vr}$ showed coefficient of determination(R2) values of $0,62(\mathrm{Sq})$ and $0,69(\mathrm{BP})$; In the post-test:0,81(Sq) and 0,77(BP).Friedman test confirmed that ds was consistent across exercises.Wilcoxon tests showed significant differences $(p<0,05)$ between dsBP(pre-test) and dsBP(post-test).Moreover, significant differences in ds were found between loads $(\mathrm{p}<0,05)$ in the BP.

Conclusions: Data reveal a more precise PV in Bp compared to $\mathrm{Sq}$ in the pre-tests and a greater improvement in the PV during Sq with training. Moreover, this study demonstrates that PV can be improved and thus improve the accuracy of the estimation. With this in mind, athletes can regulate their load based on PV even with no specific devices.

References

1. Guerriero, A.,et al., (2018). Journal of Functional Morphology and Kinesiology, 3.4: 55.

2. Bautista, I. J., et al., (2016). The Journal of Strength \& Conditioning Research, 30.2: 421-429.

3. Bautista, I. J., et al., (2014). Journal of sports science \& medicine, 13.3: 542

4. Lazarus, A., et al., (2021). Physiology \& Behavior, 231: 113,316.

\section{0}

\section{The effects of uphill and downhill high-intensity interval training on running performance in recreational runners}

\author{
Lorenzo Budel $^{1}$, Kristina Skroce ${ }^{2}$, Samuel D'emanuele ${ }^{1}$, Simone \\ Bettega $^{I}$, Federico Schena ${ }^{1}$, Cantor Tarperi ${ }^{1}$ \\ Università Degli Studi Di Verona, Dipartimento Di Neuroscenze, \\ Biomedicina E Movimento, Verona, Italy ${ }^{I}$. \\ Università Di Rijeka, Facoltà Di Medicina, Rijeka, Croazia ${ }^{2}$
}

Purpose: Despite the lack of scientific evidence, uphill high intensity interval running is considered a milestone in increasing runner's lower limb strength through specific adaptations. However, recent scientific literature proposed downhill running as a type of specific eccentric stimulus for increasing strength parameters and running economy. The aim of this study was to evaluate the effect of uphill high intensity interval treadmill training (UHIT) and downhill high intensity interval treadmill training (DHIIT) protocol on strength and physiological parameters connected with running performance.

Methods: Fourteen recreational runners were recruited to take part in the study and were randomly assigned to UHIIT ( $\mathrm{n}=7 ; 43.0 \pm$ 5.4 years; Vo2max $56.0 \pm 5.7 \mathrm{mlo} 2 \mathrm{~min}-1 \mathrm{~kg}-1)$ and DHIT $(\mathrm{n}=7$; $44.4 \pm 10.3$ yy; Vo2max $54.7 \pm 7.9$ mlo2 min-1 kg-1). Before and after a training period of 6 weeks based on 3 running sessions/week, maximal oxygen uptake (Vo2max), the velocity at Vo2max (vVo2max), maximal voluntary contraction (MVC), maximal dynamic force (1RM) and Running Economy (RE) were evaluated.

Results: The UHIIT group have showed significant increases $(\mathrm{p}<$ 0.05 ) in Vo2max, vVo2max and $2 \mathrm{~km}$ running performance, while DHIIT group showed significant increases $(p<0.05)$ only in Vo2max but significant decrease $(\mathrm{p}<0.05)$ on $2 \mathrm{~km}$ running performance. No differences were found in RE and strength parameters in any groups. Conclusions: The results of this study indicate that in recreational runners, uphill high intensity interval training (UHIIT) causes increases in metabolic but not strength parameters. Moreover, downhill high intensity interval training (DHIIT) appears to be a safe method in terms of avoiding injuries but not useful for enhancing running performance.

\section{References:}

1. Barnes, KR, Hopkins, WG, McGuigan, MR, Kilding, AE. Effects of Different Uphill Interval-Training Programs on Running Economy and Performance. Int J Sports Physiol Perform 8: 639-647, 2013.

2. Ferley, DD, Osborn, RW, and Vukovich, MD. The effects of incline and level-grade high-intensity interval treadmill training on running economy and muscle power in well-trained distance runners. J. Strength Cond. Res 28: 1298-309, 2014.

3. Li, F, Newton, RU, Shi, Y, Sutton, D, Ding, HY (2019a). Correlation of ecc.

\section{1}

\section{Could the addition of sport-specific interactions be beneficial on agility and strength performance assessment in football academies? A variation of the Illinois test}

\author{
Raffaele Zinno ${ }^{\text {, Stefano Di Paolo }}{ }^{1}$, Luca Milandri ${ }^{1}$, Erika Pinelli ${ }^{l}$, \\ Giuseppe Barone $^{I}$, Laura Bragonzoni ${ }^{I}$
}

Università Di Bologna, Dipartimento Di Scienze Per La Qualità Della Vita, Bologna, Italy

Purpose: The agility and strength performances gradually improve during the growth, though in a nonlinear way [1]. Nonetheless, such performances are increasingly required in early-age football academies, so the attention to the assessment methodologies. Currently, the assessment is often limited to sliced tests without sport-specific conditions. For example, many agility tests in football are executed without ball or opponents. The aim of the study is to assess agility and strength performances in different aged players with the introduction of a ball variable. The hypothesis of the study was that the execution time of both agility and strength tests would decrease with growth and 
that the addition of a ball variable would increase the level of detail of the agility test.

Methods: One hundred forty academy football players were enrolled. The players were grouped based on their age to under $6(n=12)$, under $8(\mathrm{n}=28)$, under $10(\mathrm{n}=25)$, under $12(\mathrm{n}=24)$, under $14(\mathrm{n}=$ $24)$, and under $16(\mathrm{n}=27)$ players. The tests performed were: 1$)$ Illinois test (IT); 2) Illinois test with ball (ITB); 3) Hexagon test. The time elapsed for each test was recorded. A repeated measure ANOVA with age category as a between-subject factor was performed to investigate the differences in Illinois test times with and without ball. A determination coefficient $\mathrm{r} 2$ was used to investigate the association between Illinois with and without ball and Hexagon test.

Results: The results comparing the Illinois tests with and without ball showed a consistent difference in the execution time up to under 8 . From the Under 10 on, the times were comparable. While, after 8 year-age the difference decreased (n.s.). When analysing the ball effect differences were found up to Under 8 without ball and up to Under 10 with ball. The coefficients of determination were $\mathrm{r} 2=0.71$ and $\mathrm{r} 2=0.79$ for IT and ITB, respectively.

Conclusions: A cut-off for agility was found at Under 8 category for both IT and ITB. This is in line with current literature on learning and developing phases in football. The ITB also showed differences for Under 10 category and was more explicative of the Hexagon test. For these reasons, the ITB variation might be useful in young football players assessment due to the combination of agility, ballistic, and strength performance.

\section{References}

1. Chiodera P, Volta E, Gobbi G, Milioli MA, Mirandola P, Bonetti A, Delsignore R, Bernasconi S, Anedda A, Vitale M. (2008) Specifically designed physical exercise programs improve children's motor abilities. Scand J Med Sci Sports. Apr;18(2):179-87.

\section{2}

\section{Differences in muscle quality between male and female firefighters}

\author{
Ludovica Cardinali ${ }^{1}$, Fabrizio Perroni ${ }^{2}$, Stefano Amatori ${ }^{2}$, Lamberto \\ Cignitti $^{3}$, Carlo Baldari ${ }^{4}$, Mario Vetrano ${ }^{5}$, Marco Bruno Luigi \\ Rocchi $^{2}$, Davide Sisti ${ }^{2}$, Sveva Maria Nusca ${ }^{5}$, Laura Guidetti ${ }^{6}$ \\ University of Rome "foro Italico", Department of Movement, Human \\ and Health Sciences, Rome, Italy ${ }^{1}$; \\ University of Urbino Carlo Bo, Department of Biomolecular \\ Sciences, Urbino, Italy ${ }^{2}$; \\ Italyn Firefighting Corp, Italyn Firefighting Corp, Rome, Italy ${ }^{3}$, \\ Ecampus University, Ecampus University, Novedrate, Como, Italy ${ }^{4}$; \\ "sapienza" University of Rome, Physical Medicine \\ and Rehabilitation Unit, Sant'andrea Hospital, Rome, Italy"; \\ University "niccolo Cusano", Department Unicusano, Rome, Italy ${ }^{6}$
}

Purpose: Muscle quality index (MQI), which is expressed as muscle strength per unit of muscle mass, has received a great attention by scientific literature as it better reflects muscular conditioning than muscle strength and mass separately [1]. Considering that male (MF) and female (FF) firefighters work side by side performing the same tasks and muscle strength is a critical factor in firefighters' duties [2], the aim of study was to analyze possible sex differences in MQI.

Methods: Anthropometric (height, weight, and BMI), body composition (Skeletal Muscle Mass, SM) and fitness (handgrip) parameters of $10(\mathrm{FF}=5 ; \mathrm{MF}=5)$ Italyn firefighters' recruits (Age: $37 \pm 3$ years; BMI: $24 \pm 3 \mathrm{~kg} / \mathrm{m} 2$ ) were measured. The MQI was calculated dividing the value obtained with the handgrip test by the SM estimated with the bioimpedance analysis. Unpaired t test was used to evaluate differences $(\mathrm{p}<0.05)$ between sex in all parameters.

Results: The results showed that there were no statistical differences in age between sexes (MF: $38 \pm 3$ years., FF: $37 \pm 3$ years.). On the contrary, significant differences $(\mathrm{p}<0.05)$ were found in anthropometric (MF: $81 \pm 13 \mathrm{~kg}, 173 \pm 5 \mathrm{~cm}, 26 \pm 2 \mathrm{~kg} / \mathrm{m} 2$; FF: $56 \pm 3 \mathrm{~kg}$, $163 \pm 4 \mathrm{~cm}, 21 \pm 2 \mathrm{~kg} / \mathrm{m} 2$ ), body composition (MF: $38 \pm 2 \mathrm{~kg}$; FF: $22 \pm 1 \mathrm{~kg}$ ) and strength (MF: $56 \pm 4 \mathrm{~kg}$; FF: $30 \pm 2 \mathrm{~kg}$ ) parameters between sex. MQI values were significantly different $(\mathrm{p}<0.0001)$ between MF $(2.4 \pm 0.1)$ and FF $(1.3 \pm 0.1)$.

Conclusions: Although muscle mass may be a major contributor to the production of strength, however muscle quantity is not synonymous with muscle quality. According to the reference values of Barbat-Artigas et al. [1], our study found low value of muscle quality in FF and normal value in MF. Implementing specific training programs is important to decrease the injury risk and increase job performance in firefighters with reference to FF.

\section{References}

1. Barbat-Artigas, S, Rolland, Y, Zamboni, M, Aubertin-Leheudre, M (2012) How to assess functional status: a new muscle quality index. The journal of nutrition, health \& aging, 16(1), 67-77.

2. Boyce, R, Ciulla, S, Jones, GR, Boone, E, Elliott, SM, Combs, CS (2008) Muscular Strength and Body Composition Comparison Between the Charlotte-Mecklenburg Fire and Police Departments. International journal of exercise science, 1, 5 .

\section{3}

\section{Evaluating intra- and inter-seasonal fitness and training load variations of elite U20 soccer players}

\author{
Massimo Saccà ${ }^{l}$, Danilo Bondi ${ }^{2}$, Fabrizio Balducci ${ }^{1}$, Giuseppe \\ Mazza $^{l}$
}

University of Firenze, School of Human Health Sciences, Firenze, Italy $^{l}$, University "G. D'annunzio" of Chieti-Pescara, Department of Neuroscience, Imaging and Clinical Sciences, Chieti, Italy ${ }^{2}$

Purpose: As a matter of fact, fitness profiling of elite football players consists of a holistic monitoring with anthropometric and physiological criteria, influenced by nature and nurture. With this study, we aimed to evaluate inter and intra-seasonal differences and the influence of playing position on a set of training and fitness metrics, during the last step to entry in professional soccer.

Methods: Sample consisted of 74 male football players (Mdn of age: 18 years; BMI: $22.4 \pm 1.3 \mathrm{~kg} / \mathrm{m} 2$ ) from the U20 team of an elite Italyn society, tested both at beginning, during and after three consecutive sport seasons. The team of coaches and trainer remained the same across the years, and the training plan and playing style were tailored to the characteristics of the players across seasons. Players underwent anthropometric measurement and were tested for a set of performance tasks; in addition, the GPS-derived measures of metabolic power (MP) and equivalent distance index of every athlete were analysed. A number of linear mixed models were conducted, and participants were considered as the random factor, with random intercept.

Results: Players of the second season performed the worst in Mognoni test $(\mathrm{p}<0.001, \mathrm{R} 2=0.20)$, and no differences emerged for counter movement jump and anthropometrics. The training load was different across seasons, as equivalent distance index decreased almost linearly $(\mathrm{p}<0.001, \mathrm{R} 2=0.53)$ from the first to the last season. The model selection criteria revealed Bosco-Vittori test achieved the best fit. BMI increased $(p<0.001$, Cohen's $f=0.45)$, and fat mass decreased $(p=0.029, f=0.18)$, from the beginning to the end of the 
sport season; the same result was achieved for counter movement jump $(\mathrm{p}=0.035, \mathrm{f}=0.16)$, but with different intra-seasonal trends $(\mathrm{p}$ $<0.001, \mathrm{f}=0.29)$. MP was almost significantly higher in midfielder $(\mathrm{p}=0.054, \mathrm{R} 2=0.12)$.

Conclusions: Differences in team characteristics and training plans emerged from several perspectives. Prospectively, a network approach in modelling performance metrics in sports team could unveil original interconnections between performance factors, likely adding novel insights for training plan and tactical strategies. In addition, authors advice for multiparametric longitudinal assessments and a huge database of sports data for paving talent identification.

\section{References}

1. Becker M, Sperlich B, Zinner C, and Achtzehn S (2020) IntraIndividual and Seasonal Variation of Selected Biomarkers for Internal Load Monitoring in U-19 Soccer Players. Front Physiol 11.

2. Carling C, Orhant E (2010) Variation in body composition in professional soccer players: interseasonal and intraseasonal changes and the effects of exposure time and player position. J Strength Cond Res 24:1332-1339.

\section{CUORE E SPORT}

\section{4}

\section{Gender specific equation to estimate peak oxygen uptake in female patients with cardiovascular disease}

\author{
Valentina Zerbini ${ }^{1}$, Andrea Raisi ${ }^{1}$, Jonathan Myers ${ }^{2}$, Tommaso Piva ${ }^{1}$, \\ Rosario Lordi ${ }^{I}$, Giorgio Chiaranda ${ }^{3}$, Gianni Mazzoni ${ }^{1}$, Giovanni \\ Grazzi ${ }^{1}$, Simona Mandini ${ }^{l}$
}

University of Ferrara, Department of Neuroscience and Rehabilitation, Ferrara, Italy ${ }^{1}$;

Stanford University, School of Medicine, Stanford, CA, USA ${ }^{2}$, Ausl Piacenza, Public Health Department, Bologna, Italy ${ }^{3}$

Purpose: Women continue to be labeled as a 'special population' in many guidelines related to cardiovascular disease (CVD), which remains the leading cause of death in women. The aim of this study was to develop a specific prediction equation for estimating peak oxygen uptake (VO2peak) based on a moderate 1-km treadmill walking test $(1 \mathrm{k}-\mathrm{TWT})$, in female patients with cardiovascular disease.

Methods: 84 female patients, aged $68 \pm 10$, completed a ramp-like protocol cardiopulmonary exercise test (CPX) for directly measured VO2peak and a 1KTWT using a self-regulated intensity of 11 to 13 of 20 on the Borg Scale to estimate CRF (eCRF). The data from the two tests were used to develop an equation for estimating VO2peak $(\mathrm{mL} /$ $\mathrm{kg} / \mathrm{min}$ ) using best subsets multiple regression analysis including age, body weight, height, body mass index, and mean and maximal walking speed during the $1 \mathrm{k}$-TWT. Data were analyzed by the D'Agostino-Pearson test for normal distribution. The equation obtained was then validated using Pearson product moment correlation, spread standard error (SEE) and paired Student t-test to compare measured and estimated VO2peak. To evaluate the agreement between the prediction equation and directly measured VO2peak, an intraclass correlation coefficient (ICC) estimate was calculated. The level of statistical significance was set at $\mathrm{P}<0.05$.

Results: Data analysis included the results of 75 subjects. Multiple regression analysis showed that age, body mass index, walking speed, were the most powerful predictors of $\mathrm{VO} 2$ peak. The comparison between measured and estimated VO2peak values yielded a multiple correlation of $\mathrm{R}=0.82(\mathrm{P}<0.001)$, and there were no significant differences between measured and estimated VO2peak.

Conclusions: The equation developed from the $1 \mathrm{k}$-TWT accurately predict VO2peak in female patients with cardiovascular disease. The model may represent a feasible, low cost, and simple tool for indirect estimations of cardiorespiratory fitness in an outpatient setting when CPX is not available. The current results may therefore be useful in providing a sex-specific approach to managing CVD in women.

\section{References}

1. Gulati M. Improving the Cardiovascular Health of Women in the Nation. Moving Beyond the Bikini Boundaries. Circulation 2017; 135:495-498.

2. Chiaranda G, Myers J, Mazzoni G, et al. Peak oxygen uptake prediction from a moderate, perceptually regulated, $1-\mathrm{km}$ treadmill walk in male cardiac patients. J Cardiopulm Rehabil Prev 2012; 32: 262-269.

\section{5}

\section{Relationship between exercise capacity and grip strength in elderly outpatients with coronary artery disease}

Andrea Raisi ${ }^{1}$, Valentina Zerbini ${ }^{1}$, Rosario Lordi ${ }^{1}$, Tommaso Piva ${ }^{l}$, Giovanni Grazzi $^{1}$, Gianni Mazzoni ${ }^{1}$, Simona Mandini ${ }^{l}$

\section{Center For Exercise Science and Sports, Università Di Ferrara /} Dipartimento Di Neuroscienze E Riabilitazione, Ferrara, Italy ${ }^{I}$

Purpose: Progressive ageing of the population has highlighted the importance of the relationship between physical function and frailty in patients with chronic disease. Among the manifestations of coronary artery disease (CAD) include reduced physical function resulting in impaired exercise tolerance, reduced capacity to perform daily activities, and higher rates of disability. The aim of this study was to assess the association between exercise capacity and grip strength (GS) in a cohort of elderly patients with CAD.

Methods: 65 outpatients (aged $75 \pm 6$ years) participating in an exercise-based secondary prevention program were included. Exercise capacity was assessed through the estimation of maximal oxygen consumption (VO2peak) and the measurement of walking speed (WS) by performing a submaximal Treadmill Walking Test. GS was evaluated in the dominant arm using a hand-held dynamometer.

Results: Subjects were subdivided into two groups based on the WS measured at enrollment. Patients with higher VO2peak and WS values exhibited significantly higher GS $(\mathrm{p}<0.01)$ and weekly Leisure Time Physical Activity (wLTPA) $(\mathrm{p}<0.01)$. Furthermore, higher GS levels were modestly correlated with higher of VO2peak values $(r=$ $0.56, \mathrm{p}<0.01)$ and WS $(\mathrm{r}=0.62, \mathrm{p}<0.01)$ and inversely associated with age $(r=-0.42, p<0.01)$. To analyze the influence of age on GS levels, the study population was subdivided into two groups (Younger $\leq 74$, Older $>74$ ) on the basis of the median age. Older FW had higher GS levels than older SW $(p<0.01)$. This observation was confirmed by multiple regression analysis $(\mathrm{p}<0.01)$, where GS was strongly associated with WS $(\mathrm{p}<0.01)$ and age $(\mathrm{p}=0.01)$, while other variables were not.

Conclusions: The main results of this study were the significant direct relationships between GS and WS and between GS and VO2peak. The assessment of these parameters was feasible and well tolerated. Preliminary results suggest that regular walking activity performed at higher average WS can help to maintain physical function in elderly patients with cardiovascular disease.

\section{References}


1. Grazzi G, et al. Improved walking speed is associated with lower hospitalization rates in patients in an exercise-based secondary prevention program. Heart 2016.

2. Seong JY et al. Association Between Aerobic Exercise and Handgrip Strength in Adults: A Cross-Sectional Study Based on Data from the Korean National Health and Nutrition Examination Survey (2014-2017). J Nutr Health Aging 2020.

\section{6}

\section{Two-month exercise-based biocircuit program increases muscle strength and cardiorespiratory fitness in cardiac patients}

\author{
Rosario Lordi ${ }^{l}$, Nicola Grazzi ${ }^{2}$, Andrea Raisi ${ }^{1}$, Valentina Zerbini ${ }^{l}$, \\ Tommaso Piva ${ }^{1}$, Simona Mandini ${ }^{l}$, Vincenzo Benedetto Flotta ${ }^{2}$, \\ Silvano Zanuso ${ }^{3}$, Antonio Sammali ${ }^{2}$, Danilo Cervellati ${ }^{2}$, Marco \\ Tesini $^{2}$, Alessandro Capucci ${ }^{2}$, Giovanni Grazzi ${ }^{1}$ \\ Center For Exercise and Sports, Dipartimento Di Neuroscienze E \\ Riabilitazione Università Degli Studi Di Ferrara, Ferrara, Italy ${ }^{I}$; \\ Abcardio Bologna Srl., Settore Scientifico, Bologna, Italy ${ }^{2}$; \\ Technogym S.p.a., Scientific Departement, Cesena, Italy ${ }^{3}$
}

Purpose: Regular practice of aerobic exercise, associated with resistance training has been highly recommended by current international guidelines as an important part of cardiovascular rehabilitation/secondary prevention $(\mathrm{CR} / \mathrm{SP})$ programs. Latest evidence demonstrate how physical activity prescription could impact on the reduction of hospitalization and mortality and on an improvement of the quality of life. Aim of this work was to assess the effect of 2-month supervised exercise-based sessions, on muscular strength (MS) and on cardiovascular fitness (CRF) in a cohort of cardiac patients.

Methods: 18 patients aged $60( \pm 11)$ years, were involved in a CR/SP program. All patients at baseline (T0) performed a moderate and perceptually regulated (11-13 on the 6-20 Borg scale) treadmill walking test to estimate oxygen uptake (VO2). Furthermore, maximum strength of upper and lower limbs was assessed through an isokinetic test. The program was carried out by using BIOCIRCUIT equipment (by Technogym), alternating aerobic and resistance training, for $45 \mathrm{~min}, 2 / 3$ times per week. BIOCIRCUIT represents an innovative training system, allowing trainers to programme and modify training loads. Patients performed a reduced eccentric contraction at $70 \%$ of the predicted concentric, evaluated during the first test. All physical evaluations were performed both at the baseline(T0) and at the end of the program (T1).

Results: Primary outcome was the improvement of MS assessed through physical tests. More in detail, there was a significative increase in all the tests: Chest Press $(\Delta 4.1 \pm 5.7 \mathrm{~kg})(\mathrm{P}<0.01)$, Low Row $(\Delta 9.5 \pm 11.2 \mathrm{~kg})(\mathrm{P}<0.01)$, Shoulder Press $(\Delta 2.9 \pm 4.9 \mathrm{~kg})(\mathrm{P}$ $<0.01)$, Leg Press $(\Delta 34.0 \pm 21.1 \mathrm{~kg})(\mathrm{P}<0.01)$, Leg Extension $(\Delta$ $15.4 \pm 10.2 \mathrm{~kg})(\mathrm{P}<0.01)$, Leg Curl $(\Delta 10.8 \pm 9.2 \mathrm{~kg})(\mathrm{P}<0.01)$. Secondary outcome was the improvement of CRF based on the walking test results, in terms of VO2 $(\Delta 1.7 \pm 2.2 \mathrm{ml} / \mathrm{kg} / \mathrm{min})(\mathrm{P}=$ $0.01)$.

Conclusions: The proposal of this circuit training was well tolerated from all the subjects. This type of physical intervention was individualized and based on the patient's perceived exertion. Results has demonstrated a general improvement of MS and CRF after 2 months. This evidence could reinforce the importance of physical activity prescription and demonstrate the efficacy of a novel validated training system even in cardiac patients.

\section{References}

1. Westcott WL,et al. Prescribing physical activity: applying the ACSM protocols for exercise type, intensity, and duration across 3 training frequencies. Phys Sportsmed. 2009 Jun;37(2):51-8.

2. Pelliccia A et al. 2020 ESC Guidelines on sports cardiology and exercise in patients with cardiovascular disease. Eur Heart J. 2021 Jan 1;42(1):17-96.

\section{7}

The effects of a $50 \mathrm{~km}$ ultramarathon race on cardiac biomarkers in highly trained athletes $\frac{\text { Marco Alfonso Perrone }}{}{ }^{1}$, Maurizio Volterrani $^{2}$, Chiara Salimei $^{l}$,

Dipartimento Di Scienze Cliniche E Medicina Traslazionale, Università Di Roma Tor Vergata, Rome, Italy ${ }^{l}$; Dipartimento Di Promozione Delle Scienze Umane E Della Qualità Della Vita, Università Telematica San Raffaele, Rome, Italy ${ }^{2}$; Dipartimento Di Scienze Motorie, Umane E Della Salute, Università Di Roma Foro Italico, Rome, Italy

Purpose: High sensitivity cardiac troponins I (hs-cTnI) and T (hs$\mathrm{cTnT}$ ) and natriuretic peptides (BNP and NT-proBNP) are universally recognized as biomarkers of cardiac damage in patients with acute coronary syndromes and heart failure respectively. The high sensitivity Methods for cardiac biomarkers have provided many answers to fundamental pathophysiological and clinical questions in patients with heart disease. Less information is available on their significance in healthy athletes after high intensity exercise. The aim of this study was to evaluate hs-cTnI and NT-proBNP in highly trained runners after a $50 \mathrm{~km}$ ultramarathon.

Methods: We have enrolled 20 highly trained male athletes who have run a $50 \mathrm{~km}$ ultramarathon. Blood samples were collected $2 \mathrm{~h}$ before the start of the race (T0) and $20 \mathrm{~min}$ after the end of the race (T1). The blood concentrations of hs-cTnI and NT-proBNP measured before the race were within reference intervals in all runners.

Results: Hs-cTnI significantly increased after the end of the race (median: 19 ng/L [IQR: $12.5-25.75$ ] versus $6 \mathrm{ng} / \mathrm{L}$ [IQR: 4.25-8.0]; P $<0.001$ ), in three cases over the upper reference limit (URL) of $34 \mathrm{ng} / \mathrm{L}$. NT-proBNP also significantly increased (median: $78 \mathrm{ng} / \mathrm{L}$ [IQR: 68.25-87.75] versus $22 \mathrm{ng} / \mathrm{L}$ [IQR: 18.25-26.75]; $\mathrm{P}<0.001$ ). Three other athletes reached concentration over the URL (125 ng/L). Conclusions: Our study showed a significant increase in hs-cTnI and NT-proBNP in highly trained athletes after a $50 \mathrm{~km}$ ultramarathon race, with $30 \%$ of runners showing values of cardiac biomarkers above URL after the race. More studies are needed to better understand the effects of intense exercise on the heart of trained athletes and their potential clinical significance.

\section{8}

\section{Cardiovascular rehabilitation: the trainer's role} $\frac{\text { Luigi Renzi }}{\text { Calcagno }^{I}}$, Germano Guerra ${ }^{I}$, Antonio Musto $^{2}$, Giuseppe

Department of Medicine and Health Science, University of Molise, Department of Medicine and Health Science, University of Molise. Campobasso (cb), Italy ${ }^{1}$;

U.o.s. Rehabilitation and Cardiac Prevention, A. Cardarelli Hospital Campobasso, U.o.s. Rehabilitation and Cardiac Prevention, A. Cardarelli Hospital Campobasso, Campobasso (cb), Italy ${ }^{2}$ 
Purpose: The aims of this study was to evaluate the long-term benefits of cardiovascular rehabilitation in patients with chronic peripheral obliterating arteriopathy (AOCP).

Methods: 17 AOCP patients were analyzed (medium age was 69,5). All of the patients underwent a treadmill test according to the Gardner Test (GT). The experimental protocol, lasting 24 weeks, was administered with the same modalities of the GT, by increasing the training load in which the speed is constant and the inclination increases of $2 \%$ every $2 \mathrm{~min}$. All patients were instructed to identify, during training sessions, the ischemic threshold and therefore to modify the working parameters to independently manage daily activities, to avoid the appearance of absolute claudication. To evaluate the speed that the patients sustained the following criteria were considered: the relative claudication (when the patient started to feel pain) and the absolute claudication (the beginning of ischemic pain). The following parameters were compared during peak physical activity during the first and last GT: the duration of physical activity, the inclination and the speed of the treadmill.

Results: Concerning the duration of the test, at the pre GT, $40 \%$ of the patients were able to reach $20 \mathrm{~min}$ of physical activity, $30 \%$ reached $30 \mathrm{~min}$ and the remaining $30 \%$ reached $40 \mathrm{~min}$. In the post GT, $100 \%$ of the patients were able to continue without reaching ischemic levels undergoing $40 \mathrm{~min}$ of physical activity. Considering the treadmill's inclination, in the pre GT, $76 \%$ used an inclination reaching 0 to 4 , $18 \%$ reached an inclination of 8 and $6 \%$ reached an inclination of 10 . However, during the post GT $12 \%$ of patients used an inclination between $0-4,76 \%$ reached an inclination of 8 and the remaining $12 \%$ reached 10 . Valuating the treadmill's speed, in the pre test $59 \%$ of the patients involved used a speed of $0-3,5 \mathrm{~km} / \mathrm{h}, 35 \%$ reached $4,5 \mathrm{~km} / \mathrm{h}$ and $6 \%$ reached $5,5 \mathrm{~km} / \mathrm{h}$. In the post test, $12 \%$ reached the speed of $3,5 \mathrm{~km} / \mathrm{h}, 26 \%$ reached $5,5 \mathrm{~km} / \mathrm{h}, 35 \%$ reached the speed of 6,5 and the remaining $27 \%$ reached $8,5 \mathrm{~km} / \mathrm{h}$.

Conclusions: The cardiac rehabilitation assumed a primary role within the therapeutic strategies and the secondary prophylaxis in vasculopathy patients. The GT was found to be effective in improving the driving range and therefore the quality of life of subjects with peripheral arterial disease, allowing them to acquire the ability to recognize and prevent any recurrence of cardiovascular symptoms.

References

1. Hirsch AT et al. ACC/AHA 2005 Practice Guidelines for the Management of Patients With Peripheral Arterial Disease (Lower Extremity, Renal, Mesenteric, and Abdominal Aortic). J Am Coll Cardiol 2006; 47: 1239-312.

2. Balady GJ et al. Recommendations for cardiovascular screening, staffing, and emergency policies at health/fitness a randomized, controlled trial. Circulation 2003; 107: 22,016.

\section{9}

\section{Cardiovascular risk prediction in football players: a machine learning framework}

\author{
Davide Barbieri ${ }^{1}$, Luciana Zaccagni ${ }^{1}$ \\ Università Di Ferrara, Dipartimento Di Neuroscienze E \\ Riabilitazione, Ferrara, Italy $^{I}$
}

Purpose: The aim of this study was to evaluate a machine learning methodology for the prediction of cardiovascular risk in a large sample of football players. In particular, we tested whether resampling could improve classification performances in an imbalanced dataset.

Methods: A large sample of Croatian male adults, playing football at a competitive level, was assessed during sport medical examinations, from both an anthropometric and clinical point of view. In particular, the athletes were examined by means of electrocardiography (ECG). In case of a positive outcome, the athlete was denied medical clearance for competitive sport practice, and eventually underwent further examinations. As expected, the large majority of the tested individuals were negative. Therefore, the collected data presented an evident class-imbalance. A set of machine learning algorithms, including resampling, was applied to the dataset, in order to predict the class of the athletes, as either positive or negative. In particular, the majority (i.e. negative) class was undersampled, while the minority (i.e. positive) class was oversampled. Results were compared with ECG outcomes. Classification performances were evaluated by means of some standard metrics.

Results: Resampling techniques determined a substantial improvement of classification performances, even if not equally in all the considered algorithms.

Conclusions: Machine learning provides a useful set of classification tools, which can be used to assist medical diagnostic, especially in the prediction of cardiovascular risk in football players. In case of class imbalance, as it is often the case with clinical dataset, where the positive (i.e. more interesting) individuals represent the minority class, resampling can effectively improve prediction performances.

\section{References}

1. Chawla NV, Bowyer KW, Hall LO, Kegelmeyer WP, SMOTE: Synthetic Minority Over-sampling Technique. J Artif Intell Res 2002, 16, 321-357.

2. Zaccagni L, Lunghi B, Barbieri D, Rinaldo N, Missoni, S, Šaric T, Šarac J, Babic V, Rakovac M, Bernardi F, Gualdi-Russo E, Performance prediction models based on anthropometric, genetic and psychological traits of Croatian sprinters Biol Sport 2019, 36, $17-23$.

\section{AMBIENTI STRAORDINARI E TECNOLOGIA}

\section{0}

\section{“Kanchenjunga exploration \& physiology" research project: trekking and high altitude hypoxia}

\section{Vittore Verratti ${ }^{1}$, Danilo Bondi ${ }^{2}$, Tiziana Pietrangelo ${ }^{2}$}

Sismes - Società Italiana Di Scienze Motorie E Sportive, University "g. D'annunzio" of Chieti-pescara,department of Psychological, Health and Territorial Sciences, Chieti, Italy ${ }^{1}$;

Sismes - Società Italiana Di Scienze Motorie E Sportive, University "g. D'annunzio" of Chieti-pescara, Department of Neuroscience, Imaging and Clinical Sciences., Chieti, Italy ${ }^{2}$

Purpose: High-altitude trekking provides a unique ecological model for exploring extreme environments and the response of humans subjected simultaneously to physical activity and hypobaric hypoxia. The "Kanchenjunga Exploration \& Physiology" project investigated several aspects of adaptive physiology to hypoxia and physical activity.

Methods: The experimental subjects completed a combined circuit of $300 \mathrm{~km}$ length, for a total of $110 \mathrm{~h}$ of walking across 19 days, along a demanding route with over $16,000 \mathrm{~m}$ of vertical displacement in the Himalayas. The project investigated adaptive physiological responses to trekking throughout low (500-2000 m), moderate (2000-3,000 m), and high $(3000-5500 \mathrm{~m})$ altitudes, by two experimental groups composed by six healthy Caucasian lowlanders and six healthy Nepalese porters.

Results: Among a plethora of results, it was found that altitude trekking caused a transient reduction in body fluids and muscle mass, 
despite adequate protein intake. Near-infrared spectroscopy (NIRS)derived parameters, i.e., Tot-Hb and tissue saturation index (TSI), were gathered at rest and after bouts of 3-min resistive exercise, both in the quadriceps and in the forearm muscles. TSI decreased with altitude, particularly in forearm muscles (from 66.9 to $57.3 \%$ ), rather than in quadriceps (from 62.5 to $57.2 \%$ ); Nepalese porters achieved greater values in thigh TSI than Italyn trekkers. Tot-Hb was increased after exercise. At altitude, such increase appeared to be higher in the quadriceps. We reported a disentanglement between serum ferritin (sFER) and Vit D temporal relationships in response to hypobaric hypoxia exposure, with respect to other pieces of evidence about inflammation or poor iron status. The observed reduction of sFER levels was related to altitude per se as inflammation or anemia were medically excluded. sFER, therefore, may act as a primary factor in the examination of hypobaric hypoxia in field studies. Nocturnal, rather than diurnal, cardiorespiratory function was affected by altitude hypoxia.

Conclusions: In the context of typical respiratory adaptation, even in the absence of a specific mechanistic analysis, we suggest that a decrease in metabolism could reverse the hypoxia-related adaptive augmentation of breathing rate. Several differential responses might be a consequence of the long-term adaptive memory due to the frequent exposures to altitude. High-altitude physiology should take advantage of ecological models for providing novel and original insights.

\section{References}

1. Bondi D et al. Feeding Your Himalayan Expedition... Nutrients. 2021 Jan 30;13(2):460.

2. Verratti V et al. Muscle Oxygen Delivery in the Forearm and in the Vastus Lateralis Muscles in Response to Resistance Exercise...Front Physiol. 2020 Nov 10;11:607,616.

3. Bondi D et al. Case studies in physiology: Nocturnal cardiorespiratory adaptive differences between an Italyn trekker and a Nepali guide. Physiol Rep. 2020 Aug;8(16).

\section{1}

\section{Independent and combined effects of hypoxia and cold on submaximal and maximal exercise}

\section{Alexa Callovini ${ }^{1}$, Alessandro Fornasiero ${ }^{1}$, Aldo Savoldelli ${ }^{1}$, Marco Decet $^{I}$, Spyros Skafidas ${ }^{I}$, Barbara Pellegrini ${ }^{1}$, Lorenzo Bortolan ${ }^{I}$, Federico Schena ${ }^{I}$}

Cerism, Centro Di Ricerca Sport, Montagna E Salute, Dipartimento Di Neuroscienze, Biomedicina E Movimento, Rovereto, Italy ${ }^{I}$

Purpose: The aim of this study was to determine the independent and combined effects of exposure to hypoxia and cold on the physiological and perceptual responses to submaximal and maximal exercise.

Methods: 10 trained male subjects (age $27.6 \pm 4$; weight $71 \pm 6 \mathrm{~kg}$, VO2max: $66 \pm 4 \mathrm{~mL} / \mathrm{kg} / \mathrm{min}$ ) randomly performed an incremental test to exhaustion on a motorized treadmill (slope: $25 \%$ ) under four different environmental conditions: Normothermic Normoxia (NN: $18{ }^{\circ} \mathrm{C}, 20.9 \% \mathrm{FiO} 2$ ), Normothermic Hypoxia (NH: $18{ }^{\circ} \mathrm{C}, 13.5 \%$ FiO2), Cold Normoxia (CN: $-20{ }^{\circ} \mathrm{C}, 20.9 \%$ FiO2) and Cold Hypoxia $\left(\mathrm{CH}:-20{ }^{\circ} \mathrm{C}, 13.5 \% \mathrm{FiO} 2\right)$. Physiological (HR, La, SpO2) and perceptual (RPE, Thermal Sensation (TS)) variables at maximal workload (WL) were evaluated, and WL, HR and RPE at the first and second lactate thresholds (LT1, LT2) determined for each condition. Results: Main effects of cold $(\mathrm{p}<0.05)$ and hypoxia $(\mathrm{p}<0.05)$, without interaction effects $(\mathrm{p}>0.05)$ were found on maximal WL $(\mathrm{NN}: 6.8 \pm 0.7, \mathrm{NH}: 5.6 \pm 0.5, \mathrm{CN}: 6.7 \pm 0.7, \mathrm{CH}: 5.4 \pm 0.5 \mathrm{~km} / \mathrm{h}$ ) and HR (NN: $191 \pm 7, \mathrm{NH}: 182 \pm 10, \mathrm{CN}: 185 \pm 8, \mathrm{CH}: 176 \pm$ $8 \mathrm{bpm})$. Both hypoxia and cold decreased TS values $(\mathrm{p}<0.05)$ at maximal exercise-intensity, whilst no differences in La and RPE were detected. WL and HR at both LT1 and LT2 significantly decreased with cold $(\mathrm{p}<0.05)$ and hypoxia $(\mathrm{p}<0.05)$, with no interaction effect $(p>0.05)$. Finally, an interaction effect $(p=0.03)$ on RPE at LT1 $(\mathrm{NN}: 49 \pm 6, \mathrm{NH}: 39 \pm 10, \mathrm{CN}: 50 \pm 7, \mathrm{CH}: 30 \pm 12)$ and a main effect of hypoxia on RPE at LT2 (NN: $65 \pm 4$, NH: $591 \pm 9$, CN: 66 \pm 7 , $\mathrm{CH}: 54 \pm 11$ ) were reported.

Conclusions: Hypoxia and cold determined an impairment in performance (decreased WL), physiological (decreased HR) and perceptual (impaired TS) variables at maximal intensities, but no amplification effect was seen when combining the two environmental stressors. Furthermore, changes in WL, HR and RPE at LT1 and LT2 suggest cautious application of lactate thresholds for exercise intensity prescription in hypoxic and cold environments.

\section{References}

1. Mugele H, Oliver SJ, Gagnon D, Lawley JS.(2021) Integrative crosstalk between hypoxia and the cold: Old data and new opportunities. Exp Physiol. 106(1):350-358.

2. Weckbach LT, Kassem S, Maier F, et al.(2019) Impact of Acute Altitude Exposure on Lactate Threshold. High Alt Med Biol. 20(3):322-323.

3. Lloyd A, Havenith G.(2016) Interactions in human performance: An individual and combined stressors approach. Temperature. 3(4):514-517.

\section{2}

\section{Effects of an acute exposure to simulated and real altitude on organ transplant recipients: the "Monte Rosa" project}

Aldo Savoldelli ${ }^{l}$, Gianluigi Dorelli ${ }^{2}$, Alessandro Fornasiero ${ }^{l}$, Alexa Callovini $^{I}$, Marco Decet ${ }^{I}$, Barbara Pellegrini ${ }^{1}$, Luigi Vanoni ${ }^{3}$, Luca Dalle Carbonare $^{2}$, Federico Schena ${ }^{1}$

University of Verona, Cerism, Sport Mountain and Health Research Centre, University of Verona, Rovereto, Italy ${ }^{1}$;

University of Verona, Department of Medicine, School of Medicine In Sports and Exercise, Verona, Italy ${ }^{2}$;

Italyn Alpine Club, National Medical Commission, Milan, Italy ${ }^{3}$

Purpose: Nowadays, patients can do almost all kind of sports after transplantation, even high altitude mountaineering [1]. We aimed to evaluate physical performance and changes in cardiopulmonary parameters (CPET) of transplant recipients during both real and simulated acute exposure to high altitude.

Methods: A group of 4 transplant (3 lung and 1 kidney) recipients and a cystic fibrosis patient were tested with a CPET during walking on an inclined $(25 \%)$ treadmill both in normoxia $(\mathrm{N})$ and in hypoxia (H) $(4050 \mathrm{~m})$. Prediction of the susceptibility to acute mountain sickness (AMS) was determined 20-30 min after exposure to the simulated hypoxia trough arterial oxygen saturation ( $\mathrm{SpO} 2)[2]$. Thereafter, the subjects attempted a two-days high altitude expedition to Capanna Margherita (4554 m), where we monitored SpO2 at different altitudes and symptoms of AMS were reported.

Results: Maximal vertical speed (N: $950 \pm 233 ; \mathrm{H}: 631 \pm 170 \mathrm{~m} * \mathrm{~h})$, maximal oxygen consumption $(\mathrm{N}: 42.2 \pm 10.8 ; \mathrm{H}: 29 \pm 6.2$ mlO2*min-1*kg-1), maximal heart rate $(\mathrm{N}: 162 \pm 24 \mathrm{H}: 149 \pm$ 26 bpm) and minimal SpO2 (N: $92 \pm 7 \mathrm{H}: 81 \pm 4 \%)$ were lower $(\mathrm{P}<$ $0.05)$ in the simulated altitude CPET. Two subjects resulted susceptible to AMS during the simulated exposure, but only one of them experienced AMS during the expedition (at $4554 \mathrm{~m}$ ). Two subjects 
reached the summit (the cystic fibrosis patient and the kidney recipient) with a mean $\mathrm{SpO} 2$ of 77.5, similar to that measured on a group of control subjects. One subject stopped at $3700 \mathrm{~m}$ due to abdominal pain while two of them withdrew at $4000 \mathrm{~m}$ due to cold related problems.

Conclusions: Contrary to other studies on transplant recipients with longer time of adaptations to high altitude [1], [3] we observed a lower rate of success during a two-days expedition. However, we can speculate that a stable patient with cystic fibrosis or after an organ transplantation, if properly trained, can achieve high altitude even with an acute exposure.

\section{References}

1. Gieszer B et al., "Lung Transplant Patients on Kilimanjaro," Transplant. Proc., vol. 51, no. 4, pp. 1258-1262, May 2019.

2. M. Burtscher, S. C, and F. M, "Prediction of the susceptibility to AMS in simulated altitude," Sleep Breath., vol. 12, no. 2, pp. 103-108, May 2008.

3. E. J. Van Adrichem et al., "Tolerance of organ transplant recipients to physical activity during a high-altitude expedition: Climbing mount Kilimanjaro," PLoS One, vol. 10, no. 11, Nov. 2015.

\section{3}

\section{Oxidative stress after administration of oxygen at different pressures and concentrations: a randomized trial}

\author{
Matteo Paganini ${ }^{1}$, Simona Mrakic-sposta ${ }^{2}$, Alessandra Vezzoli ${ }^{2}$ \\ Tommaso Antonio Giacon ${ }^{I}$, Alberto Oppio ${ }^{1}$, Cinzia Dellanoce ${ }^{2}$, \\ Tatiana Moro ${ }^{1}$, Antonio Paoli ${ }^{1}$, Gerardo Bosco ${ }^{1}$ \\ Nutrition and Exercise Physiology Laboratory, Department \\ of Biomedical Sciences, University of Padova, Padova, Italy ${ }^{I}$; \\ Institute of Clinical Physiology, National Research Council (cnr), \\ Milan, Italy ${ }^{2}$
}

Purpose: While regular training induces beneficial effects by stimulating the expression of antioxidant mechanisms, an inadequately intense exercise can be detrimental. Reactive oxygen species (ROS) increase during exercise, creating a redox imbalance towards oxidation [1] and, potentially, cellular damage. Normobaric and hyperbaric oxygen (HBO) breathed while not exercising previously showed to induce antioxidant enzymes [2], but literature is still poor. This study tested the effectiveness of oxygen breathed $(\mathrm{O} 2)$ at different mixtures and pressures in mitigating oxidative stress.

Methods: 22 well-trained, adult athletes from different disciplines were included and assigned to 5 groups: controls; $30 \%$, or $50 \% \mathrm{O} 2$; and $100 \% \mathrm{O} 2$ at 1.5 or 2.5 ATA. Subjects trained at least 3 times/ week, and a total of 20 treatments were administered every other day. Samples of venous blood, saliva, and urine were obtained at basal state (T0), at the end of treatments (T1), and 1 month after the end (T2). ROS production, antioxidant capacity (TAC), lipid peroxidation (8-isoPGF2), total and reduced aminothiols (cysteine-Cys; cysteinylglycine-CysGly; Homocysteine-Hcy; and glutathioneGSH), creatinine, neopterin and uric acid concentrations were determined.

Results: Treatments with both $30 \%$ and $50 \%$ O2 mixtures and both HBO groups showed an increase in ROS levels and lipid peroxidation (T1), along with a decrease in TAC (T1) and counterbalancing ROSrelated damages at T2. Changes in total CysGly and Hcy concentration were recorded at $\mathrm{T} 1$ after treatments of $50 \% \mathrm{O} 2$ mixtures. Furthermore, $50 \% \mathrm{O} 2$ and both $\mathrm{HBO}$ at 1.5 and 2.5 ATA showed a higher level of total and reduced GSH (T2) resulting from a positive shift in redox balance towards a more reduced state. Increase in neopterin concentration were observed at $\mathrm{T} 1$ in breathing $50 \% \mathrm{O} 2$ and HBO 2.5 ATA, followed by a slow return to basal levels, and between HBO treatment 1.5 and 2.5 ATA at T1. No differences were found in renal function biomarkers in all treatments.

Conclusions: The results suggest that $\mathrm{HBO}$ at 1.5 and 2.5 ATA similarly induce protective mechanisms against ROS, despite the latter could expose the body to higher ROS levels and neopterin concentration. The increase in total and reduced GSH indicates that redox status has been positively unbalanced towards the reduced state and capable of contrasting ROS damages. Further applications should be investigated in the future, especially targeting frail or sarcopenic subjects.

\section{References}

1. Peternelj, T.T. and J.S. Coombes, Antioxidant supplementation during exercise training: beneficial or detrimental? Sports Med, 2011. 41(12): p. 1043-69.

2. Morabito C, Bosco G, Pilla R, Corona C, Mancinelli R, Yang Z, Camporesi EM, Fanò G, Mariggiò MA. Effect of pre-breathing oxygen at different depth on oxidative status and calcium concentration in lymphocytes of scuba divers. Acta Physiol (Oxf). 2011 May;202(1):69-78.

\section{4}

\section{Analysis of fluidity of movement in parkour using one inertial measurement system}

\section{Francesco Feletti $^{1}$, Cristian Bracco ${ }^{1}$, Andrea Aliverti ${ }^{1}$, Antonio La Torre $^{2}$, Matteo Bonato ${ }^{2}$}

Politecnico Di Milano, Dept. of Bioengineering, Milan, Italy ${ }^{1}$; Università Degli Studi Di Milano Statale, Dipartimento Di Scienze Biomediche Per La Salute, Milan, Italy ${ }^{2}$

Purpose: Fluidity is a multi-parametric aspect of movement; its quantification and the evaluation of its variability may guide the performance evaluation, training and rehabilitation decisions. Therefore, this study's primary goal was to establish whether a quantitative measure of fluidity through a single wearable MIMU was accurate enough to highlight meaningful differences between advanced and novice athletes.The secondary goal was to highlight intra-individual differences of fluidity between various parts and subsequent repetitions of the same track.

Methods: Seventeen parkour participants, subdivided into two groups based on their expertise, were recruited. A wearable inertial measurement unit was worn on the back of the pelvis by each participant during three sequential repetitions of a specially created parkour routine. Two fluidity parameters, namely smoothness and hesitation, were considered. Smoothness was calculated as the number of inflexions on the so-called jerk graph. Hesitation was defined as the percentage of the drop in the CoM velocity.

Results: The smoothness is an encouraging parameter to quantitatively estimate the fluidity of movement in an acrobatic sport like parkour. In particular, it resulted significantly lower in advanced athletes (mean: 126.4; range: 36-192) than in beginners (mean: 179.37; range: 98-272) during the wall run's parkour movement (Kruskal Wallis; $\mathrm{p}=0.02$ ). Moreover, a qualitative analysis of hesitation showed that beginner athletes tend to produce more prominent velocity drops and negative deflection than more advanced athletes. Conclusions: A MIMU-based system allows measuring fluidity and assessing its variability, providing unique data about sports performance, potentially beneficial to guide and evaluate the training and rehabilitation process. 


\section{References}

1. Kerr, A., Pomeroy, V.P., Rowe, P.J., Dall, P., Rafferty, D. (2013) Measuring movement fluency during the sit-to-walk task. Gait Posture 37(4):598-602. https://doi.org/10.1016/j.gaitpost.2012. 09.026.

2. Pavei, G., Cazzola, D., La Torre, A., Minetti, A.E. (2014) The biomechanics of race walking: literature overview and new insights. Eur J Sport Sci 14(7):661-70. https://doi.org/10.1080/ 17461391.2013.878755.

\section{5}

\section{Infrared thermography technique for the evaluation of athletic task in olympic weightlifting}

\author{
Riccardo Sorrentino $^{1}$, Gabriella Epasto $^{2}$, Eugenio Guglielmino ${ }^{2}$, \\ $\overline{\text { Ludovico Magaudda }}^{l}$ \\ Università Degli Studi Di Messina, Dipartimento Di Scienze \\ Biomediche, Odontoiatriche E Delle Immagini Morfologiche E \\ Funzionali, Messina, Italy ${ }^{l}$, Università Degli Studi Di Messina, \\ Dipartimento Di Ingegneria, Messina, Italy
}

Purpose: Weightlifting technique has always been evaluated through kinematic software, by describing the movement externally. Internal load has always been described by the athlete's own sensations. The aim of this study is to focus on using Infrared Thermography (IRT) to describe the internal load of the muscle, in order to assess the athlete's technique and the thermal response of muscles in relation with workload.

Methods: Five weightlifters, $3 \mathrm{M}$ and $2 \mathrm{~F}$, were asked to perform three lifts of snatch and later three lifts of clean and jerk, at 60-70$80 \%$ of 1RM (Repetition Maximum), in front a FLIR SC540 infrared camera placed $3 \mathrm{~m}$ from the target. Participants were asked to avoid smoking and using creams that could affect thermal response of the skin. Tests were carried out in a gym respecting guidelines about the use of IRT, with an environmental temperature of $23{ }^{\circ} \mathrm{C}$ and humidity between $40-70 \%$. Male participants were asked to wear only shorts; female participants wore also training clothing. Participants who needed wrist bandages were allowed to use them. Thermal images and videos were recorded and processed with Research IR Max FLIR software.

Results: Results showed how an incremental correlation between muscle activation and 1RM load exists and is possible to analyse by IRT. The more the subjects went up with loads percentage, the more their muscle temperature raised. Moreover, results suggested that more experienced athletes (more than 4 years of weightlifting practice) with the same load, were able to increase their temperature less than the less experienced athletes. It was also shown that in a symmetric task, the presence of thermal asymmetries can lead to technical issues for athletes and, in worst cases, in pain.

Conclusions: It was possible to demonstrate that IRT is a valid tool to assess internal load as suggested from previous studies, not only in aerobic efforts but also in highly anaerobic ones, such as Olympic weightlifting and is possible to track muscle activation with it. In a symmetric effort, it is possible to look for thermal asymmetries that could describe not only a possible injury, but also a technical error. References

1. De Andrade Fernandes, A. et al. (2014) 'Measuring skin temperature before, during and after exercise: A comparison of thermocouples and infrared thermography', Physiological Measurement 48: 28-35.
2. Fernández-Cuevas, I. et al. (2015) 'Classification of factors influencing the use of infrared thermography in humans: A review', Infrared Physics and Technology 71: 28-55.

3. Marins, J. C. B. et al. (2015) 'Applications of infrared thermography in sports. A review', Revista Internacional de Medicina y Cienc.

Acknowledgements: The project was performed with the facilities of the Research Project PONa3_00422 "CERISI" ("Research and Innovation Centre of Excellence for Structure and Infrastructure of Large Dimensions").

\section{POSTERS}

\section{BIOMOLECULAR ASPECTS OF SPORTS ACTIVITIES}

\section{6 \\ Effects of long-term football training on human breast cancer cell migration}

Andreina Alfieri ${ }^{1}$, Annamaria Mancini ${ }^{1}$, Georgios Ermidis ${ }^{2}$, Daniela Vitucci $^{I}$, Stefania Orrü ${ }^{\prime}$, Malte Nejst Larsen ${ }^{3}$, Peter Krustrup ${ }^{3}$, Pasqualina Buono

1department of Movement Sciences and Wellness, University Parthenope, Naples, Italy, 2ceinge-biotecnologie Avanzate, Naples, Italy, Napoli, Italy ${ }^{1}$;

1dismeb; 3department of Sports and Clinical Biomechanics, Sport and Health Sciences Cluster, University of Southern Denmark, Odense, Denmark, 4copenhagen Centre For Team Sport and Health, Department of Nutrition, Exercise and Sports, University of Copenhagen, Copenhagen, Denmark, Copenhagen, Danimarca ${ }^{2}$; 3 department of Sports and Clinical Biomechanics, Sport and Health Sciences Cluster, University of Southern Denmark, Odense, Denmark, 4copenhagen Centre For Team Sport and Health, Department of Nutrition, Exercise and Sports, University of Copenhagen, Copenhagen, Denmark, Copenhagen, Danimarca ${ }^{3}$

Purpose: Breast cancer (BC) is the most common cancer in women worldwide responsible for 685.00 deaths in 2020 , with approximately $10 \%$ developing metastases within 5 years of early diagnosis (1). Recently, epidemiological studies reported the benefits of a regular training program, mostly aerobic, in cancer prevention; the effects of adapted football training in BC survivors were also evidenced $(2,3)$. The aim of this project was to investigate the effects of football training on $\mathrm{BC}$ cell migration in in-vitro system.

Methods: 5 older ( $>65$ years) long-term football men trained for at least 15 years (VPG) and 5 active untrained subjects (CG) matched for age were recruited from P. Krustrup' s group; blood samples at rest (VPG and CG) and immediately after a football match (VPG) were collected. Human breast adenocarcinoma cells, MCF-7, were cultured to confluence in a medium (EMEM) containing 15\% FBS. Cells were starved in $0.5 \%$ FBS for $24 \mathrm{~h}$, scratched to perform a wound healing assay, and cultured in 5\% sera from VPG or CG or FBS (as control) for $72 \mathrm{~h}$, respectively; the wound closure percentage was calculated at $72 \mathrm{~h}$ vs T0 in each group. Data are expressed as mean \pm SD from three independent experiments. Differences were considered statistically significant at $\mathrm{p}<0.05$. T-test was used for statistical analysis.

Results: Long-term football training significantly reduced the migration of MCF-7 cells in wound healing assays (VPG vs FBS at $72 \mathrm{~h}, \mathrm{p}<0.005)$; a significant reduction in the wound closure percentage was also found in VPG compared to CG $(\mathrm{p}<0.05)$ cultured cells. 
Conclusions: Our preliminary results indicate that long-term football training reduces the migration of $\mathrm{BC}$ cells. Ongoing experiments will evaluate the effect of long-term football training on MCF-7 cell invasion and metastases.

Aknowledgments: Bando di Ateneo anno 2016 (quota C) Università Parthenope, Napoli.

1. WHO, 2020.

2. Ki-Yong et al. (2020) Effects of exercise dose and type during BC chemotherapy on longer-term patient-reported outcomes and health-related fitness: A randomized controlled trial. Int J Cancer 146:150-160.

3. Uth et al. (2020) Exercise intensity and cardiovascular health outcomes after 12 months of football fitness training in women treated for stage I-III breast cancer: Results from the football fitness After BC randomized controlled trial. Prog. in Cardiovasc Dis. 63: 792-799.

\section{7}

\section{Role of skeletal muscle capillarization in muscle adaptation to resistance exercise training in young, healthy and diabetic older adults}

\author{
Giorgia Schiavinotto $^{1}$, Lisa Vigo ${ }^{1}$, Camille R. Brightwell ${ }^{2}$, Elena \\ Volpi $^{3}$, Blake B. Rasmussen ${ }^{4}$, Christopher S. Fry ${ }^{2}$, Tatiana Moro ${ }^{I}$ \\ University of Padua, Department of Biomedical Sciences, University \\ of Padova, Padova, Italy ${ }^{I}$; \\ University of Kentucky, Department of Athletic Training and Clinical \\ Nutrition, Lexington, USA ${ }^{2}$; \\ University of Texas Medical Branch, Sealy Center on Aging, \\ Galveston, Stati Uniti D’ America ${ }^{3}$; \\ University of Texas Medical Branch, Department of Nutrition \& \\ Metabolism, Galveston, USA
}

Purpose: Muscle capillarization has recently become critically important in relation to fiber hypertrophy in response to training. Older adults have shown to be capable of responding to exercise by increasing muscle size and strength, but still their response is not always sufficient. The purpose of this study was to evaluate the role of capillarization in the hypertrophic response of skeletal muscle to overloaded training in youth, old adults, and diabetic old subjects to identify potential intervention strategies to prevent functional loss and frailty in the elder population.

Methods: 17 Healthy youth $(25.2 \pm 4.7$ years $), 19$ healthy elder adults $(71.1 \pm 4.3$ years $)$ and 11 diabetic elder adults $(69.8 \pm$ 3.4 years) performed a tri-weekly frequency training program for 12 weeks. The three groups were divided in a LOW or HIGH group according to basal type II fiber capillarization. Capillarization indices (C/Fi, CFPE index, LCPF index), type I and type II fibres and CSA were calculated using 50 fibers per image.

Results: Initial values of capillarization were not affected by aging, whereas diabetics presented significantly lower capillarization than older adults $(p<0.01)$. CSA in the elder adults was significantly lower than in the youth. After a 12-week of RET CFPE index, LCPF index and CSA increased mainly in type II fibers $(p<0.01)$. When divided by the pre-RET capillarization, CFPE index increased significantly only in young subjects in the LOW group. LCPF index increased in young and diabetic subjects in both LOW $(+74.79 \%$; + $78.96 \%)$ and HIGH group $(+51.12 \%$; $+33.24 \%)$.

CSA increased only in young and older adults with HIGH basal capillarization $(+30.68 \% ;+34.07 \%)$.

Conclusions: Our results demonstrated that training improved fibers size; in particular for type II fibers in youth and older adults. The role of capillarization was confirmed to be fundamental: the group that already has a good capillarization before training significantly increases muscle size. It is therefore demonstrated that the capillarization of type II and type I muscle fibers plays an important role, especially in older subjects compared to younger ones, whilst diabetic older adults seems to be resistant to these early adaptations.

\section{References}

1. Moro T, Brightwell CR, Phalen DE et al. Low skeletal muscle capilarization limits muscle adaptation to resistance exercise training in older adults. Experimental Geronltology 127 (2019) 110,723 .

2. Snijders T, Nederveen JP, Verdijk LB et al. Muscle fiber capillarization as determining factor on indices of insulin sensitivity in humans. Physiol Rep, 5 (10), 2017, e13278.

\section{8}

\section{Role of skeletal muscle pericytes in the myogenic response to exercise in young and older adults}

$\underline{\text { Lisa Vigo }}^{1}$, Giorgia Schiavinotto ${ }^{1}$, Antonio Paoli ${ }^{1}$, Blake B. Rasmussen $^{2}$, Elena Volpi ${ }^{3}$, Christopher S. Fry ${ }^{4}$, Tatiana Morol

University of Padua, Department of Biomedical Sciences, Padova, Italy $^{1}$;

University of Texas Medical Branch, Department of Nutrition \& Metabolism, Galveston, USA ${ }^{2}$;

University of Texas Medical Branch, Department of Internal Medicine/geriatrics, Galveston, $U_{S A}^{3}$;

University of Kentucky, Department of Athletic Training and Clinical Nutrition, Lexington, USA ${ }^{4}$

Purpose: Pericytes are perivascular cells wrapped around blood capillaries. In skeletal muscle, pericytes appear to play a part in tissue regeneration, e.g. differentiation into myofibers. The aim of the present study was to establish pericytes' role on muscle capillarization and satellite cells' proliferation in response to resistance exercise (RET).

Methods: 17 healthy youths $(25.2 \pm 4.7$ years; $24.5 \pm 3.3 \mathrm{BMI}), 19$ healthy older adults $(71.1 \pm 4.3$ years; $27.6 \pm 3.1 \mathrm{BMI})$ were enrolled in the study and performed 12 weeks of whole body RET. Subjects were retrospectively divided into a LOW or HIGH group, based on their pre-RET capillarization (Ci:F). Type I and type II muscle fibre size, satellite cells (SC), capillaries (caps) content and pericytes (NG2 + ) were determined by immunohistochemistry.

Results: Neither basal pericyte density nor RET's effect showed any significant difference among groups. Basal capillarization was positively correlated with pericytes number $(r=0,41 ; p=0,02)$ and capillary linked pericytes $(r=0.41 ; p=0.02)$ when normalized by fibres' number. Basal capillarization was also correlated $(\mathrm{r}=0.35 ; \mathrm{p}=$ 0.05 ) with pericytes' response to RET. Following RET, the LOW old group increased Ci:F $(\mathrm{p}=0.021)$ and all pericytes indices $(\mathrm{NG} 2+1$ mm2: $\mathrm{p}=0.003 ; \mathrm{NG} 2+/ \mathrm{caps} / \mathrm{mm} 2: \mathrm{p}=0.004 ; \mathrm{NG} 2+/$ fibre: $\mathrm{p}=$ 0.036; $\mathrm{NG} 2+$ /caps/fibre: $\mathrm{p}=0.038$ ). LOW young group increased $\mathrm{C}: \mathrm{Fi}(\mathrm{p}=0.021)$, but did not the pericytes count. HIGH young group only increased type II fibres associated SC $(\mathrm{p}=0.0005)$ and total SC $(p=0.0032)$. HIGH old group did not show any significant difference. Conclusions: Pericytes response to RET depends on basal capillarization, while the satellite cells' response does not. Pericytes were confirmedly able to mediate angiogenic processes, particularly in subjects with a lower capillarization. Although it is unknown whether pericytes might differentiate into satellite cells in response to training, they have come across as a synergic element on the adjustments that precede muscle hypertrophy. 


\section{References}

1. Bergers G, Song S. The role of pericytes in blood-vessel formation and maintenance. Neuro-Oncology 7, 452-464, 2005.

2. Dellavalle A, Sampaolesi M, Tonlorenzi R et al. Pericytes of human skeletal muscle are myogenic precursors distinct from satellite cells. Nat Cell Biol. 2007; 9:255-267.

Motor and sports activities adapted to disabilities

129

\section{Multiple sclerosis and physical activity: a new combination to reduce fatigue?}

\section{Federica Lucarelli ${ }^{1}$}

Università Cattolica Del Sacro Cuore, Dipartimento Di Pedagogia, Milan, Italy ${ }^{1}$

Purpose: Multiple Sclerosis (MS) is an autoimmune demyelinating and degenerative disease of the central nervous system. MS is characterised by various symptoms that can occur daily or occasionally. The symptom that affects about $80 \%$ of people with this disease is fatigue, which is understood as a feeling of lack of energy, not only physical but also mental.

Objective: The research aims to show that performing a light to moderate intensity physical activity and breathing exercises during work breaks can result in a reduction of the symptom of fatigue.

Method: The research lasted 2 months and involved subjects with the following characteristics: age between 31 and 53 years, EDSS scale score between 1 and 6 and with sedentary work. Six subjects participated, four women and two men, undergoing treatment at the Don Gnocchi Institute in Milan. Every day the subjects had to perform the multicomponent physical activity prescribed by the researchers both during the work breaks (mid-morning, lunch break and mid-afternoon) and in the evening before going to rest. By filling in written and oral questionnaires and daily diaries, the progress of the research and the improvement in quality of life and reduction of fatigue perceived by each subject on a weekly basis were monitored. At the end of the month there was a comparison of the results obtained and further evaluation questionnaires were provided. At the end of the 2 months of research all the data obtained were compared in order to evaluate the progress of the activity and the onset of the symptom of Fatigue along the way.

Materials: FSS (Fatigue Scale); SF36 (Standardized Health Status Questionnaire); Oswestry Low Back Pain Disability Questionnaire; Daily Diary; Questionnaires.

Results: Only 5 patients achieved a reduction in the appearance of the symptom of fatigue, with a decrease ranging from 23 to $68 \%$ compared to the beginning of the course. The protocol was carried out with continuity and constancy by 5 subjects who showed the greatest reduction in symptoms and improvement in quality of life.

Conclusions: This research showed that the implementation of a physical activity during working time can be a method of intervention to reduce the symptom of fatigue and can lead to an improvement in the quality of life of individuals with Multiple Sclerosis.

\section{0}

Neuromuscular electrical stimulation superimposed on movement and isoinertial training for rotator cuffrelated shoulder pain: a case report and literature review

Arcangelo Parisella $^{1}$, Sebastiano Nutarelli ${ }^{2}$, Eraldo Malja $^{3}$, Andrea Macaluso $^{I}$, Arrigo Giombini ${ }^{l}$

Università, Università Degli Studi Di Roma “foro Italico”, Rome, Italy $^{1}$;

Università, School of Public Health, Physiotherapy and Sports Science, University College Dublin, Roma, Irlanda ${ }^{2}$; Università, University of Parma, Strada Dell'università, Parma, Italy $^{3}$

Purpose: Rotator cuff-related shoulder pain is a common condition in overhead athletes. Conservative treatment with exercise progression should be considered as the first-line approach but no consensus exists on preferred exercise and therapies selection. The aim of this was to assess the effectiveness of a novel exercise protocol, with neuromuscular electrical stimulation superimposed on movement (NMES + ) and isoinertial training for the treatment of rotator cuff-related shoulder pain (RCRSP) in a master swimmer.

Methods: The patient was a 44-year-old master swimmer who began complaining of shoulder pain after the Italyn Championships tournament. Following an orthopaedic examination the subject was diagnosed with supraspinatus tendinopathy and commenced a period of rest, corticosteroids and electrotherapy which led to no benefit to his shoulder symptoms. Subsequently, a structured exercise rehabilitation program including NMES superimposed on movement and isoinertial training was started with the aim of returning to sport avoiding a surgical intervention. A step-by-step rehabilitation exercise protocol was proposed focusing on load and exercise progression with the goal of strength, power and mobility recovery. NMES + was used to support early rehabilitation, while isoinertial training was used in the mid-late stage of the rehabilitation process.

Results: The protocol allowed the subject to successfully return to autonomous gym training and swimming in 10 and 11 weeks respectively, following the rehabilitation exercise program.

Conclusion: The novel NMES and isoinertial training approaches may be considered as promising tools in RCRSP rehabilitation.

References

1. Alqunaee M, Galvin R, Fahey T (2012) Diagnostic accuracy of clinical tests for subacromial impingement syndrome: A systematic review and meta-analysis. Arch Phys Med Rehabil 93:229-236.

2. Asker M, Brooke HL, Waldén M, et al. (2018) Risk factors for, and prevention of, shoulder injuries in overhead sports: A systematic review with best-evidence synthesis. Br J Sports Med 1312-1319.

\section{1}

New insight of therapeutic horse back riding in down syndrome

\author{
Simona Portaro $^{1}$, Angelo Alito ${ }^{1}$, Gisulia Leonardi ${ }^{1}$, Daniele \\ Bruschetta $^{\prime}$, Eliseo Scarcella ${ }^{I}$, Lacramioara Gina Berescu ${ }^{I}$ \\ U.o.c of Physical and Rehabilitation Medicine and Sports Medicine, \\ Policlinico Universitario G. Martino, Messina, Italy
}


Purpose: To standardize hippotherapy methodology in clinical practice, using finite elements theoretical basis, to improve gait and balance in Down syndrome.

Methods: 5 Patients affected by Down syndrome, attended a specialized hippotherapy riding school, performing a 12 months threetimes/week training. Each hippotherapy session lasted at least $30 \mathrm{~min}$, during which the patient took three different positions (face forward, side-sitting, face backward) and performed therapeutic activities on the moving horse. A finite element analysis (FEA), which is a theoretical model supporting hippotherapy clinical basis, was applied to monitor the interaction between horses and patients.

The hippotherapy responsible was a neurologist with 15-year experience in hippotherapy. During the intervention, the physiotherapist and the neurologist walked behind the horse-patient-horse handler group, providing immediate advice on horse's direction, speed, and subject's position. Static and dynamic gait analysis were assessed before starting (T0) and at the end (T1) of the 12-month hippotherapy protocol.

Results: Before and after the 12-month hippotherapy training, we collected baropodometric data, including plantar pressure and center of pressure $(\mathrm{CoP})$ parameters during stance, with either the eyes closed (EC) or opened (EO), using a platform (Diasu Ultrasensor systems) to determine participants'static evaluation. The stabilometric variables assessed were: i) $\mathrm{CoP}$, as a quantitative assessment of postural control, in quiet stance, such as postural sway and postural area; ii) mediolateral velocity of oscillations; iii) antero-posterior velocity of oscillations. Finite element analysis were also applied to continuously monitor horses' movements, adapting to the rehabilitation team approaches and treatment strategies.

Conclusions: Herein, we have objectively evaluated the 12-month hippotherapy protocol effects on stance and gait, monitored by a FEA analysis, in 5 DS males, to estimate the improvement of such therapeutic strategy on neuromotor system in DS, possibly translating such results to daily living activities. Our findings demonstrated that hippotherapy protocol led to a significant improvement in the baropodometric, stabilometric, and gait performance in DS.

References

1. Portaro S, Maresca G, Naro A, Calabrò RS, Gemelli G, Aliberti B. Role of horse-assisted therapy in the rehabilitation field: past, present, and future perspectives. Innov clin neurosci. 2020 jan 1;17(1-3):8-9. Pmid: 32,547,839; pmcid: pmc7239566.

2. Portaro S, Cacciola A, Naro A, Cavallaro F, Gemelli G, Aliberti B, De Luca R, Calabrò RS, Milardi D. Can Individuals with Down Syndrome Benefit from Hippotherapy? An Exploratory Study on Gait and Balance. Dev Neurorehabil. 2020 Aug;23(6):337-342. doi: 10.

\section{2}

\section{Sport participation in europe in individuals with down syndrome: data from the ikons study}

\author{
Maria Francesca Piacentini ${ }^{1}$, Federico Quinzi ${ }^{2}$, Valentina \\ Camomilla $^{2}$, Giuseppe Vannozzi ${ }^{2}$, Fabio Verdone ${ }^{2}$, Paola Sbriccoli ${ }^{2}$. \\ Università Degli Studi Di Roma Foro Italico, Scienze Motorie Umane \\ E Della Salute, Rome, Italy ${ }^{1}$; \\ Università Degli Studi Di Roma Foro Italico, Scienze Motorie Umane \\ E Della Salute, Rome, Italy ${ }^{2}$
}

Purpose: It is well established that physical activity and sport participation improves functional status and quality of life among people with disability. Down syndrome (DS) is the most frequent of chromosomal disorders in the world (1). Although sport participation has shown measurable physiologic and psychologic benefits in this population, data on the actual participation in structured activity programs of individuals with DS is scarce. Therefore, the present study aims to provide a clearer framework of sport participation in individuals with DS and on their actual situation in the EU.

Methods: An online survey, translated in 5 European languages, was sent through social networks and personal contacts in different European countries. The "Individual Survey" filled in by coaches, aimed to understand sport activity limitations of individuals with DS. Results: Individual Surveys: 135 individual surveys were collected. Females and males were equally represented (F: $51 \%$ M: 49\%). DS was the most represented disability $(57 \%)$ followed by Mental Impairment (20\%) and Autism spectrum disorders (6\%). $70 \%$ were regularly involved in sport activities, the most represented of which were martial arts (63\%), swimming (13\%) and team sports (10\%). Most respondents were newcomers to sport $(<$ than 3 years of practice), training less than $4 \mathrm{~h}$ per week. Although 53\% of the coaches reported that their athletes showed difficulties in applying the physical skills required for their sport, $29 \%$ reported that this difficulty no longer existed after a systematic training program. The major difficulties reported were movement sequencing and planning skills $(61 \%)$ and following coaches' instructions (36\%). No difficulty emerged in interacting with teammates.

Conclusion: Current results show that many individuals with DS participate in sport activities and support the beneficial effects of sport activity programs for individuals with DS (2). However, despite many clubs declare that athletes with DS attend their facilities, most of their coaches $(75.2 \%)$ never received specific education on how to integrate these individuals in their regular classes.

Funding: Erasmus + Sport Programme GA 2018-2512.

\section{References}

1. Al-Biltagi M. Down syndrome from Epidemiologic Point of View. EC Paediatrics 2.1 (2015): 82-91.

2. Sanyer ON. Down Syndrome and Sport Participation (2006). Current Sports Medicine Reports, 5:315- 318.

\section{3 \\ Down syndrome. Effects of psychomotor training on the structuring process of the body scheme}

\author{
$\underline{\text { Alessandra Nart }}{ }^{l}$, Cristiana Lucchetti ${ }^{1}$, Vincenzo Biancalana ${ }^{1}$ \\ University of Urbino, Department of Biomolecular Sciences, Urbino, \\ Italy $^{l}$
}

Purpose: Down Syndrome (DS) accounts for $18 \%$ of all genetically transmitted diseases, with an incidence included in the range $0.9-1$ case per 1,000 individuals born alive (World Health Organization, 2014). The psychomotor profile of the subject with DS is influenced by hypotonia and ligamentous hyperlaxity, with obvious dyspraxia and dynamic balance, coordination and body scheme disorders (Vicari, 2007). The aim of this study is to observe in subjects with DS how the image of themselves changes through psychomotor practice and how such change is expressed through the graphic representation. Methods: The project, which lasted 2 months with a 2 week follow up, involved 3 sedentary subjects ( 3 males) with DS, aged between 24 and 26 years. The physical activity protocol involved 1 lesson of $1 \mathrm{~h} /$ week and was characterized by combinations of exercises aimed at inducing the subject's awareness of his body, muscle strengthening and general dynamic coordination, with the use of various tools (i.e. mats, elastic bands, balls, sticks). In order to assess the protocol effectiveness, a drawing of the subject's body was used; the latter had to graphically represent his own body as he himself saw it 
(Biancalana, 1994). For the initial assessment -T0, the MO.V.I.T. test and the subject's own body DRAWING were used. Data were collected after 2 weeks -T1, after 3 weeks -T2, after 6 weeks -T3, after 8 weeks -T4, at the end of the follow up ( 2 weeks from end of treatment).

Results: Unfortunately, only one subject of three involved subjects completed the protocol. The results showed that, when consistently repeated, the targeted exercises favoured the perception and representation of oneself. The last drawing showed a more complete and proportionate self-image than the incoherent and incomplete one of the first drawings. The absence of exercise for only 2 weeks, however, resulted in an immediate regression.

Conclusions: In subjects with mental retardation, psychomotor practice can promote greater awareness of their own body; however, constancy and repetitiveness of actions play a key role (Hardee et al., 2017).

\section{References}

1. World Health Organization (2014) Genes and human disease Down Syndrome.

2. Vicari S. (2007) La sindrome di Down. Una disabilità diffusa, ma con una storia ricca di conquiste. Il Mulino, Bologna.

3. Biancalana V. (1994) La Pratica natatoria. Sull'ansia dell'adulto sulla personalità del bambino. Battelli, Urbino.

4. Hardee JP, Fetters L. (2017) The effect of exercise intervention on daily life activities and social participation in individuals with Down syndrome: A systematic review. Res Dev Disabil 62: 81.

\section{4}

\section{Effect of 4-week tennis training on motor skill proficiency and cognitive functions of adolescents with down's syndrome}

\author{
Silvia Boscaini $^{1}$, Valentina Cavedon ${ }^{1}$, Francesca Vitali $^{1}$, Chiara \\ Milanese $^{I}$
}

Università Di Verona, Dipartimento Di Neuroscienze, Biomedicina E Movimento, Verona, Italy ${ }^{I}$

Purpose: Down syndrome (DS) is the most frequent genetic mental disability. Individuals with DS experience a variety of cognitive, physical and motor challenges throughout the lifespan. The importance of exercise for individuals with DS has been well documented. It has been assumed that the practice of tennis may be attractive and beneficial for people with DS. This preliminary study investigated the effects of a 4-week tennis training intervention on motor skill proficiency and cognitive functions in adolescents with DS.

Methods: 8 adolescents (females, $\mathrm{n}=6$; males, $\mathrm{n}=2$ ) with DS aged 14-21 years were enrolled in this study. Participants were regularly trained 90 min twice a week for 4 consecutive weeks by an expert tennis coach. At the beginning (T0) and after the end of 4 weeks of tennis training (T1), participants were assessed for: 1) motor skill proficiency: agility (Timed Up-And-Go Test), coordination (7 items of the "upper-limb coordination" subtest within the the Bruininks Oseretsky Test of Motor Proficiency), gross manual dexterity (Box and Block Test) and reaction time (Witty SEM Reactive System); 2) cognitive functions: executive functions (Tower of London Test) and working memory (Corsi Test). Paired-samples T-test was employed for statistical analysis and the level of significance was set at $\mathrm{P}<0.05$.

Results: The results showed that 4 -week tennis training improved both reaction times and cognitive functions. In particular, the results highlighted a statistically significant reduction in the time to complete the complex reaction time test between $\mathrm{T} 0$ and $\mathrm{T} 1(-7.57 \%, \mathrm{t}=2.408$ and $\mathrm{P}=0.047)$. Moreover, the performance in the Tower of London Test and in the Corsi Test increased significantly between $\mathrm{T} 0$ and $\mathrm{T} 1$ $(+6.88$ points, $\mathrm{t}=-3.235, \mathrm{P}=0.014$ and +0.88 points, $\mathrm{t}=-3.862, \mathrm{P}=$ 0.006 , respectively).

Conclusions: 4-week tennis training seems to be able to improve complex reaction times, working memory and executive functions in adolescents with DS. The results obtained in this preliminary investigation are encouraging and suggest the potential benefits of tennis for adolescents with DS.

\section{References}

1. De la Vega, R, Ruíz, R, De la Rocha, M, and Oswaldo Rivera, JO (2013) Adaptive Behaviour and Paddle Tennis: A Case Study of Down's Syndrome. Advances in Physical Education 3(4): 187-189.

2. Regaieg, G, Kermarrec, G and Sahli, S (2020) Designed game situations enhance fundamental movement skills in children with Down syndrome. J Intellect Disabil Res 64(4): 271-279.

\section{5}

\section{Effectiveness of a home-based balance training with visual stroboscopic deprivation in chronic incomplete spinal cord injury subjects: a pilot study}

Sara Guarducci ${ }^{1}$, Ilaria Nannini ${ }^{2}$, Riccardo Bravi ${ }^{3}$, Magdalena Sicher $^{3}$, Gabriele Righi ${ }^{2}$, Giulio Del Popolo ${ }^{2}$, Giacomo Lucchesi ${ }^{2}$, Vincenzo Sorgente $^{3}$, Erez James Cohen ${ }^{3}$, Diego Minciacchi ${ }^{3}$

Università Degli Studi Di Firenze, Dipartimento Di Medicina Sperimentale E Clinica, Firenze, Italy ${ }^{1}$; Azienda Ospedaliero-universitaria Careggi, Unità Spinale, Firenze, Italy $^{2}$;

Università Degli Studi Di Firenze, Dipartimento Di Medicina Sperimentale E Clinica, Firenze, Italy ${ }^{3}$

Purpose: The maintenance of postural control is essentially a continuous and never-ending sensorimotor integration task. Central nervous system has to generate appropriate motor responses based on the efficient integration of visual, vestibular and somatosensory inputs. Research has reported that individuals with somatosensory deficits rely heavily on visual input. However, those who rely more on proprioceptive strategies have been found to be more stable. Recent studies have shown how an eyes-closed training program allows to switch off the visual mechanisms, thus determining a chronic shift from visual reliance to proprioceptive strategies for balancing. Stroboscopic visual training (SVT), by forcing individuals to perform under a condition of intermittent vision, may induce an eyes-closed level perturbation of the somatosensory system. The efficacy of SVT has never been examined in people with incomplete spinal cord injury (iSCI). Therefore, our aim was to investigate the effect of SVT on balance control in people with iSCI.

Methods: Ten patients with chronic iSCI ( 7 males, 3 females) performed a 6-week, home-based balance training, 3 days per week. Participants were randomly assigned to an experimental group $(\mathrm{n}=5)$ that performed SVT sessions, or a control placebo group $(n=5)$ that underwent SVT training with non-active strobe glasses. Before and after the intervention, subjects with iSCI were evaluated using stabilometric bipedal and unipedal stance tests, with eyes open and closed. 3 trials were performed for each condition, each lasting $30 \mathrm{~s}$. In addition, balance confidence was also evaluated by means of the Activities-specific Balance Confidence (ABC) Scale and the Visual Analogue Scales (VAS). Balance performance were also tested on six normal subjects who were enrolled as healthy control group. 
Results: Results showed that the mean changes in stability scores and balance confidence were significantly improved in both two iSCI groups. However, small differences were observable in favor of experimental group.

Conclusions: Subjects with iSCI can be trained to adopt proprioceptive strategies to maintain balance. The duration with which the adaptation response remains is yet to be determined. Therefore, the present study's results would encourage future studies to examine the effects of a longer SVT program on balance control in persons with iSCI.

\section{References}

1. Kim KM, Kim JS and Grooms DR. (2017) Stroboscopic vision to induce sensory reweighting during postural control. J Sport Rehabil 26.

2. Tamburella F, Scivoletto G and Molinari M. (2013) Balance training improves static stability and gait in chronic incomplete spinal cord injury subjects: a pilot study. Eur J Phys Rehabil Med 49: 353-364.

\section{6}

\section{Effects of stroboscopic visual training on balance and gait in subjects with incomplete spinal cord injury: a randomized pilot study}

\author{
Magdalena Sicher ${ }^{1}$, Ilaria Nannini ${ }^{2}$, Riccardo Bravi ${ }^{1}$, Sara \\ Guarducci $^{I}$, Gabriele Righi ${ }^{2}$, Giulio Del Popolo ${ }^{2}$, Giacomo \\ Lucchesi $^{2}$, Vincenzo Sorgente ${ }^{1}$, Erez James Cohen ${ }^{1}$, Diego \\ Minciacchi ${ }^{I}$
}

Università Degli Studi Di Firenze, Dipartimento Di Medicina Clinica E Sperimentale Università Di Firenze, Firenze, Italy ${ }^{l}$, Azienda Ospedaliera Universitaria Careggi, Unità Spinale, Firenze, Italy ${ }^{2}$

Purpose: Walking is considered the most important goal after an incomplete spinal cord injury (SCI). Only recently it has been demonstrated that balance is a key factor of walking recovery, but no data on the efficacy of balance training in supporting walking function in SCI subjects are available. Previous studies have shown that postural stability and walking capability can enhance by means of a balance training with additional visual feedback in individuals with iSCI. At the same time, recently, advanced technology conceived special eyewear with liquid-crystal technology in the lenses to produce intermittent vision obstruction. This special eyewear named stroboscopic eyeglasses allow individuals to perform dynamic, functional tasks that, otherwise, could be not performed under a complete visual obstruction. The purpose of this study was to assess the effect stroboscopic glasses integrated to a period of home-based balance training in influencing balance and gait in individuals with iSCI.

Methods: The pilot study enrolled 10 patients ( 7 men and 3 women) with chronic iSCI. Subjects underwent a 6-week home individual balance training program, 3 times per week. Subjects were randomly assigned to an experimental group $(n=5)$ that performed stroboscopic visual training (SVT) with active stroboscopic glasses and a placebo control group $(n=5)$ that attended the identical program with nonactivated glasses. Before and after the training subjects were evaluated regarding the gait and balance capabilities using the Timed Up and Go test, and the 6 Minute Walking Test. In addition, balance and gait confidences were also evaluated by means of the Activitiesspecific Balance Confidence (ABC) Scale and the Visual Analogue Scales (VAS). Balance and gait performances were also tested on six normal subjects who were enrolled as healthy control group.
Results: Physical activities and in specifical home based balance training and intervention of stroboscopic glasses in specific stroboscopic visual training (SVT) appears to modulate the motor capacity in subjects with iSCI.

Conclusions: Preliminary results of this pilot study encourage further research to investigate the effect of the stroboscopic intervention to improve and balance and gait performance in subjects with chronic iSCI.

\section{References}

1. Kim KM, Kim JS and Grooms DR. (2017) Stroboscopic vision to induce sensory reweighting during postural control. J Sport Rehabil 26.

2. Sayenko DG et al. (2010) Positive effect of balance training with visual feedback on standing balance abilities in people with incomplete spinal cord injury. Spinal Cord 48: 886-893.

3. Tamburella $\mathrm{F}$ et al. (2013) Balance training improves static stability and gait in chronic incomplete spinal cord injury subjects: a pilot study. Eur J Phys Rehabil Med 49: 353-364.

\section{7}

\section{Evaluating factors associated with the sprint ability in sitting volleyball athletes}

\author{
Chiara Brugnoli ${ }^{1}$, Valentina Cavedon ${ }^{1}$, Francesca Vitali $^{l}$, Carlo \\ Zancanaro $^{I}$, Chiara Milanese ${ }^{l}$
}

Università Di Verona, Dipartimento Di Neuroscienze, Biomedicina E Movimento, Verona, Italy ${ }^{I}$

Purpose: In Sitting Volleyball (SV) the ability to sprint forwards and backwards as well as the ability to change directions while sprinting are crucial factors for match success. This study investigated the relationship between anthropometry, body composition, strength, manual dexterity and the performance in sport-specific sprint test.

Methods: Several anthropometric measurements were obtained for 20 SV athletes (13 males and 7 females) aged $37.4 \pm 12.2$ years. Body composition was estimated by-means of Dual-Energy X-Ray Absorptiometry. The Chest Pass Test, the Handgrip Strength Test and the Sit-Up Test were used to assess the upper body and trunk strength. The Box and Block Test (BBT) was administered to evaluate manual dexterity. Times to complete the $5 \mathrm{~m}$ Forward Sprint Test, the $5 \mathrm{~m}$ Backward Sprint Test (BST), the Agility T-Test (ATT) and the Speed and Endurance Test were collected by-means of photocells. The degree of association between two continuous variables, accounting for sex and age, was measured by partial correlation (r_pc). Statistical significance was set at $\mathrm{P}<0.05$.

Results: Statistically significant negative associations were found between the performance in each sprint test and the sitting high (r_pc ranging from -0.679 to $-0.839, \mathrm{P}$ ranging from 0.008 to $<0.001$ ), the hand span (r_pc ranging from -0.614 to -0.782 , $P$ ranging from 0.019 to $<0.001$ ), the performance obtained in both the Sit-Up Test ( $r \_p c$ ranging from -0.553 to -0.667 , $P$ ranging from 0.040 to 0.009 ) and the BBT ( $r$ _pc ranging from -0.646 to -0.718 , $\mathrm{P}$ ranging from 0.011 to $0.004)$. The percentage of fat-free soft tissue mass in the arms was negatively associated with the BST $\left(\mathrm{r} \_\mathrm{pc}=-0.554, \mathrm{P}=0.040\right)$ and the ATT (r_pc $=0.544, \mathrm{P}=0.047$ ) only.

Conclusions: Anthropometry, body composition, trunk strength and manual dexterity are all factors associated with the sprint ability in SV. Athletes with greater values of sitting height and hand span have an advantage in the SV sprint performance. SV coaches and physical conditioners are encouraged to include in their programme exercises aimed at improving body composition in the arms, abdominal strength and manual dexterity. 


\section{References.}

1. Ahmadi, S, Uchida, MC and Gutierrez, GL (2019) Physical Performance Tests in Male and Female Sitting Volleyball Players: Pilot Study of Brazilian National Team. Asian J Sports Med 10(2):e85984.

2. Marszalek, J, Molik, B, Gomez, MA, Skučas, K, Lencse-Mucha, J, Rekowski, W, Pokvytyte, V, Rutkowska, I and KaźmierskaKowalewska, K (2015) Relationships Between Anaerobic Performance, Field Tests and Game Performance of Sitting Volleyball Players. Journal of Human Kinetics 48: 25-32.

Anatomy, sport, posture and functional recovery

\section{8}

Medical conditions treated at the ironman italy: from the epidemiology to the organization of a medical plan

$\frac{\text { Francesco Feletti }^{1}}{\text { Matteo Gaia Saini }^{2}, \text { Stefano Naldi }}{ }^{2}$, Antonio La Torre ${ }^{3}$, Matteo Bonato

Ausl Romagna, S. Maria Delle Croci Hospital, Department of Diagnostic Imaging, Ravenna, Italy ${ }^{1}$;

Ausl Romagna, S. Maria Delle Croci Hospital, Department

of Emergency, Ravenna, Italy ${ }^{2}$;

Università Degli Studi Di Milano Statale, Dipartimento Di Scienze Biomediche Per La Salute, Milan, Italy ${ }^{3}$

Purpose: Triathlon popularity is rapidly increasing, and data about the associated medical conditions' epidemiology are sparse and fragmented.

The primary goal of this study was to describe the epidemiology of injuries at an international triathlon event. The study's secondary objective was to quantify the resources and define some strategies for the organisation of medical support.

Method: This study analysed the data from the medical reports gathered during three consecutive editions of the Ironman Italy, from 2017 to 2019, which included six triathlon races over a medium to long-distance. The data contained in the registration forms and medical reports were examined.

We also analysed the data relative to the drugs and dressings used to treat any medical conditions and created a model according to Maurer's algorithm.

Results: This study covered a total of 94,611 triathlon-hours, including $80,478 \mathrm{~h}$ of long-distance, 10,336 of middle-distance and 3,797 of Olympic triathlon.

Out of 10,653 race-starters 9,165 males 1,488 females, $3.35 \%$ required medical attention and suffered from 472 medical conditions. A significantly higher risk was found for females versus males $(\mathrm{X} 2=9.78$, $\mathrm{p}$ $=0.02$ ) and in long-distance (IR: $4.09 / 1,000 \mathrm{~h}$ ) rather than in Olympic/middle distance races (IR: $1.75 / 1,000 \mathrm{~h}$ ). Most $(68.4 \%$ ) were systemic conditions, including muscular exhaustion, hypothermia, and dehydration, while only $10.2 \%$ were acute traumatic injuries. Acute and overuse injuries affecting the musculoskeletal system represented $15.2 \%(n=72)$. They prevailed to the lower limbs, most being minor injuries such as abrasion, contusion, ankle sprain, muscle strain, and plantar fasciitis. A high percentage of skin conditions, $36 \%$ $(n=56)$, were treated. On a total of 357 triathletes requiring medical assistance, $8.1 \%$ were a candidate for hospitalisation. A requirement of 7 ambulances, 30 rescuers and two medical units was calculated for a triathlon event of the same size. Ten recommendations relative to optimise the organisation of the medical assistance to a triathlon event were also formulated.
Conclusions: Although most injuries encountered in the triathlon series were minor, triathlon race organisers and medical personnel should be prepared at any time to cope with unpredictable eventualities, including the worst-case scenarios.

Specifically, a reinforced surveillance plan in the swimming fraction and the preparation of specific prevention and rescue plans are recommended.

\section{References}

1. 1.Korkia P, Tunstall-Pedoe D, Maffulli N (1994). An epidemiological investigation of training and injury patterns in British triathletes. Br J Sports Med. 1994;28:191-6.

2. 2.Egermann M, Brocai D, Lill C, Schmitt H(2003).Analysis of Injuries in Long-Distance Triathletes. Int. J. Sports Med; 24(04),271-6.

3. Gosling CM, Gabbe BJ, McGivern J, Forbes AB (2008). The incidence of heat casualties in sprint triathlon: the tale of two Melbourne race events. J Sci Med Sport.11;52-57.

\section{Sport Nutrition}

\section{9 \\ Eating disorders in strong increase in young people in developmental age}

Francesco Perrotta ${ }^{I}$

Unimol, Dipartimento Scienze Umanistiche, Campobasso, Italy ${ }^{I}$

Eating disorders, of which anorexia and bulimia nervosa are the most evident manifestations and have become a real mental health SOS in the last twenty years due to the enormous devastating effects they have on the health and life of preteens and young adults. The medical associations in the USA that deal with these phenomena define them as a real epidemic that crosses all social strata and different ethnic groups.

If not treated in time and with adequate methods, eating disorders can become a permanent condition and in severe cases lead to death, which usually occurs from suicide or cardiac arrest. According to the American Psychiatric Association (APA), they are the leading cause of death from mental illness in Western countries. It becomes necessary as it is healthy to start practicing sports in developmental age which becomes just like a form of therapy for the solution of this real social problem in the growing adolescent range. Hence the need for a full-fledged teacher of motor science who should be included in the staff of primary schools for an indefinite period. A study published in the English journal The Lancet indicates that research on treatments is much more advanced in the case of bulimia nervosa, where more than fifty studies and trials have been carried out and management according to evidence-based practices is possible. Less attention, however, has so far been devoted to research on possible treatments for anorexia nervosa and other forms of eating disorder.

Perrotta F.,Dietetica e nutrizione nella mentalità sportiva. Una ricerca scientifica e metodologica per migliorare il proprio stile di vita,Editore: Edizioni Goliardiche.

Collana: Scienze educazione motoria e sportiva.

Anno edizione: 2007.

Pagine: 158 p., Brossura.

EAN: $9,788,878,730,465$. 
140

\section{Effect of probiotics supplementation on physical performance and immunological response to overreaching: a pilot study}

\author{
Alessio Del Torto ${ }^{1}$, Anna Lirussi ${ }^{1}$, Giuseppe Marcolin ${ }^{1}$, Andrea \\ Casolo $^{I}$, Tatiana Moro ${ }^{l}$, Antonio Paoli ${ }^{1}$
}

University of Padua, Department of Biomedical Sciences, University of Padova, Padova, Italy ${ }^{l}$

Purpose: Intensified training periods are commonly incorporated into the course of a regular training season. However, on some occasions it can lead to the risk of developing symptoms related to overtraining, or overreaching syndrome. From a clinical point of view, very intense training periods are associated with a temporary immunological deficiency. From a nutritional point of view, some recent evidence has suggested that may influence recovery from fatigue, performance and immune function in athletes.

The objective of this pilot study was to determine whether supplementation with probiotics can alleviate the symptoms related to functional overreaching.

Methods: 10 Healthy national mountain bike athletes were enrolled in the study. Subjects were randomly assigned to 14 days of a placebo (PLA) or probiotics (PRO) supplementation. During the last 8 days of intervention all volunteers underwent to an intensified training protocol in order to induce an overreaching status. Performance was measured during an incremental test followed by 3 wingate bouts; blood sample were taken after an overnight fast to asses markers of inflammation and immune deficiency; state of mood was monitored with a specific questionnaire (POMS).

Results: any difference in physical performance was detected after 15 days of intervention. PRO supplementation improved mean corpuscular haemoglobin and creatinine concentration. Total T-cells $(\mathrm{CD} 3+)$ and T-helper cells $(\mathrm{CD} 4+/ \mathrm{CD} 3+)$ were significantly higher in PRO compared to PLA after treatment $(\mathrm{p}<0.05)$. The fatigue component of POMS was significantly increased only in the PLA group $(\mathrm{p}<0.05)$

Conclusions: our preliminary results suggest that probiotics seems to have a positive effect on the immune system and in the fatigue perception during high workload training, without affecting the physical performance. Their supplementation may be helpful to sustain athletes during intensified period of training and prevent immunological deficiency.

\section{1}

Effect of very low calorie ketogenic diet combined with high-intensity functional training on body composition, cardiometabolic health and sarcopenic obesity: a case report

\section{Elisabetta Camajani $^{1}$, Sabrina Basciani ${ }^{1}$, Massimiliano Caprio ${ }^{2}$}

Sapienza University of Rome, Department of Experimental Medicine, Section of Medical Pathophysiology, Food Science and Endocrinology, Rome, Italy ${ }^{1}$;

San Raffaele Roma Open University, Department of Human Sciences and Promotion of The Quality of Life, Rome, Italy ${ }^{2}$

Purpose: In subjects with sarcopenic obesity it is essential to reduce body weight preserving lean mass, in order to avoid a worsening of muscle function.
The aim of the study was to investigate safety and efficacy of a dietary intervention based on a Very Low Calorie Ketogenic Diet (VLCKD) combined with physical exercise, upon weight loss, cardiovascular risk and lean body mass, in a patient with sarcopenic obesity and metabolic syndrome.

Patient and Methods: A 56-year-old woman affected by third degree obesity, hypertension, hyperinsulinism, hypercholesterolemia, hypertriglyceridemia, psoriasis and sarcopenia, was admitted to our facility. Therefore, a nutritional regimen with a VLCKD $(\leq$ $800 \mathrm{kcal} /$ day) was started for 45 days, based on meal replacements. Seven days after the beginning of nutritional protocol, the patient started a High-Intensity Functional Training protocol (HIFT) twice a week.

Anthropometric parameters, bioelectrical impedance, blood and urine tests, muscle strength by Handgrip strength test, Chair Stand Test and SPPB (Short Physical Performance Battery), were collected before and at the end of the dietary intervention.

Results: A significant reduction of body weight (91 vs $108 \mathrm{~kg}$ ), BMI (33,8 vs $40 \mathrm{~kg} / \mathrm{m} 2)$, blood pressure (120/70 vs $140 / 90 \mathrm{mmHg}$ ), waist circumference (101 vs $115 \mathrm{~cm})$, HOMA index (2,0 vs 3,7), triglycerides (99 vs $178 \mathrm{mg} / \mathrm{dl}$ ), total cholesterol (158 vs $215 \mathrm{mg} / \mathrm{dl})$ and HDL cholesterol (70 vs $48 \mathrm{mg} / \mathrm{dl}$ ) was observed. The average weight loss was nearly $16 \%$ of initial weight, with a significant reduction in fat mass $(30,1 \%$ vs $40,9 \%)$, with a concomitant preservation of fat free mass. There was a significantly improvement of muscle strength and physical performance, as measured by handgrip (25.1 vs $15.8 \mathrm{~kg}$ ) and chair stand test (11,7 vs $16,5 \mathrm{~s})$.

Conclusions: These preliminary results show that a VLCKD protocol combined with HIFT is safe and can lead to a significant weight loss preserving fat free mass, with a marked improvement of metabolic parameters.

\section{References}

1. Cruz-Jentoft AJ, Bahat G, Bauer J, et all. Writing Group for the European Working Group on Sarcopenia in Older People 2 (EWGSOP2), and the Extended Group for EWGSOP2. Sarcopenia: revised European consensus on definition and diagnosis. Age Ageing. 2019; 16-31.

2. Caprio M, Infante $\mathbf{M}$, et all. Very-low-calorie ketogenic diet (VLCKD) in the management of metabolic diseases: systematic review and consensus statement from the Italyn Society of Endocrinology (SIE). J Endocrinol Invest. 2019.

\section{2}

Effects of an acute administration of creatine citrate on isometric muscle force expression in the biceps brachii after resistance exercise: a double-blind crossover placebo-controlled study

Giuseppe Cerullo ${ }^{1}$, Massimo Negro ${ }^{2}$, Matteo Vandoni ${ }^{3}, \underline{\text { Giuseppe }}$ $D^{\text {'antona }}{ }^{2}$

Uniparthenope, 1 Department of Movement Sciences and Wellbeing, University of Naples Parthenope, Naples, Italy, Napoli, Italy ${ }^{I}$; University of Pavia, Criams Sport Medicine Centre Voghera, Vogehra, Italy ${ }^{2}$;

University of Pavia, Department of Public Health, Experimental Medicine and Forensic Science, University of Pavia, Pavia, Italy, Pavia, Italy ${ }^{3}$

Purpose: The aim of the present study was to investigate the acute effects of a single oral administration of a Creatine Citrate (CC) on isometric muscle force parameters (Rate of Force Development 
(RFD), Maximal Voluntary Contraction (MVC), and Time to perform the Task (TtT) at 60\% MVC until exhaustion) registered before and after a Resistance Exercise Protocol (REP).

Methods: Eighteen adult males (age: $23.44 \pm 0.88$ years; body weight: $76.39 \pm 1.52 \mathrm{~kg}$; height: $1.77 \pm 0.02 \mathrm{~m}$ ) were enrolled in a double-blind crossover placebo-controlled study. Subjects were randomized to receive a single dose of CC (for a total of $3.0 \mathrm{~g}$ of creatine) or a placebo (PLA) in two successive trials 7 days apart. In both trials subjects completed a REP (a Wingate exercise followed by 3 sets of biceps curling with a dumbbell until exhaustion) $2 \mathrm{~h}$ after the ingestion of CC or PLA. Before ingestion and after REP subjects performed isometric contractions of the dominant upper limb, using an isometric-ergometer equipped with a load cell and combining a multichannel surface electromyography (sEMG) recording technique, and RFD, MVC, and $\mathrm{TtT}$ at $60 \%$ MVC until exhaustion were registered.

Results: No statistical differences were recorded of values in the prepost-REP comparison for all the outcomes considered (RFD, MVC, and $\mathrm{TtT}$ ). However, for RFD and $\mathrm{TtT}$ a positive trend was observed. Conclusions: Although $\mathrm{CC}$ did not give significant results on the outcomes considered, the improvements trends observed for RFD and $\mathrm{TtT}$ (i.e. the ability to express the maximal peak force in the shortest time, and the capacity to maintain maximal isometric force at $60 \%$ of MVC as long as possible, respectively), leads to consider that the use of creatine can have positive effects on athletic skills that engage power and muscle "high intensity" endurance, as confirmed by previous studies, but that these effects cannot be significantly explained through an acute \&quot;one shot-type" administration protocol.

References

1. Butts, J., et al. (2018) Sport. Heal. A Multidiscip. Approach 10, 31-34.

2. Chartogne, M., et al. (2021) Exp. Physiol. 105, 1758-1766.

3. D'Emanuele, S., et al. (2021). Front. Hum. Neurosci. 15. https:// doi.org/10.3389/fnhum.2021.701916.

4. Negro, M., et al. (2018). Front. Physiol. 9. https://doi.org/10. 3389/fphys.2018.01140.

\section{3}

\section{Effects of fasting duration during a time-restricted eating protocol on body composition and cardiometabolic risk factors}

\section{Alessandro Sampieri ${ }^{l}$, Edoardo Santinello ${ }^{l}$, Giorgia Schiavinotto ${ }^{l}$, Lisa Vigo $^{I}$, Antonio Paoli ${ }^{I}$, Tatiana Moro ${ }^{I}$ \\ University of Padua, Department of Biomedical Sciences, University of Padova, Padova, Italy ${ }^{1}$}

Background: The obesity epidemic, as a major risk factor for chronic diseases, overwhelms the cost of public health. To face this burden guidelines recommend changing lifestyles by increasing physical activity and inducing continuous energy restriction. However, there is a growing interest in identifying effective alternative weight loss strategies that involve in limiting food intake to certain periods of the day like the time-restricted eating (TRE). Its effectiveness in terms of weight and fat loss is well known in scientific literature, while its effects on cardiovascular risk factors and inflammatory markers is controversial in a non-trained population.

The objective of this study is on the one hand to compare the different TRE protocols in terms of body composition and on the other hand to evaluate if the benefits persist in the following months.

Methods: 42 Volunteers were recruited and randomly assigned to a TRE group $(16: 8,14: 10,12: 12)$ or a control group (ND). In the TRE group subjects consume an isocaloric food protocol in an 8- or 10- or 12-h' time window while in the ND group they could feed in anytime. Body composition was measured by dual-energy X-ray absorptiometry (DEXA) and total body water was estimated by bioelectrical impedance analysis (BIA). In addiction strength was measured by handgrip and blood was collected for evaluating the anabolic hormones, inflammatory markers, lipid profile and leptin levels. Lastly, the results of the morningness-eveningness questionnaire (MEQ) and the physical activity levels measured by the international physical activity questionnaires (IPAQ) were considered.

Results: Time restricted eating reduced body weight $(p<0.05)$ and fat mass $(\mathrm{p}<0.05)$ percentage in 16:8 while maintaining the fat-free mass. However, in the other protocols there were no significant differences between groups after 8 weeks versus ND. There was no interaction between either IPAQ or MEQ results with the effects of diet.

Conclusion: TRE with a protocol of $8 \mathrm{~h}$ feeding window could produce significant differences in reduction of fat mass versus control without calorie counting while mild effects on the blood counting. Instead, the other TRE protocols have no significant effects compare to control group. Considering other studies in the literature, our findings suggest that these protocols without adherence to a regular physical exercise practice is not effective.

\section{4}

\section{Effects of parmigiano reggiano cheese on muscle damage induced by eccentric exercise in healthy older} adults

Giacomo Mazzocco $^{1}$, Giorgia Schiavinotto ${ }^{1}$, Linda Toniolo ${ }^{1}$, Daniele Altin $^{1}$, Lisa Vigo ${ }^{1}$, Alessandro Sampieri ${ }^{1}$, Claudia Favero ${ }^{2}$, Valentina Pizzamiglio $^{3}$, Antonio Paoli ${ }^{1}$, Tatiana Moro ${ }^{1}$

University of Padua, Department of Biomedical Sciences, University of Padova, Padova, Italy ${ }^{1}$;

University of Padua, Department of Medicine, University of Padova, Padova, Italy ${ }^{2}$;

Consorzio Del Formaggio Parmigiano Reggiano, Consorzio Del Formaggio Parmigiano Reggiano, Reggio Emilia, Italy ${ }^{3}$

Purpose: Aging can affect muscle response to high intensity training. However, some nutritional aids could be helpful to assist muscle recovery. Parmigiano Reggiano contains high quality protein sources but also short chain fatty acids, which have interesting anti-inflammatory properties. Thus, the current cross-over study aimed to assess the impact of Parmigiano Reggiano supplementation on recovery of muscle function and muscle soreness response to acute eccentric exercise in healthy older adults.

Methods: A group of healthy older adults $(64.00 \pm 5.23$ years $)$ was randomized to received daily either Parmigiano Reggiano (PR; $50 \mathrm{~g} /$ day) or whey protein (WHEY; $15 \mathrm{~g} /$ day) for 15 consecutive days with 10 days of wash-out between the interventions. On day 11 of each series they performed an eccentric leg extension protocol. Muscle voluntary contraction (MVC) and soreness (DOMS) together with markers of cellular repair such as phase angle ( $\mathrm{PhA})$ measured via Bioimpedance analysis, blood cytokines and lipoprotein concentration were assessed before, $0,48,72$ and $120 \mathrm{~h}$ after performing the exercise.

Results: DOMS were significantly higher $48 \mathrm{~h}$ after exercise but significantly decreased at $72 \mathrm{~h}$ with Parmigiano Reggiano supplementation ( $\mathrm{p}<0.05)$, whilst remained higher after WHEY. A significant decrease in MVC was observed immediately after the eccentric exercise in the WHEY group but not in the PR. Phase angle, 
which is an index of cell repair ability, significantly increased after $72 \mathrm{~h}$ only in the PR group.

Conclusions: These preliminary results suggest that Parmigiano Reggiano may be an useful supplement to help older adults in muscle damage recovery after eccentric exercise.

References

1. Summer A, Formaggioni P, Franceschi P, Di Frangia F, Righi F, Malacarne M. Cheese as Functional Food: The Example of Parmigiano Reggiano and Grana Padano. Food Technol Biotechnol. 2017 Sep;55(3):277-289.

2. Peake J, Nosaka K, Suzuki K. Characterization of inflammatory responses to eccentric exercise in humans. Exerc Immunol Rev. 2005;11:64-85.

\section{5}

\section{Effects of parmigiano reggiano cheese on muscle damage induced by eccentric exercise in healthy young adults}

Daniele Altin $^{1}$, Giorgia Schiavinotto ${ }^{l}$, Giacomo Mazzocco ${ }^{l}$, Linda Toniolo $^{I}$, Lisa Vigo $^{I}$, Alessandro Sampieri ${ }^{I}$, Claudia Favero ${ }^{2}$, Valentina Pizzamiglio ${ }^{3}$, Antonio Paoli ${ }^{1}$, Tatiana Moro ${ }^{1}$

University of Padua, Department of Biomedical Sciences, University of Padova, Padova, Italy ${ }^{1}$;

University of Padua, Department of Medicine, University of Padova, Padova, Italy ${ }^{2}$;

Consorzio Del Formaggio Parmigiano Reggiano, Consorzio Del Formaggio Parmigiano Reggiano, Reggio Emilia, Italy ${ }^{3}$

Purpose: Parmigiano Reggiano cheese, due to its nutritional qualities, can be considered as "functional" food. In particular, the short chain fatty acids contained in the cheese could interact with the inflammatory response to exercise. Thus, the aim of the present study was to verify the acute effects of its consumption on exercise-induced muscle damage in healthy young adults.

Methods: Eight healthy young adults $(22.25 \pm 2.25$ years $)$ were randomized in a cross-over fashion in two groups and were asked to consume a Parmigiano Reggiano supplementation (PR) or a whey protein supplementation (WP) for ten days before an eccentric leg extension protocol (ECC). Subjects were assessed at the baseline and in the five days following the exercise (while continuing to take the supplementation) for inflammatory markers (cytokines, C-reactive protein, and cholesterol), Body Impendence Assessment (BIA), DOMS, knee extension and flexion ROM, thigh volume, handgrip and maximum voluntary contraction (MVC).

Results: Results showed a time-dependent significant variation $(\mathrm{p}<$ 0.001) in DOMS with no differences between supplementation; regarding MVC, the force reached the lowest point $48 \mathrm{~h}$ post exercise in both types of intervention $(\mathrm{p}<0.05)$, but PR supplementation seems to ameliorate the force recovery. A significant time $\mathrm{x}$ supplement interaction $(\mathrm{p}<0.05)$ emerged in CHOL and LDL levels, which remained steady in PR group, whilst decreased in WP group throughout the 5 days of follow-up.

Conclusion: The preliminary results of the present study suggest that Parmigiano Reggiano could be an useful supplement to improve strength recovery and muscle damage repair after a bout of eccentric exercise in young adults.

\section{References}

1. Summer A, Formaggioni P, Franceschi P, Di Frangia F, Righi F, Malacarne M. Cheese as Functional Food: The Example of
Parmigiano Reggiano and Grana Padano. Food Technol Biotechnol. 2017 Sep.

2. Stožer A, Vodopivc P, Križančić Bombek L. Pathophysiology of exercise-induced muscle damage and its structural, functional, metabolic, and clinical consequences. Physiol Res. 2020 Aug 31.

\section{6}

May the force be with women: effects of 9-week of a resistance training program with supplementation in untrained woman

Massimo Negro ${ }^{1}$, Giuseppe D'antona ${ }^{1}$, Vittoria Carnevale Pellino ${ }^{2}$, Nicola Lovecchio ${ }^{2}$, Matteo Vandoni ${ }^{2}$

University of Pavia, Centro Di Ricerca Interdipartimentale Nelle Attività Motorie E Sportive (criams)-sport Medicine Centre, Voghera, Italy $^{1}$;

Università Di Pavia, Laboratorio Di Attività Motoria Adattata, Dipartimento Di Salute Pubblica, Medicina Sperimentale E Forense, Pavia, Italy ${ }^{2}$

Purpose: The present study aimed to investigate the efficacy of specific resistance training program with or without protein supplementation in the prevention of loss of mass in young untrained women.

Methods: 54 Women (aged 19-35 years, healthy and resistance exercise untrained) were randomly divided in two groups. Protein Group (PG - 28 subjects), supplemented with 0.25 g. protein $/ \mathrm{kg}$ pre and post workout during training days, and Control Group (CG - 27 subjects) only exercised. Every subject performed the same training for 9 weeks (1familiarization + 8training) with 3 supervised session/ week, composed by three pre-post fatigue circuits. At the end of the first conditioning week (T0) and at the end of 8th week (T1) every subject performed 1 RM test at Leg Press machine (LP), Bench Press (BP) and Lat Machine (LM) and was evaluated on height, body weight, body composition with Bioelectrical Impedance Analyser. Differences from T0 were calculated with paired t-test while independent t-test on delta values (T1-T0) assessed differences between groups.

Results: 45 Women completed the study $(\mathrm{PG}=24, \mathrm{CG}=21)$. $\mathrm{PG}$ had a significant decrease in fat mass $(p<0.001)$ and increase in fat free mass and lean mass $(p<0.001)$. Both groups had a significant increase in 1RM in every circuit.

Conclusions: Final strength values were significant higher in both groups suggesting that it was independent from mass gain probably due to a better fiber recruitment. Moreover, results showed that protein supplementation in addition to resistance program influenced body composition likely for a lack of protein for muscle rebuild. These results should be implemented in a larger sample but could drive to new application in the field of woman resistance training.

References

1. Kraemer, W. J., Nindl, B. C., Ratamess, N. A., Gotshalk, L. A., Volek, J. S., Fleck, S. J., Newton, R. U., \& Häkkinen, K. (2004). Changes in Muscle Hypertrophy in Women with Periodized Resistance Training. Medicine and Science in Sports and Exercise, 36(4), 697-708

2. Negro M., Vandoni M., Ottobrini S., Codrons E., Correale L., Buonocore D., Marzatico F. Protein supplementation with low fat meat after resistance training: effects on body composition and strength. Nutrients 2014, August (6):3040-4. 


\section{7}

Monitoring training load in soccer: the ROMEI model

\author{
Marco Montini ${ }^{1}$, Jacopo Rocchi ${ }^{1}$ \\ Foro Italico / Figc, Foro Italico / Figc, Rome, Italy ${ }^{l}$
}

For a training organization, monitoring training load (TL) is of paramount importance. Despite this, a conclusive response on such topic is yet to be reported. The aims of this study were to investigate the relationship between TL indicators and to propose a new method for monitoring TL response and physical fitness. Twenty professional soccer players were retrospectively evaluated. The first phase of data analysis included 34 in-season training sessions. Subsequently, three microcycles (T1-T2-T3) of pre-season training were processed. A regression model was used to examine the relationships between internal TL (session rating of perceived exertion [s-RPE]) and external TL (energy expenditure, EE). The standard error of the regression equation was used to propose a new model called "ROMEI" (Relation of Ongoing Monitored Exercise in Individual). The level of statistical significance was set at $\mathrm{p}<0.05$. During the competitive season and the pre-season training camp, the average TL values were $65.8 \pm 22$ and $58.2 \pm 22 \mathrm{~min} ; 336 \pm 204$ and $228 \pm 101$ AU of s-RPE; and $29 \pm 13$ and $25 \pm 9 \mathrm{~kJ}$ of EE, respectively. In the competitive season, the collective and average individual correlation coefficients between s-RPE and EE were $r=0.888$ and $r=0.892 \pm$ 0.05 , respectively. Considering slope values $(\mathrm{m})$ of the regression line, data highlighted a significant increase of $+34.4 \pm 15.9 \%$ in T2 vs. T3 ( $<<0.001)$ and $+38.2 \pm 15.2 \%$ in T1 vs. T3 ( $<<0.001)$. Data shown in this investigation support the use of an individualized analysis to better understand the TL administered to soccer players rather than a collective analysis. This may be accomplished with the proposed ROMEI model.

\section{References}

1. Monitoring Training Load in Soccer: The ROMEI Model. Marco Montini 1, Jacopo E Rocchi J Strength Cond Res. 2020 Oct 30. https://doi.org/10.1519/JSC.0000000000003875. Online ahead of print.

\section{TRAINING and SPORT EVALUATION}

\section{8}

\section{2-km Shuttle run test for fitness evaluation in rugby union: what exactly does it measure?}

\section{Massimo Teso ${ }^{1}$, Francesca Morra ${ }^{l}$, Luca Ferrari ${ }^{l}$, Gianluca Bochicchio $^{I}$, Matteo Rizzo ${ }^{l}$, Giorgio Da Lozzo ${ }^{l}$, Silvia Pogliaghi ${ }^{l}$ \\ Università Degli Studi Di Verona, Dipartimento Di Scienze Motorie, Verona, Italy ${ }^{I}$}

The 1.2-km shuttle run test, also known as Bronco Test, was recently proposed for a time- and cost-efficiency field evaluation of aerobic fitness in rugby players. The average speed measured during the Bronco test (BT) correlates with maximal aerobic velocity and the distance covered during a match; this speed is increasingly used to guide training intensity and to monitor the effects of training over time in rugby players. However, the correspondence between this parameter and classical physiological indexes of cardiorespiratory fitness remains unclear.

Purpose: To determine the correspondance between selected variables recorded during BT and those measured during an incremental test.
Methods: 12 healthy participants (6 females; $25 \pm 3$ years) performed a ramp incremental test (RT) on a treadmill and a BT on different days and random order. Speed (V), oxygen consumption (VO2) and heart rate (HR) measured (K5, Comed, Italy) at the respiratory compensation point (RCP) and upon exhaustion (max) during $\mathrm{RT}$ were compared to the values recorded in the last 3 min of the BT by one-way RM-ANOVA and correlation analysis.

Results: HRmax and VO2max were not different and significantly correlated with HRBT and VO2BT respectively (193 \pm 7 vs $192 \pm$ $6 \mathrm{bpm}, \mathrm{p}=0.32, \mathrm{r} 2=0.72$; and $46 \pm 6$ vs $47 \pm 6 \mathrm{ml} \bullet \mathrm{kg}-1 \bullet \mathrm{min}-1, \mathrm{p}=$ $0.70, \mathrm{r} 2=0.72$ ). Data from both $\mathrm{BT}$ and max were significantly higher compared to HRRCP and VO2RCP (i.e. $177 \pm 7 \mathrm{bpm}, \mathrm{p}<0.001$, and $39 \mathrm{ml} \bullet \mathrm{kg}-1 \bullet \mathrm{min}-1, \mathrm{p}<0.001$ for all comparisons). Vmax, VBT, and VRCP were significantly different from each other (respectively, 13.2 $\pm 1.5,12.6 \pm 1.2$, and $9.8 \pm 1.3 \mathrm{~km} \bullet \mathrm{h}-1 ; \mathrm{p}<0.01$ for all comparisons) while all significantly correlated $(\mathrm{r} 2=0.86 \mathrm{Vmax}$ vs VBT; $\mathrm{r} 2=$ 0.72 Vmax vs VRCP, $\mathrm{r} 2=0.67$ VBT vs VRCP). Conclusions: During a Bronco Test VO2 and HR reach maximal values that coincide with VO2max and HRmax. However, running velocity obtained during VBT cannot be directly used as an index of either maximal velocity or velocity at RCP.

\section{References}

1. Brew D.J., Kelly V.G., The reliability of the $1.2 \mathrm{~km}$ shuttle run test for intermittent sport athletes, Journal of Australian Strength \& Conditioning, 2014.

2. Kelly V., Jackson E., Wood A., Typical scores from the $1.2 \mathrm{~km}$ shuttle run test to determine maximal aerobic speed, Journal of Australian Strength \& Conditioning, 2014.

\section{9}

\section{Comparison of power and repeated-sprint characteristics in female ultimate frisbee players of different levels and playing roles}

\author{
Ivan Galli ${ }^{1}$, Rocco Di Michele ${ }^{2}$
}

University of Bologna, Department For Life Quality Studies, Bologna, Italy ${ }^{1}$;

University of Bologna, Department of Biomedical and Neuromotor Sciences, Bologna, Italy ${ }^{2}$

Purpose: Ultimate frisbee is a growing sport, where fitness levels of competitive players are increasing over years. The purpose of this study was to compare selected physical characteristics between elite and sub-elite female players. Also, a comparison between playing roles was performed.

Methods: 23 female players (12 elite (1st Italyn League), $24.3 \pm$ 3.9 years, $168.1 \pm 5.8 \mathrm{~cm}, 61.8 \pm 6.0 \mathrm{~kg}$; and 11 sub-elite (2nd Italyn League), $20.7 \pm 1.7$ years, $164.8 \pm 7.0 \mathrm{~cm}, 58.1 \pm 3.4 \mathrm{~kg}$ ), of both playing positions (handler/cutter), were involved. The athletes performed a SJ test, a CMJ test, and a $6 \times 20+20 \mathrm{~m}$ repeated-sprint ability (RSA) test. Velocity of the fastest sprint (RSAbest), average velocity (RSAmean), and percentage decrement (RSAdec) across sprints, were examined.

Results: Elite players showed higher CMJ $(31.5 \pm 3.5 \mathrm{~cm}$ vs. $27.2 \pm$ $4.3 \mathrm{~cm}, \mathrm{~d}=1.11, \mathrm{p}<0.05)$ and $\mathrm{SJ}(29.5 \pm 3.6 \mathrm{~cm}$ vs. $26.1 \pm 3.5 \mathrm{~cm}$, $\mathrm{d}=0.97, \mathrm{p}<0.05)$ than sub-elite players. No differences between levels were observed for RSAbest (elite $18.3 \pm 0.9 \mathrm{~km} / \mathrm{h}$ vs. sub-elite $18.0 \pm 0.7 \mathrm{~km} / \mathrm{h}, \mathrm{p}>0.05$ ), RSAmean (elite $17.5 \pm 0.7 \mathrm{~km} / \mathrm{h}$ vs. subelite $17.3 \pm 0.5 \mathrm{~km} / \mathrm{h}, \mathrm{p}>0.05$ ) and RSAdec (elite $3.6 \pm 0.8 \%$ vs. sub-elite $2.9 \pm 1.3 \%, \mathrm{p}>0.05)$. In the elite group, cutters showed higher CMJ $(33.6 \pm 2.6 \mathrm{~cm})$ than handlers $(28.6 \pm 2.3 \mathrm{~cm})(\mathrm{d}=2.04$, $\mathrm{p}<0.05)$. A trend towards higher SJ of cutters $(30.0 \pm 3.9 \mathrm{~cm})$ vs. 
handlers $(28.3 \pm 2.4 \mathrm{~cm})$ was also observed $(\mathrm{d}=0.55, \mathrm{p}=0.06)$, whereas there were no differences between roles for RSA indices (all $\mathrm{p}<0.05$ ). In the sub-elite group, handlers and cutters showed similar values $(\mathrm{p}>0.05)$ for all examined variables.

Conclusions: The higher power observed in elite players reflects the high number of powerful actions (e.g. sprinting and jumping), typical of elite match play. The similar RSA between levels can be explained by the bigger roster of elite teams, resulting in shorter individual playing time and longer recoveries between stints, and by a higher ability of elite players to use their full physical skills during a match, rather than having a faster absolute running speed. Differences between roles observed for $\mathrm{CMJ}$ in the elite group are consistent with the higher number of high-intensity accelerations/decelerations performed by cutters during match play (Raya-Gonzalez et al., 2020). Sub-elite match play is instead characterized by less specialized roles, explaining the similarities between roles at that level. These findings add new insights on the physical characteristics of female ultimate frisbee players.

\section{References}

1. Palmer $\mathbf{J}$ et al. (2020) Physical demands of elite women's ultimate frisbee between halves and across matches in an international tournament. J Strength Cond Res in press.

2. Raya-Gonzalez J et al. (2020) External match load imposed upon ultimate frisbee players: a comparison between playing positions. Sci Sport 36: 160-162.

\section{0}

\section{Descriptive analysis of achilles and patellar tendon stiffness in young male basketball players}

\author{
Marco Gervasi ${ }^{1}$, Eneko Fernández-peña? ${ }^{2}$, Rosaria Gradilone ${ }^{l}$, \\ Roberto Venerandi ${ }^{l}$, Eliano Crudelini ${ }^{l}$, Valentina Natalucci ${ }^{l}$, Piero \\ Benelli $^{l}$ \\ University of Urbino, Department of Biomolecular Sciences, Urbino, \\ Italy $^{1}$; \\ University of The Basque Country (upv/ehu), Department of Phisical \\ Education and Sport, Vitoria-gasteiz, Spagna ${ }^{2}$
}

Aim: This study aims to describe age and dominant side differences in the mechanical properties of the Patellar tendon (PT) and gastrocnemius-Achilles tendon unit (GAT) in vivo in young male basketball players.

Methods: Sixty-five young male basketball players were recruited. Subjects were divided in three groups according to their age: $12-13$ (n $=18 ; 13.3 \pm 0.4$ years; $53.9 \pm 9.0 \mathrm{~kg} ; 168.0 \pm 6.4 \mathrm{~cm}), 14-15(\mathrm{n}=$ $34 ; 15.0 \pm 0.6$ years; $67.4 \pm 10.2 \mathrm{~kg} ; 179.4 \pm 7.7 \mathrm{~cm})$ and $16-17(\mathrm{n}$ $=13 ; 16.6 \pm 0.5$ years; $79.1 \pm 12.8 \mathrm{~kg} ; 187.1 \pm 7.2 \mathrm{~cm})$. The dynamic stiffness of the GAT and PT was measured on both sides using the MyotonPRO device (Myoton AS, Tallin, Estonia). For GAT assessment, participants were in prone position on a massage table with the knees in extension and feet hanging barefoot and unsupported from the edge. The measurement points were at $8,9,10$, $11,12,16$, and $20 \mathrm{~cm}$ from the plantar aspect of the heel. For the PT assessment, participants were seated with their knees flexed at $90^{\circ}$ and completely relaxed. The measurement point was at the midpoint between the distal edge of the patella and the tibial tuberosity. Dominant side was defined as the inside foot during a preferred layup. GAT data was analyzed by means of a two-way ANOVA for independent measures (age and measurement point as independent factors) and PT data was analyzed using a one-way ANOVA with age as independent factor.
Results: The analysis of GAT showed that the age significantly affected stiffness, with youngest participants having lower values and older participants having higher values from 8 to $12 \mathrm{~cm}$. These differences tended to disappear at 16 and $20 \mathrm{~cm}$. No differences were found between dominant and non-dominant sides. PT analysis showed that only the 12-13 group was significantly lower than the other groups, while no differences were found between 14-15 and 16-17 groups. The dominant side of the $12-13$ group had higher PT stiffness.

Conclusions: Stiffness can enhance athletic performance, although too much or too little stiffness may increase the risk of injury. The differences found in this study among age groups could be caused by the natural development of tendon tissue on the course of the maturation process, being unclear the effect of training. An earlier development of the preferred layup limb caused by the sport specific skill asymmetry could explain the differences found in the PT stiffness of the 12-13 age group.

\section{1}

\section{Development of mobility for the effectiveness of traditional karate skills}

Matteo Lucchini ${ }^{1}$, Matteo Quarantelli ${ }^{1}$

Università San Raffaele, Università San Raffaele, Rome, Italy ${ }^{I}$

Purpose: Adequate joint mobility favors the realization of martial arts skills. Numerous studies observe the relationship between the development of mobility and a better efficacy of sports movements. The study examines the recent literature and then tests a training protocol with athletes practicing Traditional Karate.

Methods: A retrospective analysis of the recent literature and an application of an experimental training protocol dedicated to a sample of Shotokan-style Traditional Karate athletes were conducted. The study in question promotes the use of a training card for the development of joint mobility in Traditional Karate, creating a real "warm up", with the use of functional free body training exercises of the Spartan System ${ }^{\circledR}$ FIF method. The experimental group consisted of a group of 28 athletes aged between 15 and 20 whose behaviors were monitored. Pre- and post-treatment data were collected.

Results: After 6 weeks of training in the flexibility of the posterior muscle chain and in the mobility of the ankle joint, data were found that indicate a marked improvement in joint mobility in the experimental group. The results deriving from the Sit \& Reach Test indicate a development of $+75 \%$ of flexibility of the posterior muscle chain. The Lunge Test demonstrates a $+27 \%$ variation for both ankles.

Conclusions: The Pilot study would show that the Spartan System ${ }^{\circledR}$ method exercise protocol is effective for the development of mobility of a group of Shotokan-style Traditional Karate athletes. In particular, the dynamic stretching exercises chosen for the experimental group would be effective both in the short and long term.

\section{References}

1. Warm-Up and Stretching in the Prevention of Muscular InjuryKrista Woods, Phillip Bishop and Eric Jones-Human Performance Laboratory, University of Alabama, Tuscaloosa,Alabama, USA-Sports Med 2007; 37 (12): 1089-1099 Review Article.

2. Acute Effects of Dynamic Stretching on Muscle Flexibility and Performance: An Analysis of the Current Literature-Jules Opplert, Nicolas Babault—Sports Med (2018) 48:299-325Review Article. 
152

\section{Effects of a frisbee-specific training on repeated-sprint ability in elite women's ultimate frisbee players}

\author{
Ivan Galli ${ }^{1}$, Rocco Di Michele ${ }^{2}$ \\ University of Bologna, Department For Life Quality Studies, \\ Bologna, Italy ${ }^{1}$; \\ University of Bologna, Department of Biomedical and Neuromotor \\ Sciences, Bologna, Italy ${ }^{2}$
}

Purpose: Ultimate frisbee is an invasion team sport played with a plastic flying disc, requiring high levels of agility, aerobic fitness, power, and repeated-sprint ability (RSA) (Palmer et al., 2020). The purpose of this study was to asses the effects of a training program based on sport-specific drills on RSA in elite women's players.

Methods: 14 Elite female ultimate frisbee players were involved (age: $24.2 \pm 3.3$ years; height: $166.7 \pm 5.7 \mathrm{~cm}$; weight: $61.8 \pm$ $5.6 \mathrm{~kg}$ ). All athletes completed a training program including two 5-week mesocycles, with two sessions/week. In the first mesocycle, training consisted of acceleration and speed drills performed using the frisbee, whereas the second mesocycle was based on small-sided games. RSA was tested before the program (T0), after the first mesocycle (T1), and after the second mesocycle (T2), using a $6 \times 20$ $+20 \mathrm{~m}$ sprint protocol. The velocity of the fastest sprint (RSAbest), the average velocity (RSAmean), and the percentage decrement (RSAdec) across sprints, were examined.

Results: At T1, RSAbest improved by $0.81 \mathrm{~km} / \mathrm{h}(+4.7 \%, \mathrm{ES}=1.08$, $\mathrm{p}<0.05)$ compared to T0, while there was no substantial change between $\mathrm{T} 1$ and $\mathrm{T} 2(-0.10 \mathrm{~km} / \mathrm{h} ;-0.5 \%, \mathrm{p}>0.05)$. A similar trend was observed for RSAmean $(\mathrm{T} 1 \vee \mathrm{T} 0:+0.58 \mathrm{~km} / \mathrm{h},+3.5 \%, \mathrm{ES}=$ $0.91, \mathrm{p}<0.05$; T2 v T1: $-0.14 \mathrm{~km} / \mathrm{h},-0.8 \%, \mathrm{p}>0.05)$. Conversely, RSAdec was almost unchanged across the training period, with non significant $(\mathrm{p}>0.05)$ variations between $\mathrm{T} 1$ and $\mathrm{T} 0(+1.12 \%)$, and between $\mathrm{T} 2$ and $\mathrm{T} 1(+0.25 \%)$.

Conclusions: The results suggest that frisbee-specific drills, designed for physical training with the use of the flying disc, are effective to improve speed and RSA and can be a valuable alternative to sprint training in elite female players. Moreover, small-sided games are effective to maintain RSA throughout a competitive season. These findings reflect similar observations made in other team sports (Bishop et al., 2011; Karahan, 2020), and provide useful insights for sport-specific conditioning in ultimate frisbee.

\section{References}

1. Bishop D et al. (2011) Repeated-sprint ability - part II: recommendations for training. Sports Med 41:741-756.

2. Karahan M (2020) Effect of skill-based training vs. small-sided games on physical performance improvement in young soccer players. Biol Sport 37: 305-312.

3. Palmer J et al. (2020) Physical demands of elite women's ultimate frisbee between halves and across matches in an international tournament. J Strength Cond Res in press.

\section{3}

\section{Effects of detraining in young soccer players during the COVID-19 lockdown period}

\section{Giuseppe Malizia ${ }^{1}$, Donato Formicola ${ }^{2}$}

Medical and Sport Science Department S.s. Lazio, Rome, Italy ${ }^{l}$; Department of Medical Science, Suism-University Sport Service Centre, University of Turin, Italy ${ }^{2}$
Background: In literature, the effects of detraining in elite footballer skills during COVID-19 quaran-tine were observed recently1.

Purpose: Such research analyzed the effects of detraining and home training on coordination and con-ditional skills in a population of young soccer player with age of $17 \pm 2$ years, during the COVID-19 lockdown confinement, between October and December 2020.

Methods: The Functional Movement Screen (FMS) 2 and the Counter Movement Jump (CMJ) protocols were performed to evaluate the changes in the coordination skills and the strength capacity of the low-er limbs in two groups of footballers: Under 16 (11 players, age $15 \pm 1$ years and weight $57 \pm 11 \mathrm{~kg}$ ), and Juniors, (9 players, age 18 \pm 1 years and weight $69 \pm 6 \mathrm{~kg}$ ). Data were collected in three sessions, T0, T1 and T2. The T0 session was in September 2020, the tests took place in an empty locker room, made available by the company, half an hour before the start of the first weekly training. The sessions $\mathrm{T} 1$ (post strength protocol) and T2 (post detraining / home training) were performed in an empty room at the subjects' homes due to the confinement given by the COVID-19 pandemic government disposals. These two requests were carried out at the end of October and mid-December 2020, respectively.

Results: The no-parametric ANOVA for repeated measurements Friedman's test was used to analyze the differences of CMJ results among sessions and between groups. Significant difference $(\mathrm{p}=$ 0.050) was observed in CMJ results of Juniors in detraining. Solely for Juniors, a Mann-Whitney no-parametric T-test for independent samples found a significant difference $(p=0.026)$ in the left Active Straight Leg Raise test of the FMS protocol.

Conclusions: The results of the present study suggest a significant decrement of conditioning and co-ordination skills in young soccer players after 7 weeks of detraining and home training in footballers. References

1. Cohen DD, Restrepo A, Richter C, Harry JR, Franchi MV, Restrepo C, Poletto R, Taberner M, (2020). Detraining of specific neuromuscular qualities in elite footballers during COVID-19 quaran-tine, Science and Medicine in Football, Ahead-Of-Print, 1-6, https://doi.org/10.1080/24733938.2020.1834123

2. Cook G, Burton L, Hoogenboom BJ, Voight M (2014). Functional Movement Screening: the use of fundamental movements as an assessment of function-part 1, International Journal of Sports Physical.

\section{4}

\section{Effects of isoinertial preconditioning on vertical jump and back squat}

\section{Paolo Ronco ${ }^{1}$, Matteo Romanazzi $i^{1}$}

University of Turin, School of Exercise and Sport Sciences (suism), Turin, Italy ${ }^{I}$

Purpose: Post activation potentiation (PAP) is a phenomenon that enhances neuromuscular function as a result of a high intensity submaximal effort. The eccentric action provided by isoinertial flywheel machine seems to cause a potentiating effect on subsequent exercises. The aim of this study was to verify the effects of PAP using isoinertial method on jumping performance and dynamic lower limbs muscle strength.

Methods: Sixteen experienced and resistance trained athletes $(8$ men and 8 women, height: $168.4 \pm 9.4 \mathrm{~cm}$, mass $69.6 \pm 10.6 \mathrm{~kg}$, age: $28.7 \pm 7.1$ years) were involved in a crossover study. Following tests were performed: countermovement jump (CMJ) and 1-RM half back squat (HBS) with and without isoinertial preconditioning. Preconditioning phase was performed by a half squat exercise using a flywheel 
machine and consisted of 6 reps at maximal power with plate of $3,8 \mathrm{~kg}$ and inertia of $0.048 \mathrm{~kg} / \mathrm{m}^{2}$. After $7 \mathrm{~min}$ of recovery, CMJ (ISO $+\mathrm{CMJ}$ ) or 1-RM half back squat (ISO + HBS) were assessed. Jump height, absolute power, relative power and $1 \mathrm{RM}$ weight were analyzed. Assessments were carried out on different days with a minimum recovery of $48 \mathrm{~h}$. A paired t test was performed to compare results between two conditioning phases and non-parametric Wilcoxon test was used to compare results within male and female group. aggiungere che tutti hanno fatto tutti i test in gg diversi.

Results: Results showed a significant improvement with isoinertial preconditioning in the ISO + CMJ for jump height $(\mathrm{p}=0.001)$, absolute power $(\mathrm{p}=0,001)$ and relative power $(\mathrm{p}=0.0007)$ compared to CMJ only. No significant improvement was found in the ISO + HBS test respect to HBS only. Differentiating the two groups, significant improvement were reported in all ISO $+\mathrm{CMJ}$ variables (male $\mathrm{p}=0.01$; female $\mathrm{p}=0.004$ ) but not in ISO + HBS compared to CMJ and HBS test respectively.

Conclusions: Considering the data obtained, the isoinertial flywheel protocol showed a potentiating effect on the CMJ performance, thus this pre-conditioning activity is ineffective before high intensity muscle strength performance.

\section{References}

1. Beato M., Stiff A., Coratella G. (2021). Effects of postactivation potentiation after an eccentric overload bout on countermovement jump and lower-limb muscle strength. J Strength Cond Res. 35(7), 1825-32.

2. Timon $\mathrm{R}$ et al. (2019). Post-activation potentiation on squat jump following two different protocols: Traditional vs. inertial flywheel. J Hum Kinet. 69, 271.

\section{5}

\section{Efficient squat technique: an index to estimate it}

\author{
Gianluca Bochicchio $^{I}$, Luca Ferrari ${ }^{I}$, Matteo Rizzo $^{1}$, Massimo Teso ${ }^{I}$, \\ Silvia Pogliaghi ${ }^{\text {I }}$ \\ Università Degli Studi Di Verona, Dipartimento Di Neuroscienze, \\ Biomediche E Movimento, Verona, Italy ${ }^{I}$
}

Purpose: Squat is the most common multijoint exercise used to develop strength and power of the lower limbs in different contexts. Appropriate technique grants optimal results while minimising injuries. Based on the assumption that in a correct squat the barbell moves in the vertical line while any horizontal displacement is counterproductive, our group proposed a Technical inefficiency index (TI). TI is calculated as the \% ratio of the horizontal component to the vertical component of the barbell trajectory during squatting movements. Preliminary data found higher TI in naïve vs skilled athletes. This study aims to confirm that TI is able to distinguish the quality of back squat technique in a larger and more heterogeneous group of lifters. Methods: 26 young male subjects (age: 22,5 \pm 4,3 years) were recruited and divided in beginner, intermediate and advanced groups based on experience/performance. Each performed 4 sessions in which 1RM of squat and the kinematics of barbell trajectory were evaluated (sagittal 2-dimensional motion analysis, Panasonic ${ }^{\circledR}$ Lumix FZ200, $100 \mathrm{~Hz}$ ) during three sets to failure at $60 \%, 75 \%$ and $90 \%$ of $1 \mathrm{RM}$, in a randomized order. Data were compared by twoway ANOVA (experience group and load intensity) and correlation analysis.

Result: All groups had a comparable age, weight and height. Advanced were significantly stronger and experienced (\#8, $180 \pm$ $23 \mathrm{~kg} 1 \mathrm{RM}, 4 \pm 2$ years) compared to Intermediate (\#8, $130 \pm 14 \mathrm{~kg}$ $1 \mathrm{RM}, 2.5 \pm 5$ years) and Beginners (\#10, $84 \pm 18 \mathrm{~kg} 1 \mathrm{RM}, 0.2 \pm 0.3$ years), that were different from each other. There was a significant main effect of load intensity $(\mathrm{p}=0.002)$ and experience/performance $(\mathrm{p}=0.001)$ on TI. In all groups, post-hoc analysis showed a decreased efficiency at $90 \%$ compared to $60 \%$ and $75 \%$, that were not different from each other. In addition, beginners showed higher inefficiency at all intensities compared to both intermediate and advanced lifters. Yet, the difference in TI between intermediate and advanced lifters did not reach statistical significance. A significant negative correlation was found between experience/performance and TI at all intensities (overall $r=-0.62$ ).

Conclusions: The TI index discriminates the quality of squatting technique between lifters of different experience/performance, at all tested intensities. Further research will determine if this simple index can guide the acquisition of an efficient technique and allow the monitoring of movement quality under increasing load and fatigue in back squat.

\section{References}

1. McLaughlin T., Dillman C. (1977), A_kinematic_model_of_performance_in_the_parallel squat by champion powerlifters. Med Sci Sports Exerc 9: 128-133.

2. Luca Ferrari, Gianluca Bochicchio, Silvia Pogliaghi, "A small horizontal component of the barbell trajectory is a simple index of technical efficiency in squat", Firenze, 3 - 6 Ottobre 2018, IX congresso nazionale SIAMOC: congresso nazionale della società Italiana di analisi di movimento in clinica.

\section{6 \\ Evaluating the change of direction skill in futsal referees} Luca Russomando ${ }^{I}$, Jessica Nazzaro $^{l}$, Adriano Capobianco
Orrü $^{l}$, Stefania

Università Di Napoli Parthenope, Dip. Scienze Motorie E Del Benessere, Napoli, Italy ${ }^{1}$

Purpose: The ability to perform a rapid change of direction (COD) is a critical skill for successful performances in numerous field-based sports. COD skill is often measured through agility tests as agility has relationships with trainable physical qualities such as strength, power and technique $(1,2)$. Different physical determinants affect COD performance, such as anthropometrical dispositions and technical qualities, straight sprinting speed, and leg strength qualities (3). Aim of the present pilot study is to investigate the efficacy of a novel training program, specific for futsal referees, able to optimize COD skill by addressing specific motor tasks: 1) pivot foot positioning, 2) distance between the feet and 3) body posture through arm positioning.

Methods: Four male regional futsal referees (age: $28.5 \pm 3.1$ years; weight: 74,5 $\pm 6,9 \mathrm{~kg}$; height: $176 \pm 6 \mathrm{~cm}$; BMI: $23,99 \pm 1,76 \mathrm{~kg}$ / $\mathrm{m} 2$ ), members of Italyn Association of Referees, were recruited for this pilot study. The COD specific training program was added to their weekly standard training schedule (3 days/week) for 6 weeks. It lasts 10 min during which the participants perform a specific sequence, on both sides: lateral running for $11 \mathrm{~m}$, change of direction (acoustic sound), straight sprint for $9 \mathrm{~m}$. The sequence was performed 6 times (3 times/side), focusing on the single motor tasks quoted above, separately. Then the sequence was repeated and videotaped 4 consecutive times ( 2 times/side), focusing on all motor tasks, and the videos were analyzed by three independent trainers.

We tested the efficacy of the proposed COD training by Y-Reactive Agility test. 
Results: After 6-week COD-specific training, all participants improved their score at the Y-Reactive Agility test $(2,25 \pm 0,08 \mathrm{~s}$ vs $2,16 \pm 0,11 \mathrm{~s})$. The 3 selected motor tasks were evaluated by motion analysis and, after 6week, almost all of them were properly accomplished ( $3 \pm 1$ vs $6 \pm 2$; p $<0,05)$.

Conclusions: Futsal referees could improve their COD skill by performing the proposed program which is not time-consuming while providing a match-like stimulus.

\section{References}

1. Sheppard JM, Young WB. 2006. J Sports Sci, 24: 919-932.

2. Fiorilli G, Iuliano E, Mitrotasios M, Pistone EM, Aquino G, Di Costanzo A, Calcagno G, di Cagno A. 2017. Int J Sports Sci Med, 16: 247-253.

3. Young WB, James R, Montgomery I. 2002. J Sports Med Phys Fitness, 42: 282-288.

157

\section{Factors affecting the spartan race ${ }^{\circledR}$ performance: a study protocol}

\author{
Margherita Maria Quadri ${ }^{I}$, Matteo Bonato $^{I}$, Gianluca Vernillo ${ }^{I}$ \\ Università Degli Studi Di Milano, Dipartimento Di Scienze \\ Biomediche Per La Salute, Milan, Italy ${ }^{1}$
}

Purpose: The Spartan Race ${ }^{\circledR}(\mathrm{SR})$ is one of the most famous obstacle course races, involving running from 5 to $50 \mathrm{~km}$ on different terrains with different obstacles. SR requires a variety of physical abilities to complete extreme functional movements. Accordingly, endurance, muscle strength and power, as well as flexibility and balance are all factors that may influence SR performance. To date there is no evidence regarding the physical and physiological determinants of SR performance. Therefore, we present a protocol to outline these characteristics and to quantify the relative importance of the selected attributes with the performance level.

Methods: The proposed protocol will involve 15 élite (E) and 15 amateur (A) SR athletes. Athletes will be considered as E if they are in the Italyn Élite Spartan Race ${ }^{\circledR}$ ranking or as A if the performed at least one SR. The intervention period will be planned in agreement with the involved athletes and coaches. Athletes will be familiarized with the procedures prior to the testing sessions. They will be tested for anthropometry, aerobic capacity, upper- and lower-body muscle strength, as well as dynamic balance, core-strength and flexibility over a 2-day period during a training camp. The first day, medicine ball throw (upper body muscle strength); vertical jump test (lower body strength); Y balance test (dynamic balance) and core stability test (core strength) will be performed in a randomized order. The second day athletes will perform a multi-stage fitness test (aerobic capacity) and a sit-and-reach test (flexibility) in a randomized order. Results: The primary outcomes will be anthropometric measures (i.e. height, body mass, skinfolds, segment lengths, breadths, and girths); aerobic capacity (maximum distance covered), upper- (medicine ball throw distance) and lower-body (vertical jump height) muscle strength; dynamic balance (absolute, normalized and composite reach distance), core endurance strength (time of exhaustion) and flexibility (flexion distance).

Conclusions: This study can be considered as a starting point for SR athletes and coaches to improve their training strategies and performance. Indeed, identifying the physical and physiological determinants of SR performance may give valid training recommendation, guide talent identification and development programs, as well as individualize training prescription [1].

References
1. Bishop D. (2008) An applied research model for the sport sciences. Sports Med 38(3): p. 253-63.

\section{8}

\section{Hepatic function in professional basketball athletes}

Mariarita Brancaccio $^{I}$, Cristina Mennitti ${ }^{I}$, Alessandro Gentile ${ }^{I}$, Mariella Calvanese $^{I}$, Jacopo Ulisse ${ }^{I}$, Luca Gentile ${ }^{l}$, Giovanni D’Alicandro $^{2}$, Giulia Frisso ${ }^{1}$, Olga Scudiero ${ }^{1}$

Università Degli Studi Di Napoli Federico Ii, Dipartimento Di Medicina Molecolare E Biotecnologie Mediche, Napoli, Italy ${ }^{1}$; Aorn, Santobono-pausillipon, Department of Neuroscience and Rehabilitation, Center of Sports Medicine and Disability, Napoli, Italy $^{2}$

Purpose: Constant physical activity associated with a proper diet has a positive impact on human health. However, many studies have shown that physical exercise causes an adaptive metabolic response of the body which results, in an alteration of concentration of specific biochemical parameters. This alteration may be related to the type, duration or intensity of physical exercise and can lead to the onset of cardiac diseases, liver dysfunctions and muscle damage. In our study, we have focused our attention on alanine aminotransferase (ALT), aspartate aminotransferase (AST) and alkaline phosphatase (ALP). They are biomarkers of the decline in liver functions related to the change in the liver of the cell membrane caused by lipid peroxidation induced by the presence of oxygen radicals. ALT, AST and ALP increase their serum concentration especially after a long duration exercise. Furthermore, recent studies have identified that both GSH and GGT can be used as biomarkers for hepatic and/or renal dysfunction and/or as alarm bells of prolonged oxidative stress that could cause liver damage in competitive athletes. The purpose of this study is the analysis of the alterations in the levels of GSH, GGT, AST, ALT and ALP in serum of competitive athletes before and after a specific activity, as an identification data of a possible oxidative stress caused by intense physical activity.

Methods: 15 professional basketball athletes and 15 sedentary controls were recruited at time 0 , before the start of the sports season, and at time 1,2 months later; through spectrophotometric analysis we have determined GGT, AST, ALT and ALP, instead the GSH was measured by ELISA assay.

Results: The levels of AST, ALT and ALP of the athletes are within the normal range; athletes' GGT levels are lower than sedentary but it is in the normal range; finally, the GSH levels in the serum of the athletes are slightly increased in the serum of the athletes: CTR vs time 0 or time $1(\mathrm{p}<0.001)$.

Conclusions: the levels of AST, ALT and ALP are normal; the levels of GGT in the serum of athletes are lower than in sedentary controls; this result implies that the liver function of athletes is not compromised and the metabolism of GSH works perfectly despite intense physical activity. At the same time, the increase in GSH in the serum of athletes shows that physical activity, promotes an accumulation of ROS, so athletes to counteract this accumulation produce more GSH than sedentary controls.

\section{References}

1. Lombardo B, et al. (2019). Laboratory medicine: health evaluation in elite athletes. Clin Chem Lab Med, 57:1450-1473.

2. Mennitti C, et al. (2020) Athlete's Passport: Prevention of Infections, Inflammations, Injuries and Cardiovascular Diseases. J Clin Med. 9:2540.

3. Brancaccio M, et al. (2020). Dietary Thiols: A Potential Supporting Strategy against Oxidative Stress in Heart Failure 
and Muscular Damage during Sports Activity. Int J Environ Res Public Health, 17:9424.

159

\section{An updated methodology to estimate critical velocity in front crawl swimming: a scoping review}

Luca Petrigna ${ }^{1}$, Bettina Karsten ${ }^{2}$, Anne Delextrat ${ }^{3}$, Simona Pajaujiene $^{4}$, Diba Mani ${ }^{5}$, Giuseppe Battaglia ${ }^{1}$, Marianna Bellafiore ${ }^{I}$, Antonio Palma ${ }^{l}$, Antonino Bianco ${ }^{1}$

Università Di Palermo, Sport and Exercise Sciences Research Unit, Department of Psychology, Educational Science and Human Movement, University of Palermo, Palermo, Italy ${ }^{I}$;

Università, European University of Applied Sciences (eufh), Health School, Germany, Rostock, Germania ${ }^{2}$;

Università, Faculty of Sport and Health Sciences and Social Work, Oxford Brookes University, Oxford, Regno Unito ${ }^{3}$;

Università, Department of Coaching Science, Lithuanian Sports

University, Kaunas, Lituania ${ }^{4}$;

Universita, Department of Applied Physiology and Kinesiology, University of Florida, Gainesville, Stati Uniti D' America ${ }^{5}$

Purpose: Critical velocity (CV) and its related total distance covered above CV (D') can be used to evaluate and monitor performance in swimmers. CV is used to evaluate aerobic endurance while D' can be used to evaluate the anaerobic performance. These parameters are well researched in sports such as cycling and running, but the literature on $\mathrm{CV}$ in swimming is less available. Therefore, the objectives of this review were (i) to methodologically evaluate the protocols used to obtain $\mathrm{CV}$; and (ii), eventually, to provide a standard operating procedure (SOP) for this parameter in front crawl swimming.

Methods: The review followed Preferred Reporting Items for Systematic Reviews and Meta-Analyses Extension for Scoping Reviews guidelines. PubMed, Web of Science, and Scopus were used to find original studies written in English. No exclusion criteria were adopted for the country of origin. The results were analysed through a narrative synthesis

Results: From 5,235 studies, a total of 41 studies were included. Sixteen different protocols were adopted that included distances from 50 to $1500 \mathrm{~m}$ with different number of distances (e.g. only 200-400 or $100-200-400$ or $100-200-400-800$ and so on). The tests were proposed also in swimming pool of different length. Different physiological thresholds as comparators with CV and D'.

Conclusions: $\mathrm{CV}$ and D' evaluation presents different testing protocols, making this performance marker potentially inaccurate. As a consequence, we propose a SOP based on the protocol of Wakayoshi and colleagues (1992) following the indication of the last findings. The SOP proposed suggests to calculate CV using the distances 200-400 and $800 \mathrm{~m}$ with three-data (to obtain a more precise value), to extrapolate the value from the equation of Wakayoshi and colleagues (1992).

References

1. Wakayoshi K, Yoshida T, Udo M, Kasai T, Moritani T, Mutoh Y, et al. (1992) A simple method for determining critical speed as swimming fatigue threshold in competitive swimming. Int $\mathbf{J}$ Sports Med; 13(5):367-71.

2. Bosquet L, Leger L, Legros P. (2002) Methods to determine aerobic endurance. Sports Med; 32(11):675-700.

3. Toubekis AG, Tsami AP, Tokmakidis SP. (2006) Critical velocity and lactate threshold in young swimmers. Int J Sports Med;27(2):117-23.

\section{0}

Influence of the area per player in non-professional soccer players: focus on positional roles

Annamaria Mancini ${ }^{1}$, Daniela Vitucci ${ }^{2}$, Pasquale Meo ${ }^{1}$, Adriano Capobianco $^{1}$, Domenico Martone ${ }^{1}$, Francesca Cozzolino ${ }^{1}$, Pasqualina Buono ${ }^{I}$, Stefania Orrü ${ }^{l}$

Università Di Napoli Parthenope, Dip. Scienze Motorie E Del Benessere, Napoli, Italy ${ }^{1}$;

Ceinge Biotecnologie Avanzate, Ceinge Biotecnologie Avanzate, Napoli, Italy ${ }^{2}$

Purpose: This study analyses the influence of different area per player (AP; 75, 98 and $131 \mathrm{~m} 2$ ) on the average metabolic power and other soccer-related performance variables in relation with the positional roles. Methods: 19 non-professional soccer players (attackers, midfielders and defenders) were recruited to play 3 different smallsided games (SSGs): SSG1 (5vs5; $30 \times 30 \mathrm{~m} ; 5 \mathrm{~min}$ ), SSG2 (5vs5; $35 \times 45 \mathrm{~m} ; 5 \mathrm{~min})$ and SSG3 (7vs7; $35 \times 45 \mathrm{~m} ; 8 \mathrm{~min})$. Specific playing rules were applied: i)defenders could not go beyond the midfield line; ii)attackers could not retreat behind the midfield line; iii)ball touch limit was set to 3. GPS-assessed soccer-related variables were: average metabolic power (AMP), distance covered in $1 \mathrm{~min}$ (DIS); \%time spent at high speed ( $\mathrm{v}>16 \mathrm{~km} / \mathrm{h})(\% \mathrm{hst}) ; \%$ time spent at high metabolic power $(>20 \mathrm{~W} / \mathrm{kg})(\% \mathrm{hmpt}) ; \%$ distance covered at high/negative positive speed (accelerations: $2<\mathrm{v}<4 \mathrm{~m} / \mathrm{s} 2$; decelerations; $-6<\mathrm{v}<-2 \mathrm{~m} / \mathrm{s} 2)$ (\%ACC; \%DEC); number of actions performed at high metabolic power (hmpa). One-way ANOVA was performed among training game formats on each dependent variable in relation to the position role. Results: All recorded variables differed when each SSG was compared to the others ( $p<0,05)$, but for hmpa for attackers. Most performance variables were positively associated to increasing AP $(\mathrm{p}<0,05)$, but for $\%$ ACC and \%DEC, and differed among positional roles within the same SSG $(\mathrm{p}<0,05)$. Conclusions: Here we provide qualitative and quantitative determinants, able to significantly improve soccer-specific performance variables, demonstrating that different arrays of SSGs are needed to fulfil the demands characterising each positional role, irrespective of the physical/technical level of players.

\section{1}

\section{Influence of the area per player in non-professional soccer players: focus on positional roles}

\author{
Annamaria Mancini ${ }^{1}, D$ Vitucci $^{2}$, Capobianco $^{3}$, D $_{\text {Martone }}{ }^{3}, F$ \\ Cozzolino $^{3}$, Paolina Buono ${ }^{3}$, Stefania Orru ${ }^{4}$
}

1dipartimento Di Scienze Motorie E Del Benessere, Università Degli Studi Di Napoli Parthenope, Naples, Italy ${ }^{l}$, 2ceinge-biotecnologie Avanzate, Naples, Italy ${ }^{2}$, Dipartimento Di Scienze Motorie E Del Benessere, Università Degli Studi Di Napoli Parthenope, Naples, Italy ${ }^{3}$, Ceinge-biotecnologie Avanzate, Naples, Italy ${ }^{4}$

Purpose: This study analyses the influence of different area per player (AP; 75, 98 and $131 \mathrm{~m} 2$ ) on the average metabolic power (MP) and other soccer-related performance variables in relation with the positional roles.

Methods: 19 non-professional male soccer players (25,2 \pm 6,3years; $23,7 \pm 2,3 \mathrm{~kg} / \mathrm{m} 2 ; 16,4 \pm 6,3$ years soccer experience) were recruited to play 3 different small-sided games (SSGs): SSG1 (5vs5; $30 \times$ $30 \mathrm{~m} ; 5 \mathrm{~min}), \mathrm{SSG} 2(5 \mathrm{vs} 5 ; 35 \times 45 \mathrm{~m} ; 5 \mathrm{~min})$ and SSG3 (7vs7; $35 \times$ $45 \mathrm{~m} ; 8 \mathrm{~min})$. Specific playing rules were applied. GPS-assessed soccer-related variables were: average MP (AMP), distance covered 
in $1 \mathrm{~min}$ (DIS); \%time spent at high speed (v > $16 \mathrm{~km} / \mathrm{h}$; \% hst) or MP ( $>20 \mathrm{~W} / \mathrm{kg} ; \% \mathrm{hmpt}$ ); \%distance covered at high positive/negative speed $(2<\mathrm{v}<4 \mathrm{~m} / \mathrm{s} 2, \%$ ACC; $-6<\mathrm{v}<-2 \mathrm{~m} / \mathrm{s} 2$, \%DEC); number of actions at high MP (hmpa).

Results: All recorded variables differed when each SSG was compared to the others $(\mathrm{p}<0,05)$, but for hmpa for attackers. Most performance variables were positively associated to increasing AP (p $<0,05)$, but for \%ACC and \%DEC, and differed among positional roles within the same SSG $(\mathrm{p}<0,05)$.

Conclusions: Here the general applicability of SSGs, regardless the physical/technical skills of the group of players, to enhance performance is confirmed; furthermore, quantitative advices on AMP and other performance variables are provided to achieve significant improvements in all soccer players of the team.

\section{2}

\section{Low back pain prevalence and risk factors in Italyn adolescent male soccer players: results from an online survey}

Lucia Cugusi ${ }^{1}$, Andrea Manca ${ }^{1}$, Ernest Fishback ${ }^{2}$, Claudio Secci ${ }^{3}$, Marco Bergamin ${ }^{4}$, Stefano Gobbo ${ }^{4}$, Andrea Di Blasio ${ }^{5}$, Andrea Montella $^{l}$, Pasquale Bandiera ${ }^{I}$, Franca Deriu ${ }^{1}$

University of Sassari, Department of Biomedical Sciences, Sassari, Italy $^{1}$;

University of Cagliari, Bachelor's Degree Course In Physiotherapyi, Cagliari, Italy ${ }^{2}$;

University of Cagliari, Bachelor's Degree Course In Physiotherapy, Cagliari, Italy ${ }^{3}$;

University of Padova, Sport and Exercise Medicine Division, Department of Medicine, Padova, Italy ${ }^{4}$,

G. D'annunzio University of Chieti-pescara, Endocrine Section,

Department of Medicine and Sciences of Aging, Chieti, Italy ${ }^{5}$

Purpose: Low back pain (LBP) is a widespread musculoskeletal complaint reported by athletes, often from an early age. The purpose of this retrospective survey was to analyse the prevalence of LBP among Italyn adolescent soccer players, and to identify potential risk factors.

Methods: Participants were requested to answer an online survey based on the standardized Nordic questionnaires for musculoskeletal symptoms.

Results: Data were obtained from 204 male soccer players aged 14-17 years competing at the national and regional level. More than half of the players had experienced LBP in their lives. One-way ANOVA revealed that the players with LBP were taller, heavier and with a higher BMI (all p values $<0.00001$ ). When considering the playing position, ANOVA revealed that 14-15 years-old strikers displayed higher LBP scores than all other roles (all $\mathrm{p}$ values $<0.05$ ). Accordingly, strikers were exposed to a higher risk of LBP than midfielders ( $R R=1.48 ; 95 \% \mathrm{CI}: 1.10-2.01 ; \mathrm{p}=0.01)$ and goalkeepers $(\mathrm{RR}=1.48 ; 95 \% \mathrm{CI}: 1.02-2.971 ; \mathrm{p}=0.04)$, but not defenders $(\mathrm{RR}=$ 1.23 ; 95\%CI:0.93-1.63; $\mathrm{p}=0.15)$. Within the 14-15 age-class, strikers were, again, those most exposed to LBP risk (all p values < 0.05).

Conclusions: Anthropometric and soccer-related features should be monitored to ensure early identification of potential risk factors for LBP. This information should be considered along with the specific playing position as strikers emerged as the roles most exposed to LBP risk.

Acknowledgements. Fondo di Ateneo per la ricerca 2020. Moreover, Authors gratefully thank the football clubs Cagliari Calcio, Sigma
Cagliari, ASD Futura Calcio Sales, Scuola Calcio FSD Santos and ASD Atletico Cagliari for their support in participating in this study. References

1. Tunås $P$ et al. (2015) Low back pain in female elite football and handball players compared with an active control group. Knee Surg Sports Traumatol Arthrosc 23(9):2540-7.

2. De Luigi AJ. (2014) Low back pain in the adolescent athlete. Phys Med Rehabil Clin N Am 25(4):763-788.

3. Moradi V et al. (2015) Low back pain in athletes is associated with general and sport specific risk factors: a comprehensive review of longitudinal studies. Rehabil Res Pract 2015:850,184.

\section{3}

May a cross-post activation performance enhancement (pape) exhist between upper and lower body in trained men?

$\frac{\text { Riccardo De Luca }}{\text { Bartolomei }^{2}}$,Enrico Dalolio ${ }^{1}$, Mario Morellina ${ }^{1}$, Sandro

University of Bologna, Deparment For Life Quality Studies, Bologna, Italy $^{1}$;

University of Bologna, Department of Biomedical and Neuromotor Sciences, Bologna, Italy ${ }^{2}$

Purpose: Acute performance enhancements have been associated with conditioning activities (Seitz and Haff, 2017). The aim of this study was to establish if a resistance exercise for the upper body may generate a post activation performance enhnancement (PAPE) in the lower body.

Methods: Thirteen resistance trained men (age $=26.5 \pm 3.4 \mathrm{y}$; body mass $=77.9 \pm 6.8 \mathrm{~kg}$; height $=176.6 .1 \pm 4.9 \mathrm{~cm}$ ) participated in the present investigation. Participants were tested for upper and lower body power [bench press throw (BPT) and countermovement jump power (CMJP) tests]. Participants were also tested for maximum force and electromyographic activation (EMG) of quadriceps muscles at isometric leg extension. All the assessments were performed pre and 8-min post a high intensity bench press protocol (HI: 5 sets of 1 reps at $90 \%$ of 1RM), a high power protocol (POW: 5 sets of 1 rep at $30 \%$ of 1RM with maximum explosive intent) and a control trial (CO). Participants performed all the trials in randomized order and in different days.

Results: A significant time $\mathrm{x}$ trial interaction was detected for CMJP $(p=0.049)$. This parameter was significantly increased following the HI protocol only $(p=0.025)$. A significant interaction was also noted for EMG with a significant improvement following the HI protocol ( $\mathrm{p}$ $=0.032)$ and a significant decrese post the POW protocol $(\mathrm{p}=0.020)$. No other significant effects were detected $(\mathrm{p}>0.05)$.

Conclusions: Results of this investigation indicate that a HI bench press protocol may produce a PAPE in the lower body power and increase the neuromuscular activation of leg extensor muscles. The POW bench press protocol did not show any positive effects on lower body performance. Interestingly enough, no performance enhancements were detected in the upper body following the HI protocol.

\section{References}

1. Seitz LB, Haff GG. (2016) Factors Modulating Post-Activation Potentiation of Jump, Sprint, Throw, and Upper-Body Ballistic Performances: A Systematic Review with Meta-Analysis. Sports Med 46(2):231-40. 
164

\section{Natural mineral spring waters for different sport performances}

\author{
$\underline{\text { Valentina Presta }}^{\text {, Luca Ambrosini }}{ }^{1}$ \\ Università Di Parma, Dipartimento Di Medicina E Chirurgia, Parma, \\ Italy $^{1}$
}

Purpose: Scarce scientific attention has been dedicated to the mineral composition of natural spring waters (NMSWs) as related to the average hydro-saline requirements of the different athletic performances. We asked if there was sufficient scientific data to predict that the biological properties of specific NMSWs could optimally respond to the physiological needs of athletes in relation to their specific sport activity and performance.

Methods: We reviewed the existing literature to explore the categories of NMSWs, matching mineral compositions, athletic performances, and hydro-saline requirements, trying to assign to each an at least theoretical sport-specificity.

Results: NMSWs are a natural source of water-dissolved minerals that can optimize both pre- and rehydration needs of athletes. Magnesium waters optimize anaerobic performances (short-duration events), favouring muscle strength and power in response to load effort; bicarbonate and/or alkaline waters optimize endurance sports (running, cycling, long-distance and long-duration events, etc.), where the performance is strongly dependent on efficient control of $\mathrm{pH}$ levels and fatigue. The presence of calcium ions in both magnesium and bicarbonate waters favours muscle contraction, while sodium content should be accurately controlled, according to the individual needs.

Conclusions: Although this topic deserves a higher scientific attention, available literature supports the idea that specifically chosen NMSWs can integrate athlete's diet replenishing sport-related fluid and electrolyte deficits.

\section{References}

1. Brancaccio, P, Limongelli, FM, Paolillo, I, D'Aponte, A, Donnarumma, V, and Rastrelli, L. (2012) Supplementation of Acqua Lete ${ }^{\circledR}$ (Bicarbonate Calcic Mineral Water) improves hydration status in athletes after short term anaerobic exercise. J Int Soc Sports Nutr 9(1): 35

2. Chycki, J, Kurylas, A, Maszczyk, A, Golas, A, and Zajac, A. (2018) Alkaline water improves exercise-induced metabolic acidosis and enhances anaerobic exercise performance in combat sport athletes. PloS one 13(11):e0205708.

\section{5}

\section{On the use of wearable sensors for football player performance evaluation}

\author{
Jury Taborri ${ }^{1}$, Eduardo Palermo ${ }^{2}$, Ludovica Cardinali ${ }^{3}$, Luigi \\ Febbrari $^{4}$, Fabrizio Perroni ${ }^{5}$, Stefano Rossi ${ }^{1}$ \\ University of Tuscia, Department of Economics, Engineering, Society \\ and Business Organization, Viterbo, Italy ${ }^{1}$; \\ University Sapienza of Rome, Department of Mechanical \\ and Aerospace Engineering, Rome, Italy ${ }^{2}$; \\ University of Rome "foro Italico", Department of Movement, Human \\ and Health Sciences, Rome, Italy; \\ Football Association of Albania, Performance Department, Strength \\ and Conditioning Coaches, Tirana, Albania ${ }^{4}$;
}

University of Urbino Carlo Bo, Department of Biomolecular Sciences, Section of Exercise and Health Sciences, Urbino, Italy ${ }^{5}$

Purpose: the quantitative evaluation of motor performance in athlete through wearable sensors reached a worldwide popularity $[1,2]$. This study aims at proposing an experimental protocol for the evaluation of both kinematics and muscle activation during a typical soccer team coaching session.

Methods: One professional soccer player of the Italyn major league was enrolled in the study. The player was sensorized with four inertial sensors and eight surface electromyography probes to gather the knee mobility and the lower limb muscle activation during running, sprinting, and jumping tasks. Jumping task was performed both in monopodalic and bipodalic mode. Evaluated variables included the knee flexion/extension angles, the activation time (ta) and the average rectified value (ARV) of rectus femoris, biceps femoris, tibialis anterior and gastrocnemius. Symmetry Index between two lower limb sides was computed for each variable [3].

Results: Knee angles are in line with the physiological value and no asymmetries were found in all the performed tasks. Asymmetry was instead evident between the right and left biceps femoris in running, with $\mathrm{ARV}$ equal to $145 \mu \mathrm{V}$ and $40 \mu \mathrm{V}$ for right and left side, respectively. Such difference was less evident in sprinting. For jumping task, a different behaviour was found for the rectus femoris of the two sides; the right one is more activated during the jump preparation and the left one during the flight time. In average, the ARV of lower limb muscle was higher in right monopodalic jump, allowing to strengthen the hypothesis of muscle asymmetry.

Conclusions The findings of the present abstract can open the possibility to introduce low-cost and non-invasive sensors-based assessment session during typical coaching program to quantitatively measure the performance of football players.

\section{References}

1. Camomilla, V, Bergamini, E, Fantozzi, S, Vannozzi, G (2015) Infield use of wearable magneto-inertial sensors for sports performance evaluation. In Proceedings of the 33rd International Conference on Biomechanics in Sports; pp. 1425-1428.

2. Taborri, J, Palermo, E, Rossi, S (2019) Automatic detection of faults in race walking: A comparative analysis of machinelearning algorithms fed with inertial sensor data. Sensors; 19(6): 1461

166

\section{Optimizing endurance performance by appropriate non-sport-specific strength training: a systematic review and meta-analysis}

\author{
Luca Ambrosini ${ }^{1}$, Valentina Presta ${ }^{1}$ \\ Università Di Parma, Dipartimento Medicina E Chirurgia, Parma, \\ Italy $^{1}$
}

Purpose: Non-sport-specific strength training (NST) is a way to increase endurance performance. However, exercise characteristics (maximal, plyometric, explosive or resistance strength training) that give the best Results are still under debate. We therefore metaanalysed the literature data to assess which type of NST intervention is more efficient in improving the performance in four endurance sports, i.e. Running, Cycling, Triathlon and Cross-Country Skiing.

Methods: Scientific publications were analysed according to the PRISMA Checklist and statement. The initial search yielded 500 studies, 17 of which were included in this review using the PEDro Scale. 
Results: Maximal strength training boosted the expression of strength particularly in cross-country skiing and cycling, increasing endurance performance measured as a decrease of the Time Trial or Time Trial to Exhaustion. In running, explosive strength training did not yeld advantages, whereas plyometric strength training led to improvements of Time Trial or Time Trial to Exhaustion and Work Economy.

Conclusions: In running it was possible to compare different types of NST and the plyometric strength training resulted the best methodology to enhance the endurance performance. Studies on the other sports only investigated the effects of maximal strength training that resulted more effective in cross-country skiing and in the cycling component of triathlon. On the contrary, maximal strength training induced modest effects on cyclists performance as compared to other athletes, suggesting that a different type of NST would probably be more effective, similarly to what was observed in runners.

In conclusion, each sport might optimize performance by using appropriate NST, which, however, should be studied individually.

\section{References}

1. Lum, D, Barbosa, TM. (2019) Effects of Strength Training on Olympic Time-Based Sport Performance: A Systematic Review and Meta-Analysis of Randomized Controlled Trials. Int J Sports Physiol Perform. 1-13.

2. Beattie, K, Kenny, IC, Lyons, M, and Carson, BP. (2014) The effect of strength training on performance in endurance athletes. Sports Medicine. 44(6), 845-865.

\section{7}

\section{Physical and sport activity in italyn scientific research products}

\author{
Gaetano Raiola ${ }^{1}$, Francesca D'elia ${ }^{2}$
}

Università, Università Di Salerno Dipartimento Di Studi Politi E Sociali, Fisciano Salerno, Italy ${ }^{l}$;

Università, Università Di Salerno Dipartimento Di Scienze Umane Filosofiche E Della Formazione, Fisciano Salerno, Italy ${ }^{2}$

Purpose: was to measure the scientific production of two M-EDF academic disciplines researchers relating to recruitment sectors according to scientific field: Physical and sport activity (PSA), Biomedical and Psycho-pedagogical domains.

Methods. The sample was made up of the entire population of full professors (30) plus a proportional representation of the 2 recruitment sectors ( 7 times more for 06/N2 than 11D2) of associate professors with at least one per university in M-EDF/01 or M-EDF/02 for a total of 78 researchers. For each unit of the sample, the titles of the 10 products with multiple citations in the Google Scholar database were analyzed and the full consistency of the title with the 2 scientificdisciplinary statements was assessed. ANOVA one-way was used between the 2 academic disciplines of the 2 recruitment sectors with the 3 domains to highlight the greater, average and lesser significance of the separate data.

Results. Mean and SD of 3 domains was: biomedical $=5.92 \pm 3.79$; $\mathrm{PSA}=3.38 \pm 3.30$ and psycho-pedagogical $=0.66 \pm 1.60 . \mathrm{M}-\mathrm{EDF} /$ 01 in 06/N2 had a significant difference only between biomedical and PSA and between biomedical and psycho-pedagogical $(p=0.000)$. $\mathrm{M}-\mathrm{EDF} / 02$ in 06/N2 had a significant difference only between biomedical and psycho-pedagogical and between PSA and psychopedagogical $(p=0.000)$ while it had no significant difference between biomedical and PSA ( $\mathrm{p}=0.063)$. M-EDF/01 in 11/D2 had a significant difference only between biomedical and PSA $(p=0.001)$. $\mathrm{M}-\mathrm{EDF} / 02$ in 11/D2 had a significant difference between biomedical and PSA $(\mathrm{p}=0.000)$ and between PSA and psycho-pedagogical $(\mathrm{p}=$ $0.000)$.

Discussion. The aggregate data showed a prevalence of the biomedical scientific products over the PSA one and a modest psychopedagogical scientific products in 06/N2 and biomedical in 11/D2 . The disaggregated data was the following: a) M-EDF01 in 06/N2 had a significant difference in favor of biomedical and none between PSA and Psycho-pedagogical; b) M-EDF02 in 06/N2 had only a significant difference between physical and sport activity and psycho-pedagogical in favor of PSA; c) MEDF/01 in 11/D2 had a significant difference between biomedical and PSA in favor of the latter, while between PSA and psycho-pedagogical there were no significant difference despite being PSA prevalence; d) MEDF/02 in 11/D2 had a significant difference between Biomedical and PSA, the same occurs between PSA and psycho-pedagogical.

\section{References}

1. Raiola, G. (2020) The Movement and Sport Science in Italy towards the European Research Council, Physical Culture and Sport, Studies and Research,, 86 (1).

2. Raiola, G. (2019) Complex study for an epistemology of exercise and sport sciences: A)keyconceptsofboth ERC subpanels and CUN keywords; b) physical training and sport methodology sciences academic disciplines in pedagogy recruitment sector and biomedical one: A correlationships study, Journal of Physical Education and Sport,, 19,

\section{8}

\section{Physical fitness changes during the COVID-19 lockdown in youth soccer players}

\section{Pasquale Salerno ${ }^{I}$, Cosme Franklim Buzzachera ${ }^{I}$, Luca Correale ${ }^{l}$, Mirko Bianchi ${ }^{I}$, Carlo Zanetti ${ }^{I}$}

Department of Public Health, Experimental and Forensic Medicine, University of Pavia, Pavia, Italy

Purpose: Since February 2020, multiple mitigation and containment measures were adopted to avoid spreading the COVID-19 virus in Italy. Such restrictive measures were, at least in part, harmful for many athletes, including soccer players, who stopped their training and competition activities for a relatively long period $(1,2)$. The physical and mental consequences of this adverse scenario are still uncertain, and therefore, the current study aimed to describe the physical fitness changes during the COVID-19 lockdown in young soccer players.

Materials and Methods: Fourteen-four soccer players aged 16-17 years of FIGC U17 football teams from Pavia (PV) were recruited. Each athlete was assessed before and after the regional lockdown, which lasted approximately six weeks. In each testing session, athletes performed a battery of tests, including a 30-m linear sprint, a horizontal jump (HJ), and a six-minute walking test (6MWT).

Statistical analysis: Data are expressed as mean and standard deviation. Paired t-tests were used to examine pre- vs. post-lockdown changes in physical fitness. Statistical significance was set as $\mathrm{P}<$ 0.05. Effect sizes were calculated using Cohen's D principle, with $90 \%$ confidence interval, and categorized according to the following thresholds: $<0.2$ trivial, 0.6 small, 1.2 moderate, 2.0 large, and $>2.0$ very large.

Results: Table 1 and Graph 1 summarize the main findings. In brief, athletes showed, compared to pre-lockdown, an impaired performance in all the selected tests: 6MWT $(\mathrm{p}<0.05$ Cohen's $\mathrm{d}=-2.5$, - 
3.38 to -1.56$)$, HJ ( $p<0.05$ Cohen's $d=-1.54,-2.18$ to -0.86 ), and $30-\mathrm{m}$ linear sprint ( $\mathrm{p}<0.05$, Cohen's $\mathrm{d}=-1.02,-1.55$ to -0.45 ).

Conclusions: This study revealed that restrictive measures during the COVID-19 pandemic caused a significant decrease in physical fitness in young soccer players. These findings would be helpful for coaches and physical trainers and reinforce the extreme attention to avoid physical fitness decrements during relatively long periods of lockdown.

1.Mon-López, D., García-Aliaga, A., Bartolomé, A. G., \& Solana, D. M. (2020). How has COVID-19 modified training and mood in professional and non-professional football players? Physiology \& behavior, 227, 113,148.

2.Salazar H., Svillar H., Garcia L., Gonzalez-Lago L. Castellano J. (2020). Negative Impact of COVID-19 Home Confinement On Physical Performance of Elite Youth Basketball Players. SPSR I 110 | v1.

\section{9}

Analysis of external load indicators in official matches and sport-specific training of semi-professional soccer players: focus on intensity and strength

Guglielmo Pillitteri $^{1}$, Valerio Giustino ${ }^{1}$, Marco Petrucci ${ }^{2}$, Luca Petrigna $^{I}$, Fatma Nese Sahin ${ }^{3}$, Marianna Bellafiore ${ }^{I}$, Angelo Iovane ${ }^{I}$, Ewan Thomas ${ }^{l}$, Antonino Bianco ${ }^{1}$, Antonio Palermo ${ }^{1}$, Giuseppe Battaglia $^{1}$

Sport and Exercise Sciences Research Unit, Università Degli Studi Di Palermo/department of Psychology, Educational Science and Human Movement, Palermo, Italy ${ }^{1}$;

Palermo Football Club, Fc Palermo, Palermo, Italy ${ }^{2}$;

Faculty of Sport Sciences, Ankara University/ Department of Sport and Health, Faculty of Sport Sciences, Ankara, Turchia ${ }^{3}$

Purpose: In soccer, the use of Global Positioning System (GPS) devices for monitoring external load indicators (Eli), both in official matches and training, is widely adopted to improve sport performance and reduce injuries. Hence, the aim of this study was to investigate the differences in external load indicators between Official Matches (OM) and sport-specific tasks, in semi-professional soccer players.

Methods: A number of 2186 observations among 21 semi-professional soccer players (FC Palermo, Italy, age: $25 \pm 6$ years, height: $183 \pm 6 \mathrm{~cm}$, weight: 75,2 $\pm 7 \mathrm{~kg}$ ) was collected through eight GPS devices (Qstarz BT-Q1000EX, $10 \mathrm{~Hz}$ ) and the related software (LaGalaColli V: 8.6.4.3), during the sports season 2019-2020. Participants were monitored during $\mathrm{OM}$ and the following three sportspecific tasks including: Friendly Matches (FM); Small Sided Games (SSG), and Match-Based Exercises (MBE). ELi considered for this study were: percentage of intense accelerations (\%int acc); percentage of intense decelerations (\%int dec) for sport-specific strength and Passive Recovery time / $\mathrm{min}(\mathrm{PrT} / \mathrm{m})$ as an indicator of sport-specific intensity. A one-way ANOVA was performed to detect any differences between the four tasks for each indicator. A Scheffe post-hoc test was subsequently carried out for testing multiple comparisons.

Results: Our results showed significant differences between OM and sport-specific tasks for all the ELi considered $(\mathrm{F}(3,2182)=279.28$, p $<0.001 ;(\mathrm{F}(3,2182)=627.3, \mathrm{p}<0.001 ; \mathrm{F}(3,2182)=138.96, \mathrm{p}<$ 0.001 , for \%int acc, \%int dec, PrT/m, respectively). The post-hoc multiple comparisons revealed the same significant difference for all the ELi $(p<0.001)$ between all tasks except for the comparison of the PrT/m between MBE and FM $(\mathrm{p}<0.01)$. In detail, as concern PrT/m we found the highest mean value in OM and the lowest in MBE (18.36 and 12.90 ( $\mathrm{s} / \mathrm{min})$, respectively). Conversely, regarding \%int acc and \%int dec the lowest mean values were detected in $\mathrm{OM}$ and the highest mean values in SSG (8.64 vs 13.02 and 9.25 vs 15.68 , for \%int acc and \%int dec, respectively).

Conclusions: We found that, in semi-professional soccer players, ELi are in accordance with the performance model by achieving better values in training than OM. Our outcomes suggest that the use of GPS is fundamental for monitoring external load in sport-specific training in soccer.

\section{References}

1. Akenhead R, \& Nassis GP. (2016) Training Load and Player Monitoring in High-Level Football: Current Practice and Perceptions. Int J Sports Physiol Perform 11: 587-593

2. Brito Â, Roriz P, Duarte R \& Garganta J. (2018) Match-running performance of young soccer players in different game formats. International Journal of Performance Analysis in Sport 18: 410-422

3. Varley MC, Fairweather IH, Aughey RJ. (2012) Validity and reliability of GPS for measuring instantaneous velocity during acceleration, deceler

\section{0}

Power-load relationship of bench press, ballistic bench press, and prone bench pull in italyn international canoeist and kayakers

Matteo Fortunati ${ }^{1}$, Tiziano Gemelli ${ }^{2}$, Mariangela Valentina Puci, Patrik Drid ${ }^{4}$, Julian Andrew Owen ${ }^{5}$, Antonino Bianco ${ }^{6}$, Giuseppe $D^{\prime}$ antona $^{3}$, Stefano Ramat ${ }^{7}$

University of Pavia, University of Pavia, Department of Sport and Exercise Science, Pavia, Italy ${ }^{1}$;

University of Pavia, Department of Sport and Exercise Science, Pavia, Italy ${ }^{2}$;

University of Pavia, Unit of Biostatistics and Clinical Epidemiology, Department of Public Health, Experimental and Forensic Medicine, Pavia, Italy ${ }^{3}$;

University of Novi Sad, Faculty of Sport and Physical Education, Novi Sad, Serbia ${ }^{4}$;

Bangor University, School of Sport, Health and Exercise Sciences, Bangor, Regno Unito ${ }^{5}$;

University of Palermo, Department of Psychology, Educational

Science and Human Movement, Palermo, Italy ${ }^{6}$;

University of Pavia, Department of Electrical, Computer and Biomedical Engineering, University of Pavia, Pavia, Italy ${ }^{7}$

Purpose: Athletes of kayak and canoe must possess a high level of strength, endurance and power in the upper body. The aim of our research was to describe Pavg in the guided eccentric-concentric bench press (BP), guided eccentric-concentric ballistic bench press (BBP) and guided concentric-only prone bench pull (PBP) between 20 and $90 \%$ one-repetition maximum (\% 1RM) in international canoeing and kayaking athletes.

Methods: Nine international athletes were monitored during two complementary sessions ( $48 \mathrm{~h}$ apart) of an incremental testing protocol with a $5 \mathrm{~kg}$ increase between the absolute load of $30 \mathrm{~kg}$ to $100 \mathrm{~kg}$ on the BP, $15 \mathrm{~kg}$ to $60 \mathrm{~kg}$ on the BBP, and $30 \mathrm{~kg}$ to $95 \mathrm{~kg}$ on the PBP. Instantaneous displacement was measured using a linear position transducer and average velocity and power were computed. One repetition maximum (1RM) was calculated indirectly through the load-velocity relationship in the BP and PBP with a minimum velocity of $0,15 \mathrm{~m} / \mathrm{s}$ and $0,45 \mathrm{~m} / \mathrm{s}$, respectively. The $1 \mathrm{RM}$ for the BBP press was set at $80 \%$ of the maximum weight lifted in the BP. Then, from the individual load-velocity equations the weight $[\mathrm{kg}]$ 
from 20 to $90 \% 1 \mathrm{RM}$ at each $10 \%$ interval was obtained and, subsequently, the mean power output was calculated using the individual power-load polynomial equation at the new standardized absolute load. A two-way repeated measures ANOVA was used to explore differences among the different exercises and relative load from 20 to 90\% 1RM.

Results: PBP displayed a higher average power output (Pavg) from 40 to $90 \% 1 \mathrm{RM}$ compared to BP and BBP ( $p<0,0001$ ), while no statistical difference was found between the latter at any load percentage ( $\mathrm{p}>0,05)$. In addition, the $\% 1 \mathrm{RM}$ that maximized Pavg was, respectively, $60 \% 1 \mathrm{RM}$ for $\mathrm{PBP}, 50 \% 1 \mathrm{RM}$ for BBP and $40 \%$ for BP. Post hoc analysis showed that the relative load at the maximum Pavg was not statistically different $(\mathrm{p}>0,05)$ from 40 to $80 \% 1 \mathrm{RM}$ in the $\mathrm{PBP}$, from 40 to $60 \% 1 \mathrm{RM}$ in the BBP, and from 20 to $60 \%$ in the BP. Conclusions: The main finding of our study is that in professional canoeists and kayakers the power-load relationship differs between $\mathrm{PBP}, \mathrm{BP}$ and $\mathrm{BBP}$ exercises, with the highest average power output monitored in the PBP in comparison with both, BP and BBP. Our study agrees with the one carried out by Sánchez-Medina et al., using a strength-trained population.

\section{References}

1. Mckenzie D. \& Berglund B. Handbook of Sport Medicine and Science: Canoeing. 1st ed. International Olympic Committee, editor. Oxford, UK: WILEY Blackwell, 2019.

2. Jovanovic, M, Flanagan, EP. Researched applications of velocitybased strength training. J Aust Strength Cond 22: 58-69, 2014.

3. Sánchez-Medina, L, González-Badillo, JJ, Pérez, CE, Pallarés, JG. Velocity- and power-load relationships of the bench pull vs bench press exercises. Int J Sports Med 35: 209-216, 2014.

\section{1}

\section{Relationship between muscle soreness and training load in a beach handball goalkeeper: a case study}

\author{
Alice Iannaccone $^{1}$, Andrea Fusco ${ }^{1}$, Valerio Viggiano ${ }^{1}$, Daniele \\ Conte $^{2}$, Cristina Cortis ${ }^{1}$ \\ Università Degli Studi Di Cassino E Del Lazio Meridionale, \\ Dipartimento Di Scienze Umane, Sociali E Della Salute, Cassino, \\ Italy $^{1}$; \\ Lithuanian Sports University, Institute of Sport Science \\ and Innovations, Kaunas, Lituania ${ }^{2}$
}

Purpose: To assess the relationship between the subjective perception of muscle soreness and external and internal load in a youth male beach handball goalkeeper.

Methods: One youth male goalkeeper (age: $16 \mathrm{y}$; height: $181 \mathrm{~cm}$; weight: $76 \mathrm{~kg}$ ) of the U17 Lithuanian National Beach Handball team was monitored during 14 training sessions. Muscle soreness was obtained by administering the Wellbeing scale 1 and taking into account only the item relative to the muscle soreness. External load was monitored by means of inertial movement units. Internal load was objectively assessed using the summated heart rate zones 2 whereas the subjective internal training load was assessed using the session rating of perceived exertion3. The Pearson correlation coefficient (r) was calculated to assess the relationship between muscle soreness and external and internal load, respectively.

Results: A large negative $(r=-0.51)$ relationship was found between the subjective internal load and muscle soreness, whereas a moderate negative $(r=-0.39)$ relationship was found between the objective internal load and muscle soreness. For the relationship between the external load and muscle soreness a trivial negative $(r=-0.04)$ correlation coefficient was found.
Conclusions: The present results show that muscle soreness is most strongly related with the subjective internal load. This relationship might be related to the peculiarity of the role of the goalkeeper, characterized by fewer movements than field players4. Therefore, in the case of beach handball goalkeepers, subjective internal load monitoring may be more useful than the external workload, with the aim to monitoring players' response to training stimuli and being able to understand excessive player fatigue and be able to act to prevent injuries or overtraining.

\section{References}

1. McLean et al. (2010) Neuromuscular, endocrine, and perceptual fatigue responses during different length between-match microcycles in professional rugby league players. Int J Sports Physiol Perform 5:367-383.

2. Edwards. The Heart Rate Monitor Book. 1993.

3. Foster et al. (2001) A new approach to monitoring exercise training. J Strength Cond Res 15:109-115.

4. Iannaccone et al. (2021) Relationship between external and internal load measures in youth beach handball. Int J Sports Physiol Perform.

\section{2}

\section{Serve quality analysis in tennis men at roland garros from 2003 to 2019}

\author{
Claudio Lazzaretto ${ }^{I}$, Matteo Bonato ${ }^{l}$, Carlo Rossi ${ }^{l}$ \\ Università Degli Studi Di Milano, Department of Biomedical \\ Sciences For Health, Milan, Italy ${ }^{1}$
}

Purpose: In last decades serve has become one of the most important shots of elite tennis and use of new materials and training Methods contributed to make it faster and powerful. Nowadays, the role of tennis serve becomes fundamental during match because it allowed to gain points with no or very short rallies. Therefore, the primary aim of this study was to investigate the tennis serve related parameters during the Roland Garros tournament and to compare them with the Wimbledon tournament.

Methods: The data collected refer to 17 years; in these 17 years $n=$ 2159 were played, of which $n=1632$ in the first week and $n=527$ in the second week. For each year and each group we evaluated two macro-categories of topics. The first refers in particular to the success of the serve, while the second to the performance of the same fundamental.

Results: Over the years we graphically witness a common trend of the various parameters analyzed, which consists of a rapid increase in the first years, followed by a stabilization of the same. As regards the comparison between the two weeks, highly significant $(\mathrm{p}<0.01)$ and on average significant $(\mathrm{p}<0.05)$ changes in parameters can be noted. Conclusions: The Results confirmed that serve is the most incisive shot of modern tennis on all surfaces, both at Roland Garros and both at Wimbledon.

\section{References}

1. Serve efficiency development at Wimbledon between 2002 and 2015: a longitudinal approach to impact tomorrow's tennis practice, Ralph Grambow, Craig O'Shannessy, Philipp Born, Dominik.

2. Meffert, Tobias Vogt. 
173

\section{The effects of bergamot on high sensitivity c-reactive protein and oxidized ldl in professional athletes during endurance training}

\author{
Marco Alfonso Perrone ${ }^{1}$, Bruno Donatucci ${ }^{2}$, Antonino De Lorenzo ${ }^{3}$, \\ Maurizio Volterrani ${ }^{4}$, Attilio Parisi ${ }^{5}$, Ferdinando Iellamo $^{I}$ \\ Dipartimento Di Scienze Cliniche E Medicina Traslazionale, \\ Università Di Roma Tor Vergata, Rome, Italy ${ }^{1}$; \\ Dipartimento Di Scienze Cliniche E Medicina Traslazionale, \\ Università Tor Vergata, Rome, Italy ${ }^{2}$; \\ Dipartimento Di Biomedicina E Prevenzione, Università Di Roma \\ Tor Vergata, Rome, Italy ${ }^{3}$; \\ Dipartimento Di Promozione Delle Scienze Umane E Della Qualità \\ Della Vita, Università Telematica San Raffaele, Rome, Italy ${ }^{4}$; \\ Dipartimento Di Scienze Motorie, Umane E Della Salute, Università \\ Di Roma Foro Italico, Rome, Italy ${ }^{5}$
}

Purpose: many studies have shown that endurance sports athletes have a biochemical profile of inflammation and high oxidative stress, due to the high load of training. On the other hand recent studies have shown that bergamot, in addition to the already known lipid-lowering properties, also has anti-inflammatory and antioxidant properties. The aim of the study was to determine the effects of bergamot juice on biomarkers of inflammation and oxidative stress in professional athletes who practice cross-country skiing.

Methods: 10 international-level cross-country skiing male athletes (group I) were enrolled in the study. As a control group, we enrolled 10 national-level cross-country skiing male athletes (group II). We performed a blood sample during the unloading phase of the athletic training (T0) and dosed high sensitivity C-reactive protein (hsCRP) and oxidized low-density lipoprotein (oxLDL). The internationallevel athletes took $500 \mathrm{ml} /$ day of bergamot juice, while controls did not. Afterwards, all the athletes started the training load period and after 30 days of intense training (T1) the venous sample was repeated to assess hsCRP and oxLDL. The two groups had the same training schedule but athletes of group I sustained a significantly higher training load than athletes of group II $(16,732,6 \pm 1729,3$ vs $10,756,4$ $\pm 1102,3$ METs per week respectively, $\mathrm{p}<0.05$ ).

Results: In group I there was a significant reduction in hsCRP ( $p<$ 0.05 ), while there was no significant increase in oxLDL despite the greater training load. In group II, on the other hand, there was no significant change in hsCPR levels between $\mathrm{T} 0$ and $\mathrm{T} 1$, while there was a significant increase in oxLDL $(\mathrm{p}<0.05)$.

Conclusions: Our data showed that bergamot juice in professional cross-country skiing athletes could reduce inflammation and oxidative stress due to intense training. More studies are needed to understand better the protective role of bergamot in high-level skiing athletes and in athlete's populations of different sport disciplines.

\section{4}

\section{The effects of physical training on cortisol and testosterone concentrations and physical performance in female football referees}

\author{
G. My ${ }^{1}$, Antonino Bianco ${ }^{2}, \underline{A P a l m a}^{2}$, S. Marsigliante ${ }^{1}$, Antonella \\ Muscella ${ }^{l}$
}

Department of Biological and Environmental Science and Technologies (di.s.te.b.a.), University of Salento, Lecce-italy ${ }^{1}$; Department of Psychology, Educational Science and Human
Movement, Sport and Exercise Sciences Research Unit, University of Palermo, Palermo-italy ${ }^{2}$

Although female and male youth athletes are roughly equal, a significant disparity exists in authoritative roles. Female referees are almost nonexistent at the professional level and exceedingly scarce at the local and national levels. Both male and female football referees are subjected to physical and psychological stress due to complex decision-making by dealing with players, coaches and audiences. Physical training affects the state of the hypothalamic-pituitary-gonadal axis hormones in both men and women (Hackney et al., 2003); this is particularly true for high intensity and physically demanding sports (Aldous et al., 2016; Muscella et al., 2021).

Purpose: This study aimed to determine the effects of the football season period on plasma cortisol and testosterone concentrations and female referee's physical performances.

Methods: The changes in physical parameters: $40 \mathrm{~m}$ Sprint test (s), Yo-Yo intermittent recovery tests (YYIRT1, m), and VO2max $(\mathrm{ml} \cdot \mathrm{min}-1 \cdot \mathrm{Kg}-1)$ and plasma cortisol and testosterone concentrations were evaluated at four different time points: before the beginning of the training period (T0), at the start of the pre-season (T1), at the middle of the season (T2), and the end of the season (T3), in 14 female football referees.

Results showed significant differences in hormone concentrations at the four-time points evaluated. Plasma cortisol increased during the first training period from $15.4 \pm 4.7 \mu \mathrm{g} / \mathrm{dl}$ to $20.7 \pm 6.1 \mu \mathrm{g} / \mathrm{dl}(\mathrm{p}<$ 0.01 ); then it strongly increased during mid-season (T2: $28.5 \pm$ $3.9 \mu \mathrm{g} / \mathrm{dl}, \mathrm{p}<0.001)$ and decreased at the end of the season to $16.0 \pm$ $2.4 \mu \mathrm{g} / \mathrm{dl}$.

Before the beginning of the training period, plasma testosterone concentration was $14.2 \pm 0.37 \mu \mathrm{g} / \mathrm{dl}$. Then testosterone levels increased to $57.1 \pm 3.7 \mu \mathrm{g} / \mathrm{dl}$ in $\mathrm{T} 1$ and then $47 \pm 3.7 \mu \mathrm{g} / \mathrm{dl}$, in $\mathrm{T} 2$ whilst at the end of the season, the concentration decreased to $33.5 \pm$ $2.8 \mu \mathrm{g} / \mathrm{dl}$. Thus, between the start of the training period and the end of the season, significant differences were observed in the physical performances of female referees.

Our Results showed that performance improved. Indeed, the training sessions to which the football referees were subjected resulted in good aerobic and anaerobic fitness levels, also in women. Thus, female referees possessing the required skills are potentially eligible to officiate male matches that are supposed to impose higher external loads than female competitions.

Finally, further studies including a larger population of female referees.

1. Aldous JW, Chrismas BC, Akubat I, Taylor L (2016) Hot and hypoxic environments inhibit simulated soccer performance and exacerbate performance decrements when combined. Front Physiol 6:421-427

2. Hammami MA, Kebsi W, Nebigh A, Zouhal H (2017) Hormonal (cortical-gonadotropic axis) and physical changes with 2 years intense exercise training in elite young soccer players. J Strength Cond Res 31:2388-2397

3. Muscella A, Stefàno E, Marsigliante S. (2021) The effects of training on hormonal concentratio.

175

The evolution of the 3-point shot distribution in the NBA

Samuele Paoli ${ }^{1}$, Stefano Bonnini ${ }^{2}$, Luigi Salmaso ${ }^{3}$

University of Padua, Department of Statistical Sciences, Padova, Italy $^{1}$; 
University of Ferrara, Department of Economics and Management, Ferrara, Italy ${ }^{2}$;

University of Padua, Department of Management and Engineering, Padova, Italy ${ }^{3}$

Purpose: The National Basketball Association (NBA) is the main basketball league of the United States of America and Canada, moreover, is one of the most popular basketball leagues around the world. The introduction of the 3-point (3PT) line (1979-1980) was a major change in NBA rules. The aim of the present study was to investigate how the popularity of the 3 point-shot has evolved through the years.

Methods: the 20 teams that have participated every year in the NBA from 1980 to 2019 were considered for the analysis and the following statistics from the open access NBA basketball references were taken into account: the proportion of 3 point-shot attempted over the field goal attempted (3PTA/FGA), the 3PT\% and the 2PT\%. To verify the main assumption between two consecutive years, for all the years and statistics, a multivariate permutation test, with even graphic results was employed.

Results: The graphic results showed a steady increase for the 3PTM/ 3PTA with an exponential increase in the last 10 years. A logarithmic trend for the 3PT\% and an approximately steady increased until 2010 for the $2 \mathrm{PT} \%$. The results showed that, not including the seasons in which the line was moved closer to the basket, the biggest difference of 3PTA/FGA in consecutive years was in the 2015-2016.

Conclusions: Nowadays, the NBA teams and players mostly try to get a 3 point-shot or a layup over a mid-range shot. The shot pattern did not change suddenly but is being constantly evolving.

\section{6}

\section{The relative age effect among elite female Italyn volleyball, basketball, and soccer players}

\author{
Alexandru Nicolae Ungureanu ${ }^{1}$, Corrado Lupo ${ }^{1}$, Paolo De \\ Pasquale $^{I}$, Gennaro Boccia ${ }^{2}$, Paolo Riccardo Brustio ${ }^{3}$ \\ University of Turin, Neuromuscularfunction Research Group, \\ Department of Medical Sciences, Turin, Italy ${ }^{1}$; \\ University of Turin, Department of Clinical and Biological Sciences, \\ Turin, Italy ${ }^{2}$; \\ University of Verona, Department of Neuroscience, Biomedicine \\ and Movement, Verona, Italy ${ }^{3}$
}

Aim: Relative Age Effect (RAE) is a phenomenon observed in different sport contexts and consists in an asymmetry in birth-date distribution that may affect athlete's talent identification. Nevertheless, while this phenomenon is extensively investigated in male athletes, few studies focused on female athletes especially in team sports (Smith et al., 2018). Thus, this study aimed for the first time to determine if the RAE is presented in three Italyn elite female team sports.

Methods: A total of 1,537 birthdates of elite female competing in the first and second division of Italyn federation (basketball: $n=446$; soccer: $n=670$; volleyball: $n=421$ ) were analyzed. The birth-date distributions, grouped in four quartiles (i.e., January-March, Q1; April-June, Q2; July-September, Q3; October-December, Q4), were compared to a uniform distribution using Chi-squared analysis. Odd ratios between Q1 and Q4 were calculated.

Results: The results showed an asymmetry in birth distribution in all considered sports (effect size ranged $=0.1-0.12$ ) and a large overrepresentation of players born in Q1 rather than in Q4 $(\mathrm{OR}$ ranged = 1.02-1.62). Nevertheless, RAE decreases as competition level increases (i.e., from second to first division).
Conclusions: Data underlined a bias in the selection, which may limit the identification chance of talented players born late in the second part of the year. Nevertheless, the present RAE scores report a consistent but relatively small influence on female sport participation in comparison with male sport participation (Lupo et al. 2019).

\section{References}

1. Lupo C, Boccia G, Ungureanu AN, Frati R, Marocco R, Brustio PR. The Beginning of Senior Career in Team Sport Is Affected by Relative Age Effect. Front Psychol. 2019; 10(1465).

2. Smith KL, Weir PL, Till K, Romann M, Cobley S. Relative Age Effects Across and Within Female Sport Contexts: A Systematic Review and Meta-Analysis. Sports Med. 2018:1-30.

\section{7}

\section{Anatomic distribution and classification of injuries and illnesses related to dinghy-sailing on hydrofoiling boats}

Francesco Feletti $^{1}$, Antonio La Torre ${ }^{2}$, Matteo Bonato ${ }^{2}$

Ausl Romagna, S. Maria Delle Croci Hospital, Department of Diagnostic Imaging, Ravenna, Italy ${ }^{l}$;

Università Degli Studi Di Milano Statale, Dipartimento Di Scienze Biomediche Per La Salute, Milan, Italy

Purpose: Hydrofoil technology has changed sailing, significantly increasing its speed and resulting in spectacularity and mass media interest. Although high speed can expose participants to a risk of high-energy trauma, there are no scientific studies related to trauma in dinghies that exploit this technology. Therefore, this study primarily examines the anatomical distribution and classification of most common injuries in this sport and measures the injury rate while secondarily identifies the traumatic dynamics most often involved and the main risk factors.

Methods: This descriptive epidemiology study examined data relative to injuries and illnesses suffered by 77 sailors (91\% males) from 13 nationalities during three international competitions, gathered through a specifically designed questionnaire.

Results: The prevalence of illnesses and overuse injuries during the regatta week were $6.5 \%$ and $18.2 \%$, respectively, while the incidence of acute injuries was 16/1,000 sailor-hours. Upper limbs, lower limbs and lumbar spine were involved in $34.6 \%, 26.9 \%$ and $15.4 \%$ of cases of musculoskeletal injuries, respectively. None of the acute injuries reached the maximal score of severity, while the higher score value was $63 / 100$

Most of the reported illnesses $(80 \% ; n=4)$ were upper respiratory tract infections with a prevalence in the week of the regatta of $5.2 \%$ and an incidence of 0.51/1,000 sailor-hours.

Environmental factors played a role in $77 \%$ of the incidents resulting in acute injuries.

Conclusions: This study provides valuable information for regatta organizers, boat builders, athletes, coaches, and doctors. In addition, it fosters the importance of ergonomics of boats, adequate clothing and specific physical training for injuries and illnesses prevention.

\section{References}

1.Nathanson A (2013). Sailing Injuries: A Review of the Literature. R I Med J. 2019;102:23-27.

2.Feletti F, Aliverti A (2017). Extreme sailing medicine, injuries and illnesses. In: Feletti F (Ed) Extreme Sports Medicine, Springer, pp. 275-287.

3.Clarsen B, Rønsen O, Myklebust G, Flørenes TW, Bahr R. The Oslo Sports Trauma Research Center questionnaire on health 
problems: a new approach to prospective monitoring of illness and injury in elite athletes. Br J Sports Med. 2014;48:754-60.

\section{8}

\section{The use of rhythm in track \& field: strategies for raising awareness about rhythm skills in young athletes}

\author{
Amalia Tinto $^{1}$, Emanuela Maccarani ${ }^{2}$, Elisa Fovanna ${ }^{3}$, Marta \\ Campanella ${ }^{3}$ \\ Università Degli Studi Di Torino, Suism-Centro Servizi, Torino, \\ Italy $^{1}$; \\ Fgi, Accademia Internazionale Di Ginnastica, Desio, Italy ${ }^{2}$; \\ Università Degli Studi Di Torino, Suism-Centro Servizi, Torino, \\ Italy $^{3}$
}

Purpose: The use of rhythm in track and field practice programmes (Alfonsi, 2012) allows one to achieve this result (Calavalle, 2001). In fact, it has been shown that every athlete can develop a sense of rhythm when he or she is put in the proper conditions (IAAF, 2012). The aim of the study is to understand how to utilize and implement rhythm skills in athletes ages 12-13 years old and to evaluate in which way this coordination ability influences athletic performance. Methods: The sample group was made up of 20 boys divided into a experimental group (EG) and a control group (CG). Every subject was assessed on a battery of four tests: standing long jump, $60 \mathrm{~m}$ sprint, $60 \mathrm{~m}$ hurdles and the Sargeant vertical jump test as T0 and T1. The experimental work was organized into three weekly training sessions for a period of 3 months. The targeted activity with the work group established that a general and specific warm up with hand clapping, using a wooden and an online metronome, be carried out. In the meantime, the control group continued with its usual workouts.

The GraphPad Prism programme, the Mann-White test for independent samples and the Wilcoxon test for dependent samples were all used $(\mathrm{p}<0,05)$.

Results: In T1 all the individuals of the GL showed improvements in the $60 \mathrm{~m}$ sprint, $60 \mathrm{~m}$ hurdles and the Sargeant jump, exhibiting a statistical significance. A substantial statistic with $\mathrm{p}<0.05$ was found in the individuals from both the EG and the CG groups in the standing long jump test. In this last case the performance of the CG for T0 had better results than the EG, while the results were worse for T1 with respect to the performance of the EG.

Conclusions: The experimental work that was conducted made it possible to examine the importance of rhythm based training to improve one's track and field performance. It would be useful to expand the activity of raising rhythm awareness over time. It should be extended to all areas of track and field in order to evaluate the impact of coordination skills on technical abilities. Furthermore, the introduction of rhythmic work enables training sessions to be more varied and makes it easier to correctly memorize certain steps during the learning of basic techniques.

\section{References}

1. Alfonsi, S., Swimming: Styles, Preparation, Training, Florence, Giunti, 2012;

2. Calavalle, A. R., Athletic Rhythmic Gymnastics. Conditioning and Coordination Skills in Training, Rome, Società Stampa Sportiva, 2001; IAAF, Run, Jump, Throw:

3. The Official IAAF Guide for Teaching Track and Field, Fidal Centre for Study and Research, 2000.

\section{9}

Two vs. one resistance workouts in one day: acute effects on recovery and performance

Emilia Pituelli $^{1}$, Federico Stradi ${ }^{1}$, Lucrezia Nicolai $^{1}$, Sandro

University of Bologna, Deparment For Life Quality Studies, Bologna, Italy $^{1}$;

University of Bologna, Departmente of Biomedical and Neuromotor Sciences, Bologna, Italy ${ }^{2}$

Purpose: Multiple daily training sessions have been frequently used by athletes competing in strength and power events to optimize adaptations and to increase their total training volume during highintensity training phases (Storey et al. 2012). The aim of the present study was to compare the recovery response of one resistance training session (1TRS) versus two resistance training sessions (2TRS) performed in one day, on upper body performance, muscle morphology and muscle soreness in trained men.

Methods: Twenty-four resistance trained men were randomly assigned into a $1 \mathrm{TRS}$ group $(\mathrm{N}=12$; age $=25.0 \pm 2.4 \mathrm{y}$; body mass $=$ $87.6 \pm 14.0 \mathrm{~kg}$; height $=177.1 \pm 4.9 \mathrm{~cm})$ or into a 2 TRS group $(\mathrm{N}=$ $10 ;$ age $=24.4 \pm 1.6 \mathrm{y} ;$ body mass $=81.1 \pm 5.6 \mathrm{~kg}$; height $=176.6 \pm$ $6.7 \mathrm{~cm}$ ). 1TRS performed one training session involving 8 sets of 10 reps at $70 \%$ of $1 \mathrm{RM}$ at the bench press, while $2 \mathrm{TRS}$ group divided the same training volume in 2 workouts, with a recovery time of $4 \mathrm{~h}$. Performance [bench press throw power (BTP) and isometric bench press (IBP)] and muscle thickness of pectoralis major (PECMT) were assessed at baseline (BL), 15-min, 24-h and 48-h post-exercise.

Results: Training intensity was significantly higher in 2TRS compared to 1TRS $(\mathrm{p}<0.001)$. Faster recovery rates were detected for BTP $(p=0.039)$ and PECMT ( $p=0.05)$ in 2TRS compared to 1TRS. Both BTP and PECMT were significantly more affected $(\mathrm{p}<0.05)$ in 1TRS than in 2TRS at $24 \mathrm{~h}$.

Conclusions: Results of this investigation indicate that splitting a similar training volume in two resistance exercise sessions promotes higher training intensity and facilitates the recovery of both performance and muscle morphology in trained men. This strategy may be particularly appropriate to increase maximal strength by optimizing neural adaptations.

\section{References}

1. Storey A, Wong S, Smith HK, Marshall P (2012) Divergent muscle functional and architectural responses to two successive high intensity resistance exercise sessions in competitive weightlifters and resistance trained adults. Eur J Applied Physiol 112(10):3629-3639.

\section{0}

Changes in anthropometry, body composition, HRV and muscular power performance through long period contests preparation in a natural female physique athlete

Matteo Romanazzi ${ }^{1}$, Martina Bianchini ${ }^{1}$

University of Turin, School of Exercise and Sport Sciences (suism), Turin, Italy ${ }^{I}$

Purpose: The study investigated the effect of annual training in a natural female physique athlete on markers of anthropometry, body composition, muscular power and heart rate variability (HRV). 
Methods: We carried out a prospective case study throughout preparation for 4 bodybuilding competitions over a 16 months period. Testing sessions were conducted on a monthly basis and included skinfold and circumferences assessment, vertical jump and push up height, while cardiovascular, power, resistance training parameters, besides HRV data were recorded daily. Simple linear regressions were completed to examine changes over time and to elucidate relationships between different parameters over the entire preparation period.

Results: Slope coefficients of weekly training volume tended to increase by $271.4 \mathrm{~kg}(\mathrm{R} 2=0.09)$, whereas weekly training density tended to increase by $124.8 \mathrm{~kg} / \mathrm{min}$ of recovery $(\mathrm{R} 2=0.12)$ along the analysis period. Weekly time of cardiovascular training decreased by $0.22 \min (\mathrm{R} 2=0.03)$ and power training decreased by 0.047 exercises for lower body per week $(\mathrm{R} 2=0.2)$. Inverse relationship between weekly training density and waist hip ratio (WHR) was found $(\mathrm{R} 2=$ 0.87). WHR over time revealed a decrease of 0.0005 per month $(\mathrm{R} 2=$ 0.33). For HRV slope coefficients of RMSSD and SDNN tended to increase by 0.87 and $0.79 \mathrm{~ms}$ respectively each week $(\mathrm{R} 2=0.42$ and $\mathrm{R} 2=0.40$ ), whereas low frequencies power reported a weekly increase of $60.5 \mathrm{~ms} 2(\mathrm{R} 2=0.38)$. High direct relationship between SDNN and thigh cross sectional area (tCSA) was detected, identifying an increase of $2.7 \mathrm{~ms}$ for each $\mathrm{cm} 2(\mathrm{R} 2=0.91)$.

Conclusions: In general, this prolonged contest preparation technique helped the athlete to improve anthropometric profile and cardiac autonomic function. Direct relationship between HRV and hypertrophy suggests that controlling cardiac autonomic function can be a useful way to increase muscle mass.

\section{References}

1. Schoenfeld BJ et al. (2020). Alterations in Body Composition, Resting Metabolic Rate, Muscular Strength, and Eating Behavior in Response to Natural Bodybuilding Competition Preparation: A Case Study. J Strength Cond Res. 34(11):3124-38.

2. Kistler BM et al. (2014). Case study: Natural bodybuilding contest preparation. Int $\mathbf{J}$ Sport Nutr Exerc Metab. 24(6):694-700

\section{1}

\section{Changes in sleep architecture in response to a late evening competition in team sport athletes}

\author{
Jacopo Vitale ${ }^{1}$, Andrea Galbiati ${ }^{2}$, Gaia De Giacomi ${ }^{1}$, Davide \\ Tornese ${ }^{3}$, Daniel Levendowski ${ }^{4}$, Luigi Ferini Strambi ${ }^{2}$, Giuseppe \\ Banfi $^{2}$ \\ Irccs Istituto Ortopedico Galeazzi, Laboratorio Del Movimento E \\ Scienze Dello Sport, Milan, Italy ${ }^{1}$; \\ Vita-salute San Raffaele University, Na, Milan, Italy ${ }^{2}$; \\ Synlab Cam, Na, Monza, Italy; \\ Advanced Brain Monitoring, Na, Carlsbad, Stati Uniti D' America ${ }^{4}$
}

Purpose: The aim of this study was to assess the effect of a late evening competition on sleep characteristics and perceived recovery in team sport players with the use of a validated and self-applicable multichannel device.

Methods: $\mathrm{N}=16$ team sport players (age: $25.4 \pm 1.4$ years; $\mathrm{BMI}$ : $23.6 \pm 0.5 \mathrm{~kg} / \mathrm{m} 2$ ) completed the study. Objective and subjective sleep data were collected for four consecutive nights: two nights before (PRE2 and PRE1) and the two nights (POST1 and POST2) after a late evening competition, performed between 18:00 and 23:00, with the Sleep Profiler (Advanced Brain Monitoring, California, USA) and a sleep diary. Total Quality of Recovery (TQR) values were also collected in the morning.
Results: A significantly delayed bedtime $(\mathrm{p}<0.0001 ; \eta 2 \mathrm{p}=0.68$, large) was observed in the first night after the competition $(02: 29 \pm$ 01:15) compared both to PRE2 $(+1.5 \mathrm{~h} ; \mathrm{p}<0.0001)$, PRE1 $(+1.6 \mathrm{~h}$; $\mathrm{p}<0.0001)$ and POST $2(+1.6 \mathrm{~h} ; \mathrm{p}<0.0001)$ and similar Results were observed for get-up time $(\mathrm{p}=0.033 ; \eta 2 \mathrm{p}=0.39$, large $)$ : players woke-up significantly later at POST1 $(09: 20 \pm 01: 55)$ compared to PRE2 $(+1.4 \mathrm{~h} ; \mathrm{p}=0.050)$ and POST2 $(+1.4 \mathrm{~h} ; \mathrm{p}=0.049)$. Conversely, the other objective sleep parameters, TQR values and scores of subjective sleep quality did not vary in relation to the late evening competition.

Conclusions: Team sport players had delayed bedtime and get-up time following an evening competition; however, sleep architecture, quality, duration, and subjective scores of recovery were not affected by the night match. Athletes should adopt sleep hygiene strategies following competition to promote the correct night-time sleep and they should have the opportunity to extend their sleep time the morning after the night match.

\section{References}

1. Vitale JA, Banfi G, Galbiati A, Ferini-Strambi L and La Torre A (2019). Effect of a night game on actigraphy-based sleep quality and perceived recovery in top-level volleyball athletes. Int $\mathbf{J}$ Sports Physiol Perform. 14(2):265-269.

2. Roberts SSH, Teo WP, Warmington SA (2019). Effects of training and competition on the sleep of elite athletes: A systematic review and meta-analysis. $\mathrm{Br} \quad \mathrm{J}$ Sports Med 53(8):513-522.

\section{BIOMECHANICS}

\section{2}

\section{Acute effects induced by verbal instructions in the biomechanics of the drop vertical jump performed from different drop heights}

\section{Gaetano Antonacci ${ }^{1}$, Riccardo Di Giminiani ${ }^{1}$}

Department of Biotechnological and Applied Clinical Sciences, University of L'aquila, L'aquila, Italy ${ }^{I}$

Purpose: The Drop Vertical Jump (DVJ) is commonly used in explosive strength training and physical conditioning. Recently, it has also been applied as an exercise in the rehabilitation and prevention of injuries. The purpose of this study was to assess acutely the influence of different verbal instructions on the kinematics of DVJ performed from different drop heights in two experimental groups.

Method: Sixteen young female participated voluntarily in this study; one group was composed of 8 volleyball female players (VP) participating in the Italyn national-Serie $\mathrm{C}$ (age $20.5 \pm 3.3$ years; height $1.70 \pm 0.1 \mathrm{~m}$; body mass $65.5 \pm 11.2 \mathrm{~kg}$ and BMI $22.34 \pm 2.21)$, while the other of 8 active sedentary young women (AC) (age $22.1 \pm$ 1.6 years; height $1.65 \pm 0.1 \mathrm{~m}$; body mass $58.5 \pm 4.4 \mathrm{~kg}$ and BMI $22.15 \pm 1.41 \mathrm{~kg} / \mathrm{m} 2$ ). Each participant performed $12 \mathrm{DVJ}: 3$ different heights $(20,30,40 \mathrm{~cm})$ with 4 different verbal instructions ("jump as high as possible (1A); "jump as quickly as possible" (2A); "jump as high as possible and during the landing try to damp the impact at ground contact" (1B); "jump as quickly as possible and during the landing try to damp the impact at ground contact" (2B). The range of motion (ROM) during flexion-extension of knee joint and anteriorposterior displacement of trunk were recorded by a 3D infrared camera (D-WALL TecnoBody).

Results: The ROM variation in the trials performed according to the verbal instructions $1 \mathrm{~B}$ and $2 \mathrm{~B}$ (with damping) compared to the trials $1 \mathrm{~A}$ and $2 \mathrm{~A}$ (without damping), were significantly higher in $\mathrm{VP}$ at knee ( $1 \mathrm{~B}$ and $2 \mathrm{~B}=30 \pm 1 ; 1 \mathrm{~A}$ and $2 \mathrm{~A}=22 \pm 4 ; \Delta=28 \% ; \mathrm{P}<0.05$ ) and 
trunk $(1 \mathrm{~B}$ and $2 \mathrm{~B}=15 \pm 2 ; 1 \mathrm{~A}$ and $2 \mathrm{~A}=7 \pm 2 ; \Delta=48 \% ; \mathrm{P}<0.05)$ than the $\mathrm{AS}$ at knee $(1 \mathrm{~B}$ and $2 \mathrm{~B}=37 \pm 5 ; 1 \mathrm{~A}$ and $2 \mathrm{~A}=23 \pm 5 ; \Delta=$ $37 \% ; \mathrm{P}<0.05)$ and trunk $(1 \mathrm{~B}$ and $2 \mathrm{~B}=16 \pm 2 ; 1 \mathrm{~A}$ and $2 \mathrm{~A}=10 \pm 3$; $\Delta=39 \% ; \mathrm{P}<0.05)$. The ROM variation at the knee was significant between the two groups during the push-off-phase in the trials 20-2B [VP $(72 \pm 16)$ vs. AS $(14 \pm 26) ;(\Delta \%=43 ; \mathrm{P}<0.05)], 30-2 \mathrm{~B}$ [VP $(65 \pm 26)$ vs. AS $(40 \pm 15) ;(\Delta \%=40 ; \mathrm{P}<0.05)]$ and $40-2 \mathrm{~B}$ [VP $(84$ $\pm 21)$ vs. AS $(51 \pm 17) ;(\Delta=40 \% ; \mathrm{P}<0.05)]$

Conclusion: The results of the present investigation suggest that the kinematic parameters of DVJ, during the push-off phase and landing, performed from different drop heights were influenced by the different verbal instructions given to the participants. There were significant differences within and between the two groups.

\section{References}

1. Di Giminiani, R, Petricola, S (2016) The Power Output-Drop Height Relationship to Determine the Optimal Dropping Intensity and to Monitor the Training Intervention. J Strength Cond Res:117-25.

2. Di Giminiani, R, Giovannelli, A, Capuano, L, Izzicupo, P, Di Blasio, A, Masedu, F (2020) Neuromuscular Strategies in Stretch-Shortening Exercises with Increasing Drop Heights: The Role of Muscle Coactivation in Leg Stiffness and Power Propulsion. Int J Environ Res Public Health. (22):8647.

\section{3}

\section{Markerless pose estimation of deeplabcut for shoulder motion assessment in patients with cervical spinal cord injury}

\author{
Stefano Grasso $^{1}$, Riccardo Bravi $^{1}$, Eros Quarta $^{2}$, Vincenzo Sorgente ${ }^{1}$, \\ Erez James Cohen ${ }^{1}$, Giacomo Lucchesi ${ }^{3}$, Lorenzo Mucchi ${ }^{4}$, Diego \\ Minciacchi ${ }^{l}$ \\ University of Florence, Department of Experimental and Clinical \\ Medicine, Florence, Italy ${ }^{1}$. \\ University of Rome, Sapienza, Department of Physiology \\ and Pharmacology, Rome, Italy' \\ Unità Spinale, Azienda Ospedaliero-universitaria Careggi, Firenze, \\ Italy $^{3}$; \\ University of Florence, Department of Information Engineering, \\ Florence, Italy ${ }^{4}$
}

Purpose: Residual motion of upper limbs in individuals with cervical spinal cord injury (CSCI) is a key-element in achieving functional independence and performing activities of daily living. Objective assessment of shoulder joint range of motion (ROM) is crucial to monitor CSCI patients' progress. Advancements in technological devices allowed to validate wearable Inertial Measurement Unit sensors (IMU) to evaluate shoulder movement. In addition, markerless human motion capture could also be a potential tool for studying body biomechanics. Recently, it was developed a new computer vision technology for markerless pose estimation, named DeepLabCut (DLC), based on transfer learning with deep neural networks. This study evaluated the validity of DeepLabCut contactless method in measuring active shoulder movements in CSCI patients, while seated in a wheelchair, in a clinical setting. To achieve this, we compared the accuracy of the DeepLabCut contactless method to a customized, wireless wearable IMU-based sensors system.

Methods: Eight CSCI patients and 8 healthy controls performed four shoulder movements (forward flexion, abduction, external and internal rotation at $90^{\circ}$ of shoulder abduction) with dominant upper limb. Every movement was recorded by IMU system and 2 cameras (GoPro Hero 5) placed frontally and laterally to the subject at the same time.
Two IMU sensors of the MbientLab (MetaMotion R), characterized by small dimensions and low cost, were positioned on the arm, just above the elbow, and on the wrist, respectively. For each trial, joints center locations were manually applied to 10 images from each video, and were used as training data for the neural network (ResNet-101), which is in line with recommendations when using DLC. After training, all the videos were analyzed by the DLC and the predicted joints center locations during shoulder movements were extracted. Finally, angle measurements of the extracted coordinates of the joints were calculated with Matlab R2019b.

Results: DLC reliably tracked and predicted joint center pixel locations from video recordings. Shoulder ROM measurements of DLC correlated well by comparing with IMU sensors system.

Conclusion: DeepLabCut, a new technology for markerless pose estimation, can quantify shoulder ROM measurements in CSCI patients and healthy subjects. From a conventional video recording, DLC allows for objective contactless measurements and this open up possibilities to build tele-rehabilitation.

\section{References}

1. Bravi R et al. (2021). An Inertial Measurement Unit-Based Wireless System for Shoulder Motion Assessment in Patients with Cervical Spinal Cord Injury: A Validation Pilot Study in a Clinical Setting. Sensors 2021,21,1057.

2. Mathis A et al. (2018). DeepLabCut: markerless pose estimation of user-defined body parts with deep learning. Nat Neurosci 21, $1281-1289$.

\section{4 \\ Mechanical power in semi-tethered swimming is affected by propelling efficiency}

\author{
Matteo Cortesi $^{1}$, Giorgio Gatta ${ }^{1}$, Paola Zamparo ${ }^{2}$
}

University of Bologna, Department For Life Quality Studies, Bologna, Italy ${ }^{1}$;

University of Verona, Department of Neurosciences, Biomedicine and Movement Sciences, Verona, Italy ${ }^{2}$

Purpose: During (free) swimming at constant speed propulsive power $(\mathrm{Pp}=\mathrm{Fp} * \mathrm{~V})$ must balance resistive power $(\mathrm{Pd}=\mathrm{Fd} * \mathrm{~V}$, to overcome drag). Pd is only a fraction of the total power (Ptot) a swimmer can generate in water, and propelling efficiency indicates this fraction (np1 = Pd / Ptot). Semi-tethered swimming (STS) is a training method to enhance swimming performance. In STS, a fraction of Ptot must also be utilized to overcome the external load (Pst $=$ Fst $* \mathrm{~V}$, where Fst is the semi-tethered force $)$. In this case $n p 2=((\mathrm{Pst}+\mathrm{Pd}) / \mathrm{Ptot})$. Ptot can be estimated, during full tethered tests, as Ptmax / npmax (where Ptmax is the maximal power output that can be generated in these conditions and assuming npmax $=0.40$ ). We expect that $\mathrm{np} 2<$ $\mathrm{np} 1$ since the former takes into account additional transmission losses. Methods: Fourteen male top-level sprinters $(23.1 \pm 2.0 \mathrm{y}, 189.4 \pm$ $5.3 \mathrm{~cm}, 83.6 \pm 7.1 \mathrm{~kg}, 93 \pm 3 \%$ of WR) randomly performed the following tests: 1) to measure V, Fst and Pst, five trials of $15 \mathrm{~s}$ all-out STS at different loads $(35,50,60,75,85 \%$ of individual full-tethered force), during these trials np1 was estimated based on the "paddle wheel model"; 2) to estimate Pd in active conditions, five trials of passive towing measurement (at $\mathrm{V}=1.0,1.3,1.6,1.9,2.2 \mathrm{~m} / \mathrm{s}$ ); 3 ) to assess Ptmax, a $15 \mathrm{~s}$ all-out full-tethered test and a $25 \mathrm{~m}$ all-out trial. Results: Repeated measure ANOVA revealed a significant decrease of Pd ( $<<0.001 ; \eta 2=0.840$; from 187 to $79 \mathrm{~W})$ and $\mathrm{np} 1(\mathrm{p}<0.001$; $\eta 2=0.965$; from 0.29 to 0.17 ) and a significant increase of Pst ( $\mathrm{p}<$ $0.001 ; \eta 2=0.812$; from 60 to $86 \mathrm{~W})$ and Ptot $(\mathrm{p}<0.001 ; \eta 2=0.531$; from 847 to $958 \mathrm{~W}$ ) with increasing load during STS tests. Ptmax was 
$415 \pm 39.4 \mathrm{~W}$ and $\mathrm{np} 2$ decreased from 0.23 to 0.15 (it was indeed lower than $\mathrm{np} 1$ ). The ratio $\mathrm{np} 2 / \mathrm{np} 1$ (an index of transmission efficiency) significantly increased with the external load during STS (from 0.80 to $0.90, \mathrm{p}<0.001$ ).

Conclusions: The present study highlights the role of propelling efficiency on power output in STS and suggests that the expression of power is limited by the decline in the capability to exert useful forces in water in these conditions (e.g. a decrease in propelling and transmission efficiency).

\section{References}

1. Dominguez-Castells R, and Arellano R. (2012). Effect of different loads on stroke and coordination parameters during freestyle semi-tethered swimming. J Hum Kinet 32, 33-41.

2. Gatta G, Cortesi, M, and Zamparo, P. (2016). The relationship between power generated by thrust and power to overcome drag in elite short distance swimmers. PloS One 11, 1-11.

3. Zamparo P, Cortesi M, and Gatta G. (2020). The energy cost of swimming and its determinants. Eur J Appl Phy 120, 41-66.

\section{5}

\section{The effects of four-weeks injury prevention training program on "high-risk" on-field movements biomechanics in amateur male futsal players}

\section{Lorenzo Zattoni ${ }^{1}$ \\ Università, Università Di Ferrara, Ferrara, Italy ${ }^{1}$}

Purpose: Landings, decelerations, and changing directions are key movements in recreational Futsal matches. Still, they can be associated with non-contact lower limb injuries, ranging from an ankle sprain to ACL tear, due to biomechanical risk factors, such as dynamic knee valgus. This study proposes a video-analysis methodology to identify biomechanical variables, useful to understand the biomechanical effects of a 4-week multifaceted training program intervention, which looked for modifying injury risk in sport-specific tasks.

Methods: In a randomized, controlled 4-week intervention study, a sample of 44 amateur Futsal players was equally divided into two groups: the Intervention Group (IG, N = 22), which attended a 4-week intervention program, and the Control Group ( $\mathrm{CG}, \mathrm{N}=22$ ). An onfield mobile laboratory was set to deliver a three-task test to each group: Single-Leg Hop (SLH), Deceleration (DEC), and $90^{\circ}$ Changing of Directions (COD). In each participant were video-analyzed 4 frontal stability angles (Trunk, Hip, Knee, Ankle), 2 sagittal flexion angles (Hip, Knee), the body center of mass (standing to land), the mean acceleration decelerating, and the distance between CoM and FFC in the $90^{\circ} \mathrm{COD}$; in both right and left body parts, pre-to-post intervention.

Results: IG compared to the CG at $\mathrm{T} 1$ showed a significant improved frontal stability, in COD Knee angle (right, $148^{\circ}, \mathrm{p}=0.007$ ); and a significant improved Hip (angles $<105^{\circ}, \mathrm{p}=0.015 ; \mathrm{p}=0.033$, right and left respectively) and Knee (right, $109.5^{\circ}, \mathrm{p}=0.017$ ) co-flexion during the SLH. IG, pre-to-post intervention, showed a significant improved knee frontal stability in SLH (right, $183.5^{\circ}, \mathrm{p}=0.001$ ), in DEC (right, $174.6^{\circ}, \mathrm{p}=0.027$ ); and improved significantly $\Delta \mathrm{CM}$ in SLH (right, $\mathrm{p}=0.013$; left, $\mathrm{p}=0.00)$, both Hip $\left(107.4^{\circ}, \mathrm{p}=0.007\right.$; $\left.105.8^{\circ}, \mathrm{p}=0.000\right)$ and Knee Flexion angles $\left(115.8^{\circ}, \mathrm{p}=0.027\right.$; $\left.115.2^{\circ}, \mathrm{p}=0.032\right)$ in DEC, and Knee Flexion angles $\left(115.2^{\circ}, \mathrm{p}=\right.$ $0.000 ; 118.6^{\circ}, \mathrm{p}=0.001$ ) in COD.

Conclusions: The identified biomechanical variables improved limb stability and shock absorption in recreational futsal players after a multifaceted preventive training program. This result provides coaches with a repeatable video-analysis methodology based on a field mobile laboratory, to screen football-specific movements. Future applications should be directed into other recreational sports populations' tasks, and into the youth sports population, investigating the biomechanical variables during the process of growth.

\section{References}

1. Bizzini M, Dvorak J. FIFA $11+$ : an effective programme to prevent football injuries in various player groups worldwide-a narrative review. Br J Sports Med. 2015 May;49(9):577-9.

2. Dos'Santos T. (2020). Biomechanical Determinants of Injury Risk and Performance during Change of Direction: Implications for Screening and Intervention. Article retrieved from Salford University. (Manchester, UK).

3. Hébert-Losier K, Hanzlíková I, Zheng C, Streeter L, Mayo M. The 'DEEP' Landing Error Scoring System. Appli.

\section{6}

\section{EMG analysis of planche and specific special} strengthening exercises

\section{Giuseppe Rosaci ${ }^{I}$, Simone Ciacci $^{1}$, Sandro Bartolomei ${ }^{1}$, Silvia Fantozzi $i^{2}$}

Department of Biomedical and Neuromotor Sciences, University of Bologna, Bologna, Italy ${ }^{1}$;

2department of Electrical, Electronic and Information Engineering, University of Bologna, Bologna, Italy ${ }^{2}$

Purpose: In the artistic gymnastics rings, the Iron Cross, the Swallow, and the Planche (PL) are among the most used and studied static force elements. The EMG analysis highlighted the importance of the stabilizing muscles of shoulder and trunk. The study of special training, which is fundamental for the performance, is quite investigated on the Swallow, but much less on PL. Therefore, the aim of this research is to evaluate the specificity of training comparing the muscular activity of 8 shoulder/trunk muscles during PL and 5 special exercises.

Methods: Seven elite athletes (age $23.9 \pm 4$ years; height $169.9 \pm$ $2.8 \mathrm{~cm}$; mass $65.5 \pm 3 \mathrm{~kg}$ ) were asked to perform PL and 5 exercises: dumbbells from standing position (DSP), PL with pulley (PLP), elastics in prone decubitus (EDP), PL with supported feet (PLSF) and dumbbells in supine decubitus (DSD). A wireless EMG (Cometa System, Milan, Italy, sampling frequency $2000 \mathrm{~Hz}$ ) was employed to collect surface EMG signals of pectoralis major, anterior deltoid, biceps brachii, triceps brachii, anterior dentate, infraspinatus, transverse trapezium and latissimus dorsi.

Results: All special exercises present more or less significant differences in comparison with PL regarding the activation and contribution of the various muscles. The exercise with the least significant differences seems to be DSP ( $p>0.05)$.

Conclusions: From the point of view of the shoulder flexor muscles activation, the exercise most similar to PL seems to be DSP. PLP and EDP are the most suitable exercises for technique and body attitude. PLSF and DSD, although functional for some specific aspects of PL, are the exercises that need more compensation through auxiliary and special physical preparation.

\section{References}

1. Bernasconi SM, Tor $\neg$ di NR, Parratte BM, Rouillon JRD, Monnier G.-Surface electromyography of nine shoulder muscles in two iron cross conditions in gymnastics-J Sports Med Phys Fitness 2004;44(3):240-245.

2. Bernasconi SM, Tor $\neg$ di NR, Parratte BM and Rouillon JRDCan shoulder muscle coordination during the support scale at ring 
height be re $\neg$ plicated during training exercises in gymna $\neg$ Stics?-J Strength and Cond Res 23, 2009: 2381-2388.

187

\section{Evaluation of postural stability and proprioception in subjects with severe ankle osteoarthritis}

\author{
Giuseppe Barone $^{1}$, Stefano Di Paolo ${ }^{1}$, Emanuele Vocale ${ }^{2}$, Raffaele \\ Zinno $^{I}$, Erika Pinelli ${ }^{1}$, Silvio Caravelli ${ }^{2}$, Simone Massimi ${ }^{2}$, \\ Massimiliano Mosca $^{2}$, Maria Grazia Benedetti ${ }^{3}$, Laura Bragonzoni ${ }^{1}$ \\ Università Di Bologna, Dipartimento Di Scienze Per La Qualità \\ Della Vita, Rimini, Italy ${ }^{1}$; \\ Istituto Ortopedico Rizzoli, Clinica Ortopedica E Traumatologica I, \\ Bologna, Italy ${ }^{2}$; \\ Istituto Ortopedico Rizzoli, Medicina Fisica E Riabilitativa, Bologna, \\ Italy $^{3}$
}

Purpose: Ankle osteoarthritis (OA) is a debilitating condition, which includes balance impairment, range of movements limitations and a consequence increased risk of falls. Conservative management is the first approach but for severe ankle osteoarthritis, ankle prosthesis should be taken into account. The aim of this study is to measure postural instability and proprioception in subjects with ankle osteoarthritis scheduled for primary total ankle replacement.

Methods: Subjects with ankle OA admitted in the Istituto Ortopedico Rizzoli waiting for a total ankle replacement were recruited. Delos Postural Proprioceptive System (Delos S.r.1, Turin, Italy) was used to assess postural stability and proprioception. All subjects performed two tests: The Static Riva test, which consists of maintaining a single leg stance with open and closed eyes (OE; CE), and the Dynamic Riva test, a single leg stance in a rocking board with OE. The parameters considered for these tests were the Stability Index (SI) and dynamic stability index (DSI) both are percentage scores where 100\% is a theoretical task performed with maximum stability.

Results: Subjects recruited were 23 (16 male and 7 Female) aged $56.82 \pm 10.63$ years with a body mass index of $27.51 \pm 3.05$. The SI with OE is $70.06 \pm 16.82 \%$ in the OA ankle and $85.10 \pm 10.59 \%$ in the healthy ankle $(\mathrm{p}<0.005)$. The SI with CE is $40.16 \pm 14.31 \%$ in the AO ankle and $53.89 \pm 16.51 \%$ in the healthy ankle $(\mathrm{p}=0.001)$. The DSI is $40.78 \pm 10.38 \%$ in the AO ankle and $46.57 \pm 12.74 \%$ in the healthy ankle $(\mathrm{p}<0.05)$. There is a strong correlation between the $\mathrm{OE}$ and $\mathrm{CE}$ results in the OA ankle $(\mathrm{r}=0.79 ; \mathrm{p}<0.001)$, instead the same correlation in the healthy limb is moderate $(\mathrm{r}=0.04 ; \mathrm{p}=0.014)$. The two tests are correlated just for the healthy ankle $(\mathrm{r}=0.5 ; \mathrm{p}<$ 0.05 ), instead DSI and SI results of the AO ankle are independents. Age and BMI did not influence the SI and DSI results.

Conclusion: Static and Dynamic Riva tests are indicator of postural stability (open eyes tasks) and proprioception (closed eyes and instability board). Subjects showed a consistent difference between $\mathrm{OA}$ and healthy ankle in all tests; therefore we suppose that postural stability and proprioception are strongly influenced in a severe ankle osteoarthritis condition due to pain and reduced range of motion.

\section{References}

1. Riva D et al. (2013) Single stance stability and proprioceptive control in older adults living at home: Gender and age differences. J Aging Res 2013: 561,695.

\section{8}

Free fall test to evaluate push-up landing in aerobic gymnastics: a preliminary study

\author{
$\underline{\text { Daniele Albano }}^{I}$, Cristiana D'anna ${ }^{1}$, Benedetta Romano ${ }^{I}$, Rodolfo \\ Vastola \\ Università Degli Studi Di Salerno, Dipartimento Di Scienze Umane, \\ Filosofiche E Della Formazione, Salerno, Italy ${ }^{1}$
}

Purpose: In aerobic gymnastics the push-up landing is one of the possible final phases of some difficulties, even with body rotations on the longitudinal axis before landing. Free fall has been identified as a possible test of the athlete's landing management. Therefore, the aim of the study is to understand whether indexes such as Time to Stabilization (TTS) and Asymmetry Index (AI) can provide useful information for injury prevention and performance improvement.

Methods: The study involved 39 athletes (age group 10-19 years) of Gold level in aerobic gymnastics (5 M, $34 \mathrm{~W}$ ) with average weight $(47.8 \mathrm{~kg} ; \mathrm{SD} \pm 11.8)$ and average height $(1.56 \mathrm{~cm} ; \mathrm{SD} \pm 9.4)$ belonging to the categories National Development (8), Age group (13), Junior (15) and Senior (3). Anthropometric, informative and informed consent data were collected. The athletes performed 3 free fall tests landing with their hands on two force platforms with a sampling frequency of $1000 \mathrm{~Hz}$. From the recorded force, the TTS and the AI were computed. Data were analysed using SPSS v23 statistical software. A descriptive statistic was carried out on the sample. An ANOVA was performed to compare the differences between the different groups (athletes with no previous injuries / injured athletes and competition category).

Results: The Results show that both TTS and AI decrease in value in relation to the best technical execution of free fall and therefore the best performances are recorded in the Junior and Senior categories. It has been understood that free fall being a specific basic skill of the discipline is an automated gesture by "Gold" level athletes. What is particularly interesting is that the value of AS is not only higher in those who have suffered injuries but also shows greater variability.

Conclusions: this preliminary study highlights the possibility of evaluating, through the free fall test, the AI which is particularly crucial to ensure a balanced landing in arrivals to the push-up. Future studies, on a larger sample, could deepen its validity for evaluative and preventive Purposes.

\section{References}

1. Mao, M., Yin, Y., Luo, D., Liu, H., \& Yu, B. (2021). Evaluation of dynamic postural control during single-leg landing tasks using initial impact force, landing leg stiffness and time to stabilisation. Sports Biomechanics, 1-14.

2. Jordan, M. J., Aagaard, P., \& Herzog, W. (2015). Lower limb asymmetry in mechanical muscle function: a comparison between ski racers with and without ACL reconstruction. Scandinavian journal of medicine \& science in sports, 25(3), e301-e309.

\section{9}

Kinetic and kinematic analysis of the freestyle flip turn in a national level swimmer: a case report

Giada Anastasio $^{1}$, Stefano La Greca ${ }^{1}$, Riccardo Di Giminiani ${ }^{1}$

Università, Dipartimento Di Science Cliniche E Biotecnologiche, L'aquila, Italy ${ }^{I}$ 
Purpose: The aim of the present study was to acutely examine the influence of several verbal instructions on kinematic and kinetic parameters in order to obtain the greatest final velocity (Vf) in the freestyle flip turn. The verbal instructions given to the athlete included the combination of different initial velocities (Vi) and angles of knees flexion during the push-off phase.

Methods: One national level female swimmer participated (age: 25 years, weight: $56,0 \mathrm{~kg}$, height: $164 \mathrm{~cm}$ ) in the present study. The athlete performed six flip-turns resulted from the random combination of two initial velocities (maximal: typical of a $50 \mathrm{~m}$ freestyle; submaximal: typical of a $200 \mathrm{~m}$ freestyle) and three angles of knees flexion [small $\left(60^{\circ}\right)$, medium $\left(110^{\circ}\right)$ and large $\left(150^{\circ}\right)$ angular displacement $]$. The trials were: first $=$ small, Vi max; second $=$ large, $\mathrm{Vi}$ submax; third = large, Vi max; fourth = small, Vi submax; fifth = medium, Vi submax; sixth = medium, Vi max. Initial, final velocities and knee angle were measured by using two cameras (sampling rate $=$ $60 \mathrm{~Hz}$ ). The waterproofed force platform (sampling rate $=100 \mathrm{~Hz}$ ) was fixed below the starting block to measure the reaction force over time. The videos were analyzed with the software Kinovea (opensource project version 0.8.15), while the kinetic data [maximal reaction force (MRF), impulse (I) and rate force development (RFD)] were analyzed with the software muscle-lab (Egotest Innovation, Porsgrunn, Norway).

Results: The verbal instructions did not produce the expected displacement of knee angle and the values measured ranged from $57^{\circ}$ to $74^{\circ}$. Furthermore, the maximal push-off force $(2938 \mathrm{~N})$, RFD $(17,487 \mathrm{~N} / \mathrm{s}), \mathrm{I}(475.4 \mathrm{~N} \bullet \mathrm{s})$ and the higher initial velocity $(4.67 \mathrm{~m} / \mathrm{s})$ did not increase the final velocity $(2.36 \mathrm{~m} / \mathrm{s})$. Conversely, in the trail executed with submaximal initial velocity, reduced by almost $40 \%$ $(2.52 \mathrm{~m} / \mathrm{s})$, the swimmer reached the highest final velocity by almost $10 \%(2.60 \mathrm{~m} / \mathrm{s})$. The kinematic analysis revealed differences among the shapes of the trajectory depicted by the swimmer during the flip turns.

Conclusions: The structure of motor skills of the swimmer, related to the number of training years, limited the verbal instructions effect on acute changes of the knee angle flexion during the flip turn. MRF, RFD, I, and Vi did not increase the Vf, whereas a submaximal Vi probably allowed greater coordination during the flip turn, determining an increase in the final velocity.

\section{References}

1. Puel F, Morlier J, Avalos M, Mesnard M, Cid M, and Hellard P. (2012) 3d kinematic and dynamic analysis of the front crawl tumble turn in elite male swimmers. Journal of Biomechanics 45: 510-515.

2. Araujo L, Pereira S, Gatti R, Freitas E, Jacomel G, Roesler H, and Villas-Boas J. (2010) Analysis of the lateral push-off in the freestyle flip turn. Journal of sports sciences 28: 1175-1181.

\section{0}

\section{Longitudinal functional assessment of a transfermoral amputee patient treated with osseointegration surgery}

\author{
Laura Bragonzoni ${ }^{1}$, Stefano Di Paolo ${ }^{1}$, Agostino Igor Mirulla ${ }^{2}$, \\ Domenico Alesi ${ }^{3}$, Stefano Zaffagnini ${ }^{3}$
}

University of Bologna, Department For Life Quality Studies, Bologna, Italy ${ }^{1}$;

University of Bologna, Department of Biomedical and Neuromotor Sciences, Bologna, Italy ${ }^{2}$;

Rizzoli Orthopaedic Institute, 2nd Orthopaedic and Traumatological Clinic, Bologna, Italy ${ }^{3}$
Purpose: Osseointegrated surgery is an emerging alternative to treat transfemoral amputation in patients that have problems in wearing standard socket-type prosthesis [1]. After surgery, a critical functional recovery and specific rehabilitation phase is required. Little functional assessment of these patients has been provided in the literature. The aim of the present study was to provide a functional assessment of a transfemoral amputee patient before osseointegration surgery and after rehabilitation by means of wearable sensors.

Methods: A transfemoral amputee patient (male, 47 years, time from amputation 18 years) scheduled for osseointegration surgery was enrolled. The patient was able to walk without aids and performed a gait test the day before surgery with his standard socket-type prosthesis, consisting in 10-m walking in a hospital indoor hall, two at normal self-selected speed and two at the fastest speed possible. The test was repeated 3 months after surgery, after the clearance from the rehabilitation. A set of 15 wearable inertial sensors (Awinda, Xsens Technologies) was used to collect full body kinematics. Complete gait cycles were isolated and spatiotemporal and kinematical parameters were extracted. The differences between the amputee (AL) and the sound (SL) limb and between the normal and fast gait were reported. Results: Shorter step length was noticed for the AL regardless the gait speed. A longer swing phase was noticed for the AL, with greatest differences in the midstance. At follow-up, step length and midstance differences increased, but the loading response of the AL doubled, and swing phase differences decreased. In the AL, no hip and knee abduction, peak ankle plantarflexion, in the swing phase, and of peak flexion in the stance phase were found preoperatively. A greater pelvis forward tilt and obliquity on the amputee limb, trunk forward tilt, and lateral bending were also noticed. At follow-up, asymmetries in hip abd/adduction, hip rotation, and pelvis rotation decreased, and no more trunk forward and lateral tilt were found.

Conclusion: The altered spatiotemporal and kinematical parameters found preoperatively were in line with current literature [1]. After rehabilitation, the patient showed higher time spend on the AL and lesser kinematical asymmetries. The excessive trunk and pelvis motion were curtailed. Specific pre-habilitation trainings are required also before surgery to fasten the rehabilitation period.

\section{References}

1. Cutti AG, et al., Reference values for gait temporal and loading symmetry of lower-limb amputees can help in refocusing rehabilitation targets, Journal of neuroengineering and rehabilitation, 2018 Sep 5;15(Suppl 1):61.

\section{MOTOR CONTROL and LEARNING}

\section{1}

\section{Action performance in shaping conscious behavior}

\section{Veronica Montani ${ }^{l}$, Francesco Pascucci ${ }^{l}$, Elisabetta Colombari ${ }^{1}$, Silvia Savazzi ${ }^{I}$ Paola Cesari ${ }^{1}$}

Dipartimento Di Neuroscienze, Biomedicina E Movimento, Università Di Verona, Verona, Italy ${ }^{I}$

Purpose: According to the two-streams hypothesis the visual input is transformed into perceptual representations in the ventral pathway while the visual control of actions is mediated by the dorsal pathway. Importantly, the visual processing mediated by the ventral stream supports visual awareness while this is not the case for the transformation of visual input into action. We investigated whether the online control of object grasping, which is mediated by the dorsal stream, could get access to information about an object' feature, i.e., orientation, of which the participant is not aware of. 
Methods: Participants performed a reach and grasp movement toward a briefly presented Gabor patch that could be slightly tilted either upward or downward with respect to the horizontal axis. The Gabor orientation was selected based on a previous individual threshold assessment (i.e., the orientation that participants reported they could consciously perceive about $50 \%$ of the time). Movement precision was estimated by comparing the orientations between the thumb and first digit of one hand with the orientation of the Gabor patch by means of an optical motion capture system.

Results: Results showed that participants selected the accurate hand configuration even when they reported to be not aware about the Gabor orientation, suggesting that this information could be unconsciously accessed for controlling the proper action.

Conclusions: These findings are consistent with the two-streams hypothesis: the processing of a visual feature that is usually attributed to the ventral pathway is successfully achieved without awareness through the vision for action dorsal pathway.

\section{References}

1. Goodale, M. A., \& Milner, A. D. (1992). Separate visual pathways for perception and action. Elsevier Current Trends.

\section{2}

\section{Spontaneous motor play habits and deliberate practice: implications for gross motor coordination in 5-7 years old children}

\author{
Marco Ravanelli ${ }^{1}$, Matteo Giurato $^{2}$, Valentina Biino ${ }^{2}$ \\ University of Verona, M.sc. Preventive and Adapted Motor Sciences, \\ Verona, Italy ${ }^{1}$; \\ University of Verona, Department of Neuroscience, Biomedicine \\ and Movement, Verona, Italy ${ }^{2}$
}

Purpose: Gross Motor Coordination (GMC) and physical activity (PA) in school children is essential for an adequate active lifestyle and health benefits [1]. Indeed, PA was identified as correlate of gross motor competence in typically developing children [1].

Further, Socioeconomic Status (SES) is often suggested as a factor that influences PA and associated health outcomes [2].

The present longitudinal study investigates the GMC trend in children aged 5 to 7 for 8 months concerning the Socioeconomic Status (SES) and PA-spontaneous. [2,3].

Methods: 21 subjects (boys), aged 5-7 years, were assessed across 8 months. An evaluation was recorded in October (T0), December (T1), March (T2), April (T3), May (T4). Between T3-T4, subjects were in lockdown due to COVID-19. GMC was evaluated with Körperkoordinationstest für Kinder (KTK) using age and sex-specific reference values, defined as motor quotient MQ [4]. Furthermore, it was assessed the anthropometric characteristics (Height, Body mass). Socio-Economic Status (SES) and Spontaneous Motor Play (SMP) it was recorded through a questionnaire [2,3] and divided into level bands [2,3]. Furthermore, SMP was evaluated SES using a composite score calculated as the average z-scores based on T0.

Results: GMC and anthropometric characteristics will be measured using a composite score calculated as the average z-scores based on T0. The lower level of GMC is at T3 (z-score: -0,02). Height and Body Mass, instead, increase from T0 to T4. Body Mass increases slower between T3 (z-score: 0,74) and T4 (z-score: 0,80) reaching a plateau. Most difference in PA it was showing during T3, in subjects with Low SES, showing a low level of PA (z-score: -2,37) than Medium- (z-score: -1,13) and High- (z-score: -0,95).

Conclusions: Results suggesting that during the lockdown period (T3-T4), GMC level decreasing to T0 (7 months before), indicating the difficulty of carrying out PA. These Results are highlighting the magnitude of play GMC activities during childhood. Further, medium- and high- SES subjects show a high level of PA than low- SES subjects even during the lockdown.

\section{References}

1. Barnett LM et al., Sports Med. 2016 Nov;46(11):1663-1688. https://doi.org/10.1007/s40279-016-0495-z

2. Vandendriessche JB. et al., 2012 Pediatric exercise science, 24(1), 113-128. https://doi.org/10.1123/pes.24.1.113

3. Veitch, J. et al., 2009 Journal of Science and Medicine in Sport 12 (5): 579-582. https://doi.org/10.1016/j.jsams.

4. Kiphard EJ, Schilling F. Körperkoordinationstest für Kinder. 2. Überarbeitete und ergänzte Auflage. Weinheim: Beltz Test GmbH, 2007.

\section{3}

\section{The control of postural stability through light touch imagination: disentangling the role of tactile information with the sense of agency}

\section{$\underline{\text { Yumna Ali }}{ }^{1}$, Veronica Montani ${ }^{I}$, Paola Cesari ${ }^{1}$}

University of Verona, Department of Neurosciences, Biomedicine and Movement Sciences, Verona, Italy ${ }^{I}$

Purpose: Standing in an upright position and touching a surface increases the ability to orient in space by improving postural control; this effect is so marked that even a very light touch (less than one Newton) increased body stability by reducing the area described by the center of pressure as demonstrated by several studies. The main goal of this study was to unveil the mechanism underlying this increment of stability, by disentangling the role played by the tactile information per se (surface touching) and the sense of agency (I am using the finger as a potential help for regaining stability in case of a sudden arrival of a perturbation).

Methods: Twenty healthy subjects were asked to imagine to produce either less than 15 or 1 Newton of force while thinking to press a surface with their index finger. During the imagination, no movements were allowed. Subjects were tested in two conditions: with or without lying their digits on a surface. TMS was delivered on the Primary Motor Cortex at the level of the hand muscles representation. MEPs (Motor evoked potentials) were recorded through the EMG of three hand muscles: one as a target FDI (Flexor digitorum introssei) and two as control ADM (Abductor digiti minimi) and FDS (Flexor digitorum superficialis).

Results: Results showed higher MEPs for the FDI muscle during the imagination with the digits in contact with the surface when compared with the MEPs amplitudes of ADM and FDS. Differences between the $15 \mathrm{~N}$ and the $1 \mathrm{~N}$ imagery force production were also observed, but again just for the target FDI muscle.

Conclusions: This study presents meaningful measures for detaching the role played by the sense of agency along with the tactile information when individuals are asked to maintain postural control. The light touch of the index finger, combined with the mental imagery of low and high forces, leads to the significant amplitude of the FDI MEPs as a consequence of an increment of an internal and specific body representation.

\section{References}

1. Fossataro, C., Bruno, V., Giurgola, S., Bolognini, N., \& Garbarini, F. (2018). Losing my hand. Body ownership attenuation after virtual lesion of the primary motor cortex. European Journal of Neuroscience, 48(6), 2272-2287. 
2. Jeka, J. J., \& Lackner, J. R. (1994). Fingertip contact influences human postural control. Experimental Brain Research, 79(2), 495-502.

194

\section{The effect of different visual instructions for enhancing motor performance during childhood}

\author{
Vincenzo Sorgente $^{I}$, Erez James Cohen ${ }^{2}$, Riccardo Bravi ${ }^{2}$, Diego \\ Minciacchi $^{2}$ \\ Univesity of Florence, Department of Experimental, Clinical \\ and Biomedical Sciences, Florence, Italy ${ }^{1}$; \\ University of Florence, Department of Experimental and Clinical \\ Medicine, Florence, Italy ${ }^{2}$
}

Purpose: Two approaches have shown to be successful in improving children's motor performances: one is focusing on movement technique, another is focusing on the goal of the task. To date, a comparison of effectiveness between these two approaches is yet to be investigated in a population of children. In this study, we sought to investigate if and how technique-focus and goal-focus visual instructions possibly differ in improving motor skills across late childhood in a throwing task.

Methods: 180 boys and girls (ages 6-11) participated in this study and were divided into technique-focus, goal-focus, and control groups. The task was to throw balls at a target aiming at the bull's eye. The experiment was divided into pretest $(5$ throws $)$, practice $(7 \times$ 3 throws $)$, and retention $(7 \times 3$ throws $)$ phases. Following the pretest phase, the technique-focus and goal-focus groups were shown a video of how to perform the experimental task. For the technique-focus group, the video showed the correct movement preparation (i.e., feet/ hand placement, stance, grip), but not the movement execution (i.e., the ball throwing). For the goal-focus group, the same video was cropped in such a way that only the movement execution was visible. Retention tests were executed the following day. The control group did not watch the video.

Results: Regarding the ball throwing scores, preliminary findings show that the technique-focus group performed significantly better than both the goal-focus group and the control group $(\mathrm{p}<0.05)$. Specifically, the difference in performance is significant at 10-11 years old. The same results are visible in the retention tests. Conclusions: When it comes to motor skills enhancing strategies, focusing on the correct movement technique is more advantageous than concentrating on the goal of the task. This appears to be particularly true towards the end of the late childhood developmental stage. These results could allow teachers and youth trainers to efficiently maximize children's motor experience. Further studies are needed to clarify whether a longer retention effect is present and whether specific visual instructions also influence motor performances during adolescence.

\section{References}

1. Bekkering, H., Wohlschlager, A., \& Gattis, M. (2000). Imitation of gestures in children is goal-directed. The Quarterly Journal of Experimental Psychology: Section A, 53(1), 153-164.

2. Palmer, K. K., Matsuyama, A. L., Irwin, J. M., Porter, J. M., \& Robinson, L. E. (2017). The effect of attentional focus cues on object control performance in elementary children. Physical Education and Sport Pedagogy, 22(6), 580-588.

\section{5}

\section{The effects of general and local muscle fatigue on anticipatory and compensatory postural adjustments under an external mechanical perturbation}

\author{
Mauro Nardon $^{1}$, Francesco Piscitelli $^{1}$, Enrico Tam ${ }^{1}$, Matteo \\ Bertucco \\ Università Di Verona, Department of Neurosciences, Biomedicine \\ and Movement Sciences, Verona, Italy ${ }^{1}$
}

Purpose: Neuromuscular fatigue is a physiological phenomenon caused by peripheral and central factors. To date, little is known about its effects on the neural processes regulating human postural control and adaptation (1). When facing an expected external perturbation, feedforward (anticipatory) and feedback (compensatory) mechanisms are both involved (2). The aim of the present study was to assess the effects of different fatiguing protocols on the neural processes that control posture by measuring the postural adjustments (EPAs, APAs and CPAs) to an external perturbation.

Methods: Fourteen healthy male subjects were exposed to pendulumlike mechanical perturbation before and after three different protocols of fatiguing exercise. Sessions were randomized and performed on separate days. During one session (Loc) participants were exposed to local muscle fatigue via sustained isometric contractions of a muscle involved in the task (m. tibialis anterior), while the other consisted in general fatigue (Gen): subjects performed an incremental arm-ergometer test until exhaustion. The latter session (Con) - serving as the control condition - consisted in sustained isometric contractions of a muscle not involved in the task ( $\mathrm{m}$. flexor carpi ulnaris). Subjects were exposed to a total of 41 perturbation trials: 12 before- (PRE) and 29 in the $30 \mathrm{~min}$ following the exercise (POST). Cardio-respiratory parameters were recorded throughout the test using a portable system (K5, Cosmed, Rome, Italy). Surface EMG signals from ventral and dorsal muscles of the dominant side were collected using a wireless system (Zerowire, Aurion, Italy).

Results: Cardiorespiratory and metabolic parameters were higher during the Gen session, while Con and Loc session elicited a similar cardiorespiratory response. In each session, we observed an increase of EPAs, APAs and CPAs for the m. tibialis anterior, especially for the first POST trials. For the Loc condition, we observed an increase of CPAs in the first trial POST.

Conclusions: Despite the differences across protocols, the three fatiguing protocols caused a similar effect on postural control. Mechanisms within the central nervous system (CNS) allow to compensate for disturbances due to neuromuscular fatigue (3). Local fatigue seems to challenge the vestibular/somatosensory component of the CNS to a lager extent. Recovery of postural adjustment parameters seems to be faster than the cardio-metabolic recovery.

\section{References}

1. Paillard T, (2012) Effects of general and local fatigue on postural control: A review. Neurosci Biobehav Rev 36 162-176.

2. Massion, J. (1992) Movement, posture and equilibrium: Interaction and coordination. Prog Neurobiol 38(1), 35-56.

3. Monjo, F., Terrier, R., \& Forestier, N. (2015) Muscle fatigue as an investigative tool in motor control: A review with new insights on internal models and posture-movement coordination. Hum Mov Sci 44, 225-233. 


\section{6}

Development of gross and fine motor competences and the effect of gross motor training on fine motor skills in primary school children

\author{
Vincenzo Sorgente $^{I}$, Erez James Cohen ${ }^{I}$, Riccardo Bravi ${ }^{1}$, Diego \\ Minciacchi \\ University of Florence, Department of Experimental and Clinical \\ Medicine, Florence, Italy ${ }^{1}$
}

Purpose: It is known that development of gross and of fine motor competence are tightly linked during childhood, being domains that share the same higher order neuromotor processes. However, interactions between gross motor practice and fine motor skills have not been adequately addressed to date. In this study we investigate the effects of short gross motor interventions on fine motor skills in primary school children. Specifically, we examined changes in fine motor proficiency following three different sets of gross motor practice sessions.

Methods: 240 boys and girls (ages 6-11) participated in this study. Fine motor skills were assessed at the baseline and post gross motor intervention, using the Box and Blocks Test. The gross motor practice sessions were based on the Test of Gross Motor Development-3rd Edition.

Results: Our findings show that gross and fine motor skills proficiencies correlate across all years of primary school, both improving significantly with age. Furthermore, significant differences were found for both gross and fine motor performances between children age 6-8 and children age $9-11$. Finally, it appears that short gross motor interventions do not influence significantly fine motor skills.

Conclusions: During the late childhood developmental stage, overall motor development is continuous but non-linear. From age 9 onwards there seems to be a major step-up in both gross and fine motor competence, which teachers/educators should be aware of to shape accordingly motor educational programs. Moreover, while gross and fine motor domains might be functionally integrated for enhancing children's motor performances, further research is needed to better clarify the effects of gross motor practice on fine motor skills enhancement.

\section{References}

1. Morgan P, Barnett L, Cliff D, Okely A, Scott H, Cohen K, and Lubans D. (2013) Fundamental movement skill interventions in youth: A systematic review and meta-analysis. Pediatrics, 132(5), e1361-e1383.

2. Dayem T, Salem E, and Hadidy E. (2015) Correlation between gross motor activities and hand writing skills in elementary school children. Trends in Applied Sciences Research, 10(5), 259.

197

\section{Does strength level influence the cognitive abilities of elementary school children?}

\section{Federico Abate Daga ${ }^{l}$, Samuel Agostino ${ }^{I}$, Luca Beratto ${ }^{l}$, Michela Calandretti ${ }^{1}$}

Adapted Training and Performance-Research Group, Università Degli Studi Di Torino, Torino, Italy ${ }^{l}$

Purpose: In the last decades, the population intelligence quotients declined and led to the Flynn effect's inversion (Bratsberg 2018). On the other hand, strength training promotes the creation and development of neuromuscular synapses (Peña-González et al., 2019). This study investigates the relationship between cognitive abilities and strength levels in prepubertal children attending elementary school.

Methods: 75 Children from an elementary school in the metropolitan city of Turin were recruited for this study. General strength was estimated based on the overhead medicine ball toss test, while cognitive abilities were evaluated using the digit span test, coming from the Wechsler Intelligence Scale for Children (WISC) (Kaufman, 1994).

Results: Demographic and anthropometric characteristics were: age 8 \pm 1 years, height $132,87 \pm 8,84 \mathrm{~cm}$, weight $29,45 \pm 5,88 \mathrm{~kg}$, BMI $16,89 \pm 2,22$. Finally, boys represented $60 \%$ of the sample, while female the $40 \%$. The results of the regression indicated that the model explained $54 \%$ of the variance and that the relationship between age and flexibility is powerful $\mathrm{F}(1,73)=30,039, \mathrm{p}<0,0001 ; \mathrm{R} 2=0,292$. Averagely, digit span score increases of 1,741 levels each unit of strength gained. Conclusion: Strength training could be an interesting way to increase the cognitive abilities of prepubertal children attending elementary school.

\section{References}

1. Bratsberg B, Rogeberg O. Flynn effect and its reversal are both environmentally caused. Proc Natl Acad Sci U S A. 2018 Jun 26;115(26):6674-6678.

2. Peña-González I, Fernández-Fernández J, Cervelló E, MoyaRamón M. Effect of biological maturation on strength-related adaptations in young soccer players. PLoS One. 2019;14(7):e0219355.

3. Alan S. Kaufman. Intelligent Testing with the WISC-III, New York, Wiley, 1994, ISBN 978-0-471-57,845-1.

\section{8}

Effects of closed- and open-skills sport practice on proactive and reactive motor inhibition via a mouse response-registration system

Riccardo Bravi ${ }^{l}$, Stefano Grasso ${ }^{1}$, Viola Benedetti ${ }^{2}$, Gioele Gavazzi ${ }^{2}$, Fabio Giovannelli ${ }^{2}$, Maria Pia Viggiano ${ }^{2}$, Diego Minciacchi ${ }^{1}$

University of Florence, Department of Experimental and Clinical Medicine, Florence, Italy ${ }^{1}$;

University of Florence, Section of Psychology-department of Neuroscience, Psychology, Drug Research and Child's Health (neurofarba), Florence, Italy ${ }^{2}$

Purpose: Human sport performance is a showcase of skilled cognitive-motor control. A key component of cognitive functions employed in a variety of sport context-related situations is the inhibitory motor control, defined as the ability to retain 'prepotent' response tendency and suppress inappropriate ongoing actions. Recently, research focused on evaluating influences on cognitive function exerted by different sport categories. Indeed, to understand how sport experience modulate the cognitive functioning of athletes might have implications not only for talent identification and development in sport but also encourage specific disciplines as potential intervention for populations with cognitive deficits. In this study we investigated the effect of open- and closed-skills sport practice on proactive and reactive inhibition. To serve this goal we employed for the first time a behavioral method based on a mouse response-registration system. Methods: We compared inhibitory control across tennis players $(\mathrm{n}=$ $15)$, swimmers $(n=16)$ and sedentary non-athletic controls $(n=16)$ for a total of 47 participants. Proactive and reactive inhibition was estimated by employing a non-sport-specific cued Go No-Go (GNG) 
and a Stop stop-signal task (SST), respectively, in which participants inhibited categorizing left/right arrows. For cued GNG, the accuracy rate in inhibiting action was estimated to assess proactive inhibitory performance; for SST, the Stop Signal Reaction Time (SSRT) was calculated to evaluate reactive inhibitory performance. Finally, the new proposed methodology gave the opportunity to explore whether the practice of sport would influence dynamic aspects of inhibitory control by introducing a one-shot and non-one-shot velocity profiles analysis.

Results: Accuracy rate in inhibiting action during No-Go trials showed no differences between tennis players and swimmers or athletes and non-athletes. Moreover, SSRTs were significant shorter for the tennis players and swimmers compared to non-athletes. However, no differences between athletes of different sport categories were observed. Finally, in both cued GNG and SST, velocity profiles analysis evidenced a higher proportion of non-one-shot movements in No-Go/Stop conditions in athletes than non-athletes.

Conclusions: Our findings confirmed that sport practice improves inhibitory motor control mechanisms. However, inhibitory functions do not benefit differently from different types of sport practice.

\section{References}

Duque J, Greenhouse I, Labruna L, Ivry RB. Physiological markers of motor inhibition during human behavior. Trends Neurosci. 2017, 40, 219-236.

Benedetti V, Gavazzi G, Giovannelli F, Bravi R, Giganti F, Minciacchi D, Mascalchi M, Cincotta M, Viggiano MP. Mouse Tracking to Explore Motor Inhibition Processes in Go/No-Go and Stop Signal Tasks. Brain Sci. 2020, 10, 464.

\section{9}

\section{Exploring the enjoyment impact on school performance promoted by active breaks in primary school}

Giulia Di Martino ${ }^{1}$, Alessandra Di Cagno ${ }^{2}$, Marco Centorbi ${ }^{3}$, Andrea Buonsenso, Sebastiano Riccio ${ }^{3}$, Giuseppe Calcagno ${ }^{3}$, Giovanni Fiorilli $^{3}$

Universita' Degli Studi Di Roma Foro Italico, Dipartimento Di Scienze Motorie, Umane E Della Salute, Rome, Italy ${ }^{1}$;

Universita' Degli Studi Di Roma "foro Italico", Dipartimento Di

Scienze Motorie, Umane E Della Salute, Rome, Italy ${ }^{2}$;

Universita' Degli Studi Del Molise, Dipartimento Di Medicina E

Scienze Della Salute, Campobasso, Italy ${ }^{3}$

Purpose: The increasing need to face the problem of sedentarism, especially in COVID-19 era, induced teachers and researchers to find new useful intervention methodologies in school context. Active Breaks (ABs) include brief periods of physical activity as a part of the curriculum. A new proposal of different active breaks between academic instruction periods in primary school has been evaluated in terms of attentive and mathematic skill improvements and enjoyment in a primary school.

Methods: A total of 141 children (aged $9.61 \pm 0.82$ ), divided in 6 classes, participated in this study. Each class was randomly assigned to three groups based on the type of protocol performed during the three ABs scheduled in a school day: fitness experimental group (FIT), creativity experimental group (CREAT) and control group $(\mathrm{CON})$. At baseline and at the end of interventions all participants underwent the Stroop Color and Word test (SCWT) and the Math test (MATH) to assess the level of attention and academic achievement, respectively. The degree of enjoyment was evaluated through the modified Physical Activity Enjoyment Scale (PACES).

Results: The Factorial ANOVA showed significant differences between the FIT and CON in MATH test $(p=0.023)$ and SCWT $(p=$
0.034). CREAT and FIT groups showed higher degree of enjoyment than CON one (both ps $<0.001$ ). Significant differences were found in MATH, where medium and high enjoyment groups had higher scores than low group ( $\mathrm{p}=0.040$ and $\mathrm{p}<0.001$, respectively); significant differences were found for time of execution in SCWT, where medium and high groups had better scores than low group $(\mathrm{p}=0.010$ and $\mathrm{p}<0.001$, respectively).

Conclusion: This study showed a positive acute impact of $\mathrm{ABs}$ interventions. These effects are more pronounced when these interventions enhance children's motivation and higher level of enjoyment [1]. FIT positively influenced attentive and math performances more than the CREAT, probably due to the correct work/rest ratio and executive rhythm which allowed children to reach a good level of exertion.

\section{References}

1.Chatzistamatiou, M., Dermitzaki, I., Efklides, A., \& Leondari, A. (2015). Motivational and affective determinants of self-regulatory strategy use in elementary school mathematics. Educational Psychology, 35(7), 835-850.

\section{0}

\section{How absolute and relative exercise intensities impact on quantitative and qualitative running gait indexes}

\author{
Laura Simoni $^{1}$, Alessandra Scarton ${ }^{2}$, Filippo Gerli $^{3}$, Filippo Gori $^{2}$, \\ Guido Pasquini ${ }^{3}$, Silvia Pogliaghi ${ }^{4}$ \\ University of Verona-Irccs Fondazione Don Gnocchi Onlus, \\ University of Verona, Firenze, Italy ${ }^{1}$; \\ Microgate, Microgate, Bolzano, Italy ${ }^{2}$; \\ Irccs Fondazione Don Gnocchi Onlus, Fondazione Don Gnocchi \\ Firenze, Firenze, Italy $^{3}$; \\ University of Verona, Unversity of Verona, Verona, Italy ${ }^{4}$
}

Purpose: Running gait analysis for clinical and training purposes is usually performed at a submaximal self-selected intensity to reduce tests' duration. If performed in a large range of absolute/relative intensities (i.e., speed and \% VO2max), it may provide relevant data on running patterns adaptations to the increase in running intensity. This study investigates running adaptations during a maximal incremental running test, in function of the increase in speed/\% VO2max. Methods: 29 runners $(27 \pm 11$ years, VO2max $51.6 \pm 9.3 \mathrm{~mL} / \mathrm{kg}$ / min) performed a maximal incremental test on treadmill. The $\%$ VO2max at each 1-min step was calculated by using a mobile ergospirometric device. Quantitative (QNTi) and qualitative (QLi) gait indexes were recorded each step by a photosensor system (Optogait, Microgate, Italy) and a video-based system (Graal, Microgate) 1,2. QNTis were: contact and flight time (CT, FT), cadence, step length (SL). QLis were: cadence and step length variability (vCad, vSL - by Optogait), Harmony and Synchrony Index (HI, SI-Graal). HI provides a synthetic measure of the global body movements variability, SI of the inter/intra-segmental coordination during gait. Indexes were linearly interpolated as a function of speed and \%VO2max. One-way ANOVA and Pearson's correlations were used to study gait indexes vs speed/\% VO2max relation.

Results: significant differences were found in all QNTis with the increase in speed/\%VO2max $(\mathrm{p}<0.0001)$ : CT decreased, FT, cadence, SL increased. For QLis, the increase in speed was related with a decrease of SI $(\mathrm{p}=0.017)$.

CT correlated positively with speed/\%VO2max while FT, Cadence, SL negatively $(\mathrm{p}<0.0001)$. vCad and vSL were negatively correlated with speed/\% VO2max ( $p<0.0001)$, HI did not correlate with them ( $\mathrm{p}$ $>0.05)$ and SI correlated negatively only with speed $(\mathrm{p}<0.0001)$. 
Conclusions: in our sample \% VO2max and speed influenced gait almost in the same way. Hence, in short duration runs even at severe intensities, fatigue may not affect gait patterns.

To run at high intensities, runners linearly decreased CT and increased FT, Cadence, SL. Speed/\%VO2max correlated significantly with $\mathrm{vCad}$ and $\mathrm{vSL}$ but a main effect was not found, reinforcing the hypothesis that the relation gait variability vs running intensity is not linear but U-shaped. The increase in speed lead to a decrease in SI, probably because the higher mechanical charge on body structures at high speeds makes more difficult to maintain a well-coordinated gai. References

1. Simoni, L., Scarton, A., Gerli, F., Macchi, C., Gori, F., Pasquini, G., \& Pogliaghi, S. (2020). Testing the Performance of an Innovative Markerless Technique for Quantitative and Qualitative Gait Analysis. Sensors, 20(22), 6654.

2. Simoni, L., Scarton, A., Macchi, C., Gori, F., Pasquini, G., \& Pogliaghi, S. (2021). Quantitative and Qualitative Running Gait Analysis through an Innovative Video-Based Approach. Sensors, 21(9), 2977.

\section{HEARTH HEALTH and SPORT}

\section{1}

\section{Extended sports cardiology screening reveals myocardial bridge in a symptomatic young athlete}

\author{
Mariarita Brancaccio ${ }^{1}$, Cristina Mennitti ${ }^{1}$, Arturo Cesaro $^{2}$, Valeria \\ D'argenio $^{3}$, Giorgio Casaburi ${ }^{4}$, Ferdinando Barretta ${ }^{1}$, Fabiana \\ Uomo $^{1}$, Cristina Mazzaccara ${ }^{1}$, Giovanni D'alicandro ${ }^{5}$, Emanuele \\ Monda ${ }^{6}$, Giuseppe Limongelli, ${ }^{6}$ Paolo Calabro ${ }^{2}$, Daniela \\ Terracciano $^{7}$, Barbara Lombardo ${ }^{1}$, Giulia Frisso ${ }^{1}$, Olga Scudiero ${ }^{1}$ \\ University of Naples Federico Ii, Department of Molecular Medicine \\ and Medical Biotechnology, Napoli, Italy ${ }^{I}$; \\ Università Degli Studi Della Campania, Luigi Vanvitelli, Department \\ of Translational Medical Sciences, Napoli, Italy ${ }^{2}$; \\ Ceinge Biotecnologie Avanzate, S.c.a.r.l, Napoli, Italy; \\ Prescient Metabiomics, San Diego County, California, Stati Uniti D' \\ America $^{4}$; \\ Aorn, Santobono-pausillipon, Centro Di Medicina Dello Sport E \\ Delle Disabilità, Dipartimento Di Neuroscienze E Riabilitazione, \\ Napoli, Italy; \\ Università Degli Studi Della Campania, Luigi Vanvitelli, Department \\ of Cardio-thoracic and Respiratory Sciences, Napoli, Italy ; \\ University of Naples Federico Ii, Department of Translational \\ Medical Sciences, Napoli, Italy
}

Laboratory medicine, along with genetic investigations, is playing an increasingly crucial role in sports medicine to monitor the athlete's health condition. Acute or intense exercise can lead to metabolic imbalances, muscle injuries or can emphasizes cardiovascular disorders. The aim of this study was to monitor the health status of a basketball player both through biochemical and genetic investigations, to shed light on the causes of repeated fainting that occurred during training. The results obtained showed that the athlete had abnormal iron, ferritin and bilirubin levels. However, the Multilayer Computed Tomography was the key investigation, showing the presence of an intramyocardial bridge. To understand if it could be caused by an inherited genetic condition, both whole exome sequencing (WES) and array-CGH were performed. WES highlighted the genotype compatible with the Gilbert syndrome the athlete suffered from; no pathogenic mutation related to the patient's cardiological phenotype was detected. The array-CGH showed two intronic macrodeletions, in the MACROD2 and COMMD10 genes.
Both genes are expressed in human embryonic cardiac cells, endothelial and smooth muscle cells. Furthermore, MACROD2 polymorphisms have been associated to development of Transposition of the Great Arteries. Therefore, we could hypothesize a role for MACROD2 and COMMD10 genes in the heart developing, thereby contributing, at least in part, to the development of congenital heart disorders, including myocardial bridge.

In conclusion, this case report highlights the need to perform a thorough clinical / laboratory / instrumental evaluation in athletes. Moreover, in the presence of suspected signs of myocardial ischaemia. The current finding must push to plan more adequate prevention paths to create ad personam training and treatments, thus avoiding the appearance of diseases and injuries which, if underestimated, can become irreversible disorders leading, in some cases, to the death of the athlete.

PHYSICAL EDUCATIONA and SPORT PEDAGOGY.

\section{2}

"Moving in the woods" - a preliminary observational study of physical activity in green space

\author{
Nicola Rovetti ${ }^{1},{\text { Alessandro } \text { Ongaro }^{2}, \text { Massimo Lanza }^{1}, \text { Matteo }}^{1}$ \\ Bertucco
}

University of Verona, Department of Neurosciences, Biomedicine and Movement Sciences, Verona, Italy ${ }^{1}$;

University of Verona, Department of Human Sciences, Verona, Italy ${ }^{2}$

Purpose: It is well known that exercising in the natural environments is associated with positive effects for children and youth. Particularly, access to green spaces Results with improved mental and general well-being. The aim of the current study was to investigate the effects of physical activity where children practiced the fundamental motor skills in complex and unpredictable natural environments. The objective was to have children exploiting motor skill in unusual and novel contexts that are scarcely experienced in indoor environments, such as gym or schools.

Methods: 148 children aged between 8 to 12 years were recruited for the study during the period 2018-2021. They exercised either $2 \mathrm{~h}$ twice a week for a year or during an intensive week daily for $8 \mathrm{~h}(8: 30$ am-4:40 pm). During the activity the children were required to perform diversified motor skills, such as tree climbing, jumping over the rocks, sloth crawls, quadrupedal movements etc., in green space context as woods, brushwood, undergrowth, and scrubs. Children were asked to perform and complete the motor tasks through motor explorations and problem-solving approaches. Physical Activity Enjoyment Scale was used to evaluate the level of appreciation the activity.

Results: Children showed a meaningful level of satisfaction of the activity, which was also confirmed by a large rate of enrollment of the children to the next season. Namely, $85 \%, 74 \%$ and $85 \%$ of the children chose to take part to the following season classes between 2018 and 2019, 2019 and 2020 and 2020 and 2021 respectively.

Conclusions: The promising preliminary results of this observational study allow us to envision future research to quantify the level of satisfaction, levels of physical activity, self-esteem and self-efficacy while exercising diversified nature-based learning environments, and eventually to investigate its effect on psychosocial and physiological health outcomes.

\section{References}

1. Monti F, Farné R, Crudeli F, Agostini F, Minelli M, and Ceciliani A. (2017) The role of Outdoor Education in child development in Italyn nursery schools. Early Child Dev Care 189(6): 867-882. 
2. Mnich C, Weyland S, Jekauc D, Schipperijn J. (2019) Psychosocial and Physiological Health Outcomes of Green Exercise in Children and Adolescents-A Systematic Review. (2019) Int J Environ Res Public Health 16(21):4266.

203

\section{PE teacher and kinesiologist profile and relationship to coherence and congruence of european credits transfer system and educational goal of degree courses in exercise and sports science}

\section{Francesca D'elia ${ }^{1}$, Gaetano Raiola ${ }^{2}$}

Università Degli Studi Di Salerno, Dipartimento Di Scienze Umane, Filosofiche E Della Formazione, Fisciano (sa), Italy ${ }^{1}$;

Università Degli Studi Di Salerno, Dipartimento Di Studi Politici E Sociali, Fisciano (sa), Italy ${ }^{2}$

Purpose: The sport reform has established the new professional kinesiologist profile, access to which is allowed only to graduates in exercise and sports sciences from L22, LM67 and LM68 degree courses. Study plans of the 3 degree courses, in addition to being consistent with the educational objectives of the physical education teacher, also need to be consistent with the profile of the kinesiologist. The problem is, therefore, to evaluate overall the quantity of European credits transfer system (ECTS) of the 3 most significant educational areas for its coherence to educational objectives: Physical and sport activity (PSA), Biomedical and Psycho-pedagogical. The aim is to verify, on the entire population of the degree courses, the relationship between ECTS of the PSA, psycho-pedagogical and biomedical educational areas, to assess whether they are sufficient to achieve the educational objectives as well as the physical education teacher, also of the kinesiologist and are part of a systemic action of the degree courses.

Methods: The population of the degree courses consists of $n^{\circ} 41$ of L22, $n^{\circ} 31$ of LM67 and ${ }^{\circ} 20$ of LM68. After verifying the normality of data with the Shapiro Wilk test, central tendency and dispersion indices were calculated to analyze the number of credits of the 3 educational areas. Multiple regression was used to verify the relationship between degree courses and the ECTS of the three educational areas of the complexity of the degree courses. Data were analyzed using SPSS.

Results: The result is statistically significant between degree courses and PSA-biomedical areas $(\mathrm{p}<0.05)$, but not between degree courses and psycho-pedagogical ones $(\mathrm{p}>0.05)$. The ECTS in PSA, biomedical and psycho-pedagogical areas are 2132, 2552 and 855 in L22; 844, 1096 and 315 in LM67; 718, 539 and 172 in LM68 with mean, median, mode, as well as different SD.

Discussion: ECTS in the psycho-pedagogical area, not depending on degree course as it is for the other two educational areas, are not part of the system's formation. The other 2 areas, on the other hand, have a homogeneous distribution and therefore fall within it. The lower overall number of ECTS in the PSA educational area compared to the biomedical one makes the training path not congruent with respect to the qualifying educational objectives and the fluctuation of the average, median and mode values as well as DS, makes the training even less congruent with respect to the 2 professional profiles.

References

1. D'Elia F. (2019). The core curriculum of University training to teach physical education in Italy, JOURNAL of PHYSICAL EDUCATION and SPORT. Vol. 19 (Supplement issue 5), $1755-1758$.
2. Sanseviero, I.; Cassese, F. P.; Fonzo, E.; Altavilla, G.; D’Elia, F. (2019). Study on the master's degree in sciences of sports evaluation and sport for disabled at the University of Salerno. JOURNAL of HUMAN SPORT and EXERCISE, 14(Proc2), S239-S244.

\section{4}

\section{Physical education and integrated digital teaching in accordance with the global approach}

\author{
Sara Sofia Odisio ${ }^{1}$, Stefania Cazzoli ${ }^{2}$
}

Federazione Internazionale Di Educazione Fisica -fiep, Liceo Sportivo P. Levi Torino, Torino, Italy ${ }^{I}$;

Federazione Internazionale Di Educazione Fisica -fiep, Università Degli Studi Torino Suism- Dfe, Torino, Italy ${ }^{2}$

Purpose: Physical education was a subject of curriculum schoolbased. The aim was to support the student (0-19 aged) in motor learning. They could move effectively, efficiently and understanding what they did, from school to life (empowerment, individual decision making for active lifestyle and long-life learning) in accordance with the International Charter of Physical Education, Physical Activity and Sport (UNESCO, 2015). In the global pandemic COVID19 emergency, education and PE need to rethinking and replanning the teaching and learning processes.

Methods: Analysis of international general education documents for guarantee right and access at quality PE in accordance with OECD (2020). Remote learning has become a fundamental tool for education. Digital technology was a temporary solution during a crisis. Distance learning was a revolution for speed and pervasiveness in a global world. In this period the use of technologies digital-based became in short time opportunity for learning and teaching in remote, but it needs the design of the new Integrated Digital Education (IDE) scenarios. (OECD, 2020).

Results: Showed a higher percentual of teachers they belive quite bit in the support student by use of the technologies 65\% (OECD media) and lower percentual of teachers use frequently or always the ITC in the classwork or in homeschooling 50\% (OECD media) Fig. 1 OECD Library.

The OECD guidelines (2020) for emergency management showed to rethink the didactic choices and the organization of the curriculum: adoption of collaborative, multidisciplinary, cross-curricular, interdisciplinary learning/teaching models in accordance with the step: prepare; cope; recover; evaluate/reflect; Plan of transformation (Azzi and Shims, 2020) Fig. 2.

Conclusions: Considering the whole range of Changement of education in the global pandemic COVID19, the PE by ITC were important to support at the teaching/learning processes for guarantee the right at education in presence or hybrid or remote educational setting. The rethinking and re-planning PE items were: a. space was in accordance with the social distance (presence or remote modality, indoor or outdoor); the time was blended in synchrony and asynchrony; the content became a learning task with the mobilization of the personal and collaborative activities; teachers were facilitators of new learning setting based on the needs of the students. (learning personalization).

\section{References}

1. Azzi-H (2020), Managing the impact of COVID-19 on education systems around the world: How countries are preparing, coping, and planning for recovery World Bank Education COVID-19 Monitoring. 
2. OECD (2020) Supporting the continuation of teaching and learning during the COVID-19 Pandemic - Annotated resources for online learning \& The impact of Covid-19 on education Insights from Education at a glance 2020.

3. UNESCO (2015) International Charter of Physical Education, Physical Activity and Sport.

\section{5}

\section{Physical education and integrated digital teaching: sustainable and ecological proposal}

\section{Stefania Cazzoli \\ Federazione Internazionale Di Educazione Fisica -fiep, Università Degli Studi Di Torino- Suism E Dfe, Torino, Italy ${ }^{I}$}

Purpose: Physical education was a subject of curriculum schoolbased. The aim was to support the student (0-19 aged) in motor learning and to move effectively, efficiently and understanding what they did, from school to life (empowerment, individual decision making for active lifestyle and long-life learning) in accordance with the International Charter of Physical Education, Physical Activity and Sport (UNESCO, 2015) and the international general education documents for guarantee right and access at quality PE in accordance with OECD (2020). In the global pandemic COVID19 emergency, education and PE need to rethinking and replanning the teaching and learning processes.

Methods: from the theoretical paradigm of the Integrated Digital Teaching (IDT) (OECD, 2020) to quality PE in accordance with OECD Learning Compass 2030 (2016) Fig. 1. The metaphor learning compass showed the new students competencies: knowledge, skills, attitudes, and values... for to reach the goal of well-being with PE school-based. The IDT for PE need rethinking and replanning sustainable and ecological proposal based on the movement learning in new space (from the gym space to the social space in according with the social distancing $2 \times 2 \mathrm{~m}$ ) for guarantee the access at PE in presence/remote/hybrid setting; new time (synchrony or asynchrony); new learning setting based on short, cooperative learning, projectbased learning, flipped classroom, Debate (OECD, 2020; MIUR 2020). Figure 2

Results showed the planning the PE setting from sport-based pratice versus a. Starting from deliberate practice, moving towards theory (project-based learning) (EF laboratory activity; b. Starting from experience (movement) towards the core of knowledge, skills, and competence, attitude, and value (Flipped classroom); c. With reflections and discussions (debate, cooperative learning); $d$. the teacher was facilitator and organizes the new learning settings by preparing new learning situations and start from the affordance of the environment, tools, situations of the groups of students integrated by digital technologies.

Conclusion: Considering the whole range of Changement of education in the global pandemic COVID19, the PE by ITC was important to support the teaching/learning processes and the teacher flexibility to rethinking and replanning the PE school-based and centered on the learning needs of the person and all the students for learning and arrive at the standard goals of the national curricula.

\section{References}

1. OECD (2020) Supporting the continuation of teaching and learning during the COVID-19 Pandemic - Annotated resources for online learning \& The impact of Covid-19 on education Insights from Education at a glance 2020.

2. MIUR (2020) Linee guida per la Didattica digitale integrate.
3. UNESCO (2015) International Charter Physical Education, Physical activity and Sport.

\section{6}

\section{Physical education in the age of COVID-19: a narrative} review of the literature

\author{
$\frac{\text { Attilio Carraro }^{1}}{\text { Lanza }^{4}}$, Alessandra Colangelo ${ }^{2}$, Dario Colella $^{3}$, Massimo
}

Libera Università Di Bolzano, Facoltà Di Scienze Della Formazione, Bressanone, Italy ${ }^{1}$, Università Di Padova, Fisppa, Padova, Italy ${ }^{2}$, Università Di Foggia, Department of Humanities, Cultural Heritage, Education Sciences, Foggia, Italy ${ }^{3}$, Università Di Verona,

Department of Neurosciences, Biomedicine and Movement Sciences, Verona, Italy ${ }^{4}$

Background: Physical education (PE) has been usually considered as a practical school subject, where close proximity and body contact represents the "norm". The COVID-19 pandemic has dramatically changed PE delivery and routines, modifying teaching contents and strategies, with online teaching that often replaced traditional face-toface modalities.

Purpose: The aim of this study is to present the results of a narrative review of studies regarding the teaching of school PE during the COVID-19 pandemic, reporting the main issues tackled and research methods adopted.

Methods: A search with EBSCO, ERIC, Human Kinetics Journals, PubMed, Scopus, SPORTDiscus, Web of Science was conducted, using "Physical Education" \& "Covid-19" as keywords.

Results: A total of 52 documents which contained in the title the two key-words was found. Six papers were excluded because not related to PE; other 20 were excluded since authors did not consider questions directly connected with $\mathrm{PE}$ teaching (6 regarded higher education in PE, $4 \mathrm{PE}$ teacher education, $4 \mathrm{PE}$ teachers health, 2 the role of $\mathrm{PE}$ for health, 2 research on PE during the Covid-19 pandemic, and 2 out-of-school PE). Twenty-six papers were finally included in the review because they were directly linked to school PE teaching/ learning and particularly to online teaching. Twenty articles out of the 26 were journal articles, the rest were abstracts (3), commentary (1), author's position (1), editorial (1). The majority of the studies used qualitative methods (14), followed by quantitative method (11). Online survey (9) was the most frequent method used for quantitative data collection, while semi-structured interview (4) was the most popular method to collect qualitative data, followed by case study (3). Other methods used were focus group (1), pedagogical cases (1), reflective journals (1), discussion groups (1), photovoice (1), interviews (1), web-based analysis of blog concerning PE (1), PRISMA literature review (1), participant-produced drawings (1).

Conclusions: Throughout school closures and social restrictions imposed by the Covid-19 pandemic, PE teachers and students faced the enormous challenge to replace traditional in-presence teaching with online teaching, radically transforming discipline contents, methodologies, practices, and communication strategies. The implications of these changes could significantly contribute to redesign future PE.

A list of references will be shared with conference attendants. 


\section{7}

\section{Preliminary survey on the feasibility} of the "Körperkoordinations test für kinder" (KTK) for the assessment of gross-motor coordination in young people over $14 y$.

\author{
Massimo Lanza ${ }^{1}$, Ludovica Fiocco ${ }^{2}$, Matteo Giuriato $^{3}$, Valentina \\ Biino $^{4}$
}

Department of Neuroscience, Biomedicine and Movement., University of Verona, Verona, Italy ${ }^{I}$;

School University of Medicine and Surgery, University of Verona,

Verona, Italy ${ }^{2}$;

Faculty of Physical Culture, Department of Health and Natural

Sciences, Unit of Molecular Biology, University of Physical

Education and Sport, Gdansk, Polonia ${ }^{3}$;

Department of Human Sciences, University of Verona, Verona, Italy ${ }^{4}$

Purpose: Gross motor coordination significantly influences the adoption of an active lifestyle and development at a young age. KTK (Kiphard, 1974) is a validated tool for its assessment, but it has reference values only up to 14 years. The main goal is to verify the feasibility of KTK in young people over 14 years of age. Secondary goals are to collect preliminary data on the differences in results between genders and in the different age groups investigated.

Methods: The KTK test was performed by 231 subjects ( 123 females; 108 males), 206 secondary school students and by 25 other more adults.

Results: The qualitative evaluation of the test highlighted its feasibility without adverse events and within the times provided by the authors (15-20 min per subject) even in the conditions of hygienic safety imposed by the COVID-19 pandemic. For some subjects, it was necessary to have the tests performed away from their peers to reduce anxiety and fear of making mistakes. Quantitative measurements showed that the scores of three out of four KTK's sub-tests and the overall value (Raw score-RS) are positively related with age (WB: $r$ $=0.18, \mathrm{p}<0.01 ; \mathrm{MS}: \mathrm{r}=0.20, \mathrm{p}<0.01$;H: $\mathrm{r}=0.16, \mathrm{p}<0.05$; RS: $\mathrm{r}$ $=0.24, \mathrm{p} .001)$. The RS shows significant differences between age groups $(\mathrm{p}<0.001)$, between genders $(\mathrm{p}<0.001)$ and interaction between genders and age groups $(\mathrm{p}<0.001)$. In particular, in males, age groups show no differences, while females over 20 significantly worsen performance. Overall, males show better results than females in the RS $(\mathrm{M}=280.10 \pm 25.70 ; \mathrm{F}=256.52 \pm 29.19 ; \mathrm{p}<0.001)$ and in three of the four sub-tests (JS, MS, HH-for all $\mathrm{p}<0.001$ ) while females perform better than males in the MB test $(\mathrm{p}<0.001) .12 .12 \%$ and $38.96 \%$ of the subjects obtained the highest possible score in the WB and $\mathrm{HH}$ sub-tests respectively, highlighting a significant "ceiling effect" at least for $\mathrm{HH}$.

Conclusions: The data seems to indicate that the KTK can also be used for individuals over the age of 14. Further data collection is underway to create a database with validated reference parameters for these age groups. Attention should be paid to the monopodalic jump test $(\mathrm{HH})$, where a "ceiling effect" was found for a significant part of the subjects. In these age groups, the hypothesis of using the "KTK 3" (Coppens, 2021), which does not adopt the HH subtest, can be considered. The small number of subjects makes the results by gender and by age only indicative.

References

1. Kiphard, E. J., \& Schilling, F., 1974. Körperkoordinationstest für Kinder. Weinheim: Beltz Test GmbH.

2. Coppens E., Laureys F. Mostaert M., D’Hondt E., Deconinck F.J. A., Lenoir M., 2021. Validation of a Motor Competence Assessment Tool for Children and Adolescents $($ KTK3 +) With Normative Values for 6- to 19-Year-Olds Front. Physiol., 23 June 2021 https://doi.org/10.3389/fphys.2021.652952
208

Relationship among specific learning disability, sport and academic achievement in middle and high school. An explorational study in the school of veneto

\author{
Massimiliano Marino $^{1}$, Andrea Bano ${ }^{1}$
}

University of Padua, Department of Biomedical Sciences, Padova, Italy $^{I}$

Purpose: Specific Learning Disabilities (SLD) are conditions that regard both cognitive and motor development. The relationship between SLD and motor development is evident in literature and it is well known that physical activity can be an important factor to support cognitive development. Thereafter, literature underlines that students with SLD that are involved in sport could achieve better academic Results than their sedentary peers. The aim of this study is to explore relationship among academic performance, SLD and sport in middle (MS) and high school (HS) students.

Methods: This study analysed school grades of 7912 students in Veneto (2844 MS; 5068 HS) crossing data with presence of SLD and sport practice. The data were collected using Google Form asking students to indicate their first quarter school report, information about the sport they practice and presence of SLD.

Results: Results showed a higher percentage of student with SLD compared to the year 2017-18 in Veneto. The data revealed that students with SLD were quite less involved in sport than their peers and that, during the lockdown period, the percentage of students with SLD who practiced sport decreased from $67 \%$ to ca $41 \%$. Moreover, we found an important role of gender, SLD, and sport practice on school grades. In both school grades, female, students without SLD and students who practice sport showed better grades in several school subject (e.g.: Italyn, Math, PE, Technology) than, respectively, male, students with SLD and non-active students $(p<0.001)$. Furthermore, MS students with SLD that practice sport showed better grades than students without SLD in Italyn $(\mathrm{p}<0.05)$ and Math, PE, Story, Geography and Science $(\mathrm{p}<0.001)$. HS students with SLD that practice sport showed better grades in Story $(\mathrm{p}<0.05)$ and Math and PE $(\mathrm{p}<0.001)$.

Conclusions: This study is aligned with the hypothesis, widely spread in literature, that motor and SLD development are strictly connected. Results underlined the important role of sport on the school path of all students, included those with SLD, in obtaining better academic performance. However, students with SLD seems to had more barriers in sport participation in general and during the lockdown period.

\section{References}

Carson V, et al. (2016) Systematic review of physical activity and cognitive development in early childhood. J Sci Med Sport 19(7): 573-578.

Fedewa AL, and Ahn S. (2011) The effects of physical activity and physical fitness on children's achievement and cognitive outcomes: a meta-analysis. Res Q Exerc Sport 82(3): 521-535.

\section{9}

\section{Rhythmic education: a proposal for an exercise} programme for young gymnasts

\author{
Amalia Tinto $^{1}$, Beatrice Bolla ${ }^{2}$, Emanuela Maccarani ${ }^{3}$, Marta \\ Campanella ${ }^{4}$ \\ Università Degli Studi Di Torino, Suism- Centro Servizi, Torino, \\ Italy $^{1}$; \\ Università Degli Studi Di Torino, Suism-Centro Servizi, Torino,
}


Italy $^{2}$;

Fgi, Accademia Internazionale Di Ginnastica, Desio, Italy ${ }^{3}$; Università Degli Studi Di Torino, Suism-Centro Servizi, Torino, Italy $^{4}$

Purpose: The aim of the work is to examine the effects of a training workout with specific rhythmic proposals for a group of young athletes who practice Rhythmic Gymnastics (GR). The GR group requires a perfect partnership between movement and music (FGI, 2017-2020) and to succeed in making the gymnasts aware by increasing their rhythmic skill level becomes a necessary prerequisite in order for them to be good interpreters of the discipline.

Methods: The sample group was made up of 9 gymnasts from 8 to 14 years old, who were involved in regional Italyn Gymnastic Federation competitions. The work protocol involved carrying out a workout plan, characterized by the use of rhythms in binary and ternary time. In the last two phases of the exercise programme two small pieces of equipment (a ball and a rope) were also used. The rhythmic performance was marked by a metronome. The validity of the proposal was explored through the execution of 5 types of tests that included a rhythmic part and a part without a rhythmic base to evaluate the internalization of the rhythm on the part of the athlete. Results: Some differences between the values of the parameters of T0 and $\mathrm{T} 1$ were registered. All of the gymnasts reduced the number of errors in the final tests, demonstrating an excellent command in their handling of rhythm achieved during the workout.

Conclusions: Working on rhythm with specific drills is the key to creating stable technical skills and allows for the continuing evolutionary process in perfecting movements over time. The GR underlines this process, but the workouts should always be designed by starting from a rhythmic base and, above all, the same should represent the majority of the work with young gymnasts.

Scoring Code, 2017-2020, Gymnastic Federation of Italy.

\section{0}

\section{A longitudinal and allometric approach of gross motor coordination during growth in male children}

\author{
Matteo Giuriato $^{1}$, Nicola Lovecchio ${ }^{2}$, Valentina Biino ${ }^{1}$ \\ University of Verona, Department of Neurosciences, Biomedicine \\ and Movement Sciences, University of Verona, Verona, Italy ; \\ University of Bergamo, Dep. of Human and Social Science, \\ University of Bergamo, Bergamo, Italy ${ }^{2}$
}

Purpose: Longitudinal study was important to have a robust approach to consider the variation of growth. Further, allometric modeling permits to avoid incorrect conclusions and misinterpretation of data, accounting separate the developmental growth and maturation factors from other components. The goal of the study was to assess Gross Motor Coordination (GMC) longitudinally, considering anthropometric factors and physical activity (PA) through allometric scaling methodology.

Methods: 50 children (only boys) were recruited from North-East Italy. Subjects were assessed from 2017 to 2020. GMC was analyzed with KTK3 while the anthropometric characteristics were, simultaneously, collected. PA was evaluated with a questionnaire (PAQ-C) during 2017, -18 , and -20 .

Results: Allometric modeling suggested nonlinear associations with GMC across the children's developmental years. Further, ectomorph children seem to perform better because of lean body mass. PAQ-C was significantly associated with GMC from 8 to 13 years of age.

Conclusions: Growth does not respect linear trends. Lean body mass could be evaluated as a reliable predictor of GMC in children. Gold period to improve GMC seems in 8-10 years. Indeed from 10 to $13-\mathrm{y}$, it was marked maintenance.

\section{References}

1. Nevill, A. M., Holder, R. L., Baxter-Jones, A., Round, J. M., \& Jones, D. A. (1998). Modeling developmental changes in strength and aerobic power in children. Journal of applied physiology (Bethesda, Md.: 1985), 84(3), 963-970. https://doi.org/10.1152/ jappl.1998.84.3.963

2. Novak, A. R., Bennett, K. M., Beavan, A., Pion, J., Spiteri, T., Fransen, J., Lenoir, M. (2017) The Applicability of a Short Form of the Körperkoordinationstest für Kinder for Measuring Motor Competence in Children Aged 6 to 11.

\section{1}

\section{Soft skills and sports practice in the childhood: an explorative survey}

\section{Pasqualina Forte $^{1}$, Valeria Minghelli $^{2}$, Cristiana D'anna ${ }^{2}$}

Universita’ Degli Studi Del Molise, Dipartimento Di Medicina E Scienze Della Salute, Campobasso, Italy ${ }^{1}$, Universita' Degli Studi Di Salerno, Dipartimento Di Scienze Umane, Filosofiche E Della Formazione, Salerno, Italy ${ }^{2}$

Purpose: Although there is no exhaustive definition of soft skills, they are described by the European Union and the OECD (2006) as those skills that everyone needs for personal development and fulfillment, active citizenship social inclusion and employment.

Soft skills are the result of a combination of knowledge, skills and attitudes and represent for young people indispensable tools to successfully face the transition to adulthood.

One of the learning contexts that is absolutely fertile in promoting the development of these skills can be found in the physical education and sport activities that promote the integrated development of the individual, physical, emotional, intellectual, social and productive human capital (Bailey et al., 2013).

The objective of this study is to carry out an exploratory survey that focuses on the development of social and emotional skills, in relation to sports practice, to understand if there are differences between who conducts a sedentary lifestyle, and who plays a sport. Methods: The survey was conducted through the administration of the Big Five Questionnaire for Children (BFQ-C) (Barbanarelli et al., 2003), internationally validated tool for the evaluation of social and emotional learning: a taxonomy that crosses the skills, measuring, through 65 items, five macro factors such as Extraversion/Energy, Agreeableness, Conscientiousness, Neuroticism/Emotional instability, and Openness/Intellect.

The study examined a sample of students $(\mathrm{N}=118)$ aged from 8 to 14. The self-completed questionnaire was sent to the students via the school's digital platform.

Results: The data were processed with SPSS v.23 statistical software; the five factors of the BFQ-C, analyzed through an ANOVA with comparison between groups (gender, school grades, sports practice), tend to have higher mean values in subjects who practice sport at a competitive level, highlighting the need to understand more this trend through a survey on a larger sample, analyzing the differences between individual and team sports.

Conclusions: Participation in sports have been positively associated with the development of personal and social skills by numerous studies. It is necessary to continue to investigate the role played by sport in promoting soft skills which represent not only the opportunity to learn more successfully, but also the possibility to overcome the transition phase into adult life. 


\section{References}

1. Bailey, R., Hilman, C., Arent, S. \& Petitpas, A. (2013). Physical activity: an Understimated investement in human capital? Journal of Physical Activity and Health, 10, pp. 289-308.

2. Barbaranelli, C., Caprara, G.V., Rabasca, A. (2003). BFQ-C. Big Five Questionnaire-Children. Manuale. Firenze: Giunti O.S.

3. OECD (2006). Uno sguardo sull'educazione. Gli indicatori OCSE 2006. Roma: Armando.

\section{2}

\section{Sport intended as a pedagogical speech and not only competitive}

Francesco Perrotta ${ }^{l}$

Unimol, Dipartimento Scienze Umanistiche, Campobasso, Italy ${ }^{I}$

The sport practiced in Italy involves millions of young people with passion and dedication to train, play and compete under the constant guidance of physical education teachers, coaches and instructors. From my reflection, I wonder why sport is not education in itself, like all other human educational activities. Being based on the physical and athletic difference that is increasingly exalted and emphasized in sports competition, sport is not for everyone and anyone who has had experience in athletics such as racing knows that the important thing is not to participate, but that the very structure of the sports is about looking for victory and you play to win. The task of the instructors, coaches is to lightly move accents where sport is practiced at a youth level: youth sport has its dynamics and its specificity if it wants to be educational, and therefore it must be something more and something different from just sport. Then it should be possible for a teacher or coach who works for the practice of a sports education, focusing on the material elements of the sporting event, such as the rules of the game, the materials, the field, the opponents and transforming them into options education.

\section{References}

1. Muzio, M. (1988).Psicopedagogia dello sport. Roma: Edi Ermes.Norman, D.A. (1981).Memoria e attenzione. Roma: Franco Angeli Editore

2. Oliverio, A. (1986). Biologia e comportamento. Roma: Zanichelli

3. Oserov, P. (1984). Sviluppo psicomotorio degli atleti. Roma: Società Stampa Sportiva

4. Petter, G. (1984). Lo sviluppo mentale nelle ricerche di Jean Piaget. Roma: Giunti Barbera.

5. Piaget, J. (1967)

\section{3}

\section{The effects of physical activity on motor and psychomotor development in childhood}

\author{
Rosanna Perrone ${ }^{l}$, Lucia Pallonetto ${ }^{l}$, Carmen Palumbo ${ }^{1}$
}

Università Degli Studi Di Salerno, Dipartimento Di Scienze Umane, Filosofiche E Della Formazione, Fisciano(sa), Italy ${ }^{1}$

Purpose: Motor activity in developmental age is essential in order to consolidate targeted behaviors that will allow each individual to be able to act in a planned and conscious manner (Goodway J.D., 2003). Sports practice is the basis of psychomotor and organic development, a means of promoting learning and self-learning, (Lapierre A., 2001) whether it occurs by imitation or by exploration. Movement is at the basis of personality development, to respond to the natural needs of socialization, personalization, the establishment of symmetrical and complementary relationships (Miato A., 2007). The objective of the research was born from the intent to investigate the actual relevance of children who practice physical activity or sports in developmental age.

Methods: The research was conducted in the field and is of a qualitative-quantitative type and the sample investigated is made up of 281 children between 6 and 8 years of age. The sample was given a questionnaire to find out how many practiced sports; after obtaining this data, the same sample was administered the APCM-2 protocol (Sabbadini L., 2018), a specific psychomotor screening tool at the service of the clinician for the diagnosis of movement disorder or psychomotor development atypia.

Results: From the two micro-samples detected, following the qualitative data obtained with respect to whether physical activity was carried out, differences in both functional and psychomotor performance can be deduced. In fact, the average of children who practice sports is fairly higher than children who do not practice it, a result that confirms how much movement contributes to improving praxic-motor performance.

Conclusions: Physical activity and motor education allow you to counter the difficulties that natural morphofunctional changes entail. Motor activity produces significant effects on motor patterns, contributing to the general motor development of the individual, but to promote physical and sporting activity, especially among the youngest, educators must act in this sense with innovative and diversified teaching proposals. The school needs to redefine the objectives and disciplinary contents of physical education, starting with good practices, also according to the desirable inclusion of the figure of the specialized teacher (Colella D., 2020).

\section{References}

1. Ceciliani, A. (2020) L'educazione motoria nella scuola primaria. Roma: Carocci Editore;

2. Lapierre, A., (2001) Dalla psicomotricità relazionale all'analisi corporea della relazione, Roma: Armando editore;

3. Sabbadini, L., (2018) Manuale APCM-2, Abilità Prassiche e della Coordinazione Motoria 2a Edizione, Firenze: Hogrefe Editore;

4. Secli, P., Ceciliani, A., (2014) Metodi e strumenti per l'insegnamento e l'apprendimento delle scienze motorie. I quaderni della didattica, Napoli: Edises.

\section{4}

The formative value of motor education through bodily expression activities for an inclusive body

\section{Lucia Pallonetto ${ }^{1}$, Carmen Palumbo ${ }^{1}$}

Istituzione, Universita' Degli Studi Di Salerno/dipartimento Di Scienze Umane, Filosofiche E Della Formazione, Fisciano, Italy ${ }^{l}$

Purpose: Motor education offers many training opportunities, therefore, it is essential to have theoretical tools capable of demonstrating its aims and developing appropriate programs.

If we start from the assumption of a knowledge based on the possibilities of the body, the study of motor prasseology shows us the communicative-relational nature of body practices, that we must consider as experiences that allow an individual to express himself in the relationship with others and towards the physical world (Bortolotti, 2020).

The new way that allows the school to teach young people the centrality of the body and perceptions is above all the dance 
movement (Ceciliani, 2020), through which the being manifests itself through the ways of moving, seeing, perceiving and doing (Husserl, 1950). Studying dance also means putting the body in the spotlight, as the performing body of a performative art that includes carnal practices and knowledge widely incorporated and that must therefore be scientifically observed how to act bodily.

Method: 320 Children between the ages of 9 and 10 were given the Oseretzky-Guilman General Dynamic Coordination and Equilibrium Test in order to assess whether the psychomotor age corresponds to the chronological age and the degree of motor maturation.

Results: It has emerged that to investigate the psychomotor development it is necessary to analyze the movement of the body. Children who practice bodily expression activities, develop greater not only motor skills compared to others, but also logical, creativity, intuition and design.

Conclusions: With the expressive activities the child is placed in a situation of search, that starting from the lived experience of the body will lead him, through progressive adjustments, to the discoveries of new praxis, ensuring overall body ease in relation to the environment of action.

By moving and playing, each child becomes the protagonist of his own inner (Bruner, 2001) and outer world, activating himself in the choice and selection of control and harmonization of one or more movements, both in terms of effectiveness and continuity (Palumbo et al., 2019). Bodily experiences become communicative and expressive actions of the subject, therefore make him more aware of his own possibilities.

\section{References}

Bortolotti, A. (2020) Le attività ludico-motorie all'aria aperta come espressione di cultura inclusiva, in Ceciliani, A. (2020) L'educazione motoria nella scuola primaria. Roma: Carocci editore.

Bruner, J. (2001). La cultura dell'educazione. Milano: Feltrinelli.

Palumbo C., Ambretti A., Sgambelluri R. (2019). Psicomotricità infantile: implicazioni didattiche secondo una prospettiva prasseologica. Formazione \& Insegnamento XVII - 3 - 2019 Pensa MultiMedia Editore https://doi.org/10.7346/XVII-03-19_13

\section{5}

\section{Active breaks at primary school. A pilot experience of including physical practice in school lifetime during the COVID-19.}

\author{
Luciano Bertinato $^{1}$, Dino Mascalzoni ${ }^{2}$, Donatella Donati ${ }^{1}$, Francesca \\ Vitali $^{I}$, Alina Klonova ${ }^{1}$, Valentina Biino ${ }^{1}$, Nicola Schena ${ }^{3}$, Ilaria \\ Giuffrida $^{l}$, Sara Bigardi ${ }^{l}$, Federico Schena ${ }^{l}$ \\ University of Verona, Department of Neuroscience, Biomedicine \\ and Movement, Verona, Italy ${ }^{1}$; \\ Ufficio Scolastico Territoriale Xii, Direzione Scolastica Regionale \\ Del Veneto, Verona, Italy; \\ Dna Sport Consulting, Dna Sport Consulting, Verona, Italy ${ }^{3}$
}

Purpose: The EU Guidelines on "Recommended Policy Actions in the Support of Health-Friendly Physical Activity" (2008) invite to give special attention to physical health and mental issues due to the decreased physical activity in children and adolescents and the related dissemination of sedentary lifestyles and obesity. School time is the ideal place to reach this target.

Aims: This project aims at: a) to change the "structural" organization in the school timetable inserting breaks during the typical frontal lessons; b) to educate teachers on the benefits of this "methodological" novelty, c) to improve behavioral climate and psychophysical well-being of the pupils and all the stakeholders that are involved in school life.
Methodology: Three "Active breaks" were inserted in the daily school lifetime and additional breaks introduced in the afternoon at school or at home during homework. The teachers were oriented to the choice of the best "Active break" to be inserted at the beginning, at the end or during their own class lesson. Taking into consideration COVID-19 limitations, the "Active breaks" included 3 levels: 1 . "Light" to be carried out in the classroom, at own desk; 2 . "Moderate" to be held in the classroom or in the hall; 3 . "Intense" to take place in the school yard or gym. A website was created to collect comments, experiences and questionnaires from the schools.

Results. In 2020/2021 edition 25 Schools, 415 classes and 7221 pupils and families joined the project. 70 teachers participated to the project; about 20 of them actively contributed to the website. The involvement of the school councils, class teachers and families was respectively $88 \%, 92 \%, 86 \%$. About $60 \%$ of the participants did the activity 1 to 3 times a week; $15 \%$ reported a daily activity. Pupil satisfaction reached the level 'high' or 'very high' for $86 \%$ of the responders. Questionnaire reported criticisms in the level of motor coordination and time of attention of the children while education support obtained a great appreciation.

Conclusion: This study indicates that enlarging time of physical practice in school lifetime can be feasible even during a challenging period as COVID-19. Educational intervention for the teachers and regular supervision by experts in physical education appear to be key tools to support this kind of projects. A deep renovation of teaching/ learning process, as it has traditionally thought to implement the teaching calendar, is required to apply th.

\section{6}

\section{Development of cognitive, emotional, and interpersonal skills through the paddle}

Davide Sisti ${ }^{1}$, Fabrizio Perroni ${ }^{1}$, Stefano Amatori ${ }^{1}$, Erica Gobbi ${ }^{1}$, Federica Pulcini ${ }^{2}$, Damiano Stefanini ${ }^{3}$, Marco Rocchi ${ }^{1}$, Claudia Maulini $^{4}$

University of Urbino Carlo Bo, 61029 Urbino, Italy, Department of Biomolecular Sciences, Urbino, Italy ${ }^{1}$; Eclepta, Medial and Sport Pertformance Center, Rome, Italy ${ }^{2}$; H2o Sparta Paddle, Performance Center, Teri, Italy ${ }^{3}$;

University of Naples "parthenope“., Department of Sport Sciences and Wellness, Naples, Italy ${ }^{4}$

Purpose: Literature showed a positive relationship between paddle playing and a person's subjective well-being (Villena-Serrano et al., 2020; Cayetano et al., 2020). The aim of the present study was to analyze the development of cognitive, emotional and interpersonal skills through the paddle.

Methods: We administered to 252 players (male: 174; female: 78) an ad hoc 26 items survey with 5 point "Likert Scale" questions $(0=$ not at all; $5=$ very much). In order to identify a small number of possible skills, an Ascending Hierarchical Classification procedure was used. The groupings are highlighted by the high level of homogeneity of the groups expressed by the variability (variance) within them and assessable on the dissimilarity axis at the node where the elements of a cluster converge. All analysis were performed using Excel 365 or SPSS 20.0

Results: The educational degree of the sample (age: $40.8 \pm 11.3$ years) was as follows: $21(8.3 \%)$ lower and $126(50.0 \%)$ upper secondary school diploma; $101(40.0 \%)$ degree; and 4 (1.6\%) Ph.D. Clustering analysis finds 4 classes in which the 26 items are associated (total variance explained $=72.9 \%$ ): the first cluster $(\mathrm{n}=5$ items) identifies self-awareness; the second cluster $(\mathrm{n}=7$ items) highlights the ability to positively interact with a group; the third cluster $(n=9$ 
items) defines the dimension of self-esteem, while the last one refers to the positive management of stress. Participants showed scores for all clusters above the median, showing an overall beneficial effect of Padel.

Conclusions: The Results show a positive correlation between the practice of padel and the development of interpersonal, intrapersonal and emotional skills (Cayetano et al., 2020). Specifically, participants indicate an improvement in their self-esteem and self-awareness in addition to highlighting how the practice of padel contributes to a more effective stress management, corroborating the Results of recent padel research (Villena-Serrano et a., 2020) and in accordance with the literature that shows the close correlation between psychosocial well-being and sports.

\section{References}

1. Cayetano, A. R., Muñoz, S. P., Ramos, J. M. M., Beneitez, N. C., \& Muñoz, A. S. (2020). Motivos de participación deportiva y satisfacción intrínseca en jugadores de pádel. Retos: nuevas tendencias en educación física, deporte y recreación, (38), 35.

2. Villena-Serrano, M, Castro-López, R, Zagalaz-Sánchez, ML, and Cachón-Zagalaz, J (2020). Analysis of the subjective well-being of the paddle player of paddle. Revista de Psicología del Deporte (Journal of Sport Psychology), 29(1): 29-38.

\section{7}

\section{Evaluation of kinetic chain intervention in a throwing assessment}

\section{Paolo Moisè $^{l}$, Giorgia Baratella ${ }^{2}$, Luca Russomando ${ }^{2}$}

Suism, Università, Torino, Italy ${ }^{1}$;

Università Telematica Pegaso, Dipartimento Scienze Umanistiche, Napoli, Italy ${ }^{2}$

Purpose: Throwing movements are used in training protocols for strength, core stability and special strength in many sports disciplines. The same exercises with the med ball find space in numerous test assessment in different areas, where it is important to evaluate physical efficiency, in particular the strength of the upper body and the ability to express strength involving the entire kinetic chain.

The purpose of the study is to understand whether the ability to express explosive strength can be influenced by the intervention of the entire kinetic chain.

Methods: The sample consists of a group of students from the middle school $(\mathrm{N}=171)$ aged $12.34 \pm 1.24$ years, 89 males of 12.46 years \pm 1.24 , stature $154.11 \pm 11.12 \mathrm{~cm}$, weight $45.16 \pm 10.75 \mathrm{~kg}$ and BMI $18.90 \pm 3.53,82$ females of years $12.21 \pm 1.23$, stature $152.45 \pm$ $8.41 \mathrm{~cm}$, weight $43.76 \pm 10.17 \mathrm{~kg}$ and BMI $18.67 \pm 3.24$ The data was collected using the Office Excel program through person's correlation index, significance was set at $\mathrm{p}<0.05$.

The two tests chosen for the study are "seated medicine ball throw" commonly used to measure the explosive strength of the upper limbs and the throwing of the med ball from the chest (Forward Chest Throw).

Results: The analysis of the results shows that there is a significant correlation between the strength expressed analytically by the upper limbs to that expressed by the entire kinetic chain ( $p>0.001)$. If the correlation is analyzed separately for males and females there is significance for both genders, but with higher values for males $(\mathrm{r}$ $0,77)$ than females $(\mathrm{r} 0,56)$. The study confirms what is present in the literature, regarding the correlation between height and performance in throws, in the sample taken into consideration they are dependent in both throws, both in that from the erect station $(\mathrm{p}<0.001)$ and in the seated one $(\mathrm{p}<0.001)$. However, there is no correlation between body mass index (B.M.I.) and Forward Chest Throw.

Conclusions: In conclusion, it is possible to say that the study shows that, in the structuring of an assessment protocol, the physical education teacher who needs to evaluate the explosive strength of a subject can choose one of the throwing tests.

\section{References}

1. Palao JM, Valadés D. (2013) "Testing protocol for monitoring upper-body strength using medicine balls.” J. Hum. Sport Exerc. Vol.8, No. 2, pp. 334-341.

2. Ruiz J.R. et al. (2010) Field-based fitness assessment in young people: the ALPHA health-related fitness test battery for children and adolescents. Br J Sports Med May;45(6):518-24.

\section{8}

\section{Lifelong learning and physical education teachers}

\author{
Paolo Moise $^{I}$, Valentina Chianura ${ }^{2}$, Ubaldo Fadini ${ }^{2}$
}

Suism, Università, Torino, Italy ${ }^{1}$;

Iul Università Telematica Degli Studi, Università, Firenze, Italy ${ }^{2}$

Purpose: The speed with which society evolves has made lifelong learning a necessity in all professions, not excluding the teachers. The researchers call "Lifelong Learning " a real way of life. The aim of the work is to investigate the training priorities and the ways in which teachers of physical education access the updating from childhood to university.

Methods: Teachers were offered a questionnaire electronically with some questions aimed at constructing the sample profile and investigating the characteristics of continuing training. 975 teachers participated in the study, $8 \%$ in kindergarten, $18 \%$ in primary school, $36 \%$ in middle school, $35 \%$ in high school and $10 \%$ at university. The sample is made up of $51.9 \%$ of women and $40.9 \%$ of men. The age of the participants is heterogeneous with a greater representativeness in the 51-60 age range (37.1\%).

Results: From the answers of the teachers it emerges that the areas of greatest interest are the methodological- didactic (80\%), the communicative-relational (55\%) and disciplinary (37\%). Among the ways in which physical education teachers prefer to use the training courses provided by sports agencies that issue certifications (59\%), courses provided by the Ministry of Education and territorial school bodies (51\%), observation of colleagues (45\%), webinars (39\%) and support to experienced colleagues $(31 \%)$. It is important to note that $97 \%$ consider lifelong learning very important or important.

Conclusions: The questionnaire was administered during the COVID 19 emergency and this may have influenced teachers' choices. It is certainly important to note that teaching and reporting play a fundamental role for teachers and that formal learning is important for them in terms of how they approach updating, but informal learning is also available through the relationship with colleagues.

Finally, most teachers $(73.2 \%)$ believe that the experience lived during the COVID-19 emergency will change their approach to updating. What emerges from the research can certainly be useful to those who deal with continuing training in schools by orienting their contents and ways in which to plan proposals.

\section{References}

1. Gola G. (2008) "L'apprendimento informale degli insegnanti. Prospettive di ricerca sulle pratiche didattiche" Metodički obzori 31 Pregledni rad Review article UDK: 37.013.77 Primljeno: 4. 5. 2007. https://hrcak.srce.hr/file/40726 
2. Tovkanet H. (2018) "Lifelong Learning in Enhancing Professional Teacher Training in the European Countries" Comparative Professional Pedagogy 8(2).

\section{9}

\section{Multicomponent interventions for health promotion in primary school. The sbam project! Motor performance and related factors}

\author{
$\underline{\text { Dario Colella }}^{1}$, Cristina D'arando ${ }^{1}$, Benedetto Pacifico ${ }^{2}$, Domenica \\ Cinquepalmi $^{3}$, Domenico Monacis ${ }^{1}$ \\ University of Foggia, Department of Humanities, Cultural Heritage, \\ Education Sciences, Foggia, Italy ${ }^{1}$; \\ Puglia Region, "sport For All" Office, Bari, Italy ${ }^{2}$, \\ Puglia Region-"sport For All" Office, "sport For All" Office, Bari, \\ Italy
}

Purpose: The rates of overweight and obesity in childhood have increased across countries and are associated with dangerous health implications. Italy, globally, ranks 61st (females) and 46th (males) for the percentage of obese children and adolescents. According to data from Okkio alla Salute (2019), in Italy there are $20.4 \%$ overweight children aged $8-9$ and $9.4 \%$ obese.

The school is the privileged setting in which to promote an interdisciplinary curriculum to reduce the sedentary habits of children, increasing awareness of the benefits of daily motor activities. SBAM! is the multicomponent project promoted by the Puglia Region aimed at 8-year-old children and includes the following integrated measures: Physical education; Education in correct eating habits; Education for active transport and safe home-school-home journeys.

Methods: The monitoring carried out by the University of Foggia (2019), highlighted quantitative and qualitative data on motor development and related factors, referring to 8-year-old children from the Puglia Region. The regional sample is 10255 primary school students divided into three groups according to gender and BMI differences (Nw vs OW vs Ob); Males, Overall $\mathrm{n}=4333$; Nw 3168 (60.47\%), Ow 1165 (22.24\%), Ob 906 (17.29\%); Females, Overall n = 5016; Nw 3023 (60.27\%), Ow 1146 (22.85), Ob 847 (16.89\%). In addition to the descriptive statistics $(\mathrm{M} \pm \mathrm{SD}$ ), ANOVA 2 (group, $\mathrm{Nw}$ vs $\mathrm{Ow}$ vs $\mathrm{Ob}) \times 2$ (gender) was performed, in relation to the variables considered. All the students performed 4 motor tests (standing long jump; $1 \mathrm{~kg}$ medicine ball throw; shuttle run $10 \times 4 ; 6$ min WT) and two self-reports: PACES and PSP_C.

Results: The results show that the motor performance and self-report scores of overweight and obese children are lower in both sexes than in the normal weight groups $(\mathrm{p}<0.05)$.

Conclusions: Sedentary habits influence the motor development and related psychological factors of children, limiting the preventive and protective effects of motor activities. Low levels of motor abilities and motor skills are associated with low physical activity levels. Multicomponent institutional interventions are required, to promote health, fight overweight and obesity, through experimental teaching models that involve the community and public health.

\section{References}

1. Duncan et al. (2020). The Sport and Exercise Scientist, 66, 6-7. https://www.epicentro.iss.it/okkioallasalute/indagine-2019

2. Mead et al. (2017). The Cochrane database of systematic reviews, 6(6)

3. Yuksel et al. (2020). International Journal of Environmental Research and Public Health, 17(1).

Exercise, prevenction and therapy

\section{0}

"Zero hour" daily program: effects on fitness and body composition in AA.VV.F. - Italyn firefighter

Annamaria Mancini ${ }^{1}$, Luca Cenni ${ }^{2}$, Alessia Terracciano ${ }^{1}$, Daniela
Vitucci $^{1}$, Tarquinia Mastroianni ${ }^{3}$, Michele Maria La Veglia Pasqualina Buono ${ }^{1}$

University Parthenope, Naples, Italy, Department of Movement Sciences and Wellness, Napoli, Italy';

Polo Didattico Vigili Del Fuoco Campania, Polo Didattico Vigili Del Fuoco Campania, Napoli, Italy ${ }^{2}$;

Ufficio Per Le Politiche Di Tutela Della Sicurezza Sul Lavoro Del Personale Del Corpo Nazionale Dei Vigili Del Fuoco, Ufficio Per Le Politiche Di Tutela Della Sicurezza Sul Lavoro Del Personale Del Corpo Nazionale Dei Vigili Del Fuoco, Rome, Italy ${ }^{3}$

Purpose: Firefighters work activities require heavy physical effort1. The present pilot study aims to evaluate the importance of "Zero hour" daily program to improve firefighters fitness.

Methods: 53 male cadets from the 89th AA.VV.F. course were evaluated from August 2020 to February 2021. All the cadets must underwent to 7-month-daily "Zero hour" training program (45-60 min, 5day/weeks); they have no food restrictions or nutritional advices. "Zero hour" (adapted from Gunnarsson et al. 2012), program is a circuit training like HIIT comprising warm-up, stretching and breathing exercises; the program was supervised by an expert trainer and was performed in the gymnasium of the Fire Brigade early in the morning before the training activities. Anthropometry (Weight, Height, BMI), cardiorespiratory (1-Mile Jogging Test), musculoskeletal fitness (Push-Up and Jump Test) and flexibility (YMCA S\&R and Back Scratch Test) were evaluated at T0 (September), T1 (January) and T2 (March) in all cadets.

Results: After 3 and 6 months of "zero hour" program an improvement in 1-Mile Jogging Test (T2 vs T1 and T2 vs T0; $<<0.0001$ ); in Jump Test (T1 vs T0: $\mathrm{p}<0.0001$; T2 vs T0: $\mathrm{p}<0.0001$ ); in Push-up Test at (T2 vs T0: $\mathrm{p}<0.001$; T1 vs T0: $\mathrm{p}<0.05$ ) and in S\&R Test at T2 (T2 vs T0: $p<0.0001$ ) were observed; no differences in anthropometry were observed $(\mathrm{p}>0.05)$.

Conclusions: Six months of "Zero hour" supervised, structured exercise training improves aerobic and muscular fitness, and flexibility in cadets firefighter, reducing risk of musculoskeletal injuries and cardiac acute events.

Acknowledgments: Direzione Regionale Vigili del Fuoco Campania; Ufficio per le politiche di tutela della sicurezza sul lavoro del personale del Corpo Nazionale dei Vigili del Fuoco; Polo Didattico Vigili del Fuoco Campania; Ufficio Formazione Motoria Professionale Campania.

\section{References}

1. M. Sokoloski et al. (2020) Changes in Health and Physical Fitness Parameters After 6 Months of Group Exercise Training in Firefighters. Sports, 8: 143.

2. Gunnarsson et al. (2012) The 10-20-30 training concept improbe performance and health profile in moderately trained runners. J Appl Physiol(1985) 113:16-24.

\section{1}

Adapted physical activity service in subjects with alzheimer's disease: esercizio vita pilot project during the SARS-COV-2 pandemic period

Luca Innella $^{l}$, Michele Felisatti $^{1}$, Piero Fumarola ${ }^{1}$, Luca Pomidori ${ }^{1}$ 
Esercizio Vita Medical Fitness, R\&s, Ferrara, Italy ${ }^{1}$

Purpose: Non-pharmacological therapy such as Adapted Physical Activity is still poorly applied in individuals with Alzheimer's disease. The following pilot project aims to provide a structural and objectively feasible model of administering physical activity to patients with Alzheimer's, in order to evaluate the impact of physical exercise on the functional capabilities of the participants.

Method: The sample was identified among the members of the Alzheimer Association of Ferrara who joined the "Exercise for people with Alzheimer's disease" project.

All participants, 17 users, performed functional evaluations at the beginning of the project (T0) and after 6 months of activity (T1), exactly 2- Minute Walking Test (2MWT), Short Physical Performance Battery (SPPB),Timed Up and Go (TUG), and an indirect test for the muscle strength one-repetition maximum (1-RM) for lower limbs, evaluated with leg press (LP) and upper limbs chest press (CP).The proposed activity was supervised twice a week in specialized centers.

Results: Activity was interrupted in March and restarted in June. N. 16 subjects $(12 \mathrm{M} \& 5 \mathrm{~F})$ with an average age of $79.1 \pm 6.71$ achieved some improvements: 2MWT T0:138,7 \pm 19,91(mt);T1:151,3 \pm 15,21(mt.)(p = 0.05),TUG:T0:11,41 $\pm 1,02 \mathrm{~s} ; \mathrm{T} 1: 9,17 \pm 3,02 . \mathrm{sec}$; LP:T0:77,11 \pm 16,41(kg);T1:92,42 \pm 31,51(kg); CP:T0:22,22 \pm 9,08(Kg);T1:42,18 $\pm 16,67(\mathrm{Kg}$.). Other Results are being processed. Conclusion: This work affirms the importance of the interaction between exercise under supervision in a specialized physical activity center, different professional figures and local associations, in order to ensure a qualitative and innovative service in the territory.

Recent studies (1) show a slowdown on cognitive impairment associated with an improvement of daily activities (ADL) induced by the practice of physical exercise(2), placating aggressive attitudes at particular times of the day and giving benefits in managing for the caregiver.

\section{References}

1. Forbes D, Thiessen EJ, Blake CM, Forbes SC, Forbes S. Exercise programs for people with dementia. Cochrane Database Syst Rev. 2013 Dec 4;(12):CD006489. https://doi.org/10.1002/14651858. CD006489.pub3.

2. Meng Q, Lin MS, Tzeng IS. Relationship Between Exercise and Alzheimer's Disease: A Narrative Literature Review. Front Neurosci. 2020 and https://doi.org/10.3389/fnins.2020.00131, 14:131. Published 2020 Mar 26.

\section{2}

\section{An exercise-telehealth intervention in a glioblastoma patient during covid-19 outbreak}

\author{
Paolo Frada $^{1}$, Ilaria Trestini ${ }^{2}$, Tregnago Daniela ${ }^{2}$, Michele Milella $^{2}$, \\ Massimo Lanza ${ }^{1}$, Sara Pilotto ${ }^{2}$, Alice Avancini ${ }^{2}$ \\ University of Verona, Department of Neuroscience, Biomedicine \\ and Movement Science, Verona, Italy ${ }^{l}$; \\ University of Verona, Department of Oncology, Verona, Italy ${ }^{2}$
}

Purpose: The (COVID-19) has caused an unprecedented global pandemic, in which social distancing and quarantine were the only ways to keep the spread of the infection under control. In this context, delivering exercise (EX) resulted difficult, especially in vulnerable patients, such as those with cancer. Glioblastoma is a rare cancer type with a low survival rate. Due to the possible side effects, as falls, epileptic seizures, or bleeding, (EX) is rarely offered and tested in this kind of patient. Here, we report the feasibility and the effectiveness of a home-based EX program in a case of a glioblastoma patient undergoing chemotherapy, during the COVID-19 outbreak.

Methods: The EX-program started in March 2020 and lasted 12-week. A tailored instruction manual detailing the program to perform twice a week and a diary to log exercise sessions were provided. EX prescription included: aerobic training and resistance training. The program consisted of aerobic activity (walking or cycling), in which the load slowly increased from 10 to $20 \mathrm{~min}$ at a constant intensity of 3-5 on the CR10 Borg Scale of perceived exertion (CR10). Exercises with bodyweight and resistance bands were proposed to increase strength. Each resistance exercise comprised two-three sets of 8-12 repetitions at 3-4 intensity of CR 10 , which were progressively increased. Evaluations included: the "Six minutes walking test" (6MWT), handgrip and leg press strength tests, anthropometric values. Moreover, quality of life (QoL) using the European Organization of Research and Treatment in Cancer questionnaire and the Godin Leisure-Time Exercise Questionnaire about exercise level, were collected.

Results: The adherence rate to the intervention was $100 \%$, while four adverse events (epileptic seizures) were recorded. A decrement in 6MWT was registered $(-33,8 \mathrm{~m})$, while lower limb $(+18,48 \mathrm{~kg})$ and upper limbs strength (right hand $+5.0 \mathrm{~kg}$; left hand $+5 \mathrm{~kg}$ ) increased. Regarding QoL domains, physical function improved, whereas a worsening in fatigue, pain, social and cognitive functions was found. Anthropometric values remained stable.

Conclusion: A home-based EX intervention during the COVID-19 pandemic, was found feasible in a patient with glioblastoma undergoing chemotherapy. A combined EX program was shown efficacious to improve strength, while the effects on functional capacity and QoL, remain to be elucidated in further studies.

\section{References}

1. Avancini A, Trestini I, Tregnago D, Wiskemann J, Lanza M, Milella M, Pilotto S. Physical Activity for Oncological Patients in COVID-19 Era: No Time to Relax. JNCI Cancer Spectr. 2020 Aug 24;4(6):pkaa071. https://doi.org/10.1093/jncics/pkaa071. PMID: 33,385,107; PMCID: PMC7499670.

\section{3}

\section{Beneficial effects of a Judo program on the fear of falling (FOF) on young subjects}

Matteo Campanella ${ }^{l}$, Valentina Mancuso ${ }^{l}$, Dafne Ferrari $^{l}$, Lavinia Falcioni $^{2}$, Ludovica Cardinali ${ }^{2}$, Davide Curzi ${ }^{3}$, Laura Guidetti ${ }^{3}$, Carlo Baldari ${ }^{1}$

University, Department of Theoretical and Applied Sciences, Ecampus University, Novedrate (co), Italy ${ }^{1}$;

University, Department of Movement, Human and Health Sciences, Foro Italico University, Rome, Italy ${ }^{2}$;

University, Niccolò Cusano University, Rome, Italy ${ }^{3}$

Purpose: Fear of falling (FOF) is a potentially serious problem for the population. The major risk factors for developing a fear of falling are mainly having experienced at least one fall and minor predisposition to physical activity. VAS-FOF (visual analogue scale-fear of falling) is a simple and easy-to-use instrument for measuring the fear of falling and requires minimal effort by the subjects. VAS-FOF uses a numerical scale from one to 10 to measure perceived fear of falling, with one indicating absence of fear and 10 representing extreme fear. The aim of the present study was to verify whether teaching judo falls could reduce the fear of falling.

Methods: 77 subjects (56 males and 21 females) (age $=21.7 \pm$ 1.4 years) participated in the study. The subjects were divided into 
three groups: combat sports (CS), individual sports (IS) and team sports (TS). Subjects who had been practicing judo were excluded from the study. The subjects took part in a 6-month judo course focused on teaching falls. VAS-FOF was administered to subjects at the beginning of the course, in the middle of the course and at the end of the course.

Results: The Results showed that significant differences $(p=0.04)$ were found among the three sport groups: in particular, subjects who had been playing combat sports had significantly $(p=0.00)$ lower FOF values than those who had been playing individual or team sports. In all groups FOF values significantly decreased $(p=0.001)$ over time $(\mathrm{T} 1=4.2 \pm 2.2 ; \mathrm{T} 2=3.2 \pm 1.8 ; \mathrm{T} 3=2.8 \pm 1.6)$. No significant sex differences were found.

Conclusions: In conclusion, the Results show that taking part to a judo program focused on teaching falls can help reducing the fear of falling. Therefore, judo would appear to be a valid instrument to include in physical activity programs in order to promote injury prevention.

References

2. Scheffer AC. (2011) Psychometric properties of the VAS-FOF, a visual analogue scale for fear of falling in older persons. International Journal Geriatric Psychiatry. 26(3):284-91.

3. Toronjo-Hornillo L, Castañeda-Vázquez C, Campos-Mesa MDC, González-Campos G, Corral-Pernía J, Chacón-Borrego F, and DelCastillo-Andrés Ó. (2018) Effects of the Application of a Program of Adapted Utilitarian Judo (JUA) on the Fear of Falling Syndrome (FOF) for the Health Sustainability of the Elderly Popula.

\section{4}

\section{Effect of individualized whole-body vibration exercise on postural control in a person with multiple sclerosis: a 2-years case report}

\author{
Stefano La Greca ${ }^{I}$, Rocco Totaro ${ }^{2}$, Federico Passeri ${ }^{1}$, Francesco \\ Masedu ${ }^{l}$, Riccardo Di Giminiani ${ }^{I}$ \\ Università, Dipartimento Di Science Cliniche E Biotecnologiche, \\ L'aquila, Italy ${ }^{1}$; \\ Asl 1 Avezzano-l'aquila-sulmona, Ospedale San Salvatore- Centro \\ Malattie Demielelinizzanti, L'aquila, Italy ${ }^{2}$
}

Purpose: Whole-body vibration (WBV) has been considered as an alternative exercise to attenuate the processing functional and neuromuscular degeneration of postural control in persons with multiple sclerosis (PwMS). To date no studies have considered the use of individual protocols defined in terms of intensity, volume and other methodological parameters. This study aimed to investigate the acute and long-term effects, by applying individualized WBV, on postural control during a follow-up of 2 years including also the interruption period due to COVID-19.

Method: A PwMS (age: 62 years; stature: $180 \mathrm{~cm}$; body mass: $65 \mathrm{~kg}$; body mass index: $20.1 \mathrm{~kg} / \mathrm{m} 2$ ) voluntarily took part in this study. The optimal WBV magnitude was determined by recording the surface electromyographic activity (sEMG) as reported in the literature. During the two-years WBV intervention, the acute and long-term effects on the postural control were assessed 3-times: July 2019 (T1), December 2019 (T2) and July 2021 (T3). The postural control was assessed by measuring the body sway on the force platform during bipodalic standing position. The body sway was measured in different conditions: bipodalic static (BS) and bipodalic dynamic (BD) (inducing a standardized external perturbation); open (OE) and closed eyes (CE). The acute and long-term differences between pre-post
WBV intervention was calculated in the following way: $\Delta=(($ PostPre)/Pre)*100.

Results: The acute effects induced by individualized WBV on mediolateral body sway at $\mathrm{T} 1$ decreased during BD-CE $(\Delta=-16 \%)$, BD-OE $(\Delta=-28 \%)$, BS-CE $(\Delta=-27 \%)$ and BS-OE $(\Delta=-30 \%)$. Conversely, the medio-lateral sway increased during BD-CE $(\Delta=6 \%)$, BD-OE $(\Delta$ $=38 \%)$, BS-CE $(\Delta=72 \%)$ and BS-OE $(\Delta=7 \%)$.Similarly, at T3 the medio-lateral sway increased during BD-CE $(\Delta=8 \%)$, BD-OE $(\Delta=$ $19 \%)$ and BS-OE $(\Delta=71 \%)$ with the exception during BS-CE that decreased $(\Delta=-10 \%)$, The long-term effects induced by individualized WBV on medio-lateral body sway between T2-T1 decreased during BD-CE $(\Delta=-8,5 \%)$, BD-OE $(\Delta=-44 \%)$, BS-CE $(\Delta=-32 \%)$, BS-OE $(\Delta=-26 \%)$.Similarly, medio-lateral sway between T3-T2 decreased during BD-CE $(\Delta=-21 \%)$, BS-OE $(\Delta=-53 \%)$, whereas increased during BD-OE $(\Delta=33 \%)$, BS-CE $(\Delta=12 \%)$.

Conclusions: WBV increased the postural control after 22 sessions (T1 vs. T2) but decreased between $\mathrm{T} 3$ and $\mathrm{T} 2$ as the WBV intervention was interrupted (1-year). Anyway, when comparing the results of postural control between $\mathrm{T} 3$ and $\mathrm{T} 1$ emerges that the beneficial effects of WBV are still mantained.

\section{References}

1. Kantele, S, Karinkanta, S, and Sievänen H (2015) Effects of longterm whole-body vibration training on mobility in patients with multiple sclerosis: A meta-analysis of randomized controlled trials. J Neurol Sci 358(1-2):31-7.

2. Di Giminiani, R, Masedu, F, Tihanyi, J, Scrimaglio, R, and Valenti M (2013). The interaction between body position and vibration frequency on acute response to whole body vibration. J Electromyogr Kinesiol 23(1):245-51.

\section{5}

Effect of pilates and boxing in patients with parkinson disease: a case study

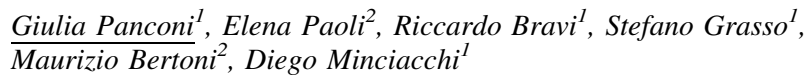

University of Florence, Department of Experimental and Clinical Medicine, Florence, Italy ${ }^{1}$, Training Lab, Firenze, Florence, Italy ${ }^{2}$

Purpose: The most important predictors of the quality of life in patients with Parkinson disease (PD) are balance and gait. Treatments with levodopa aim to compensate for dopaminergic deficits, reducing bradykinesia and rigidity, but causing dyskinesia and greater speed of movement which increases the falls percentage. In addition to pharmacological and surgical therapy, rehabilitation through physical exercise has been re-evaluated in recent years and many studies showed how rehabilitation, through balance and gait exercises, reduces falls and improves the quality of life. Pilates was shown to improve axial stability and core muscle function through a performance of coordinated movement sequences, but the effect on balance and gait wasn't evaluated with markerless pose estimation. Moreover, we combined Pilates with boxing, which has shown to improve functional mobility. In these case study we investigated the effect of Pilates and boxing on balance control and gait through Time Up and Go test (TUG) and computerized static stabilometry, combined with markerless pose estimation of DLC.

Methods: Three patients with PD performed Mat Pilates and boxing during two weekly session of 60 min for 6 weeks. Patients performed TUG test and static stabilometry at the beginning and end of the intervention. In the static stabilometry the subjects stood on a force plate, where signals were sampled at $200 \mathrm{~Hz}$, and performed two trials of $30 \mathrm{~s}$ : with open and closed eyes. With the platform we 
calculated the descriptive center of pressure (COP) in time and frequency domain. During each test kinematic analysis was evaluated through markerless pose estimation of DLC.

Results: The subjects showed an improvement in TUG, performing a reduced time and better gait parameters. In static stabilometry the balance control and the pattern of postural sway were improved.

Conclusions: Pilates protocol showed some improvement on balance control including postural and dynamic components. Mat Pilates combined with boxing may be an effective strategy of rehabilitation to improve the quality of life in PD patients. Therefore, exercise in combination with pharmacologic and surgical therapy may be the most effective form of treatment for Parkinson disease.

\section{References}

1. Ellis T, Cavanaugh JT, Earhart GM, Ford MP, Foreman KB and Dibble LE. Which measures of physical function and motor impairment best predict quality of life in Parkinson's disease? Parkinsonism \& Related Disorders 2011;17(9):693e7.

2. Morris S, Morris Me and Iansek R. Reliability of Measurements Obtained With the Timed "Up \& Go" Test in People With Parkinson Disease. Phys Ther. 2001 Feb;81(2):810-8.

\section{6}

Effects of exercise typologies among breast cancer patients and survivors on cardiorespiratory fitness, strength, fatigue and quality of life: a systematic review

\author{
Salvatore Ficarra ${ }^{l}$, Ewan Thomas $^{I}$, Antonino Bianco ${ }^{l}$, Ambra \\ Gentile $^{I}$, Petra Thaller ${ }^{2}$, Fulvio Grassadonio ${ }^{3}$, Sofia \\ Papakonstantinou ${ }^{4}$, Thorsten Schulz ${ }^{5}$, Nils Olson ${ }^{5}$, Alexandra \\ Martin $^{6}$, Christian Wagner ${ }^{7}$, Anna Nordström ${ }^{8}$, Hande Hofmann ${ }^{5}$ \\ Sport and Exercise Sciences Research Unit, Department \\ of Psychology, Educational Science and Human Movement, \\ University of Palermo, Palermo, Italy ${ }^{I}$; \\ Oac-Outdoor Against Cancer, Europe-wide Outdoor Sport \\ and Exercise Network For Cancer Prevention, Munich, Germania ${ }^{2}$; \\ International Centre For The Promotion of Education \\ and Development, (ceipes), Palermo, Italy ${ }^{3}$; \\ Creative Thinking Development, (crethidev), Rafina, Grecia ${ }^{4}$; \\ Institute of Preventive Pediatrics, Department of Sport and Health \\ Sciences, Technical University of Munich, Munich, Germania ${ }^{5}$; \\ Tum, Department of Sport and Health Sciences, Technical University \\ of Munich, Munich, Germania ${ }^{6}$; \\ Naturfreunde, Bundesorganisation, Vienna, Austria ${ }^{7}$; \\ Umea School of Sport Sciences, Umea University, Umeå, Svezia ${ }^{8}$
}

Purpose: Female breast cancer is the most frequently diagnosed cancer. Complementary approaches, and particularly exercise, may help to avoid the adverse effects and symptoms exacerbation and to reduce sedentary lifestyle that limit Quality of Life (QoL) in breast cancer patients (BCP) and survivors (BCS).

In order to identify the effects of different exercise interventions in BCP and BCS on cardiorespiratory fitness (CRF), strength (ST), fatigue (F) and QoL we carried out a systematic review.

Methods: The current review protocol was registered in the PROSPERO database [Reg.ID CRD42021237917]. A comprehensive literature search was conducted using two databases: Medline and Scopus. Randomized controlled trials with isolated exercise interventions in women $\mathrm{BCP}$ and $\mathrm{BCS}(<5$ years from therapy completion) were included. The risk of bias assessment was conducted using the Cochrane RoB-2-tool. Variables regarding CRF, ST, $\mathrm{F}$ and QoL were collected and discussed.
Results: Twenty-two studies met all the inclusion criteria and were deemed eligible. The Risk of Bias assessment indicated that the studies were predominantly "some concerns" or had "low Risk of Bias", with only 3 studies presenting a "high Risk of Bias". The mean duration and frequency of exercise interventions were 19 weeks and 3 sessions/week, performed at moderate intensity $(65 \% \mathrm{VO} 2 \mathrm{max}$ and $66 \% 1 \mathrm{RM}$, for aerobic and resistance-training interventions, respectively).

Conclusions: Exercise interventions are a beneficial strategy in breast cancer patients to avoid decline of major components of physical fitness, fatigue and QoL. Conversely, improvements among breast cancer survivors were observed for the same variables. Resistance training and combined resistance training + aerobic interventions provide the most encouraging variations of the selected outcomes. Specialized trainers should consider our Results in order to design appropriate interventions for this populations.

\section{References}

1. Sung H, Ferlay J, Siegel RL, Laversanne M, Soerjomataram I, Jemal A, et al. Global cancer statistics 2020: GLOBOCAN estimates of incidence and mortality worldwide for 36 cancers in 185 countries. CA: a cancer journal for clinicians. 2021 Feb 4.

2. McNeely ML, Campbell KL, Rowe BH, Klassen TP, Mackey JR, Courneya KS. Effects of exercise on breast cancer patients and survivors: a systematic review and meta-analysis. CMAJ: Canadian Medical Association journal $=$ journal de l'Association medicale.

\section{7}

Electromyographic analysis of core training exercises performed with stable and unstable surfaces in people with adoloscent idiopathic scoliosis (AIS)

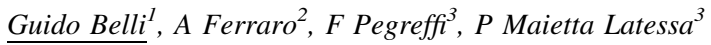 \\ Epartment of Sciences For Quality of Life, University of Bologna, \\ Italy $^{1}$; \\ Master Degree In Sport Sciences, University of Bologna ${ }^{2}$; \\ Department of Sciences For Quality of Life, University of Bologna, \\ Italy $^{3}$
}

Purpose: Adolescent Idiopathic Scoliosis (AIS) is one of the most common spinal disease during growth. Although the role of physical exercise has been previously demonstrated in AIS treatment, a debate still exists about the most efficient exercise setting to approach this problem. Recently, several studies demonstrated that self-correction, core training, instability and balance training exercises decrease Cobb angle and improve AIS. Since trunk muscle activity evidences differences between convex and concave side of the spine, asymmetric exercises seem to obtain better result in improving muscular balance. The aim of this study is to investigate paraspinal muscles activity during core training and balance exercises performed in stable (MAT) and unstable condition (BOSU) in people with AIS.

Methods: Five people (2 M and $3 \mathrm{~F}$; age range: $11-15$ years) with clinical diagnosis of AIS were recruited in a physiotherapy centre. Muscle activity (EMG) has been recorded with surface electromiography (BTS Free EMG) during 2 balance training and 3 core training exercises using MAT and BOSU. Electrodes were placed bilaterally in T5 (T: Trapezius Ascendens), T10 (ES: Erector Spinae) and L5 (M: Multifidus) area. Balance exercises were 1 leg standing balance with right (MBR) and left (MBL) foot. Core training exercises were Prone Plank (PP), Side Plank on right side (SPR) and left side (SPL). All exercises were performed in static condition using MAT and then BOSU. Data were recorded and analyzed in order to describe EMG 
differences between MAT and BOSU for left (L) and right (R) side of the body (reference condition: MAT).

Results: Global EMG during BOSU exercises showed greater activity compared to MAT for MBR, MBL, PP and SPR (respectively, $213,55 \%, 230,67 \%, 107,5$ and $120,5 \%$ ), while SPL evidenced lower values (88\%). Higher differences between $\mathrm{R}$ and $\mathrm{L}$ have been found during BOSU MBL and MBR for M, T and ES muscles related to spine curvature and body perturbation, while PP, SPR and SPL showed differences specially in T muscle using BOSU.

Conclusion: Balance and core exercises using unstable surface (BOSU) increase muscle activity in people with AIS. Since 1 leg standing balance requires stability and neuromuscular control, MBR and MBL exercises could enhance asymmetric EMG of paraspinal muscles in order to oppose against trunk sway and perturbation. Consequently, BOSU can be a useful tool for specific training in scoliotic people.

\section{References}

1. Czaprowsky D, Afeltowicz A, Gebicka A, Pawiowska P, Kedra A, Barrios C, Hadala M (2014), Abdominal muscle EMG-activity during bridge exercise on stable and unstable surfaces, Physical Therapy in Sport, 15(3): 162-168.

2. Farahpour N, Ghasmi S, Allard P, Sadegh Saba M (2014), Electromyographic responses of erector spinae and lower limb's muscles to dynamic postural perturbations in patients with adolescent idiopathic scoliosis, Journal of Electromyography and Kinesiology, 24(5): 645-651.

\section{8}

\section{Evaluating the feasibility of physical activity at a distance in older adults during covid-19 lockdown: a pilot study in the framework of S.T.E.P.S. - shared time enhances people solidarity}

\author{
Alins Klonova ${ }^{l}$, Carlotta Chiari ${ }^{1}$, Paolo Rossi ${ }^{l}$, Paolo Riccardo \\ Brustio $^{I}$, Doriana Rudi ${ }^{1}$, Juris Grants ${ }^{2}$, Federico Schena ${ }^{l}$ \\ Universita Di Verona, Dipartimento Di Neuroscienze, Biomedicina E \\ Movimento, Verona, Italy ${ }^{1}$, Latvian Academy of Sport Education, \\ Latvian Academy of Sport Education, Riga, Lettonia ${ }^{2}$
}

Purpose: The S.T.E.P.S. project was born with the purpose to envisage actions through physical activity interventions aimed to improve the general well-being and to reduce the state of loneliness, also through intergenerational activities. Nevertheless, the COVID-19 pandemic imposed an adjustment of the initial project, proposing physical activity at a distance that however guarantees a process of interaction between the subjects involved. In particular, due to the lack of physical exercise during COVID-19 lockdown and the related negative consequences observed in older adults (e.g., reduced mobility function, increased falls and frailty, and decreased quality of life) this first pilot study of the project aimed to verify the adherence and feasibility of a remote exercise program.

Methods: Seven older adults (mean age: $68 \pm 5$ years; $38 \%$ females) parteciped in the study. The exercise program consisted of a guided training sessions via telecommunication device (i.e., tablet: Hamlet model) through video calls with Zoom meeting platform. The exercise program was composed of two 40-min sessions per week for 5 consecutive weeks. At the conclusion of each training session, both partecipants and physical teacher completed a questionnaire designed to investigate the acceptability and feasibility of the exercise program..
Results: All participants engaged in the training completed the study and attended at least $90 \%$ of the total exercise sessions. From the beginning to the end of the training the partecipants perceived to increase their ability to perform the exercises $(F=2.64 ; p=0.015)$. Similarly, the physical teacher reported that the ability to connect with the device $(\mathrm{F}=3.27 ; \mathrm{p}=0.004)$, the understanding $(\mathrm{F}=2.24 ; \mathrm{p}=$ 0.037), the adaptation $(\mathrm{F}=1.00 ; \mathrm{p}=0.008)$ and execution $(\mathrm{F}=2.96 ; \mathrm{p}$ $=0.001)$ of the exercise, as well as the relationship among the participants $(F=3.27 ; p=0.004)$ increased throughout the lessons.

Conclusions: The preliminary results of this pilot study showed the feasibility of implementing a remote exercise program in older adults. This type of exercise program could represent a valid alternative to the traditional face-to-face training session, especially considering the future evolution of the pandemic condition. Further studies need to verify if this type of intervention can have a similar effect then general physical activity program in improving physical wellbeing or/ and reduce possible negative outcomes related to physical inactivity.

\section{9}

\section{"LAMA JUNIOR": online supervised training program in children with obesity. A preliminary study}

Vittoria Carnevale Pellino ${ }^{l}$, Alessandro Gatti ${ }^{1}$, Agnese Pirazzi ${ }^{l}$, Virginia Maron $^{\prime}$, Enrico Repossi ${ }^{1}$, Ilaria Grimoldi ${ }^{I}$, Vincenzo Rizzo ${ }^{l}$, Annalisa Barone ${ }^{1}$, Elisa Peretti ${ }^{1}$, Valeria Tranfaglia ${ }^{2}$, Valeria Calcaterra $^{2}$, Matteo Vandoni ${ }^{1}$

University of Pavia, Laboratory of Adapted Motor Activity (lama), Department of Public Health, Experimental Medicine and Forensic Science, University of Pavia, Pavia, Italy ${ }^{l}$;

Pediatric Department, "vittore Buzzi" Children's Hospital, Milan, Italy $^{2}$

Purpose: Since childhood, obesity and weight excess are connected to increased cardiovascular and metabolic disorders in adult age. However, physical activity improves the obesity-related risk factors, with a recommended $60 \mathrm{~min} / \mathrm{die}$ of moderate to vigorous intensity exercise for children. Online training showed benefits such as the possibility to perform directly at home enhancing positive responses to intensity, effort and pleasure. This preliminary study aims to evaluate the effort, pleasure and intensity of an online supervised training program developed for children with obesity.

Methods: 14 children with obesity $(6$ females; $10+1.8$ years; +2.35 $+0.25 \mathrm{BMI} z$-score) were enrolled in this study. They were involved in a 8-week training program with three supervised sessions per week. All the sessions included both aerobic and resistance exercises adapted to the young age of participants. Each training session lasted $1 \mathrm{~h}$ and the heart rate trend (HR-measured through Fitbit), perceived effort (Cert Scale) and pleasure (feeling scale) were recorded two times during workout (after 30 and $45 \mathrm{~min}$ ) and the mean of these values was calculated for each session. The means of the three initial and final sessions were considered to perform the analysis. Comparison between HR trend, Cert and Feeling Scale was calculated through non-parametric U-Mann Whitney test.

Results: Data revealed a significant decrease in HR and CERT scale between the initial and final sessions of the training $(-11.7+4.23$ Bpm;-0.88 + 1.17; $\mathrm{p}<0.05)$. While we did not report a significant change in the Feeling Scale, there was an increase of better mood states during training ("good" and "very good" pleasure).

Conclusion: Preliminary findings showed a positive effect of supervised online training for intensity and perceived effort indicating an enhancement of children in performing different exercises. The feeling scale reported an increase of pleasure during training with 
possible benefits on maintaining an active lifestyle through an online supervised exercise program.

\section{References}

1. DiCesare,M.;Soric,M.;Bovet,P.;Miranda,J.J.;Bhutta,Z.;Stevens,G.A.;Laxma-

iah,A.;Kengne,A.P.;Bentham,J.The epidemiological burden of obesity in childhood: A worldwide epidemic requiring urgent action. BMC Med. 2019, 17, 1-20.

2. Darren E R Warburton and Shannon S D Bredin (2017) Health benefits of physical activity: a systematic review of current systematic reviews. Curr Opin Cardiol. 32(5):541-556.

\section{0}

\section{Evaluation of body composition and strength in overweight / obese young people}

\author{
Roberto Pippi $^{1}$, Gabriele Mascherini ${ }^{2}$, Cristina Aiello ${ }^{1}$, Claudia \\ Ranucci $^{I}$, Carmine Fanelli ${ }^{l}$ \\ C.u.r.i.a.mo., Dipartimento Di Medicina E Chirurgia, Università \\ Degli Studi Di Perugia, Perugia, Italy, Perugia, Italy ${ }^{1}$; \\ Dipartimento Di Medicina Sperimentale E Clinica, Università Degli \\ Studi Di Firenze, Firenze, Italy ${ }^{2}$
}

Purpose: Previous studies have shown that exercise improves body composition and has a positive impact on health status in obese children and adolescents. While it is the most recommended exercise, however, aerobic exercise alone may not be the most effective exercise modality to counteract pediatric obesity. Muscle strength are an important protective factor against chronic disease and all-cause mortality. For this reasons, current physical activity guidelines for young suggest performing also muscle-strengthening activities 3 times per week. We propose the integration of body composition and the assessment of muscle strength in overweight young subjects following an exercise program.

Methods: From October 2013 to May 2017, a total sample of 138 overweight/obese young people (75 girls aged $11.6 \pm 2.6$ years and 63 boys aged $11.1 \pm 2.7)$ were recruited at the C.U.R.I.A.Mo. Center: 88 children (6-12 years) and 50 adolescent (13-17 years). Children were involved in a 6 month lasting exercise intervention program, while adolescents participated in a 3-month intervention. Body composition were assessed by BMI, waist circumference (WC), waist-to-height-ratio (WHTR) and Air Displacement Plethysmography (ADP). In children group strength of the lower and upper limbs were assessed by submaximal functional test (Sargent test, or ST, and medicine ball throw, or MBT), while for adolescents group we provided test with isotonic machine. Moreover, in both group strength was measured through Handgrip (HG) test for right and left hand. The effectiveness of the exercise program was established by comparing the baseline parameters with those at the end of the exercise program. Results: In children group we observe an improvements of body composition (BMI, $\mathrm{p}=0.016$; WC, $\mathrm{p}<0.001$; WHTR, $\mathrm{p}=0.002$; fat mass and lean mass, $\mathrm{p}<0.001$, measured by ADP) and strength (ST, $\mathrm{p}$ $<0.001$; MBT, $\mathrm{p}<0.001$; HG, $\mathrm{p}<0.005)$. Similar effects were observed in the adolescent group: body composition (BMI, WC, WHTR, $p<0.001$; fat mass, $p<0.00$, and lean mass, $p=0.043$, measured by ADP) and strength (for test with isotonic machine test, $\mathrm{p}$ $<0.001$; and $\mathrm{HG}, \mathrm{p}<0.005$ ).

Conclusions: A multidimensional approach based on the combination of strength test and the study of body composition trough ADP can represent an effective tool for controlling the state of health and assessing the efficacy of the exercise program, in young people with overweight/obesity.
Comparison of body composition methods in overweight and obese children.

References

1. Gately PJ, Radley D, Cooke CB, Carroll S, Oldroyd B, Truscott JG, Coward WA, Wright A. J Appl Physiol (1985). 2003 Nov;95(5):2039-46.

2. Kouwenhoven SMP, Antl N, Twisk JWR, Koletzko BV, Finken MJJ, van Goudoever JB. Methods to Assess Fat Mass in Infants and Young Children: A Comparative Study Using Skinfold Thickness and Air-Displacement Plethysmography. Life. 2021; 11(2):75. https://doi.org/10.3390/life11020075.

\section{1}

Exercise oncology: the use of three-dimensional cell culture models for the translational research of cancer recurrence and dormancy

Mauro De Santi ${ }^{1}$, Giosuè Annibalini ${ }^{1}$, Giulia Baldelli ${ }^{1}$, Matteo Bocconcelli ${ }^{1}$, Valentina Natalucci ${ }^{1}$, Carlo Ferri Marini ${ }^{1}$, Francesco Lucertini ${ }^{l}$, Marco Gervasi ${ }^{l}$, Davide Sisti ${ }^{1}$, Roberta Saltarelli ${ }^{1}$, Andrea Rocco Panico ${ }^{1}$, Marco Bruno Luigi Rocchi ${ }^{1}$, Giorgio Brandi ${ }^{1}$, Rita Emili $^{2}$, Elena Barbieri ${ }^{1}$

University of Urbino Carlo Bo, Department of Biomolecular Sciences, Urbino, Italy ${ }^{I}$;

U.o.c. Oncologia Medica, Asur Area Vasta 1, Ospedale Santa Maria Della Misericordia Di Urbino, Urbino, Italy ${ }^{2}$

Purpose: The use of cell culture models in exercise oncology has recently received growing interest [1]. Particularly, three-dimensional (3D) cell culture models are useful to translational research, mimicking the biological processes related to cancer recurrence and dormancy. We propose a double 3D cell culture approach to evaluate the effects of exercise-conditioned human serum (HS) in both single cell-derived microtumor formation and cancer cell spheroids.

Methods: Exercise-conditioned sera were obtained from 12 healthy women before (t0) and immediately after (t1), after $4 \mathrm{~h}(\mathrm{t} 2)$, and $24 \mathrm{~h}$ (t3) of a high-intensity endurance cycling (HIEC) session. HIEC consisted of four 5-min stages at $50 \%, 55 \%, 60 \%$, and $70 \%$ of individual maximal power (Pmax), which were followed by ten 90 -s sprints at $90 \%$ Pmax separated by 180 -s at $55 \%$ Pmax. Breast cancer (BC) cells were cultured in semisolid medium (soft agar) and U-shaped low attachment microplates to form microtumors and spheroids, respectively.

Results: All of the HIEC-conditioned sera (t1, t2, and t3) markedly impacted the proliferative and the microtumor-forming capacity of BC cells in semisolid medium, while the HS collected from the subjects at rest (t0) did not [2]. The microtumors-forming capacity of HIEC-conditioned HS was less than $30 \%$ compared to that induced by HS taken at rest. In addition, the BC spheroids were affected by postHIEC human sera, showing a decrease in cell viability and spheroidization compared to spheroids cultured with HS taken at rest. Conclusions: These results demonstrated the potential of HIEC bouts in tumor progression control and tumor morphological changes, highlighting the importance of 3D models in advancing knowledge about the mechanisms through which exercise acts on cancer dormancy and recurrence. Moreover, the precise evaluation of the effects induced by exercise at different intensities could identify physiological predictors of cancer progression inhibition, ultimately improving our understanding of the relationship between physical exercise and cancer progression control, allowing us to optimize recommendations and exercise protocols for cancer recurrences prevention.

References 
1. Metcalfe RS, Kemp R et al. (2021) Anti-carcinogenic effects of exercise-conditioned human serum: evidence, relevance and opportunities. Eur J Appl Physiol 121(8):2107-2124.

2. Baldelli G, De Santi M et al. (2021) The effects of human sera conditioned by high-intensity exercise sessions and training on the tumorigenic potential of cancer cells. Clin Transl Oncol 23(1):22-34.

\section{2}

\section{Functional capacity in patients with obesity before and after sleeve gastrectomy}

\author{
Valentina Bullo $^{I}$, Stefano Gobbo ${ }^{1}$, Gioia Bianchini ${ }^{2}$, Eleonora \\ $\overline{\text { Doria }}^{2}$, Federica Duregon ${ }^{1}$, Andrea Ermolao ${ }^{1}$, Marco Bergamin ${ }^{1}$ \\ Università Degli Studi Di Padova, Dimed, Padova, Italy ${ }^{1}$, Gymhub \\ S.r.l., Spin-off of The University of Padova, -, Padova, Italy ${ }^{2}$
}

Purpose: Obesity is a chronic multifactorial pathology caused by environmental, behavioral and genetics factors, with negative impact on general health, quality of life, and increased risk of disability and morbidity. Non-surgical management of obesity consists on a multicomponent approach, including behavioral therapy, pharmacotherapies, and lifestyle change to reduce the energy intake with diet, and increase physical activity. Because few patients achieved an important weight loss with lifestyle change, many undergo to bariatric surgery.

Methods: The aim of this study is to evaluate physical functional of patients with obesity before and after sleeve gastrectomy to analyzed changes of maximal oxygen consumption, muscular strength, postural control, level of physical activity and quality of life. One hundred and seventy-four patients with obesity (48 males, 126 females) were recruited and evaluated 1 month before and 6 months after sleeve gastrectomy. Physical functioning evaluation consisted on: cardiopulmonary capacity, muscle strength (handgrip, quadriceps and hamstrings), postural control (static balance), level of physical activity (Global Physical Activity Questionnaire), and quality of life (Short Form Health Survey 36-item).

Results: After surgery were recorded significant reduction of body weight (males $-28.4 \%, \mathrm{p}<0.001$; females $-26.1 \%, \mathrm{p}<0.001$ ), body mass index and waist circumference (males $-20.9 \%, \mathrm{p}<0.001$; females $-18.8 \%, \mathrm{p}<0.001)$. Absolute muscular strength of upper and lower limb decreased, while strength adjusted by body weight increased significantly. Quality of life and level of physical activity increased after surgery.

Conclusions: After sleeve gastrectomy, all patients reduced body weight, with higher percentage recorded in man. Functional capacity evaluation showed a decreased of absolute cardiopulmonary capacity and muscular strength, the increased of muscular strength corrected by body weight, and partially improved postural control. The level of physical activity increased especially in activity of leisure time and active transport, suggesting the initial change in life-style. Quality of life improved in physical composite domain.

\section{3}

Functional scores improvement after 6-month of an exercise program for women with osteoporosis: a randomized trial

\author{
Laura Bragonzoni ${ }^{1}$, Erika Pinelli ${ }^{1}$, Giuseppe Audino ${ }^{1}$, Claudio \\ Ripamonti $^{2}$, Francesco Benvenuti ${ }^{1}$, Laura Dallolio ${ }^{3}$, Sofia Marini ${ }^{3}$, \\ Pasqualino Maietta Latessa ${ }^{I}$, Raffaele Zinno ${ }^{1}$, Giuseppe Barone ${ }^{I}$ \\ University of Bologna, Department of Life Quality Studies, University \\ of Bologna, Bologna, Italy ${ }^{1}$; \\ Istituto Ortopedico Rizzoli, Istituto Ortopedico Rizzoli, Bologna, \\ Italy $^{2}$; \\ University of Bologna, Department of Biomedical and Neuromotor \\ Sciences, Bologna, Italy ${ }^{3}$
}

Purpose: Osteoporosis (OP) is a systemic disease of the skeleton characterized by a reduced bone mass and deterioration of the microarchitecture. There is a consensus on the effectiveness of physical activity in preventing bone loss, falls and, consequently, fractures. The main aim of the study is to evaluate modifications of quality of life $(\mathrm{QoL})$. The second aims concern functional score.

Methods: Forty women with OP were enrolled in the randomized trial. The women were divided into 2 groups: OGT and IHT. Both groups performed a specific exercise protocol for 6-month in 2-days/ week 1-h sessions. Moreover, all women were requested to choose an additional third day of the week. The OGT was followed by a trainer through online supervision, while the IHT performed the protocol at home without supervision. The participants were assessed at the baseline and after 6-month of training. The assessed outcomes were: QoL measured through ECOS-16, fear of fall by short FES-I, aerobic capacity and endurance assessed by 6MWT, joints mobility by sit and reach and stick test, muscles force by handgrip and $30 \mathrm{~s}$ chair stand test.

Results: ECOS-16 showed no changes at 6-month follow-up for both groups and no difference between groups. 6MWT and $30 \mathrm{~s}$ chair stand test showed a statistically significant improvement in both groups. The distance travelled of the 6MWT changed in the OGT, from $420 \pm 25 \mathrm{~m}$ to $457 \pm 46 \mathrm{~m}(\mathrm{p}<0.005)$, and from $369 \pm 85 \mathrm{~m}$ to $415 \pm 66 \mathrm{~m}(\mathrm{p}<0.05)$ in the IHT. The repetition number of the $30 \mathrm{~s}$ chair stand test changed in the OGT from $12 \pm 3$ to $15 \pm 3$ (p $<$ $0.001)$ and from $12 \pm 2$ to $13 \pm 3(\mathrm{p}<0.005)$ in the IHT. Sit and reach and stick test showed not a significant improvement.

Conclusions: Despite the difficulty due to the SARS Covid-2 pandemic, the study results showed that participants had maintained the same quality of life after 6-month of training. Moreover, the functional outcomes have increased after the training. These results proved the efficacy of the exercise training in both settings.

\section{References}

1. Choi, M.; Prieto-Merino, D.; Dale, C.; Nüesch, E.; Amuzu, A.; Bowling, A.; Ebrahim, S.; Casas, J.P. Effect of changes in moderate or vigorous physical activity on changes in healthrelated quality of life of elderly British women over 7 years. Qual. Life Res. 2013, 22, 2011-2020. 


\section{4}

Impact of BMI, physical activity and sedentariness levels on health-related measures in a group of overweight and obese adults with and without type 2 diabetes

Roberto Pippi ${ }^{1}$, Vittorio Bini ${ }^{2}$, Carmine Fanelli ${ }^{1}$, Riccardo Spitale $^{3}$, Lucia Cugusi $^{4}$,Marco Bergamin ${ }^{5}$, Andrea Di Blasio ${ }^{6}$

C.u.r.i.a.mo., Dipartimento Di Medicina E Chirurgia, Università Degli Studi Di Perugia, Perugia, Italy ${ }^{1}$;

Dipartimento Di Medicina E Chirurgia, Università Degli Studi Di

Perugia, Perugia, Italy ${ }^{2}$;

Corso Di Studio Magistrale Interclasse In Scienze E Tecniche Dello Sport E Delle Attività Motorie Preventive E Adattate, Università Degli Studi Di Perugia, Perugia, Italy ${ }^{3}$;

Dipartimento Di Scienze Biomediche, Università Degli Studi Di

Sassari, Sassari, Italy ${ }^{4}$;

Dipartimento Di Medicina, Università Di Padova, Padova, Italy;

Dipartimento Di Medicina E Scienze Dell'invecchiamento, Università Di Chieti-pescara, Chieti, Italy 6

Purpose: This study aimed to evaluate the effects of BMI, physical activity levels (PAL) and sitting time (SIT) on the variation of healthrelated measures, in a group of overweight and obese adults with and without type 2 diabetes following an intensive exercise program.

Methods: From January 2011 to February 2014, 293 overweight/ obese adults ( 165 women and 128 men, mean age of $51.9 \pm 9.5$ years and $54.6 \pm 8.3$, respectively), with and without type 2 diabetes mellitus (DM2), participated in a 3-month intensive exercise program. Before start, participants were allocated in 3 subgroups (overweight, $\mathrm{BMI}=25-29.9$; class 1 of obesity, BMI $=30-34.4$; class 2 (or superior) of obesity, BMI > 35). International physical activity questionnaire (IPAQ-it) has been used to evaluate participants' baseline sitting time (SIT) and physical activity level (PAL). Stratified multiple analyses were performed using 4 subgroups of SIT level according to Ekelund et al. 2016 (low, $<4 \mathrm{~h} /$ day; medium, 4-5.9 h/day; high, 6-8 h/day; very high, $>8 \mathrm{~h} /$ day of SIT) and using 3 subgroups for PAL (high, moderate and low). Health-related measures as anthropometric variables (weight, BMI, waist circumference), body composition (fat mass and lean mass), blood tests (glycemia, glycated haemoglobin, total cholesterol, HDL and LDL lipoproteins, triglycerides), blood pressure values and functional capacities (strength of the lower and upper limbs, flexibility and VO2max) were studied at the beginning and at the end of the training period.

Results: An overall improvement of PAL was observe in all sample following the 3-month intensive exercise program together with a general improvement in several health-related measures. BMI group factor influenced variations of $\mathrm{VO} 2 \mathrm{max}$, leg press values, triglycerides and anthropometric variables, while SIT group factor impacts on variations of sitting time, $\mathrm{VO} 2 \mathrm{max}$, glycemia and fat mass.

Conclusions: In this study, baseline PAL -unlike BMI and SIT- does not seem to influence the effects of an exercise intervention. The characteristics of our educational program, also including physical exercise, allowed to obtain positive Results.

\section{References}

1.Ekelund U, Steene-Johannessen J, Brown WJ, Fagerland MW, Owen N, Powell KE, Bauman A, Lee IM. (2016) Does physical activity attenuate, or even eliminate, the detrimental association of sitting time with mortality? A harmonised meta-analysis of data from more than 1 million men and women. Lancet 388(10,051):1302-10.
Mannocci A, Thiene DD, Cimmuto AD, Masala D, Vito E, and Torre G. (2010). International Physical Activity Questionnaire: validation and assessment in an Italyn sample. Ital J Public.

\section{5}

\section{Lifestyle and physical fitness in adolescents with type 1 diabetes}

Francesca Cozzolino $^{l}$, Giuliana Valerio ${ }^{l}$, Alessia Terracciano ${ }^{l}$, Patrizia Calella $^{I}$, Dario Iafusco ${ }^{2}, F$. Casaburo ${ }^{2}$, A. $_{\text {Chianese }}^{2}$, A. Gentile $^{2}$, A. Piscopo ${ }^{2}$, S. Rollato ${ }^{2}$, V. Testa ${ }^{2}$, A. Troncone ${ }^{2}$, A. Zanfardino $^{2}$, F. Zanfardino ${ }^{2}$, Francesca Galle ${ }^{l}$, Giorgio Liguori ${ }^{l}$, Annamaria Mancini ${ }^{3}$, Stefania Orrü, Pasqualina Buono ${ }^{3}$

Università Degli Studi Di Napoli Parthenope, Dipartimento Di Scienze Motorie Per La Prevenzione Ed Il Benessere, Napoli, Italy ${ }^{1}$; Università Luigi Vanvitelli, Dipartimento Della Donna, Del Bambino E Di Chirurgia Generale E Specialistica, Napoli, Italy ${ }^{2}$; Ceinge, Biotecnologie Avanzate, Napoli, Italy ${ }^{3}$

Purpose: The first aim of the present study was to evaluate the levels of physical activity (PA), sedentary activities and physical fitness in adolescents with type 1 diabetes (T1D) in relation to body mass index (BMI) categories. The second aim was to investigate the association between PA level, physical fitness and glycaemic control.

Methods: 74 adolescents with T1D (39 females and 35 males) with a mean age of $14.67 \pm 1.57$ were enrolled: 22 were normal-weight, 37 overweight and 15 obese. The short form of International Physical Activity Questionnaire in adolescents (IPAQ-A) was used to assess PA. The Health Behavior in School Children (HBSC) questionnaire was used to assess time spent in sedentary activities (TV, internet and video games). Physical fitness was assessed by: handgrip strength; 2-min step test; 3-m up and go test. Glycosilated haemoglobin (HbA1c) was measured in the same day when the tests were performed.

Results: Time spent in overall PA (sum of walking, moderate and vigorous PA) was $9.3 \pm 6.3 \mathrm{~h} /$ week conversely, time spent in sedentary activities was $8.7 \pm 3.2 \mathrm{~h} /$ day. Normal-weight spent a major amount of time walking $(446 \pm 343 \mathrm{~min} / \mathrm{week}, \mathrm{p}=0.053)$ compared to overweight $(276 \pm 205 \mathrm{~min} /$ week $)$ and obese $(293 \pm$ $272 \mathrm{~min} /$ week) adolescents. Normal-weight adolescents performed better in the timed up and go test compared to obese $(\mathrm{p}=0.025)$; no differences were found in the other fitness tests. In the whole sample, moderate PA, no physical fitness, correlated with $\mathrm{HbA} 1 \mathrm{c}(\mathrm{r}=-0.253$; $\mathrm{p}=0.029$ ).

Conclusions: Adolescents with T1D meet the WHO recommendations for PA but had high levels of sedentary behaviors, regardless to BMI categories. Moderate PA positively correlates with better glycaemic control. Further investigations are in progress to clarify the interactions among lifestyle, physical fitness, BMI and glycaemic control.

\section{References}

1. Wiśniewski A, Poliszczuk T, Pańkowska E. (2010) Assessment of physical fitness in children and teenagers with type 1 diabetes. Pediatric Endocrinology, Diabetes, and Metabolism 16(3):171-175.

2. Mannocci A, Di Thiene D, Del Cimmuto A, et al. (2010) International Physical Activity Questionnaire: Validation and assessment in an Italyn sample. Ital J Public Health 7:369-376. https://doi.org/10.2427/5694 


\section{6}

\section{Lifestyle effects of activity tracker-based counseling and live-web exercise on breast cancer. Survivors during italy covid-19 lockdown. the "angel project"}

Gianluca Viscioni ${ }^{l}$, Andrea Di Blasio ${ }^{l}$, Teresa Morano ${ }^{l}$, Federica Lancia $^{l}$, Angelo Di Iorio ${ }^{1}$, Simona Grossi ${ }^{2}$, Ettore Cianchetti ${ }^{2}$, Lucia Cugusi $^{3}$, Stefano Gobbo ${ }^{4}$, Marco Bergamin ${ }^{4}$, Anna D'eugenio ${ }^{1}$, Laura Masini $^{l}$, Massimo Rinaldi ${ }^{1}$, Maria Teresa Scognamiglio ${ }^{2}$, Francesca Di Giandomenico ${ }^{2}$, Antonino Grassadonia ${ }^{2}$, Giorgio Napolitano ${ }^{1}$

G. D'annunzio University of Chieti-pescara, Department of Medicine and Ageing Sciences, Chieti, Italy ${ }^{1}$;

Eusoma Breast Centre, "g. Bernabeo" Hospital, Asl02 Lancianovasto-chieti, Ortona, Italy ${ }^{2}$;

University of Sassari, Department of Biomedical Sciences, Sassari, Italy $^{3}$;

University of Padova, Department of Medicine, Padova, Italy ${ }^{4}$

Purpose: Both traditional interventions and new strategies are important for breast cancer survivors' (BCS) health to elicit the positive effects of physical activity and exercise on their health. During the first period of therapy, it is particularly important to a continuous feedback, especially in those women not having a proper lifestyle. The aim of the study was to compare the effects of weekly personal feedback, based on objectively measured physical activity, on daily sedentary time, physical activity and sleep of BCS (Egroup) with those of an intervention also including online supervised physical exercise sessions ( $\mathrm{E}+$ group), considering the effects of COVID-19 lockdown.

Methods: A total of 51 BCS were studied over an 18-week period and had objective registration of day-to-day sedentary time, physical activity and sleep. Both subsamples received weekly or fortnight personal feedback. Data were analysed considering four key periods, according to the COVID-19 emergency steps.

Results: The E group had a high overall sedentary time and a different trend of light-to vigorous and light-intensity physical activities, with a reduction from pre to first month of national lockdown, showing a significant rise just at the end of the national restrictions. E + group, from pre to first month of national lockdown, experienced the increase of both total and restorative sleeping time, inverting its trend from the first month of lockdown to its end.

Conclusions: The use of an activity tracker and its accompanying app, with the reception of weekly tailored advice and supervised online physical exercise sessions, can elicit proper physical activity recomposition and sleep improvement in BCS, also in the presence of personal restrictions. In those cancer survivors experiencing personal limitations, our Results are particularly important because allowed to better program effective physical activity interventions.

\section{References}

1. Di Blasio A et al. (2021). Effects of activity tracker-based counselling and live-web exercise on breast cancer survivors during Italy COVID-19 lockdown. J Funct Morphol Kinesiol $6: 50$

\section{7}

Palestra della salute for health: an opportunity for the long-term active ageing for stroke survivors

Piero Fumarola $^{l}$, Michele Felisatti ${ }^{1}$, Luca Innella ${ }^{1}$, Luca Pomidori ${ }^{1}$

Esercizio Vita Medical Fitness Center, R\&s, Ferrara, Italy ${ }^{I}$
Purpose: Supervised Exercise Training (SEXT) immediately after a rehabilitation therapy is fundamental for maintaining an active lifestyle and reducing the chances of hypokinesia and clinical flare-ups (1). The following observational study aimed to assess the effectiveness of the rule of a Palestra della Salute (PS) through the longterm effects of a SEXT program developed, managed and followed up by Exercise Physiologists and aimed at maintaining the residual abilities in stroke survivors.

Methods: A study sample has been identified and analyzed among 111 customers $(80 \mathrm{M})$ with stroke outcomes who attended the Esercizio Vita PS from 2015 to today. For this study, only those who regularly attended at least two consecutive years have been chosen. Functional assessments such as 6 Minute Walking Test (6MWT), Timed Up and Go (TUG), Sit to Stand (STS), Short Physical Performace Battery (SPPB) and Berg Balance Scale (BBS) have been performed at the beginning (T0), then regularly every 6 months until 2 years (Tf). SEXT program was based on mobility, static and dynamic balance, dexterity, strengthening and aerobic exercises with a minimum frequency of 2 times per week for each 60-min session(1). Results: 14 subjects completed the program. After 2 years of follow up and regular SEXT we found: $6 \mathrm{MWT}$ (T0 $=228,3 \mathrm{mt} \square 96,7$; $\mathrm{Tf}=$ $288,6 \mathrm{mt} \square 121^{*}$ ), TUG (T0 $=14,2 \mathrm{~s} \square 5,8$; $\left.\mathrm{Tf}=10,8 \mathrm{~s} \square 5,2^{*}\right)$, STS ( $\mathrm{T} 0=17,2 \mathrm{~s} \square 7,7$; $\mathrm{Tf}=13,3 \mathrm{~s} \square 4,3^{*}$ ), SPPB (T0 = 7,6 $\square 3,2$; $\mathrm{Tf}=$ 9,7 $\square 3,0 *$ ), BBS (T0 = 49,4 $\square 5,6$; Tf $=51,6 \square 4,9 \mathrm{~ns}$ ).

$*=\mathrm{p}<0,005$.

ns $=$ not significant.

Conclusions: As already shown in literature, we observed that SEXT is important to reduce the vicious circle of disability and physical inactivity after a stroke [2]. Palestra della Salute (PS) is effective in maintaining the residual motor skills through physical activity and improving physical fitness and quality of life of stroke survivors. Furthermore, it encourages socialization if practiced in a group. [3] PS acts as a link between healthcare facilities and the territory in the field of chronic diseases, with programs offered by highly-trained and qualified professionals in the field of exercise physiology.

References

2. C. J. Winstein, Guidelines for Adult Stroke Rehabilitation and Recovery A Guideline for Healthcare Professionals From the American Heart Association/American Stroke Association, Stroke, 2016.

3. D. H. S. Mead, Physical fitness training for stroke patients, 24 March 2016, Cochrane Systematic Review.

4. P. Belfiore, J Sports Med Phys Fitness. 2018 Dec;58(12):1867-1875. https://doi.org/10.23736/S0022-4707.17. 07749-0. Epub 2017 Oct 24. Adapted Physical Activity and Stroke: A Systematic Review.

\section{8}

Physical activity and sleep habits in BRCA 1/2 women

Letizia Galasso ${ }^{I}$, Lucia Castelli $^{1}$, Antonino Mule ${ }^{I}$, Eleonora Bruno $^{2}$, Patrizia Pasanisi $^{2}$, Fabio Esposito ${ }^{1}$, Angela Montaruli ${ }^{1}$, Eliana Rovedal

Dipartimento Di Scienze Biomediche Per La Salute, Università Degli Studi Di Milano, Milan, Italy ${ }^{1}$;

Dipartimento Di Ricerca, Fondazione Irccs Istituto Nazionale Dei Tumori Di Milano, Milan, Italy ${ }^{2}$

Purpose: The BRCA 1/2 mutations are the most known typologies of hereditary breast cancer (BC). In women carry these mutations, studies have provided preliminary evidence of a protective role of physical activity (PA) against BC, particularly during adolescence or 
early adulthood. Data from the German LIBRE study confirmed a significantly lower BC prevalence in BRCA $1 / 2$ women who reported higher PA during their adolescence. In addition, physical activity has also been shown to improve or have a protective effect on sleep in women with a diagnosis of $\mathrm{BC}$. Aim of the present study was to investigate the role of PA and sleep on BC risk factors in BRCA 1/2 women.

Methods: Data analysis involved 63 women $(47.6 \pm 12.4$ years $)$ with BRCA 1/2 mutations in care at Fondazione IRCCS Istituto Nazionale dei Tumori, Milan. The participants filled in Godin-Shepard LeisureTime Physical Activity Questionnaire and in Pittsburgh Sleep Quality Index for the evaluation of the PA levels and sleep, respectively. Moreover, they underwent to anthropometric, metabolic, and blood sample evaluations. Data were analyzed with SPSS version 27.

Results: Through the questionnaires, the women were classified as active $(n=22)$ and inactive $(n=41)$, and as $\operatorname{good}(n=26)$ and bad $(n$ $=37$ ) sleepers. Insulin levels were found significantly lower in active compared to the inactive women $(\mathrm{p}<0.05)$; the active women were found to be good sleepers while the inactive women were bad sleepers. Indeed, there was a statistically significant difference for the PSQI score between the two groups $(\mathrm{p}<0.01)$. Referring to the sleep behavior, the good sleepers were classified as active while the bad sleepers were classified as inactive. Indeed, there was a statistically significant difference in the PA levels between the two groups $(\mathrm{p}<$ 0.001). In addition, the PA intensities, heavy, moderate and light, were found significantly higher in good sleepers compared to bad sleepers ( $p<0.01 ; \mathrm{p}<0.01 ; \mathrm{p}<0.05$, respectively).

Conclusions: These findings suggest that the active BRCA 1/2 women has lower insulin levels and better sleep compared with inactive BRCA $1 / 2$ women. In this population PA can play an important and protective role against BC.

\section{References}

1. Kiechle, M, et al., (2017) Feasibility of structured endurance training and Mediterranean diet in BRCA1 and BRCA2 mutation carriers-an interventional randomized controlled multicenter trial (LIBRE-1). BMC Cancer 17:752.

2. Mustian, KM, et al., (2013) Multicenter, Randomized Controlled Trial of Yoga for Sleep Quality Among Cancer Survivors. J Clin Oncol 31(26):3233-3241.

\section{9}

A field-test for force-velocity profiling in older adults: feasibility and relationship with appendicular lean mass index

\section{Luca Ferrari $^{1}$, Gianluca Bochicchio ${ }^{I}$, Valentina Cavedon ${ }^{1}$, Chiara Milanese ${ }^{I}$, Massimo Teso ${ }^{I}$, Matteo Rizzo ${ }^{l}$, David Tacconi ${ }^{2}$, Stefano E. Lenzi $^{1}$, Silvia Pogliaghi ${ }^{1}$}

Dipartimento Di Neuroscienze, Biomedicina E Movimento, Università Degli Studi Di Verona, Verona, Italy ${ }^{l}$; Corehab, Euleria, Trento, Italy ${ }^{2}$

Purpose: The Appendicular lean mass (ALM) adjusted for height (i.e. ALM index, ALMI kg/m2) is used for the diagnosis of sarcopenia, a highly prevalent medical condition in older adults. The purpose of this study was to validate an easy field-test for the characterisation of muscle function in older adults and to assess its possible association with ALMI.

Methods: Forty-seven older adults (47\% females, $67 \pm 7$ years, $73 \pm$ $12 \mathrm{~kg}, 1,67 \pm 0,99 \mathrm{~m})$ were asked to perform five Sit-to-stand movements (STS) on a force platform at Body weight (BW), BW + $12,5 \%$ and $\mathrm{BW}+25 \%$, in randomised order. Two subjects $(5 \%)$ were unable to complete the trials $>$ BW and were excluded. For each subject and trial, the mean velocity was calculated from the vertical displacement and the time of the concentric portion of the STS. Then, the individual force-velocity profile of the lower limbs was determined for the extrapolation of the maximal isometric force (L0), corresponding to the null velocity. In addition, ALMI, was calculated based on the quantification of appendicular muscle mass with the Dual-energy X-ray Absorptiometry (DXA). Simple correlation between individual characteristics, L0 and ALMI were determined; then a step-forward multiple linear regression model was identified to estimate ALMI (est ALMI). The correspondence between est ALMI and measured ALMI was evaluated by paired t-test, correlation, and Bland-Altman analysis.

Results: The group mean value of L0 was $174,8 \pm 43,97 \mathrm{~kg}$ and that of ALMI was 7,37 $\pm 1,23 \mathrm{~kg} / \mathrm{m} 2$. Only BW, Sex, and L0 were significantly correlated with AMLI $(r=0,78, r=0,68, r=0,66$, respectively). The step-forward multiple linear regression excluded sex as a predictor and the following final formula was identified: ALMI $=0,209+(0,0789 * \mathrm{BW})+(0,00,793 * \mathrm{~L} 0),(\mathrm{r} 20.83$, SEE $0.5)$. estALMI $(7,37 \pm 1,18 \mathrm{~kg} / \mathrm{m} 2)$ was not significantly different from $(\mathrm{p}=0.985)$ and highly correlated with $(\mathrm{r} 2=0.83)$ ALMI, with a non-significant bias $(-0.002 \mathrm{~kg} / \mathrm{m} 2)$ and an imprecision of $0.5 \mathrm{~kg} / \mathrm{m} 2$ (7\% of ALMI).

Conclusion: The modified STS test proposed in our study is a feasible, simple, submaximal, time-efficient test to characterise muscle function of the lower limbs in older adults. Moreover, maximal isometric force, as estimated from STS (i.e. L0), in conjunction with body weight, allows an accurate and precise prediction of ALMI, one of the reference markers of the sarcopenic condition in older adults. References

1. Alcazar J, Losa-Reyna J, Rodriguez-Lopez C, et al. The sit-tostand muscle power test: An easy, inexpensive and portable procedure to assess muscle power in older people. Exp Gerontol. 2018;112(August):38-43. https://doi.org/10.1016/j.exger.2018. 08.006

2. Cruz-Jentoft AJ, Sayer AA. Sarcopenia. Lancet. 2019;393(10,191):2636-2646. https://doi.org/10.1016/S01406736(19)31138-9.

240

Reduction of sarcopenia through a home-based, APPmonitored, physical exercise intervention. A study protocol of a randomized controlled trial

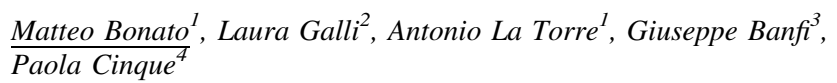

Università Degli Studi Di Milano, Department of Biomedical Sciences For Health, Milan, Italy ${ }^{1}$;

Irccs San Raffaele Scientific Institute, Department of Infectious Diseases, Milan, Italy'

Irccs Istituto Ortopedico Galeazzi, Lamss-Laboratory of Movement and Sport Sciences, Milan, Italy ${ }^{3}$;

Irccs San Raffaele Scientific Institute, Department of Infectious Diseases, Milan, Italy ${ }^{4}$

Purpose: Sarcopenia is a pathophysiological process of aging, caused by reduction of muscle strength and quality, and associated with reduced physical performance, increased risk of falls and fractures, physical disability and premature death. Aims of this study will be to assess the benefit of a home-based, app-monitored resistance-training program on muscle health and other measures in elderly and people living with HIV (PLWH) with sarcopenia. 
Methods: We plan to enroll 98 elderly and 98 PLWH in a multicentric, 48-week, randomized, parallel-group, superiority trial comparing the effect of a home-based and app-monitored strength exercise intervention versus no intervention. Inclusion criteria will be being sedentary; $>60$ year-old in the elderly or $>50$ year-old in the PLWH group; with sarcopenia, as defined by both relative loss of muscle mass by bioimpedentiometry (BIA) and low muscle strength by handgrip. Participant will be randomized 1:1, separately in each group (elderly and PLWH), to either 1) Exercise group (EG), where participants will perform a home-based, app-monitored resistancetraining program; 2) Control group (CG), without exercise prescription. At baseline (BL), week 12 (W12) and end of study (W48) participants will be tested for muscle strength (handgrip, leg extension), balance and gait (Mini-BESTest), leg function (chair standing, 6 min walking test); body composition by dual energy X-ray absorptiometry (DEXA, W48 only) and leg muscle volume by magnetic resonance imaging (MRI, W48 only); quality of life and profile of mood states (standard questionnaires); blood lipids and soluble and cell biomarkers of inflammation and muscular function (immunoenzymatic assays and flow cytometry).

Results: The primary endpoint will be the proportion of subjects with improvement from BL of $>20 \%$ of handgrip muscle strength at W48. Secondary endpoints will be the changes at W12 of handgrip muscle strength and at W12 and W48 of muscle strength as measured by leg extension, balance, gait and leg function; body composition and leg muscle volume by MRI; quality of life and state of mood; blood lipids; soluble and cell biomarkers. We expect that EG participants of both groups will improve their parameters and that improvements will be higher than in CG.

Conclusions: Our approach may be a feasible and immediately available strategy for home-based exercise to reduce sarcopenia in elderly and PLWH.

\section{1}

\section{Resistance training improves physical fitness and reduces pain perception in a group of female workers with upper limb work-related musculoskeletal disorders: a pilot study}

\section{Angelo Ferrillo $^{1}$, Stefano Gobbo ${ }^{2}$, Valentina Bullo ${ }^{2}$, Barbara Vendramin $^{2}$, Martina Pizzichemi ${ }^{1}$, Andrea Ermolao ${ }^{2}$, Marco Bergamin $^{2}$}

Università Degli Studi Di Padova, Padova, Italy ${ }^{1}$; Università Degli Studi Di Padova Dimed, Padova, Italy ${ }^{2}$

Purpose: Work related musculoskeletal disorders (WRMDs) are a cause of productivity losses and disability. Resistance training (RT) and stretching seems to relief pain, reducing the relative workload via range of motion improvement.

Methods: Sixteen women (age: $48.69 \pm 5.88$ years old, working career: $22.75 \pm 2.18$ ) were recruited to participate to a 14 weeks work-based RT and stretching program. Specific exercise training (SET) targeting all body area affected by WRMDs was performed after week 6. Physical fitness was measured via the 2 mins step test (2MST), the back scratch test and the handgrip test (HG). To evaluate the level of pain in cervical spine, shoulder, elbow and wrist the visual analogue scale (VAS) was used.

Results: Differences were verified with $\mathrm{T}$ test. Cervical spine $(\mathrm{p}=$ $0.02)$ and left wrist $(p=0.04)$ VAS decreased, while HG for both right $(\mathrm{p}=0.01)$ and left $(\mathrm{p}=0.01)$ hands and 2MST $(\mathrm{p}=0.01)$ improved. Participants with WRMDs affecting the cervical spine reported a 3.72 higher VAS score for the neck at the beginning of the protocol $(\mathrm{p}=$ $0.03)$.
Conclusions: The protocol improved the physical fitness of participants but showed a limited effect on WRMDs pain. SET could reduce the perception of effort reaching the same benefit as compound movement.

\section{2}

\section{Response to long term supervised exercise training (sext) combined with physical activity in copd: a case report}

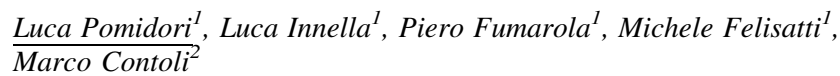

Esercizio Vita Medical Fitness Center, R\&s, Ferrara, Italy ${ }^{1}$, Department of Morphology, Surgery and Experimetal Medicine, University of Ferrara, Ferrara, Italy ${ }^{2}$

Purpose: Pulmonary rehabilitation (PR) and specific exercise training are highly effective treatments for patients with chronic obstructive pulmonary disease (COPD)(1) but remains underuse across the word. Many patients do not access or complete PR, and long-term exercise maintenance is difficult to achieve after $\mathrm{PR}(2)$.

Here we present and report clinical outcomes of a case of long-term (8-years) exercise training adherence. The training consisted of regular supervised exercise combined with videoconferencing exercise sessions and telemonitoring of physical activity provided by a center qualified for SEXT to people affected by chronic disease the "Palestra della Salute".

Methods: Patient's characteristics: 69-year-old female with very severe COPD. She has a 70 pack-year smoking history (stop 2011) and experienced movement-related dyspnea. The patient is regularly visited at a local respiratory unit where she also undergoes pulmonary function tests. The patient attends the center for exercise program twice a week since 2013.The subject received the first indication of structured exercise (2012) in relation to a musculoskeletal problem in 2012. She was also advised to follow a course of SEXT specific for people with chronic diseases.

When the patient started the SEXT, (T0) FEV1 was 33\% predicted; in the 6MWT, the patient walked a total distance of $297 \mathrm{~m}$ without rest; mMRC score was 3 .

After the initial exercise capacity assessment and data collection, the subject started the exercise program consisting of aerobic and resistance training as prescribed by American Thoracic Society (ATS)(2).

The patient underwent 738 SEXT in the gym, 36 videoconferencing exercise sessions and daily regular spontaneous physical activity.

Results: After 8 years of follow up and regular SEXT we found: no change in mMRC and walked distance at the 6MWT. These data suggest that the exercise program resulted in stability of the disease rather than progression.

Interestingly, the number of exacerbations was reduced from a mean number of 6 events/yr before exercise program to 2 events/yr during follow up. The adherence to exercise remained very high trough the $8 \mathrm{yr}$ of follow up.

Conclusions: Long-term exercise maintenance in COPD is feasible. Results suggest that active lifestyle supported by supervised exercise training can prevent deterioration and maintain over time physical performance, health status and quality of life even in fragile subjects such as COPD patients.

References

1. Pedersen BK, Saltin B. Exercise as medicine-evidence for prescribing exercise as therapy in 26 different chronic diseases. Scand J Med Sci Sports. dicembre 2015;25 Suppl 3:1-72. 
2. Holland AE et al. Defining Modern Pulmonary Rehabilitation. An Official American Thoracic Society Workshop Report. Ann Am Thorac Soc. maggio 2021;18(5):e12-29.

\section{3}

\section{Rowing training for the correct somato-postural development in adolescents: a case report}

\author{
Domiziana Minissale $^{1}$, Gianluigi Vaccarino ${ }^{2}$, Riccardo Sorrentino ${ }^{3}$ \\ Università Degli Studi Di Messina, Dipartimento Di Scienze \\ Chimiche, Biologiche, Farmaceutiche Ed Ambientali, Messina, Italy ${ }^{1}$; \\ Direttore Sanitario, Medicalsport, Vaprio D'adda, Italy ${ }^{2}$; \\ Università Degli Studi Di Messina, Dipartimento Di Scienze \\ Biomediche, Odontoiatriche E Delle Immagini Morfologiche E \\ Funzionali, Messina, Italy ${ }^{3}$
}

Purpose: Body modifications during the growth process are many and they can lead adolescents to assume new and sometimes incorrect positions. The alteration of muscle forces during growth, could create some postural and muscular imbalances up to hypotonia and hypotrophy. Postural gymnastic is the most known method to counter these paramorphism; in this study postural exercises were mixed together with rowing practice. The choice of rowing has been made due to its features, since that it is an effort using almost all muscles of posterior back chain and moreover, it stimulates rhytm, coordination and self control.

Methods: In this study, a participant (15 years old, $67 \mathrm{~kg}, 180 \mathrm{~cm}$ ), previously assessed through a postural and radiological examination, diagnosing lumbar scoliotic attitude to left convexity (D10-L4, $8^{\circ}$ ), limbs asymmetry, kyphosis attitude and a shortening of the hamstring muscles, went under a program, combining some postural exercises and rowing sessions. Three weekly technical rowing sessions at a moderate intensity (55-65\% of RPE) with back muscles stretching sessions and two weekly postural and proprioceptive sessions, were performed by the participant for a period of six months. Two postural and medical evaluations were made, one before starting the protocol and one at the end of it.

Results: Results showed how after the mix of rowing and postural exercises, the participant perfomed better at several postural and clinical evaluations. There was an improvement of lumbar and dorsal curvatures, an improvement on mantaining for longer period of time the correct rachid posture and an improvement on controlling hip muscles. There was a slight improvement of the shorter harmsting muscles, but there was still some rigidity after the last assessment. Conclusions: It possible to suggest that rowing, as a total body effort, is actually a valid tool to use in association with postural exercises, in order to control and improve some muscle and postural imbalances. Acknowledgements: The protocol was performed with the facilities of S.C. Thalatta Rowing Club in Messina.

\section{References}

1. Cororve, MB, et al. (2001) 'Body dysmorphic disorder: a review of conceptualizations, assessment, and treatment strategies', Clinical Psychology Review 21: 949-970.

2. Zangla, D.,et al. (2005) 'Modificazioni posturali indotte dal canottaggio in giovani atleti ben allenati', Italyn Journal of Sport Sciences 12: 150-154.

3. Bell, G., et al. (2013) 'A Physiological and Kinematic Comparison of two Different Lean Back Positions During Stationary Rowing on a Concept II Machine', J Hum Kinet 37: 99-108.

\section{4}

Safety and feasibility of a home-based respiratory muscle training in patients recovered from critical covid-19 infection

\author{
Michele Franchi ${ }^{l}$, Matteo Pizzolato ${ }^{l}$, Antonio Argentoni ${ }^{l}$, Giorgio \\ Gandolfi, $^{1}$ Nicola Lamberti ${ }^{2}$, Fabio Manfredini ${ }^{2}$, Annalisa Cogo ${ }^{3}$
}

Scuola Di Specializzazione In Medicina Dello Sport E Dell'esercizio Fisico, Department of Neuroscience and Rehabilitation, University of Ferrara, Italy, Ferrara, Italy ${ }^{1}$;

Department of Neuroscience and Rehabilitation, University of Ferrara, Ferrara, Italy ${ }^{2}$;

Biomedical Sport Studies Center, University of Ferrara, Ferrara, Italy ${ }^{3}$

Purpose: After COVID-19 infection respiratory function may be impaired. Previous studies found that respiratory muscle training can improve ventilatory pattern and reduce oxygen desaturation during endurance exercise testing in patients with pulmonary diseases. The study aim was to analyse safety and feasibility of a 4-week homebased respiratory muscle training in patients who recovered from critical COVID-19 infection.

Methods: Five patients aged 57-71 were enrolled in the study. They all recovered from a severe COVID-19 infection, with a medium length of hospital stay of 24 days. A control group evaluation was conducted with 3 participants of similar characteristics. Spirometry parameter and indices of respiratory muscle strength as maximal inspiratory and expiratory pressures (MIP e MEP) were measured at baseline and after the training. Each patient was administered a 6-min walk test (6MWT) at baseline and after the intervention. Respiratory muscle training was prescribed at the hospital after a brief instructional session during control visits, and conducted at home. Purposely, a positive pressure and volume exerciser (Respi-in-out ${ }^{\circledR}$, Medinet Srl) was delivered to each patient. The program was based on two daily 10-min sessions of respiratory muscle training, every day for 4 weeks, with a progressive increase of air resistance.

Results: No adverse events were observed in both exercise and control groups. Each patient completed the training, with a mean adherence of $80 \%$ respect to the prescribed sessions. At baseline respiratory parameters were similar to predicted values. After training, MVV shows an average increase of 52,36 $1(\mathrm{p}=0.10)$, MIP of $13,00 \mathrm{cmH} 2 \mathrm{O}(\mathrm{p}=0.18)$, MEP of $17,40 \mathrm{cmH} 20(\mathrm{p}=0.17)$. The distance covered during 6MWT increased by $49 \mathrm{~m}(\mathrm{p}=0.13)$. No correlations were observed between respiratory muscles function and 6MWT. No changes were observed in the control group, and no between-group differences were noted due to the limited sample size. Conclusions: In a preliminary observation home-based respiratory muscle training appears to be safe and feasible in patients who recovered from a critical Covid-19 infection, with a respiratory improvement concomitant to a functional benefit. Further evaluations are required to assess the impact of a respiratory muscle training program on respiratory parameters of patients who recovered from severe Covid-19 infection, in order to assess if it could be a part of standard rehabilitation protocols.

\section{References}

1. Pollard C, Morran M, Nestor-Kalinoski A. (2020) The COVID19 pandemic: a global health crisis. Physiol Genomics Nov 1;52(11):549-557.

2. Sales AT. (2016) Respiratory muscle endurance after training in athletes and non-athletes: A systematic review and meta-analysis Phys Ther Sport. Jan;17:76-86.

3. Bernardi E, Cogo A. (2015) Respiratory muscle training with normocapnic hyperpnea improves ventilatory pattern and 
thoracoabdominal coordination, and reduces oxygen desaturation during endurance exercise.

\section{5 \\ SARS-COV-2: supervised exercise training (SEXT) as strategy for improving health and exercise capacity after hospitalization}

\author{
Piero Fumarola $^{1}$, Michele Felisatti $^{1}$, Luca Innella ${ }^{1}$, Luca Pomidori ${ }^{1}$
}

Esercizio Vita Medical Fitness, R\&s, Ferrara, Italy ${ }^{1}$

Purpose: Not all individuals having access to hospital care undergo rehabilitation that includes. functional recovery sessions. Home-based exercise is recommended in these cases, following given instructions. Social isolation may also represent a worsening factor. Therefore, certified structures such as Palestre della Salute (PS) can operate as an ally in functional recovery and social reintegration.

Methods: This work takes into consideration subjects with symptomatic outcomes of Sars-Covid-19 infection, discharged from the hospital without specific rehabilitation program for the recovery of exercise capacity. Subjects in possession of a prescription of SExT from the pulmonologist were selected among the clients of a certified PS. They performed an initial functional assessment (TI) and a final functional assessment after 6 months (TF) consisting of: 6-Minute Walking Test (6-MWT), Berg Balance Scale(BBS), Sit To Stand (STS), Timed up and go(TUG), 10-Meter Walk Test (10-MWT) and Short Physical Performance Battery (SPPB). SExT is based on aerobic capacity, mobility, static and dynamic balance and muscle strengthening with a frequency of at least 2 weeks and a duration of 60 min per session.

Results: To this day two subjects have been selected (1F aged 53 and $1 \mathrm{M}$ aged 71), they are completing a training period of 6 months. No adverse events have been reported and they have resumed their usual work activities. The Results are being processed and will be presented directly.

at the congress.

Conclusions: SExT can be a keystone not only to improve or maintain impaired physical abilities but also for the socializing aspect in subjects who have suffered a long period of isolation during hospitalization. A non-healthcare territorial service, conducted by certified "PS" such as the model structured by Esercizio VIta capable of administering scheduled and supervised physical exercise (1)(2) may be essential for the recovery of impaired functionality in subjects who have encountered a Sars-Covid-19 infection. Although gyms are not generally considered as functional recovery in this issue, we believe they are useful to increase the APA.

\section{References}

1. Returning to physical activity after covid-19.: BMJ,372:m4721.

2. American College of Sports Medicine. ACSM's Guidelines for Exercise Testing and Prescription, 10th ed.; Wolters. Kluwer: Alphen aan den Rijn, The Netherlands, 2017; ISBN $9,788,578,110,796$.

\section{6}

Scoliosis online: feasibility study of a video exercise program administered during lockdown

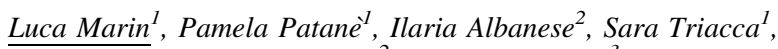
Federica Letizia Lucia Gentile ${ }^{2}$, Luisella Pedrotti ${ }^{3}$, Sara Ottobrini ${ }^{4}$
Laboratory of Adapted Motor Activity (lama), University of Pavia, Department of Public Health, Experimental and Forensic Medicine, Pavia, Italy ${ }^{1}$;

Phd School, Tor Vergata University Department of Industrial Engineering, Rome, Italy ${ }^{2}$;

Section of Pathologies of The Musculoskeletal System, Orthopedics Unit, University of Pavia Department of Pediatric Diagnostic Clinical Science, Pavia, Italy ${ }^{3}$;

Laboratory For Rehabilitation Medicine and Sport (larms), Department of Special Abilities, Rome, Italy ${ }^{4}$

Purpose: In Italy, the pandemic of COVID-19 imposed the interruption of non-urgent health services. Tele-exercise (TE) has proved to be an effective and satisfactory alternative in supporting the administration of exercise in subjects with Adolescent Idiopathic Scoliosis (AIS). During the lockdown period, the Department of Pediatric Orthopedics of the University Hospital "Città di Pavia" (Pavia, Italy) supported AIS patients with the project "Scoliosis Online", to reduce negative effects on the biopsychological component. The aim of the study was to evaluate adherence, perceived pleasantness, and level of satisfaction with the technology used to administer the TE in a group of subjects with AIS.

Methods: Between 14 and 20th April 2020, 11 adolescents with AIS were recruited. From 28th April (T0) to 26th June (T1) they autonomously carried out a bi-weekly exercise program using a web platform (YouTube, Google LLC, Mountain View, CA, USA). Each participant accessed to a personal channel, containing videos of the self-correction movement and six exercises, tailored to their type of scoliotic curve. For each exercise video showed execution and number of repetitions to be done. Every 15 days participants received an e-mail encouraging them to give a feedback and to report any problems. To assess adherence, participants wrote date and time of each completed session in a diary. Alike, at the end of each session, Feeling Scale (FS) was filled to express the perceived pleasantness. At T1 the Technology Acceptance Model (TAM) was administered to evaluate how participants accepted and used the technology employed and their attitude to use it again.

Results: Results, expressed as mean and standard deviation, were: completed sessions $13.25 \pm 2.19$ out of 15 , FS total score $1.02 \pm 1.34$ out of \pm 5 and TAM total score $21.78 \pm 1.09$ out of 28 .

Conclusions: Adherence to the program was very high as were the scores related to the technology. Results could lead to the children's habit of using social platforms and technologies. Perceived pleasantness achieved positive, but low, values. This could be related to solitary training and the many hours forcedly spent at the computer for distance learning. Limits of the study are the small number of the sample and the self-compilation of the number of sessions performed. Face-to-face work remains the gold standard but in periods of social distancing a TE program could help support the exercise practice of children with AIS.

\section{References}

1. Negrini S, Donzelli S, Negrini A, Negrini A, Romano M, Zaina F. (2020) Feasibility and acceptability of telemedicine to substitute outpatient rehabilitation services in the COVID-19 emergency in Italy: an observational everyday clinical-life study. Arch Phys Med Rehabil S0003-9993(20)30,506-2. Online ahead of print.

2. L Marin Editor, (2021) Attività Motoria Adattata: dalla teoria alla pratica. Calzetti \& Mariucci Editors, Ferriera di Torgiano, Perugia, Italy. ISBN 978-88-6028-457-0. 


\section{7}

\section{The effect of exercise training on postural control and proprioception in women with osteoporosis}

\author{
Giuseppe Barone $^{1}$, Erika Pinelli ${ }^{1}$, Maria Grazia Benedetti ${ }^{2}$, Raffaele \\ $\overline{\text { Zinno }}^{I}$, Giuseppe Audino ${ }^{1}$, Laura Bragonzoni ${ }^{1}$
}

Università Di Bologna, Dipartimento Di Scienze Per La Qualità Della Vita, Rimini, Italy ${ }^{1}$, Istituto Ortopedico Rizzoli, Medicina Fisica E Riabilitativa, Bologna, Italy ${ }^{2}$

Background: Osteoporosis (OP) is a skeleton systemic disease characterized by a reduced bone mass and deterioration of microarchitecture. OP is often accompanied by an increased risk of fall and consequently a high risk of fracture. Since postural control and proprioception are two of the most significant factors in falls and injury prevention, exercise training, including specific balance and proprioceptive exercises, could be the keys to reducing the risk of fall and fractures. This study was aimed at assessing proprioception and postural control, using Delos Postural Proprioceptive System (DPPS; Delos, Turin, Italy), in persons with osteoporosis, before and after an exercise training.

Methods: A cohort of 29 women with osteoporosis aged $66.20 \pm 5.80$ were involved in the study. The participants will perform training, structured in 2-days per week, to improve joint mobility, muscle force and balance. Moreover, an additional activity between walking, cycling, or swimming was requested to improve endurance and reach the weekly exercise recommended by World Health Organization. Finally, every six weeks, the trainer upgraded the exercise program following the principle of frequency, intensity, time and type. DPPS was used to assess postural control and proprioception in single-limb stance, respectively, with open and closed eyes. The parameter considered is the stability index (SI; percentage score where $100 \%$ is a theoretical task performed with maximum stability).

Results: No significant differences were found in terms of dominant and non-dominant limbs. Thus, the analysis was performed on the average Results of two limbs. The SI improved from $84.14 \% \pm 10.10$ to $86.96 \% \pm 5.60$ with opened eyes $(\mathrm{p}<0.05)$ and from $52.22 \% \pm$ 13.87 to $56.25 \% \pm 17.60$ with closed eyes $(\mathrm{p}<0.05)$.

Conclusion: In people with osteoporosis, fall prevention is essential to decrease the risk of fall, build confidence for performing daily-life activities and improve the quality of life. Moreover, preventing falls decrease the costs for the health care systems. Physical activity should be integrated with pharmacotherapy in osteoporosis treatment since it benefits bone tissue and improves global fitness. In particular, including balance exercises in a training program is effective in improving postural control and proprioception. The latter seems to have greater benefit and is very important in poorly lighting places or conditions of sudden instability.

\section{References}

1. Riva D et al. (2013) Single stance stability and proprioceptive control in older adults living at home: Gender and age differences. J Aging Res 2013: 561,695.

2. WHO, WHO guidelines on physical activity and sedentary behaviour. World Health Organization; 2020: Geneva, 2020.
Università Degli Studi Di Palermo, Dipartimento Scienze Psicologiche, Pedagogiche, Dell'esercizio Fisico E Della Formazione, Palermo, Italy ${ }^{I}$;

Università Degli Studi Di Palermo, Dipartimento Di Biomedicina, Neuroscienze E Diagnostica Avanzata, Palermo, Italy ${ }^{2}$;

Università Degli Studi Di Palermo, Dipartimento Scienze E Tecnologie Biologiche Chimiche E Farmaceutiche, I Palermo, Italy ${ }^{3}$

Purpose: the aim of this study was to investigated the improvement of physical health status and reduce the chronic fatigue associated with that pathology in people with multiple sclerosis following lactacid anaerobic training protocol.

Methods: 8 subjects (age: $34,88 \pm 4,45$ ) affected by multiple sclerosis (EDSS $<3.5$ ) were involved in our study. An anaerobic lactacid training was administered for 12 weeks, 2 times a week, an hour and a half for each session. At the beginning (T0), at the end of the study (T1) and after 9 months from the end of the study (Follow-up, FU) physical parameters (body compositions analysis; strength, balance and reaction tests; Visual Analog Fatigue Scale, VAFS) and hematochemicals parameters (lactate and neurotrophins BDNF and DHEAS) were tested.

Results: Body compositions analysis showed an increase in total body water and in basal metabolic rate $(\mathrm{p}<0.05)$. Furthermore, an improving trend was detected on the percentages of lean mass and a tendency to decrease was detected on the percentages fat mass $(\mathrm{p}=$ $0.05)$. BDNF levels significant increase from $\mathrm{T} 0$ to $\mathrm{T} 1$ as well as DHEA-S levels $(p<0.05)$ but the latter significant decrease from T0 and FU $(\mathrm{p}<0.05)$ and from T1 and FU $(\mathrm{p}<0.01)$. About strength, balance and reaction tests no significant differences was found after lactacid anaerobic training protocol; moreover Magnetic Resonance Imaging and EDSS evaluation show no progression of disease for all the subjects. Furthermore, baseline lactate levels showed a decrease after the protocol training $(\mathrm{p}<0.05)$ which could be best re-used as energy substrate. VAFS showed statistically significant decrease in fatigue level between T0 and T1 (p < 0.01).

Conclusions: The results obtained have confirmed that lactacid exercise can influence physical health. Our protocol may increase brain-derived neurotrophic factors and DEHA-S concentrations and their functions that are related to decrease in fatigue level. It's known that fatigue symptom is associated with low levels of DHEA-S in subjects with multiple sclerosis. Lactacid physical exercise appear to act with synergic positive effects on inflammation, neural plasticity, and neuroprotection, by reducing lactate basal level and it can reduce MS symptoms progression.

\section{References}

1. Azevedo L, Pereira JR, Santos RMS, Rocha NP, Teixeira AL, Christo PP, Scalzo PL. (2021) Acute exercise increases BDNF serum levels in patients with Parkinson's disease regardless of depression or fatigue. Eur J Sport Sci, 1-21.

2. Angelova G, Skodova T, Prokopiusova T, Markova M, Hruskova N, Prochazkova M, Rasova K. (2020) Ambulatory Neuroproprioceptive Facilitation and Inhibition Physical Therapy Improves Clinical Outcomes in Multiple Sclerosis and Modulates Serum Level of Neuroactive Steroids: A

\title{
248
}

\section{The efficacy of anaerobic training on multiple sclerosis symptoms management}

\author{
Alessandra Amato ${ }^{1}$, Paolo Ragonese ${ }^{2}$, Gabriella Schiera ${ }^{3}$, Sara
}

Baldassano $^{3}$, Italy Di Liegro ${ }^{2}$, Patrizia Proia ${ }^{1}$ 
249

The exercise facilitator in the dialysis unit promoting attitude and ability to exercise to improve quality of life in the patients with end-stage kidney disease

\author{
Giovanni Piva $^{1}$, Nicola Lamberti ${ }^{2}$, Maria Donadi $^{3}$, Yuri Battaglia ${ }^{3}$, \\ Roberto Manfredini ${ }^{4}$, Fabio Fabbian ${ }^{4}$, Alda Storari ${ }^{3}$, Fabio \\ Manfredini $i^{2}$ \\ Università Degli Studi Di Ferrara, Studi Umanistici, Ferrara, Italy ${ }^{1}$; \\ Università Degli Studi Di Ferrara, Neuroscienze E Riabilitazione, \\ Ferrara, Italy ${ }^{2}$; \\ Azienda Ospedialiero-universitaria Di Ferrara, Medico Specialistico, \\ Ferrara, Italy ${ }^{3}$; \\ Università Degli Studi Di Ferrara, Scienze Mediche, Ferrara, Italy ${ }^{4}$
}

Purpose: End-stage kidney disease is associated to fatigue, sedentary behavior and high risk of morbidity and mortality. The study (NCT04282616 [1]) aims to test in a pragmatic implementation trial the presence of an exercise facilitator (EF) in a dialysis center. This exercise specialist has to motivate and initiate patients to the preferred training modality to obtain physical and psychological benefits.

Methods: End-stage kidney disease patients undergoing hemodialysis without contraindication to exercise after the provision of informed consent were asked to choose between four training programs proposed by the EF: i) in-hospital supervised with the EF before/after the dialysis session (IN); ii) structured home-based [2] (HB); iii) advices to increase spontaneous physical activity [3] (AD); iv) physical performance assessment only (PE). Patients underwent testing sessions at baseline and at the end of the programs (3-month) that included: 6-min walking test (6MWT), 10-m gait speed, 5-time sit-to-stand test (5STS). Quality of life and psychosocial outcomes are also explored. Results: Since late 2019, 300 patients were contacted, and 222 addressed the inclusion criteria. of those, $180(81 \%)$ were contacted but 95 refuse to take part in the program reporting: "poor interest" (38\%), "already active" (34\%) or "fatigue" (12\%). Within the 85 patients $(47 \%)$ that agreed to participate, $43(51 \%)$ chose the IN program, $28(33 \%)$ the $\mathrm{HB}, 7(8 \%) \mathrm{AD}$ and $7 \mathrm{PE}$. Patients choosing the IN program were significantly older respect to $\mathrm{HB}$ and AD (70 vs 59 vs 54 years, respectively; $p<0.001$ ), more frail (Charlson Index 7 vs 4 of other groups) and with impaired mobility (6-min walking distance $202 \mathrm{~m}$ vs $321 \mathrm{~m}$ of the HB). Up to now, 40 patients $(\mathrm{IN} \mathrm{n}=$ 25; $\mathrm{HB} \mathrm{n}=15$ ) have concluded the program without any adverse event. Both groups significantly improved all outcomes respect to baseline: 6MWT (IN + $31 \mathrm{~m}$; HB $+35 \mathrm{~m}$ ); 10-m speed (IN + $0.6 \mathrm{~km} / \mathrm{h} ; \mathrm{HB}+0.7 \mathrm{~km} / \mathrm{h}$ ); $5 \mathrm{STS}$ (IN $-2.7 \mathrm{~s}$; HB $-1.9 \mathrm{~s}$ ). No betweengroup differences were observed between IN and $\mathrm{HB}$, even after the correction for the baseline covariates.

Conclusion: The constant presence of the EF in the dialysis center highlighted a satisfactory attitude of renal patients towards exercise and favored the adherence to the training (compared to the 23\% compliance rate reported in literature) with positive functional outcomes. The continue of the trial will possibly confirm these preliminary findings.

References

1. Manfredini F, et al. A Personalized Patient-Centered Intervention to Empower through Physical Activity the Patient in the Dialysis Center: Study Protocol for a Pragmatic Nonrandomized Clinical Trial. Methods Protoc. 2020 Dec 6;3(4):83.

2. Manfredini F, et al. Exercise in Patients on Dialysis: A Multicenter, Randomized Clinical Trial. J Am Soc Nephrol. 2017 Apr;28(4):1259-1268.

3. Johansen KL. Exercise and chronic kidney disease: current recommendations. Sports Med. 2005;35(6):485-99.
250

The potential impact of physical activity on muscle fatigue in patients with parkinson's disease

Camilla Martignon $^{l}$, Fabio Giuseppe Laginestra ${ }^{l}$, Gaia Giuriato ${ }^{l}$, Anna Pedrinolla $^{I}$, Chiara Barbi ${ }^{l}$, Federico Schena ${ }^{l}$, Massimo Venturelli $^{l}$

Università Di Verona, Università Di Verona, Verona, Italy ${ }^{I}$

Purpose: Given the increased level of fatigue frequently reported by patients with Parkinson's disease (PD), this study investigated the interaction between central and peripheral components of muscle fatigue (MF) in this population, compared to healthy peers.

Methods: Changes in maximal voluntary activation $(\triangle \mathrm{MVA}$, central fatigue) and potentiated twitch force ( $\Delta$ Qtw,pot, peripheral fatigue) from pre to post exercise were determined via the interpolated twitch technique in 10 patients with PD and 10 healthy controls (CTRL) matched for age, sex and physical activity. Pulmonary gas exchange, femoral blood flow (FBF), and quadriceps electromyography (iEMG) were measured during a fatiguing exercise $(85 \%$ of peak power output (PPO)). For a specific comparison, on another day, CTRLs performed an additional fatiguing test matching the time to failure (TTF) and PPO of PDs.

Results: Comparing the groups at $85 \%$ of PPO (PD $=21 \pm 7 \mathrm{~W}$; CTRL $=37 \pm 22 \mathrm{~W})$, TTF ( $\sim 5.9 \mathrm{~min})$, pulmonary gas exchange, FBF, and iEMG were similar. After this exercise, the maximal voluntary contraction force (MVC) and Qtwpot decreased equally in both groups $(-16 \%, \mathrm{p}=0.483 ;-43 \%, \mathrm{p}=0.932)$, while PD exhibited a greater reduction in MVA compared to CTRL $(-3.8 \%$ Vs $-1.1 \%, \mathrm{p}=$ $0.081)$. At the same PPO and TTF of PDs (21 W; $5.4 \mathrm{~min})$, CTRLs showed a constant drop in MVC, and Qtwpot $(-14 \%, \mathrm{p}=0.854 ;-39 \%$, $\mathrm{p}=0.540)$, instead MVA decreased more in PD than in CTRL $(-3.8 \%$ Vs $-0.7 \%, p=0.028)$.

Conclusion: In PDs, central MF seems exacerbated by the fatiguing task which, however, does not alter peripheral fatigue. This, besides the TTF like CTRLs, suggests that physical activity may limit MF and counterbalance PD-induced degeneration through peripheral adaptations.

\section{References}

1. Garber, C. E. \& Friedman, J. H. Effects of fatigue on physical activity and function in patients with Parkinson's disease. Neurology 60, 1119-1124 (2003).

2. Kelly, N. A. et al. Effects of aging and Parkinson's disease on motor unit remodeling: Influence of resistance exercise training. J. Appl. Physiol. 124, 888-898 (2018).

\section{1}

Tolerability and preliminary efficacy of a supervised exercise program in cancer patients with metastatic disease

Giulia Benato $^{1}$, Lorenzo Belluomini ${ }^{2}$, Ilaria Trestini ${ }^{2}$, Daniela Tregnago $^{2}$, Michele Milella $^{2}$, Massimo Lanza $^{1}$, Sara Pilotto ${ }^{2}$, Alice Avancini $^{2}$

University of Verona, Department of Neuroscience, Biomedicine, and Movement, Verona, Italy ${ }^{1}$;

University of Verona, Department of Oncology, Verona, Italy ${ }^{2}$

Purpose: exercise (EX) is an attractive strategy to adopt in the oncology setting due to its benefits and potential impact on patients' prognosis. Unfortunately, most of the available data derive from trials 
on patients in the early stage of disease, whereas the evidence in the advanced stages is still limited. This feasibility study aims to explore the tolerability and preliminary efficacy of an EX-program in metastatic cancer patients.

Methods: A 12-week supervised EX-program was offered to patients with metastatic cancer recruited at the Oncology Unit in the Verona twice a week was proposed. The aerobic activity progressively increased from 10 to $30 \mathrm{~min}$ at moderate intensity, i.e., 3-5 of the 10-point Borg Rating of the Perceived Exertion Scale (RPE). The strength component consisted of body weight or elastic-bands exercises, performed in 2-3 sets of 8-12 repetitions at moderate intensity, i.e., 3-5 of the RPE. Tolerability of the intervention was measured by the program, and the drop-out rate. Safety was assessed through the number of serious and non-serious adverse events that occurred during the training sessions. Among secondary outcomes, cardiorespiratory fitness was evaluated with the "Six minutes walking test" (6MWT), strength by the leg press test and handgrip test, and quality of life (QoL) with the European Organization for Research and Treatment of Cancer Quality of Life and Core Questionnaire.

Results: Seven patients with cancer at stage IV were included in the intervention study. The main tumor sites were lung $(n=3)$, pancreas $(n=2)$, ovary $(n=1)$, and breast $(n=1)$. No drop-out was registered. Adherence to the training sessions was $81.7 \%$, whereas compliance at assessments was $81.7 \%$. Grade 1 (mild) adverse events were registered: delayed onset muscle soreness $(n=3)$ and dizziness $(n=1)$. Positive trends were observed for 6MWT $(+29.1 \mathrm{~m})$, for upper $(+$ $2.9 \mathrm{~kg})$ and lower $(+21.0 \mathrm{~kg})$ limb strength, and lower limbs flexibility, although the results were not significant. Upper limb flexibility improved significantly $(\mathrm{p}<0.003)$. Concerning QoL, the "social function" domain increased significantly $(\mathrm{p}<0.04)$.

Conclusion: A supervised EX intervention is well-tolerated by cancer patients with metastatic disease. A future randomized controlled trial will be able to verify the efficacy.

\section{References}

1. Campbell KL, Winters-Stone KM, Wiskemann J, May AM, Schwartz AL, Courneya KS, et al. Exercise Guidelines for Cancer Survivors: Consensus Statement from International Multidisciplinary Roundtable. Med Sci Sports Exerc. 2019 Nov; 51(11):2375-90.

2. Nadler, M. B., Desnoyers, A., Langelier, D. M., \& Amir, E. (2019). The effect of exercise on quality of life, fatigue, physical function and safety in advanced solid tumor cancers: a metaanalysis of randomized control trials. Journal of Pain and Symptom. Hospital. A multicomponent (aerobic + strength) training to perform adherence to baseline and post-intervention assessments, adherence to

Asl02 Lanciano-vasto-chieti, Eusoma Breast Centre, "g. Bernabeo" Hospital, C.da S. Liberata, 66026, Ortona, Italy, Ortona, Italy ${ }^{3}$

Purpose: Pharmacological therapies of breast cancer can negatively affect quality of life of breast cancer survivors (BCS). Increasing literature focus the attention on the importance of physical exercise to improve the recovery of BCS, especially for the cardiovascular system, and both aerobic and strength trainings are suggested to optimize the intervention. As it is not common to completely comply international guidelines, the aim of the study was to investigate the effects of Walking (W), Nordic Walking (NW) and Circuit Training (CT) programs on cardiovascular variables (i.e. mean arterial blood pressure (MABP), augmentation index (ABI), pulse wave velocity (AI), intima media thickness (IMT)) of BCS.

Methods: Sixty BCS (52.35 $\square 7.59$ ), after a battery of psychophysical and behavioural tests, and after having obtained medical eligibility, were randomly assigned to one of the 3 training groups. After the training period, the tests were repeated. Here, we focus the attention on MABP, measured through electronic spygmomanometer (Omron M6 Comfort, Kyoto, Japan) and on ABI, AI, and IMT, measured through Vascular Explorer (enverdis GmbH, Dusseldorf, Germany). Participants were trained for 12 weeks, 3 times per week. Each lesson lasted $75 \mathrm{~min}$. W and NW group had the following scheme of training, from 1 st to 4 th week at RPE $10-11$, from 5 to 8 th week at RPE 12-13, and from 9 to 12th week at RPE 13-14. CT group was trained according to Schmitz et al. (2010) findings.

Results: At the end of the study, independently from age, body recomposition and pharmacological treatment, MABP, AI and PWV have been shown modified according to the discipline. W determined the worse reduction of MABP, while it not elicited a significant modification of both AI and PWV. NW determined the best improvement of MABP, AI and PWV. IMT reduction has been shown inversely correlated with the volume of physical exercise.

Conclusions: In BCS, physical exercise can improve vascular compliance, reducing arterial stiffness. According to our Results, NW elicits the best improvement of MABP, AI and PWV. Even if this is not the only explanation, this is due to the contemporaneous aerobic use of big muscle masses, eliciting a major production of DHEA-s, adiponectin, VEGF, and nitric oxyde, as showed in our published (Izzicupo et al. 2017) and unpublished researches. On the contrary, after 3 months of training, the IMT reduction seems linked to the volume of training, instead of to the discipline.

\section{References}

1. Schmitz et al. (2010). Weight lifting for women at risk for breast cancer-related lymphedema. JAMA 304:2699-2705.

2. Izzicupo et al. (2017). Nordic Walking increases circulating VEGF more than traditional walking training in postmenopause. Climacteric 20:533-539.

\section{2}

\section{Vascular effects of walking, nordic walking and circuit training in breast cancer survivors}

Federica Lancia ${ }^{l}$, Andrea Di Blasio ${ }^{1}$, Teresa Morano ${ }^{1}$, Sabina Gallina $^{2}$, Valentina Bucciarelli ${ }^{2}$, Francesco Bianco ${ }^{2}$, Alessandra Di Marco $^{1}$, Luca Innella ${ }^{1}$, Ines Bucci ${ }^{1}$, Saveria Tavoletta ${ }^{3}$, Ettore Cianchetti $^{3}$, Simona Grossi ${ }^{3}$, Giorgio Napolitano ${ }^{1}$

"g. D’annunzio" University of Chieti-pescara, Department of Medicine and Ageing Sciences, Via Polacchi L. 11, 66100, Chieti, Italy, Chieti, Italy ${ }^{I}$;

"g. D'annunzio" University of Chieti-pescara, Department of Neuroscience and Imaging, Via Polacchi L. 11, 66100, Chieti, Italy, Chieti, Italy ${ }^{2}$;

\section{3}

\section{Walking kinematics and functional mobility in patients wearing a brace after shoulder surgery}

\author{
Francesca Greco $^{1}$, Alessandro Barone ${ }^{2}$, Olimpio Galasso ${ }^{2}$, Filippo \\ Familiari $^{2}$, Antonio Aversa ${ }^{1}$, Giorgio Gasparini ${ }^{2}$, Gian Pietro \\ Emerenziani ${ }^{I}$ \\ “magna Graecia” University of Catanzaro, Italy, Department \\ of Experimental and Clinical Medicine, Catanzaro, Italy ${ }^{1}$; \\ "magna Graecia” University of Catanzaro, Italy, Department \\ of Orthopaedics and Traumatology, Catanzaro, Italy ${ }^{2}$
}


Purpose: Falls are especially relevant in patients undergoing elective orthopedic procedures. Orthopedic research has been focused predominantly on the risk of falls after lower extremity surgical procedures. On the contrary, little is known regarding the effects of wearing a brace after elective upper extremity surgery on gait performance and falls. Therefore, the aim of the present study was to investigate the influence of a $15^{\circ}$ shoulder abduction brace (SAB) on walking kinematics and functional mobility in patients undergoing shoulder arthroplasty and rotator cuff repair.

Methods: Non-invasive analyses were carried out on a sample of twenty-three patients (mean age: $56.4 \pm 9.9$ years) using a wearable inertial device for gait analysis (G-Walk System by BTS Bioengineering). Each patient underwent a gait and functional mobility evaluation performing the $10-\mathrm{m}$ walk test and Timed Up and Go (TUG) test before (T0) and $24 \mathrm{~h}$ (T1) after the surgery.

Results: In the $10-\mathrm{m}$ walk test, pelvic rotation $(\mathrm{p}<0.05)$ and symmetry index $(\mathrm{p}=0.01)$ were significantly lower at $\mathrm{T} 1$ than T0. No significant differences for time to perform 10-m test, cadence, speed, pelvic tilt and obliquity were found. Time to perform TUG was significantly higher $(\mathrm{p}<0.05)$ at $\mathrm{T} 1$ than T0. Moreover, the duration of the sitting phase was significantly higher $(\mathrm{p}<0.05)$ at $\mathrm{T} 1$ than T0. No significant differences for the duration of the lift and turn-around phases were found during TUG.

Conclusions: The use of SAB after shoulder surgery could influence some walking kinematics and patients' functional mobility. It is not known if changes in gait parameters may persist after the rehabilitation process and if these changes may be linked to the risk of falls. References

1. Sridharan, M. J., Everhart, J. S., Frantz, T. L., Samade, R., Neviaser, A. S., Bishop, J. Y., \& Cvetanovich, G. L. (2020) High prevalence of outpatient falls following elective shoulder arthroplasty. J Shoulder Elbow surg 29:699-706.

2. Sonoda, Y., Nishioka, T., Nakajima, R., Imai, S., Vigers, P., \& Kawasaki, T. (2018) Use of a shoulder abduction brace after arthroscopic rotator cuff repair: A study on gait performance and falls. Prosthetics and orthotics international, 42:136-143.

\section{4}

\section{Adapted physical activity for subject with parkinson disease: a case study}

\author{
Erika Pinelli $^{I}$, Giuseppe Barone ${ }^{1}$, Eugenia Capitanelli ${ }^{1}$, Raffaele \\ $\overline{\text { Zinno }}^{\text {, Laura Bragonzoni }}{ }^{1}$ \\ University of Bologna, Department of Life Quality Studies, University \\ of Bologna, Italy, Bolgona, Italy ${ }^{l}$
}

Purpose: The study aims to evaluate the effects of an adapted physical activity program on a woman with Parkinson's. An 81-yearold woman also presents with arrhythmic heart disease with episodes of paroxysmal atrial fibrillation being treated, osteoporosis, and right hip replacement.

Methods: The woman underwent a 3-month adapted physical activity program, a 45-min program two/three times a week; the objectives of the program were to increase joint mobility, muscle strength, static and dynamic balance, motor coordination, and endurance. The subject was evaluated at baseline and after 3 months of activity. The tests performed were: Four Test Balance Scale (FTBS), Chair Stand Test (CST), Timed Up and Go (TUG), evaluation of the range of motion with the goniometer. At the end of the 3 months, in addition to the functional tests mentioned above, the subject completed a questionnaire on the satisfaction of the proposed approach or protocol.
Results: The 3-month follow-up revealed improved right hip mobility ( $85^{\circ}$ to $90^{\circ}$ flexion, $5^{\circ}$ to $8^{\circ}$ extension, $8^{\circ}$ to $10^{\circ}$ external rotation), on the FTBS (for position standing in tandem at baseline: failure, at follow up: 10 "), in Timed Up and Go (walking without a walker test at baseline: failure, at follow up: 23 ") Finally, from the questionnaire, it emerged that the subject has acquired awareness of the importance of physical exercise in her state of health and reported an improvement in the quality of sleep in the days in which the activity was practiced, and also a decrease in tremor until from the first sessions.

Conclusions: The right amount of physical activity on a person with Parkinson's disease can slow the progression of the disease and its symptoms. It was also observed that exercise participates in a better emotional state of the subject, also improving the quality of life.

\section{References}

1. Kim, Y., Lai, B., Mehta, T., Thirumalai, M., Padalabalanarayanan, S., Rimmer, J. H., \& Motl, R. W. (2019). Exercise Training Guidelines for Multiple Sclerosis, Stroke, and Parkinson Disease. American Journal of Physical Medicine \& Rehabilitation, 98(7), 613-621.

2. Ramaswamy B, Jones J, Carroll C Exercise for people with Parkinson's: a practical approach Practical Neurology 2018;18:399-406.

\section{5}

\section{Adapted physical activity online for tertiary prevention in chronic non-communicable diseases: preliminary feasibility study}

\author{
Ilenia Fracca ${ }^{1}$, Omar Piccinin ${ }^{2}$, Massimo Lanza ${ }^{2}$
}

Studio Prevenzione E Salute Di Fracca Dott.ssa Ilenia-Palestra Della Salute, Università Degli Studi Di Verona, Arzignano-Vicenza, Italy $^{1}$;

Universita', Università Degli Studi Di Verona, Verona, Italy ${ }^{2}$

Purpose. During the lockdown period caused by Sars-Cov-2, the "Palestra Della Salute" proposed adapted physical activity (APA) online. The study main aim was to verify the feasibility of postural online training in two subjects suffering from osteoporosis (OP) and ankylosing spondylitis (SA). The second aim was to verify if this kind of training was maintainable and effective.

Methods. We used questionnaires assessing the general state of health (SF-36), the feeling of disability induced by the pathologies (NDI, RDQ), the level of satisfaction of the physical activity (PACES). Sit and reach, back scratch, lateral head flexion and arm curl tests were adopted to measure flexibility and strength. Tracker Online (https://physlets.org/tracker/) Orthotoolkit.com, Zoom, Excel and Adobe Premiere have been adopted for the analysis of tests images and data.

Results. Compliance at assessments and adherence to the training sessions was $100 \%$. No adverse events were registered. Both participants reported a better feeling of general health (OP: SF-36 $=79.2 \%$; SA: SF-36 $=75.14 \%$ ) and a lower perception of disability due to problems cervical (OP: 12\% PRE, 10\% POST; SA: 14\% PRE, $6 \%$ POST) and lumbar (OP: 1/24 PRE, 0/24 POST; SA: 8/24 PRE, 4/24 POST). Furthermore, from this type of intervention, at the level of joint mobility, flexibility and muscle strength, no worsening was observed, but rather, several observable improvements at the scapulohumeral joint level (OP: $+1.87 \mathrm{~cm}$ and $+3.70 \mathrm{~cm}$; SA: $+2.63 \mathrm{~cm}$ and $+1.13 \mathrm{~cm}$ ) and arms strength (SA: +4 rep and +3 rep).

Conclusions. According to the study carried out APA online seems to be feasible, safe and tolerable over time. This kind of methodology 
can be helpful to counteract inactivity. The distance tests execution and analysis, however, must be preceded by training with specific executive indications.

\section{References}

1. Aytekin, E. e c. (2012). Home-based exercise therapy in patients with ankylosing spondylitis: Effects on pain, mobility, disease activity, quality of life, and respiratory functions. Clinical Rheumatology, 31(1), 91-97.

2. Senderovich, H e c. (2017). The Role of Exercises in Osteoporotic Fracture Prevention and Current Care Gaps. Where Are We Now? Recent Updates. Rambam Maimonides Medical Journal, 8(3), 0032.

\section{EXERCISE AND SPORT PHYSIOLOGY}

\section{6}

\section{Comparison between arterial blood gases, $\mathrm{SPO} 2$ and ORI in scuba diver with air breathing apparatus. A pilot study}

\author{
Fabio Di Pumpo ${ }^{I}$, Matteo Paganini ${ }^{1}$, Tommaso Antonio Giacon ${ }^{1}$ \\ Luca Martani ${ }^{I}$, Danilo Cialoni ${ }^{I}$, Giacomo Garetto ${ }^{I}$, Ennio \\ Talamonti $^{l}$, Gerardo Bosco ${ }^{1}$
}

Master Level Ii In Diving and Hyperbaric Medicine, Department of Biomedical Sciences, University of Padova, Padova, Italy ${ }^{1}$

Purpose: The conditions of hypoxia and hyperoxia represent two of the problems to which a scuba diver is exposed. Hypoxia while diving represents a potentially fatal event for the diver as it quickly leads to the loss of brain functions and consequent loss of breathing control, all in the absence of warning symptoms. However, hyperoxia in immersion as well represents, a deadly event. Various diving tests with blood gas analysis have been conducted. The pulse oximeter has already been used to work on hypoxia $[1,2]$. In a non-invasive and real-time way, $\mathrm{SpO} 2$ and ORi (Oxygen Reserve index) monitoring could highlight the oxygen status of a diver while diving to avoid hypo-hyperoxia.

Methods: The radial artery of one diver diver was cannulated, and blood sampled during one dive at $-15 \mathrm{~m}$ (at rest; at the bottom, after $15 \mathrm{~min}$ of pedaling on a scuba-bike; and at the end of the dive). Arterial $\mathrm{SaO} 2$ and $\mathrm{PO} 2$ levels were measured using a point-of-care blood gas analyzer. Two different marinized device for pulse oximetry was applied to the diver, to measure heart rate, $\mathrm{SpO} 2$ and ORi (with a forehead sensor inside a mask and through the use of a special sensor inside a waterproof glove).

Results: The diver was normoxic before the dive. After pedaling, at the time of the second sampling, the diver was slightly hyperoxic. On the surface, as soon as he emerged, the diver was again normoxic. $\mathrm{SaO} 2$ from sampling and $\mathrm{SpO} 2$ from pulse oximeter were comparable in the sampling on the bottom. The increase in $\mathrm{PaO} 2$ on the bottom is correlated with an increase in the ORi index during physical exercise on the bottom. Even the ORi on the surface has normalized.

Conclusions: These preliminary results seem to confirm that $\mathrm{SaO} 2$ and $\mathrm{SpO} 2$ in immersion are comparable to avoid hypoxia, that ORi can be used to get information on the diver's initial hyperoxia, but above all that it will be possible to use only non-invasive methods for the study of hypoxia and hyperoxia in scuba divers. Future experiments will try to standardize the method by increasing the number of subjects investigated.

References
1. Lance RM, Natoli MJ, Dunworth SAS, Freiberger JJ, Moon RE. The Dewey monitor: Pulse oximetry can independently detect hypoxia in a rebreather diver. Undersea HyperbaricMed 44(6):569-580.

2. Di Pumpo F, Ruffino G, Malacarne P. Pulse oximeter to detect peripheral oxygen saturation in underwater rebreather ECCR diver: a preliminary study. Undersea HyperbaricMed 46(1):1-6.

257

Comparison of metabolic power, energy cost and EMG in a case report in a different sprinting conditions: flat, uphill, downhill and sand.

\section{Gabriele Grassadonia ${ }^{1}$, Michele Bruni ${ }^{2}$}

1ucam, 2upss, 3upm, 4miu, 1catholic University of Murcia, 2popular University of Sport Sciences of Rome, 3popular University of Milan, 4miami International University, Murcia, Spagna ${ }^{1}$; Independent Researcher, Independent Researcher, Rome, Italy ${ }^{2}$

Purpose: The aim of this case report was investigated sprints via EMG of lower limbs (detecting quadriceps, hamstrings, and gluteus), metabolic power and energy cost recalculated by EMG with an innovative approach (Colli R. unpublished and Grassadonia G. 20,211) in different conditions: flat, uphill, downhill and sand, to study possible differences.

Methods: Data were acquired in a collegiate athlete (25 years old, mass $69.7 \mathrm{~kg}$, height $170 \mathrm{~cm}$ ) with GPS-IMU SpinItaly v2 and EMG Myontec M-Body 3 resampled at $100 \mathrm{~Hz}$. All sprints were performed in ecological (field) conditions over $50 \mathrm{~m}$. The slope of the uphill and downhill terrain was the same $\left(4.3^{\circ} \pm 0.4 \approx 7.5 \% \pm 0.7\right)$. Flat, uphill and downhill all three had asphalt surface. EMG was normalized respect to the peak value in $\mathrm{CMJ}$, in accordance with Kyröläinen $\mathrm{H}$. et al. 20,052.

Results: In the flat sprint the metabolic power and energy cost were $50.19 \pm 5.82(\mathrm{~W} \mathrm{Kg}-1)$ and $7.23 \pm 3.24(\mathrm{~J} \mathrm{~m}-1 \mathrm{~kg}-1)$ taking a time of 6.90 ', in the uphill $46.56 \pm 4.37$ and $7.38 \pm 2.70$ with 7.77 ' $(\Delta$ $12.61 \%$ than flat), in downhill $52.68 \pm 2.98$ and $7.10 \pm 3.00$ with 6.63 ' $(\Delta-3.91 \%$ than flat $)$, and in sand $46.62 \pm 23.73$ and $6.80 \pm 2.51$ with 7.16 " ( $\Delta 3.77 \%$ than flat). In the flat sprint the EMG was $93.98 \% \pm 10.70$, in the uphill $87.30 \% \pm 8.03$, in the downhill $98.57 \%$ \pm 5.49 , and in the sand $87.41 \% \pm 6.86 \%$. Regarding muscle distribution, compared to the flat condition, our results are statistically significant in quadriceps between flat and sand $(p=0.00$ and $d=1.23)$ and in the gluteus, between flat and other conditions (respectively $\mathrm{p}=$ 0.00 and $\mathrm{d}=1.68$ in uphill, $\mathrm{p}=0.02$ and $\mathrm{d}=1.19$ in downhill and $\mathrm{p}=$ 0.00 and $d=1.53$ in sand).

Conclusions: These preliminary results indicate the importance of studying surface changes during running, as temporal and mechanoenergetic changes can induce significant differences in the expression of metabolic power, energy cost, and EMG.

\section{References}

1. Grassadonia G. (2021) A comparison of metabolic power and energy cost by GPS with the "Colli Method" of profiling and recalculation by EMG detected. NSCA 2021 National Conference, 7-10 July Orlando, Fl. (USA)

2. Kyröläinen H, Avela J, Komi PV. Changes in muscle activity with increasing running speed. J Sports Sci. 2005 Oct;23(10):1101-9. 


\section{8}

\section{Effect of maximal strength training in a traumatic brain injured young male: a case study}

\author{
Anna Pedrinolla $^{1}$, Fabio La Ginestra ${ }^{2}$, Massimo Venturelli $^{2}$ \\ Università Degli Studi Di Verona, Neuroscienze, Biomedicina E \\ Scinze Del Movimento, Verona, Italy ${ }^{1}$; \\ Università Degli Studi Di Verona, Neuroscienze, Biomedicina E \\ Scienze Del Movimento, Verona, Italy ${ }^{2}$
}

Methods: A 26-years old male who suffered a traumatic brain injury with consequent diffuse axonal damage at the age of 24 was included in the study. Subject (height $175 \mathrm{~cm}$, weight $84.5 \mathrm{~kg}$ ) exhibits a lefthanded lower limb hemiplegia. Pre and post a 4-week training program the following evaluations tests were performed: quadriceps maximal voluntary contraction (MVC), rate of force development (RFD), voluntary activation (VA), and potentiated twitch (Qtw,pot); 3 -speed $(80 \%, 100 \%$, and $120 \%$ of self-selected speed) energy cost of walking $(\mathrm{CW})$, functional tests such as: 6-min walking test (6-MWT), time up and go test (TUG), and 30" sit-to-stand test. Also, lower limbs fat and lean mass were measured by means of DXA. The 4-week program, performed 3 times a week, included MST added to the standard training using a seated leg press with 4 sets of 4 repetitions at $\sim 90 \%$ of maximal strength of $1 \mathrm{RM}$ and maximal intended velocity in the concentric phase. MST was performed exclusively with the hemiplegic leg, while quadriceps strength testing procedures were performed on both, left and right legs.

Results: Hemiplegic, trained leg exhibited an increase in the following quadriceps strength parameters: MVC, $+12.5 \%$; RFD + $35.8 \%$; Qtw,pot, $+18.4 \%$, while no change was detected in VA. Nontrained leg did not exhibit change in any variable. Energy cost of walking greatly improved at each tested speed $(-15.8 \%,-10 \%,-14 \%$ respectively). Functional tests also improved: $6 \mathrm{MWT},+18 \%$, TUG $2.1 \%$, and 30 " sit-to-stand $+29 \%$. No changes in lower limbs fat and lean mass were detected.

Conclusions: Data from this case study suggests that MST positively affects strength components in the trained leg of a traumatic brain injured young subject, but no crossover-effect has been detected on the non-trained leg. However, most importantly seems that MST performed exclusively with hemiplegic leg leads to great improvement of $\mathrm{Cw}$ and functional tasks contributing to improve the quality of life and independence of this individual.

Wang E, Nyberg SK, Hoff J, Zhao J, Leivseth G, Tørhaug T, Husby OS, Helgerud J, Richardson RS. (2017) Impact of maximal strength training on work efficiency and muscle fiber type in the elderly: Implications for physical function and fall prevention. Exp Gerontol. 91:64-71.

\section{9}

\section{Is the human body able to travel on mars? A review}

\author{
$\underline{\text { Luca Petrigna }}^{l}$, Antonio Paoli $^{2}$, Antonio Palma $^{l}$, Antonino Bianco $^{l}$
}

Università Di Palermo, Sport and Exercise Sciences Research Unit, Department of Psychology, Educational Science and Human Movement, University of Palermo, Palermo, Italy ${ }^{l}$; Università, Department of Biomedical Sciences, University of Padova, Padova, Italy ${ }^{2}$

Purpose: The first fly in space has been in 1961, in 1969 the Apollo 11 mission landed on the Moon, from 1998 humans can live in the space in the International Space Station but now the new goal is to travel to Mars. The Mars mission will be of at least 1 year and half a and the human physical reaction is unknown. Therefore, the question of this review is to understand if humans are ready to fly to Mars from a physical point of view.

Methods: A review of literature was performed. The results were discussed narratively. Only English written manuscripts were included despite the Country of origin. Physical training was the main inclusion criteria.

Results: A total of 31 studies concerning long-duration space travels were analyzed. A total of 467 space travelers of both genders were recruited in the study and they were from different Countries. Muscle mass aerobic performance, flexibility and body mass were trained with different methodologies, but from all the space travels, people returned with compromised physical fitness functions.

Conclusions: The main finding is that, up to now, the technologies and the training of space travelers are still not enough to prevent muscle and bone loss, and a decrease in aerobic capacities and muscle flexibility. Consequently, up to now, humans body is still not ready to fly to Mars and come back to the Earth.

\section{References}

1. Blue RS, Jennings RT, Antunano MJ, Mathers CH. (2017) Commercial spaceflight: Progress and challenges in expanding human access to space. REACH 7-8, 6-13.

2. Ramachandran V, Dalal S, Scheuring RA, Jones JA. (2018) Musculoskeletal Injuries in Astronauts: Review of Pre-flight, Inflight, Post-flight, and Extravehicular Activity Injuries. Current Pathobiology Reports 6, 149-158.

3. Korth DW, Reeves JM. (2015) Exercise countermeasure hardware evolution on iss: The first decade. Aerospace medicine and human.

\section{0}

Monte rosa exploration \& physiology 2021: preliminary evidence

Danilo Bondi ${ }^{I}$, Carmen Santangelo ${ }^{1}$, Pamela Pignatelli ${ }^{2}$, Agnese Sbrollini $^{3}$, Laura Burattini ${ }^{3}$, Lucio Lobefalo ${ }^{2}$, Federica Ciampini ${ }^{1}$, Sofia Bonan ${ }^{1}$, Giorgio Paglia ${ }^{4}$, Raffaela Piccinelli ${ }^{5}$, Giulia Prete ${ }^{6}$, Tiziana Pietrangelo ${ }^{1}$, Stefania Fulle ${ }^{1}$, Vittore Verratti ${ }^{6}$

University "G. D'annunzio" of Chieti-Pescara, Department of Neuroscience, Imaging and Clinical Sciences, Chieti, Italy ${ }^{I}$; University "G. D'annunzio " of Chieti-Pescara, Department of Medical, Oral and Biotechnological Sciences, Chieti, Italy ${ }^{2}$; Marche Polytechnic University, Department of Information Engineering, Ancona, Italy ${ }^{3}$;

University "G. D'annunzio " of Chieti Pescara, Department of Engineering and Geology, Chieti, Italy ${ }^{4}$;

Council For Agricultural Research and Economics, Research Centre For Food and Nutrition, Rome, Italy ${ }^{5}$;

University "G. D'annunzio " of Chieti Pescara, Department of Psychological, Health and Territorial Sciences, Chieti, Italy ${ }^{6}$

Purpose: Trekking at high altitude represents an excellent ecological model to deepen and understand the physiological acute response, with possible translational insights for those disease characterized by hypoxia. In addition, high altitude expeditions represent intriguing field conditions to evaluate the dynamic interconnectedness of physiological systems and sub-systems.

Methods: A multidisciplinary expedition at Monte Rosa (Alps, Italy) has been set, from August 29 to September 2, 2021. The group of expeditioners ( 15 healthy adults, 8 males and 7 females) will trek up to $4,554 \mathrm{~m}$ of altitude (Capanna Margherita, the European highest mountain hut). Several scholars of the University of Chieti, partnered 
with other Universities and private companies, designed the study. The topics addressed will encompass environmental and exercise physiology, oral medicine, myology, redox biology, microbiota, ophthalmology, bioengineering, human nutrition, psychology, geology, and climatology.

Results: We hypothesize that combined stressors of physical exercise and altitude hypoxia will trigger acute cardiorespiratory and inflammatory response and a disruption in the multilayer interaction. These responses may be revealed by both local, systemic and dynamic biomarkers, linked to altered inter-tissue crosstalk and interlocking of biological oscillators. The findings will be controlled by climate condition, nutritional intake, and baseline status of participants.

Conclusions: A set of preliminary Results will be presented during the conference, accounting for a multidisciplinary perspective for defining the acute response to the environmental and physical stressors. As altitude traveling has become popular in the last couple of decades, original insights will be provided for both facing medical emergent problems and allowing novel research possibilities.

1. Métrailler P, Greiser J, Dietrich G, et al. (2019) Swiss Mountain Guides: Medical Education, Knowledge, and Practice. High Alt Med Biol 20:251-261.

2. West JB (2016) Early history of high-altitude physiology. Annals of the New York Academy of Sciences 1365:33-42.

\section{1}

\section{Neuromuscular fatigue in individuals with cerebral palsy and healthy peers: a pilot study}

\section{Mauro Nardon $^{1}$, Luca Martini $^{1}$, Federico Ruzzante ${ }^{1}$, Massimo Venturelli $^{l}$, Matteo Bertucco ${ }^{I}$}

Università Di Verona, Department of Neurosciences, Biomedicine and Movement Sciences, Verona, Italy ${ }^{I}$

Purpose: Cerebral Palsy (CP) is a neurological disease caused by a perinatal injury (1). Physical fatigue is a main limitation in this population (2). The underlying neurophysiological mechanisms are still debated (3). The aim of the study was to assess the contribution of central and peripheral components of neuromuscular fatigue (NMF) in young adults with CP and typically developing (TD) peers. We hypothesized the neural drive to the muscle to be modified in $\mathrm{CP}$, exacerbating the level of central fatigue.

Methods: Participants performed six maximal isometric contractions of the quadriceps muscle before- and after a dynamic leg-extension exercise to exhaustion (TL). Central and peripheral components of NMF were assessed via interpolated twitch technique. Maximal voluntary contractions (MVC), electrically evoked resting twitch (Qtw) and voluntary muscle activation (VA) were calculated. During the fatiguing test, metabolic and cardiovascular parameters were recorded. TD subjects performed an additional session, this time exercising with same workload of their CP-match (TLiso), allowing for additional comparisons.

Results: Preliminary Results of four CP-TD matches (8 subjects) are discussed. MVC (CP: $323.6 \pm 68.4$, TD: $454.3 \pm 18.8$ N), VA (CP: $68.5 \pm 15.8$, TD: $79.4 \pm 9.9 \%$ ) and Qtw (CP: $127.8 \pm 38.5$, TD: $223.2 \pm 91.6 \mathrm{~N}$ ) pre-exercise values were lower in individuals with CP. Peak single-leg exercise workload (CP: $34 \pm 23$, TD: $85 \pm$ $21 \mathrm{~W})$ and metabolic efficiency (CP: $2.7 \pm 1.8$, TD: $5.1 \pm 1.1 \%)$ were lower in $\mathrm{CP}$ group. Oxygen consumption (VO2) at rest and during exercise was similar between groups. Time to exhaustion (CP: $861 \pm 480$, TD: $311 \pm 117$ s) was higher in participants with $\mathrm{CP}$. During TLiso session, TD participants did not exhaust within $30 \mathrm{~min}$. Fatigue-induced changes in MVC and Qtw were similar in CP and TD in session TL (MVC: -23 and -19\%; Qtw: -21 and $-45 \%$, respectively), whereas VA was reduced only in CP group (CP: -8 , TD: + $3 \%$ ). The same parameters did not change in TD after the TLiso session.

Conclusions: Findings support our hypothesis. Individuals with $\mathrm{CP}$ are weaker than TD peers and characterized by sub-optimal muscle activation. Reduced metabolic efficiency might be explained by muscle co-contractions and alterations in muscle properties. However, when compared to $\mathrm{TD}$, subjects with $\mathrm{CP}$ exercise for longer time at a relative high intensity (3). This is likely due to an adaptation of their neuromuscular system.

\section{References}

1. Graham, H. K., Rosenbaum, P., Paneth, N., Dan, B., Lin, J.-P., et al. (2016). Cerebral palsy. Nat Rev Dis Primers 2(1), 15,082.

2. Opheim, A., Jahnsen, R., Olsson, E., \& Stanghelle, J. K. (2009). Walking function, pain, and fatigue in adults with cerebral palsy: A 7-year follow-up study. Dev Med Child Neurol 51(5), 381-388.

3. Brunton, L. K., \& Rice, C. L. (2012). Fatigue in cerebral palsy: A critical review. Dev Neurorehabil 15(1), 54-62.

262

\section{Profiling of stress and motivation biomarkers in adolescent rowing athletes during indoor and outdoor competitions}

\section{Giovanni Ficarra $^{l}$, Daniela Caccamo $^{I}$, Caterina Saija ${ }^{1}$, Alessandra Bitto $^{I}$, Fabio Trimarchi ${ }^{l}$}

Università Degli Studi Messina, Department of Biomedical Sciences, Dental Sciences, and Morpho-functional Imaging,, Messina, Italy ${ }^{I}$

Purpose: There is a lack of data regarding the stress and motivation response in adolescent athletes during competitions. The athletic performance can be highly influenced by stress rather than appropriate training, at this age. The aim of this investigation is to evaluate the level of stress markers in adolescent rowers in different competition settings that might alter their stress status and performance. Methods: Adolescent rowing athletes (12-18 years) have been tested for determining saliva content of stress biomarkers, cortisol (C) and testosterone $(\mathrm{T})$, before and after competitions that have been performed indoor and outdoor. Specifically samples have been taken in the morning, before and after the race in 2 different settings: 1) an indoor rowing competition with an ergometer, 2) an outdoor rowing competition on boats.

Results: A general reduction in cortisol levels has been observed in athletes right before the race both during indoor and outdoor performances, while testosterone levels increased at the same time point only before the boat competition and kept rising at the end of the race, especially in those who reported a winning score. Significant differences have been found comparing the pre-race with early morning data, and between indoor and outdoor data.

Conclusion: Despite the age differences among athletes might have an influence on their hormone levels, these data suggest that rowing athletes subjected to different kind of competitions show a different stress and motivation response profile that might influence their performance.

\section{References}

1. Wood RI, Stanton SJ. Testosterone and sport: current perspectives. Horm Behav. 2012;61(1):147-155. https://doi.org/10.1016/ j.yhbeh.2011.09.010 


\section{3}

\section{Purely explosive contractions induce primarily central fatigue}

\author{
Gennaro Boccia $^{1}$, Samuel D'emanuele ${ }^{2}$, Paolo Riccardo Brustio ${ }^{2}$, \\ Alberto Rainoldi $^{3}$, Federico Schena ${ }^{2}$, Cantor Tarperi ${ }^{1}$ \\ University of Turin, Department of ClinicSsecal and Biological \\ Sciences, Torino, Italy ${ }^{P}$; \\ University of Verona, Department of Neurosciences, Biomedicine \\ and Movement Sciences, Verona, Italy ${ }^{2}$; \\ University of Turin, Department of Medical Sciences, Torino, Italy ${ }^{3}$
}

Purpose: The early phase of explosive contractions is mainly dependent on central mechanisms (e.g. motor unit recruitment and firing rate). Nevertheless, it is unknown if repeated explosive isometric contractions induce primarily central fatigue, as it is reasonable to hypothesize (D'Emanuele et al. 2021). Thus we aimed to characterize the central and peripheral fatigue caused by 100 purely explosive, i.e. without a plateau phase, isometric contractions.

Methods: 30 physically active young adults performed 100 short (i.e. $<200 \mathrm{~ms}$ ) maximally explosive isometric knee extensions interspersed by $3 \mathrm{~s}$ of rest. High-density surface electromyographic (HDEMG) signals were recorded from the vastus medialis and lateralis muscles. During the first (PRE) and last (POST) 10 contractions, we calculated the rate of force development (RFD) over the first 50, 100, and $150 \mathrm{~ms}$ of contractions. We also assessed the contractile and EMG responses to electrically-evoked single and octet stimuli. Neural efficacy was calculated as the ratio between octet-evoked and voluntary force during the first $50 \mathrm{~ms}$ of contraction.

Results: Voluntary RFD decreased by $12 \%$ at $50 \mathrm{~ms}$ and by and $6 \%$ at $100 \mathrm{~ms}$ (all $\mathrm{P}$ values $<0.01$ ). The root mean square of RMG signal (normalized to M-wave amplitude) decreased by around $20 \%$ in all time intervals from 0 to $150 \mathrm{~ms}$ (all $\mathrm{P}$ values $<0.001$ ); muscle fiber conduction velocity (normalized to $\mathrm{M}$-wave conduction velocity) decreased by $2.5 \%$ at 100 and $150 \mathrm{~ms}(\mathrm{P}<0.05)$. Neural efficacy also declined by $15 \%(\mathrm{P}<0.01)$. Unexpectedly, some of the metrics related to peripheral function increased. Indeed, the RFD in the first $50 \mathrm{~ms}$ of the electrically-evoked octet increased by $3 \%(\mathrm{P}<0.01)$ and the amplitude and conduction velocity of the $\mathrm{M}$-wave increased by $7 \%(\mathrm{P}<0.05)$.

Conclusions: Repeated explosive contractions decreased the neural efficacy. In the absence of unequivocal marks of peripheral fatigue, the impairment in explosive capacity has been primarily driven by central fatigue.

\section{References}

1. D'Emanuele S, Maffiuletti NA, Tarperi C, Rainoldi A, Schena F and Boccia G (2021) Rate of Force Development as an Indicator of Neuromuscular Fatigue: A Scoping Review. Front. Hum. Neurosci. 15:701,916

\section{4}

\section{Saliva testing as non-traditional, non-invasive way for monitoring exercise intensity-dependent stress response in teenage elite water polo players}

\section{Debora Di Mauro ${ }^{l}$, Francesco Naccari ${ }^{2}$, Nadia Ferlazzo ${ }^{l}$, Monica Curro $^{I}$, Daniela Caccamo $^{I}$, Riccardo Ientile ${ }^{I}$, Fabio Trimarchi ${ }^{I}$}

Università Degli Studi Di Messina, Dipartimento Di Scienze Biomediche, Odontoiatriche E Delle Immagini Morfologiche E
Funzionali, Messina, Italy ${ }^{1}$,

Cus Unime Asd, Università Degli Studi Di Messina, Messina, Italy ${ }^{2}$

Purpose: Excessively increased training volume and/or intensity and competition can lead to the development of overtraining syndrome, causing a performance decrement in athletes. Thus, tracking individual response to exercise intensity is crucial for establishing appropriate recovery strategies.

Methods: Here we assessed the exercise intensity-dependent variability of stress response biomarkers, namely cortisol (C), testosterone (T), sIgA and advanced oxidation protein products (AOPP), in saliva samples of teenage male water polo players. Saliva was collected using specific devices on a day of training match (T1) and a day of competitive match (T2), at morning, before and after match. Salivary protein content was quantified in order to take into account different salivary flow rate and normalize the concentrations of stress biomarkers. Non-parametric Kruskal Wallis test and Mann-Whitney $\mathrm{U}$ test were used for comparison of the three data sets and the two-bytwo comparisons between groups. The Spearman correlation test was applied.

Results: Cortisol/proteins and testosterone/proteins concentrations decreased throughout day T1, while increased throughout day T2. The highest values were measured after match on day $\mathrm{T} 2$. sIgA/proteins and AOPP/proteins concentrations increased throughout both days and were higher after T2 match than T1 one. Significant differences between concentrations of different biomarkers recorded on $\mathrm{T} 1$ and T2 were found only for AOPP, suggesting an increased oxidative stress after match on day T2. T:C ratio after match on day $\mathrm{T} 2$ was lower than that at morning, indicating an increased catabolic response after competitive match. A highly significant positive correlation was found between Cortisol/Proteins and Testosterone as well as $\operatorname{IgA} /$ Proteins on day T1, and between Cortisol/Proteins and AOPP on day T2.

Conclusion: Our findings confirm the usefulness of saliva testing as non-invasive way for monitoring the individual response to changes in exercise intensity in teenage elite water polo players.

\section{References}

1. Steckl AJ, Ray P. (2018) Stress Biomarkers in Biological Fluids and Their Point-of-Use Detection. ACS Sens 3:2025-2044.

2. Lee EC, Fragala MS, Kavouras SA, Queen RM, Pryor JL, Casa DJ. (2017) Biomarkers in Sports and Exercise: Tracking Health, Performance, and Recovery in Athletes. J Strength Cond Res 31:2920-2937.

\section{5}

Sex differences in autonomic responses to post-exercise muscle metaboreflex isolation

\section{Thomas Favaretto $^{1}$, Fabio Laginestra $^{I}$, Alessandro Cavicchia ${ }^{1}$, Massimo Venturelli ${ }^{l}$ \\ Università Degli Studi Di Verona, Dipartimento Di Neuroscienze, Biomedicina E Movimento, Verona, Italy ${ }^{I}$}

Background: Physiological sex influences the autonomic adjustments to exercise. In women, metaboreflex isolation result in attenuated increase in blood pressure (BP) and sympathetic activity then men. Since exercise-induced metabolites accumulation is paramount when assessing metaboreflex-mediated responses, previous studies that utilised a percentage of maximal voluntary contraction for intensity prescription may have resulted in confounding findings.

Purpose: The aim of the study was to investigate whether the autonomic responses to post-exercise metaboreflex isolation are 
influenced by sex when exercise intensity is matched based on critical metabolic intensity. A secondary aim was to explore the potential of using the high-frequency (HF) power component of heart rate variability (HRV), calculated from the time-frequency method, to assess the cardiac autonomic response to metaboreflex isolation.

Methods: Eighteen subjects ( 8 men/10 women) performed two identical exercise bouts consisting in $300 \mathrm{~s}$ of intermittent $(3 \mathrm{~s}$ on/ $2 \mathrm{~s}$ off) isometric knee-extension at $110 \%$ of the previously determined critical force $(\mathrm{CF})$. At the end of the exercise bout, recovery was performed either with (PECO) or without (CON) suprasystolic circulatory occlusion of the exercised limb. Central hemodynamics and blood pressure were continuously recorded throughout the trial. Cardiac autonomic responses were assessed by HRV, and blood pressure variability (BPV). Data were analysed using three-way ANOVA.

Results: In PECO the same mean arterial pressure (MAP) was achieved (men:107 \pm 7 , women: $99 \pm 8 \mathrm{mmHg}$, p(sex vs.session $)=$ 0.762), men presented an overall increased total peripheral resistances (TPR) $($ men: $1.3 \pm 0.4$, women: $1.1 \pm 0.3 \mathrm{mmHg} \bullet \mathrm{s} / \mathrm{ml}, \mathrm{p}(\mathrm{sex})=$ 0.040), while women presented a HR-driven adjustment (men:77 \pm 10 , women:94 $\pm 19 \mathrm{bpm}$, p(time vs.sex $)<0.001)$. However, no significant sex differences were found in any of the calculated HRV and BPV indices in response to metaboreflex isolation ( $\mathrm{p}(\mathrm{sex}$ vs.session) $>0.05$ ) between men and women. Significant differences were found in MAP, HRV, and BPV between sessions ( $\mathrm{p}$ ( sex vs.session) $<0.05$ ), indicating successful metaboreflex isolation. The correlation test between RMSSD and HF showed a significant positive relationship (CON: $\mathrm{r}=0.72 \mathrm{p}=0.016$, PECO: $\mathrm{r}=0.88 \mathrm{p}<$ $0.001)$.

Conclusion: These findings suggest that the autonomic responses to metaboreflex isolation are similar in men and women, even though the strategy to accomplish these adjustments may be different.

\section{References}

1. Joyner MJ, et al. (2015). Neural control of the circulation: How sex and age differences interact in humans. Compr Physiol 5, 193-215.

2. Peçanha T, et al. (2017). Methods of assessment of the postexercise cardiac autonomic recovery: A methodological review. Int J Cardiol 227, 795-802.

3. Smith JR, et al. (2019). Influence of Sex, Menstrual Cycle, and Menopause Status on the Exercise Pressor Reflex. Med Sci Sports Exerc 51, 874-881.

\section{6}

Are incremental exercise relationships between rating of perceived exertion and oxygen uptake or heart rate reserve valid during steady-state exercises?

${ }_{\text {Carlo Ferri Marini }}{ }^{l}$, Lorenzo Micheli ${ }^{1}$, Ario Federici ${ }^{1}$, Giovanni Piccoli ${ }^{I}$, Francesco Lucertini ${ }^{1}$, Vilberto Stocchi ${ }^{2}$, Luca Zoffoli $^{3}$, Luca Correale $^{4}$, Stefano Dell'anna ${ }^{4}$, Carlo Alberto Naldini ${ }^{5}$, Matteo Vandoni $^{5}$

University of Urbino Carlo Bo, Department of Biomolecular Sciences - Division of Exercise and Health Sciences, Urbino, Italy ${ }^{1}$; University San Raffaele Roma, Department of Human Sciences For The Promotion of Quality of Life, Rome, Italy ${ }^{2}$; Technogym S.p.a, Scientific Research \& Innovation Department, Cesena, Italy ${ }^{3}$;

University of Pavia, Sports Science Unit, Department of Public Health, Experimental and Forensic Medicine, Pavia, Italy ${ }^{4}$; University of Pavia, Laboratory of Adapted Motor Activity (lama),
Department of Public Health, Experimental and Forensic Medicine, Pavia, Italy ${ }^{5}$

Purpose: Rating of perceived exertion (RPE) scale (6-20) is used to prescribe prolonged aerobic steady-state exercise (SSE) intensity due to its correlations with physiological indicators of exercise intensity (e.g., oxygen uptake [VO2] or heart rate [HR]), which were found during graded exercise tests (GXT). This study aims to verify if a) the associations between RPE and HR or VO2 derived from GXTs are valid during SSEs and b) SSE intensity and duration affect these associations.

Methods: Eight healthy and physically active males $(22.6 \pm 1.2$ years) were enrolled. On the first day, pre-exercise and maximal HR and VO2 were measured during 20-min standing and a GXT. In the following 4 days, SSEs were performed by running at $60 \%$ and $80 \%$ of HRR for 15 and 45 min (random order). Post-SSE maximal HR and VO2 were assessed after SSEs. HR and VO2 of GXTs (last $30 \mathrm{~s}$ of each 1-min stage) and SSEs (last 5 min of each SSE) were converted into percentages of the reserves (HRR and VO2R) using preexercise and, respectively, maximal and post-SSE maximal values. Individual linear regressions (ILR) were performed between GXTs' RPE (recorded $15 \mathrm{~s}$ before the end of each stage-dependent variable) and \%VO2R or \%HRR (independent variables). Then, RPEs corresponding to SSE \%HRR (RPEhrr) and \%VO2R (RPEvo2r) were predicted using GXT's ILRs. For each relation (RPE-\%HRR and RPE-\%VO2R), a 3-way RM-ANOVA $(\alpha=0.025)$ was used to assess if RPE was affected by exercise Modality (RPE recorded during SSE [RPEsse] or GXT-predicted), Intensity (60\% or $80 \% \mathrm{HRR})$ and Duration (15 or $45 \mathrm{~min}$ ).

Results: RPEsse and RPEhrr were not different (no significant effect of Modality or its interactions with SSE Intensity and Duration). When RPEsse was compared with RPEvo2r, Modality or its interactions with Intensity or Intensity x Duration were not significant, whereas the Modality $x$ Duration interaction was significant $(\mathrm{p}=$ 0.020 ). RPEsse did not change (mean difference $[\mathrm{MD}]=-1.4 \pm 1.8, \mathrm{p}$ $=0.054)$ from SSE of $15(12.1 \pm 2.0)$ to $45(13.5 \pm 2.1) \mathrm{min}$, while RPEvo2r decreased significantly $(\mathrm{MD}=1.3 \pm 1.5, \mathrm{p}=0.022)$ from SSE of $15(13.7 \pm 3.2)$ to $45(12.4 \pm 2.8) \mathrm{min}$. This yielded a dissociation between RPEsse and RPEvo2r, with RPEsse lower at 15-min SSE (MD = -1.6 \pm 3.0 ) and higher at 45-min SSE $(\mathrm{MD}=1.1$ \pm 2.8 ) compared to RPEvo2r.

Conclusions: When SSE intensity is prescribed using \%HRR, GXTs' RPE-\%VO2R associations may under- or over-estimate SSE intensity (VO2R), whereas GXTs' RPE-\%HRR associations seem to be preserved during SSE.

\section{References}

1. Swain DP, American College of Sports Medicine. (2014). ACSM's Resource Manual for Guidelines for Exercise Testing and Prescription (7th ed.). Philadelphia: Wolters Kluwer Health/ Lippincott Williams \& Wilkins, pp. 475-476.

2. Ferri Marini C, Correale L, Carnevale Pellino V, Federici A, Vandoni M, Lucertini F. (2021). Assessing Maximal Oxygen Uptake: Creating Personalized Incremental Exercise Protocols Simply and Quickly. Strength Cond J 43: 86-92.

\section{7}

\section{Sex differences in sympathetic blunting of vasodilation}

Alessandro Gentilin $^{I}$, Antonio Cevese $^{I}$, Cantor Tarperi $^{2}$, Federico $^{\prime}$ Schena

University of Verona, Department of Neuroscience, Biomedicine, and Movement Sciences, Verona, Italy ${ }^{l}$; 
University of Turin, Department of Clinical and Biological Sciences, Torino, Italy ${ }^{2}$

Purpose. Skeletal muscle blood flow (BF) is precisely matched to energy demand at rest and during exercise through the modulation of vascular conductance (VC) by constantly balancing sympathetic vasoconstriction and metabolic vasodilation. The current understanding of how the control mechanisms differ between the sexes in controlling skeletal muscle $\mathrm{BF}$ is limited and suggests that muscle sympathetic nerve activity (MSNA) is lower in young females than males and that it correlates to peripheral VC in males only, suggesting blunted transduction of sympathetic activity into vasoconstriction in females. However, most evidence comes from studies where sympathetic vasoconstriction has been investigated independently and where the effects of metabolic vasodilation were not accounted for. This study assessed whether sympathetic restraining of vasodilation is likewise blunted in young females than males.

Methods. 12 males (age $25.7 \pm 3.6$ yo) and 13 females (age $23.0 \pm$ 2.5 yo) underwent $\mathrm{VC}$ and brachial artery (BA) flow-mediated vasodilation (FMD) assessment after a 5-min forearm circulatory occlusion, not preceded or preceded by a 3-min cold pressor testmediated acute sympathetic activation (SYMP). Beat-by-beat brachial $\mathrm{BF}$, mean arterial pressure, and BA diameter within $90 \mathrm{~s}$ after the cuff release were collected to assess percent BA FMD and cumulative arm $\mathrm{VC}$ increase.

Results. BA baseline diameter and baseline VC were $20.4 \%$ and $36.6 \%$ higher in males than females, respectively. BA FMD without SYMP was higher $(4.96 \pm 2.07$ vs $7.26 \pm 2.36 \% ; \mathrm{p}=0.001)$ in females than males, but similar $(3.61 \pm 1.99$ vs $4.96 \pm 1.88 \%$; $\mathrm{p}=$ $0.23)$ during SYMP, which blunted FMD more in females $(-1.35 \pm$ 1.08 vs $-2.34 \pm 1.08 \% ; \mathrm{p}=0.03)$. However, any sex differences disappeared by normalizing for baseline BA diameter and shear rate. Regardless of SYMP, cumulative VC increases were always higher ( $\mathrm{p}$ $<0.001$ ) in males than females when expressed in absolute values, but similar $(\mathrm{p}=0.98)$ between sexes when normalized for baseline VC. Thus, SYMP blunted the \%VC increase similarly $(-16.4 \mathrm{~K} \pm$ $6.9 \mathrm{~K}$ vs $-16.4 \mathrm{~K} \pm 8.8 \mathrm{~K} \%$; $\mathrm{p}=0.98$ ) between sexes.

Conclusions. This data show the artery and body size accounting for sex differences, but not evident sex differences on neurovascular modulation when VC is assessed dynamically. Indeed, any lower sympathetic vasoconstriction in females may be associated with relatively reduced shear rate and vasodilation function, resulting in similar effects on VC than males.

\section{References}

1. Dyson KS, Shoemaker JK, Hughson RL. (2006) Effect of acute sympathetic nervous system activation on flow-mediated dilation of brachial artery. Am J Physiol Heart Circ Physiol. 290(4):H1446-53.

2. Buckwalter JB, and Clifford PS. (2001) The paradox of sympathetic vasoconstriction in exercising skeletal muscle. Exerc. Sport Sci. Rev. 29(4):159-163.

3. Lind L, Johansson K, Hall J. (2002) The effects of mental stress and the cold pressure test on flow-mediated vasodilation. Blood Press. 11(1):22-27.

268

\section{Sex differences in the TMS-induced relaxation rates of unfatigued and fatigued knee extensors}

\author{
Chiara Barbi ${ }^{1}$, Massimo Venturelli ${ }^{2}$, Gaia Giuriato ${ }^{2}$, Fabio \\ Laginestra $^{2}$, Camilla Martignon ${ }^{2}$, Federico Schena ${ }^{2}$, Gianluca \\ Vernillo
}

Università Degli Studi Di Verona, Dipartimento Di Neuroscienze Biomedicina E Movimento, Verona, Italy ${ }^{1}$;

Università Degli Studi Di Verona, Dipartimento Di Neuroscienze, Biomedicina E Movimento, Verona, Italy ${ }^{2}$;

Università Degli Studi Di Milano, Dipartimento Di Scienze Biomediche Per La Salute, Milan, Italy ${ }^{3}$

Purpose: Muscle relaxation rate (mRR) refers to the speed at which muscle fibers relax after a contraction. The assessment of the transcranial magnetic stimulation (TMS)-induced $\mathrm{mRR}$ is an indicator of muscle relaxation proprieties [1] and reveals changes with fatigue [1]. Sex differences in $\mathrm{mRR}$ were studied for the elbow flexors, showing a faster mRR in men than in women [1]. However, it has not been reported for the knee extensors (KE). It is possible that $\mathrm{mRR}$ behaves differently for KE since KE presents different somatotopic organization and recruitment thresholds, functional role, as well as neuromuscular aspects [1]. Hence, this study evaluated the sex-related differences in TMS-induced $\mathrm{mRR}$ in unfatigued and fatigued KE.

Methods: Peak mRR (pmRR) was assessed with TMS during maximal voluntary isometric contractions (MVICs) in 10 men and 12 women before (PRE), after (POST) a 2-min sustained MVIC, and 4 more times within 8 min of recovery. pmRR was determined from the decrease in KE force during the TMS-induced silent period and calculated as the negative slope over a 10 -ms interval (5 ms either side of the steepest instantaneous slope) and normalized to the total force (voluntary plus evoked) prior to the silent period.

Results: Men were stronger than women $(577 \pm 119$ vs. $366 \pm 79 \mathrm{~N}$; $\mathrm{P}<0.001)$ and MVIC declined for men and women at POST $(\mathrm{P}<$ $0.001)$. The relative reduction was similar $(26 \pm 10 \%$ vs. $27 \pm 7 \%$ of PRE values; $\mathrm{P}=0.743)$. At 8 min recovery, MVIC similarly recovered for men and women $(89 \pm 11 \%$ vs. $93 \pm 11 \%$; $\mathrm{P}=0.491)$. At PRE, absolute $(-7412 \pm 1806$ vs. $-3956 \pm 987 \mathrm{~N} / \mathrm{s} ; \mathrm{P}<0.001)$ and normalized ( $-13 \pm 1$ vs. $-11 \pm 2 \mathrm{~s}-1 ; \mathrm{P}=0.005) \mathrm{mRR}$ were faster for men than women. At POST, absolute and relative pmRR declined for both sexes ( $\mathrm{P}<0.001)$, but more so for men than women (Absolute: $13 \pm 6 \%$ vs. $20 \pm 12 \% ; \mathrm{P}=0.030$; Relative: $44 \pm 21 \%$ vs. $60 \pm 24 \%$; $\mathrm{P}=0.010$ ). At $8 \mathrm{~min}$ recovery, absolute and relative pmRR similarly recovered for men and women $(\mathrm{P} \geq 0.548)$.

Conclusions: Men had faster mRRs prior to fatigue and slowed more after the fatiguing task than women. It is hypothesized that these results are due to a different distribution of fiber types in men and women $\mathrm{KE}$, with more fast twitch fibers in men [2]. Indeed, when compared to slow-twitch fibers, fast-twitch fibers present a faster KE $\mathrm{mRR}$ [3], as well as higher muscle glycolytic enzyme activities and lower oxidative capacity [2], which makes them less fatigue-resistant.

\section{References}

1. Vernillo G. et al. (2021), Use of transcranial magnetic stimulation to assess relaxation rates in unfatigued and fatigued kneeextensor muscles. Exp Brain Res, 239(1): p. 205-216.

2. Haizlip, K.M. et al. (2015), Sex-based differences in skeletal muscle kinetics and fiber-type composition. Physiology (Bethesda), 30(1): p. 30-9.

3. Wiles, C.M., et al. (1979), Relaxation rate of constituent musclefibre types in human quadriceps. Clin Sci (Lond), 56(1): p. $47-52$. 


\section{9}

The effect of prior exercise involving central motor drive on subsequent high-intensity knee-extensors endurance performance

\section{Fabio Giuseppe Laginestra ${ }^{l}$, Alessandro Cavicchia ${ }^{1}$, Jennifer Vanegas-lopez ${ }^{2}$, Chiara Barbi ${ }^{I}$, Anna Pedrinolla ${ }^{I}$, Markus Amann ${ }^{3}$, Thomas Hureau ${ }^{2}$, Massimo Venturelli ${ }^{1}$ \\ Universita' Di Verona, Neuroscienze, Biomedicina, E Movimento, Verona, Italy ${ }^{1}$; \\ University of Strasbourg, Medicine, Mitochondria, Oxidative Stress and Muscular Protection Laboratory,, Strasburgo, Francia ${ }^{2}$; \\ University of Utah, Anaesthesiology, Salt Lake City, Stati Uniti D' America $^{3}$}

Purpose: Endurance performance and neuromuscular fatigue development are affected by a plethora of central and peripheral factors. Moreover, a complex interaction of feedforward (e.g., corollary discharge) and feedback mechanisms contribute to the achievement of the sensory tolerance limit, ultimately limiting exercise tolerance. The aim of this study was to determine whether the involvement of central motor drive during fatiguing exercise plays a role on the subsequent endurance exercise performance and fatigue development.

Methods: Nine healthy young males completed 3 single-leg knee extension dynamic time to exhaustion (TTE) tests at $80 \%$ of the peak power output on 3 different days. While in one visit the subjects only performed the dynamic fatiguing task $(\mathrm{CON})$, on the other two occasions fatigue was pre-induced on the exercising leg either by voluntary (VOL) or electrically-evoked (EVO) isometric contractions at $\sim 20 \%$ maximal voluntary contraction (MVC). Neuromuscular fatigue was assessed via MVC, voluntary activation (VA), quadriceps potentiated twitch force (Qtw,pot), performed pre-post exercise, but also after every minute of the pre-fatiguing protocol. The two prefatiguing protocols were matched for peripheral fatigue development and stopped when Qtw,pot declined by $\sim 35 \%$. Corticospinal excitability was also assessed pre-post exercise while electromyography, hemodynamic, ventilatory, and perceptual responses were recorded during exercise.

Results: TTE was significantly shorter in EVO $(4.4 \pm 1.4 \mathrm{~min})$ and VOL $(4.7 \pm 1.6 \mathrm{~min})$ compared to CON $(11.2 \pm 3.7 \mathrm{~min})(\mathrm{p}<0.01)$. No difference in TTE was found between VOL and EVO $(\mathrm{p}>0.05)$. $\Delta$ Qtw,pot (EVO: $-59 \pm 7 \%$, VOL: $-55 \pm 14 \%$, CON: $-61 \pm 9 \%, \mathrm{p}>$ $0.05)$ and $\triangle$ VA (EVO: $-9 \pm 7 \%$, VOL: $-8 \pm 5 \%$, CON: $-7 \pm 5 \%, \mathrm{p}>$ 0.05 ) at the end of the dynamic task were not different between conditions. $\triangle \mathrm{MVC}$ was lower in CON compared to the both the prefatigue conditions (EVO: $-45 \pm 8 \%$, VOL: $-44 \pm 8 \%$, CON: $-53 \pm$ $8 \%, \mathrm{p}<0.05)$. Rate of perceived exertion was higher $(\mathrm{p}<0.05)$ at the beginning of the dynamic task in VOL (12.2 \pm 1.0$)$ than EVO (10.6 \pm $1.7)$ and $\mathrm{CON}(6.8 \pm 0.8)$, with no difference at exhaustion between conditions ( $\mathrm{p}>0.05)$.

Conclusions: Our Results suggest that the involvement of central motor drive during prior exercise play a negligible role on the subsequent high-intensity endurance performance. Also, these findings support the importance of the peripheral fatigue threshold in achieving the sensory tolerance limit and determining exercise tolerance.

References

1. Hureau TJ, Romer L, and Amann M (2018). The "sensory tolerance limit": A hypothetical construct determining endurance performance. Eur J Sport Sci 18:1, 13-24.

2. Amann M, Wan HY, Thurston TS, Georgescu VP, Weavil JC (2020). On the influence of group III/IV muscle afferent feedback on endurance exercise performance. Exerc Sport Sci Rev 48(4): 209-216.

\section{0}

The role of different warm-up protocols on cardiovascular and metabolic responses in dynamic apnoea

Luca Vitali $^{1}$, Milena Raffi ${ }^{2}$, Alessandro Piras ${ }^{2}$

Department For Life Quality Studies, University of Bologna, Rimini, Italy $^{1}$;

Department of Biomedical and Neuromotor Sciences, University of Bologna, Bologna, Italy ${ }^{2}$

Purpose: The aim of the present study was to assess the physiological response to different warm-up protocols before, during and after a dynamic apnoea performance. We compared the traditional approach, which includes a series of short-mid dives in water (WET warm-up), with a more recent strategy, that consists in exercises performed exclusively outside the water (DRY warm-up).

Methods: Nine freedivers were tested in two different sessions, with the two warm-up protocols executed before a $75 \mathrm{~m}$ of dynamic apnoea with fins. Heart rate variability and baroreflex sensitivity were recorded to evaluate the cardiac autonomic control, haemoglobin concentrations for evaluating the effect of spleen contraction, blood lactate levels to quantify the intensity of physical effort and the recovery process. To quantify the individuals' perception of the physical demand we collected the rate of perceived exertion.

Results: DRY exercises exhibited lower lactate level after the warmup ( 1.93 vs. $2.60 \mathrm{mmol} / \mathrm{L}, \mathrm{P}=0.006)$, higher autonomic parameters and higher bradycardia during the subsequent dynamic apnoea in comparison to WET protocol. We found a significant correlation between the lactate produced after WET warm-up and the duration of the successive dynamic apnoea, indicating that higher lactate level could reduce the diving performance ( 72 vs. $70 \mathrm{~s}, \mathrm{P}=0.028$ ). The haemoglobin concentration and the rate of perceived exertion were not significantly different between protocols.

Conclusions: Before the dynamic apnoea performance, the use of the DRY warm-up seems to be a better strategy respect to the WET warm-up since it induces a more pronounced diving response, avoids lactate accumulation pre-apnoea, and reduces the time needed to complete the dive. Finally, the rating of perceived exertion, widely used in many sports, should be well examined and appropriate scales for apnoea disciplines should be developed.

\section{References}

1. Ostrowski A, Strzała M, Stanula A, et al. (2012) The role of training in the development of adaptive mechanisms in freedivers. J Hum Kinet 32:197-210.

2. Schagatay E (2010) Predicting performance in competitive apnea diving. Part II: Dynamic apnoea. Diving Hyperb Med 40:11-22.

3. Schagatay E, Andersson JPA, Nielsen B (2007) Hematological response and diving response during apnea and apnea with face immersion. Eur J Appl Physiol 101:125-132.

\section{1}

Are muscle and tendon "Sensitive" to the direction of a mechanical stimulus? Adaptations to moderateload concentric vs. eccentric training in young and older men

Martino Franchi ${ }^{1}$, Jonathan I Quinlan ${ }^{2}$, Phil Atherton ${ }^{2}$, Ken Smith $^{2}$, $\overline{\text { Paul Greenhaff }}^{2}$, Costantinos Maganaris ${ }^{3}$, Marco Narici ${ }^{1}$ 
University of Padova, Department of Biomedical Sciences, Padova, Italy $^{1}$;

University of Nottingham, Mrc-aruk Centre of Excellence

For Musculoskeletal Ageing, Nottingham, Regno Unito ${ }^{2}$; Liverpool John Moores University, School of Sports Science, Liverpool, Regno Unito ${ }^{3}$

Aim: Resistance training (RT) is known to counteract negative agerelated changes in both muscle and tendon tissue. Moderate eccentric (ECC) loading has been advocated as an efficient way to achieve increases in muscle mass and tendon mechanical properties. Indeed, isolated ECC contractions are metabolically less demanding; thus, may be more suitable for older populations. However, whether submaximal $(60 \% 1 \mathrm{RM})$ concentric $(\mathrm{CON})$ or ECC contractions differ in their effectiveness is relatively unknown This study aimed to establish the time course of muscle and tendon adaptations to submaximal CON and ECC RT.

Methods: 20 healthy young ( $24.5 \pm 5$.1years) and 17 older males $(68.1 \pm 2.4$ years) were randomly allocated to either leg-press based isolated CON or ECC RT which took place 3/week for 8 weeks. Patellar tendon biomechanical properties, vastus lateralis (VL) muscle morphology and architecture, and maximal voluntary contraction were assessed every 2 weeks, quadriceps and VL muscle volume every 4 weeks.

Results: Positive changes in tendon Young's modulus were observed after 4 weeks in all groups. Since then, adaptations in young males plateaued, whereas they continued to increase in older males, suggesting a dampened rate of tendon remodelling with age. However, both CON and ECC presented similar overall changes in tendon Young's modulus, in all groups. Muscle hypertrophy and strength increases were similar between CON and ECC in all groups. Nonetheless, contraction-specific adaptations were observed for pennation angle (i.e., greater increases after CON compared to ECC) and fascicle length (i.e., greater increases after ECC compared to CON).

Conclusions: Tendon mechanical properties respond equally to CON and ECC RET in both young and elderly groups, suggesting that the patellar tendon may be blind to the loading modality used. However, it may require a longer period for the elderly tendon to fully adapt; albeit this may be due to a lower starting point. Noteworthy, muscle and tendon adaptations appeared to occur in synergy; presumably to maintain the efficacy of the muscle-tendon unit. On a practical perspective, considering the characteristics of ECC loading modality (greater load imposed at a lower metabolic cost), and the greater early hypertrophic responses than of concentric training, moderate-intensity ECC exercise can be recognised as a very efficient training modality for counteract the loss of muscle mass in older individuals.

\section{2}

Use of heart rate variability during an incremental test, for the evaluation of baroceptor and respiratory activity

\section{Mosè Mondonico $^{1}$, Schillaci Giacomo ${ }^{1}$ \\ Isec, R\&d, Montecatini Terme, Italy ${ }^{l}$}

Purpose: The use of HRV heart rate variability has long been used as a reliable tool for measuring and consequently evaluating the state of activity of the central nervous system. But while all most of the studies have focused on the assessment of HRV in a resting state, little is known about the potential of this investigation on subjects during training. In this preliminary study we wanted to investigate how to use the parameters deriving from the "frequency domain " to identify the activity of baroreceptors Mayer Waves (MW) during an incremental test and the consequent interaction with the respiratory system. Mayer Waves can be defined as arterial blood pressure (AP) oscillations at frequencies slower than respiratory rate and which show significant association with efferent sympathetic nervous activity (ANS).

Methods: Ten male subjects with good training experience performed an incremental test on a cycle ergometer, the protocol provided for 6 min at 80 watts as a warm-up and subsequent increases of 40 watts every 4 min until exhaustion. The heart rate was measured with the Polar H10 belt and subsequently downloaded in R-R format from the Polar Flow web application. The time domain data were processed by analyzing each single predicted fraction with Kubios 3.4.1 software. Results: In this preliminary study we found an activation of MW between 0.08 and $0.2 \mathrm{~Hz}$ at submaximal exercise intensities up to $85 \%$ of maximal heart rate. The respiratory frequencies are instead very individual, not giving statistically significant results.

Conclusion: The appearance of MW indicates a response activity of the peripheral vascular system in response to physical exercise, at intensities close to the maximum the MW gradually disappear. The analysis of AP adaptation capacity could be used as an individual training parameter. The analysis of respiratory rates can instead be useful for assessing the effectiveness of the respiratory action and the impact this has on the fatigue of the ANS system.

\section{References}

1. Elkaranshawy HA, Ali AME, Abdelrazik IM. An effective heterogeneous whole-heart mathematical model of cardiac induction system with heart rate variability. Proc Inst Mech Eng H. 2021 Mar;235(3):323-335.

2. Elstad M, Walløe L, Chon KH, Toska K. Low-frequency fluctuations in heart rate, cardiac output and mean arterial pressure in humans: what are the physiological relationships? J Hypertens. $2011 \mathrm{Jul} ; 29(7): 1327-36$.

3. Jafarnia-Dabanloo N, McLernon DC, Zhang H, Ayatollahi A, Johari-Majd V. A modified Ze.

\section{3}

\section{Behaviour of eye movements in a targeting task: the basketball free throw}

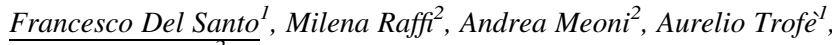 \\ Alessandro Piras ${ }^{2}$
}

Università Di Bologna, Dipartimento Di Scienze Per La Qualità Della Vita, Bologna, Italy ${ }^{1}$;

Università Di Bologna, Dipartimento Di Scienze Biomediche E Neuromotorie, Bologna, Italy ${ }^{2}$

Purpose. In targeting tasks, the function of the gaze behaviour and attention system is to locate a target in space and control the aiming of an object to a specific target area. In these tasks an object is pushed with the hands or feet away from the body in an aiming movement toward a target (Vickers, 2007). Accuracy and consistency in performance are the ultimate goal of the task. The aim of the present study was to investigate the effective role of gaze behaviour during the execution of basketball free throw.

Methods. 24 basketball players $(21.04 \pm 3.1$ years $)$, subdivided in 12 experts (E) and 12 near-experts (NE) were involved in the study. Participants wore an eye-tracker (EyeLink II), and an inertial sensor (Cometa Systems, Italy) placed on their throwing hand, for both eye and hand movements recording. They stand in a basketball field, behind the free-throw line, and shoots a ball into a hoop of $45 \mathrm{~cm}$ located at a height of $3.05 \mathrm{~m}$ from the floor. Response accuracy and gaze behaviour were recorded for analysis. 
Results. There was no significant difference between the E (67\%) and $\mathrm{NE}(70 \%)$ for response accuracy $(\mathrm{p}=0.63)$. The free throw duration was shorter in NE than E group (2658 vs. $2990 \mathrm{~ms} ; \mathrm{p}=0.002)$. Regarding gaze behaviour, E made more microsaccades $(0.98$ vs. 0.47 $\mathrm{n} / \mathrm{sec} ; \mathrm{p}<0.001)$ and less saccades $(2.42$ vs. $2.95 \mathrm{n} / \mathrm{sec} ; \mathrm{p}=0.009)$ than NE. Moreover, microsaccade and saccade durations were higher in $\mathrm{E}$, meanwhile saccade and microsaccade peak velocities exhibited higher values in NE group. Finally, the angular distributions of saccade and microsaccade directions have not shown significant differences between groups ( $\mathrm{p}>0.05$ ).

Conclusions. The different subtle eye movements found between E versus NE group during an aiming task did not show a direct effect on performance accuracy. These results suggest that the high variability associated with this skill (Button et al., 2003) could be not directly influenced by the athletes visual search strategy (Piras et al., 2020).

\section{References}

1.Button et al. (2003). Examining movement variability in the basketball free-throw action at different skill levels. Research quarterly for exercise and sport, 74(3), 257-269.

2.Piras et al. (2020). Understanding the underlying mechanisms of Quiet Eye: The role of microsaccades, small saccades and pupilsize before final movement initiation in a soccer penalty kick. European Journal of Sport Science, 1-10.

3.Vickers (2007). Perception, Cognition \& Decision Training: The Quiet Eye in Action.

\section{4}

\section{Blood gases analysis during breath-hold diving activities: the normoxemia at depth paradox}

Gerardo Bosco ${ }^{1}$, Matteo Paganini ${ }^{1}$, Tommaso Antonio Giacon ${ }^{1}$, Luca Martani $^{I}$, Danilo Cialoni ${ }^{1}$, Giacomo Garetto ${ }^{1}$, Ennio Talamonti ${ }^{1}$, Jacopo Lion ${ }^{1}$, Chris Mcknight ${ }^{2}$, Enrico Camporesi ${ }^{3}$, Richard Moon ${ }^{4}$

Master Level Ii In Diving and Hyperbaric Medicine, Department of Biomedical Sciences, University of Padova, Padova, Italy ${ }^{1}$; Sea Mammal Research Unit, University of St. Andrews, St. Andrews, Regno Unito ${ }^{2}$;

Teamhealth Research Institute, Tampa General Hospital, Tampa, Florida, Stati Uniti D' America ${ }^{3}$.

Center For Hyperbaric Medicine and Environmental Physiology, Department of Anesthesiology, Duke University Medical Center, Durham, North Carolina, Stati Uniti D' America ${ }^{4}$

Purpose: Based on theory and experiments conducted in hyperbaric chambers, arterial $\mathrm{PO} 2$ and $\mathrm{PCO} 2$ in breath-hold $(\mathrm{BH})$ divers progressively increase during descent. While at depth, due to metabolic $\mathrm{O} 2$ consumption, $\mathrm{PCO} 2$ continues to rise while $\mathrm{PO} 2$ falls $[1,2]$. However, new findings during in-water $\mathrm{BH}$ diving have demonstrated that (1)PCO2 at maximum depth is highly variable; (2) PO2 does not consistently rise in all subjects and in some individuals may remain at concerning low levels [3, 4]. Further experiments were then conducted at different depths while evaluating cerebral perfusion.

Methods: the radial artery of 6 well-trained BH divers was cannulated, and blood sampled during breath $\mathrm{BH}$ dives at $-15 \mathrm{~m}$ and $-42 \mathrm{~m}$ (at rest; immediately before the dive; at the bottom; and at the end of the dive, before the resumption of breathing). Arterial PO2 and $\mathrm{PCO} 2$ levels were measured using a point-of-care blood gas analyzer. A marinized NIRS apparatus was applied to the forehead to measure heart rate, cerebral volume and cerebral blood oxygen saturation.

Results: At $-15 \mathrm{~m}$, one subject demonstrated a rise in arterial PO2 as expected $(235.6 \mathrm{mmHg})$ and one a slight rise at depth $(155.4 \mathrm{mmHg})$. Surprisingly, the remaining subjects showed a nonsignificant $\mathrm{PaO} 2$ rise. Hypoxemia was detected at the end of the dive in all the subjects - slightly blunted in the subject hyperoxemic at the bottom. Similarly, only one subject showed high arterial PO2 at $-42 \mathrm{~m}$ while the others showed less extreme values. All the subjects except one were hypoxemic at the surface. Arterial PCO2 showed slighter variations, with similar trends across the two depths. NIRS confirmed bradycardia and cerebral blood volume reduction during the dives, with significant drops in cerebral oxygen saturation, especially in the $42 \mathrm{~m}$ dive.

Conclusions: These preliminary Results seem to confirm that a nonnegligible amount of subjects manifest normoxemia at depth, resulting in hypoxemia while resurfacing and important changes in cerebral hemodynamics and blood oxygen saturation. Further experiment will explore the mechanisms underlying the normoxemic paradox at depth, potentially originating from lung atelectasis.

\section{References}

1. Lindholm P, Lundgren CE. The physiology and pathophysiology of human breath-hold diving. J Appl Physiol. 2009;106(1):284-92.

2. Linér $\mathrm{MH}$, Ferrigno M, Lundgren CE. Alveolar gas exchange during simulated breath-hold diving to $20 \mathrm{~m}$. Undersea HyperbaricMed 1993;20(1):27-38.

3. Bosco G, Rizzato A, et al. Environmental physiology and diving medicine. Front Psychol. 2018;2(9):72.

4. Bosco G, Rizzato A, et al. Arterial blood gas analysis in breathhold divers at depth. Front Physiol. 2018Nov5; 9:1558.

\section{5 \\ Cardiovascular stress during resistance exercise: effect of age and protocol}

Massimo Teso ${ }^{l}$, Luca Ferrari $^{\text {I, Gianluca Bochicchio }}{ }^{1}$, Tobia Bertuzzo $^{I}$, Amedeo Benedetti ${ }^{1}$, Alessandro Colosio ${ }^{2}$, Silvia Pogliaghi ${ }^{I}$

Università Degli Studi Di Verona, Dipartimento Di Scienze Motorie, Verona, Italy ${ }^{I}$;

University of Ghent, Department of Movement and Sport Science, Ghent, Italy ${ }^{2}$

A supposedly high acute cardiovascular stress during resistance exercise (e.g. squat) discourages the use of this training modality in ageing adults. Moreover, protocols involving high fractions of 1 repetition maximum (\%1RM) are typically avoided to minimize cardiovascular stress.

Purpose: To quantify the cardiovascular stress during squat exercise in relation to participant's age and training intensity.

Methods: 8 Middle-age (MA, 4 females, $50 \pm 6$ years) and 16 young (Y, 8 females, $26 \pm 2$ years) participants performed: indirect 1-RM determination in squat; a low-intensity (SPlow, 55\%1RM, 12 repetitions, 3 series) and a high-intensity (SPhigh, 80\%1RM, 5 repetitions, 5 series) squat protocol. During the exercise phase in both protocols we measured average HR (K5, Cosmed), systolic (SBP) and diastolic blood pressure (DBP) (Tango blood pressure monitor, Suntech); moreover, we expressed $\mathrm{HR}$ as $\%$ of the previously determined maximal value and calculated rate-pressure product (RPP). Data were compared with t-test and two-way ANOVA (protocols and agegroup).

Results: 1RM was significantly lower in MA $(76 \pm 26$ vs $94 \pm 41 \mathrm{~kg}$, $\mathrm{p}<0.001)$. HR was significantly lower in MA $(136 \pm 18$ vs $153 \pm$ $13 \mathrm{bpm}$, main effect of age $\mathrm{p}=0.008$ ) with no differences among protocols for both age groups (main effect of protocol $p=0.08$ ). For both age groups $(\mathrm{p}=0.08)$ and both intensities $(\mathrm{p}=0.16)$, HR represented a comparable fraction of the maximal value (overall mean 
MA $78 \pm 8$ vs Y $83 \pm 8 \%$ HRmax, indicative of a vigorous intensity). DBP was significantly higher in MA $(84 \pm 13$ vs $63.0 \pm 10 \mathrm{mmHg}$, main effect of age $\mathrm{p}<0.001$ ), with no main effect of protocol ( $\mathrm{p}=$ 0.608). No differences were detected in either SBP (overall average $155 \pm 5 \mathrm{mmHg}$ ) or RPP (overall average 22,493 $\pm 4463 \mathrm{bpm} \mathrm{mmHg}$, indicative of an intermediate hemodynamic response) in relation to either age group ( $\mathrm{p}=0.795$ and 0.118 for SBP and RPP, respectively) or protocol ( $\mathrm{p}=0.402$ and 0.257 for SBP and RPP, respectively).

Conclusion: In middle-age and young individuals alike, squat exercises of either low or high intensity are associated with a similar cardiovascular response. Individuals of both sexes with a healthy blood pressure display hemodynamic responses that are comparable to those of a vigorous aerobic exercise; these responses can be classified as intermediate, based on RPP.

\section{References}

1. Lemmer, J. T. et al. Age and gender responses to strength training and detraining. Med. Sci. Sports Exerc. 32, 1505-1512 (2000).

2. Lakatta, E. G. Age-associated cardiovascular changes in health: Impact on cardiovascular disease in older persons. Heart Fail. Rev. 7, 29-49 (2002).

\section{POSTURE and FUNCTIONAL RECOVERY}

\section{6}

\section{A balance matter: postural differences determined by the exposure to neutral and negative stimuli}

\author{
Ambra Gentile ${ }^{l}$, Giustino Valerio ${ }^{l}$, Xhuliano Kosta $^{l}$, Ewan Thomas ${ }^{1}$, \\ Antonino Bianco $^{l}$, Antonio Palma ${ }^{1}$, Giuseppe Messina ${ }^{1}$, Stefano \\ Boca $^{l}$ \\ Università Degli Studi Di Palermo, Dipartimento Di Scienze \\ Psicologiche, Pedagogiche, Dell'esercizio Fisico E Della \\ Formazione, Palermo, Italy ${ }^{I}$
}

Purpose. The current study proposes a new attitude measurement technique based on postural reactions determined by the exposure to stimuli with a clear affective tonality. We hypothesize that people will change their baropodometric (weight distribution between heel, first and fifth metatarsal heads) and stabilometric parameters (sway surface Centre of Pressure [CoP], sway length CoP, Length as Function of Surface ratio [LFS], displacement of $\mathrm{CoP}$ on the $\mathrm{Y}$ axis, sway frequency of the $\mathrm{Y}$ axis, maximum speed of $\mathrm{CoP}$ displacement) in front of neutral and negative images.

Methods. Eighty-three adult participants were involved in the data collection. Each participant was invited to remove the shoes and step on the platform (Podata, Chinesport S.P.A., Udine, Italy), distanced $3 \mathrm{~m}$ from a screen, and to undergo a baropodometric and stabilometric exam of $1 \mathrm{~min}$. Five conditions were created, each one lasting $20 \mathrm{~s}$ : baseline open eyes, baseline closed eyes, visual target (red point on the screen), neutral stimulus on the screen, negative stimulus on the screen (the last two conditions were randomized).

Results. A repeated measures MANOVA (baseline open eyes vs. baseline closed eyes vs. visual target vs. neutral stimulus vs. negative stimulus) was implemented to detect differences across the five conditions. The preliminary results showed that there was a difference among conditions concerning the weight distribution on the heels $(\mathrm{F} 328,4=3.23, \mathrm{p}<0.05)$, the sway length $\mathrm{CoP}(\mathrm{F} 328,4=31.71, \mathrm{p}<$ $0.001)$, the sway surface $\operatorname{CoP}(\mathrm{F} 328,4=11.07, \mathrm{p}<0.001)$, displacement of center of pressure on the $\mathrm{Y}$ axis $(\mathrm{F} 328,4=7.87, \mathrm{p}<$ $0.001)$, LFS (F328,4 = 8.34, p < 0.001), and sway frequency on the $Y$ axis $(\mathrm{F} 328,4=3.39, \mathrm{p}<0.001)$. No difference was detected on maximum speed of $\mathrm{CoP}$ displacement.
Conclusion. Differences in baropodometric and stabilometric parameters were found in the neutral and negative conditions, indicating that posture might change in relation to visual stimuli. Our findings can open new scenarios concerning attitude measurement.

\section{7}

\section{Postural strategies for action anticipation and action performance}

Francesco Pascucci ${ }^{1}$, Matteo Cristani $^{2}$, Matteo Ricci $^{3}$, Paola Cesari ${ }^{1}$

University of Verona, Department of Neuroscience, Biomedicine and Movement, Verona, Italy ${ }^{1}$;

University of Verona, Department of Computer Science, Verona, Italy $^{2}$;

Azienda Ospedaliera Universitaria Integrata, Department of Orthopaedic Surgery, Verona, Italy ${ }^{3}$

Purpose: Measurements of postural system are critical for figuring out predictors of performance, evaluate musculoskeletal injuries and estimate the effectiveness of physical training and rehabilitation treatments. The present study has the specific purpose of determining the measures to be taken to appraise an individual's behaviour at different conditions of instability.

Methods: The proposed approach allows us to investigate the anticipatory strategies that people apply when asked to release a sphere while maintaining postural control on different proprioceptive boards. To capture these strategies, Center of Pressure (CoP) migration and the anticipatory postural adjustments (APAs) parameters were recorded and analyzed. Healthy people and individuals that have undergone anterior cruciate ligament (ACL) reconstruction, are compared in their ability to cope with eight experimental conditions presenting different levels of instability, from a low-level to a highlevel balance task difficulty.

Results: preliminary results show that, when the tasks were the easiest ones, the ACL group presented the tendency to anticipate more the action when compared to the control group, as expressed by the early detection of the APA onset.

Conclusions: For the two groups different motor strategies emerged: in action anticipation ACL group showed an early onset for compensating the inability to quickly detect sudden changes in muscle length due to the muscle damage suggesting a specific training interventions.

\section{References}

1. Aruin AS, Latash ML. The role of motor action in anticipatory postural adjustments studied with self-induced and externally triggered perturbations. Exp Brain Res 1995;106(2):291-300

2. Aruin AS et al. Anticipatory postural adjustments in conditions of postural instability. Electroencephalogr Clin Neurophysiol 1998;109(4): 350-9

3. Labanca L et al. Early compensatory and anticipatory postural adjustments following anterior cruciate ligament reconstruction. Eur J Appl Physiol 2015:115(7):1441-51. 


\section{8}

\section{Sleep loss, circadian rhythm, and postural control:} a systematic review

\author{
Mohammad Izadi ${ }^{1}$, Ewan Thomas ${ }^{2}$, Abbey Thomas ${ }^{3}$, Guglielmo \\ Pillitteri $^{I}$, Salvatore Ficarra ${ }^{l}$, Alessandra Amato ${ }^{l}$, Daniele Zangla ${ }^{2}$, \\ Antonio Palma ${ }^{2}$, Antonino Bianco ${ }^{2}$, Marianna Bellafiore ${ }^{2}$ \\ Phd Student In Health Promotion and Cognitive Sciences, Sport \\ and Exercise Research Unit, Department of Psychology, Educational \\ Sciences and Human Movement, University of Palermo, Palermo, \\ Italy $^{1}$; \\ Sport and Exercise Research Unit, Department of Psychology, \\ Educational Sciences and Human Movement, University of Palermo, \\ Palermo, Italy ${ }^{2}$; \\ $\mathrm{Na}$, Department of Kinesiology, University of North Carolina At \\ Charlotte, Charlotte, United States of America ${ }^{3}$
}

Purpose: While postural control (PC) assessment is a way to detect those who are susceptible to injuries (e.g., risk of fall), circadian rhythm may change the result of the measurement. That is, physical and mental functions are altered in accordance with the time of day; thus, it is expected individuals experience optimal PC at a specific time of day (1). However, there are contradictory results surrounding this issue in the literature. Similarly, sleep deprivation can alter PC. Specifically, a decrease in normal sleep period and an increase in time of wakefulness may result in a reduction in cognitive function, which in turn can lead to difficulty in maintaining PC. However, the mechanism underlying this process is not well understood. Whereas PC measurement is widely used to detect neuromuscular deficits in addition to specifying sleepiness, which can help to prevent occupational accidents, there is no systematic review in the literature to critically assess the relevant studies to comprehensively understand the effect of circadian rhythm and sleep loss on PC; hence, the aim of the study was to systematically review the studies to understand the effect of circadian rhythm and sleep loss on PC.

Method: This systematic review was carried out based on the PRISMA statement (2) in which PubMed, Web of Science, Scopus, and Embase were used to detect relevant studies. Only studies that examined the effect of time of day and/or sleep loss on postural control among healthy individuals were included in this systematic review.

Results: Forty-nine studies (1296 healthy participants) were included in accordance with the inclusion and exclusion criteria. Both circadian rhythm and sleep loss had a significant effect on postural control; however, there are contradictory results for optimal postural control in regard to time of day. In terms of sleep loss, all studies reported that sleep deprivation worsens postural control.

Conclusion: This systematic review revealed a negative and meaningful effect of sleep deprivation on PC. Time of day was also determined as a contributory factor that can lead to changes in PC. Hence, it is suggested that clinicians and researchers consider both factors when measuring PC.

\section{References}

1.Gribble PA, Tucker WS, White PA. Time-of-day influences on static and dynamic postural control. Journal of athletic training. 2007;42(1):35.

2.Moher D, Shamseer L, Clarke M, Ghersi D, Liberati A, Petticrew M, et al. Preferred reporting items for systematic review and meta-analysis protocols (PRISMA-P) 2015 statement. Systematic reviews. 2015;4(1):1-9.

EXERCISE and SPORT PSICOLOGY.

\section{9}

Detection of cognitive differences between rowing
athletes and sport practitioners by using big five test

$\underline{\text { Riccardo Sorrentino }}^{1}$, Valerio $^{\text {Caruso }}{ }^{2}$, Rosa Angela Fabio $^{3}$, Debora Di Mauro

Università Degli Studi Di Messina, Dipartimento Di Scienze Biomediche, Odontoiatriche E Delle Immagini Morfologiche E Funzionali, Messina, Italy ${ }^{1}$;

Università Di Pisa, Dipartimento Di Medicina Clinica E

Sperimentale, Pisa, Italy ${ }^{2}$;

Università Degli Studi Di Messina, Dipartimento Di Medicina

Clinica E Sperimentale, Messina, Italy ${ }^{3}$

Purpouse: Cognitive analysis of high-level athletes has always been an interesting topic about sport psychology. The aim of this study was to assess cognitive differences between Rowing athletes (RA) and Sport Practitioners (SP) by using Big Five Questionnaire Test (BFQ). Methods: Thirty-one RA, representing the case group, and thirty SP, representing the control group, were asked to complete the BFQ. Both RA and SP groups have been selected randomly from a population, respectively, of several rowing Sicily sport clubs and several Sport Science classes of University of Messina. Due to COVID-19 pandemic event, BFQ was transposed electronically, with an additional file describing all the procedures and examples about the test. Each participant signed a informed consent explaining aim and duties of the research.

Results: Results were assessed with test- $t$ for independent samples with an additional Holm- Bonferroni correction test. SPSS version 20. was used to manage data and tests. Results showed how RA present significative differences than SP, on Energy $(\mathrm{p}<0.0006)$, Conscientiousness $(p=0.01)$, Dominance $(p<0.03)$. On the contrary, SP scored higher on Agreeableness $(\mathrm{p}<0.05)$ and Cooperation $(\mathrm{p}=$ $0.01)$. No differences were registered on the other scales.

Conclusions: From results emerges a precise description of high-level athletes, in this case rowers. It is possible claiming that, as suggested from previous studies, that there are cognitive traits characterizing high level athletes. Despite BFQ it is not a gold standard method for psychological assessment, however it remains a valid tool to seek for cognitive differences, in this case between RA and SP.

Acknowledgements: The authors thank the director of the Sport Sciences degree courses for providing us with the subjects of the control group and Domiziana Minissale (Department of Pharmacy, University of Messina) for providing us the contacts of rowing societies.

1. Laborde, S., Guillén, F. and Mosley, E. (2016) Positive personality-trait-like individual differences in athletes from individualand team sports and in non-athletes Psychology of Sport and Exercise 26:9-13.

2. Steca, P. et al. (2018) Associations between personality, sports participation and athletic success. A comparison of Big Five in sporting and non-sporting adults Personality and Individual Differences 121:176-183.

\section{0}

Self-efficacy correlates with perceived but not objective physical ability in football players

\author{
Fabio Ballini ${ }^{1}$ \\ Unibo, Scienze Biomediche E Neuromotorie, Bologna, Italy ${ }^{l}$
}


Purpose: A high self-efficacy (SE) is beneficial to sport performance and knowing its correlates may identify targets to increase self-efficacy in athletes. The aim of this study was to test the hypotheses that SE is associated with high levels of both objective and perceived physical ability in football players.

Methods: Before the tests of physical ability, 17 male amateur football players (age $21.1 \pm 3.3$ years) were given questionnaires to measure general SE and role-specific SE, and performance-oriented physical ability (PPA) and motor-control oriented physical ability (MPA). Objective physical ability was measured with various test in the following order: counter movement jump (CMJ), the Illinois agility test and the Yo-Yo test of endurance. Pearson or Spearman correlation coefficients were calculated depending on the normality of the data.

Results: There were significant correlations between PPA and both general SE $(r=0.535, p<0.05)$ and role-specific SE $(r=0.490, p<$ $0.05)$. No significant correlations were found between the objective measures of physical ability and the measures of SE. Moreover, there were no significant correlations between measures of objective and perceived physical ability.

Conclusion: As hypothesized, perceived physical ability was significantly correlated with SE in football players. However, objective measures of physical ability did not correlate with SE in this population. The results suggest that his may be due to an incorrect perception of their own abilities possibly due to reduced training and competitions due to COVID-related lockdown in 2020 when the study was performed. Strategies to increase perceived physical ability may improve SE and performance in football players and need to be further investigated.

\section{References}

1. Bortoli, L. and Robazza, C. (1997) 'Italyn Version of the Perceived Physical Ability Scale', Perceptual and Motor Skills, 85(1), pp. 187-192. https://doi.org/10.2466/pms.1997.85.1.187.

2. Costa, S., Greco, A. and Steca, E. P. (2018) 'Una scala per la misurazione delle convinzioni di efficacia personale e collettiva nel calcio', p. 9 .

\section{FITNESS and SPORT NUTRITION}

\section{1}

Active breaks study (ABS): reducing physical inactivity and improving the quality of school life in primary school children using an active breaks intervention during COVID-19 pandemic

\section{Alice Masini ${ }^{1}$, Sofia Marini ${ }^{2}$, Stefania Toselli ${ }^{1}$, Laura Bragonzoni ${ }^{2}$, Gerardo Astrorino ${ }^{3}$, Maurizio Liberti ${ }^{3}$, Andrea Ceciliani ${ }^{2}$, Laura Dallolio ${ }^{1}$}

University of Bologna, Department Od Biomedical and Neuromotor Science, Bologna, Italy ${ }^{I}$;

University of Bologna, Department For Life Quality Studies, Rimini, Italy $^{2}$;

Ausl, Department of Public Health, Ausl Bologna, Bologna, Italy ${ }^{3}$

Purpose: Active Breaks (AB) are emerging as a possible classroom based Physical Activities (PA) strategy to help children in accumulating the required amount of $\mathrm{PA}$. $\mathrm{AB}$ are characterised by $10 / 15 \mathrm{~min}$ of PA led by the teachers also during distance learning due to Covid19 pandemic. The aim of the Active Breaks study (ABS) was to evaluate the feasibility and the efficacy of $\mathrm{AB}$ intervention in primary school children in improving the levels of physical activity, the health-related quality of life and classroom behaviour during Covid19 pandemic.

Methods: Study design: pre-post study in primary schools (children from 6 to 9 years, from 1st to 5th grade) in Emilia Romagna region, 10 min of $\mathrm{AB}$ were performed 3 times per day for 10 weeks.

The assessed variables were: self-reported physical activity level (PAQ-c) health related quality of life (Peds-QL) and classroom behaviour (ad Hoc questionnaire). Statistical analyses were performed using SPSS.

Results: We calculated the pre-post difference using paired T-test. Results showed an improvement in self-reported PA levels measured with PAQ-c questionnaire $(\mathrm{n}=82 ; \mathrm{T} 0=2.55 \pm 0.73$ to $\mathrm{T} 1=2.73 \pm$ $0.74 ; \mathrm{p}<0.01)$. The health-related quality of life's results were not statistically significant. Considering children's classroom behaviour after the $\mathrm{AB}$ intervention: $66.4 \%$ of student perceived positive school wellbeing, $59.7 \%$ felt more focused, $48.5 \%$ learned more easily and $62.7 \%$ perceived less difficulty in sitting. Almost the entire sample (n $=134)$ reported having fun $(84.3 \%)$ and enjoying $(82.8 \%)$ the $\mathrm{AB}$ intervention; the $86.6 \%$ of student are willing to continue bthis programme.

Conclusion: There was a significant positive improvement in selfreported levels of $\mathrm{PA}$ confirming the effect of $\mathrm{AB}$ in reducing physical inactivity. Our findings suggested that $A B$, based on children' perception produce positive effect towards student's well-being and time on task. Active Breaks intervention showed to be feasible also during distance learning.

\section{References}

1. Calella, P. et al. (2020) Classroom active breaks: a feasibility study in Southern Italy. Health promotion international, 35(2), 373-380.

2. Masini, A. et al. (2020) Evaluation of school-based interventions of active breaks in primary schools: A systematic review and meta-analysis. Journal of science and medicine in sport, 23(4), $377-384$.

\section{2}

Motor performance effects of sensory deprivation in elderly

Elvira Padua ${ }^{I}$, Michele Panzarino ${ }^{1}$, Francesca Campoli ${ }^{1}$, Cristian Romagnoli $^{2}$, Anas Alsharam ${ }^{3}$, Vincenzo Manzi ${ }^{4}$, Virginia Tancredi ${ }^{5}$, Mauro Lombardo ${ }^{6}$, Giuseppe Annino ${ }^{5}$

Università Telematica San Raffaele Roma, Dip. Di "promozione Delle Scienze Umane E Della Qualità Della Vita”, Rome, Italy' Università "alma Mater Studiorum” Di Bologna, Dipartimento Di Scienze Per La Qualità Della Vita, Rimini, Italy²

Isra University, Department of Physiotherapy, Amman, Giordania ${ }^{3}$; Università Telematica Pegaso, Dipartimento Di Scienze Umanistiche, Napoli, Italy ${ }^{4}$;

Università Di Roma Tor Vergata, Dipartimento Di Medicina Dei Sistemi, Rome, Italy

Università Telematica San Raffaele, Dip. Di "promozione Delle Scienze Umane E Della Qualità Della Vita", Rome, Italy 6

Purpose: Aim of this study was to investigate the effect of a motor activity program for elderly, based on the use of a mask that "partially " limits sight.

Methods: 114 subjects (76,3 \pm 6 years), 16 men, divided into three groups. An experimental group ExG (51 subjects) that practices regular physical training (PT) with sensory deprivation and a PT group (as control group) CG (63 subjects) that practices general fitness training without the mask. In ExG all exercises are carried out 
ensuring maximum safety to avoid injuries. The two gym groups practice activities twice a week (50'per session) for 5 months. The tests evaluate the back muscle flexibility, balance, abdominal and legs strength. The groups, in baseline, are homogeneous in all motor skills analyzed. Descriptive statistical procedures are presented as mean and the percentage changes $(\Delta \%)$ were calculated for each study group. A multivariate between-within groups ANOVA was conducted to assess any significant difference in mean variables used. Tukey's post hoc test of critical difference was used to locate significance between means. The significance level was fixed at $\mathrm{p}<0.05$.

Results: Results of this study showed that the abdominal $(p<0,05)$ and lower limb strength $(\mathrm{p}<0,01)$ improved more in CG than in ExG: lower limb strength: CG $\Delta 10 \%$ (p < 0.001) CG $\Delta 21 \%$ (p < $0.001)$; abdominal strength SG $\Delta 14 \%$ (p < 0.001$)$ GG $\Delta 21 \%$ (p $<$ $0.001)$. Important results are in flexibility $(\mathrm{p}<0,05)$ and balance test $(\mathrm{p}<0,01)$ where the ExG showed a greater improvement than CG: Flexibility: ExG $\Delta 31 \%(\mathrm{p}=0.001) \mathrm{CG} \Delta 24 \%(\mathrm{p}<0.001)$; balance test ExG $\Delta 93 \%(\mathrm{p}<0.0001)$ and CG $\Delta 11 \%(\mathrm{p}=0.1)$.

Conclusions: In light of the results observed on both groups it seems that the sensory deprivation condition is suitable in old people training programs overall in conditioning the balance performance that play a key role on the expectance and quality of life of this special population related to the risk of falls.

\section{References}

1. Riemann B. L.; Lephart S. M., The Sensorimotor System, Part II: The Role of Proprioception in Motor Control and Functional Joint Stability (2002), Journal of Athletic Training; 37(1):80-84.

2. Springer BA, Marin R, Cyhan T, Roberts H, Gill NW. (2007). Normative values for the unipedal stance test with eyes open and closed. J Geriatr Phys Ther.

3. Rose DJ. Fallproof. A comprehensive balance and mobility program. Champaign, IL. Human Kinetics, 2003.

\section{3}

\section{Observational study on physical activity and sleep during Covid-19 pandemic}

\author{
$\underline{\text { Ausilia Elce }}^{1}$, Ilaria Loperto ${ }^{2}$, Lucia De Coppi ${ }^{2}$, Armando \\ Sangiorgio $^{2}$, Angelina Vivona ${ }^{2}$, Clorinda Sorrentino ${ }^{2}$, Lucia \\ Martiniello $^{2}$, Ersilia Nigro ${ }^{3}$, Aurora Daniele ${ }^{4}$
}

Ceinge-biotecnologie Avanzate Scarl, Napoli, Dipartimento Di Scienze Umanistiche, Università Telematica Pegaso, Napoli, Italy ${ }^{1}$; Università Telematica Pegaso, Università Telematica Pegaso/ Dipartimento Di Scienze Umanistiche, Napoli, Italy ${ }^{2}$; Ceinge-biotecnologie Avanzate Scarl, Dipartimento Di Scienze E Tecnologie Ambientali, Biologiche, Farmaceutiche, Università Della Campania, Napoli, Italy ${ }^{3}$;

Ceinge-biotecnologie Avanzate Scarl, Dipartimento Di Medicina Molecolare E Biotecnologie Mediche, Università Degli Studi Di Napoli Federico Ii, Napoli, Italy ${ }^{4}$

Purpose: Multiple mental alterations were described in Chinese population during COVID-19 pandemic, among these, sleep deprivation as a consequence of social isolation (Sci L. et al., 2020; Gualano M.R. et al., 2020).

In Italy, COVID pandemic spread out at the beginning of 2020 and determined severe lockdown periods until May 2021.

The aim of our study was to observe the effects of lockdown on sleep and mood in 25 sedentary and 36 trained subjects who continued to train even during the lockdown period for at least 5 weekly hours).
Methods: All the 61 subjects of the study were aged from 19 to 35 years. In April 2021, we administered an online semi structured questionnaire consisting of psychometric and anthropometric sections, physical activity levels and lifestyle habits questionnaire.

Three categories have been defined: sedentary, non-competitive athletes, elite athletes. All items in the questionnaires were evaluated in percentages and the chi-square test / Fisher's exact test was performed, as appropriate.

Results: Preliminary analysis shows that in the three groups we found the same prevalence of difficulty falling asleep (over 30\%), tendency of nocturnal awakenings (over 30\%) and anxiety (over 38\%). 72,73\% of the elite athletes declared snoring during sleep vs $42,86 \%$ of noncompetitive athletes and $37,50 \%$ of sedentary subjects $(p=0,043)$. No other statistical significative differences were found for trained and sedentary subjects except for perception of being constant in daily activity, which is significantly more reported by trained subjects $(\mathrm{p}<$ 0,005).

Conclusions: Our data show a similar scenario of anxiety and sleep disturbances for the two groups of trained and sedentary subjects, suggesting that lockdown by COVID-19 pandemic has partially reverted the known beneficial effects of physical activity on mental health. Further analysis, using appropriate scores, are necessary to better define the phenomenon and the associated risk factors.

\section{References}

1. Shi L, Lu ZA, Que JY, Huang XL, Liu L, Ran MS, Gong YM, Yuan K, Yan W, Sun YK, Shi J, Bao YP, Lu L., 2020 JAMA Netw Open. 2020 Jul 1;3(7):e2014053. https://doi.org/10.1001/ jamanetworkopen.2020.14053.2).

2. Gualano MR, Lo Moro G, Voglino G, Bert F, Siliquini R. Effects of Covid-19 Lockdown on Mental Health and Sleep Disturbances in Italy. Int J Environ Res Public Health. 2020 Jul; 17(13): 4779. https://doi.org/10.3390/ijerph17134779 3).

\section{4}

\section{Physical exercise, dietary habits and psychological states of Italyn university students during COVID-19 lockdown}

Stefano Amatori ${ }^{1}$, Sabrina Donati Zeppa ${ }^{1}$, Antonio Preti ${ }^{2}$, Marco Gervasi $^{I}$, Erica Gobbi $^{1}$, Fabio Ferrini ${ }^{1}$, Marco Bruno Luigi Rocchi ${ }^{l}$, Carlo Baldari ${ }^{3}$, Fabrizio Perroni ${ }^{1}$, Giovanni Piccoli ${ }^{1}$, Vilberto Stocchi $^{1}$, Piero Sestili ${ }^{1}$, Davide Sisti ${ }^{1}$

Università Degli Studi Di Urbino Carlo Bo, Dipartimento Di Scienze Biomolecolari, Urbino, Italy ${ }^{1}$;

Università Di Torino, Dipartimento Di Neuroscienze, Torino, Italy ${ }^{2}$; Università Ecampus, Facoltà Di Psicologia, Rome, Italy ${ }^{3}$

Purpose: Restriction measures due to COVID-19 pandemic strongly affected people lifestyle, reducing the possibilities to practice physical activity, influencing nutritional habits and mental health. The aim of this longitudinal study was to explore the effects of physical exercise and mood states on nutritional choices of a sample of university students during the COVID-19 lockdown.

Methods: 176 students $(\mathrm{M}=92, \mathrm{~F}=84,23 \pm 4$ years old $)$ voluntarily took part in the study. Subjects' nutrition (food diary) and exercise sessions (duration and intensity) were daily monitored for 21 days, and psychological states (health-related quality of life, depression, positive and negative affect scales) were assessed. Relationship among these lifestyle factors were investigated using a multivariate general linear model and a mediation approach.

Results: Exercise practice was positively associated with fish, vegetables and fruit consumption $(\mathrm{p}=0.004)$. Cereals, legumes and low- 
fat meat intake were positively and negatively associated with depression and perceived quality of life, respectively $(\mathrm{p}<0.05$ for all). Exercise played a role as a mediator between mood states and fruit, vegetables and fish consumption, respectively accounting for $4.2 \%$ and $1.8 \%$ of the total variance.

Conclusions: Poorer psychological states during the COVID-19 lockdown, possibly led to unhealthy dietary habits, which can themselves be associated to negative mood states, as in a vicious circle. Conversely, exercise practice was associated to healthier nutritional choices, mediating the worsening effect of negative mood states.

\section{References}

1. Chirico, A, Lucidi, F, Galli, F, Giancamilli, F, Vitale, J, Borghi, S, La Torre, A, and Codella, R. (2020) COVID-19 Outbreak and Physical Activity in the Italyn Population: A Cross-Sectional Analysis of the Underlying Psychosocial Mechanisms. Front. Psychol 11: 2100.

\section{5}

\section{Rapid weight loss practices in italyn boxers: exploring factors of influence}

$\underline{\text { Stefano Amatori }}{ }^{1}$, Oliver Barley ${ }^{2}$, Erica Gobbi ${ }^{1}$, Diego Vergoni ${ }^{l}$, Attilio Carraro $^{3}$, Carlo Baldari ${ }^{4}$, Laura Guidetti, ${ }^{5}$,Marco Bruno Luigi Rocchi $^{l}$, Fabrizio Perroni ${ }^{l}$, Davide Sisti ${ }^{l}$

Università Degli Studi Di Urbino Carlo Bo, Dipartimento Di Scienze Biomolecolari, Urbino, Italy ${ }^{l}$;

Edith Cowan University, Centre For Exercise and Sports Science Research, School of Medical and Health Sciences, Joondalup, Australia ${ }^{2}$;

Libera Università Di Bolzano, Facoltà Di Scienze Della Formazione, Bolzano, Italy ${ }^{3}$;

Università Ecampus, Facoltà Di Psicologia, Rome, Italy ${ }^{4}$;

Università Telematica Unicusano, Area Scienze Motorie, Rome, Italy ${ }^{5}$

Purpose: It is common practice for combat athletes to lose significant body weight in the days/weeks leading up to the competition to gain an advantage by being paired with a smaller opponent: this practice is colloquially referred to as 'weight-cutting', and can be achieved by several different strategies. The primary aim of this cross-sectional study was to investigate the common weight-cutting strategies used by a sample of professional and amateur boxers competing in Italy. Methods: A total of 164 subjects (144 males and 20 females) completed an online survey, based on the validated "Rapid Weight Loss questionnaire" (RWLQ). The RWLQ provides a validated score that allows a quantitative measure of the aggressiveness of the weightcutting behaviors. Hierarchical clustering analysis was performed on the subjects, and a chi-square test has been used to test differences in weight-cutting strategies used and in participants characteristics between the two clusters.

Results: of the 164 athletes, only 20 (12\%) reported to have not ever applied acute weight loss strategies before a match. Two clusters have been found to be significant different: Cluster 1 comprised 133 subjects $(81 \%)$, while 31 subjects (19\%) belonged to Cluster 2 . The two clusters significantly differ on all the strategies analyzed $(\chi 2$ test; $\mathrm{p}<$ $0.05)$, except for the 'Gradual Weight Loss' $(p=0.529)$. With the practices becoming more dangerous to health, the difference between the two clusters became more visible. The rapid weight loss score was significantly higher in the Cluster 2, respect to the Cluster 1.

Conclusions: The cluster analysis put a focus on the characteristics of fighters who apply more severe practices (i.e., losing greater amounts of body weight as well as using methods such as fluid restriction, heat exposure and gut content manipulation), showing that professionals and higher-level fighters are more prevalent in Cluster 2. Fighters pertaining to this cluster also cut their weight more frequently during a competitive season, accompanied by a higher number of kilograms lost each time, and then regained to a greater extent after the match. References

1. Artioli, GG, Scagliusi, F, Kashiwagura, D, Franchini, E, Gualano, B, Junior, AL. (2010) Development, validity and reliability of a questionnaire designed to evaluate rapid weight loss patterns in judo players. Scand. J. Med. Sci. Sports 20, e177-e187.

2. Barley, OR, Chapman, DW, Abbiss, CR, (2019) The Current State of Weight-Cutting in Combat Sports-Weight- Cutting in Combat Sports. Sports 7, 123.

\section{6 \\ Self-reportsed physical fitness in children and adolescents with obesity: a cross-sectional analysis on the level of alignment with multiple adiposity indexes}

Matteo Vandoni ${ }^{1}$, Nicola Lovecchio ${ }^{2}$, Vittoria Carnevale Pellino ${ }^{1}$, Roberto Codella ${ }^{3}$, Alessandro Gatti ${ }^{1}$, Valentina Fabiano $^{4}$, Virginia Rossi $^{4}$, Matteo Giuriato ${ }^{5}$, Gian Vincenzo Zuccotti ${ }^{4}$, Valeria Calcaterra $^{4}$

University of Pavia, Laboratory of Adapted Motor Activity (lama), Department of Public Health, Experimental Medicine and Forensic Science, University of Pavia, Pavia, Italy ${ }^{1}$;

University of Bergamo, Department of Human and Social Science, University of Bergamo, Bergamo, Italy ${ }^{2}$;

University of Milan, Department of Biomedical Science For Health, University of Milan, Milan, Italy ${ }^{3}$;

Pediatric Department, "vittore Buzzi” Children's Hospital, Milan, Italy $^{4}$;

Department of Neurosciences, Biomedicine and Movement Sciences, University of Verona, Verona, Italy ${ }^{5}$

Purpose: Obesity has been associated with several alterations that could limit physical activity (PA) practice. In pediatrics, some studies have highlighted the importance of enjoyment as a motivation to begin and maintain adherence in PA. Since self-reported physical (SRPF) fitness was related to motivation, the aim of this study was to investigate the existence of differences between SRPF in children with obesity (OB) compared to normal weight (NW).

Methods: The International Fitness Enjoyment Scale (IFIS) questionnaire was administered to $200 \mathrm{OB}$ and $200 \mathrm{NW}$ children. In all the subjects, height, weight, and BMI and in OB children adiposity indexes including waist circumference, body shape index, triponderal mass index and fat mass were measured.

Results: The NW group showed higher IFIS item scores than the OB group ( $\mathrm{p}<0.01$ ), except in muscular strength. In OB, the anthropometric outcomes were inversely correlated to SRPF outcomes except for muscular strength. OB children reported a lower perception of fitness that could limit participation in PA/exercise programs.

Conclusions: OB children reported a lower perception of fitness that could limit participation in PA/exercise programs. The evaluation of anthropometric patterns may be useful to prescribe a tailored exercise program considering individual better self-perception outcomes to obtain an optimal PA adherence.

\section{References}

1. Ortega, F.B.; Ruiz, J.R.; España-Romero, V.; Vicente-Rodriguez, G.; Martínez-Gómez, D.; Manios, Y.; Béghin, L.; Molnar, D.; Widhalm, K.; Moreno, L.A.; et al. The International Fitness Scale 
(IFIS): Usefulness of self-reported fitness in youth. Int. J. Epidemiol. 2011, 40, 701-711.

2. Carissimi, A.; Adan, A.; Tonetti, L.; Fabbri, M.; Hidalgo, M.P.; Levandovski, R.; Natale, V.; Martoni, M. Physical self-efficacy is associated to body mass index in schoolchildren. J. Pediatr. 2017, 93, 64-69.

287

Teachers' perceptions of the effects of a primary school active breaks intervention during COVID-19 pandemic in terms of classroom behaviour: results from the "active breaks study (ABS)"

\author{
Andrea Ceciliani ${ }^{1}$, Alice Masini ${ }^{2}$, Sofia Marini ${ }^{2}$, Daniele Coco ${ }^{3}$, \\ Laura Bragonzoni $^{1}$, Stefania Toselli ${ }^{2}$, Maurizio Liberti ${ }^{4}$, Gerardo \\ Astorino $^{4}$, Laura Dallolio ${ }^{2}$
}

Universittà Di Bologna, Dipartimento Scienze Per La Qualità Della Vita Unibo, Bologna, Italy ${ }^{P}$;

Universtità Di Bologna, Dipartimento Di Scienze Biomediche E Neuromotorie, Bologna, Italy ${ }^{2}$;

Università Di Roma 3, Dipartimento Scienze Dell'educazione, Rome, Italy $^{3}$;

Ausl, Dipartimento Sanità Pubblica, Bologna, Italy ${ }^{4}$

Purpose: Active breaks (AB) are short bouts of physical activity led by teachers inside the classroom. Many studies, focused on $A B$ interventions, showed a general positive effect that requires further analysis on the specific behavioural domains most sensitive to $\mathrm{AB}$. The aim of our study was to evaluate the teachers' perception toward children classroom behaviour after performing $\mathrm{AB}$.

Methods: We conducted a pre-post multicentre study, from January to June 2021, in 1118 primary school children performing AB for ten weeks. An online questionnaire (five-points Likert scale) was administered to a female sample of 45 teachers (mean age $50.7 \pm 7.9$ ) from different cities (centre-northern Italy), to assess possible differences in perceptions about students' behaviours before and after the $\mathrm{AB}$ intervention. Specifically, the 15 items proposed were related to the following domains: student's classroom wellbeing, student time on task and teaching's benefits. Statistical analysis was conducted using SPSS.22.

Results: The pre-post differences $(\Delta)$ were calculated using paired T-test and ANOVA was performed to analyse group differences. Results show a significant improvement in students' classroom wellbeing $(+0.95 \pm 3.16 ; \mathrm{p}=0.048)$ and children's time on task $(+1.39$ $\pm 3.12 ; \mathrm{p}=0.005)$. We reported a trend of improvement in the total score even if not-statistically significant $(+1.30 \pm 9.58)$. No differences were found for children' or teachers' age and social context (city and suburban).

Conclusions: Our findings suggest that $\mathrm{AB}$ according to teachers' perception produce positive effects on student well-being and time on task behaviour. This intervention appears to be feasible and manageable for all age groups and school-based settings. The $\mathrm{AB}$ perceived effects seem to be independent from both children and teachers age, and from the social context. Results support the idea that $\mathrm{AB}$ can be applied in all schools and by all teachers without any particular issues or negative effects.

References

1. Infantes-Paniagua Á, Silva AF, Ramirez-Campillo R, et al. Active School Breaks and Students' Attention: A Systematic Review with Meta-Analysis. Brain Sci. 2021 May 21;11(6):675.
2. Masini A, Marini S, Leoni E, et al. Active Breaks: A Pilot and Feasibility Study to Evaluate the Effectiveness of Physical Activity Levels in a School Based Intervention in an Italyn Primary School. Int J Environ Res Public Health. 2020 Jun 17;17(12):4351.

\section{8}

The cut-off value for classifying italyn active children using the corresponding national version of the physical activity questionnaire

Corrado Lupo $^{1}$, Gennaro Boccia ${ }^{2}$, Alexandru Nicolae Ungureanu ${ }^{1}$, $\overline{\text { Anna Mulasso }}^{1}$, Paolo De Pasquale ${ }^{1}$, Alberto Rainoldi ${ }^{I}$, Paolo Riccardo Brustio ${ }^{3}$

Neuromuscularfunction Research Group, School of Exercise \& Sport Sciences, Suism, Departement of Medical Science, University of Turin, Turin, Italy ${ }^{1}$;

Neuromuscularfunction Research Group, School of Exercise \& Sport Sciences, Suism, Department of Clinical and Biological Sciences, University of Turin, Turin, Italy ${ }^{2}$;

Neuromuscularfunction Research Group, Department

of Neuroscience, Biomedicine and Movement, University of Verona, Verona, Italy ${ }^{3}$

Purpose: Regular physical activity is considered a milestone for health promotion and wellbeing (WHO; 2020). In this regard, part of the project "ACTLIFE: Is active lifestyle enough for health and wellbeing?" (PRIN2017-prot. 2017RS5M44) aims: i) to firstly determine the PAQ-C-it sedentary-active cut-off value; and ii) to consequently evaluate the differences between sedentary and active children in term of physical fitness.

Methods: One-hundred-thirty-four children (5 Piedmont schools; $47.8 \%$ female; mean age $=9.8 \pm 0.3$ years) worn an accelerometer wGT3X-BT (ActiGraph, Pensacola, Florida, USA) to objectively assess physical activity during one-week. Afterwards, PAQ-C-it (Gobbi et al., 2016) was filled by participants. Thus, ROC curves procedure was applied to obtain sedentary-active cut-off point.

Data on participants' anthropometric (i.e., body weight, height, waist circumference) and physical fitness (i.e., flexibility: sit and reach; balance: single leg stance; upper and lower strength; handgrip and long jump; sprint: 20-m linear sprint; cardiovascular fitness: shuttle run) indicators were collected. Controlling for gender and age, a multivariate analysis of covariance was used to assess the differences between sedentary and active groups.

Results: ROC analysis fixed PAQ-C-it cut-off point value at 2.75 to discriminate active children (area under the curve $=0.61$; standard error $=0.049 ; \mathrm{p}=0.03$; coefficient intervals $=0.514-0.707$ ), determining that 66 participants $(49 \%)$ were sedentary (mean PAQ-C-it value $=2.2 \pm 0.4$; active mean PAQ-C-it value $=3.3 \pm 0.4$ ). The active group reported better values for anthropometric indicators (higher stature, $\mathrm{p}=0.004$; lower body mass, $\mathrm{p}=0.03$; lower waist circumference, $p=0.002)$ as well as for flexibility $(p=0.02)$, lower strength hand $(\mathrm{p}=0.004)$, and sprint $(\mathrm{p}=0.024)$ performances (better performance also for cardiovascular fitness, $\mathrm{p}=0.057$, and handgrip, dominant hand, $\mathrm{p}=0.058$, despite only approaching significance).

Conclusions: According to the determined PAQ-C-it cut off, the present study reported a higher occurrence of active children with respect to the same-age European (Konstabel et al., 2014) and national (Ministero della Salute, 2014) individuals, also highlighting how specific tests demonstrated to be able to discriminate sedentary from active children.

References 
1. Gobbi et al. (2016). Psychometric Properties of the Physical Activity Questionnaire for Older Children in Italy. PLoS One, 11, $\mathrm{e} 0156354$.

2. Konstabel et al. (2014). Objectively measured physical activity in European children: The IDEFICS study. Int. J. Obes, 38, S135S143.

3. Ministero della Salute (2014). Okkio alla salute. www.salute.gov. it.

4. World Health Organization (WHO; 2020). Guidelines on Physical Activity and Sedentary Behaviour: Web Annex Evidence Profiles. www.apps.who.int.

\section{9}

\section{The effect of COVID-19 in physical activity levels and its relationship to living environments in kosovan youth}

\section{Kaltrina Feka ${ }^{1}$, Masar Gjaka ${ }^{2}$, Faton Tishukaj ${ }^{3}$, Giuseppe Battaglia ${ }^{1}$, Antonio Palma $^{1}$, Antonino Bianco ${ }^{1}$ \\ University of Palermo, Department of Psychology, Educational Science and Human Movement, Palermo, Italy ${ }^{I}$; \\ University For Business and Technology, Department of Sport and Movement Science, Pristina, Kosovo ${ }^{2}$; \\ University of Pristina, Faculty of Physical Education and Sport, Pristina, Kosovo}

Purpose: The purpose of the current study was twofold; firstly, was to evaluate the effect of COVID-19 lockdown in PA levels expressed as energy expenditure (MET-minutes/week) and sedentary behavior among Kosovo youth population, having the living environment as the main factor, and secondly, to investigate the differences in PA levels between males and females considering the living area.

Methods: 1130 participants (505 males and 625 females) under the age of 18 years old were enrolled in the current study. A cross-sectional study design was implemented using an online questionnaire, including an adapted version of the International Physical Activity Questionnaire-Short Form (IPAQ-SF), created on the Google Forms platform during the COVID-19 pandemic outbreak in Kosovo. The data concerning PA levels during pandemic were collected under the restriction measures of the government, whereas, concerning PA levels before the pandemic were collected retrospectively. To analyze the data Mann-Whitney non-parametric t-test was used.

Results: No significant differences in PA levels pre-COVID-19 between urban e rural living populations were found $(p=0.55)$. The results showed significant reductions in PA levels in both living environments (urban and rural) as a result of pandemic restrictions ( $p$ $<0.001$ ), with higher PA levels decrease among urban living young individuals. With respect to within gender differences, there were no significant differences between pre-COVID-19 in rural vs urban in both, males $(\mathrm{p}<0.58)$ and females $(\mathrm{p}<0.25)$, while significant differences in PA levels during-COVID-19 restrictions were observed, showing a decreased PA levels among rural and urban cohorts in both genders $(\mathrm{p}<0.001)$.

Conclusion: COVID-19 restrictions decreased the PA levels and MET-min/week among Kosovan youth, in rural and urban environments, with a higher decrease among the urban living population, while increasing sedentary behavior.

\section{References}

1. Gjaka, M., Feka, K., Bianco, A., Tishukaj, F., Giustino, V., Parroco, A. M., ... \& Battaglia, G. (2021). The Effect of COVID19 Lockdown Measures on Physical Activity Levels and
Sedentary Behaviour in a Relatively Young Population Living in Kosovo. Journal of Clinical Medicine, 10(4), 763.

2. Moore, S. A., Faulkner, G., Rhodes, R. E., Brussoni, M., ChulakBozzer, T., Ferguson, L. J., ... \& Tremblay, M. S. (2020). Impact of the COVID-19 virus outbreak on movement and play behaviours of Canadian chil.

\section{0}

The effect of physical activity changes and musculoskeletal pain onset among italyn university students after 1 year of COVID-19 pandemic

\author{
Federico Roggio ${ }^{1}$, Bruno Trovato ${ }^{2}$, Silvia Ravallit ${ }^{2}$, Antonino \\ Bianco $^{3}$, Antonio Palma ${ }^{3}$, Giuseppe Musumeci ${ }^{4}$
}

Phd Program In Healt Promotion and Cognitive Sciences, Sport and Exercises Sciences Research Unit, University of Palermo,

University of Catania, Research Center On Motor Activitties (cram), Catania, Italy ${ }^{1}$;

University of Catania, Department of Biomedical and Biotechnological Sciences, Human Anatomy and Histology Section, School of Medicine, Catania, Italy ${ }^{2}$;

University of Palermo, Department of Psychology, Educational Science and Human Movement, Palermo, Italy ${ }^{3}$;

University of Catania, Research Center On Motor Activities (cram), Department of Biomedical and Biotechnological Sciences, Human Anatomy and Histology Section, School of Medicine, Catania, Italy ${ }^{4}$

Purpose: COVID-19 quarantine caused a reduction to physical activity levels, PA, all over the Italyn population [1]. The high amount of hours spent seated in a wrong posture expose students to musculoskeletal overload [2]. The outbreak restrictions to daily activities and the increase of seated time due to distance learning might determine a higher predisposition to experience pain. The aim of this study was to evaluate physical activity levels changes and their correlation to musculoskeletal pain among Italyn university students after 1 year of pandemic.

Methods: An online survey addressed to students from Italyn universities was developed to investigate the effects of a sedentary lifestyle and presence of musculoskeletal pain due to pandemic restriction. A total of 2044 Italyn university students responded to the survey. The survey was divided into four sections: 1 sociodemographic data, 2 and 3 cervical and lumbar pain aspects, 4 adherence to face-mask usage.

Results: Physical activity, PA, levels drastically changed. Before the pandemic $50 \%$ of participants had PA $\geq 150 \mathrm{~min} /$ week, while $19.9 \%$ were physically inactive. During the pandemic PA $\geq 150 \mathrm{~min} /$ week reduced to $21.4 \%$ while physical inactivity increased to $30.6 \%$. Sedentary behavior was present in $38.8 \%$ of participants; with regard to musculoskeletal pain, $43.5 \%$ and $33.5 \%$ reported neck pain and low back pain presence, respectively. A statistically significant correlation between PA levels, Verbal Descriptive Scale, and pain frequency was observed for both neck and low back pain.

Conclusion: After 1 year of restrictions due to COVID-19 pandemic, physical activity levels considerably reduced. Those who had PA $<$ $150 \mathrm{~min} /$ week or were physically inactive showed a higher incidence of neck and low back pain. Severe daily restrictions due to pandemic, negatively affected Italyn university students.

\section{References}

1.Musumeci, G., Physical Activity for Health-An Overview and an Update of the Physical Activity Guidelines of the Italyn Ministry of Health. Journal of functional morphology and kinesiology 2016, 1 (3), 269-275. 
2.Caromano, F.; Amorim, C.; Rebelo, C.; Contesin, A.; Favero, F.; Costa, J.; Kawai, M.; Voos, M., Prolonged sitting and physical discomfort in university students. Acta Fisiátrica 2015, 22, 176-180.

\section{1}

\section{The effects of a park-based physical activity intervention on active behavior and wellbeing: the Italyn project "moving parks"}

\author{
Stefania Toselli ${ }^{1}$, Laura Bragonzoni ${ }^{2}$, Laura Dallolio ${ }^{I}$, Alessia \\ Grigoletto $^{1}$, Alice Masini ${ }^{l}$, Marini Sofia ${ }^{l}$, Giuseppe Barone ${ }^{2}$, Erika \\ Pinelli $^{2}$, Raffaele Zinno ${ }^{2}$, Mario Mauro ${ }^{2}$, Gherardo Astorino ${ }^{3}$, Pietro \\ Loro Pilone ${ }^{3}$, Simona Galli ${ }^{3}$, Pasqualino Maietta Latessa ${ }^{2}$
}

Università Di Bologna, Dipartimento Di Scienze Biomediche E Neuromotorie, Bologna, Italy ${ }^{1}$;

Università Di Bologna, Dipartimento Di Scienze Per La Qualità

Della Vita, Rimini, Italy ${ }^{2}$;

Dipartimento Di Salute Pubblica Ausl Di Bologna, Dipartimento Di Salute Pubblica Ausl Di Bologna, Bologna, Italy ${ }^{3}$

Purpose: Obesity and physical inactivity are global health problems responsible for the risk increment of non-communicable diseases. Sedentary behavior has been identified as an important risk factor for mortality, representing a challenge for public health. To overcome these problems, interventions aimed at increasing physical activity (PA) are necessary, to change the lifestyle of the subjects and improve physical and psychological well-being. The environment in which PA takes place can influence adherence and it is known that green space can have a positive influence in promoting PA, increasing its total amount. The aim of the present study was to assess the effectiveness of the project 'The Moving Parks project', which provides for the administration of PA by qualified instructors to citizens within Bologna's parks. This project was carried out for 3 months during the summer period and involved fourteen sports associations offering different types of activities to the citizens.

Methods: Ad hoc questionnaire was created to investigate the participants' habits and their health status and was administered before and after the 3 months of outdoor PA among an adult sample from Bologna. Paired sample Wilcoxon test and the chi-squared test were carried out to value the differences (between the two measurements). Results: A total of 329 (257 women and 72 men) adult subjects (> 18 years) participated to the survey. Weight status did not show any significant differences between baseline and follow-up. Men generally presented a higher percentage of overweight and obese subjects than women, while women showed also underweight subjects. At follow-up, the importance given by the participants to PA practice has increased. All psychosocial parameters showed an improvement after following the 3 months PA program, with a reduction of the state of tension, sadness and fatigue, and an improvement of the state of energy, serenity, and vitality.

Conclusions: The impact of the interventions carried out in the "Moving Parks project" was positive and appears to be a good strategy for improving health outcomes. In fact, the results confirmed that the urban green plays an important role in promoting PA. A third of the participants reported to have started the practice of PA thanks to this project, so it is important to involve qualified personnel to conduct activities and encourage an increase in participation, creating trust and continuity for the local citizens.

\section{References}

1. López-Valenciano, XM, et al. (2020). Changes in sedentary behaviour in European Union adults between 2002 and 2017. BMC Public Health: 20: 1206.
2. Derose, KP, et al. (2021). Effects of park-based interventions on health-related outcomes: a systematic review. Prev Med:147, 106,528 .

3. Mytton, OT, et al. (2012). Green space and physical activity: An observational study using Health Survey for England data, Health \& Place: 18 (5), 1034-1041.

\section{2}

The influence of age, gender, and weight status on different motor skills in sicilian children aged 6-13 years old

Valerio Giustino $^{1}$, Giuseppe Battaglia ${ }^{1}$, Garden Tabacchi ${ }^{1}$, Massimo Lanza $^{2}$, Federico Schena ${ }^{2}$, Valentina Biino ${ }^{2}$, Matteo Giuriato ${ }^{2}$, Maria Chiara Gallotta ${ }^{3}$, Laura Guidetti ${ }^{4}$, Carlo Baldari ${ }^{5}$, Antonio Gennaro $^{6}$, Antonio Palma ${ }^{1}$, Marianna Bellafiore ${ }^{I}$

University of Palermo, Department of Psychology, Educational Science and Human Movement, Palermo, Italy ; University of Verona, Department of Neuroscience, Biomedicine and Movement, Verona, Italy ${ }^{2}$;

Sapienza University of Rome, Department of Physiology and Pharmacology "vittorio Erspamer", Rome, Italy ${ }^{3}$.

University, Niccolò Cusano, Rome, Italy ${ }^{4}$;

Ecampus University, Department of Theoretical and Applied Sciences, Rome, Italy ${ }^{5}$;

Aou "g. Martino", Department of Biomedical, Dental Sciences and Morphofunctional Imaging, Messina, Italy ${ }^{6}$

Purpose: Although various studies have reported that children with higher weight status show a lower level of motor skills, this relationship remains inconclusive. Furthermore, there is no evidence on the influence of age and gender on motor coordination. Thus, this study aimed to investigate the association between different motor skills and variables such as age, gender, and weight status in a large sample of Sicilian children.

Methods: For this cross-sectional study, 647 Sicilian schoolchildren (319 girls) were recruited and stratified in three age groups (6-7, 8-10, 11-13 years) and four weight status categories (underweight, normal weight, overweight, obese). The Körperkoordinationstest für Kinder (KTK) was used to assess the following motor skills: 1) walking backward (WB) on a balance beam of decreasing widths; 2) two-legged jumping sideways (JS) from side to side over a beam for $15 \mathrm{~s} ; 3$ ) one-legged hopping for height $(\mathrm{HH})$ over a foam obstacle of increasing height; 4) moving sideways (MS) on the floor in $20 \mathrm{~s}$. The score of each motor skill test was standardized for age and gender. One-way ANOVA for each motor skill test was performed separately to detect any differences between age groups, gender classes, and weight status categories.

Results: The level of the measured motor skills decreased significantly with increasing age $(\mathrm{F}=22.87, \mathrm{p}<0.001$ for $\mathrm{WB} ; \mathrm{F}=3.18, \mathrm{p}$ $<0.05$ for JS; $F=17.90, p<0.001$ for MS). Boys had significantly better JS and $\mathrm{HH}$ levels than girls $(\mathrm{F}=114.68, \mathrm{p}<0.001$ and $\mathrm{F}=$ $60.82, \mathrm{p}<0.001$, respectively). Overweight and obese children showed significantly lower levels than underweight and normal weight children in all the motor skills $(\mathrm{F}=16.09, \mathrm{p}<0.001$ for $\mathrm{WB} ; \mathrm{F}$ $=4.94, \mathrm{p}<0.01$ for JS; $\mathrm{F}=19.88, \mathrm{p}<0.001$ for $\mathrm{HH} ; \mathrm{F}=8.17, \mathrm{p}<$ 0.001 for MS).

Conclusions: Our outcomes denote a sharp decline in motor coordination that is associated with late childhood, female gender, and overweight/obesity. Therefore, to contrast this phenomenon it would be appropriate that schools and families adopt educational strategies to promote an active and healthy lifestyle during childhood. 


\section{References}

1. Barnett LM, Lai SK, Veldman SLC, Hardy LL, Cliff DP, Morgan PJ, et al. (2016) Correlates of Gross Motor Competence in Children and Adolescents: A Systematic Review and MetaAnalysis. Sports Med 46(11):1663-88.

2. D'Hondt E, Deforche B, Vaeyens R, Vandorpe B, Vandendriessche J, Pion J, et al. (2011) Gross motor coordination in relation to weight status and age in 5- to 12 -year-old boys and girls: a crosssectional study. Int J Pediatr Obes 6(2-2):e556-64.

\section{3}

\section{COVID-19 and remote training: evaluation of three different strategies}

\section{Michael Daveri $^{1}$, Gabriele Mascherini ${ }^{1}$}

Università Degli Studi Di Firenze, Dipartimento Di Medicina Sperimentale E Clinica, Firenze, Italy ${ }^{I}$

Purpose: The purpose of this work is to evaluate three different training strategies adopted during the period of the Covid-19 pandemic. The assumption of the study is whether three different training strategies can lead to different levels of adherence, differences in the increase in levels of physical activity and therefore in the results of physical performance.

Methods: Twenty-one male subjects, aged 18-25, randomly assigned to three different groups. One group received instruction about the physical activity program from the instructor by a live stream (LS) for each workout, one received instruction by a video recording (VR) for each workout, and the last received instruction by a schedule (S) for each workout. Each group's training program lasted five weeks with a weekly frequency of three sessions. Every participant is subjected to evaluation tests for anthropometric parameters, resting heart rate, muscle strength, balance, mobility and flexibility before the start of the program at baseline and at the end of the training program. Statistical analysis were performed by ANOVA test and T test in order to compare the differences between baseline with final results.

Results: At the end of the program show that the LS group reveal greater adherence to the physical activity program (LS $=93.3 \%$; VR $=$ $86 \% ; \mathrm{S}=74 \%$ ) and show how all three strategies were effective in increase physical activity levels $(\mathrm{LS}=+91.39 \%$; VR $=+89.43 \%$; $\mathrm{S}$ $=+68.74 \%)$ and performance parameters, with a preference for the live stream one, which revealed greater improvements in the measured values such as the waist hip ratio with the most decrease (LS = $1,59 \% ; \mathrm{VR}=-1,00 \% ; \mathrm{S}=-0,53 \%$ ), the effort tolerance with an higher reduction $(\mathrm{LS}=-21.72 \% ; \mathrm{VR}=-16.76 \% ; \mathrm{S}=+6.12 \% ; \mathrm{p}<0.05)$, the maximal push up with the bigger increase $(\mathrm{LS}=+37.65 \%$; $\mathrm{VR}=+$ $29,78 \% ; \mathrm{S}=+22.17 \% ; \mathrm{p}<0.05)$, the one leg test with the greater increase $(\mathrm{LS}=+200,97 \%$; VR $=+80.71 \% ; \mathrm{S}=+135,10 \% \mathrm{p}<$ $0.05)$ and the Laseguè test with the larger increase (LS $=+12.45 \%$; $\mathrm{VR}=+8.05 \% ; \mathrm{S}=+6.31 \% ; \mathrm{p}<0.05)$.

Conclusions: The use of technology that allowed the supervision of the instructor during training together with the possibility of training in company, showed the greatest increases in the measured parameters, indicating how one strategy was the most incisive, the live stream one. The schedule strategy seems to be the least incisive, while the video recording showed the intermediate improvements compared the other two.

\section{References}

1. Gentil P, de Lira CAB, Souza D, et al. Resistance Training Safety during and after the SARS-Cov-2 Outbreak: Practical
Recommendations. Biomed Res Int. 2020;2020:3,292,916. Published 2020 Sep 23. https://doi.org/10.1155/2020/3292916

2. Maugeri G, Castrogiovanni P, Battaglia G, et al. The impact of physical activity on psychological health during Covid-19 pandemic in Italy. Heliyon. 2020;6(6):e04315. Published 2020 Jun 24. https://doi.org/10.1016/j.heliyon.2020.e04315

\section{4}

Effects of aging on muscle damage induced by eccentric exercise in healthy adults

$\underline{\text { Linda Toniolo }}^{l}{ }^{\text {, Giorgia Schiavinotto }}{ }^{1}$, Giacomo Mazzocco ${ }^{l}$, Daniele Altin $^{I}$, Lisa Vigo ${ }^{I}$, Alessandro Sampieri ${ }^{1}$, Claudia Favero ${ }^{2}$, Antonio Paoli $^{1}$, Tatiana Moro ${ }^{1}$

University of Padua, Department of Biomedical Sciences, University of Padova, Padova, Italy ${ }^{1}$;

University of Padua, Department of Medicine, University of Padova, Padova, Italy ${ }^{2}$

Purpose: Sarcopenia is a multifactorial syndrome that involves a reduction in strength, changes in muscle architecture and reduced functionality. Eccentric exercise is a model that can be employed with older adults, due to the muscle's ability to combine high muscle force production with a low energy cost. However eccentric protocols normally require greater time to fully recover from the exercise-induced muscle damage. The aim of the study is to compare the recovery process after eccentric exercise between young and older adults.

Methods: Eight young $(22.33 \pm 2.12$ years $)$ and eight older adults $(64.00 \pm 5.23$ years) were asked to perform the only eccentric phase of seven set of ten repetition in leg extension. The load employed was the $110 \%$ of the one repetition maximum; sets were separated by 3 min of rest. Participants were asked to consume Whey Protein supplementation for 10 days before eccentric exercise and during the 5 days of follow-up. Main inflammatory markers, phase angle for lower limb via BIA (Body Impendence Assessment), Delay-Onset Muscle Soreness (DOMS), Range of Motion (ROM) of the knee extension/flexion, handgrip and maximum voluntary contraction (MVC) were evaluated at baseline and following 0, 48, 72 and $120 \mathrm{~h}$ from exercise.

Results: A significant effect of age $(\mathrm{p}<0.05)$ was observed for ROM, MVC, cholesterol and phase angle measured via BIA. In particular, MVC decreased after $48 \mathrm{~h}$ in young $(\mathrm{p}<0.05)$ and after $72 \mathrm{~h}$ from exercise in older adults $(\mathrm{p}<0.05)$. Markers of cellular repair as total cholesterol and LDL significantly decreased after exercise only in the young volunteers. Also the phase angle, which indicate the ability of a cell to recover, significantly increased at $48 \mathrm{~h}$ and $120 \mathrm{~h}$ only in the young group.

Conclusions: These preliminary results suggest that muscle recovery after eccentric exercise is impaired in older adults compared to younger pairs. Moreover, protein ingestion doesn't seem to reduce this gap. 


\section{5}

Four weeks of supervised online burpees training during COVID-19 quarantine improve quality of life, strength and heart rate variability in healthy young adults

Salvatore Ficarra ${ }^{l}$, Ewan Thomas ${ }^{I}$, Guglielmo Pillitteri ${ }^{l}$, Davide Migliore $^{I}$, Angelo Iovane ${ }^{1}$, Daniele Zangla ${ }^{l}$, Marianna Bellafiore ${ }^{1}$, Giuseppe Battaglia ${ }^{1}$, Manuel Gómez-lópez ${ }^{2}$, Antonio Palma ${ }^{1}$, Antonino Bianco ${ }^{l}$

Università Degli Studi Di Palermo, Sport and Exercise Sciences Research Unit, Department of Psychology, Educational Science and Human Movement, Palermo, Italy ${ }^{I}$;

University of Murcia, Department of Physical Activity and Sport, Faculty of Sports Sciences, Murcia, Spagna ${ }^{2}$

Purpose: The COVID-19 pandemic caused the population confinement and increased people sedentary level. In order to maintain physical fitness during the quarantine, we designed a short-term intervention with one body-weight exercise (burpee). Therefore, the aim of this study is to understand the effects of a progressive online burpees training in young adults, during the COVID-19 quarantine.

Methods: A 4-weeks intervention was administered to 13 young adults (age $22.5 \pm 1.39$ years, Weight $71.8 \pm 10.1 \mathrm{~kg}$ ) using a telecommunication software to implement a fully supervised online approach. Training sessions presented 3 phases: 1. Wam-up (joint mobility exercises and 10 push-up, 5 squat and 5 squat jump), 2. Main phase, and 3. Cool-down (10 min static stretching exercises). The main phase of each session was implemented trough burpees, a calisthenics body-weight exercise. One minute rest period was administered between each set. The training was administered daily, 5 days a week. Data regarding quality of life (QoL), body composition, posture, Heart Rate Variability (HRV), cardiovascular health, and strength were collected before and after the intervention period. Results: Participants QoL significantly increased after 4 weeks ( $\mathrm{p}=$ 0.025). Also participants strength improved during the push-up test ( $\mathrm{p}$ $=0.017$ ). No clear results were found about Systolic blood pressure in pre to post measures, while a significant reduction was found in Diastolic blood pressure. The HRV assessment showed increased mean RR $(p=0.005)$ and RMSSD $(p=0.014)$ and decreased mean HR $(p=0.004)$ (in the time-domain). For the frequency-domain variables we were not able to determine certain conclusions. Also no evident results were noted regarding body composition, posture, handgrip strength and countermovement squat jump height.

Conclusions: Our results suggested that a 4-week daily online burpees intervention improves quality of life, upper body strength and Heart Rate Variability in young adults. This non-time consuming approach could be easily administered, in order to promote a healthy living and counteract physical inactivity, during COVID-19 restrictions, thanks to its feasibility, short duration and low cost.

\section{References}

1. Giustino V, Parroco AM, Gennaro A, Musumeci G, Palma A, Battaglia G. Physical Activity Levels and Related Energy Expenditure during COVID-19 Quarantine among the Sicilian Active Population: A Cross-Sectional Online Survey Study. Sustainability. 2020;12(11):4356.

2. Burpee RH. Seven quickly administered tests of physical capacity and their use in detecting physical incapacity for motor activity in men and boys: Teachers College, Columbia University; 1940.

\section{6}

Influence of grip strength and age on manual dexterity in adult woman

Simone Cuomo $^{1}$, Anna Mulasso ${ }^{1}$, Gennaro Boccia ${ }^{2}$, Samuel Agostino $^{3}$, Alberto Rainoldi ${ }^{1}$

University of Turin, Department of Medical Sciences, Turin, Italy ${ }^{1}$; University of Turin, Department of Clinical and Biological Sciences, Turin, Italy ${ }^{2}$;

University of Turin, School of Exercise and Sport Sciences, Turin, Italy $^{3}$

Purpose: A number of studies have highlighted the relationship between age and hand-grip strength as well as between age and manual dexterity, showing that increasing the age there is a decrease in strength and dexterity. However, studies that have investigated the relationship among age, hand-grip strength, and manual dexterity are scarce. Dominance and gender and their effects on the aforementioned variables have never been examined (Martin et al., 2015). The aim of the present study is to evaluate the relationship among age, hand-grip strength, and manual dexterity in a sample of women, considering the dominance as a factor.

Methods: A total of 291 women $(47 \pm 10$ years $)$ participated in the project Wellness@Work for UniTO and were included in the analyses. One hand-grip strength (Mathiowetz et al., 1984) was measured from both hands, standing upright with the shoulder adducted and the elbow at $180^{\circ}$. Manual dexterity was tested from each hand with the Finger Tapping test (FTT; Reitan \& Wolfson, 1993) performed for $10 \mathrm{~s}$. Number of touches and variability among touches at the FTT were the outcomes of the study. Data were treated through linear regression analyses.

Results: Hand-grip strength with dominant limb $(\beta=0.421 ; p<$ 0.001 ) influenced the number of taps performed at the FTT more than age $(\beta=-0.108 ; p=0.044)$. The model explained $19.2 \%$ of the variance. Similar results were obtained for non-dominant, with a variance of $18.4 \%$. Differently, only age (dominant: $\beta=0.152 ; p=$ 0.012; non-dominant: $\beta=0.189 ; \mathrm{p}=0.002$ ), and not hand-grip strength, explained tap variability at the FTT. Results were similar for dominant and non-dominant limbs, with an explained variance of $1.5 \%$ and $3.4 \%$, respectively.

Conclusion: Hand-grip strength has a major role in explaining the number of tap, while age is the most important feature explaining the variability among touches during FTT. Dominant and non-dominant were influenced by the same variables.

\section{References}

1. Martin JA, Ramsay J, Hughes C, Peters DM and Edwards MG. (2015). Age and grip strength predict hand dexterity in adults. PLoS One. 10(2):e0117598.

2. Mathiowetz V, Weber K, Volland G and Kashman N. (1984). Reliability and validity of grip and pinch strength evaluations. J Hand Surg Am. 9(2):222-226.

3. Reitan RM and Wolfson D. (1993). The Halstead-Reitan Neuropsychological Test Battery: Theory and Clinical Interpretation. 2nd Edition, Neuropsychology Press, Tucson.

\section{7}

\section{Lockdown period effects on elderly motor skills}

$\underline{\text { Elvira Padua }}^{l}$, Michele Panzarino ${ }^{l}$, Francesca Campoli $^{l}$, Cristian Romagnoli $^{2}$, Anas Alsharam ${ }^{3}$, Vincenzo Manzi ${ }^{4}$, Virginia Tancredi ${ }^{5}$, Mauro Lombardo ${ }^{6}$, Giuseppe Annino ${ }^{5}$ 
Università Telematica San Raffaele Roma, Dip. Di "promozione Delle Scienze Umane E Della Qualità Della Vita”, Rome, Italy" Università "alma Mater Studiorum" Di Bologna, Dipartimento Di Scienze Per La Qualità Della Vita, Rimini, Italy ${ }^{2}$;

Isra University, Department of Physiotherapy, Amman, Giordania ${ }^{3}$; Università Telematica Pegaso, Dipartimento Di Scienze

Umanistiche, Napoli, Italy;

Università Di Roma Tor Vergata, Dipartimento Di Medicina Dei

Sistemi, Rome, Italy ${ }^{5}$;

Università Telematica San Raffaele, Dip. Di "promozione Delle

Scienze Umane E Della Qualità Della Vita", Rome, Italy ${ }^{6}$

Purpose: This study aims to investigate the effects on motor skills performance in elderly individuals during lockdown period for Covid 19 pandemic conditions.

Methods: 83 healthy subjects (15 men and 68 women), free from injury and without any physical problem that could affect the test results were enrolled for this study. The sample was divided into two groups: the old group OG (54 subjects, 73,3 $\pm 6,6$ years) who practiced regularly physical activity (PA) for more than 5 years and the new group NG (29 subjects, 70,4 $\pm 7,1$ years) who practiced for less than 5 years' regular PA. The baseline and the post pandemic battery tests were performed in November 2019 and in May 2021. The battery tests performance consisted on back muscle flexibility, balance, abdominal, legs and handgrip strength assessment. Descriptive statistical procedures are presented as mean and the percentage changes $(\Delta \%)$ for each group. A multivariate between-within groups ANOVA was conducted to assess any significant difference in mean variables used. Tukey's post hoc test of critical difference was used to locate significance between means. The significance level was fixed at $\mathrm{p}<$ 0.05 .

Results: The results of this study show no difference in motor skills investigated relative to years of regular physical activity practice. Statistically significant differences were observed over time in both groups where all motor skill outcomes worsened after the lockdown period: Flexibility: $\Delta-23 \%(\mathrm{p}=0.01)$ in both group; balance $\Delta-19 \%$ $(\mathrm{NG} \mathrm{p}=0.08$, OG $\mathrm{p}=0.05)$; in lower limb strength NG $\Delta 1 \%(\mathrm{p}=0.4)$ OG $\Delta-1 \%(\mathrm{p}=0.2)$; abdominal strength NG $\Delta-4 \%(\mathrm{p}=0.1)$ OG $\Delta-$ $7 \%(\mathrm{p}=0.08)$ and handgrip test: right hand NG $\Delta-11 \%(\mathrm{p}<0.001)$ OG $\Delta-10 \%(\mathrm{p}<0.001)$, left hand NG $\Delta-9 \%(\mathrm{p}<0.001)$ OG $\Delta-7 \%(\mathrm{p}$ $<0.001)$.

Conclusions: In light of the results observed on both groups, it is possible conclude that the sedentary life associated to the pandemic condition, caused a decline of all recorded physical capacities without any significant difference between the starting physical practice conditions. The percentage differences over time observed in each test on both groups could be related to the starting elderly biological differences participant to this study. These results underline that an imposed long lockdown period could accelerate the decrease of the neuromuscular efficiency in the elderly population with serious consequences for their quality of life.

\section{References}

1. Melchiorri G., Annino G., Panzarino M., Viero V., Campoli F., Lombardo M., Tancredi V., Padua E. (2017) "Proposal for a physical fitness evaluation test battery of an Italyn elderly population. MED SPORT. https://doi.org/10.23736/S0025-7826. $17.03098-8$

\section{8}

Motivation, physical activity and active commuting in park: an observational study in arcoveggio park, boslogna

\author{
$\frac{\text { Alessia Grigoletto }}{1}$, Pasqualino Maietta Latessa ${ }^{2}$, Alberto Loi ${ }^{3}$, \\ Stefania Toselli ${ }^{I}$
}

Università Di Bologna, Dipartimento Di Scienze Biomediche E Neuromotorie, Bologna, Italy ${ }^{1}$;

Università Di Bologna, Dipartimento Di Scienze Per La Qualità Della Vita, Rimini, Italy ${ }^{2}$;

Università Di Bologna, Dipartimento Di Farmacia, Biotecnologie E Scienze Motorie, Bologna, Italy ${ }^{3}$

Purpose: The World Health Organization guidelines on physical activity and sedentary behaviour support that many of the benefits of physical activity (PA) are seen within average weekly volumes of $150-300 \mathrm{~min}$ of moderate intensity or $75-100 \mathrm{~min}$ of vigorous intensity or an equivalent combination of the two. It is estimated that 3.3 million people die annually worldwide as a result of physical inactivity. Promoting PA is a well-established priority. It was reported that the presence of green spaces helps people to achieve the right quantitative of PA. Even if there is an increasing literature about the practice, the motivation and the intervention related to the increase outdoor PA, few studies have been carried out in Italy.

The present study had two aims: (1) to understand the motivations that drive people to reach the park and (2) to value if the practice of PA and the active commuting are enough to meet the minimum levels of PA.

Methods: A new questionnaire was created and administered to visitors of the Arcoveggio park in Bologna ( 9 hectares): it included information about the motivation to use the park, the way they use to reach it and the quality and quantity of the PA carried out in the park. Results: Three hundred and eighty-three subjects taken part in the survey. The main motivations for using the park were related to relaxation $(\mathrm{n}=151,39.42 \%)$, to perform physical activity $(\mathrm{n}=171$, $44.64 \%)$ or both $(n=61,15.92 \%)$. Considering only training time, only few people achieved the minimum levels of physical activity ( $=7,3.02 \%)$. But, considering that the largest part of the participants reached the park walking, running or bicycling $(n=331,86.42 \%)$, when the time of active commuting was added to the minutes of training, $50,9 \%$ of people achieved the goal of $150 \mathrm{~min} /$ week.

Conclusion: The results of the present study showed that the park represents an important element to reach the recommended levels of PA. Even if PA alone was not sufficient for this purpose, adding active transport to reach the park increased the activity levels, making them adequate. All the initiatives to promote activities in the park can represent an important aspect for improving the health of the community.

\section{References}

1. World Urbanization Prospects-Population Division-United Nations. Available online: https://population.un.org/wup/ (ac cessedon 20 November 2020).

2. Evenson, KR, Wen, F, Hillier, A, and Cohen, DA (2013). Assessing the Contribution of Parks to Physical Activity Using GPS and Accelerometry. Med Sci Sports Exerc: 45, 1981-1987.

3. Veitch, J, Carver, A, Abbott, G, Giles-Corti, B, Timperio, A, and Salmon, J (2015). How Active Are People in Metropolitan Parks? An Observational Study of Park Visitation in Aus. 
SPECIAL ENVIRONMENT and TECHNOLOGY

299

\section{E-kayak: a wireless system for real time performance analysis in flatwater kayaking.}

Cristian Romagnoli $^{1}$, Giorgio Gatta
Ghelardini $^{3}$, Vincenzo Bnas Alashram $^{4}$, Elvira $^{3}$ Padua $^{5},{ }^{2}$, Gucaseppe Annino $^{6}$

University of Bologna, Department For Life Quality Studies, Rimini, Italy $^{l}$;

"tor Vergata" University of Rome, Sport Engineering Lab, Dept. Industrial Eng, Rimini, Italy';

Italyn Canoe/kayak Federation (fick), Italyn Canoe/kayak Federation (fick), Rome, Italy ;

Isra University, Department of Physiotherapy, Amman, Giordania ${ }^{4}$; San Raffaele Open University of Rome, Department of Human Science and Promotion of Quality of Life, Rome, Italy;

"tor Vergata" University of Rome, Department of Medicine Systems, Rome, Italy ${ }^{6}$

Purpose: On-water measurement systems are required to properly evaluate the effectiveness of the paddling technique by professional kayak athletes. These systems have to be able to measure kinematic (speed, acceleration, roll and pitch of the boat) as well as dynamic parameters (stroke frequency, force acting on paddle and foot brace) by using inertial and force sensors properly synchronized.

Methods: We present a wireless multi-node real time data logger customized for flatwater sprint kayaks. The system presents a modular hardware architecture, based on a central unit and wireless sensor nodes, which have been designed to be scaled for use on K1, K2 and K4 boats. It consists of a high frequency $(20 \mathrm{~Hz}) \mathrm{GPS}$, an Inertial Motion Unit (IMU) with nine DOF and two force channels, respectively placed on the paddle and footrest, for each of the athletes belonging the crew. Data from all the channels are synchronously acquired, processed by a microprocessor unit, wirelessly sent via a Wi-Fi link to client terminals (PC, tablet or smartphone) and also stored in a local memory for future offline analyses.

Results: Some results about training sessions of K1 and K2 crews will be presented with the aim of highlighting the features of the system together with its capabilities to obtain specific parameters related to the effectiveness of the paddling technique.

Conclusion: Athletes and coach can visualise the training data on a suited web page. It provides real time feedback on some of the main parameters (time, speed, stroke frequency, force symmetry) and, at the end of the training session. Moreover, these can be downloaded to a PC for further deep analysis.

References

1. Bonaiuto V et al. (2018) A Multiprotocol Wireless Sensor Network for High Performance Sport Applications. Applied System Innovation.

2. Bonaiuto, V et al. (2020). A Pilot Study on the e-Kayak System: A Wireless DAQ Suited for Performance Analysis in Flatwater Sprint Kayaks. Sensors.

3. Camomilla V et al. (2018) Trends supporting the in-field use of wearable inertial sensors for sport performance evaluation: A systematic review. Sensors.

\section{0}

\section{Experimental approach to evaluate foot warmth in a cold environment while wearing mountaineering boots}

Chiara Zoppirolli ${ }^{l}$, Marco Decet ${ }^{l}$, Aldo Savoldelli ${ }^{l}$, Francesca $\overline{\text { Salaorni }}^{I}$, Federico Schena ${ }^{l}$, Loreenzo Bortolan ${ }^{l}$, Barbara Pellegrini $^{1}$

Cerism, Università Degli Studi Di Verona, Rovereto, Italy ${ }^{I}$

Purpose: More and more frequently, sports companies are turning to specialized research centers to obtain scientific data characterizing their products, to compare and improve different prototypes. Here, we present a pilot study showing the methodological approach used to evaluate foot warmth when wearing mountaineering boots in a cold environment.

Methods: Experimental sample and protocol were organized as follows. Five expert alpinists were recruited for the measurements. When testing different boots, they were asked to use the same types of socks and sport wear, perform at the same time of the day standardizing exercise, meals and sleeping routines. To simulate an icefall approach, alpinists entered in a climatic chamber with $-15^{\circ} \mathrm{C}$ and $30 \%$ humidity and performed 60 min hiking on a treadmill at $20 \%$ of incline and self-selected speed, anchored to the 25 th value of the Borg' CR-100 scale $(2,5 \pm 0.5 \mathrm{~km} / \mathrm{h})$. Their right foot was instrumented with 4 temperature probes (sized $2 \times 1 \times 1 \mathrm{~mm}$, SHT75, Sensirion, Staefa, Switzerland) at the level of the internal end external foot sides, big toe and little finger. Data were acquired continuously via Bluetooth $(1 \mathrm{~Hz}$ frequency acquisition) by a Raspberry and sent wirelessly to a PC that stored the data. Perception of foot thermal comfort was assessed every $10 \mathrm{~min}$ through the scale ANSI/ASHRAE Standard 55.

Results: Skin temperatures of specific foot zones and thermal comfort trends increased significantly during 60 min hiking at $-15^{\circ}$ but showing a plateau. Internal side, big teo, little finger and external side temperature raised on average $8,9,6$ and $6{ }^{\circ} \mathrm{C}$ respectively during the walking phase, and decreased of about $4,6,3$ and $3{ }^{\circ} \mathrm{C}$ during the sitting. The site with the highest temperature changes was the big toe, while both the fingers started with a lower temperature respect to the rest of the foot. When comparing different boot prototypes, no differences in foot thermal perceived comfort were reported, even though punctual differences were highlighted by the probes.

Discussion: The protocol proposed was helpful in detecting foot thermal differences in specific zones when wearing different boot' models. Thermal perception represents the average thermal status of the foot when walking and resting in a cold environment but our data showed that it is important to measure punctual temperature in addition to thermal comfort to characterize different mountain footwear from a thermal point of view.

\section{References}

1. Zhang Y, Zaho R (2006). Overall thermal sensation, acceptability and comfort. Building and environment 43:44-50.

\section{1}

Goal kick in the serie a 2019/2020 championship: report based on the score situation and finalization.

\author{
Dante Pertosa ${ }^{l}$ \\ Università Degli Studi Di Milano, La Statale (unimi), Milan, Italy ${ }^{l}$
}


Purpose: In 2019, the rulebook of football has undergone an important modification of the 16 rule regarding the goal kick: the ball is in play from the first touch which can also take place inside the penalty area. The primary purpose of this study is to evaluate which type of goal kick, standardized in short (GKs), short-long (GKs-l) and long (GKl), has been most used in the Serie A 2019-2020 football championship in relation to the score situation (advantage, disadvantage, draw). Secondly, the percentage of finalization was assessed in relation to the three types of goal kick.

Methods: Through the video analysis, conducted with the Wyscout platform (www.wyscout.com), 380 matches of the 20 Serie A football teams were analyzed, which have resulted in a total of 6160 goal kicks.

Results: Statistical analysis showed significant differences on the goal kicks in advantage situations (GKl $>$ GKs, $\mathrm{p}=0.028$; Gkl $>$ GKs-l, $\mathrm{p}$ $<0.0001$; GKs $>$ GKs-1, $\mathrm{p}=0.009$ ), disadvantage (GKl $<$ GKs, $\mathrm{p}=$ 0.018; GKl < GKs-1, p = 0.007; GKs $>$ GKs-1, p < 0.0001) and draw (GKs $>$ GKs-1, $\mathrm{p}<0.0001$; GKs-1 $<$ GKl, $\mathrm{p}=0.0001$ ). Regarding the finalization, significant differences were found between GKs $>$ GKs-1 $(\mathrm{p}=0.0002)$ and GKs $>$ GKl $(\mathrm{p}<0.0001)$.
Conclusions: In advantage situations the choice of GKl prevails, at disadvantage GKs prevails and in a draw GKs and GKl prevail in equal numbers. The highest percentage of finalization was recorded with GKs (10\%) compared to GKs-1 (5\%) and GKl (4\%). Since the change to rule 16 was made, this can be considered the first study that research the relationship between different types of goal kicks with the score situation and finalization in the 2019/2020 Serie A championship.

\section{References}

1. Rodrigo Aquino, Guilherme $\mathrm{H}$ Munhoz Martins, Luiz H Palucci Vieira, Rafael P Menezes; Influence of Match location, Quality of Opponents, and Match Status on Movements Patterns in Brazilian Professional Football Players; J Strenght Cond Res.; 2017 Aug; https://doi.org/10.1519/JSC.0000000000001674

Publisher's Note Springer Nature remains neutral with regard to jurisdictional claims in published maps and institutional affiliations. 